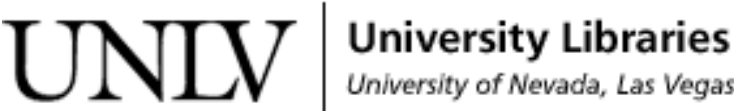

UNLV Theses, Dissertations, Professional Papers, and Capstones

$8-1-2016$

\section{Patterns of Synorogenic Sedimentation Associated with the Unroofing of the Willard-Paris-Meade Thrust Sheets, Sevier Fold-Thrust Belt}

Amanda Gentry

University of Nevada, Las Vegas, amanda.l.gentry@gmail.com

Follow this and additional works at: https:// digitalscholarship.unlv.edu/thesesdissertations

Part of the Geology Commons

\section{Repository Citation}

Gentry, Amanda, "Patterns of Synorogenic Sedimentation Associated with the Unroofing of the Willard-Paris-Meade Thrust Sheets, Sevier Fold-Thrust Belt" (2016). UNLV Theses, Dissertations, Professional Papers, and Capstones. 2779.

https://digitalscholarship.unlv.edu/thesesdissertations/2779

This Thesis is brought to you for free and open access by Digital Scholarship@UNLV. It has been accepted for inclusion in UNLV Theses, Dissertations, Professional Papers, and Capstones by an authorized administrator of Digital Scholarship@UNLV. For more information, please contact digitalscholarship@unlv.edu. 


\title{
PATTERNS OF SYNOROGENIC SEDIMENTATION ASSOCIATED WITH THE UNROOFING OF THE WILLARD-PARIS-MEADE THRUST SHEETS, SEVIER FOLD-THRUST BELT
}

By

\author{
Amanda Leigh Gentry \\ Bachelor of Science - Geology \\ Weber State University, Ogden, UT \\ 2012
}

\begin{abstract}
A thesis to be submitted in partial fulfillment
of the requirements for the

Master of Science - Geoscience

Department of Geoscience

College of Sciences

The Graduate College
\end{abstract}

University of Nevada, Las Vegas

August 2016 
Copyright by Amanda L. Gentry, 2016

All Rights Reserved 


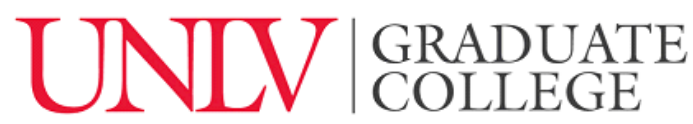

This thesis prepared by

Amanda Leigh Gentry

entitled

Patterns of Synorogenic Sedimentation Associated with the Unroofing of the WillardParis-Meade Thrust Sheets, Sevier Fold-Thrust Belt

is approved in partial fulfillment of the requirements for the degree of

Master of Science - Geoscience

Department of Geoscience

Michael Wells, Ph.D.

Examination Committee Chair

Joshua Bonde, Ph.D.

Examination Committee Member

Adolph Yonkee, Ph.D.

Examination Committee Member

George Rhee, Ph.D.

Graduate College Faculty Representative

\section{Thesis Approval}

The Graduate College

The University of Nevada, Las Vegas

May 11, 2016 


\section{Abstract}

The Willard-Paris-Meade thrust is the oldest and western-most sheet to develop in the Wyoming salient of the Sevier fold-thrust belt. The $10-15 \mathrm{~km}$ thick thrust sheet was emplaced 60 km eastward and included Jurassic-Triassic strata, mixed siliciclastic-carbonate upper Paleozoic strata, carbonate-rich lower Paleozoic strata, and quartzite-rich basal Cambrian to Neoproterozoic strata. Each stratigraphic interval has a distinctive detrital zircon (DZ) age signature, which can be used in provenance analysis of foreland basin fill. The thrust system had a long deformation history recorded by westward thickening and coarsening synorogenic strata. DZ U-Pb geochronology of 27 synorogenic samples collected from two transects reveals an unroofing sequence with stratigraphically consistent changes in DZ patterns. DZ spectra for the Gannett Group in the lower part of the basin fill are consistent with erosion of Mesozoic to upper Paleozoic strata. Maximum depositional ages (MDA) from limited euhedral and likely volcanic grains for the foreland basin sequence show the following for the northern transect: basal Ephraim Formation, $150 \pm 2.8 \mathrm{Ma}(\mathrm{n}=10)$; upper Ephraim, 115.2 $\pm 1.8 \mathrm{Ma}(\mathrm{n}=13)$; lower Bechler, 116.2 $\pm 2.3 \mathrm{Ma}(\mathrm{n}=9)$; upper Bechler 107.3 $\pm 3.3 \mathrm{Ma}(\mathrm{n}=4)$. These data clarify that there is not a significant unconformity at the base of the Bechler, and that the Bechler conglomerate facies at Red Mountain spans the depositional interval including the $\sim 112$ Ma Drainey Limestone, which is absent at Red Mountain but present elsewhere. The southern transect yields the following MDAs: Upper Gannett, $109 \pm 6.1 \mathrm{Ma}(n=5)$ and $108.3 \pm 3.4 \mathrm{Ma}(n=10)$; Cokeville, 101.6 \pm 0.7 Ma ( $n=32)$; Lower Sage Junction 101.55 $\pm 0.5 \mathrm{Ma}(\mathrm{n}=67)$; Upper Sage Junction, 101.3 $\pm 0.6 \mathrm{Ma}(\mathrm{n}=29)$; Aspen, $98.8 \pm 0.4 \mathrm{Ma}(\mathrm{n}=51)$; lower Frontier, $99.93 \pm 0.6 \mathrm{Ma}$ ( $n=21)$; upper Frontier, 95.68 $\pm 1.4 \mathrm{Ma}(\mathrm{n}=38)$. DZ spectra for the Bear River and Aspen 
formations in the middle part of the basin fill are consistent with erosion of mostly Paleozoic bedrock. DZ spectra in the Frontier Formation in the upper part of the basin fill are consistent with increased erosion of basal Cambrian and Neoproterozoic quartzites. The early history of deposition is elusive due to the potential bias from the early Cretaceous Sierran magmatic lull and a lack of datable materials related to corollary studies. 
Acknowledgements

I am grateful for the guidance and advice from my advisor, Dr. Michael Wells, whose comprehensive knowledge of the development of the western U.S. helped make my journey as a master's student fun and rewarding.

My warm thanks also go out to the professors that helped guide me down the path of geoscience. In particular, I want to thank Dr. Adolph Yonkee for finding time in his busy schedule to take me on as an undergraduate researcher, and continuing to help me by being an advisor on my master's committee. His passion and knowledge of geology is awe inspiring and I shall always be grateful to have been his student. Dr. Jeff Eaton was also a great inspiration in my geoscience career. Dr. Eaton also took me on as an undergraduate research student, and it was during his classes and his excursions in Tropic, UT, that I knew I wanted to be a geoscientist.

My research would also not have been possible without the help and friendship of Dr. Josh Bonde, whose knowledge of all things sediment related got me through some tough spots.

I also want to thank the faculty, staff, and my friends in the UNLV Geoscience Department for their companionship and guidance. I especially want to thank those who let me tag along on their field trips and who let me ask questions of all sorts at all times of the day. The knowledge you shared with me is an invaluable resource that I will cherish forever. 
Last but not least, I would like to thank my husband, Michael, for believing in me and supporting me in everything I do. Words cannot express my ultimate gratitude. This research would not have been possible without his support, knowledge, friendship and strength. 
Table of Contents

Title Page...

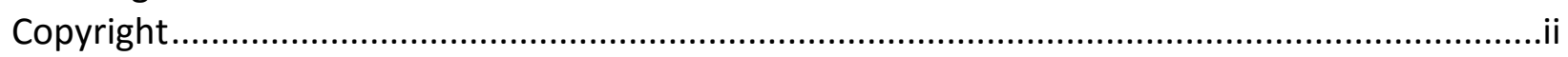

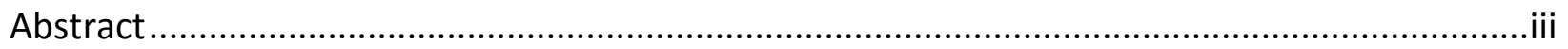

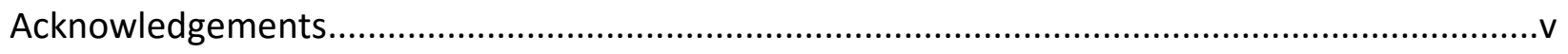

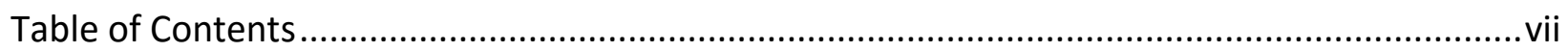

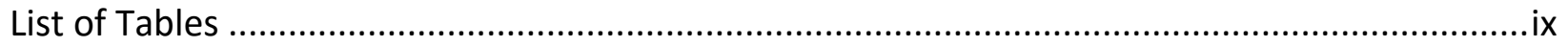

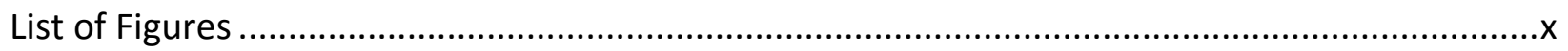

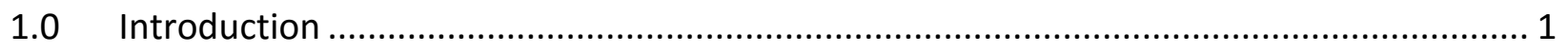

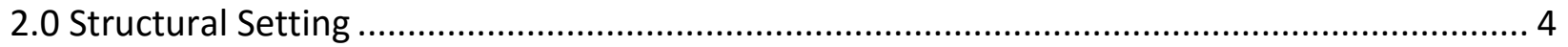

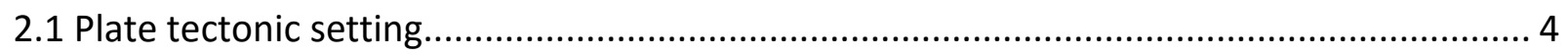

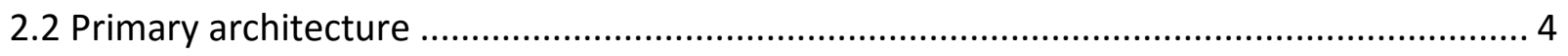

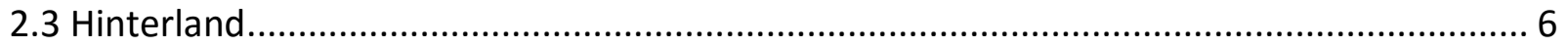

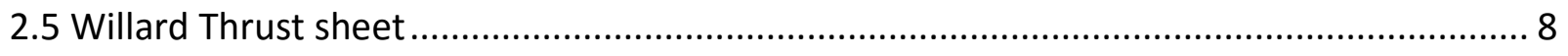

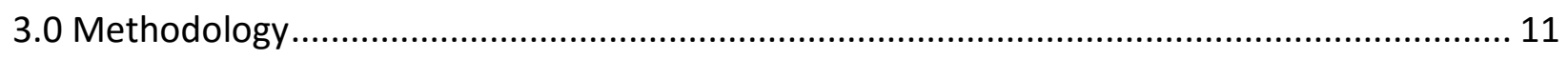

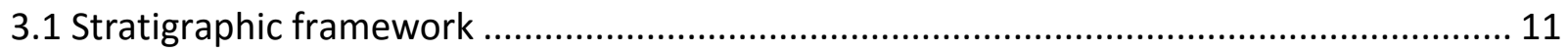

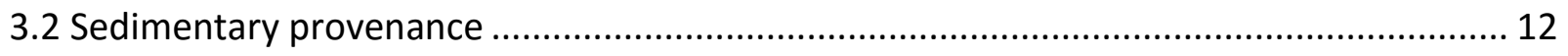

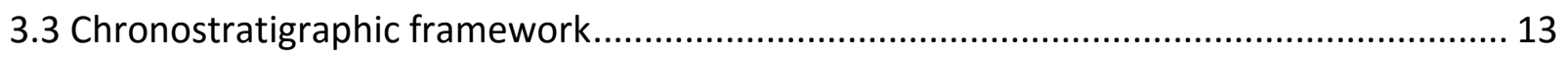

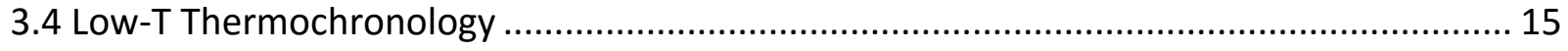

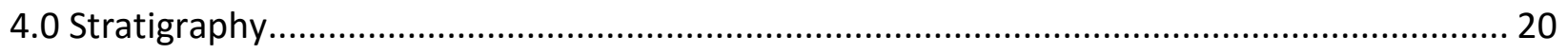

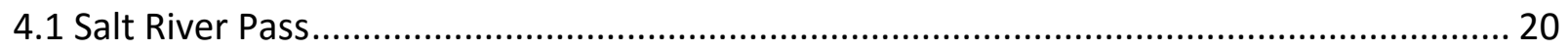

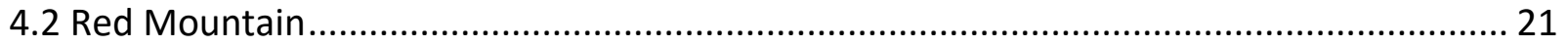

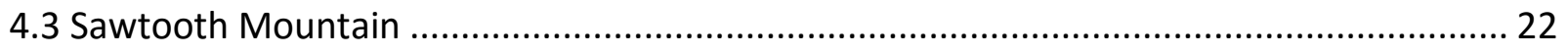

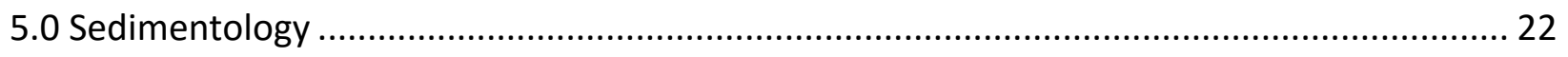

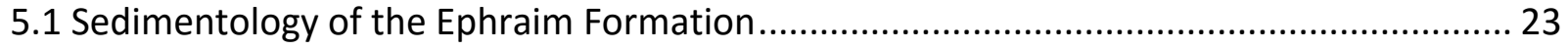

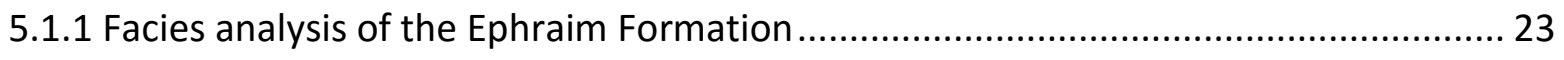

5.1.2 Description and interpretation: major conglomerate (FA1) .................................. 23

5.1.3 Description and interpretation: minor conglomerate (FA2) ................................ 24

5.1.4 Description and interpretation: minor tabular to lenticular sandstone (FA3) ............ 26

5.1.5 Description and interpretation: minor tabular to lenticular siltstone/mudstone (FA4) 
5.1.6 Description and interpretation: massive siltstone/mudstone (FA5) ....................... 28

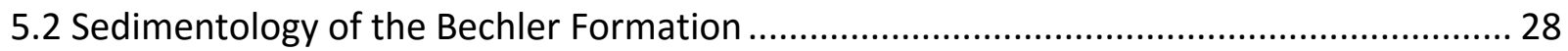

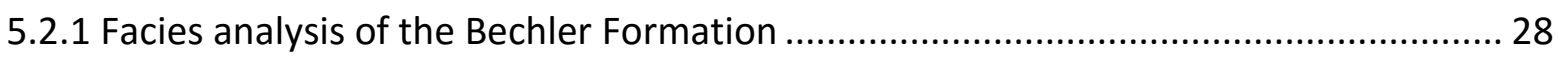

5.2.2 Description and interpretation: major conglomerate (FA1) ................................... 29

5.2.3 Description and interpretation: minor conglomerate (FA2) ................................ 30

5.2.4 Description and interpretation: minor tabular to lenticular sandstone (FA3) ........... 31

5.2.5 Description and interpretation: minor tabular to lenticular siltstone/mudstone (FA4) .

5.2.6 Description and interpretation: massive siltstone/mudstone (FA5) ....................... 32

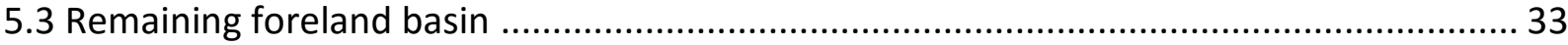

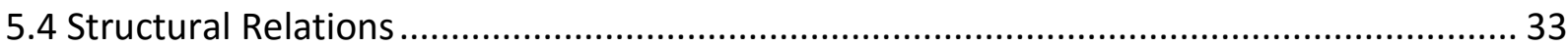

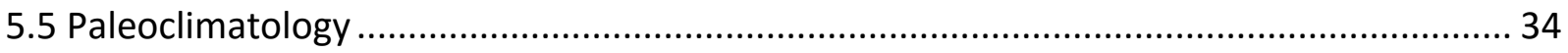

6.0 Detrital Zircon studies of Lower Cretaceous synorogenic strata....................................... 35

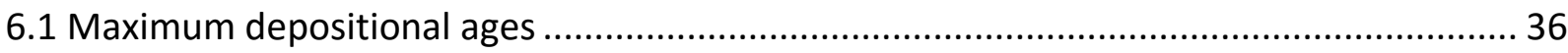

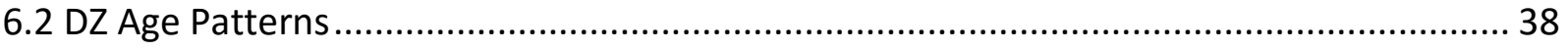

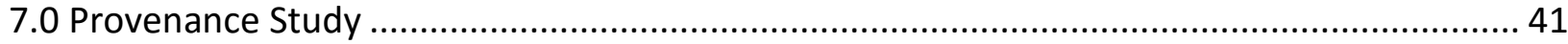

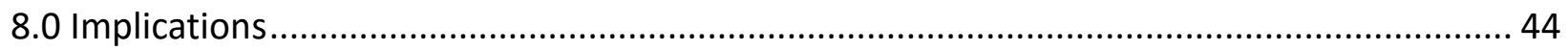

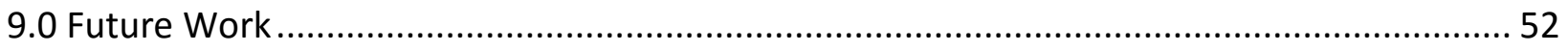

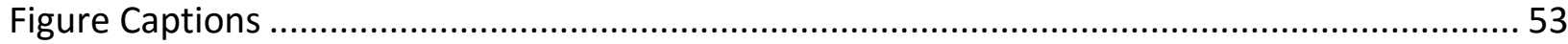

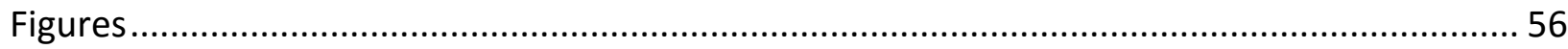

Table 1. Lithofacies and Facies Associations of Ephraim and Bechler formations ..................... 80

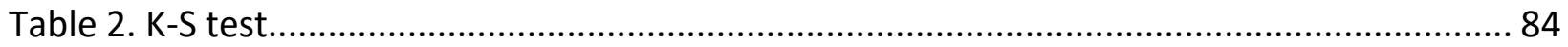

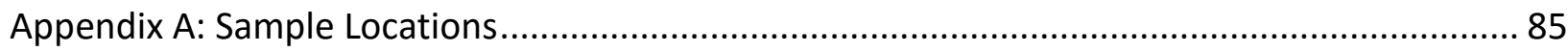

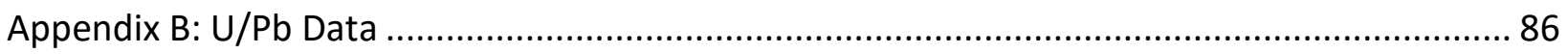

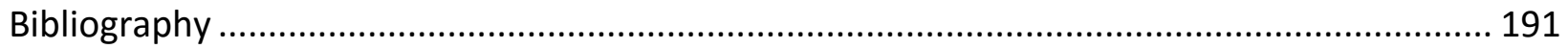

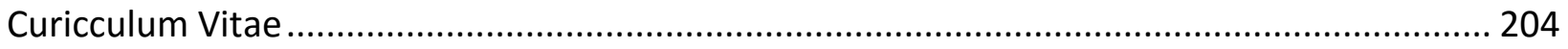


List of Tables

Table 1. Lithofacies and Facies Associations of the Ephraim and Bechler formations.................77

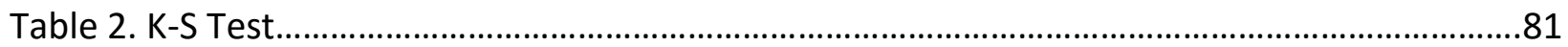


List of Figures

Figure 1 Regional Map of the Sevier Fold-Thrust Belt...... .64

Figure 2 Synorogenic Sedimentary Record of Basins Adjacent to the Sevier Orogenic

Belt .65

Figure 3 Proterozoic Source Terranes with Corresponding DZ Patterns. .66

Figure $4 \quad$ ZHe Age vs. Paleodepth from Samples Collected Along Wellsville Transect of Willard Thrust Sheet. 67

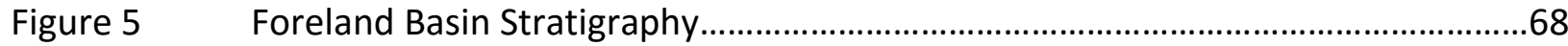

Figure $6 \quad$ Schematic Map of Field Area with Localities........................................................69

Figure $7 \quad$ Correlation of Regional Stratigraphic Sections.....................................................70

Figure $8 \quad$ Bedding Present within the Ephraim Formation..................................................71

Figure $9 \quad$ Paleocurrent Data for the Ephraim Formation....................................................72

Figure 10 QFL Plot and Photomicrograph of the Ephraim Formation..................................73

Figure 11 Crevasse Channel Deposit within the Ephraim Formation.....................................74

Figure 12 Architectural Elemental Analysis of Bechler Formation........................................75

Figure 13 Representative Measured Section of the Bechler Formation at Salt River Pass

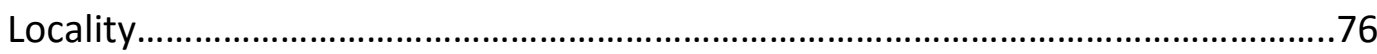

Figure $14 \quad$ Example of Bechler Formation Major Conglomerate Lithofacies...........................77

Figure 15 Paleocurrent Direction Measurements of Imbricated Clasts of the Bechler

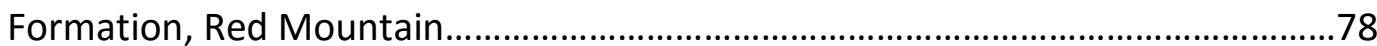

Figure 16 QFL Plot of the Bechler Formation......................................................................79

Figure 11 Paleocurrent Directions Measured within the Bechler Formation.........................80

Figure 12 Stratigraphic Relations between Foreland Basin Units of the WPM Thrust Sheet. .81

Figure 19 Age of Apparent Intrusive Influx and Whole Rock Age of Sierra Nevada. .82

Figure 20 MDA's For Stratigraphic Units within the Foreland Basin. $.83-84$

Figure 21 Stratigraphy of the Willard Thrust Sheet with Unique DZ Age Patterns................85

Figure 22 DZ Age Patterns Found within Specific Foreland Basin Units. .86 
Figure 23 DZ Patterns from the WPM Thrust Sheet Compared With DZ Patterns from

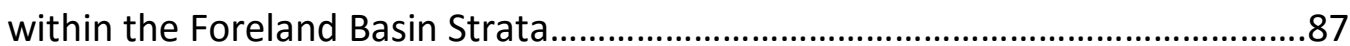




\subsection{Introduction}

Subduction along the Cordilleran margin of North America during Jurassic to Paleogene time resulted in intra-plate deformation that propagated into the continental interior with development of a retro-arc fold-thrust belt spanning $>4000 \mathrm{~km}$ up the western margin from Canada to Mexico (Armstrong, 1968; Oldow et al., 1989; Price and Mountjoy, 1970; Burchfiel et al., 1992; Dickinson, 2004; DeCelles, 2004; Yonkee and Weil 2015). The Sevier fold-thrust belt, the easternmost contractile belt, is characterized by $>200 \mathrm{~km}$ of overall E-directed shortening of passive margin sedimentary rocks above a regional decollement (DeCelles, 1994). While the structural geometry and the kinematic evolution of the Sevier fold-thrust belt are well understood, the initial timing of deformation is poorly resolved. Regional tectonic questions remain including whether deformation propagated eastward in a continuous fashion as an orogenic wedge (Camilleri et al., 1997; DeCelles, 2004), or whether deformation was a twostaged process, with Middle to Late Jurassic deformation followed by mid- to Late Cretaceous deformation (e.g., Heller et al., 1986; Smith et al., 1993). Additionally, questions remain regarding the driving mechanism for intraplate deformation at non-collisional plate boundaries (DeCelles, 1994; Yonkee and Weil, 2015). The Willard-Paris-Meade (WPM) thrust sheet is the westernmost and oldest thrust sheet to develop within the Wyoming Salient of the Sevier foldthrust belt.

By understanding the timing of thrust initiation, as well as controlling structures and composition of provenance sources, a better picture of the evolution of foreland basin fill related to motion of the WPM thrust sheet can be developed. In addition, synorogenic 
sedimentation can be used to interpret exhumation history and flexural loading patterns to help construct a more detailed shortening history.

The WPM thrust sheet displays a ramp-flat geometry, with ramps developing in the basal Neoproterozoic strata and Middle Cambrian quartzite and limestone, and flats developing in weaker intervals of shale and evaporite. Overall, $>60 \mathrm{~km}$ of eastward motion of its hanging wall relative to its footwall was accomplished (Yonkee, 2005; Weil and Yonkee, 2012). Uplift of the thrust sheet over ramps resulted in local faulting, folding and erosional unroofing. Unroofing during thrusting is expected to result in progressively older rocks being eroded with time, which should be recorded in synorogenic strata deposited in the adjacent foreland basin to the east of the thrust front in northern Utah and southern Idaho to southwest Wyoming (Figure 2).

The initial timing of displacement and development of the WPM thrust sheet has received much study and yet remains poorly constrained, with age estimates of initiation ranging from 150 to $115 \mathrm{Ma}$ (Heller et al., 1986; Yonkee et al., 1989; DeCelles et al., 1995; Yonkee et al., 1997; Currie, 2002; DeCelles, 2004; Yonkee and Weil, 2015). This study utilizes U$\mathrm{Pb}$ analysis of detrital zircon (DZ) grains as well as sedimentologic analysis to address the timing of initiation, timing and development of an unroofing sequence, and changes in paleogeography related to the early kinematic development of the fold-thrust belt. These data will be coupled with recent results from systematic (U-Th)/He zircon low temperature thermochronology through the Willard thrust sheet, which identifies a period of enhanced cooling interpreted to represent uplift-triggered erosion of the Willard thrust sheet beginning 
130 Ma (Eleogram, 2014) to evaluate the paired syntectonic erosional and depositional history. 


\subsection{Structural Setting}

\subsection{Plate tectonic setting}

Regionally, the Sevier orogenic belt represents Andean-style thin-skinned retroarc deformation that propagates far into the North American interior. The response of the western margin of North America to variations in plate subduction parameters (e.g., rate, direction, dip) resulted in the diverse evolution of the Cordillera. Before the development of the Sevier orogenic belt, apparent polar wander (APW) paths of North America showed a NW drift during the early Jurassic that shifted to orthogonal convergence with a W-directed drift during the middle Jurassic. In the Early Cretaceous, convergence shifted from orthogonal to sinistral oblique (Dickinson and Lawton, 2001). Magnetic anomalies, fracture zones that developed during the opening of the Atlantic spreading center, as well as tracking of hot spot positions demonstrate increasing convergence rates along the western margin in the early Mid Cretaceous (Torsvik et al., 2008). Humphreys (1995) and Madsen et al, (2006) show that relative convergence rates from $145-130 \mathrm{Ma}$ are estimated to be $\sim 5 \mathrm{~cm} / \mathrm{yr}$ with an increase to $8-12$ $\mathrm{cm} / \mathrm{yr}$ from $~ 120-50 \mathrm{Ma}$. The spreading rate and directions of the Pacific, Atlantic and Indian Oceans experienced significant changes from $\sim 147-145$ and could be a result of the eruption of the Shatsky Rise at the Izanagi-Farallon-Pacific triple junction (Engebretson, 1985; Seton et al., 2012).

\subsection{Primary architecture}

The primary crustal architecture of the western margin of North America is essential in understanding the development of deformation within the Cordillera, specifically within the Sevier fold-thrust belt. Basement rocks, composed mostly of Proterozoic and Archean rocks, 
were not only important in providing the foundation for initial rifting of the supercontinent Rodinia, but also provided detritus for sedimentary cover with the eventual development of the Paleozoic passive margin. In addition, DZ grains originating within Proterozoic volcanic and metamorphic terranes bracket the age of crustal formation while also acting as a provenance signature for sedimentary sinks, which incorporate sediment from these terranes through paleotranscontinental drainage systems. The basement rocks include granitoid intrusions, supracrustal belts and gneiss of the Wyoming Province (Frost et al., 2006; Mueller and Frost, 2006), granitoids, amphibolite and schist of the Grouse Creek block (Strickland et al., 2011; Isakson, 2012), paragneiss and orthogneiss of the Farmington zone (Bryant, 1988; Mueller et al., 2011), paragneiss and orthogneiss of the Mojave province (Wooden and Miller, 1990; Shufeldt et al., 2010) and juvenile volcanic arc rocks intruded by granitic plutons of the Yavapi and Mazatzal provinces (Bennett and DePaolo, 1987; Karlstrom and Bowring, 1988). Figure 3 from Yonkee et al. (2014) details the location and ages of each Precambrian province based on previously determined DZ age patterns.

Carbonate-rich Paleozoic strata represent development of the passive margin formed from the breakup of Rodinia, quartzarenite of lower Cambrian to upper Neoproterozoic strata represent the transition from rifting to passive margin sedimentation, and a lower level of micaceous Neoproterozoic strata that include diamictite and volcanic deposits is related to protracted rifting of Rodinia. Within the Sevier fold-thrust belt, the stratigraphy provides the mechanical stratigraphic framework that influenced the structural geometry of the fold-thrust belt and erosional unroofing that is recorded in the sedimentary sequence observed in the 
foreland basin (Mitra, 1994; Dickinson, 2004; Yonkee, 2005; Yonkee and Weil, 2010; Yonkee and Weil, 2015).

The western thrust system of the Sevier fold-thrust belt is composed of the Willard, Paris and Meade thrust sheets, which incorporate Neoproterozoic through early Mesozoic strata. The eastern system includes the Crawford, Absaroka, and Hogsback thrusts, which incorporate only thinner Paleozoic strata that were deposited on the North American craton. The western and eastern systems exhibit ramps developed in thicker, stronger carbonate and sandstone beds, and flats developed in weaker intervals of shale and evaporite.

Micaceous strata of the Neoproterzoic Perry Canyon and correlative formations acted as a failure plane for the basal decollement of the western system. Eastern thrusts had basal decollements that formed in the Middle Cambrian shale and limestone, and higher decollements in Jurassic evaporite.

\subsection{Hinterland}

The hinterland in Nevada, westernmost Utah and southern Idaho is important to initial development of the Sevier fold-thrust belt, as well as subsequent deformation of the Cordillera. Forces from plate coupling at the western margin and the subducting slab, as well as crustal rheology and lower lithosphere influences resulted in significant thickening of crustal lithosphere (Harris et al., 2007; Cruz-Uribe et al., 2015; Kelly et al., 2015; Bonde et al., 2015). In addition, studies have shown that the hinterland was able to provide some of the initial detritus incorporated into the foreland basin from long range run-out gravels before WPM thrusting began (Heller and Paola, 1989; Benvenuti and Martini, 2002; Heller et al., 2003; Pierson, 2005). Specifically, the basal Ephraim Formation contains a unique collection of red and black cherts 
that are thought to have originated from the Antler orogeny derived from the Roberts Mountain allochthon (Gehrels et al., 2000). Due to uplift of the hinterland and thrusting of the Sevier orogenic belt, there are limited preserved deposits from the Early Cretaceous in any hinterland localities.

The Lower Cretaceous Newark Canyon Formation and Upper Cretaceous Sheep Pass Formation are some of the few preserved deposits that record how the evolution of the hinterland either led to further deformation, or helped accommodate the thin-skinned thrusting of the Sevier orogenic belt. Comparison of fossil assemblages of Newark Canyon to those in a stratigraphically correlative position within the developing foreland basin, namely the Cedar Mountain Formation, show that there was minimal elevation differences when the onset of development of thrust-related sedimentary basins first began 120 Ma (Bonde et al, 2015). Analysis of clumped C-O isotopes found within subsequently deposited primary carbonates of the Sheep Pass Formation show there was uplift of $2-3 \mathrm{~km}$ of the hinterland by $70 \mathrm{Ma}$ (Snell et al., 2014). With overall shortening of $\sim 200 \mathrm{~km}$ within the Sevier orogenic belt, uplift of $2-3 \mathrm{~km}$ is consistent with a crustal thickness of $\sim 50-60 \mathrm{~km}$ beneath the hinterland as a result of basement underthrusting, basement-cored nappes, or lower crustal duplexing (Yonkee and Weil, 2015).

Thrusting of the WPM sheet may have effectively cut off western sediment sources for the foreland basin due to formation of topographic barriers. This effect is recorded in the Sevier foreland basin with replacement of hinterland derived radiolarian chert within the basal Ephraim unit with more locally derived chert from Carboniferous and Permian units from the WPM sheet deposited in the upper Ephraim and into the Bechler formations. 


\subsection{Willard Thrust sheet}

Thrust belts in the Cordillera are largely controlled by the Neoproterozoic to Cambrian stratigraphic architecture laid down on the passive western margin of Laurentia (Yonkee and Weil, 2015). The western system of the Sevier fold-thrust belt is composed of the WPM thrust sheets that incorporated Neoproterozoic through Paleozoic strata initially formed on a passive margin as well as Triassic and Jurassic strata related to deformation of the western margin. The eastern system includes the Crawford, Absaroka, and Hogsback thrust sheets and incorporate thinner Paleozoic strata, deposited on the North American craton.

The WPM thrust sheet represents initial propagation of the westernmost thrust system within the Wyoming salient (Camilleri et al., 1997). The Willard, and associated Paris-Meade thrusts to the north, had a protracted deformation history during the Early Cretaceous based on relations of synorogenic strata (DeCelles, 1993; DeCelles, 1994), although the chronology of that basal strata is poorly understood. Synorogenic deposits from the WPM thrust sheet are preserved across a large region stretching over $300 \mathrm{~km}$ north to south from eastern Idaho to northeast Utah, and over $60 \mathrm{~km}$ west to east from present day Montpelier, ID past Kemmerer, WY (Figure 6). Sediment transport of this magnitude is influenced by the paleogeography of the area and may represent initial uplift of the Willard and development of a flexural basin. Overall, the large-scale structural geometry of the belt is well constrained (DeCelles, 1994). The nature of previously deposited stratigraphic packages have significant control on the structural deformation of thrust sheets (Protzman and Mitra, 1990).

The Willard thrust sheet and its deformed footwall are well exposed in northern Utah where younger uplift and erosion have exhumed a wide range of structural levels. The thrust 
sheet includes up to $13 \mathrm{~km}$ of passive margin strata which include Jurassic to Triassic strata that have been mostly eroded, an upper level of carbonate-rich Paleozoic strata, a middle level of quartzarenitic lower Cambrian to upper Neoproterozoic strata, and a lower level of micaceous Neoproterozoic strata; thin slices of Archean to Paleoproterozoic basement rocks were locally incorporated into the base of the sheet. Upper levels of the sheet exhibit relatively little deformation, but internal deformation increases downward and westward, with development of cleavage, minor folds, and vein arrays (Crittenden, 1972; Camilleri, 1998; Yonkee, 2005). ${ }^{40} \mathrm{Ar} /{ }^{39} \mathrm{Ar}$ ages of muscovite from syntectonic veins near the base of the Willard sheet vary mostly from 140 to $110 \mathrm{Ma}$, recording internal deformation that overlapped with major thrust slip (Yonkee et al., 1989).

The Paris and Meade thrusts have been shown to have a complex interaction with emplacement of the Paris sheet resulting in layer-parallel shortening within the proto-hanging wall of the Meade sheet, as well the Paris sheet being passively transported during movement of the Meade sheet (DeCelles et al., 1993). The Meade sheet is a footwall imbricate of the Paris sheet (DeCelles et al., 1993). While initial movement of the Paris sheet occurs before movement of the Meade sheet, it remains unclear if there was synchronous movement of the Meade and Willard sheets.

The Paris sheet has a basal decollement in the micaceous Neoproterozoic to early Cambrian quartzarenite and exposes Proterozoic through Mississippian strata; Pennsylvanian through Upper Jurassic strata were probably once part of the sheet and have subsequently been eroded (Coogan, 1992). 
The thrust front of the Meade sheet is $\sim 30 \mathrm{~km}$ east of the thrust front of the Paris sheet and has Mississippian through middle Jurassic rocks exposed with detachment within the Pruess Formation. There are imbricates with an overturned section of Triassic and Jurassic strata as well as local development of a structural ramp through Jurassic units (Protzman and Mitra, 1990). The basal decollement formed at the level of the Proterozoic strata of the Paris sheet with total movement of the Meade thrust $\sim 45 \mathrm{~km}$ (Coogan, 1992). Initial displacement of the Meade thrust sheet has previously been dated to Aptian time ( 118-113) based on structural relations and paleontologic data at Red Mountain (DeCelles et al., 1993). 


\subsection{Methodology}

Early Cretaceous sedimentary strata in the foreland basin east of the WPM thrust sheet may provide evidence for the timing of development of structurally induced topographic relief and erosional exhumation of the thrust sheet. To better date motion of the WPM thrust sheet and to establish the link between motion and deposition, a stratigraphic framework must be established, incorporating sediment provenance analysis and an improved chronostratigraphic framework. This can then be used to provide an integrated record of active erosional exhumation of the WPM thrust sheet as well as basin deposition.

Integrated studies were completed to determine stratigraphic relations, provenance, maximum depositional ages, and subsidence history of Cretaceous synorogenic strata along the leading edge and east of the Willard-Paris-Meade thrust system. Fieldwork involved description

of sedimentary features, clast counts within conglomerate layers, paleocurrent measurements, and collection of samples for petrographic and geochronologic analyses.

\subsection{Stratigraphic framework}

In order to establish a stratigraphic framework, unit thicknesses were determined at several locations based on best available outcrops and proximity to the WPM thrust sheet. Due to the general incomplete nature of outcrops, rather than measure stratigraphic sections by Jacob's staff, unit thicknesses were determined by interpretation of Google Earth imagery and measured bedding attitudes. One section of the Bechler Formation and one section of the Thomas Fork Formation were exposed in road cuts that allowed detailed measurement for use in architectural elemental analysis inferred in other covered sections. One section was measured near Salt Creek Pass in SE Idaho (Figure 1) that included the Ephraim Formation 
through the Thomas Fork Formation. This locality was chosen based on both stratigraphic completeness, and its proximity $(<30 \mathrm{~km})$ to the leading edge of the WPM thrust sheet. In addition, this locality provided road cut access to the Bechler Formation and the Thomas Fork Formation. A second section was measured near Red Mountain, ID and represents the most proximal sediment to the thrust sheet, with exposures adjacent to the Paris-Meade section of the thrust. This section only included the Ephraim and Bechler formations, with the Peterson, Draney and subsequent foreland basin stratigraphy absent. A third section was measured near Sawtooth Mountain in SW Wyoming, and included the Ephraim Formation through the Frontier Formation. While this southern section is not proximal to the WPM thrust sheet, this locality lies east of the previously collected thermochronologic data (Eleogram, 2014).

\subsection{Sedimentary provenance}

Small cobble to pebble sized clasts were identified based on lithology, texture, and fossil assemblage and an effort was made to correlate them to possible source strata in the Willard sheet including Early Mesozoic carbonates and sandstones, Paleozoic carbonate and quartzite, lower Cambrian to upper Neoproterozoic quartzarenite, and Neoproterozoic micaceous quartzite clasts; possible source areas further west were also evaluated.

The provenance of sandstones were studied by point counting (300 points per thin section) to estimate volume fractions of monocrystalline quartz, polycrystalline quartz, chert, plagioclase, orthoclase, limestone, dolostone, detrital lithic fragments, volcanic lithic fragments, and matrix (<50 $\mu$ m grains). 27 samples were processed, with 15 thin sections stained for calcite. The Gazzi-Dickinson technique was necessary due to variation in grain lithology, producing grains of different sizes. Gazzi-Dickinson method used in this study utilized randomly 
selected thin sections from sandstone samples. An automated stage was used to randomly select points on a slide with a petrographic microscope. A minimum of 300 representative points were collected with composition of each point determined by the operator. These counts are then converted to percentages and graphed on a QFL ternary diagram in TriPlot (Graham and Midgley, 2015) and used for compositional comparisons in provenance studies. This technique normalizes each sample with respect to grain size (Ingersoll et al., 1984).

Paleocurrent data were collected by locating distinguishable cross bedding or troughs with good three-dimensional control. Orientation data were measured using a Brunton compass. Paleocurrent data were corrected for bedding tilts by restoring bedding back to horizontal, and paleocurrent directions were plotted on Rose diagrams using Stereonet 9 (Allmendinger, 2015).

\subsection{Chronostratigraphic framework}

Due to the relative ease in which zircon grains are incorporated and preserved within sandstone, sandstone-bearing units are the main focus of this study. Identification of sites for collection was based on previously published studies (Eyer, 1969; M'Gonigle and Dover, 1992;

DeCelles et al., 1993), as well as remote sensing techniques (Google Earth) and field identification. Stratigraphic sections for collecting samples were selected based on completeness of stratigraphic section, degree of exposure, proximity to depocenter (northern transect) and proximity to previous thermochronological transects (southern transect) through the Willard thrust sheet. For each unit, nine-kilogram samples were collected. In total, 27 samples from well exposed sections of Cretaceous synorogenic strata were collected, focusing 
on the basal Lower Cretaceous Ephraim Conglomerate of the Gannet Group and working up section through the Turonian Frontier Formation (Figure 6).

Zircon grains were extracted from samples by traditional methods of crushing, followed by Wilfley table, heavy-liquid, and magnetic separation. Representative splits of zircon fractions were incorporated into epoxy mounts along with fragments of zircon standards, which were polished and high-resolution BSE images were collected on a Hitachi ZZ SEM. U-Pb analysis of grains was done using the laser ablation-inductively coupled plasma mass spectrometry (LAICPMS) system at the Arizona LaserChron Center (ALC). Analysis followed procedures given by Gehrels et al. (2006, 2008). Analytical uncertainties were typically less than $\pm 1 \%$ at a 1 -sigma level ( $\pm 3 \mathrm{Ma}$ for a $300 \mathrm{Ma}$ grain). U-Pb data were plotted on age-probability diagrams using the software package Isoplot (Ludwig, 2008). The ${ }^{206} \mathrm{~Pb} /{ }^{207} \mathrm{~Pb}$ age was used for grains older than $900 \mathrm{Ma}$ and the ${ }^{206} \mathrm{~Pb} /{ }^{238} \mathrm{U}$ age was used for younger grains. Analyses $>10 \%$ discordant based on comparison of ${ }^{206} \mathrm{~Pb} /{ }^{238} \mathrm{U}$ and ${ }^{206} \mathrm{~Pb} /{ }^{207} \mathrm{~Pb}$ ages for grains older than $400 \mathrm{Ma}$ were not plotted; discordance is poorly constrained for young grains. For all SYN 12 samples, grain analysis was performed on up to 100 grains per sample. For all SYN 14 samples, grain analysis was performed on up to 300 grains per sample. The increased number of analyses during the second round of data collection was due to an alternative collection procedure that reduced overall sample collection time.

Methods used to determine the similarity of DZ age spectra as a means of provenance identification have included visual comparison of age-distribution curves and age-bin histograms (Dickinson and Gehrels, 2009; Dickinson and Gehrels, 2009; Lawton et. al., 2010; Leier and Gehrels, 2011). These methods, however, do not provide the high resolution 
necessary to visually compare expected DZ patterns eroding from the WPM thrust sheet, with those found within the adjacent foreland sediment. Other workers have resorted to using the Kolmorgorov-Smirnov test (K-S test), as a way to quantitatively compare the age-distribution curves between two samples (Gehrels et. al., 2006; Dickinson and Gehrels, 2008; Konstantinou et. al., 2012). The K-S test, as used in this study, is a nonparametric analysis that quantifies the distance between the empirical distribution functions of two samples. The Excel program was provided from the Arizona Laserchron Center (available at www.geo.arizona.edu/alc). The K-S program takes the calculated ages and associated errors from two samples and determines a Pvalue, which is the calculated probability of one sample being statistically similar to another. If $P$ is greater than 0.05 , there is $95 \%$ confidence that the two samples are from the same age populations.

\subsection{Low-T Thermochronology}

Although understanding the sedimentologic history of the foreland basin provides insight into the exhumation history of the Willard-Paris-Meade thrust sheet, it does not provide detailed information on timing of slip and slip rates. This study aims to address the erosional exhumation history of the thrust sheet by coupling the sedimentologic data from this study with previous work using detrital zircon (U-Th)/He thermochrometry (ZHe) within the Willard thrust sheet (Eleogram, 2014) (Figure 4).

ZHe studies allow rock cooling ages to be determined by measuring the radiometric production and subsequent trapping within the crystal lattice of the daughter isotope, ${ }^{4} \mathrm{He}$, from the decay of ${ }^{238} \mathrm{U},{ }^{235} \mathrm{U}$, and ${ }^{235} \mathrm{Th}$. Within each thermochronologic system, a closure temperature (Tc) exists, above which radiogenic daughter isotopes can diffuse out of the crystal 
lattice, preventing overall accumulation. The amount of diffusion of the daughter product from the crystal depends on a number of factors, including cooling rate and grain size. At temperatures below the Tc daughter particles are unable to freely escape, allowing complete accumulation of all daughter products within the crystal lattice (Dodson, 1973; Farley, 2002). The ZHe system has a Tc of $180^{\circ}$ to $200^{\circ}$, which is exceeded at depths greater than $8-10 \mathrm{~km}$, assuming typical geothermal gradients (Farley, 2002; Reiners, 2005). The range of temperatures at which ${ }^{4} \mathrm{He}$ becomes fully to partially locked within the crystal lattice is termed the partial retention zone (PRZ), where Tc can lie within the PRZ at the base of the section and rocks above the PRZ have cooled through Tc, recording earlier tectonic events (Reiners, 2005). This can be seen when rapid uplift and exhumation from thrust faulting is preserved as a fossilized PRZ (Braun, et al., 2006).

Inverse time-temperature (t-T) modeling was performed for the Bear River to Browns Hole and Wellsville vertical transects, which was then compared with measured ZHe ages, using sample depths and user defined geothermal gradients that provide thermal histories that fit with measured data (Eleogram, 2014).

ZHe studies were performed on 40 prescreened zircon samples, based on similar U-Pb ages and $\mathrm{U} / \mathrm{Th}$ contents, with 6 grains run per sample. Slow cooling related to slip on the Willard thrust sheet began $\sim 130$ Ma with progressively larger exhumation rates from the eastern leading edge of the sheet $(0.12 \mathrm{~mm} / \mathrm{yr})$ increasing to the western exposures $(1.7$ $\mathrm{mm} / \mathrm{yr}$ ) from 125 to $90 \mathrm{Ma}$ (Eleogram, 2014).

Application of ZHe is particularly useful within the Willard thrust sheet due to the extensive exposure of stratigraphic levels, the latitudinal extent as well as the significant 
structural displacement (Eleogram, 2014). Dates of exhumation determined from specific stratigraphic levels within the thrust sheet provide timing of uplift that is corroborated by depositional ages within the foreland basin. As the thrust sheet is uplifted, the top stratigraphic levels are eroded into the adjacent basin and combined with any available MDA DZ grains. At the same time within lower levels of the thrust sheet, zircon grains are recording an internal uplift history. When the two histories are compared, a detailed uplift and unroofing history can be determined.

By coupling the synorogenic sedimentary history with the exhumation history of the Willard-Paris-Meade thrust sheet, flexural subsidence in western North America will be better understood and can be applied to recognize the driving mechanisms behind intraplate deformation and overall timing of the shortening history of the Sevier orogenic belt.

\subsection{Foreland basin framework}

Adjacent to the WPM thrust sheet, the foreland basin stratigraphy (Figure 4) includes the basal Gannett group, the Smith Formation, Cokeville Formation, Thomas Fork Formation, Sage Junction Formation, Frontier Formation and Hilliard Formation. The Aspen shale is an eastern equivalent of the Sage Junction Formation and the Bear River Formation is an eastern equivalent of the Smiths/Cokeville/Thomas Fork formations. 
Within the study area there are no sections that preserve the complete stratigraphy from the bottom of the basin to the top due to erosion, faulting and changes in depositional environments. Sections were chosen for sampling and measurement from localities that had the most complete exposures (Figure 1).

The Aptian to Albian ( 125 to $100 \mathrm{Ma}$ ) Gannett Group is comprised of the basal Ephraim Formation, Peterson Limestone, Bechler Formation and capping Draney Limestone. Locally, the Ephraim and Bechler formations include thick proximal conglomerates, the presence of which record major slip on the thrust (DeCelles et al., 1993). Westward thickening and coarsening Cenomanian to Turonian ( 100 to $90 \mathrm{Ma}$ ) strata record continued slip and unroofing of the thrust system. However, details of the slip history, including timing of initiation, are debated and exhumation history is poorly constrained.

The development of foreland basins provides information on rates and magnitudes of thrust loading, dynamic subsidence controls and crustal strength. Traditional basin modeling is based on thrust loading of orogenic wedges (Flemings and Jordan, 1990; Paola, 2000). Models that invoke both crustal loading, as well as flexural and dynamic subsidence, have been created to explain the extent of deposition related to orogenic events as well as the influence of subduction of an oceanic plate (Gurnis, 1992; Liu and Nummendal, 2004; Xie and Heller, 2009; Liu et al., 2011; Painter and Carrapa, 2013; Liuet al., 2014; Fan and Carrapa, 2014). However, the initiation of flexural and dynamic subsidence is difficult to predict without accurate data regarding the initiation and rates of thrust slip as well as depositional characteristics controlling synorogenic sedimentation. 
The foreland basin strata of the Sevier orogen have been studied by many (Armstrong, 1968; Heller et al., 1988; Heller and Paola, 1989; Flemings and Jordan, 1990; Schmitt and Steidtmann, 1990; DeCelles et al., 1993; Currie, 1998; DeCelles, 1994; DeCelles and Currie, 1996; DeCelles and Giles, 1996; Stewart et al., 2001; Currie, 2002; DeCelles, 2004; DeCelles and Coogan, 2006; Zaleha, 2006; Dickinson and Gehrels, 2009; Lawton et al., 2010; Hunt et al., 2011; Aschoff and Steel., 2011; Aschoffand Steel, 2011; Laskowski et al., 2013; May et al., 2013). However, most work has focused on the stratigraphic intervals younger than the initiation of slip on the WPM thrust sheet, both in terms of basin fill and provenance. 


\subsection{Stratigraphy}

Three complementary stratigraphic sections (Figure 7) were chosen based on stratigraphic completeness, proximity to WPM thrust sheet, and proximity to thermochronology data. These include two northern locations at Red Mountain, ID and Salt River Pass, WY; and one southern section at Sawtooth Mountain, WY.

\subsection{Salt River Pass}

The locality at Salt River Pass, Wyoming preserves the most complete stratigraphy with over $2000 \mathrm{~m}$ of section exposed (Figure 7). This section represents part of the early foreland basin that is proximal $(<30 \mathrm{~km})$ to the WPM thrust sheet and includes the Ephraim through Thomas Fork formations. The complete foreland basin section includes Ephraim through Frontier formations (Figure 5). A $100 \mathrm{~m}$ section of the Bechler Formation and a $50 \mathrm{~m}$ section of the Thomas Fork Formation were coherent enough for detailed measurement at this locality. The majority of the Bechler Formation at this location is composed of a reddish brown finegrained mudstone. The bottom $\sim 70 \mathrm{~m}$ consists of the reddish brown mudstone that has minor amounts of calcite concretions present in float. The remaining $30 \mathrm{~m}$ demonstrates a transition to reddish brown mudstone interbedded within sub meter fine to medium grained fluvial sandstones. Dips are consistent with a local syncline. The Thomas Fork Formation consists of almost entirely reddish grey to light grey mudstone with calcite concretions present in float throughout the road cut. There are few intervals of fine-grained fluvial sandstone on a sub meter scale (Figure 11). Salt River Pass also provides easy access to multiple outcrops that allow for increased data acquisition for paleocurrents, clast counts and bedding orientation. 


\subsection{Red Mountain}

Red Mountain, Idaho preserves a dominantly conglomeratic section of the Gannett Group, encompassing the unconformity at the base of the Ephraim with the Stump Formation, to the latest Bechler Formation (Figure 7). Over 300 meters of Ephraim and over 850 meters of Bechler are preserved in what appears to be a significant localized, proximal structural basin (DeCelles et al., 1993). This locality is at the current leading edge of the Meade section of the WPM thrust sheet and shows evidence for basin development during thrust sheet emplacement. At Red Mountain, there is significant accumulation and preservation of what is assumed to be a structural basin, accounting for the thick section of Bechler that is preserved (Eyer, 1969; DeCelles et al., 1993). In most localities, the Bechler is only $400 \mathrm{~m}$ thick; at Red Mountain, the Bechler is over 850 m thick and is dominated by coarse conglomerates (DeCelles et al., 1993). The Peterson Limestone is absent at this locality either due to erosion or lack of deposition. The Ephraim and Bechler conglomerates are inferred to be in contact beneath a 50 m covered interval at this locality.

DeCelles et al. (1993) has suggested that the main reason for a coarse conglomerate lithology at the Red Mountain locality is because this section is the only locality that preserves a localized depocenter related to thrusting of the Meade sheet. This project did not address the reasons for the localized sediment accumulation and preservation, however, it may be due to a combination of flexural loading from the encroaching thrust sheet and a structural basin formed during development of a syncline in the footwall of the Meade thrust (DeCelles et al., 1993); growth stratal relationships associated with the syncline document syntectonic sediment 
accumulation. In any case, data were collected for the purposes of timing, provenance study and paleocurrent analysis.

\subsection{Sawtooth Mountain}

The section at Sawtooth Mountain was chosen for sedimentologic and stratigraphic study based on relatively good exposure of a complete section from the basal Ephraim to Thomas Fork formations (Figure 7). The Sawtooth Mountain section has the additional benefit of lying east of, and potentially recording the accumulation of, the erosional record of the Willard sheet captured in the thermochronological study of Eleogram (2014). To further understand the early development of the WPM emplacement, evidence of thrust initiation and unroofing from this study will be combined with the previously determined thermochronology data. While part of the foreland basin has been preserved adjacent to these localities, this preserved section has been passively transported $\sim 20 \mathrm{~km}$ eastward as a result of lying on the Crawford thrust sheet and being transported in the late Cretaceous with movement of the Absaroka thrust sheet. As a result, samples were taken at Sawtooth Mountain to determine timing and provenance; however, additional sections from Red Mountain and Salt River Pass must be used to help place the samples in context. 


\subsection{Sedimentology}

\subsection{Sedimentology of the Ephraim Formation}

\subsubsection{Facies analysis of the Ephraim Formation}

A suite of five lithofacies (Miall, 1977) have been identified for the Ephraim Formation within the Gannet Group. Rudimentary facies associations were defined based on detailed analysis of internal and external geometries and bounding surfaces and sections with significant outcrop (but not complete sections) present. Five primary facies associations were identified for the Ephraim Formation including: major conglomerate (FA1), minor conglomerate (FA2), minor tabular to lenticular sandstone (FA3), tabular to lenticular siltstone/mudstone (FA4), and massive siltstone/mudstone (FA5) (Table 1).

\subsubsection{Description and interpretation: major conglomerate (FA1)}

Major conglomerates in the Ephraim Formation are defined as laterally discontinuous, matrix supported conglomerate sequences. They may represent single thick units, or multiple thinner, stacked beds that are dominated by conglomerate lithofacies $\left(\mathrm{G}_{1} \mathrm{~mm}\right.$, Table 1$)$. Isolated major conglomerate outcrops are typically several meters thick. Thin, tabular to lenticular lenses of lithofacies $S_{1} p$ and $S_{1} t$ (Table 1 ) are present throughout the conglomerate and do not exceed $1 \mathrm{~m}$ in thickness.

FA1 is dominated by massive outcrops composed of pebble-sized, poorly sorted, subangular to subrounded clasts of extraformational red chert, light grey to grey carbonate, tan sandstone, white and grey quartzite. The matrix is reddish brown to reddish tan poorly sorted medium to coarse sand. Outcrops are generally well indurated and tend to be ridge formers. Pebble counts were conducted on conglomerate outcrops by counting and classifying 100 clasts 
within a half-meter grid. Red and black cherts tend to dominate the classified lithofacies, along with a moderate amount of light grey to grey carbonate and minor amounts of tan sandstone, white and grey quartzite.

Major conglomerates are found intermittently within the middle to top sections of the Ephraim Formation. Contacts between lithologic facies are difficult to identify in the field, so it is unclear if most contact surfaces are erosional or continuous. Based on clast size and composition, sorting, percentage of matrix support and the presence of trough and planar cross bedding, FA1 is interpreted to represent an alluvial fan deposit. FA1 units are only found at the Red Mountain locality and can be interpreted as unmodified debris flows based on the disordered, $60 \%$ matrix-supported nature of the deposits that include larger, angular to subangular clasts located almost directly adjacent to the WPM thrust front.

These observations indicate that alluvial fans and low-grade tributary systems transitioned into an anastamosing river system draining off the eastward propagating thrust front. The alluvial fans would have provided the gravel-sized sediment input seen in the distal fluvially-dominated deposits.

FA1 deposits in the Ephraim Formation are not found in the first $\sim 50 \mathrm{~m}$ of the unit, suggesting that uplift and development of the thrust front was not immediate in relation to the formation of perhaps an initially dynamically driven flexural basin, or a flexural basin that formed in response to internal stress regimes.

5.1.3 Description and interpretation: minor conglomerate (FA2)

FA2 is found at one locality, Red Mountain, and represents one of the most proximal synorogenic deposits related to the WPM thrust front. Minor conglomerates of the Ephraim 
Formation at Red Mountain are defined as laterally discontinuous, matrix supported conglomerate sequences. They may represent single thick units, or multiple thinner, stacked beds that are dominated by conglomerate lithofacies $\left(G_{2} m m\right.$, Table 1$)$. Isolated major conglomerate outcrops are typically several meters thick. Thin, tabular to lenticular lenses of lithofacies $S_{1} p$ are present throughout the conglomerate and do not exceed $1 \mathrm{~m}$ in thickness.

FA2 is dominated by massive outcrops composed of small boulder to pebble-sized, poorly sorted, subangular to rounded clasts of extraformational red chert, light grey to grey carbonate, tan sandstone, white and grey quartzite (Figure 8). The matrix is a reddish brown to reddish tan, poorly sorted medium to coarse moderately sorted sand. Outcrops are generally well indurated and tend to be ridge formers. Pebble counts were conducted on conglomerate outcrops by counting and classifying 100 clasts within a half-meter grid. Red and black cherts tend to dominate the classified lithofacies, along with a moderate amount of light grey to grey carbonate and minor amounts of tan sandstone, white and grey quartzite.

FA2 sequences found at Sawtooth Mountain and Salt River Pass are $40 \%$ matrix supported. Clasts are distinctly smaller in size and are subangular to rounded and overall sorting is better suggesting distally derived channel avulsions within an anastomosing river system.

Paleocurrent data collected for the Ephraim Formation (Figure 9) at Red Mountain indicate a dominant W-E flow direction accompanied by a collection of low dispersion flow directions. E-W flow directions are consistent with the presence of a topographic high in an eastern position, while N-S flow directions are consistent with the development of a foreland basin axis running parallel to the thrust front. 
The distribution of Lower Cretaceous gravels throughout the North American Cordillera are of particular interest due to the fact that there is far traveled sediment found along the eastern margin. In particular, the Gannett Group is of interest due to the presence of far traveled sediment within units that are thicker than expected. Although previous studies and modeling have attempted to explain these thin, long run-out gravels, the presence of a thicker (>500 m) Ephraim conglomerate ranging from southwest Wyoming to northeastern Utah remains controversial (Heller and Paola, 1989).

These observations indicate that alluvial fans and low-grade tributary systems were likely incorporated into anastamosing river systems draining off the eastward propagating thrust front. The alluvial fans would have provided the gravel-sized sediment input seen in the distal, fluvially-dominated deposits.

5.1.4 Description and interpretation: minor tabular to lenticular sandstone (FA3)

Within the FA3 facies association, minor tabular to lenticular sandstones $\left(\mathrm{S}_{1} \mathrm{p}, \mathrm{S}_{1} \mathrm{t}, \mathrm{Table}\right.$ 1) found within the Ephraim Formation are only found interbedded within the conglomerate lithofacies $\mathrm{G}_{1} \mathrm{~mm}$. Typical bedding thickness is no greater than $1 \mathrm{~m}$.

Modal assemblages of the sandstone within the Ephraim Formation were plotted on a ternary diagram with an emphasis on QFL percentages (Figure 16). Feldspar grains are absent in all samples collected. Furer (1970) point counted 88 samples from the Ephraim Formation and identified similar modal QFL percentages, again with a distinct lack of feldspar grains. The cement is typically $30 \%$ and is composed of either calcite or chert, with minimal evidence of chemical dissolution or major deformation. 
Overall paleocurrent directions measured from both planar- and three-dimensionally exposed trough-cross-bedding demonstrate an overall E-W drainage direction. Paleocurrent data collected for the Ephraim Formation (Figure 9) at Salt River Pass and Sawtooth Mountain indicate a dominant W-E flow direction consistent with the presence of a topographic high in an eastern position. Paleocurrent data support clast provenance data in that both proximal and distal clasts can be correlated to stratigraphy within the WPM thrust (discussion in Provenance section below).

S1p, S1t, S1m lithofacies have been identified within FA3. S1t is the most common lithology and consists of uniform bed set thickness of $\sim 20-70 \mathrm{~cm}$ thick and a mean set thickness of $\sim 40 \mathrm{~cm}$.

FA3 units within the Ephraim Formation are interpreted as crevasse channel deposits (Figure 11). Sedimentary characteristics supporting this interpretation include planar and trough cross bedding as well as architectural elements such as channels and channel fill. The discontinuous nature of sand bodies within the thick, massive FA5 mudstone and siltstone coupled with low dispersion paleocurrents indicate avulsive channel fill deposits that may be part of an anastomosing system.

\subsubsection{Description and interpretation: minor tabular to lenticular siltstone/mudstone (FA4)}

Minor tabular to lenticular siltstone and mudstone are found interbedded within the minor tabular to lenticular sandstone and conglomerate units, and typically do not exceed 0.5 $m$ in thickness. Fine grained lithofacies include $F_{1} l$ and $F_{1} m$ with little preservation of fine laminations due to the nature of the outcrop. $F_{1} l$ and $F_{1} m$, when interbedded, consist of fine silt and muds with a dark reddish brown, pale red purple and brown color. 
The tabular to lenticular nature of FA4, in addition to the fine to medium grained sands within the deposits, are interpreted as overbank flood and channel fill deposits within an anastomosing river system.

5.1.6 Description and interpretation: massive siltstone/mudstone (FA5)

The massive siltstone and mudstone, FA5, dominates the Ephraim Formation, representing over $70 \%$ of the stratigraphic sequence. FA5 is highly variable in overall character and lithofacies composition. Deposits are typically massive, with no discrete sedimentary structure present, and range in thickness from $1 \mathrm{~m}$ to over $15 \mathrm{~m}$. The fine grained silt and mud is dark reddish brown, pale red purple and brown. Calcareous nodules are found within float in several locations.

The thick, fine grained nature of FA5 represents significant overbank deposits related to the observed anastomosing drainage system. The lack of reactivation surfaces and massive nature of FA5 indicates fast generation of accommodation space (Schumm, 1981).

\subsection{Sedimentology of the Bechler Formation}

\subsubsection{Facies analysis of the Bechler Formation}

A suite of five lithofacies (Miall, 1977) have been identified for the Bechler Formation within the Gannet Group. Rudimentary facies associations were defined based on detailed analysis of internal and external geometries, bounding surfaces and architectural elemental analysis (Figure 12) of measured section (Figure 13) and sections with significant outcrop (but not complete sections). Five primary facies associations were identified for the Bechler Formation including: major conglomerate (FA1), minor conglomerate (FA2), minor tabular to 
lenticular sandstone (FA3), tabular to lenticular siltstone/mudstone (FA4), and massive siltstone/mudstone (FA5) (Table 1).

\subsubsection{Description and interpretation: major conglomerate (FA1)}

Major conglomerates in the Bechler Formation are defined as laterally discontinuous, matrix supported conglomerate sequences. They may represent single thick units, or multiple thinner, stacked beds that are dominated by conglomerate lithofacies $\left(\mathrm{G}_{2} \mathrm{~mm}\right.$, Table 1$)$. Isolated major conglomerate outcrops are typically several meters thick. Thin, tabular to lenticular lenses of lithofacies $S_{1} p$ and $S_{1} t$ are present throughout the conglomerate and do not exceed 1 $m$ in thickness.

FA1 is dominated by massive outcrops composed of small boulder to pebble-sized, poorly sorted, subrounded to rounded clasts of extraformational clear quartzite, red and tan sandstone, carbonate, and red and black chert. The matrix is a reddish brown to reddish light grey, medium to coarse grained, moderately well-sorted sand. Outcrops are generally well indurated and tend to be ridge formers. Pebble counts were conducted on conglomerate outcrops by counting and classifying 100 clasts within a half meter grid. Clear quartzites, red and tan sandstone and carbonates tend to dominate the classified lithofacies, along with subordinate red and black chert.

Major conglomerates are found intermittently throughout the entire Bechler Formation. Contacts between lithologic facies are difficult to identify in the field, so it is unclear if most contact surfaces are erosional or gradational.

Based on clast size and composition, sorting, percentage of matrix support and the presence of interbedded trough and planar cross bedding, FA1 is interpreted to represent an 
alluvial fan deposit. FA1 units (Table 1) are only found at the Red Mountain locality and can be interpreted as unmodified debris flows based on the disordered, 60\% matrix-supported nature of the deposits that include larger, angular to subangular clasts located almost directly adjacent to the WPM thrust front.

These observations indicate that alluvial fans and steep-grade tributary systems were likely incorporated into an anastamosing river system draining off the eastward propagating thrust front. The alluvial fans would have provided the gravel-sized sediment input seen in the distal fluvially-dominated deposits. Figure 15 shows paleocurrent directions determined by DeCelles et al. (1993) based on imbricated clasts, which demonstrate a NE-E flow direction accompanied by a collection of low dispersion flow directions.

\subsubsection{Description and interpretation: minor conglomerate (FA2)}

FA2 is found at one locality, Red Mountain, and represents one of the most proximal synorogenic deposit related to the WPM thrust front. Minor conglomerates of the Bechler Formation at Red Mountain are defined as laterally discontinuous, matrix supported conglomerate sequences. They may represent single thick units, or multiple thinner, stacked beds that are dominated by conglomerate lithofacies $\left(\mathrm{G}_{2} \mathrm{~mm}\right.$, Table 1$)$. Isolated major conglomerate outcrops are typically several meters thick. Thin, tabular to lenticular lenses of lithofacies $S_{1} p$ are present throughout the conglomerate and do not exceed $1 \mathrm{~m}$ in thickness.

FA2 is dominated by massive outcrops composed of cobble to pebble-sized, poorly sorted, subangular to rounded clasts of extraformational clear quartzite, red and tan sandstone, carbonate, and red and black chert. The matrix is a reddish brown to reddish light grey, medium to coarse grained, moderately well sorted sand. Outcrops are generally well indurated and tend 
to be ridge formers. Pebble counts were conducted on conglomerate outcrops by counting and classifying 100 clasts within a half meter grid. Clear quartzites, red and tan sandstone and carbonates tend to dominate the classified lithofacies, along with subordinate red and black chert.

5.2.4 Description and interpretation: minor tabular to lenticular sandstone (FA3)

Minor tabular to lenticular sandstones $\left(S_{2} p, S_{2} t\right.$, Table 1) found within the Bechler Formation are only found interbedded within the conglomerate lithofacies $\mathrm{G}_{2} \mathrm{~mm}$. Typical bedding thickness is no greater than $1 \mathrm{~m}$.

Modal assemblages of the sandstone within the Bechler Formation were plotted on a ternary diagram with an emphasis on QFL percentages QFL (Figure 16). Feldspar grains are absent in all samples. The cement is typically $30 \%$ and is composed of either calcite or chert, with minimal evidence of chemical dissolution or deformation.

Overall paleocurrent directions measured from both 2D- and three-dimensionally exposed trough-cross-bedding demonstrate an overall E-W drainage direction with minor dispersal patterns. Paleocurrent data collected for the Bechler Formation (Figure 17) at Red Mountain indicate a dominant W-E flow direction accompanied by a collection of low dispersion flow directions. W-E flow directions are consistent with the presence of a topographic high in an eastern position, while N-S flow directions are consistent with axial flow and development of a foreland basin running parallel to the thrust axis. Poor exposures of the Bechler Formation at Sawtooth Mountain did not provide adequate exposures to obtain reliable paleocurrent measurements. 
S1p, S1t, S1m lithofacies have been identified within FA2. S1t is the most common lithology and consists of uniform bed set thickness of $\sim 20-70 \mathrm{~cm}$ thick and a mean set thickness of $\sim 40 \mathrm{~cm}$.

5.2.5 Description and interpretation: minor tabular to lenticular siltstone/mudstone (FA4)

Minor tabular to lenticular siltstone and mudstone are found interbedded within the minor tabular to lenticular sandstone, and typically do not exceed $0.5 \mathrm{~m}$ in thickness. Fine grained lithofacies include $F_{2} I$ and $F_{2} m$ with little preservation of fine laminations due to the nature of the outcrop. $F_{2} I$ and $F_{2} m$, when interbedded, consist of fine silt and muds with a dark reddish brown, pale red purple and brown color.

The tabular to lenticular nature of FA4, in addition to the fine to medium grained sands within the deposits, are interpreted as overbank flooding and channel fill deposits within an anastomosing river system. Subsequent incision and scouring by overlaying fluvial channels is a possible mechanism that removed any paleosol development that is seen in the more massive siltstone and mudstone deposits.

5.2.6 Description and interpretation: massive siltstone/mudstone (FA5)

The massive siltstone and mudstone, FA5, dominates the Bechler Formation, representing over $70 \%$ of the stratigraphic sequence (Figure 13). FA5 is highly variable in overall character and lithofacies composition. Deposits are typically massive, with no discrete sedimentary structure present, and range in thickness from $1 \mathrm{~m}$ to over $15 \mathrm{~m}$. The fine grained silt and mud is dark reddish brown, pale red purple and brown. Calcareous nodules are found within float in several locations. 
The thick, fine grained nature of FA5 in the Bechler Formation represents significant overbank deposits related to an inferred anastomosing drainage system. As with the massive overbank deposits of the Ephraim Formation, the lack of reactivation surfaces and massive nature of FA5 in the Bechler Formation indicates fast generation of accommodation space (Schumm 1981). Presence of carbonate nodules indicates that these deposits were not buried rapidly, allowing for the development of soils before the next flooding event.

\subsection{Remaining foreland basin}

The remaining units within the foreland basin include the Frontier, Sage Junction, Cokeville, Aspen, and Bear River formations (Figure 5). They represent a collection of marine and terrestrial sandstone and shale units that not only preserve the continued thrusting and erosional unroofing of the WPM thrust sheet, but also record, to a first order effect, the lithospheric response to flexural loading and dynamic subsidence.

\subsection{Structural Relations}

At Salt River Pass, overall bedding of the Bechler Formation dips $\sim 20^{\circ} \mathrm{E}$, and the Thomas Fork Formation dips $\sim 5^{0} \mathrm{E}$. These dips are consistent with the Bechler Formation representing the western limb of a local syncline, while the Thomas Fork Formation is located near the axis of the syncline.

At Red Mountain, the Ephraim Formation has an overall dip of $\sim 55^{\circ} \mathrm{W}$ while the overlying Bechler dips at only $26^{0} \mathrm{~W}$. This is consistent with development of growth strata within the Ephraim Formation followed by semi-static and proximal deposition of the Bechler Formation. 
At Sawtooth Mountain, bedding within the Ephraim and Bechler formations range from

$\sim 45-70^{\circ} \mathrm{W}$. While this may represent development of growth strata, it is important to recognize that this section of synorogenic strata related to WPM development lies on the mid-late Cretaceous Crawford thrust sheet and was passively transported on the late Cretaceous Absaroka sheet.

\subsection{Paleoclimatology}

Paleoclimate studies on the Early Cretaceous of Western North America demonstrate a warm, humid environment. Paleoclimatology studies of $\delta \mathrm{O}^{18}{ }_{\mathrm{p}}$ within mammals and herbivorous dinosaurs from within the stratigraphically equivalent Cedar Mountain Formation show that the relative humidity during this time was $\sim 58 \%$ and had a range of $\sim 42-76 \%$ (Suarez et al., 2012). In addition, megaflora fossils found within the Aspen Shale of southwestern Wyoming record climatic conditions most similar to Subzone IIB of the Potomac Group of the eastern United States (Peppe et al., 2008). These, combined with evidence of significant uplift of the Nevada hinterland of up to $4 \mathrm{~km}$ (Bonde, 2015; Snell et. al., 2014) demonstrate conditions condusive to the hyperconcentratd flows found within the foreland of the Sevier fold and thrust belt (Heller and Paola, 1989). 


\subsection{Detrital Zircon studies of Lower Cretaceous synorogenic strata}

Here we report over 10,000 DZ U-Pb ages from 27 sandstone samples collected from syntectonic sedimentary units representing the early history of the Sevier foreland basin. In addition, data from previously published studies are incorporated to further validate timing and erosional patterns. Grain ages provide information on the age of the deposits in their youngest age populations as maximum depositional ages (MDA), and information on their sedimentary provenance in their DZ age components. The sandstone collected belong to terrestrial fluvial systems that developed in response to topographic relief developed from thrusting over ramps as well as formation of a foreland basin system. Deposition of DZ grains interpreted as MDAs in this study are thought to have been deposited in either of two different ways. (1) Sierran arcderived airfall zircon grains were deposited in the foreland basin almost immediately after their formation via travel as volcanic ash in the airstream. This interpretation is based on the preservation of a common euhedral crystal structure of the zircon grains indicating little terrestrial transport. In contrast, it is possible that zircon grains were ejected and deposited relatively close to a magmatic source or at an intermediate position and were subsequently remobilized during fluvial transport events into the foreland basin; in this scenario large distance transport occurred with little abrasion to the zircon grains, but deposition still occurs within a relatively short time interval.

The main magmatic source for the western margin of North America during the Mesozoic and Early Cenozoic was the Sierra Nevada magmatic arc. Detailed dating of magmatic pulses related to arc magmatism show a distinctive lull between 140-120 Ma (Irwin and 
Wooden, 2001; DeCelles et al., 2009). Figure 19 shows the main pulses of magmatic activity in the Sierra Nevada.

\subsection{Maximum depositional ages}

Isoplot is a program run on Windows Excel that is used to perform mathematical and graphical analysis of radiogenic-isotopic data (Ludwig, 2008). Using this software, MDA's can be determined using assorted parameters and methods. Previous work has defined various ways of determining MDA's of geologic samples (Dickinson and Gehrels, 2009; Barbeau et al., 2009) and include using 1) the youngest distinct age peak within an age-probability density plot, 2) the youngest single grain with $1 \sigma$ uncertainty, 3) a weighted mean age of the youngest age group of two or more grains within $1 \sigma$ uncertainty, 4) the Unmixing routine within Isoplot that estimates the maximum likelihood of the age of grains within the youngest age group, and 5) the TuffZirc algorithm (Ludwig and Mundil, 2002). Within this study, the age-probability density plot was used to identify the youngest distinct age group. TuffZirc was then used to obtain coherent groups of grains in which their age overlapped by $1 \sigma$. A minimum of eight grains were required for this program to determine a stratigraphically robust age.

MDA's from limited euhedral, and likely volcanic, grains show the following for the northern transect: basal Ephraim Formation, $150 \pm 2.8 \mathrm{Ma}(\mathrm{n}=10)$; upper Ephraim, $115.2 \pm 1.8$ Ma (n=13); lower Bechler, 116.2 $\pm 2.3 \mathrm{Ma}(\mathrm{n}=9)$; and upper Bechler 107.3 $\pm 3.3 \mathrm{Ma}(\mathrm{n}=4)$ (Figure 20). Schwanke and others (2008) dated zircon from reworked volcanic ash that was deposited in shale interbedded in the Draney Limestone. A maximum depositional age of $112.5 \pm 2.6 \mathrm{Ma}$ based on a coherent group of $20 \mathrm{DZ}$ grains was determined (Schwanke et al., 2008). The MDA of $150 \pm 2.8 \mathrm{Ma}(n=10)$ for the Ephraim Formation might be reflecting recycling of sediment 
between the upper Pruess/Morrison formations and lower Ephraim Formation, or it may reflect the depositional age of the lower Ephraim Formation, consistent with earlier but limited biostratigraphy (Eyer, 1989).

These data clarify that there is not a significant haitus at the base of the Bechler, and that the Bechler conglomerate facies at Red Mountain spans the depositional interval including the 112 Ma Drainey Limestone, which is absent at Red Mountain but present elsewhere. If there is an angular unconformity at the base of the Bechler Formation at Red Mountain as suggested by DeCelles et al. (1993), then folding of the strata is constrained between $115.2 \pm$ 1.8 Ma (upper Ephraim) and, 116.2 $\pm 2.3 \mathrm{Ma}$ (lower Bechler), or between 117 and 113.9 Ma.

The southern transect yields the following MDAs: Pruess Formation, $169.33 \pm 1.5 \mathrm{Ma}$ $(n=10)$; Upper Gannett, $109 \pm 6.1 \mathrm{Ma}(n=5)$ and $108.3 \pm 3.4 \mathrm{Ma}(\mathrm{n}=10)$. The Morrison Formation, which is stratigraphically equivalent to the Pruess Formation, is well constrained with a basal age of $154.8 \pm 0.5 \mathrm{Ma}$ in the Tidwell member (Kowallis et. al., 1998), and a capping age of $148 \pm$ 0.5 Ma within the Brushy Basin member (Kowallis, 1991).

The remaining foreland strata in the southern transect, from the Bear River to Frontier formations, represent subsequent movement on the WPM thrust and foreland basin development. MDA's for the remaining foreland strata include: Cokeville, 101.6 $\pm 0.7 \mathrm{Ma}$

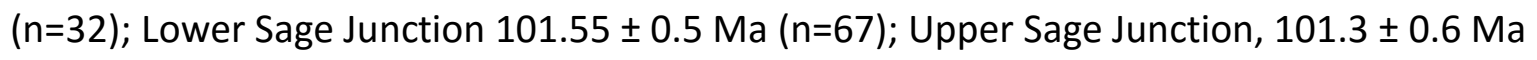
$(n=29)$; Aspen, $98.8 \pm 0.4 \mathrm{Ma}(n=51)$; lower Frontier, $99.93 \pm 0.6 \mathrm{Ma}(n=21)$; and upper Frontier, $95.68 \pm 1.4 \mathrm{Ma}(\mathrm{n}=38)$ (Figure 20). 


\subsection{DZ Age Patterns}

By analyzing DZ age patterns through the stratigraphic succession within the sedimentary basin, chronofacies can be identified that correspond to specific strata with in the adjacent thrust sheet (Lawton et al., 2010). These chronofacies represent a unique collection of age patterns, which can be sourced from multiple places. As a result, when erosion of the thrust sheet reaches each exposure gate, and those unique age pattern collections are re-transported to the corresponding basin in a predictable unroofing succession that establishes the chronofacies. For instance, strata near the top of the WPM thrust sheet would include DZ grains that include Jurassic populations as well as a collection of older DZ ages, depending on the drainage systems that existed, whereas basal strata would include only older DZ grains. As a result, the top strata have younger and more variable DZ age patterns and basal strata have DZ patterns with older grain populations.

Comparison of the WPM DZ age spectra with the adjacent foreland basin sediment DZ spectra, shows that a clear expression of a progressive unroofing sequence is preserved (Table 2). P values in comparison of the Nugget Formation with foreland strata include strong correlations with the Ephraim (0.977), Bechler (0.877), Bear Valley (0.187) and Hilliard (0.353) formations. The Moenkopi Formation had no influence on older Cretaceous foreland basin units, and had little influence on the Upper Frontier (0.001) and Hilliard (0.001). The Oquirrh Formation had significant influence on the Cokeville (0.365), Frontier (0.480), Upper Frontier (0.185), Aspen (0.551) and Hilliard (0.139) formations and minor influence on the Sage Junction (0.003) and Bear Valley (0.042) formations. The Swan Peak and Worm Creek, formations appear to have no influence on any of the still preserved foreland basin sediment. The Geertsen 
influenced the Upper Frontier Formation (0.159) and had minor influence on the Sage Junction Formation (0.004). The Mutual Formation strongly influenced the Bear Valley (0.067), Frontier (0.198), Upper Frontier (0.076), and Aspen (0.712) formations, with minor influence on the Cokeville (0.002) and Hilliard (0.022) formations. The Caddy Canyon Formation had minor influence on the Ephraim (0.001), Frontier (0.002), Upper Frontier (0.004), and Aspen (0.002) formations. Figure 20 shows the evolution of DZ patterns through time and stratigraphic section.

The unique age of the $D Z$ grains can provide an ultimate source that originated on the North American craton when there was a passive western margin. Grains that range in age from 2.6-3.0 Ga are sourced from the Wyoming province (Frost et al., 2006; Fan et al., 2011). A distinct peak at $2.55 \mathrm{Ga}$ is attributed to the Grouse Creek block (Strickland et al., 2011; Isakson, 2012). There is a mix of 1.7-1.9 Ga grains that belong to the Farmington zone, Mojave province, and Selway terrane, as well as reworked Archean grains (Shufeldt et al., 2010; Mueller et al., 2011; Yonkee et al., 2013). Grains yielding a 1.7-1.8 Ga peak belong to the Yavapai and Mazatzal provinces (Bickford et al., 2008). Eriksson et al. (2003) report ages of 1.3-1.5 Ga for mid-continent granite and A-type granite intrusions in the southwest U.S., 1.0-1.3 Ga ages for the Grenville-Llano province, as well as $\sim 1.1$ Ga late granite intrusions in the southwest United States. Relatively younger grains with peaks around 650-470 Ma have been attributed to the Idaho batholith area (Lund et. al., 2009) and peaks 300 Ma are sourced from the Appalachians (Dickinson and Gehrels, 2009). 
Figure 3 shows the distribution of source terranes for Paleozoic- early Mesozoic DZ grains that could have been incorporated into the WPM thrust sheet. In addition, the age range of these source terranes are superimposed on DZ patterns found within the Willard thrust sheet in Figure 21 in order to make more effective correlations between patterns found within the thrust sheet and within the foreland basin sediment.

In the analysis of an unroofing sequence in the early Sevier foreland basin, the sedimentological history is congruent with DZ patterns correlated directly with the WPM thrust sheet (Figure 22). Within the basal deposits of the Ephraim Formation, clasts include a unique collection of red and black chert thought to originate from the Roberts Mountain allochthon in the hinterland of Nevada (Gehrels et al., 2000). Clasts of the Twin Creek limestone and Wells quartzite that were shed off the top of the WPM thrust sheet when uplift initiated were identified within upper levels of the Ephraim Formation. 


\subsection{Provenance Study}

Point counting was performed on 27 petrographic sandstone thin sections, with 15 being calcium stained for carbonates. Clast counts were performed in the field on conglomerate intervals of the Ephraim and Bechler Formations where identification of clast composition was possible. Clast counts were collected at 10 sites for the Ephraim Formation and 3 sites for the Bechler Formation. Petrographic thin section counts were plotted on a QFL diagram, with the quartz apex including monocrystalline and polycrystalline grains, the lithic apex including silicic detritus, carbonate fragments and chert fragments, and the feldspar apex including orthoclase and plagioclase compositions. Samples analyzed follow similar patterns found within typical Gannett samples (DeCelles et al., 1993), compositions of average Gannett samples are plotted against collected samples shown in Figure 10.

Field clast-count data for the Ephraim Conglomerate were collected at all three localities (Figure 1). At Sawtooth Mountain, the conglomerate is composed of red and black chert, white and grey quartzite, carbonate and minor amounts of tan sandstone, with red chert and grey quartzite dominant. At Salt River Pass, the Ephraim conglomerate is composed of white and grey quartzite, red chert, brick red and tan sandstone, and minor amounts of black chert and carbonate clasts. Here the conglomerate is dominated by grey and white quartzite clasts. At Red Mountain, the Ephraim conglomerate is composed of carbonate, brick red sandstone, grey to brownish grey quartzite and minor amounts of red chert, with carbonate and brick red sandstone grains dominant.

Field clast-count data for the Bechler Conglomerate were also collected at three localities (Figure 1): Sawtooth Mountains, Salt River Pass, and Red Mountain. At Sawtooth 
Mountain, the Bechler is composed of carbonate, white and grey quartzite, and minor amounts of red and black chert and tan sandstone. At Salt River Pass, the Bechler Conglomerate is composed of grey and white quartzite, red chert, and minor amounts of carbonate, and tan and red sandstone. The Bechler at Red Mountain includes carbonate, red chert, brick red sandstone, grey quartzite and minor amounts of black chert, tan sandstone, and tan quartzite. Carbonate grains are most likely derived from the Devonian Hyrum Dolomite, the Mississippian Lodgepole or the Monroe Canyon Limestone. Several clasts were found with crinoid fossils. Sandstone clasts were probably derived from Pennsylvanian Wells Formation as well as Jurassic Nugget Formation. Basal Ephraim Ordovician through Devonian aged chert clasts were most likely derived from the Roberts Mountain allochthon or in Mississippian flysch deposits derive from the allochthon (Diamond Peak Formation and correlative units).

Subsequent chert clasts were derived from the Mississippian Lodgepole (brown colored chert), Monroe Canyon Formation (yellowish-grey colored chert) or Permian Phosphoria Formation (black colored chert). White-grey quartzite clasts were derived from the Ordovician aged Swan Peak Formation.

Within each unit, overall DZ age data present unique patterns that can be analyzed for provenance. Within samples, paleogeograpghic and depositional age groups were determined to identify major provenance trends in addition to any local variability introduced during deposition (Figure 23).

The geomorphic breaching of Jurassic sediments in this region represents initiation of uplift and thrusting of the WPM sheet over footwall ramps. This development also marks 
cessation of fluvial sediment transport from western hinterland sources, with the WPM sheet acting as a geographic barrier to previous drainage systems.

It is important to keep in mind that DZ patterns can vary through time based on changes in the paleogeography of drainage basins and resulting fluvial systems. These variations can be in both provenance and in basin sediment architecture. 


\subsection{Implications}

The depositional environments of the Ephraim and Bechler formations are dominated by deposits of an anastamozing river system that demonstrates periods of hyperconcentrated flows containing both proximal and distal source units (Nadon, 1994). These flows are characterized by subrounded to rounded pebble clasts and overall planar bedding. Because the Ephraim is interpreted to represent the initial formation of a foreland basin related to emplacement of the WPM thrust sheet, similarities between northern and southern sections are important in determining overall thrust development. Within both sections, locally laterally continuous distal conglomerate facies have been identified, while proximal conglomerate facies related to the WPM have been found only at the Red Mountain locality. Although the Sawtooth Mountain locality lies on the Crawford thrust sheet and was passively transported on the late Cretaceous Absaroka sheet, similarities to the Salt River Pass section including bedding, clast size and composition and sedimentary features such as cross bedding, allow comparisons to be made related to thrust emplacement in terms of depositional environments.

Results from previous thermochronology studies of the Willard thrust sheet show the Wellsville transect yields a thermal history with cooling starting at $134 \mathrm{Ma}$, with an average exhumation rate of $0.11 \mathrm{~mm} / \mathrm{yr}$ (Eleogram, 2014). These cooling ages are interpreted to broadly represent the initiation of slip, onset of thrust-related uplift and erosion along the Willard thrust.

Development of deformation within the WPM thrust sheet is expected to be recorded within multiple structural and depositional features. MDA's of DZ grains within the earliest Sevier foreland basin are expected to coincide with ZHe ages within the Willard thrust sheet 
that record cooling due to erosional exhumation. Emplacement of a significant load would result in a lithospheric flexural response, creating accommodation space capable to preserving and DZ grains that may have been present 130 Ma (Heller and Paola, 1989). In addition, the presence of marine fossils within the basal Ephraim suggests that conditions were favorable for preservation of any DZ grains brought to the depositional system at this time (Eyer, 1989; Rogers, 1998).

An MDA of $115.2 \pm 1.8$ Ma within the Ephraim Formation and $116.2 \pm 2.3$ Ma within the lower Bechler, represents the earliest preserved DZ grains related to basin formation from uplift of the WPM thrust sheet. The main pulse of synorogenic deposition related to uplift of the WPM thrust sheet is well bracketed within units of the Gannett Group in the northern transect. Deposition within the basin began no later than 115.2 \pm 1.8 Ma within the Ephraim Formation. The Draney Limestone represents the final depositional unit within the Gannett Group. Previous geochronologic work done on the Draney Limestone near Tincup Canyon, ID, show an MDA of (112.5 $\pm 2.6 \mathrm{Ma}$ ) (Schwanke et al., 2008). While absent at the Red Mountain locality, the age of the Draney Limestone suggests that the Bechler Formation may have locally spanned the depositional age of the Draney, shutting down the carbonate factory in the process. However, given one sigma error for MDA of the Bechler and the Draney Limestone, the apparent span could also be the result of an overlap of errors by $\sim 4 \mathrm{Ma}$.

At Red Mountain, the contact between the between the Ephraim and Bechler formations was previously interpreted an angular unconformity (DeCelles et al., 1993). MDA's show that there is at most little time missing and perhaps continuous deposition, as the upper 
Ephraim yields an MDA of 115.2 \pm 1.8 Ma whereas the lower Bechler yields an MDA of $116.2 \pm$

2.3 Ma. These data clarify that there is not a significant unconformity at the base of the Bechler. Furthermore, the upper Bechler yields an MDA of $107.3 \pm 3.3 \mathrm{Ma}$, indicating that the Bechler conglomerate facies at Red Mountain spans the depositional interval including the 112 Ma Draney Limestone, which is present elsewhere but absent at Red Mountain.

The lack of 115 Ma DZ grains within the Ephraim Formation in the southern section, as well as $130-115$ Ma grains that would be coeval with the early erosional history of the Willard thrust sheet (as evident in the ZHe cooling ages), could result from several factors. In one case, it could be the result of early local uplift that would prevent depositional preservation. ${ }^{40} \mathrm{Ar} /{ }^{39} \mathrm{Ar}$ UV laser ablation ages of muscovite from the western margin of the Willard thrust sheet show that early alteration and internal deformation of the Willard thrust sheet may have begun as early as $140-134 \mathrm{Ma}$ (Giallorenzo, 2013). ${ }^{40} \mathrm{Ar} /{ }^{39} \mathrm{Ar}$ ages from muscovite found along the basal contact of the Willard thrust sheet demonstrate slip ages of 140-110 Ma (Yonkee et. al., 1989). Locally, The Wasatch Anticlinorium represents a structural feature that may have contributed to early uplift and erosion, however, it is thought to have experienced uplift beginning $90 \mathrm{Ma}$ (Naeser et. al., 1983) which is significantly later than the suggested 134 Ma uplift history (Eleogram, 2014). Furthermore, uplift of the Wasatch Anticlinorium caused folding within the overlying Willard thrust sheet (DeCelles, 2004), which would require movement of the Willard thrust sheet before uplift of the anticlinorium. Alternatively, uplift of the early foreland basin or propagation of the forebulge may have resulted in erosion of the initial foreland deposits into the adjacent backbulge basin and propagation of the forebulge/backbulge system to the east as lithospheric forces developed (DeCelles and Giles, 1996). The Cloverly Formation, which 
is an eastern stratigraphic and latitudinal equivalent of the basal Ephraim Formation, has evidence of palynomorphs ranging in age from 139-126 Ma (DeCelles and Burden, 1992; Painter et. al., 2014). With the dominance of east directed river systems in both the Ephraim and Cloverly formations, the wet and humid climatic conditions, as well as evolution of the forebulge; it is possible that DZ grains, as well as palynomorph fossils, were eroded and redeposited farther out on the basin, thereby eliminating the Early Cretaceous record in proximal parts of the foreland (Fleming, 1994).

Alternatively, the absence of young grains could be the result of a different drainage system that did not bring young DZ grains into the basin at that location. Although ZHe data indicate that structures may have initiated by $134 \mathrm{Ma}$ (Eleogram, 2014), it is possible that significant topographic relief took longer to develop, allowing the sediments to get deposited in identical environments (Vandervoort, 1987; Vandervoort and Schmitt, 1990). Alternatively, evidence of distal cherts and Jurassic DZ age signatures within lower-, mid-, and upper levels of the early foreland basin (Figure 20), suggests that distally-derived sediment was successfully navigating around the WPM thrust sheet through extensive drainage systems perpendicular to the thrust axis, or within axial flow drainages. Paleocurrent directions within troughs of the Ephraim and Bechler formations (Figures 9 and 17 respectively) show dominant W-E directed flows, while the planar cross bedding data indicate variability within the paleocurrent directions, suggesting that the distal deposits can be arriving through either depositional.

Distinct populations of 150-175 Ma DZ grains combined, with identical lithofacies within both the northern and southern section of the foreland basin, represent a complex erosional history along the western margin. The Morrison Formation, which spans the Jurassic 
Kimmeridgian (157-152 Ma) - Tithonian (152-145.5 Ma) stages is stratigraphically equivalent to the Pruess Formation. The Morrison contains units that have been previously dated including a basal age of $154.8 \pm 0.5 \mathrm{Ma}$ for the Tidwell member (Kowallis, 1998), and a capping age of $\sim 148$ \pm 5 Ma within the Brushy Basin member (Kowallis 1991). The MDA of $150 \pm 2.8$ Ma for the Ephraim Formation within the northern section could be reflecting recycling of provenance material from the upper Pruess/Morrison formations. Alternatively, during the mid to late Jurassic, magmatism related to subduction of the Farallon plate resulted in emplacement of several plutons in the Nevada hinterland that were possible source units for DZ grains found within the early Sevier foreland basin (DeCelles et. al., 2009). Combined with evidence of distally-derived red and black chert pebbles found locally within specific lithologic units of the Ephraim and Bechler formations as well as stratigraphic equivalents in the Cedar Mountain Formation (Heller and Paola, 1989), DZ grains between 150-175 Ma are interpreted to have source areas within the Nevada hinterland, Sierra Nevada, Klamath Mountains and Mojave Desert (Armstrong and Ward, 1993; Wyld, 1996).

Furthermore, the absence of grains in the 140-117 Ma age range could be related to the Sierran magmatic lull that was prominent during this time period (DeCelles et. al., 2009). Several corollary studies that have attempted to identify DZ grains within regionally temporal equivalent deposits of the basal Ephraim Formation as well as slightly older deposits that fall within the magmatic lull, but have not been able to locate any such evidence (Dickinson and Gehrels, 2008; Druschke et. al., 2011; Johnston and Kylander-Clark, 2016).

In comparison of WPM U-Pb DZ age spectra with the adjacent foreland basin sediment DZ spectra, a progressive unroofing sequence is preserved (Table 2). The strongest correlation 
between the WPM thrust sheet and foreland strata include the Nugget Formation and the Ephraim (0.977), Bechler (0.877), Bear Valley (0.187) and Hilliard (0.353) formations. The Oquirrh Formation had the second largest influence of the Cokeville (0.365), Frontier (0.480), Upper Frontier (0.185), Aspen (0.551) and Hilliard (0.139) formations. The Swan Peak and Geertsen formations appear to have no influence on any of the foreland basin sediment. The Geertsen and Mutual formations show a distinct influence on sediment within the upper part of the foreland basin (Geertsen - Upper Frontier Formation (0.159); Mutual - Bear Valley (0.067), Frontier (0.198), Upper Frontier (0.076), and Aspen (0.712)). The Caddy Canyon Formation had minor influence on the Ephraim (0.001), Frontier (0.002), Upper Frontier (0.004), and Aspen (0.002) formations.

These data indicate a generalized unroofing pattern with DZ grains from the upper part of the thrust sheet becoming incorporated into basal sediment units of the foreland basin, as well as DZ grains from the mid to lower part of the WPM thrust sheet being incorporated into middle to upper units of the foreland basin. The absence of influence from the Worm Creek or Swan Peak formations may be due to the discontinuous nature of preserved early foreland basin deposits. The sedimentary units that may have contained signatures may simply have been eroded away. A fault propagation fold related to initiation of thrusting of the WPM could have also influenced the signatures, with the production of topographic relief capable of diverting any existing drainage system. Units within the thrust sheet such as the Wells/Weber Formation, and subsequent carbonate units lack a rich DZ signature, which would lead to an apparent lack of DZ influence within the foreland strata. Furthermore, the variation in DZ signatures may be related to out of sequence thrusting of the WPM, in which varying levels of 
the thrust sheet provide a complex combination of DZ signatures that are not sequentially recorded in one location within the foreland.

The reappearance of DZ grains with a Nugget Formation age pattern has implications for timing of deformation of the Paris and Meade thrust sheets. Shortening within the northern part of the WPM thrust system began with early deformation of the Paris thrust sheet, followed by movement of the Meade sheet that incorporated and passively transported the Paris sheet (DeCelles et. al., 1993). The topmost Nugget Formation experienced erosion as the Paris sheet was uplifted, and shed DZ grains with a unique age pattern into the adjacent foreland basin. As deeper levels of the thrust sheet were breached by erosion, the DZ pattern changed to reflect the exposed stratigraphic level. Table 2 shows that this initial pulse of Nugget erosion was preserved within the foreland sediment with $\mathrm{P}$ values in the Ephraim, Bechler and Bear Valley formations all above $95 \%$ certainties. The Hilliard Formation also experienced a strong influence from the Nugget Formation, with a P value of 0.353 . The presence of this pattern within the upper stratigraphy of the foreland basin suggests that secondary movement of the Meade thrust sheet reintroduced an erosional surface containing the Nugget Formation. Since uplift and erosion of the Nugget is synorogenic with deposition of the Hilliard based on the P value, then the MDA $(94.1 \pm 2.3 \mathrm{Ma}(\mathrm{n}=4))$ from DZ grains within the Hilliard represents a maximum age of deformation related to uplift of the Meade thrust sheet.

In addition, MDA's found from within stratigraphy of the foreland basin demonstrate a well-defined temporal depositional progression. Stratigraphy within the southern transect represent Albian to Cenomanian foreland basin deposits and yield the following MDAs: Upper 
Gannett, $109 \pm 6.1 \mathrm{Ma}$ and 108.3 $\pm 3.4 \mathrm{Ma}$; Cokeville, 101.6 \pm 0.7 Ma; Lower Sage Junction 101.55 \pm 0.5 Ma; Upper Sage Junction, 101.3 \pm 0.6 Ma; Aspen, $98.8 \pm 0.4 \mathrm{Ma}$; lower Frontier, $99.93 \pm 0.6 \mathrm{Ma}$; and upper Frontier, $95.68 \pm 1.4 \mathrm{Ma}$. 


\subsection{Future Work}

To gain a better understanding of the kinematic evolution of the WPM thrust sheet, thermochronologic studies within the Paris and Meade sheets should be performed to identify initiation of cooling, differences in exhumation rates between each sheet and within each sheet. These studies should then be compared to the now resolved exhumation history of the Willard thrust sheet. Vertical transects like those taken in Eleogram, 2014 should be identified based on robust stratigraphic sections as well as take advantage of previously existing data.

In addition, geochemistry of collected DZ grains should be investigated to understand where the young volcanic grains providing maximum depositional ages were sourced from. This would not only help identify Early Cretaceous volcanic centers effecting the western North American Cordillera, but may also provide insight into plate boundary subduction conditions and a different proxy for magmatic flux that may highlight volcanic over plutonic activity, providing further insight into possible driving mechanisms behind crustal deformation. 
Figure Captions

Figure 1. Regional map of the Sevier fold-thrust belt, with Willard-Paris-Meade, Crawford, Absaroka and Hogsback thrust sheets outlined (modified from Yonkee and Weil, 2011). Red stars shows sample locations. Yellow bars show locations of previously collected thermochronologic data (Eleogram, 2014)

Figure 2. Synorogenic sedimentary record of basins adjacent to the Sevier orogenic belt with depositional equivalents along a stratigraphic section (Yonkee and Weil 2011)

Figure 3. Proterozoic source terranes with DZ patterns representing age of formation. These DZ patterns are then used in provenance studies to determine features such as paleocurrent directions and paleotopography (Yonkee et. al., 2014).

Figure 4. ZHe age vs. paleodepth from samples collected along Wellsville transect of Willard thrust sheet. Large red circles are sample average ZHe ages, small circles are ZHe ages for individual grains. Grey lines are exhumation curves generated from HeMP modeling software.(Eleogram, 2014).

Figure 5. Foreland basin stratigraphy which includes the Gannett Group, which is composed of the Ephraim, Peterson, Bechler and Draney formations); Smiths Formation; Thomas Fork Formation; Bear River Formation which is laterally equivalent with the Cokeville and Sage Junction formations; Frontier Formation; Aspen Formation and Hilliard Formation.

Figure 6. Schematic map with localities. Northern section includes Red Mountain and Salt River Pass localities, and southern section includes Sawtooth Mountain locality.

Figure 7. Correlation of regional stratigraphic sections. (Modified from Eyer, 1969 and DeCelles, 1994). 
Figure 8. Bedding present within the Ephraim Formation. Planar bedding and planar cross bedding are present in this figure.

Figure 9. Paleocurrent data for the Ephraim Formation. Trough cross bedding are shown at Sawtooth Mountain and Salt River Pass respectively. Planar cross bedding are shown at Sawtooth Mountain and Salt River Pass respectively.

Figure 10. A. Ternary QFL plot showing modal assemblages of quartz, feldspar and lithics within the Ephraim Formation. Base plot includes data from Furer, 1970. Red stars represent counts within the Bechler Formation. Blue starts represent counts within the Ephraim Formation. Chert is included within lithic fragments. B. Photomicrograph of the Ephraim Formation demonstrating high percentage of quartz grains cemented with either calcite or chert.

Figure 11. Crevasse channel deposit within the Ephraim Formation.

Figure 12. Architectural elemental analysis, road cut, Bechler Formation.

Figure 13. Representative measured section of the Bechler Formation at Salt River Pass locality. Figure 14. Example of Bechler Formation major conglomerate lithofacies. Note size of boulder clast within outcrop.

Figure 15. Paleocurrent direction measurements of imbricated clasts of the Bechler Formation at Red Mountain (DeCelles et al., 1993).

Figure 16. QFL diagram showing modal percentages of quartz, feldspar and lithic grains within the Bechler Formation Ternary QFL plot showing modal assemblages of quartz, feldspar and lithics within the Ephraim Formation. Base plot includes data from Furer. 
Figure 17. Paleocurrent directions measured within the Bechler Formation. Trough cross bedding measured at Salt River Pass and Red Mountain respectively. Planar cross bedding measured at Salt River Pass, Red Mountain and Ham's Fork (near Sawtooth Mountain.

Figure 18. Stratigraphic relations between foreland basin units of the WPM thrust sheet. Note the stratigraphic relations between the western units and eastern units (modified from Yonkee and Weil, 2015).

Figure 19. Age of apparent intrusive influx and whole rock age of Sierra Nevada (modified from DeCelles et. al., 2009).

Figure 20. MDA's for stratigraphic units within the foreland basin. A) MDAs plotted against the stratigraphic column. B) 95\% confidence plots showing coherent groups within each collection of MDAs for each sample.

Figure 21. Stratigraphy of the Willard thrust sheet with unique DZ age patterns plotted in stratigraphic order. Wyoming Province (WY); Grouse Greek Block (GC); Farmington ZoneMojave province and Selway terrane (F-Mo-Se); 2.45 GA grains from Fe-rich granitic pluton (asterisk); Yovapai and Mazatzal provinces (Y-M); mid-continent granite (MG); Grenville-Llano province (GR) (modified from Yonkee and Weil, 2015).

Figure 22. DZ age patterns found within specific foreland basin units.

Figure 23. Column shows source rock types in WPM thrust sheet. Distinctive DZ age-probability density distributions of different source units are shown on the right. Modified from (9), with DZ data from (8) and (3). B) DZ age-probability density distributions of samples of synorogenic strata; formation name and number of analyzed grains for each sample listed. 


\section{Figures}

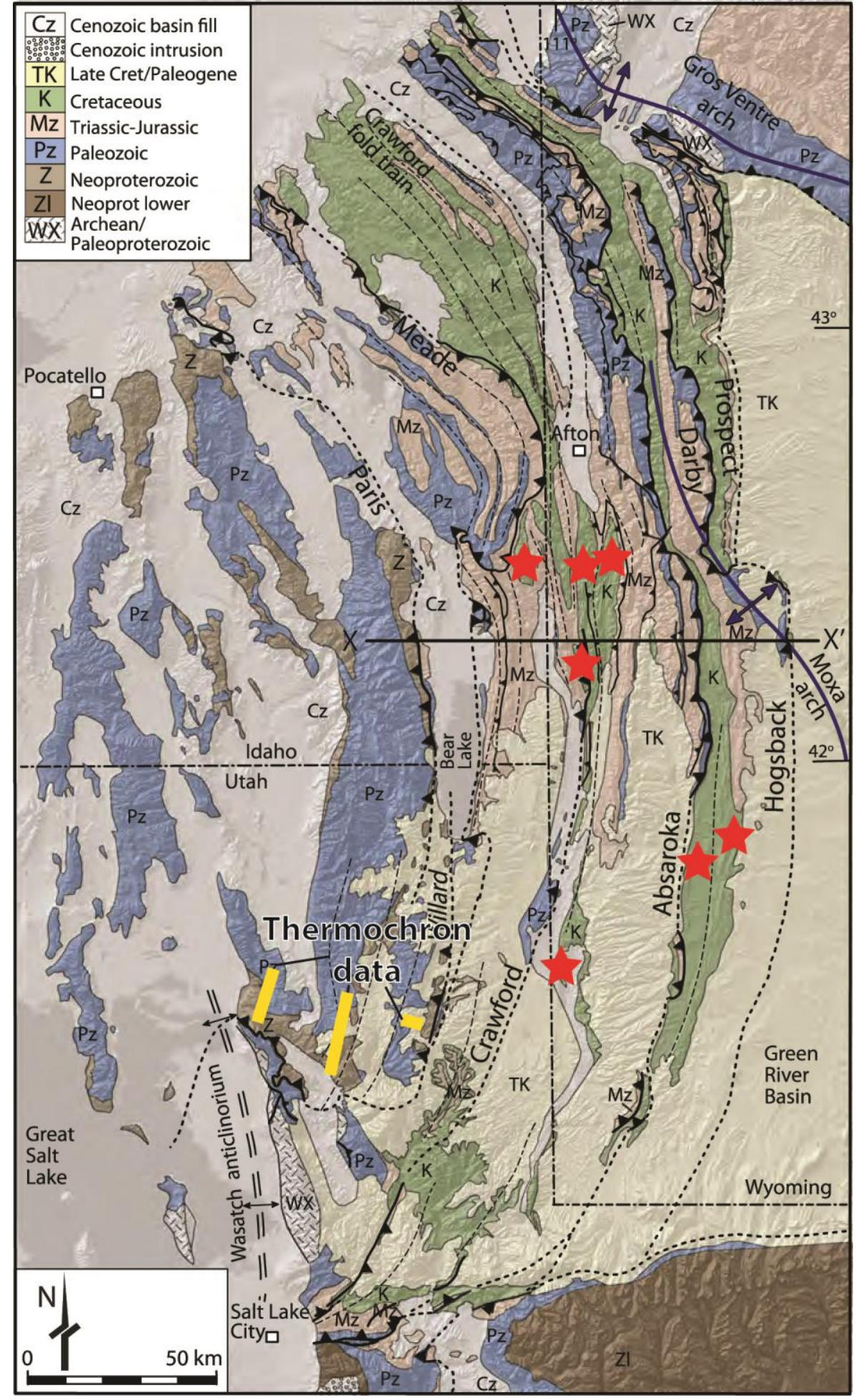

Figure 1. Regional map of the Sevier fold-thrust belt 


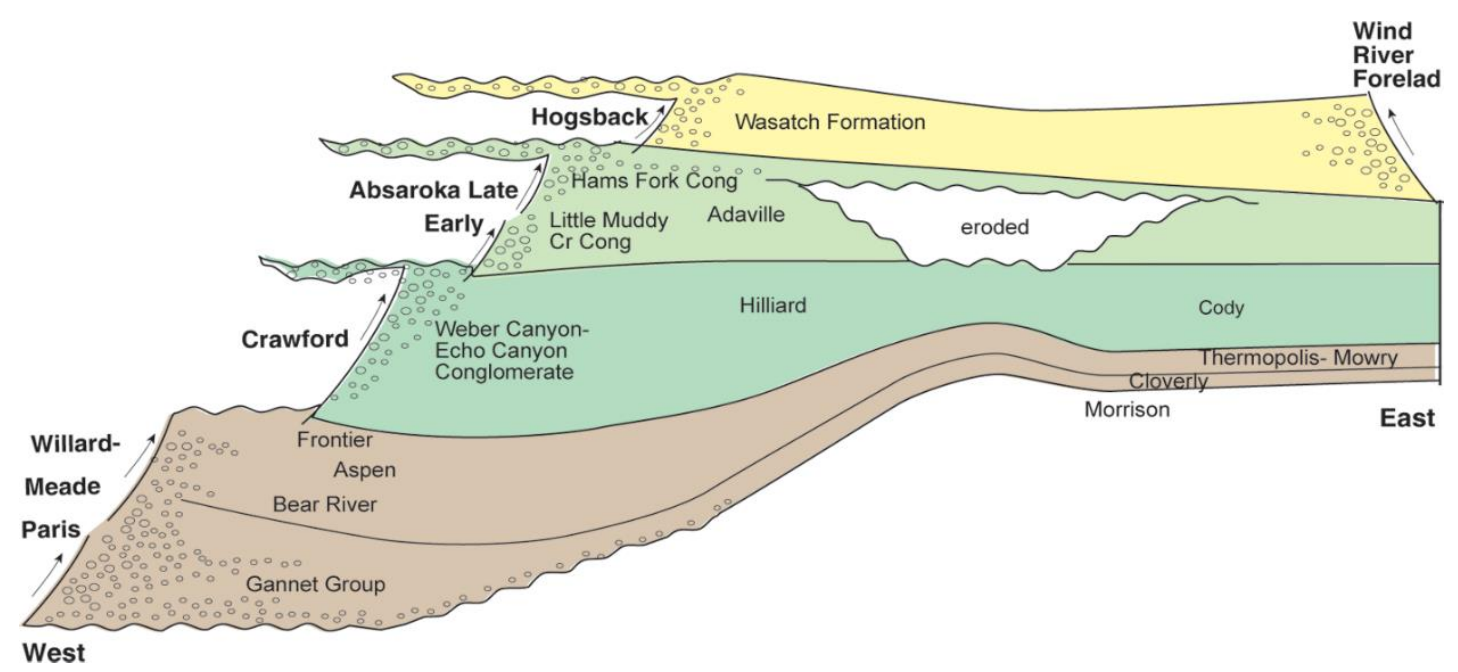

Figure 2. Synorogenic sedimentary record of basins adjacent to the Sevier orogenic belt. 


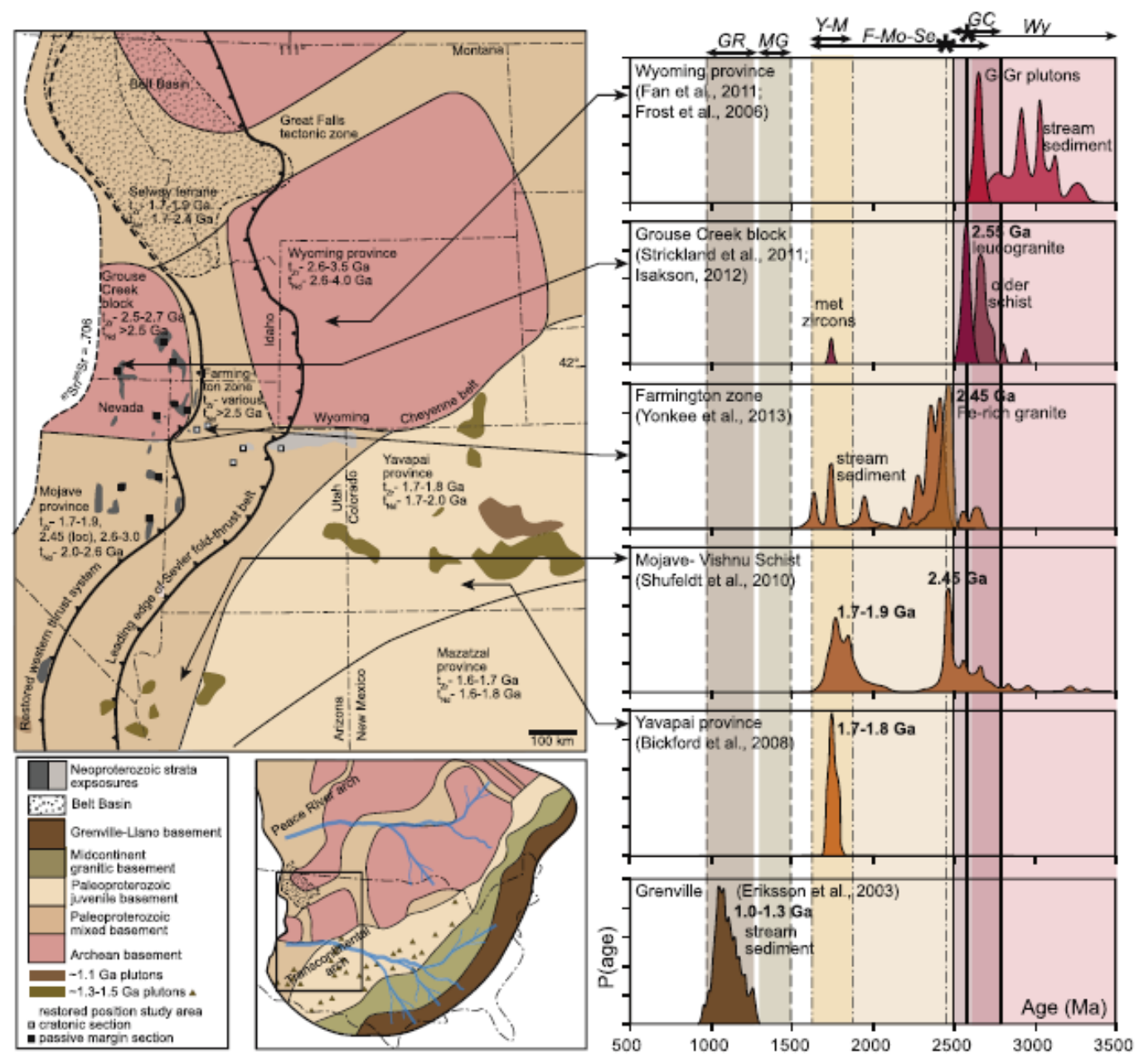

Figure 3. Proterozoic source terranes with corresponding DZ patterns. 


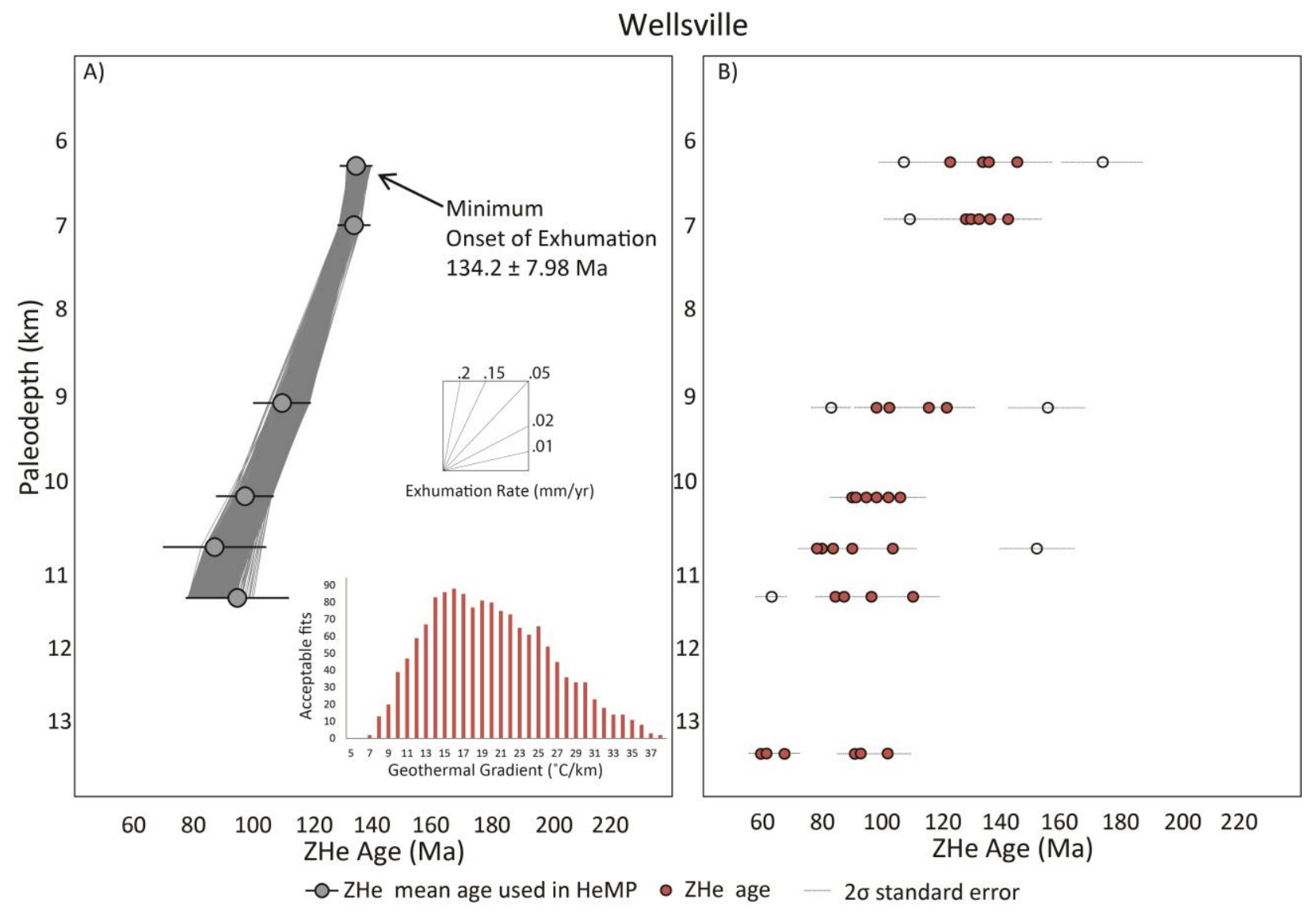

Figure 4. ZHe age vs. paleodepth from samples collected along Wellsville transect of Willard thrust sheet 


\section{Foreland Basin Stratigraphy}

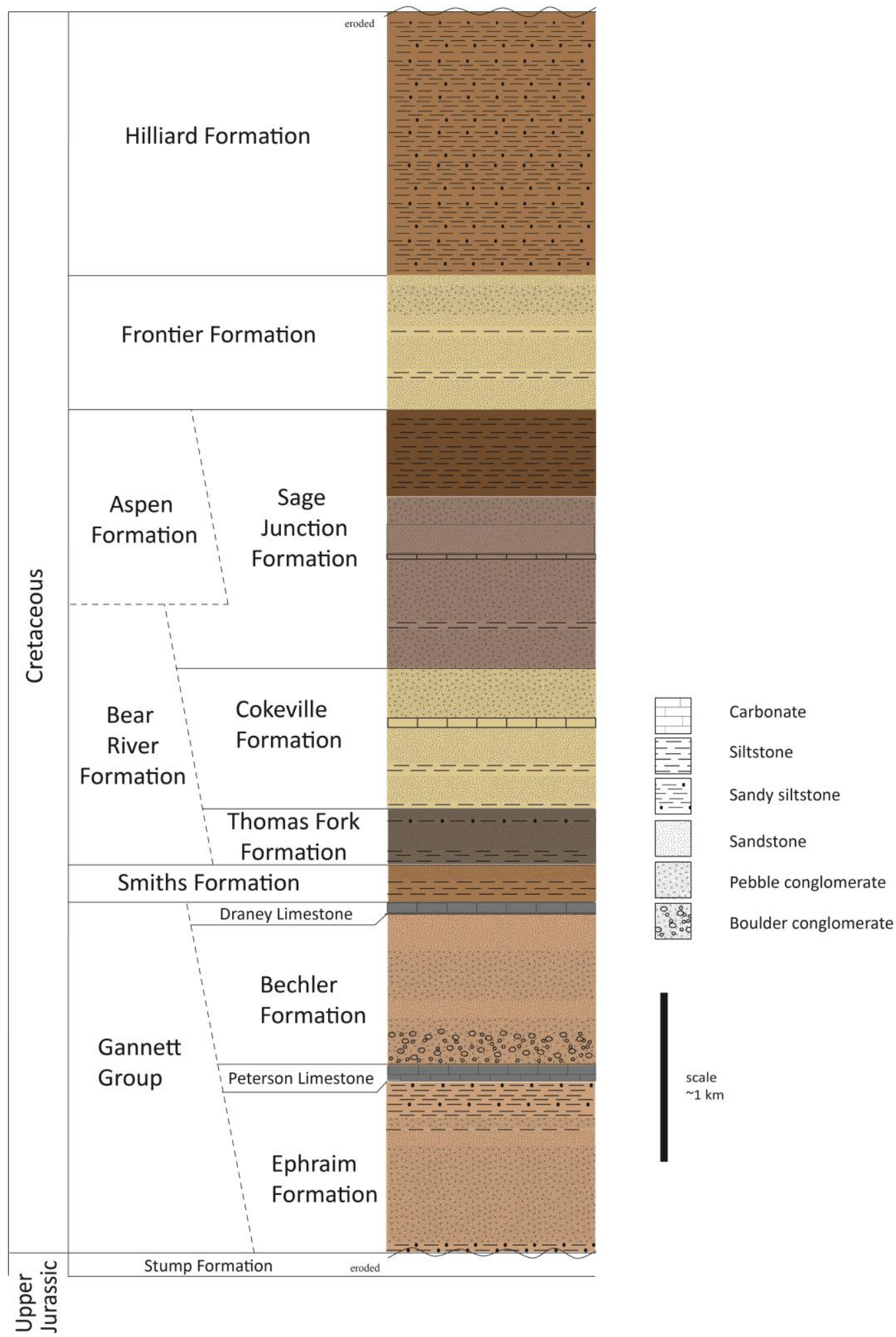

Figure 5. Foreland basin stratigraphy 


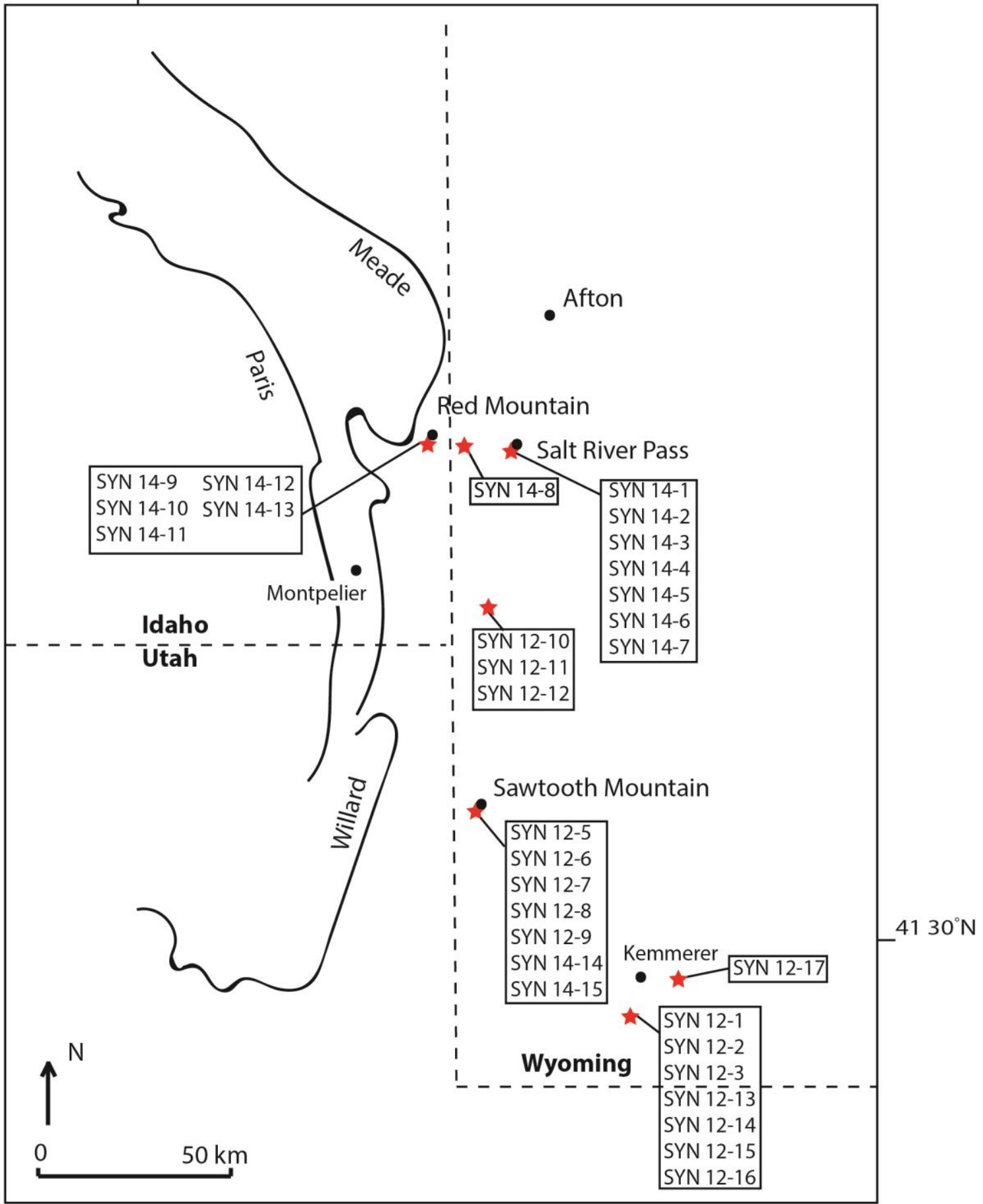

Figure 6. Schematic map of field area with localities 


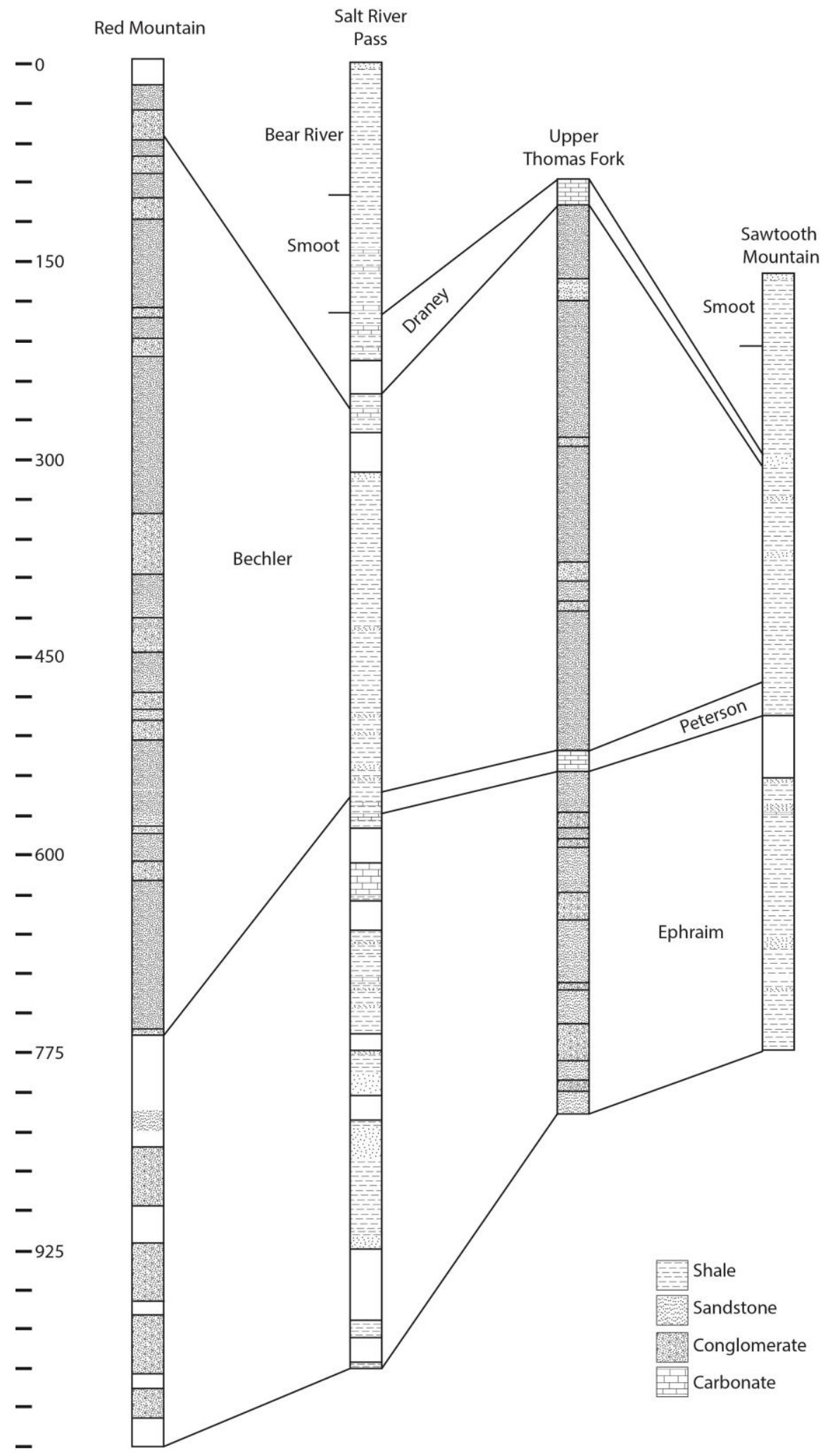

Figure 7. Correlation of regional stratigraphic sections 


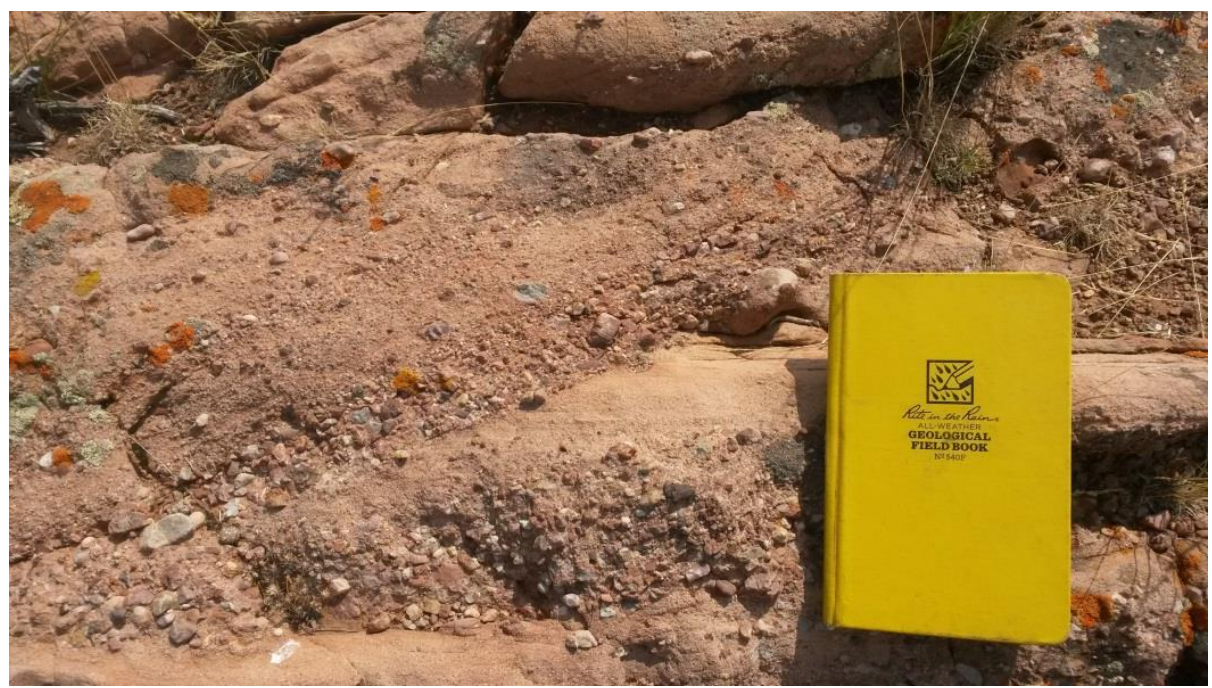

Figure 8. Bedding present within the Ephraim Formation 


\section{Ephraim Paleocurrent Directions}

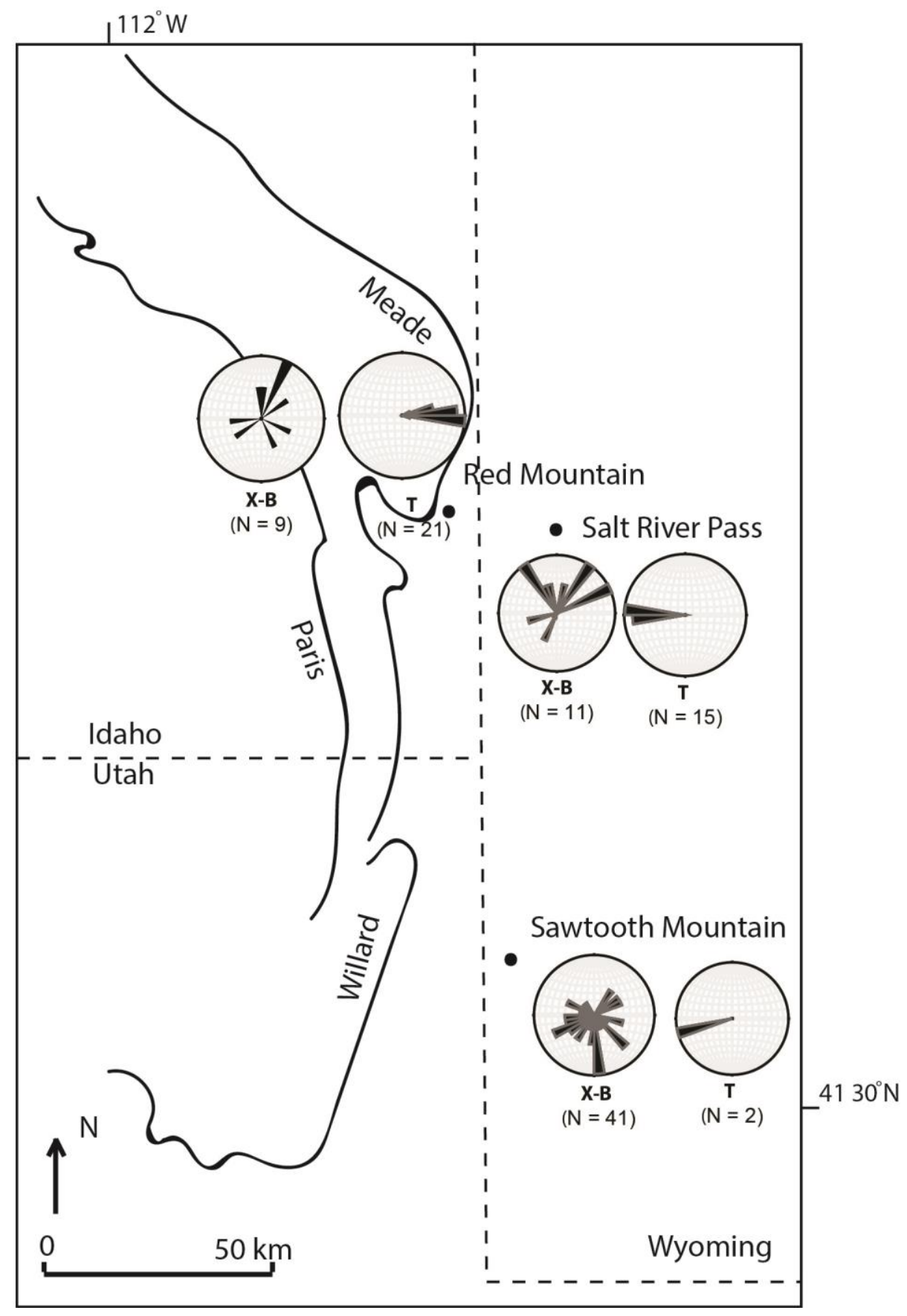

Figure 9. Paleocurrent data for the Ephraim Formation 
A)

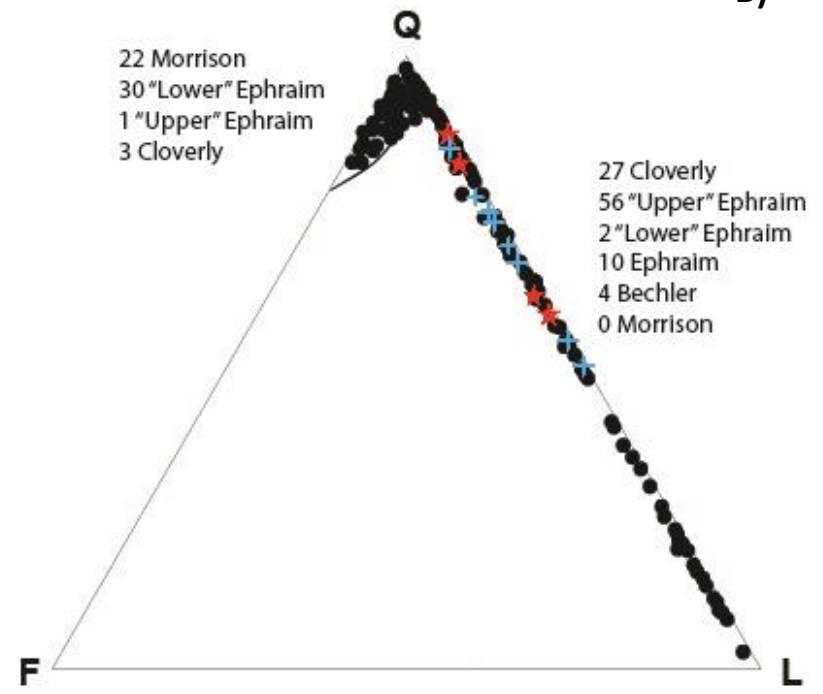

B)

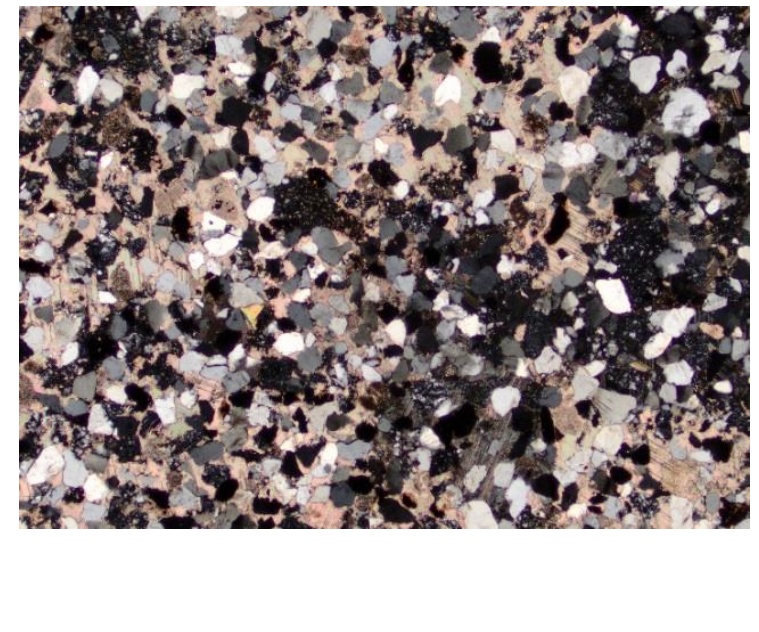

Figure 10. QFL plot and photomicrograph of the Ephraim Formation 


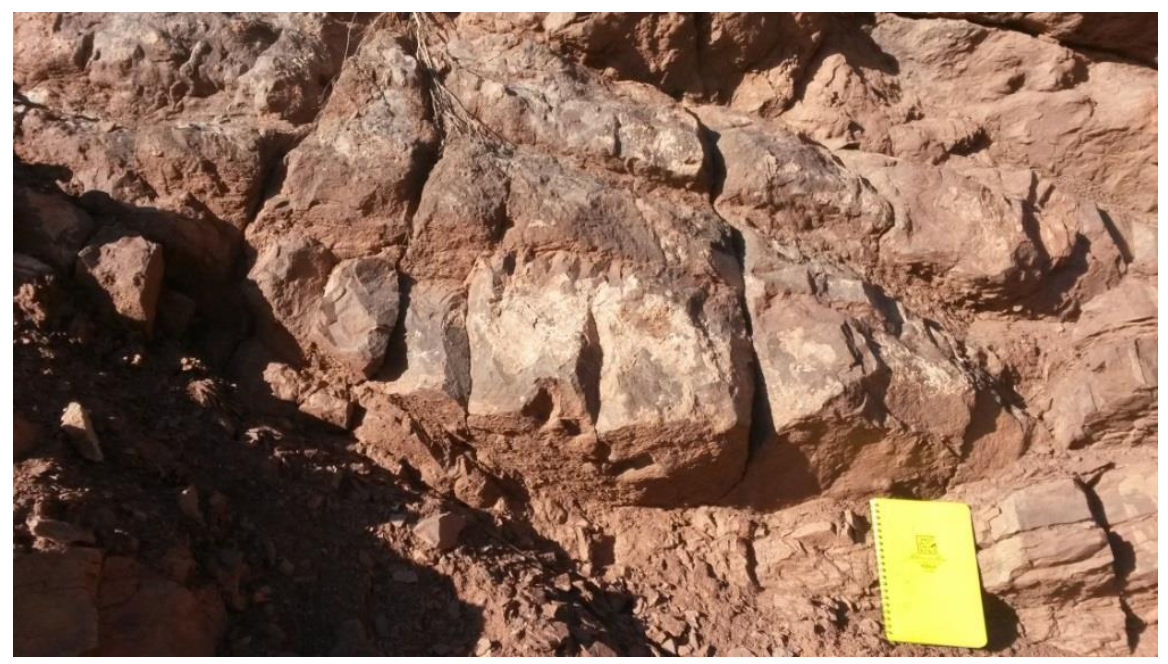

Figure 11. Crevasse channel deposit within the Ephraim Formation 


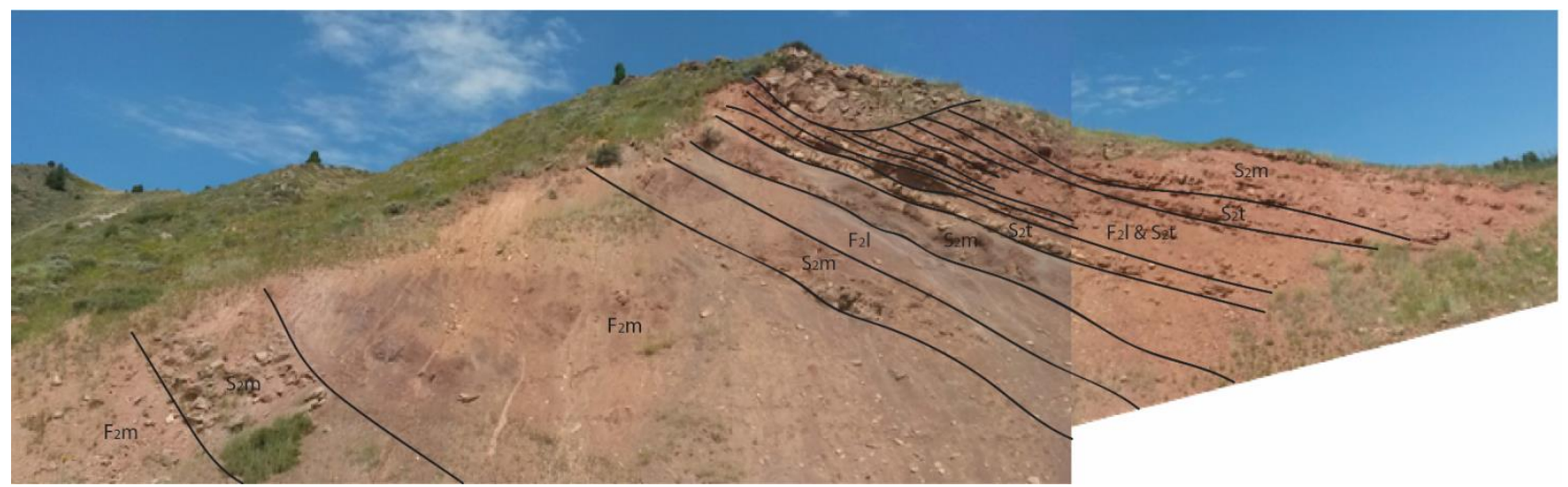

Figure 12. Architectural elemental analysis of Bechler Formation 


\section{Bechler Formation Road Cut}

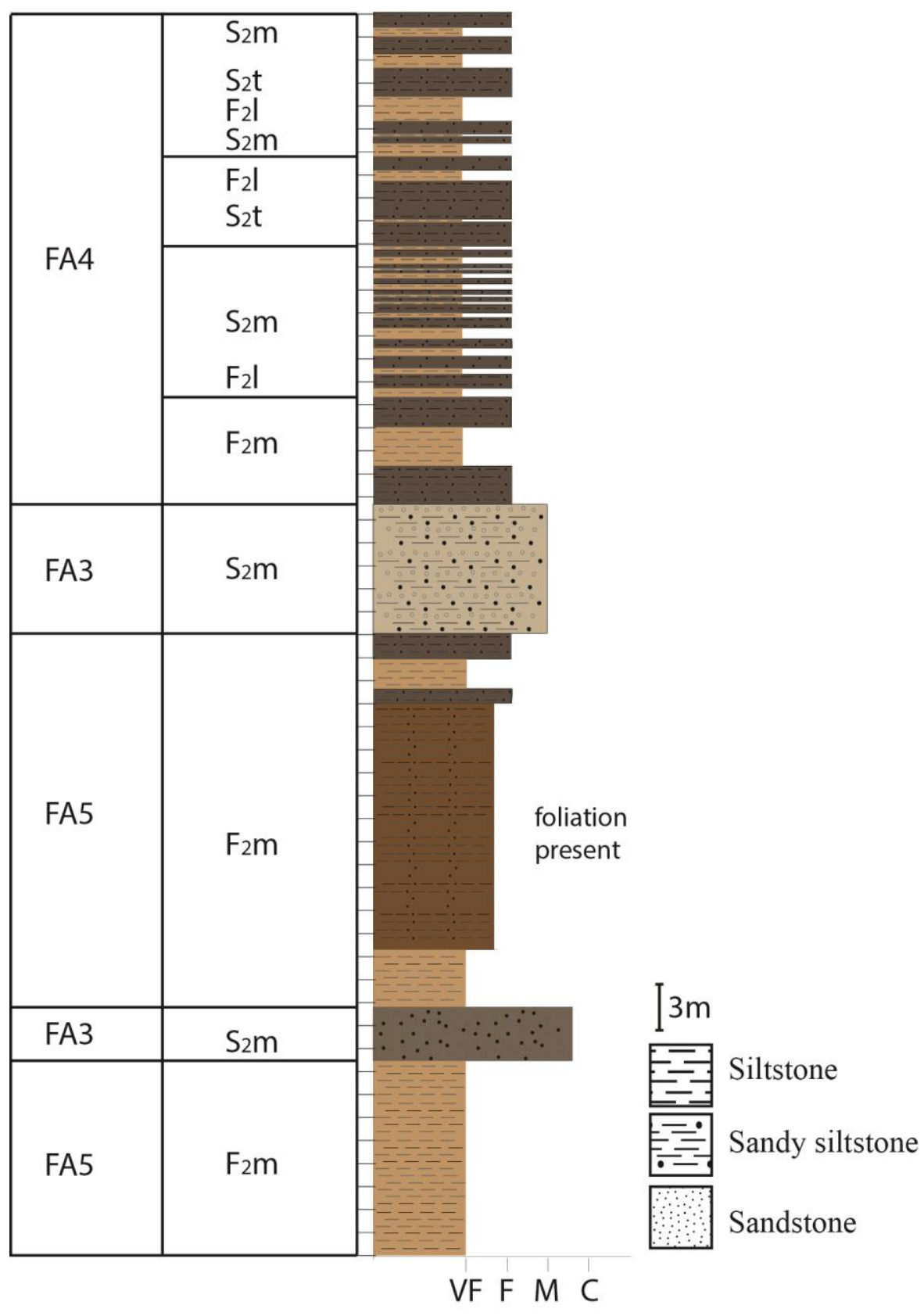

Figure 13. Representative measured section of the Bechler Formation at Salt River Pass locality 


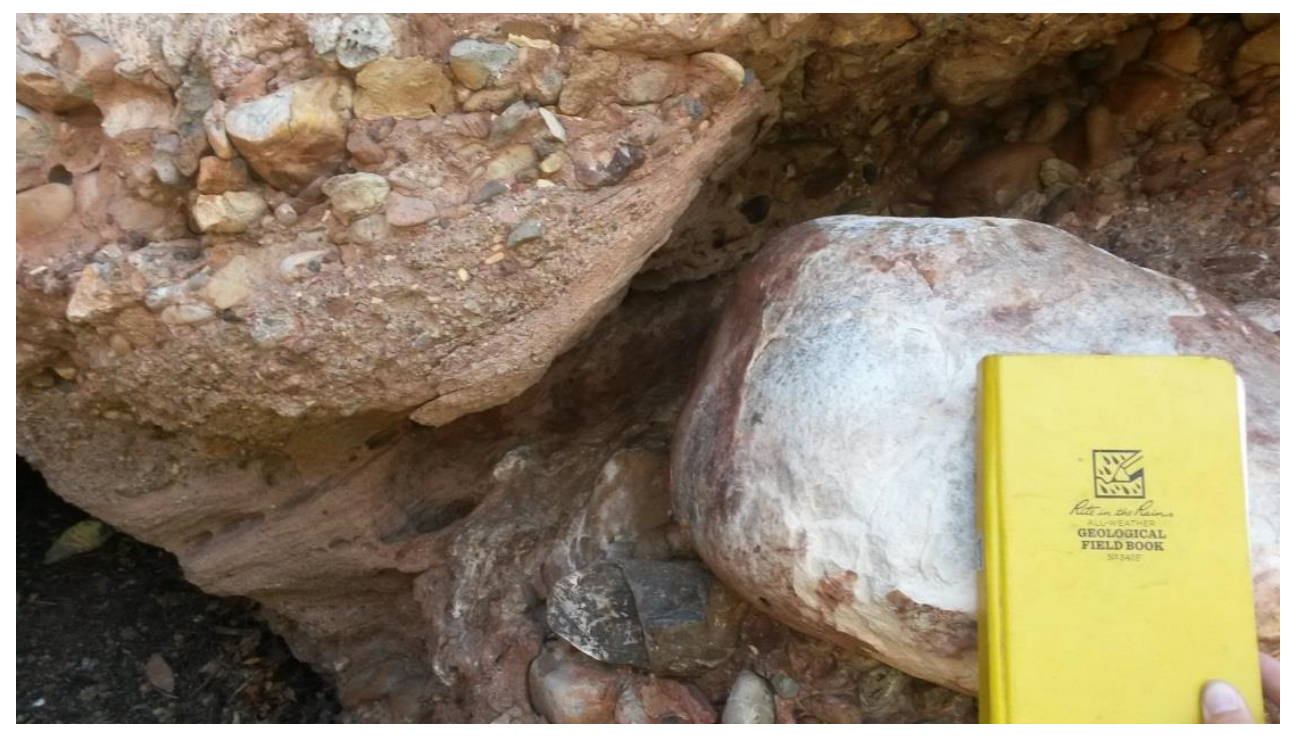

Figure 14. Example of Bechler Formation major conglomerate lithofacies 


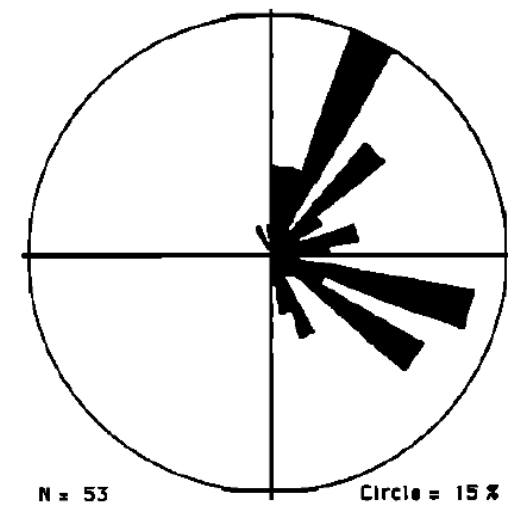

Figure 15. Paleocurrent direction measurements of imbricated clasts of the Bechler Formation at Red Mountain 


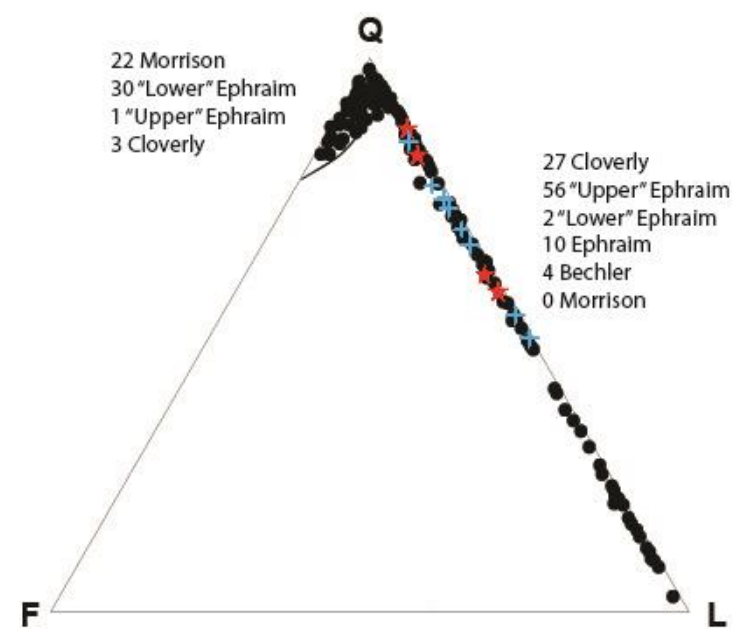

Figure 16. QFL plot of the Bechler Formation 


\section{Bechler Paleocurrent Directions}

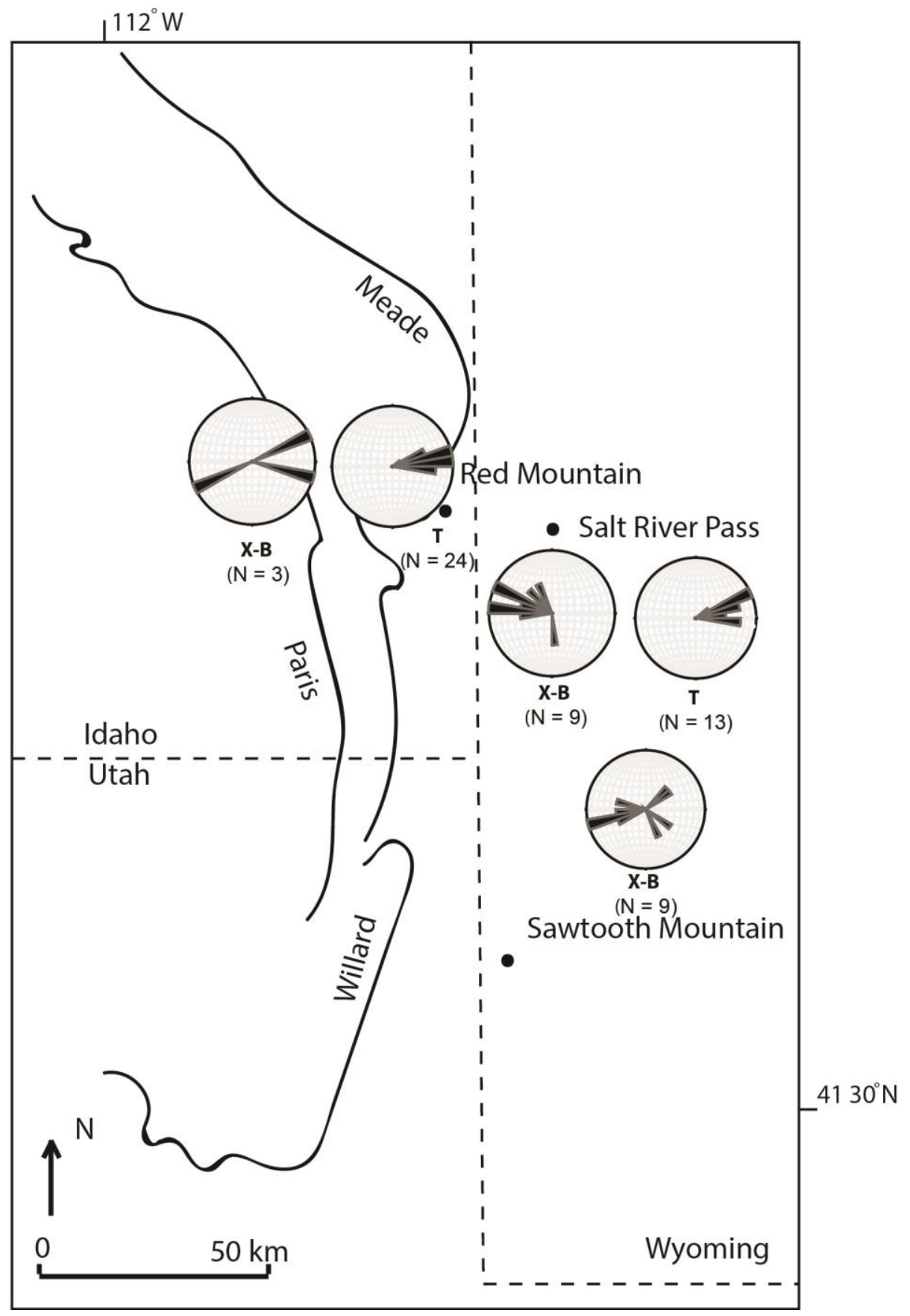

Figure 17. Paleocurrent directions measured within the Bechler Formation 


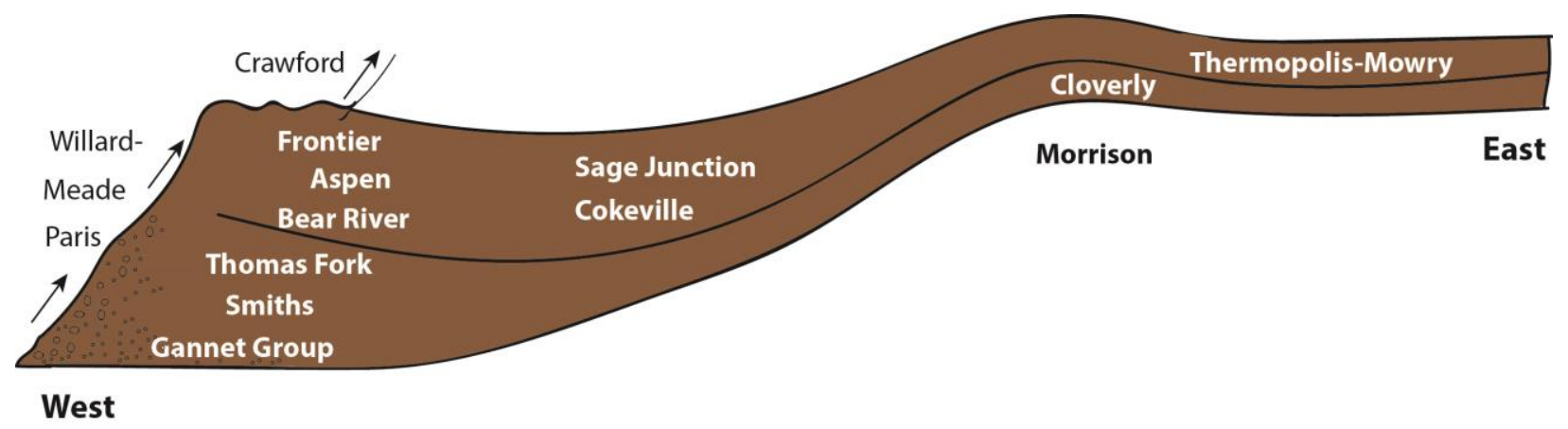

Figure 18. Stratigraphic relations between foreland basin units of the WPM thrust sheet 


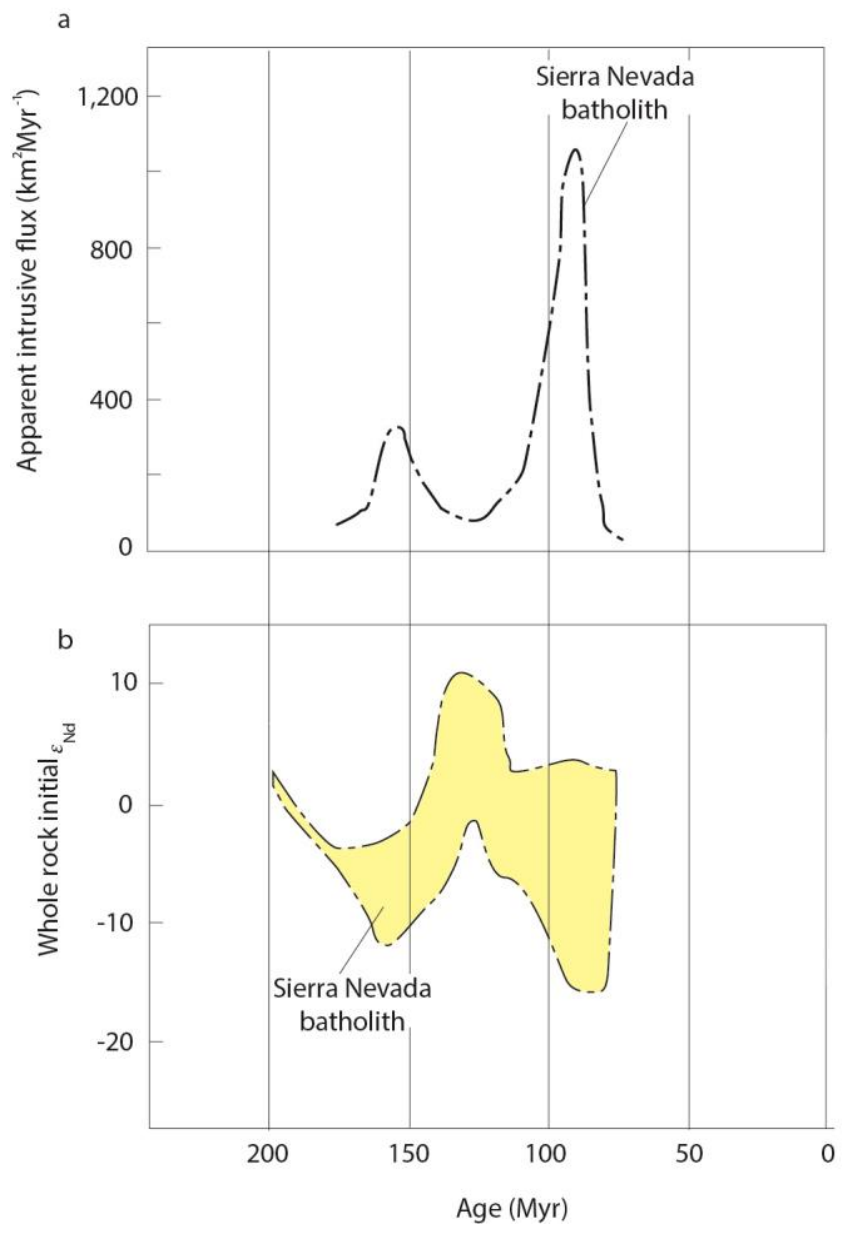

Figure 19. Age of apparent intrusive influx and whole rock age of Sierra Nevada 
A)

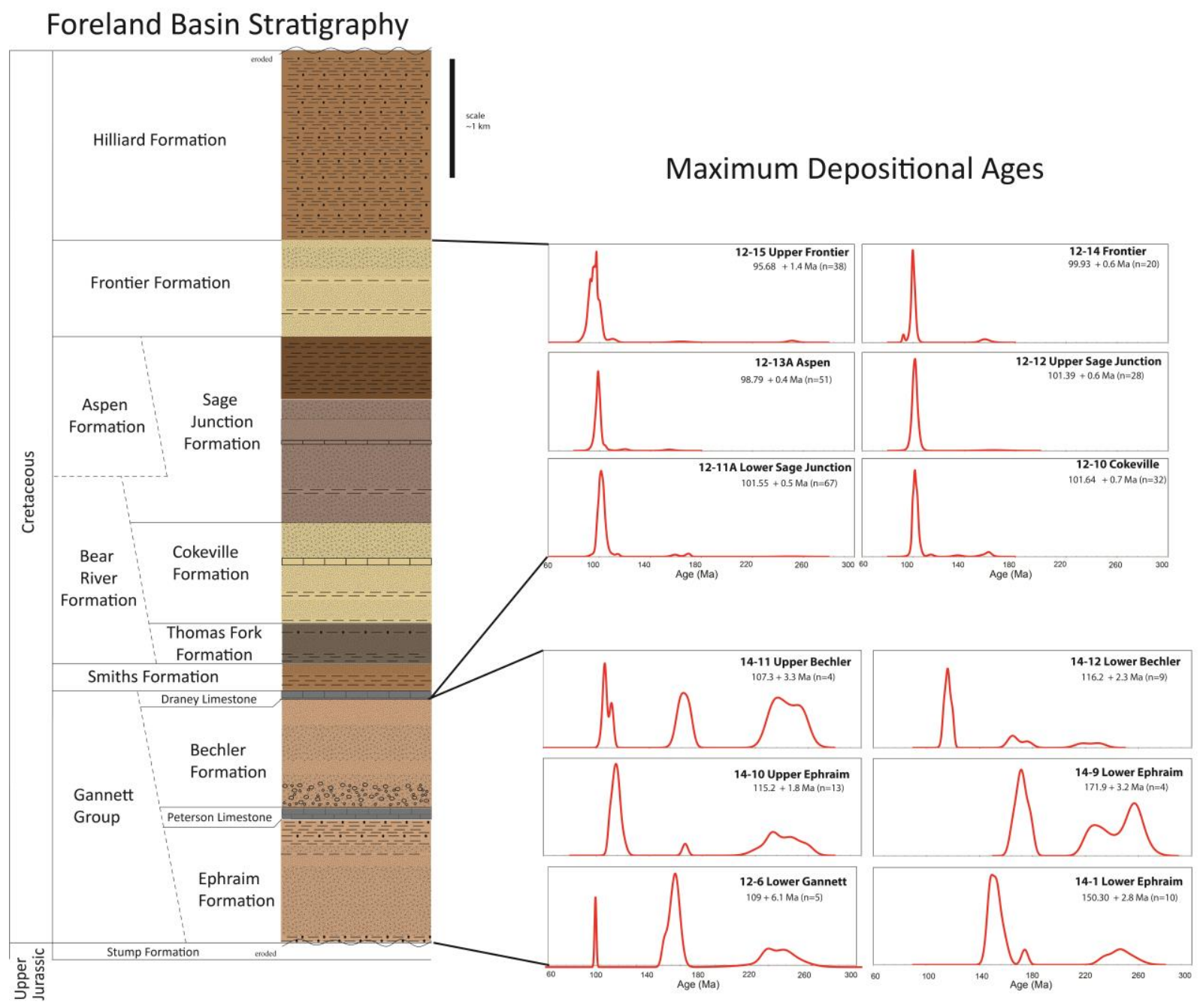

Figure 20. MDA's for stratigraphic units within the foreland basin 
B)
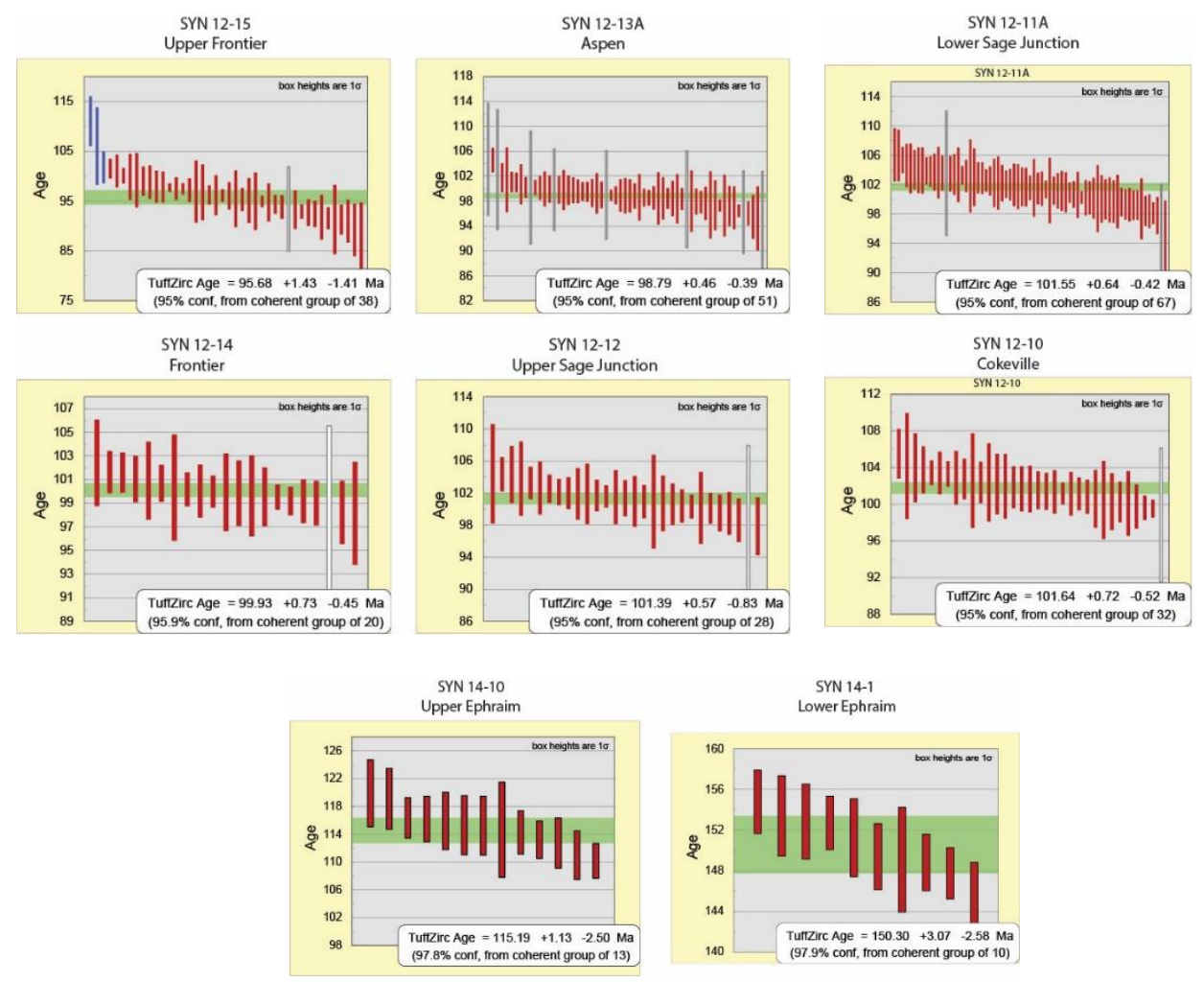

Figure 20. MDA's for stratigraphic units within the foreland basin 
Willard Thrust Sheet

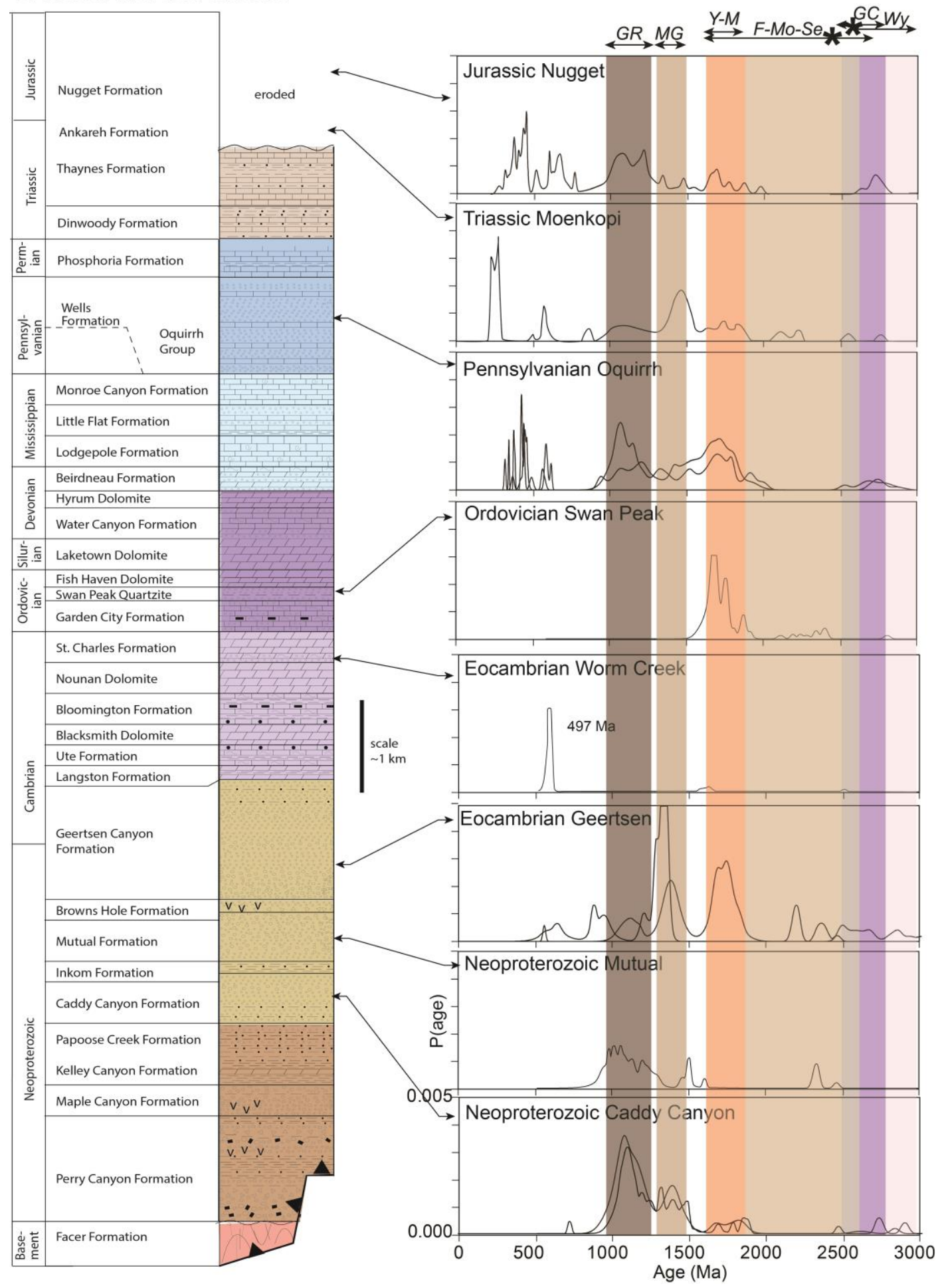

Figure 21. Stratigraphy of the Willard thrust sheet with unique DZ age patterns 


\section{Foreland Basin Stratigraphy}

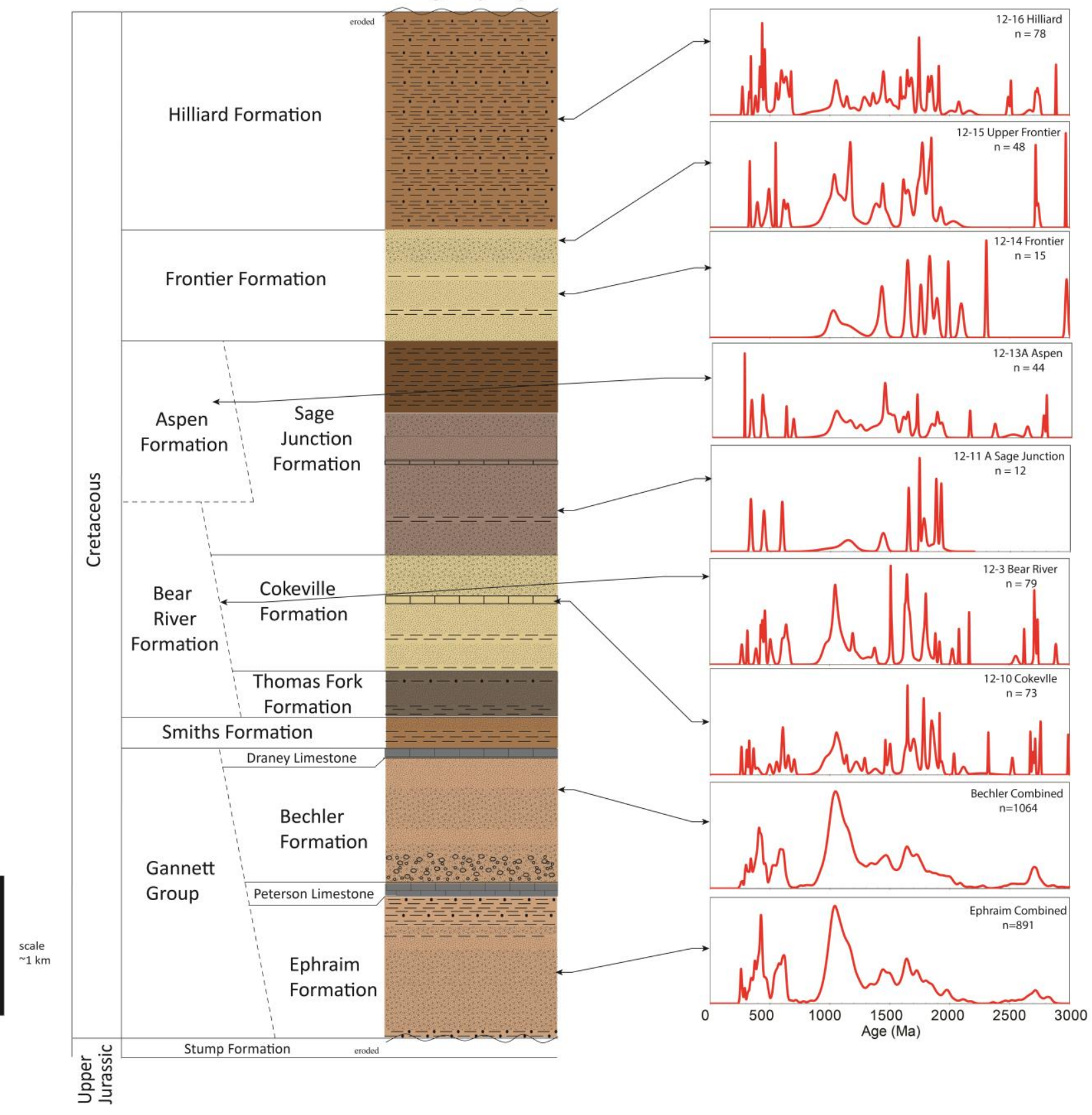

Figure 22. DZ age patterns found within specific foreland basin units 


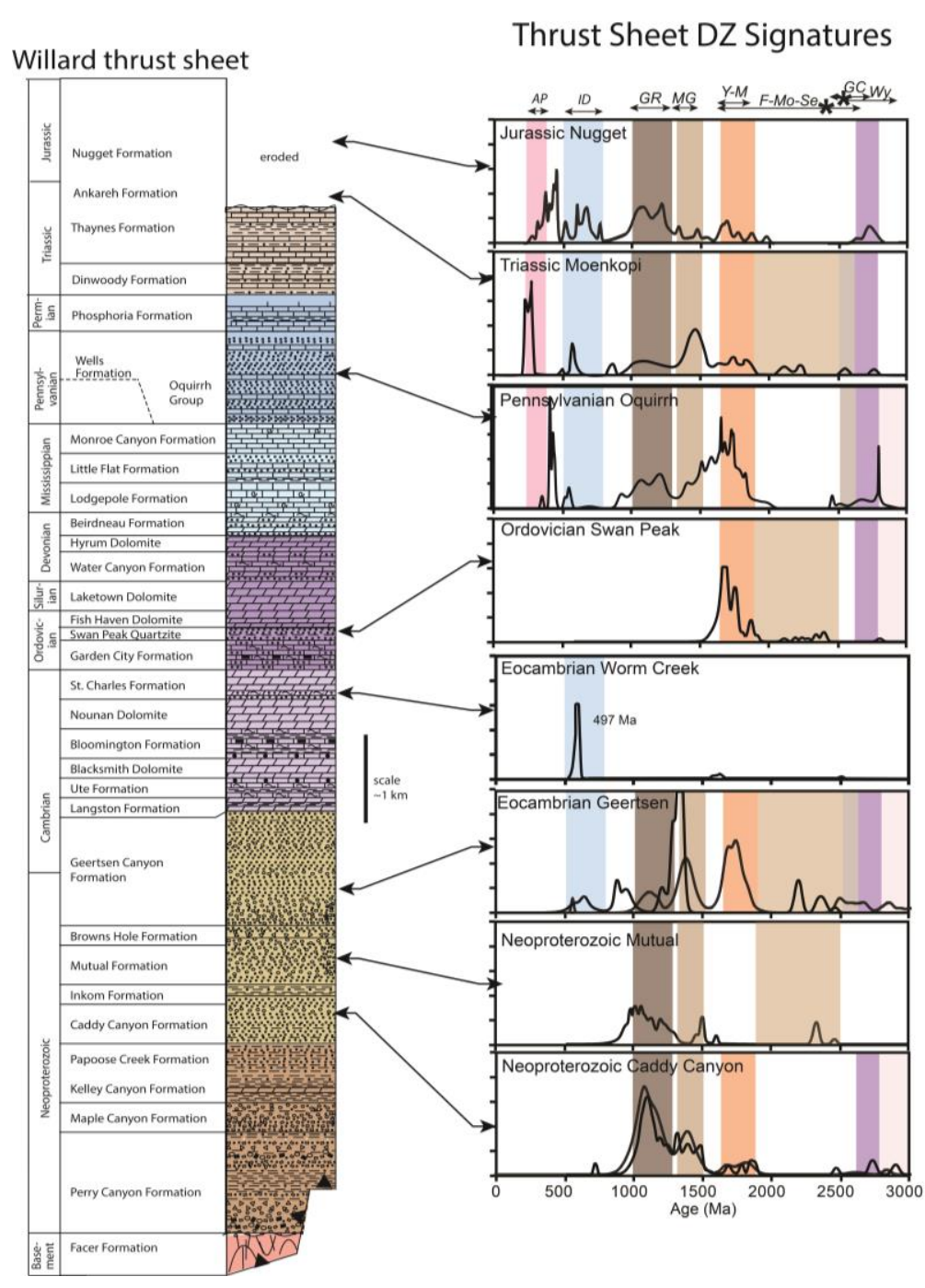

Foreland Basin DZ Signatures

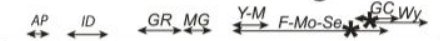
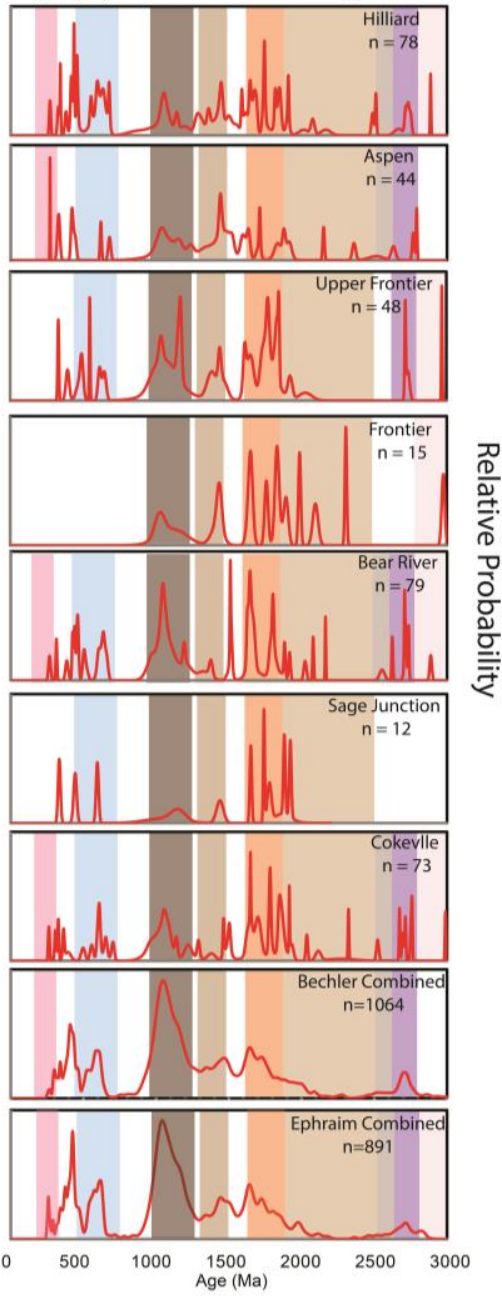

Figure 23. DZ patterns from the WPM thrust sheet compared with DZ patterns from within the foreland basin strata 
Table 1. Lithofacies and Facies Associations of Ephraim and Bechler formations Lithofacies in the Ephraim Formation

\begin{tabular}{|c|c|c|c|c|c|}
\hline Code & Lithofacies & Texture & $\begin{array}{l}\text { Structures and } \\
\text { features }\end{array}$ & Color & Interpretation \\
\hline $\mathrm{G}_{1} \mathrm{~mm}$ & $\begin{array}{l}\text { Matrix- } \\
\text { supported } \\
\text { conglomerate }\end{array}$ & $\begin{array}{l}\text { Matrix: poorly sorted } \\
\text { medium to coarse sand. } \\
\text { Clasts: pebble-sized, poorly } \\
\text { sorted, subrounded to } \\
\text { rounded, dominated by } \\
\text { extraformational red chert, } \\
\text { but also include light grey to } \\
\text { grey carbonate, and minor } \\
\text { amounts of tan sandstone, } \\
\text { white and grey quartzite }\end{array}$ & $\begin{array}{l}\text { Typically massive, 1- } \\
2 \text { m scale bedding } \\
\text { where present, clast } \\
\text { orientation within } \\
\text { proximal depositsto } \\
\text { the thrust front, no } \\
\text { grading to weak } \\
\text { normal grading }\end{array}$ & $\begin{array}{l}\text { Reddish } \\
\text { brown, } \\
\text { reddish } \\
\text { tan }\end{array}$ & Debris flows \\
\hline $\mathrm{S}_{1} \mathrm{p}$ & $\begin{array}{l}\text { Planar cross- } \\
\text { bedded } \\
\text { sandstone }\end{array}$ & $\begin{array}{l}\text { Fine- to medium-grained } \\
\text { sand, moderately well sorted }\end{array}$ & $\begin{array}{l}\text { Planar tabular } \\
\text { crossbeds; Coset } \\
\text { thickness: ranges } \\
\text { from } 0.1 \text { to } 0.4\end{array}$ & $\begin{array}{l}\text { Red to } \\
\text { reddish } \\
\tan \end{array}$ & $\begin{array}{l}\text { Lower flow } \\
\text { regime 2D } \\
\text { dunes within } \\
\text { channels }\end{array}$ \\
\hline $\mathrm{S}_{1} \mathrm{t}$ & $\begin{array}{l}\text { Trough cross- } \\
\text { bedded } \\
\text { sandstone }\end{array}$ & $\begin{array}{l}\text { Fine- to medium-grained } \\
\text { sand, moderately well sorted }\end{array}$ & $\begin{array}{l}\text { Trough crossbeds: } \\
\text { Coset thickness } 0.1 \\
\text { to } 1.5 \mathrm{~m}\end{array}$ & & $\begin{array}{l}\text { Lower flow } \\
\text { regime 3D } \\
\text { dunes within } \\
\text { channels }\end{array}$ \\
\hline $\mathrm{S}_{1} \mathrm{~m}$ & $\begin{array}{l}\text { Massive } \\
\text { sandstone }\end{array}$ & $\begin{array}{l}\text { Fine- to medium-grained } \\
\text { sand, moderately well sorted }\end{array}$ & Massive & $\begin{array}{l}\text { Reddish } \\
\text { brown, } \\
\text { reddish } \\
\text { tan }\end{array}$ & $\begin{array}{l}\text { Rapid } \\
\text { sedimentation }\end{array}$ \\
\hline $\mathrm{F}_{1} \mathrm{I}$ & $\begin{array}{l}\text { Finely } \\
\text { laminated } \\
\text { siltstone and } \\
\text { mudstone }\end{array}$ & Very fine grained silt and muc & Very fine laminations & $\begin{array}{l}\text { Dark } \\
\text { reddish } \\
\text { brown, } \\
\text { s pale red } \\
\text { purple, } \\
\text { brown }\end{array}$ & $\begin{array}{l}\text { Suspension } \\
\text { load deposits }\end{array}$ \\
\hline
\end{tabular}




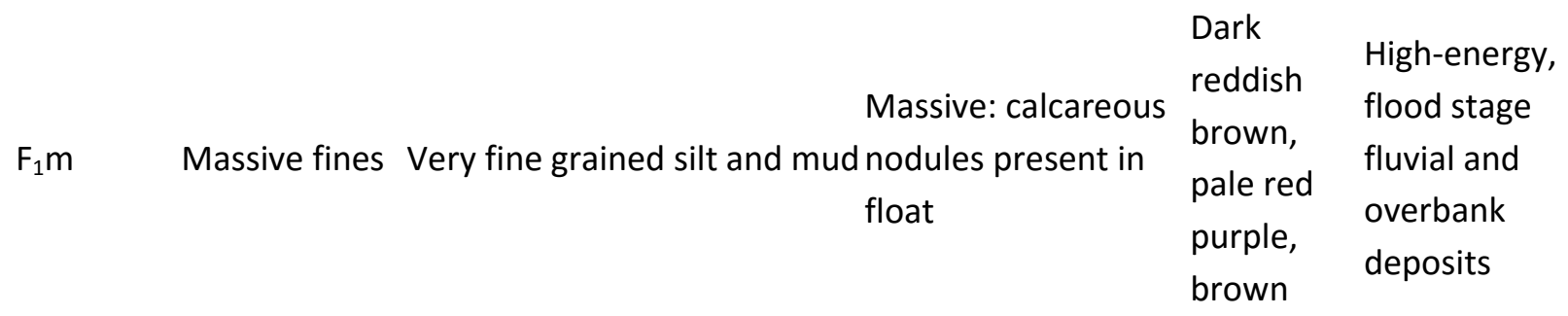

Lithofacies in the Bechler Formation

\begin{tabular}{|c|c|c|c|c|c|}
\hline Code & Lithofacies & Texture & $\begin{array}{l}\text { Structures and } \\
\text { features }\end{array}$ & Color & Interpretation \\
\hline $\mathrm{G}_{2} \mathrm{~mm}$ & $\begin{array}{l}\text { Matrix- } \\
\text { supported } \\
\text { conglomerate }\end{array}$ & $\begin{array}{l}\text { Matrix: poorly sorted } \\
\text { medium to coarse sand. } \\
\text { Clasts: dominately } \\
\text { extraformational clear } \\
\text { quartzite, red and tan } \\
\text { sandstone, minor amounts of } \\
\text { carbonate, red and black } \\
\text { chert }\end{array}$ & $\begin{array}{l}\text { Typically massive, 1- } \\
2 \mathrm{~m} \text { scale bedding } \\
\text { where present, no } \\
\text { grading to weak } \\
\text { normal grading }\end{array}$ & $\begin{array}{l}\text { Reddish } \\
\text { brown, } \\
\text { reddish } \\
\text { light grey }\end{array}$ & Debris flows \\
\hline$S_{2} p$ & $\begin{array}{l}\text { Planar cross- } \\
\text { bedded } \\
\text { sandstone }\end{array}$ & $\begin{array}{l}\text { Fine- to medium-grained } \\
\text { sand, moderately well sorted }\end{array}$ & $\begin{array}{l}\text { Planar tabular } \\
\text { crossbeds; Coset } \\
\text { thickness: ranges } \\
\text { from } 0.1 \text { to } 0.5 \mathrm{~m}\end{array}$ & Light grey & $\begin{array}{l}\text { Lower flow } \\
\text { regime 2D } \\
\text { dunes within } \\
\text { channels }\end{array}$ \\
\hline $\mathrm{S}_{2} \mathrm{t}$ & $\begin{array}{l}\text { Trough cross- } \\
\text { bedded } \\
\text { sandstone }\end{array}$ & $\begin{array}{l}\text { Fine- to medium-grained } \\
\text { sand, moderately well sorted }\end{array}$ & $\begin{array}{l}\text { Trough crossbeds: } \\
\text { Coset thickness } 0.1 \\
\text { to } 1.5 \mathrm{~m}\end{array}$ & $\begin{array}{l}\text { Light grey; } \\
\text { reddish } \\
\text { tan, tan }\end{array}$ & $\begin{array}{l}\text { Lower flow } \\
\text { regime 3D } \\
\text { dunes within } \\
\text { channels }\end{array}$ \\
\hline $\mathrm{S}_{2} \mathrm{~m}$ & $\begin{array}{l}\text { Massive } \\
\text { sandstone }\end{array}$ & $\begin{array}{l}\text { Fine- to medium-grained } \\
\text { sand, moderately well sorted }\end{array}$ & $\begin{array}{l}\text { Massive: possible } \\
\text { crude stratification }\end{array}$ & $\begin{array}{l}\text { Reddish } \\
\text { tan, tan }\end{array}$ & $\begin{array}{l}\text { Rapid } \\
\text { sedimentation }\end{array}$ \\
\hline $\mathrm{F}_{2} \mathrm{I}$ & $\begin{array}{l}\text { Finely } \\
\text { laminated } \\
\text { siltstone and } \\
\text { mudstone }\end{array}$ & Very fine grained silt and mud & Very fine laminations & $\begin{array}{l}\text { Dark } \\
\text { reddish } \\
\text { brown, } \\
\text { pale red } \\
\text { purple, } \\
\text { brown }\end{array}$ & $\begin{array}{l}\text { Suspension } \\
\text { load deposits }\end{array}$ \\
\hline
\end{tabular}




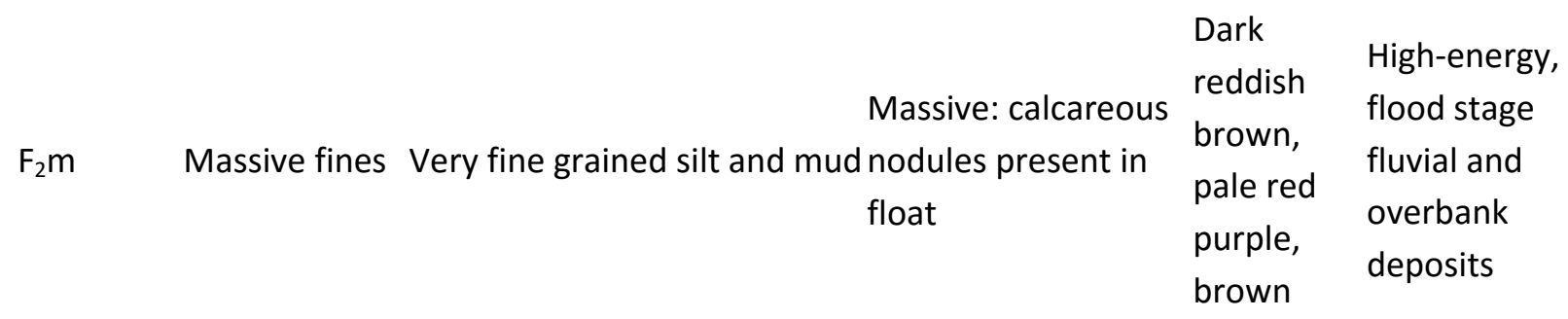

Facies associations identified in the Gannet Group

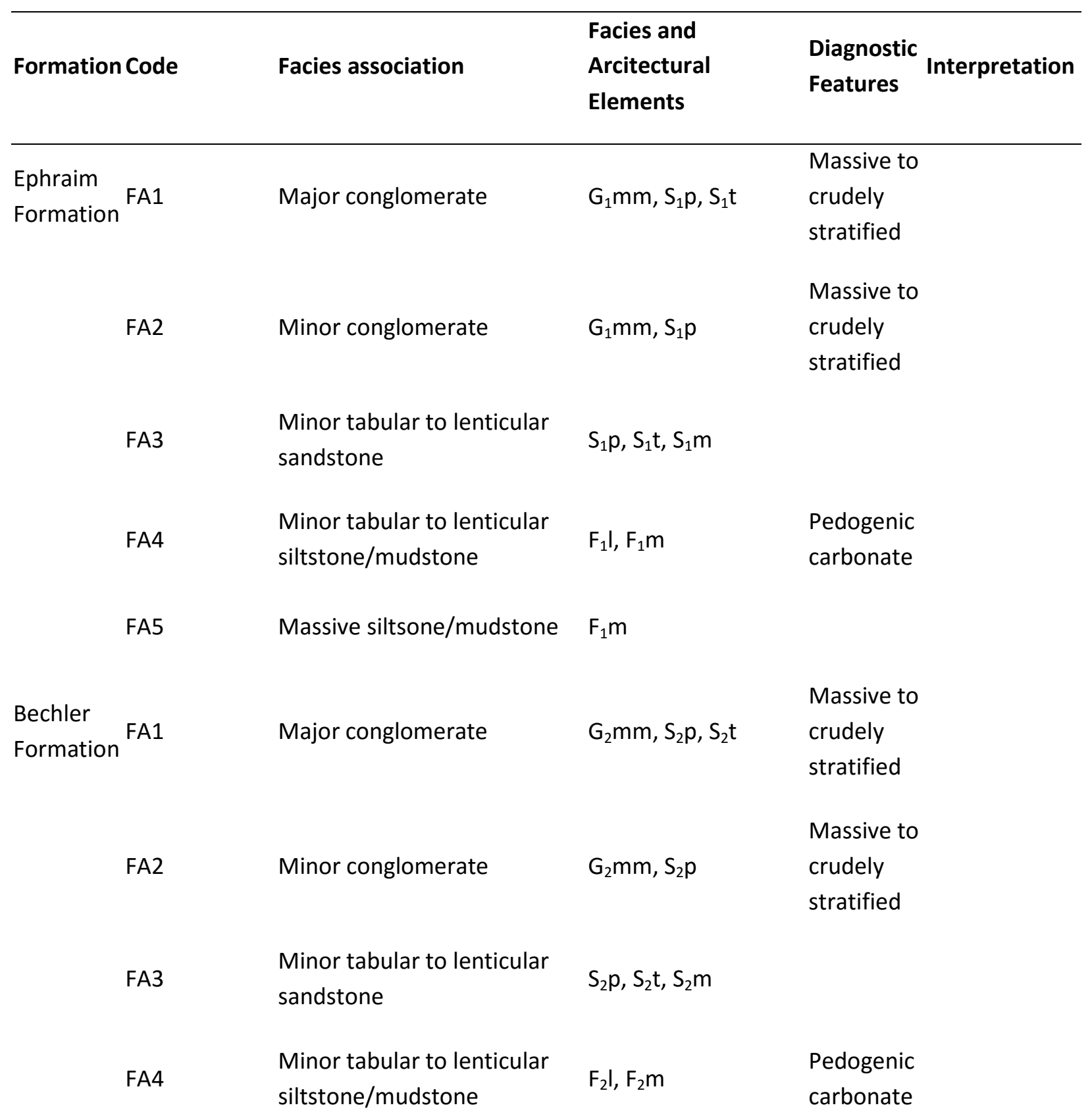


Massive siltstone/mudstone $F_{2} m$ 
Table 2. K-S test

K-S P-values using error in the CDF

\begin{tabular}{|c|c|c|c|c|c|c|c|c|}
\hline & $\begin{array}{l}\text { Caddy } \\
\text { Canyon }\end{array}$ & Mutual & Geertsen & $\begin{array}{l}\text { Worm } \\
\text { Creek }\end{array}$ & $\begin{array}{l}\text { Swan } \\
\text { Peak }\end{array}$ & Oquirrh & Moenkopi & Nugget \\
\hline Ephraim & 0.001 & 0.000 & 0.000 & 0.000 & 0.000 & 0.000 & 0.000 & 0.977 \\
\hline Bechler & 0.000 & 0.000 & 0.000 & 0.000 & 0.000 & 0.000 & 0.000 & 0.877 \\
\hline $\begin{array}{l}\text { Cokeville } \\
\text { Sage }\end{array}$ & 0.000 & 0.002 & 0.000 & 0.000 & 0.000 & 0.365 & 0.000 & 0.006 \\
\hline $\begin{array}{l}\text { Junction } \\
\text { Bear }\end{array}$ & 0.000 & 0.000 & 0.004 & 0.000 & 0.000 & 0.003 & 0.000 & 0.000 \\
\hline Valley & 0.000 & 0.067 & 0.000 & 0.000 & 0.000 & 0.042 & 0.000 & 0.187 \\
\hline $\begin{array}{l}\text { Frontier } \\
\text { Upper }\end{array}$ & 0.002 & 0.198 & 0.000 & 0.000 & 0.000 & 0.480 & 0.000 & 0.000 \\
\hline Frontier & 0.004 & 0.076 & 0.159 & 0.000 & 0.000 & 0.185 & 0.001 & 0.000 \\
\hline Aspen & 0.002 & 0.712 & 0.000 & 0.000 & 0.000 & 0.551 & 0.000 & 0.000 \\
\hline Hilliard & 0.000 & 0.022 & 0.000 & 0.000 & 0.000 & 0.139 & 0.001 & 0.353 \\
\hline
\end{tabular}


Appendix A: Sample Locations

\begin{tabular}{|c|c|c|c|c|}
\hline Sample & Unit/Formation & Latitude (N) & Longitude (W) & Elevation (m) \\
\hline SYN 12-1 & Ephraim & $41^{\circ} 35^{\prime} 58.11^{\prime \prime}$ & $110^{\circ} 41^{\prime} 00.00^{\prime \prime}$ & 2071 \\
\hline SYN 12-2 & Bechler & $41^{\circ} 35^{\prime} 56.94^{\prime \prime}$ & $110^{\circ} 40^{\prime \prime} 49.43^{\prime \prime}$ & 2077 \\
\hline SYN $12-3$ & Bear River & $41^{\circ} 35^{\prime} 49.11^{\prime \prime}$ & $110^{\circ} 40^{\prime} 53.87^{\prime \prime}$ & 2072 \\
\hline SYN 12-5 & Pruess & $41^{\circ} 36^{\prime} 01.10^{\prime \prime}$ & $110^{\circ} 57^{\prime} 40.15^{\prime \prime}$ & 2307 \\
\hline SYN 12-6 & Ephraim & $41^{\circ} 36^{\prime} 00.85^{\prime \prime}$ & $110^{\circ} 57^{\prime} 43.78^{\prime \prime}$ & 2329 \\
\hline SYN 12-8 & Bechler & $41^{\circ} 36^{\prime} 00.50^{\prime \prime}$ & $110^{\circ} 58^{\prime} 12.87^{\prime \prime}$ & 2228 \\
\hline SYN $12-10$ & Cokeville & $41^{\circ} 54^{\prime} 38.92^{\prime \prime}$ & $110^{\circ} 56^{\prime} 30.37^{\prime \prime}$ & 1915 \\
\hline SYN 12-11 & Lower Sage Junction & $41^{\circ} 54^{\prime} 32.52^{\prime \prime}$ & $110^{\circ} 56^{\prime} 39.21^{\prime \prime}$ & 1918 \\
\hline SYN $12-12$ & Upper Sage Junction & $41^{\circ} 54^{\prime} 32.52^{\prime \prime}$ & $110^{\circ} 56^{\prime} 39.21^{\prime \prime}$ & 1918 \\
\hline SYN $12-13$ & Aspen & $41^{\circ} 34^{\prime} 57.05^{\prime \prime}$ & $110^{\circ} 30^{\prime} 54.25^{\prime \prime}$ & 2051 \\
\hline SYN 12-14 & Frontier & $41^{\circ} 35^{\prime} 19.08^{\prime \prime}$ & $110^{\circ} 33^{\prime} 39.99^{\prime \prime}$ & 2025 \\
\hline SYN 12-15 & Upper Frontier & $41^{\circ} 53^{\prime} 18.82^{\prime \prime}$ & $110^{\circ} 34^{\prime} 10.69^{\prime \prime}$ & 2004 \\
\hline SYN 12-16 & Hilliard & $41^{\circ} 34^{\prime} 43.45^{\prime \prime}$ & $110^{\circ} 35^{\prime} 30.58^{\prime \prime}$ & 2018 \\
\hline SYN 12-17 & Ephraim & $41^{\circ} 45^{\prime} 45.53^{\prime \prime}$ & $110^{\circ} 27^{\prime} 29.09^{\prime \prime}$ & 2123 \\
\hline SYN 12-18 & Nugget & & & 0 \\
\hline SYN $12-19$ & Ankareh & & & 0 \\
\hline SYN 14-1 & Ephraim & $42^{\circ} 28^{\prime} 54.66^{\prime \prime}$ & $110^{\circ} 53^{\prime} 47.56^{\prime \prime}$ & 2405 \\
\hline SYN 14-3 & Ephraim & $42^{\circ} 28^{\prime} 55.04^{\prime \prime}$ & $110^{\circ} 54^{\prime} 02.33^{\prime \prime}$ & 2290 \\
\hline SYN $14-4$ & Bechler & $42^{\circ} 28^{\prime} 52.71^{\prime \prime}$ & $110^{\circ} 54^{\prime} 11.73^{\prime \prime}$ & 2222 \\
\hline SYN 14-5 & Bechler & $42^{\circ} 29^{\prime} 00.95^{\prime \prime}$ & $110^{\circ} 54^{\prime} 21.68^{\prime \prime}$ & 2206 \\
\hline SYN 14-9 & Ephraim & $42^{\circ} 36^{\prime} 41.23^{\prime \prime}$ & $111^{\circ} 00^{\prime} 38.77$ & 2227 \\
\hline SYN $14-10$ & Ephraim & $42^{\circ} 29^{\prime} 42.11^{\prime \prime}$ & $111^{\circ} 08^{\prime} 45.66^{\prime \prime}$ & 2302 \\
\hline SYN 14-11 & Bechler & $42^{\circ} 28^{\prime} 45.88^{\prime \prime}$ & $111^{\circ} 07^{\prime} 55.88^{\prime \prime}$ & 2555 \\
\hline SYN 14-12 & Bechler & $42^{\circ} 28^{\prime} 52.80^{\prime \prime}$ & $111^{\circ} 087^{\prime} 58.25^{\prime \prime}$ & 2621 \\
\hline SYN 14-13 & Bechler & $42^{\circ} 28^{\prime} 57.21^{\prime \prime}$ & $111^{\circ} 08^{\prime} 09.73^{\prime \prime}$ & 2972 \\
\hline SYN 14-14 & Ephraim & $41^{\circ} 35^{\prime} 55.23^{\prime \prime}$ & $110^{\circ} 57^{\prime} 24.54^{\prime \prime}$ & 2234 \\
\hline
\end{tabular}


Appendix B: U/Pb Data

\begin{tabular}{|c|c|c|c|c|c|c|c|c|c|}
\hline Analysis & $206 \mathrm{~Pb} *$ & \pm & 207Pb* & \pm & $206 \mathrm{~Pb}^{*}$ & \pm & Best age & \pm & Conc \\
\hline & $238 U^{*}$ & (Ma) & $235 \mathrm{U}$ & (Ma) & $207 \mathrm{~Pb}^{*}$ & (Ma) & (Ma) & (Ma) & $(\%)$ \\
\hline & & & & & & & & & \\
\hline & & & & & & & & & \\
\hline SYN12-1-74 & 160.7 & 7.3 & 129.9 & 30.0 & -403.2 & 638.6 & 160.7 & 7.3 & NA \\
\hline SYN12-1-63 & 160.9 & 3.8 & 164.7 & 10.3 & 220.1 & 147.4 & 160.9 & 3.8 & $\mathrm{NA}$ \\
\hline SYN12-1-102 & 167.4 & 1.7 & 165.9 & 2.9 & 143.5 & 36.9 & 167.4 & 1.7 & NA \\
\hline SYN12-1-18 & 170.5 & 3.5 & 159.7 & 22.6 & 2.4 & 366.1 & 170.5 & 3.5 & NA \\
\hline SYN12-1-32 & 247.3 & 5.3 & 229.6 & 28.3 & 51.1 & 325.3 & 247.3 & 5.3 & NA \\
\hline SYN12-1-104 & 254.3 & 2.8 & 254.6 & 5.8 & 257.8 & 53.1 & 254.3 & 2.8 & NA \\
\hline SYN12-1-39 & 255.5 & 3.7 & 267.6 & 13.6 & 375.5 & 126.2 & 255.5 & 3.7 & NA \\
\hline SYN12-1-08 & 256.7 & 4.0 & 253.1 & 4.8 & 220.7 & 33.8 & 256.7 & 4.0 & NA \\
\hline SYN12-1-43 & 258.3 & 4.1 & 261.3 & 7.4 & 288.3 & 63.4 & 258.3 & 4.1 & NA \\
\hline SYN12-1-01 & 288.8 & 4.9 & 288.2 & 8.7 & 283.5 & 68.6 & 288.8 & 4.9 & NA \\
\hline SYN12-1-05 & 298.8 & 4.6 & 295.3 & 4.7 & 267.8 & 20.9 & 298.8 & 4.6 & $\mathrm{NA}$ \\
\hline SYN12-1-62 & 307.4 & 4.9 & 305.8 & 5.6 & 293.0 & 30.9 & 307.4 & 4.9 & NA \\
\hline SYN12-1-16 & 314.9 & 7.9 & 318.1 & 21.9 & 341.2 & 172.0 & 314.9 & 7.9 & NA \\
\hline SYN12-1-17 & 322.1 & 6.2 & 335.1 & 23.0 & 426.5 & 174.4 & 322.1 & 6.2 & NA \\
\hline SYN12-1-21 & 369.5 & 5.4 & 372.7 & 8.4 & 392.6 & 50.0 & 369.5 & 5.4 & NA \\
\hline SYN12-1-30 & 380.0 & 5.0 & 381.9 & 10.9 & 393.0 & 70.7 & 380.0 & 5.0 & $\mathrm{NA}$ \\
\hline SYN12-1-90 & 380.3 & 10.2 & 385.4 & 29.3 & 415.8 & 194.6 & 380.3 & 10.2 & NA \\
\hline SYN12-1-92 & 386.2 & 4.6 & 383.2 & 18.5 & 365.0 & 127.9 & 386.2 & 4.6 & NA \\
\hline SYN12-1-101 & 387.4 & 5.1 & 384.6 & 9.5 & 368.1 & 59.4 & 387.4 & 5.1 & NA \\
\hline SYN12-1-65 & 411.8 & 5.8 & 413.3 & 6.9 & 421.1 & 31.6 & 411.8 & 5.8 & 97.8 \\
\hline SYN12-1-59 & 424.1 & 5.1 & 422.9 & 6.8 & 416.1 & 34.5 & 424.1 & 5.1 & 101.9 \\
\hline SYN12-1-49 & 428.8 & 7.4 & 422.6 & 12.7 & 388.8 & 71.8 & 428.8 & 7.4 & 110.3 \\
\hline SYN12-1-61 & 456.6 & 6.0 & 454.9 & 5.9 & 446.6 & 18.7 & 456.6 & 6.0 & 102.2 \\
\hline SYN12-1-20 & 463.4 & 6.0 & 473.1 & 18.0 & 520.2 & 100.3 & 463.4 & 6.0 & 89.1 \\
\hline SYN12-1-76 & 468.6 & 7.4 & 464.5 & 12.9 & 444.3 & 67.6 & 468.6 & 7.4 & 105.5 \\
\hline SYN12-1-29 & 491.5 & 5.3 & 496.9 & 14.1 & 521.6 & 75.0 & 491.5 & 5.3 & 94.2 \\
\hline SYN12-1-79 & 518.6 & 21.9 & 495.4 & 29.3 & 389.9 & 135.9 & 518.6 & 21.9 & 133.0 \\
\hline SYN12-1-23 & 523.3 & 5.3 & 520.5 & 16.4 & 508.1 & 85.7 & 523.3 & 5.3 & 103.0 \\
\hline SYN12-1-86 & 552.1 & 5.1 & 550.4 & 6.0 & 543.3 & 23.0 & 552.1 & 5.1 & 101.6 \\
\hline SYN12-1-40 & 555.5 & 6.8 & 556.8 & 6.5 & 562.0 & 17.7 & 555.5 & 6.8 & 98.8 \\
\hline SYN12-1-91 & 556.0 & 7.8 & 548.7 & 23.9 & 518.5 & 119.5 & 556.0 & 7.8 & 107.2 \\
\hline SYN12-1-50 & 563.6 & 7.6 & 562.7 & 6.3 & 558.9 & 9.4 & 563.6 & 7.6 & 100.8 \\
\hline SYN12-1-87 & 586.0 & 12.9 & 576.2 & 16.7 & 537.8 & 66.1 & 586.0 & 12.9 & 109.0 \\
\hline
\end{tabular}




\begin{tabular}{|c|c|c|c|c|c|c|c|c|c|}
\hline SYN12-1-103 & 611.3 & 10.9 & 619.0 & 18.8 & 647.4 & 77.5 & 611.3 & 10.9 & 94.4 \\
\hline SYN12-1-46 & 640.8 & 12.8 & 638.8 & 27.3 & 631.8 & 115.6 & 640.8 & 12.8 & 101.4 \\
\hline SYN12-1-81 & 650.0 & 13.9 & 651.5 & 29.4 & 656.7 & 122.2 & 650.0 & 13.9 & 99.0 \\
\hline SYN12-1-22 & 1027.8 & 12.6 & 1019.1 & 17.9 & 1000.5 & 49.8 & 1000.5 & 49.8 & 102.7 \\
\hline SYN12-1-24 & 997.1 & 10.1 & 1002.5 & 8.0 & 1014.5 & 12.2 & 1014.5 & 12.2 & 98.3 \\
\hline SYN12-1-96 & 1024.4 & 14.4 & 1022.3 & 10.1 & 1017.9 & 7.4 & 1017.9 & 7.4 & 100.6 \\
\hline SYN12-1-82 & 1020.7 & 18.6 & 1020.4 & 16.2 & 1019.8 & 31.7 & 1019.8 & 31.7 & 100.1 \\
\hline SYN12-1-54 & 1040.8 & 12.3 & 1035.3 & 9.3 & 1023.8 & 13.2 & 1023.8 & 13.2 & 101.7 \\
\hline SYN12-1-41 & 1035.9 & 15.7 & 1032.4 & 17.3 & 1025.2 & 42.5 & 1025.2 & 42.5 & 101.0 \\
\hline SYN12-1-77 & 1048.7 & 15.7 & 1042.7 & 13.8 & 1030.1 & 27.7 & 1030.1 & 27.7 & 101.8 \\
\hline SYN12-1-06 & 1045.8 & 32.8 & 1045.0 & 25.5 & 1043.2 & 38.8 & 1043.2 & 38.8 & 100.2 \\
\hline SYN12-1-31 & 1074.2 & 15.2 & 1064.6 & 16.3 & 1045.0 & 39.2 & 1045.0 & 39.2 & 102.8 \\
\hline SYN12-1-11 & 1058.5 & 30.6 & 1056.4 & 23.5 & 1052.1 & 34.9 & 1052.1 & 34.9 & 100.6 \\
\hline SYN12-1-27 & 1069.6 & 11.0 & 1070.2 & 10.4 & 1071.3 & 22.3 & 1071.3 & 22.3 & 99.8 \\
\hline SYN12-1-100 & 1089.0 & 16.1 & 1084.7 & 11.5 & 1075.9 & 12.7 & 1075.9 & 12.7 & 101.2 \\
\hline SYN12-1-35 & 1025.0 & 17.5 & 1052.8 & 14.7 & 1111.0 & 25.1 & 1111.0 & 25.1 & 92.3 \\
\hline SYN12-1-93 & 1116.7 & 17.9 & 1115.0 & 15.9 & 1111.6 & 31.5 & 1111.6 & 31.5 & 100.5 \\
\hline SYN12-1-25 & 1115.7 & 13.0 & 1118.3 & 28.9 & 1123.5 & 81.0 & 1123.5 & 81.0 & 99.3 \\
\hline SYN12-1-75 & 1129.4 & 21.0 & 1128.0 & 15.7 & 1125.4 & 22.1 & 1125.4 & 22.1 & 100.4 \\
\hline SYN12-1-14 & 1152.9 & 12.9 & 1146.5 & 9.1 & 1134.5 & 10.6 & 1134.5 & 10.6 & 101.6 \\
\hline SYN12-1-99 & 1142.0 & 16.9 & 1146.2 & 15.5 & 1154.1 & 31.0 & 1154.1 & 31.0 & 99.0 \\
\hline SYN12-1-13 & 1142.8 & 15.7 & 1147.6 & 10.8 & 1156.5 & 9.2 & 1156.5 & 9.2 & 98.8 \\
\hline SYN12-1-55 & 1180.2 & 17.4 & 1172.0 & 19.7 & 1156.9 & 46.2 & 1156.9 & 46.2 & 102.0 \\
\hline SYN12-1-69 & 1176.4 & 16.4 & 1175.4 & 11.8 & 1173.6 & 14.5 & 1173.6 & 14.5 & 100.2 \\
\hline SYN12-1-58 & 1135.8 & 22.2 & 1162.1 & 15.5 & 1211.4 & 13.2 & 1211.4 & 13.2 & 93.8 \\
\hline SYN12-1-73 & 1221.4 & 23.3 & 1226.1 & 19.9 & 1234.5 & 36.4 & 1234.5 & 36.4 & 98.9 \\
\hline SYN12-1-36 & 1259.7 & 19.0 & 1251.7 & 12.1 & 1237.8 & 5.4 & 1237.8 & 5.4 & 101.8 \\
\hline SYN12-1-71 & 1045.4 & 33.5 & 1117.5 & 25.0 & 1260.7 & 24.4 & 1260.7 & 24.4 & 82.9 \\
\hline SYN12-1-83 & 1335.2 & 17.1 & 1320.7 & 21.0 & 1297.3 & 48.0 & 1297.3 & 48.0 & 102.9 \\
\hline SYN12-1-26 & 1269.5 & 60.4 & 1297.9 & 67.5 & 1345.1 & 146.2 & 1345.1 & 146.2 & 94.4 \\
\hline SYN12-1-67 & 1345.4 & 19.6 & 1348.7 & 16.2 & 1354.0 & 28.0 & 1354.0 & 28.0 & 99.4 \\
\hline SYN12-1-78 & 1352.3 & 22.2 & 1359.8 & 19.9 & 1371.6 & 37.1 & 1371.6 & 37.1 & 98.6 \\
\hline SYN12-1-85 & 1309.0 & 17.9 & 1339.9 & 15.5 & 1389.5 & 27.7 & 1389.5 & 27.7 & 94.2 \\
\hline SYN12-1-03 & 1500.7 & 31.7 & 1472.9 & 21.0 & 1433.0 & 24.6 & 1433.0 & 24.6 & 104.7 \\
\hline SYN12-1-56 & 1450.5 & 16.5 & 1446.0 & 10.7 & 1439.3 & 10.5 & 1439.3 & 10.5 & 100.8 \\
\hline SYN12-1-95 & 1463.7 & 12.5 & 1454.7 & 12.3 & 1441.4 & 24.3 & 1441.4 & 24.3 & 101.5 \\
\hline SYN12-1-97 & 1478.6 & 20.1 & 1474.0 & 13.3 & 1467.5 & 14.8 & 1467.5 & 14.8 & 100.8 \\
\hline SYN12-1-94 & 1502.5 & 18.5 & 1498.4 & 10.9 & 1492.5 & 2.6 & 1492.5 & 2.6 & 100.7 \\
\hline SYN12-1-45 & 1602.3 & 22.5 & 1597.0 & 13.5 & 1590.1 & 10.3 & 1590.1 & 10.3 & 100.8 \\
\hline
\end{tabular}




\begin{tabular}{|l|l|l|l|l|l|l|l|l|l|}
\hline SYN12-1-53 & 1643.4 & 35.2 & 1635.9 & 21.4 & 1626.4 & 19.2 & 1626.4 & 19.2 & 101.0 \\
\hline SYN12-1-37 & 1669.5 & 21.5 & 1651.7 & 12.3 & 1629.2 & 6.5 & 1629.2 & 6.5 & 102.5 \\
\hline SYN12-1-105 & 1377.3 & 29.9 & 1480.3 & 18.9 & 1631.1 & 4.6 & 1631.1 & 4.6 & 84.4 \\
\hline SYN12-1-48 & 1674.4 & 19.8 & 1663.7 & 12.6 & 1650.1 & 14.3 & 1650.1 & 14.3 & 101.5 \\
\hline SYN12-1-64 & 1672.1 & 26.6 & 1665.8 & 16.4 & 1657.8 & 16.1 & 1657.8 & 16.1 & 100.9 \\
\hline SYN12-1-10 & 1684.5 & 16.8 & 1673.0 & 9.4 & 1658.6 & 3.1 & 1658.6 & 3.1 & 101.6 \\
\hline SYN12-1-19 & 1648.7 & 24.0 & 1656.3 & 19.3 & 1666.0 & 31.2 & 1666.0 & 31.2 & 99.0 \\
\hline SYN12-1-60 & 1742.3 & 19.6 & 1742.4 & 13.2 & 1742.6 & 16.9 & 1742.6 & 16.9 & 100.0 \\
\hline SYN12-1-88 & 1762.5 & 14.5 & 1754.8 & 9.8 & 1745.6 & 13.0 & 1745.6 & 13.0 & 101.0 \\
\hline SYN12-1-07 & 1776.8 & 14.8 & 1768.0 & 8.1 & 1757.7 & 3.4 & 1757.7 & 3.4 & 101.1 \\
\hline SYN12-1-12 & 1781.2 & 21.3 & 1771.5 & 14.0 & 1760.1 & 17.4 & 1760.1 & 17.4 & 101.2 \\
\hline SYN12-1-04 & 1812.1 & 32.7 & 1802.2 & 22.9 & 1790.7 & 31.9 & 1790.7 & 31.9 & 101.2 \\
\hline SYN12-1-98 & 1843.4 & 19.1 & 1824.3 & 10.7 & 1802.6 & 7.7 & 1802.6 & 7.7 & 102.3 \\
\hline SYN12-1-47 & 1886.3 & 26.9 & 1891.8 & 14.2 & 1897.9 & 3.5 & 1897.9 & 3.5 & 99.4 \\
\hline SYN12-1-02 & 1975.4 & 21.7 & 1952.2 & 12.6 & 1927.6 & 12.6 & 1927.6 & 12.6 & 102.5 \\
\hline SYN12-1-09 & 1757.8 & 23.5 & 1851.8 & 13.6 & 1959.0 & 8.6 & 1959.0 & 8.6 & 89.7 \\
\hline SYN12-1-33 & 1979.1 & 26.5 & 1972.6 & 13.7 & 1965.8 & 4.3 & 1965.8 & 4.3 & 100.7 \\
\hline SYN12-1-38 & 1976.0 & 18.1 & 1983.0 & 10.7 & 1990.4 & 11.1 & 1990.4 & 11.1 & 99.3 \\
\hline SYN12-1-68 & 2419.6 & 66.5 & 2401.6 & 39.4 & 2386.4 & 46.6 & 2386.4 & 46.6 & 101.4 \\
\hline SYN12-1-28 & 2587.1 & 34.3 & 2590.4 & 15.3 & 2593.0 & 4.5 & 2593.0 & 4.5 & 99.8 \\
\hline SYN12-1-15 & 2757.7 & 33.1 & 2720.9 & 14.1 & 2693.6 & 2.8 & 2693.6 & 2.8 & 102.4 \\
\hline SYN12-1-52 & 2811.1 & 42.1 & 2822.4 & 17.8 & 2830.5 & 5.3 & 2830.5 & 5.3 & 99.3 \\
\hline SYN12-1-34 & 2920.1 & 29.6 & 2871.8 & 12.4 & 2838.0 & 5.4 & 2838.0 & 5.4 & 102.9 \\
\hline SYN12-1-84 & 3060.6 & 173.0 & 3096.4 & 69.7 & 3119.7 & 18.4 & 3119.7 & 18.4 & 98.1 \\
\hline
\end{tabular}

\begin{tabular}{|l|l|l|l|l|l|l|l|l|l|}
\hline Analysis & $206 \mathrm{~Pb}^{*}$ & \pm & $207 \mathrm{~Pb}^{*}$ & \pm & $206 \mathrm{~Pb}^{*}$ & \pm & Best age & \pm & Conc \\
\hline & $238 \mathrm{U}^{*}$ & $(\mathrm{Ma})$ & $235 \mathrm{U}$ & $(\mathrm{Ma})$ & $207 \mathrm{~Pb}^{*}$ & $(\mathrm{Ma})$ & $(\mathrm{Ma})$ & $(\mathrm{Ma})$ & $(\%)$ \\
\hline & & & & & & & & & \\
\hline & & & & & & & & & \\
\hline & & & & & & & & & \\
\hline SYN12-2-101 & 98.2 & 12.1 & 107.6 & 17.0 & 321.2 & 250.3 & 98.2 & 12.1 & NA \\
\hline SYN12-2-29 & 104.4 & 3.0 & 106.7 & 4.9 & 156.8 & 89.5 & 104.4 & 3.0 & NA \\
\hline SYN12-2-23 & 105.5 & 4.0 & 118.8 & 15.9 & 393.8 & 308.0 & 105.5 & 4.0 & NA \\
\hline SYN12-2-20 & 106.1 & 3.5 & 113.0 & 17.7 & 261.6 & 373.9 & 106.1 & 3.5 & NA \\
\hline SYN12-2-31 & 108.0 & 1.8 & 105.6 & 11.7 & 52.8 & 275.5 & 108.0 & 1.8 & NA \\
\hline SYN12-2-42 & 108.6 & 1.9 & 104.5 & 9.4 & 13.5 & 225.0 & 108.6 & 1.9 & NA \\
\hline SYN12-85 & 109.7 & 4.1 & 121.2 & 12.2 & 353.0 & 226.8 & 109.7 & 4.1 & NA \\
\hline SYN12-2-107 & 109.9 & 1.7 & 110.3 & 4.1 & 119.3 & 84.3 & 109.9 & 1.7 & NA \\
\hline SYN12-88 & 111.2 & 3.8 & 110.4 & 9.1 & 94.9 & 190.1 & 111.2 & 3.8 & NA \\
\hline
\end{tabular}




\begin{tabular}{|c|c|c|c|c|c|c|c|c|c|}
\hline SYN12-2-106 & 116.6 & 3.5 & 110.4 & 11.5 & -21.3 & 256.9 & 116.6 & 3.5 & NA \\
\hline SYN12-2-108 & 120.5 & 3.0 & 101.1 & 30.4 & -334.6 & 826.8 & 120.5 & 3.0 & NA \\
\hline SYN12-81 & 122.3 & 4.3 & 113.7 & 13.2 & -62.5 & 287.4 & 122.3 & 4.3 & NA \\
\hline SYN12-2-109 & 122.7 & 1.4 & 120.9 & 10.0 & 84.5 & 205.7 & 122.7 & 1.4 & NA \\
\hline SYN12-2-110 & 144.2 & 1.5 & 142.6 & 4.8 & 116.3 & 81.6 & 144.2 & 1.5 & NA \\
\hline SYN12-2-47 & 149.6 & 3.6 & 147.8 & 12.3 & 119.1 & 203.1 & 149.6 & 3.6 & NA \\
\hline SYN12-95 & 150.9 & 7.9 & 151.8 & 30.7 & 166.9 & 497.8 & 150.9 & 7.9 & NA \\
\hline SYN12-70 & 154.1 & 2.7 & 172.2 & 15.3 & 427.5 & 211.6 & 154.1 & 2.7 & NA \\
\hline SYN12-61 & 155.4 & 7.4 & 157.7 & 11.2 & 191.7 & 137.5 & 155.4 & 7.4 & NA \\
\hline SYN12-2-8 & 161.0 & 4.6 & 152.6 & 12.2 & 23.9 & 195.8 & 161.0 & 4.6 & NA \\
\hline SYN12-58 & 161.3 & 3.0 & 160.4 & 6.8 & 147.2 & 97.5 & 161.3 & 3.0 & NA \\
\hline SYN12-93 & 162.0 & 2.8 & 166.4 & 5.1 & 228.8 & 66.1 & 162.0 & 2.8 & NA \\
\hline SYN12-96 & 167.5 & 1.8 & 166.3 & 4.9 & 150.0 & 69.9 & 167.5 & 1.8 & NA \\
\hline SYN12-2-9 & 168.0 & 4.5 & 172.4 & 13.0 & 233.2 & 179.3 & 168.0 & 4.5 & NA \\
\hline SYN12-67 & 170.6 & 3.2 & 180.6 & 15.4 & 314.5 & 207.8 & 170.6 & 3.2 & NA \\
\hline SYN12-98 & 170.9 & 8.8 & 142.0 & 30.5 & -317.8 & 581.0 & 170.9 & 8.8 & NA \\
\hline SYN12-2-19 & 172.3 & 3.3 & 175.6 & 6.2 & 220.9 & 76.7 & 172.3 & 3.3 & NA \\
\hline SYN12-2-103 & 172.4 & 6.1 & 189.1 & 53.7 & 402.8 & 707.7 & 172.4 & 6.1 & NA \\
\hline SYN12-2-102 & 173.6 & 2.4 & 168.3 & 7.7 & 93.9 & 113.4 & 173.6 & 2.4 & NA \\
\hline SYN12-73 & 173.8 & 3.9 & 174.6 & 8.5 & 185.5 & 111.1 & 173.8 & 3.9 & NA \\
\hline SYN12-2-49 & 178.0 & 1.8 & 176.4 & 3.7 & 153.8 & 48.5 & 178.0 & 1.8 & NA \\
\hline SYN12-2-44 & 180.5 & 2.2 & 179.9 & 6.1 & 171.4 & 81.6 & 180.5 & 2.2 & NA \\
\hline SYN12-82 & 181.8 & 3.2 & 182.9 & 12.1 & 197.7 & 162.8 & 181.8 & 3.2 & NA \\
\hline SYN12-2-18 & 192.4 & 6.8 & 196.6 & 8.2 & 248.3 & 65.4 & 192.4 & 6.8 & NA \\
\hline SYN12-2-16 & 240.9 & 9.2 & 259.0 & 22.3 & 426.5 & 199.8 & 240.9 & 9.2 & NA \\
\hline SYN12-2-104 & 303.0 & 6.1 & 328.0 & 43.1 & 510.2 & 336.2 & 303.0 & 6.1 & NA \\
\hline SYN12-2-37 & 307.7 & 5.2 & 304.4 & 10.3 & 279.7 & 80.9 & 307.7 & 5.2 & NA \\
\hline SYN12-64 & 364.7 & 4.8 & 360.3 & 15.5 & 332.0 & 111.5 & 364.7 & 4.8 & NA \\
\hline SYN12-2-43 & 367.6 & 7.7 & 373.9 & 13.5 & 413.0 & 84.0 & 367.6 & 7.7 & NA \\
\hline SYN12-63 & 395.5 & 4.4 & 394.7 & 6.0 & 390.4 & 32.0 & 395.5 & 4.4 & NA \\
\hline SYN12-2-34 & 413.4 & 4.7 & 409.4 & 6.1 & 386.6 & 30.6 & 413.4 & 4.7 & 106.9 \\
\hline SYN12-2-1 & 417.4 & 8.0 & 418.3 & 9.1 & 423.0 & 39.5 & 417.4 & 8.0 & 98.7 \\
\hline SYN12-75 & 420.3 & 3.5 & 413.6 & 17.5 & 376.1 & 114.0 & 420.3 & 3.5 & 111.8 \\
\hline SYN12-89 & 421.7 & 11.0 & 403.2 & 39.6 & 298.2 & 264.9 & 421.7 & 11.0 & 141.4 \\
\hline SYN12-2-13 & 433.5 & 11.8 & 431.2 & 14.1 & 419.0 & 64.1 & 433.5 & 11.8 & 103.5 \\
\hline SYN12-2-51 & 434.4 & 8.1 & 438.1 & 19.0 & 458.0 & 110.4 & 434.4 & 8.1 & 94.8 \\
\hline SYN12-99 & 435.8 & 7.8 & 438.1 & 9.1 & 450.3 & 39.2 & 435.8 & 7.8 & 96.8 \\
\hline SYN12-65 & 438.5 & 8.0 & 435.0 & 21.0 & 416.3 & 125.8 & 438.5 & 8.0 & 105.3 \\
\hline SYN12-68 & 440.7 & 16.8 & 444.9 & 20.1 & 466.2 & 87.0 & 440.7 & 16.8 & 94.5 \\
\hline
\end{tabular}




\begin{tabular}{|c|c|c|c|c|c|c|c|c|c|}
\hline SYN12-2-3 & 446.1 & 11.1 & 444.4 & 14.3 & 435.7 & 67.3 & 446.1 & 11.1 & 102.4 \\
\hline SYN12-2-4 & 469.3 & 8.2 & 473.8 & 11.8 & 495.6 & 56.0 & 469.3 & 8.2 & 94.7 \\
\hline SYN12-2-46 & 499.2 & 6.4 & 561.2 & 10.5 & 821.1 & 42.5 & 499.2 & 6.4 & 60.8 \\
\hline SYN12-87 & 558.4 & 7.9 & 561.8 & 10.9 & 575.3 & 44.4 & 558.4 & 7.9 & 97.1 \\
\hline SYN12-2-24 & 559.6 & 11.7 & 553.6 & 17.2 & 529.3 & 74.2 & 559.6 & 11.7 & 105.7 \\
\hline SYN12-2-55 & 572.1 & 12.6 & 575.8 & 20.3 & 590.6 & 86.8 & 572.1 & 12.6 & 96.9 \\
\hline SYN12-2-38 & 603.7 & 11.3 & 600.7 & 28.5 & 589.4 & 129.7 & 603.7 & 11.3 & 102.4 \\
\hline SYN12-2-22 & 609.9 & 6.6 & 609.3 & 7.4 & 607.3 & 24.5 & 609.9 & 6.6 & 100.4 \\
\hline SYN12-2-105 & 615.0 & 7.0 & 613.3 & 8.2 & 606.6 & 28.5 & 615.0 & 7.0 & 101.4 \\
\hline SYN12-2-12 & 622.5 & 8.1 & 619.9 & 11.1 & 610.4 & 42.3 & 622.5 & 8.1 & 102.0 \\
\hline SYN12-2-32 & 632.7 & 13.9 & 642.4 & 37.4 & 676.8 & 160.4 & 632.7 & 13.9 & 93.5 \\
\hline SYN12-2-40 & 1018.8 & 9.5 & 1019.3 & 11.8 & 1020.4 & 31.0 & 1020.4 & 31.0 & 99.8 \\
\hline SYN12-78 & 1030.2 & 20.0 & 1027.7 & 14.9 & 1022.3 & 18.9 & 1022.3 & 18.9 & 100.8 \\
\hline SYN12-2-27 & 1007.2 & 24.9 & 1013.2 & 48.3 & 1026.2 & 142.4 & 1026.2 & 142.4 & 98.1 \\
\hline SYN12-2-14 & 1041.9 & 16.9 & 1041.6 & 15.3 & 1040.8 & 31.3 & 1040.8 & 31.3 & 100.1 \\
\hline SYN12-2-33 & 1017.4 & 8.7 & 1026.3 & 17.9 & 1045.3 & 52.8 & 1045.3 & 52.8 & 97.3 \\
\hline SYN12-2-54 & 1096.6 & 23.9 & 1081.2 & 21.8 & 1050.4 & 45.7 & 1050.4 & 45.7 & 104.4 \\
\hline SYN12-62 & 1069.1 & 15.3 & 1064.4 & 14.1 & 1054.9 & 29.8 & 1054.9 & 29.8 & 101.3 \\
\hline SYN12-2-50 & 1080.5 & 26.7 & 1072.6 & 18.7 & 1056.7 & 18.1 & 1056.7 & 18.1 & 102.3 \\
\hline SYN12-2-45 & 1068.1 & 9.7 & 1066.3 & 8.1 & 1062.5 & 14.6 & 1062.5 & 14.6 & 100.5 \\
\hline SYN12-90 & 1090.1 & 15.5 & 1084.8 & 14.1 & 1074.1 & 29.1 & 1074.1 & 29.1 & 101.5 \\
\hline SYN12-2-21 & 1065.2 & 20.2 & 1069.9 & 14.9 & 1079.5 & 18.5 & 1079.5 & 18.5 & 98.7 \\
\hline SYN12-97 & 1063.1 & 10.2 & 1069.3 & 8.2 & 1082.1 & 13.2 & 1082.1 & 13.2 & 98.2 \\
\hline SYN12-92 & 1101.8 & 9.7 & 1097.5 & 7.6 & 1089.0 & 12.3 & 1089.0 & 12.3 & 101.2 \\
\hline SYN12-83 & 1145.8 & 23.4 & 1132.2 & 19.3 & 1106.2 & 34.7 & 1106.2 & 34.7 & 103.6 \\
\hline SYN12-2-7 & 1099.9 & 23.2 & 1104.8 & 25.3 & 1114.6 & 59.5 & 1114.6 & 59.5 & 98.7 \\
\hline SYN12-60 & 1146.7 & 27.3 & 1136.1 & 23.6 & 1116.1 & 45.0 & 1116.1 & 45.0 & 102.7 \\
\hline SYN12-71 & 1101.3 & 19.3 & 1107.9 & 12.9 & 1120.8 & 4.2 & 1120.8 & 4.2 & 98.3 \\
\hline SYN12-2-6 & 1119.4 & 30.4 & 1123.3 & 20.7 & 1130.7 & 14.0 & 1130.7 & 14.0 & 99.0 \\
\hline SYN12-57 & 1148.4 & 19.4 & 1142.9 & 13.3 & 1132.5 & 12.4 & 1132.5 & 12.4 & 101.4 \\
\hline SYN12-77 & 1140.8 & 19.1 & 1143.0 & 12.8 & 1147.3 & 7.5 & 1147.3 & 7.5 & 99.4 \\
\hline SYN12-2-48 & 1147.1 & 26.0 & 1153.9 & 18.7 & 1166.7 & 22.0 & 1166.7 & 22.0 & 98.3 \\
\hline SYN12-2-28 & 1189.6 & 19.0 & 1182.4 & 13.8 & 1169.2 & 18.3 & 1169.2 & 18.3 & 101.8 \\
\hline SYN12-72 & 1184.6 & 42.9 & 1184.3 & 28.9 & 1183.7 & 23.8 & 1183.7 & 23.8 & 100.1 \\
\hline SYN12-2-2 & 1182.3 & 10.9 & 1182.9 & 9.9 & 1183.9 & 19.6 & 1183.9 & 19.6 & 99.9 \\
\hline SYN12-2-10 & 1088.2 & 37.0 & 1122.9 & 32.6 & 1190.7 & 60.2 & 1190.7 & 60.2 & 91.4 \\
\hline SYN12-100 & 1237.6 & 24.6 & 1229.6 & 16.1 & 1215.8 & 11.0 & 1215.8 & 11.0 & 101.8 \\
\hline SYN12-2-39 & 1215.7 & 42.0 & 1217.2 & 28.1 & 1219.8 & 22.1 & 1219.8 & 22.1 & 99.7 \\
\hline SYN12-56 & 1218.9 & 23.7 & 1233.5 & 26.1 & 1259.0 & 57.9 & 1259.0 & 57.9 & 96.8 \\
\hline
\end{tabular}




\begin{tabular}{|l|l|l|l|l|l|l|l|l|l|} 
SYN12-91 & 1294.7 & 29.9 & 1310.8 & 22.0 & 1337.1 & 30.3 & 1337.1 & 30.3 & 96.8 \\
\hline SYN12-84 & 1322.7 & 15.6 & 1328.7 & 18.1 & 1338.5 & 39.9 & 1338.5 & 39.9 & 98.8 \\
\hline SYN12-2-26 & 1346.9 & 29.8 & 1355.3 & 19.0 & 1368.5 & 12.6 & 1368.5 & 12.6 & 98.4 \\
\hline SYN12-86 & 1396.5 & 13.5 & 1393.8 & 11.4 & 1389.7 & 20.0 & 1389.7 & 20.0 & 100.5 \\
\hline SYN12-2-25 & 1351.4 & 14.1 & 1366.5 & 10.1 & 1390.2 & 13.4 & 1390.2 & 13.4 & 97.2 \\
\hline SYN12-2-36 & 1471.7 & 31.9 & 1459.5 & 19.6 & 1441.8 & 14.2 & 1441.8 & 14.2 & 102.1 \\
\hline SYN12-79 & 1451.1 & 14.6 & 1457.1 & 26.9 & 1465.8 & 62.6 & 1465.8 & 62.6 & 99.0 \\
\hline SYN12-66 & 1576.2 & 49.6 & 1576.2 & 28.8 & 1576.2 & 11.0 & 1576.2 & 11.0 & 100.0 \\
\hline SYN12-69 & 1559.6 & 14.1 & 1588.1 & 8.3 & 1626.1 & 4.1 & 1626.1 & 4.1 & 95.9 \\
\hline SYN12-2-53 & 1706.5 & 16.8 & 1706.4 & 10.7 & 1706.2 & 12.1 & 1706.2 & 12.1 & 100.0 \\
\hline SYN12-76 & 1719.8 & 24.4 & 1748.8 & 13.6 & 1783.6 & 3.1 & 1783.6 & 3.1 & 96.4 \\
\hline SYN12-2-52 & 1817.7 & 18.1 & 1812.6 & 10.0 & 1806.8 & 5.3 & 1806.8 & 5.3 & 100.6 \\
\hline SYN12-2-15 & 1861.6 & 23.0 & 1840.5 & 14.8 & 1816.7 & 18.3 & 1816.7 & 18.3 & 102.5 \\
\hline SYN12-2-11 & 1845.1 & 12.0 & 1836.5 & 7.0 & 1826.8 & 6.3 & 1826.8 & 6.3 & 101.0 \\
\hline SYN12-74 & 1780.6 & 49.7 & 1814.8 & 27.2 & 1854.3 & 6.0 & 1854.3 & 6.0 & 96.0 \\
\hline SYN12-2-17 & 1830.1 & 28.3 & 1881.6 & 15.3 & 1938.9 & 4.4 & 1938.9 & 4.4 & 94.4 \\
\hline SYN12-94 & 2104.7 & 23.5 & 2099.3 & 21.8 & 2094.1 & 36.6 & 2094.1 & 36.6 & 100.5 \\
\hline SYN12-2-41 & 2539.3 & 21.9 & 2535.1 & 11.9 & 2531.6 & 12.2 & 2531.6 & 12.2 & 100.3 \\
\hline SYN12-59 & 2754.1 & 41.5 & 2766.4 & 18.0 & 2775.4 & 7.3 & 2775.4 & 7.3 & 99.2 \\
\hline SYN12-2-35 & 2827.6 & 47.3 & 2843.9 & 19.7 & 2855.4 & 1.6 & 2855.4 & 1.6 & 99.0 \\
\hline SYN12-80 & 2930.7 & 222.6 & 2955.0 & 91.3 & 2971.5 & 12.5 & 2971.5 & 12.5 & 98.6 \\
\hline
\end{tabular}

\begin{tabular}{|l|l|l|l|l|l|l|l|l|l|}
\hline Analysis & $206 \mathrm{~Pb}^{*}$ & \pm & $207 \mathrm{~Pb}^{*}$ & \pm & $206 \mathrm{~Pb}^{*}$ & \pm & Best age & \pm & Conc \\
\hline & $238 \mathrm{U}^{*}$ & $(\mathrm{Ma})$ & $235 \mathrm{U}$ & $(\mathrm{Ma})$ & $207 \mathrm{~Pb} *$ & $(\mathrm{Ma})$ & $(\mathrm{Ma})$ & $(\mathrm{Ma})$ & $(\%)$ \\
\hline & & & & & & & & & \\
\hline & & & & & & & & & \\
\hline & & & & & & & & & \\
\hline SYN12-3-68 & 111.8 & 1.4 & 111.2 & 4.5 & 97.5 & 96.4 & 111.8 & 1.4 & NA \\
\hline SYN12-3-15 & 181.7 & 2.5 & 180.7 & 5.2 & 167.7 & 65.0 & 181.7 & 2.5 & NA \\
\hline SYN12-3-34 & 267.2 & 7.6 & 267.7 & 10.0 & 272.7 & 70.9 & 267.2 & 7.6 & NA \\
\hline SYN12-3-95 & 314.0 & 4.5 & 312.7 & 9.2 & 303.5 & 70.7 & 314.0 & 4.5 & NA \\
\hline SYN12-3-100 & 385.7 & 9.5 & 382.9 & 10.5 & 366.0 & 47.4 & 385.7 & 9.5 & NA \\
\hline SYN12-3-37 & 425.0 & 4.1 & 430.3 & 5.0 & 458.5 & 22.2 & 425.0 & 4.1 & 92.7 \\
\hline SYN12-3-39 & 438.5 & 4.5 & 435.5 & 13.9 & 419.6 & 84.2 & 438.5 & 4.5 & 104.5 \\
\hline SYN12-3-35 & 446.2 & 11.7 & 442.1 & 48.4 & 420.9 & 296.3 & 446.2 & 11.7 & 106.0 \\
\hline SYN12-3-1 & 457.8 & 5.3 & 456.2 & 5.2 & 448.2 & 16.2 & 457.8 & 5.3 & 102.1 \\
\hline SYN12-3-52 & 464.0 & 5.6 & 471.2 & 12.3 & 506.7 & 66.0 & 464.0 & 5.6 & 91.6 \\
\hline SYN12-3-23 & 503.0 & 8.4 & 500.5 & 10.5 & 489.2 & 44.5 & 503.0 & 8.4 & 102.8 \\
\hline SYN12-3-28 & 516.9 & 13.6 & 492.8 & 28.9 & 382.2 & 154.9 & 516.9 & 13.6 & 135.2 \\
\hline
\end{tabular}




\begin{tabular}{|c|c|c|c|c|c|c|c|c|c|}
\hline SYN12-3-60 & 604.4 & 9.7 & 602.1 & 10.5 & 593.4 & 34.2 & 604.4 & 9.7 & 101.9 \\
\hline SYN12-3-38 & 607.3 & 17.7 & 606.9 & 14.2 & 605.3 & 11.9 & 607.3 & 17.7 & 100.3 \\
\hline SYN12-3-5 & 624.8 & 9.2 & 627.2 & 20.1 & 635.9 & 86.1 & 624.8 & 9.2 & 98.3 \\
\hline SYN12-3-44 & 637.9 & 7.5 & 634.9 & 7.0 & 624.1 & 17.3 & 637.9 & 7.5 & 102.2 \\
\hline SYN12-3-49 & 646.4 & 12.1 & 645.4 & 15.3 & 642.1 & 54.2 & 646.4 & 12.1 & 100.7 \\
\hline SYN12-3-36 & 659.6 & 10.6 & 656.9 & 12.5 & 647.4 & 42.4 & 659.6 & 10.6 & 101.9 \\
\hline SYN12-3-4 & 947.1 & 20.1 & 950.3 & 17.4 & 957.7 & 33.8 & 957.7 & 33.8 & 98.9 \\
\hline SYN12-3-7 & 935.2 & 26.1 & 944.0 & 21.0 & 964.4 & 33.3 & 964.4 & 33.3 & 97.0 \\
\hline SYN12-3-85 & 999.5 & 7.1 & 991.5 & 8.4 & 974.0 & 22.1 & 974.0 & 22.1 & 102.6 \\
\hline SYN12-3-19 & 1030.7 & 48.1 & 1016.2 & 36.1 & 985.0 & 50.2 & 985.0 & 50.2 & 104.6 \\
\hline SYN12-3-88 & 1035.9 & 12.2 & 1031.6 & 10.3 & 1022.6 & 19.1 & 1022.6 & 19.1 & 101.3 \\
\hline SYN12-3-40 & 1058.6 & 11.4 & 1047.8 & 11.0 & 1025.2 & 24.7 & 1025.2 & 24.7 & 103.3 \\
\hline SYN12-3-41 & 1031.9 & 11.6 & 1032.8 & 10.4 & 1034.5 & 21.3 & 1034.5 & 21.3 & 99.7 \\
\hline SYN12-3-74 & 1070.9 & 9.2 & 1061.9 & 7.6 & 1043.5 & 14.0 & 1043.5 & 14.0 & 102.6 \\
\hline SYN12-3-45 & 1093.4 & 14.8 & 1076.9 & 18.6 & 1043.8 & 48.1 & 1043.8 & 48.1 & 104.7 \\
\hline SYN12-3-12 & 1071.6 & 10.5 & 1062.8 & 7.6 & 1044.9 & 9.2 & 1044.9 & 9.2 & 102.6 \\
\hline SYN12-3-31 & 1087.2 & 19.1 & 1076.8 & 14.3 & 1055.6 & 20.2 & 1055.6 & 20.2 & 103.0 \\
\hline SYN12-3-21 & 1074.3 & 23.5 & 1069.7 & 16.4 & 1060.4 & 14.6 & 1060.4 & 14.6 & 101.3 \\
\hline 61 & 1081.4 & 14.9 & 1075.8 & 13.7 & 1064.4 & 28.8 & 1064.4 & 28.8 & 101.6 \\
\hline SYN12-3-62 & 1089.5 & 9.9 & 1081.5 & 10.2 & 1065.3 & 23.6 & 1065.3 & 23.6 & 102.3 \\
\hline SYN12-3-94 & 1075.9 & 14.7 & 1077.1 & 28.7 & 1079.6 & 81.5 & 1079.6 & 81.5 & 99.7 \\
\hline SYN12-3-89 & 1092.2 & 12.0 & 1089.9 & 13.0 & 1085.4 & 30.9 & 1085.4 & 30.9 & 100.6 \\
\hline SYN12-3-58 & 1137.3 & 13.1 & 1122.6 & 21.3 & 1094.3 & 57.4 & 1094.3 & 57.4 & 103.9 \\
\hline SYN12-3-26 & 1112.9 & 16.8 & 1107.2 & 21.5 & 1096.1 & 54.7 & 1096.1 & 54.7 & 101.5 \\
\hline SYN12-3-25 & 1070.1 & 21.2 & 1079.2 & 18.2 & 1097.7 & 34.1 & 1097.7 & 34.1 & 97.5 \\
\hline SYN12-3-87 & 1093.9 & 25.2 & 1100.4 & 19.8 & 1113.2 & 31.1 & 1113.2 & 31.1 & 98.3 \\
\hline SYN12-3-54 & 1086.0 & 79.1 & 1105.3 & 61.4 & 1143.6 & 89.7 & 1143.6 & 89.7 & 95.0 \\
\hline SYN12-3-51 & 1236.1 & 19.7 & 1219.0 & 28.3 & 1188.8 & 70.6 & 1188.8 & 70.6 & 104.0 \\
\hline SYN12-3-16 & 1194.9 & 17.7 & 1192.8 & 16.7 & 1189.2 & 34.2 & 1189.2 & 34.2 & 100.5 \\
\hline SYN12-3-55 & 1248.7 & 19.0 & 1228.3 & 12.3 & 1192.5 & 8.6 & 1192.5 & 8.6 & 104.7 \\
\hline SYN12-3-97 & 1215.4 & 21.7 & 1217.4 & 17.3 & 1220.8 & 28.7 & 1220.8 & 28.7 & 99.6 \\
\hline SYN12-3-10 & 1269.4 & 16.5 & 1290.3 & 23.0 & 1325.2 & 54.2 & 1325.2 & 54.2 & 95.8 \\
\hline SYN12-3-22 & 1328.7 & 21.0 & 1329.0 & 19.4 & 1329.5 & 37.5 & 1329.5 & 37.5 & 99.9 \\
\hline SYN12-3-2 & 1396.7 & 13.6 & 1388.7 & 9.4 & 1376.5 & 11.7 & 1376.5 & 11.7 & 101.5 \\
\hline SYN12-3-18 & 1529.7 & 19.2 & 1519.4 & 13.5 & 1505.0 & 18.4 & 1505.0 & 18.4 & 101.6 \\
\hline SYN12-3-3 & 1561.9 & 15.0 & 1539.9 & 8.7 & 1509.9 & 2.7 & 1509.9 & 2.7 & 103.4 \\
\hline SYN12-3-73 & 1508.7 & 11.2 & 1509.9 & 7.1 & 1511.7 & 6.6 & 1511.7 & 6.6 & 99.8 \\
\hline SYN12-3-64 & 1592.4 & 23.8 & 1560.5 & 13.8 & 1517.7 & 7.1 & 1517.7 & 7.1 & 104.9 \\
\hline SYN12-3-48 & 1670.0 & 17.7 & 1651.0 & 10.1 & 1626.9 & 5.2 & 1626.9 & 5.2 & 102.7 \\
\hline
\end{tabular}




\begin{tabular}{|c|c|c|c|c|c|c|c|c|c|}
\hline SYN12-3-96 & 1661.1 & 13.5 & 1647.7 & 16.9 & 1630.5 & 34.4 & 1630.5 & 34.4 & 101.9 \\
\hline SYN12-3-30 & 1669.7 & 25.4 & 1656.0 & 15.9 & 1638.6 & 16.9 & 1638.6 & 16.9 & 101.9 \\
\hline SYN12-3-24 & 1654.7 & 18.5 & 1648.5 & 11.5 & 1640.6 & 11.4 & 1640.6 & 11.4 & 100.9 \\
\hline SYN12-3-77 & 1688.8 & 34.3 & 1668.6 & 19.3 & 1643.2 & 8.7 & 1643.2 & 8.7 & 102.8 \\
\hline SYN12-3-42 & 1686.7 & 40.6 & 1668.2 & 22.6 & 1645.1 & 6.2 & 1645.1 & 6.2 & 102.5 \\
\hline 81 & 1677.9 & 18.0 & 1663.9 & 12.8 & 1646.2 & 18.4 & 1646.2 & 18.4 & 101.9 \\
\hline SYN12-3-67 & 1641.9 & 36.0 & 1648.5 & 21.0 & 1657.0 & 12.9 & 1657.0 & 12.9 & 99.1 \\
\hline SYN12-3-82 & 1715.1 & 46.1 & 1692.7 & 25.6 & 1665.0 & 10.4 & 1665.0 & 10.4 & 103.0 \\
\hline SYN12-3-53 & 1707.5 & 58.5 & 1689.3 & 33.4 & 1666.6 & 20.3 & 1666.6 & 20.3 & 102.5 \\
\hline SYN12-3-91 & 1692.3 & 18.9 & 1685.1 & 11.4 & 1676.2 & 10.4 & 1676.2 & 10.4 & 101.0 \\
\hline SYN12-3-56 & 1676.2 & 30.5 & 1693.0 & 21.5 & 1713.9 & 29.3 & 1713.9 & 29.3 & 97.8 \\
\hline SYN12-3-83 & 1816.8 & 28.4 & 1801.3 & 17.5 & 1783.4 & 19.0 & 1783.4 & 19.0 & 101.9 \\
\hline SYN12-3-27 & 1714.5 & 31.6 & 1746.5 & 18.1 & 1785.1 & 9.7 & 1785.1 & 9.7 & 96.0 \\
\hline SYN12-3-33 & 1811.0 & 73.1 & 1804.7 & 39.4 & 1797.4 & 11.1 & 1797.4 & 11.1 & 100.8 \\
\hline SYN12-3-8 & 1796.5 & 22.6 & 1799.4 & 12.3 & 1802.8 & 5.0 & 1802.8 & 5.0 & 99.7 \\
\hline SYN12-3-14 & 1837.7 & 11.7 & 1823.3 & 7.2 & 1806.8 & 8.0 & 1806.8 & 8.0 & 101.7 \\
\hline SYN12-3-76 & 1866.8 & 23.5 & 1846.4 & 13.7 & 1823.5 & 12.7 & 1823.5 & 12.7 & 102.4 \\
\hline SYN12-3-11 & 1844.9 & 59.4 & 1850.5 & 33.7 & 1856.8 & 25.2 & 1856.8 & 25.2 & 99.4 \\
\hline SYN12-3-69 & 1910.1 & 10.3 & 1897.2 & 5.9 & 1883.1 & 5.5 & 1883.1 & 5.5 & 101.4 \\
\hline SYN12-3-66 & 1943.6 & 30.1 & 1931.0 & 15.8 & 1917.6 & 6.7 & 1917.6 & 6.7 & 101.4 \\
\hline SYN12-3-70 & 2071.4 & 27.6 & 2048.0 & 14.5 & 2024.5 & 9.6 & 2024.5 & 9.6 & 102.3 \\
\hline SYN12-3-90 & 2099.1 & 39.9 & 2089.3 & 19.8 & 2079.7 & 4.2 & 2079.7 & 4.2 & 100.9 \\
\hline SYN12-3-75 & 1961.4 & 23.8 & 2062.2 & 12.5 & 2164.6 & 2.9 & 2164.6 & 2.9 & 90.6 \\
\hline SYN12-3-13 & 2597.8 & 54.3 & 2573.1 & 25.7 & 2553.7 & 17.6 & 2553.7 & 17.6 & 101.7 \\
\hline SYN12-3-43 & 2694.3 & 48.2 & 2654.4 & 20.7 & 2624.1 & 4.3 & 2624.1 & 4.3 & 102.7 \\
\hline SYN12-3-29 & 2757.0 & 27.2 & 2728.8 & 11.6 & 2708.1 & 2.8 & 2708.1 & 2.8 & 101.8 \\
\hline SYN12-3-63 & 2807.9 & 34.3 & 2753.7 & 15.8 & 2714.3 & 11.6 & 2714.3 & 11.6 & 103.4 \\
\hline SYN12-3-6 & 2547.0 & 55.5 & 2642.3 & 24.9 & 2716.1 & 4.9 & 2716.1 & 4.9 & 93.8 \\
\hline SYN12-3-17 & 2745.2 & 24.6 & 2740.2 & 10.6 & 2736.5 & 3.6 & 2736.5 & 3.6 & 100.3 \\
\hline SYN12-3-57 & 2945.8 & 74.7 & 2911.8 & 30.6 & 2888.4 & 7.4 & 2888.4 & 7.4 & 102.0 \\
\hline
\end{tabular}

\begin{tabular}{|l|l|l|l|l|l|l|l|l|l|}
\hline Analysis & $206 \mathrm{~Pb}^{*}$ & \pm & $207 \mathrm{~Pb}^{*}$ & \pm & $206 \mathrm{~Pb}^{*}$ & \pm & Best age & \pm & Conc \\
\hline & $238 \mathrm{U}^{*}$ & $(\mathrm{Ma})$ & $235 \mathrm{U}$ & $(\mathrm{Ma})$ & $207 \mathrm{~Pb}^{*}$ & $(\mathrm{Ma})$ & $(\mathrm{Ma})$ & $(\mathrm{Ma})$ & $(\%)$ \\
\hline & & & & & & & & & \\
\hline & & & & & & & & & \\
\hline & & & & & & & & & \\
\hline SYN12-5-16 & 165.2 & 3.7 & 161.9 & 14.4 & 113.6 & 221.8 & 165.2 & 3.7 & NA \\
\hline SYN12-5-25 & 168.0 & 4.1 & 161.1 & 12.2 & 60.9 & 186.8 & 168.0 & 4.1 & NA \\
\hline SYN12-5-76 & 168.7 & 3.3 & 177.9 & 14.4 & 302.4 & 196.4 & 168.7 & 3.3 & NA \\
\hline
\end{tabular}




\begin{tabular}{|c|c|c|c|c|c|c|c|c|c|}
\hline SYN12-5-58 & 168.8 & 2.8 & 164.3 & 21.0 & 100.5 & 325.7 & 168.8 & 2.8 & NA \\
\hline SYN12-5-46 & 169.3 & 5.7 & 165.7 & 15.9 & 114.0 & 232.5 & 169.3 & 5.7 & NA \\
\hline SYN12-5-101 & 169.3 & 2.3 & 168.6 & 20.8 & 158.6 & 312.9 & 169.3 & 2.3 & NA \\
\hline SYN12-5-23 & 170.8 & 5.7 & 171.8 & 11.9 & 186.2 & 156.2 & 170.8 & 5.7 & NA \\
\hline SYN12-5-9 & 171.0 & 13.6 & 168.2 & 17.2 & 128.9 & 180.1 & 171.0 & 13.6 & NA \\
\hline SYN12-5-99 & 171.1 & 11.0 & 144.3 & 63.9 & -276.3 & 1256.3 & 171.1 & 11.0 & NA \\
\hline SYN12-5-66 & 176.2 & 16.4 & 181.4 & 71.5 & 249.9 & 1005.9 & 176.2 & 16.4 & NA \\
\hline SYN12-5-26 & 218.0 & 7.4 & 228.6 & 25.3 & 339.1 & 269.3 & 218.0 & 7.4 & NA \\
\hline SYN12-5-80 & 224.4 & 9.6 & 248.6 & 45.1 & 483.7 & 445.5 & 224.4 & 9.6 & NA \\
\hline SYN12-5-75 & 237.8 & 7.6 & 257.6 & 16.8 & 442.8 & 147.6 & 237.8 & 7.6 & NA \\
\hline SYN12-5-20 & 239.9 & 4.4 & 239.1 & 18.1 & 230.8 & 191.6 & 239.9 & 4.4 & NA \\
\hline SYN12-5-32 & 240.8 & 4.8 & 242.1 & 30.2 & 255.3 & 320.1 & 240.8 & 4.8 & NA \\
\hline SYN12-5-11 & 241.1 & 4.8 & 228.3 & 39.9 & 97.5 & 462.8 & 241.1 & 4.8 & NA \\
\hline SYN12-5-62 & 242.8 & 3.8 & 239.6 & 18.4 & 208.6 & 196.5 & 242.8 & 3.8 & NA \\
\hline SYN12-5-36 & 243.5 & 15.2 & 258.7 & 19.5 & 399.2 & 128.1 & 243.5 & 15.2 & NA \\
\hline SYN12-5-8 & 307.0 & 4.3 & 304.4 & 11.9 & 284.6 & 98.1 & 307.0 & 4.3 & NA \\
\hline SYN12-5-12 & 324.2 & 13.3 & 322.4 & 72.3 & 309.5 & 595.7 & 324.2 & 13.3 & NA \\
\hline SYN12-5-85 & 385.6 & 5.9 & 384.2 & 11.3 & 376.1 & 71.4 & 385.6 & 5.9 & NA \\
\hline SYN12-5-105 & 388.6 & 4.0 & 385.9 & 13.4 & 369.6 & 90.8 & 388.6 & 4.0 & NA \\
\hline SYN12-5-1 & 402.1 & 30.4 & 291.4 & 92.8 & -523.1 & 980.5 & 402.1 & 30.4 & NA \\
\hline SYN12-5-35 & 413.1 & 11.1 & 423.3 & 21.9 & 479.5 & 125.5 & 413.1 & 11.1 & 86.1 \\
\hline SYN12-5-104 & 421.2 & 2.7 & 428.0 & 6.1 & 464.8 & 35.7 & 421.2 & 2.7 & 90.6 \\
\hline SYN12-5-92 & 433.9 & 5.2 & 430.9 & 9.6 & 414.9 & 54.7 & 433.9 & 5.2 & 104.6 \\
\hline SYN12-5-49 & 458.3 & 9.2 & 458.4 & 30.3 & 458.8 & 176.4 & 458.3 & 9.2 & 99.9 \\
\hline SYN12-5-37 & 472.1 & 5.5 & 455.0 & 20.4 & 369.4 & 122.3 & 472.1 & 5.5 & 127.8 \\
\hline SYN12-5-90 & 525.0 & 8.5 & 524.3 & 27.2 & 521.6 & 140.8 & 525.0 & 8.5 & 100.7 \\
\hline SYN12-5-50 & 529.6 & 5.5 & 502.3 & 27.5 & 380.0 & 154.5 & 529.6 & 5.5 & 139.4 \\
\hline SYN12-5-14 & 539.8 & 7.2 & 544.4 & 20.1 & 563.9 & 99.2 & 539.8 & 7.2 & 95.7 \\
\hline SYN12-5-63 & 540.3 & 11.1 & 555.5 & 25.7 & 618.5 & 121.4 & 540.3 & 11.1 & 87.3 \\
\hline SYN12-5-55 & 543.5 & 4.2 & 542.9 & 8.2 & 540.1 & 38.7 & 543.5 & 4.2 & 100.6 \\
\hline SYN12-5-43 & 547.5 & 4.6 & 538.2 & 15.4 & 498.9 & 78.9 & 547.5 & 4.6 & 109.7 \\
\hline SYN12-5-81 & 571.6 & 9.1 & 568.2 & 16.5 & 554.5 & 74.5 & 571.6 & 9.1 & 103.1 \\
\hline SYN12-5-39 & 588.0 & 7.2 & 584.1 & 19.0 & 568.9 & 88.6 & 588.0 & 7.2 & 103.4 \\
\hline SYN12-5-95 & 588.3 & 10.7 & 604.9 & 33.1 & 667.7 & 150.3 & 588.3 & 10.7 & 88.1 \\
\hline SYN12-5-17 & 592.6 & 5.8 & 599.6 & 13.5 & 626.2 & 60.2 & 592.6 & 5.8 & 94.6 \\
\hline SYN12-5-34 & 596.7 & 15.9 & 560.3 & 67.4 & 415.0 & 345.7 & 596.7 & 15.9 & 143.8 \\
\hline SYN12-5-40 & 627.1 & 6.2 & 622.9 & 18.5 & 607.7 & 83.1 & 627.1 & 6.2 & 103.2 \\
\hline SYN12-5-74 & 631.3 & 9.1 & 638.9 & 15.7 & 665.8 & 63.1 & 631.3 & 9.1 & 94.8 \\
\hline SYN12-5-82 & 646.7 & 9.1 & 649.1 & 16.1 & 657.3 & 64.7 & 646.7 & 9.1 & 98.4 \\
\hline
\end{tabular}




\begin{tabular}{|c|c|c|c|c|c|c|c|c|c|}
\hline SYN12-5-79 & 756.3 & 18.3 & 750.9 & 44.1 & 735.0 & 167.5 & 756.3 & 18.3 & 102.9 \\
\hline SYN12-5-30 & 820.1 & 15.0 & 816.6 & 22.1 & 807.0 & 71.8 & 820.1 & 15.0 & 101.6 \\
\hline SYN12-5-4 & 1027.1 & 8.7 & 1012.3 & 20.8 & 980.4 & 63.5 & 980.4 & 63.5 & 104.8 \\
\hline SYN12-5-77 & 1020.6 & 12.4 & 1011.3 & 24.5 & 991.0 & 73.0 & 991.0 & 73.0 & 103.0 \\
\hline SYN12-5-22 & 1020.3 & 22.9 & 1011.3 & 31.7 & 991.9 & 87.9 & 991.9 & 87.9 & 102.9 \\
\hline SYN12-5-98 & 1042.2 & 29.3 & 1031.2 & 22.3 & 1007.9 & 32.3 & 1007.9 & 32.3 & 103.4 \\
\hline SYN12-5-45 & 1032.5 & 7.8 & 1027.7 & 11.4 & 1017.6 & 31.7 & 1017.6 & 31.7 & 101.5 \\
\hline SYN12-5-7 & 1024.9 & 9.5 & 1023.0 & 8.1 & 1019.0 & 15.5 & 1019.0 & 15.5 & 100.6 \\
\hline SYN12-5-52 & 1066.6 & 19.0 & 1052.3 & 45.7 & 1022.6 & 135.6 & 1022.6 & 135.6 & 104.3 \\
\hline SYN12-5-28 & 1030.7 & 16.6 & 1029.2 & 18.0 & 1026.0 & 44.1 & 1026.0 & 44.1 & 100.5 \\
\hline SYN12-5-3 & 1000.3 & 9.5 & 1008.4 & 19.2 & 1026.0 & 57.0 & 1026.0 & 57.0 & 97.5 \\
\hline SYN12-5-72 & 1021.1 & 10.3 & 1023.2 & 8.0 & 1027.6 & 12.0 & 1027.6 & 12.0 & 99.4 \\
\hline SYN12-5-91 & 1042.3 & 6.2 & 1038.4 & 12.2 & 1030.1 & 35.7 & 1030.1 & 35.7 & 101.2 \\
\hline SYN12-5-57 & 1017.2 & 15.0 & 1022.2 & 10.6 & 1033.1 & 8.4 & 1033.1 & 8.4 & 98.5 \\
\hline SYN12-5-70 & 1036.2 & 5.1 & 1035.3 & 8.9 & 1033.4 & 25.5 & 1033.4 & 25.5 & 100.3 \\
\hline SYN12-5-83 & 1032.1 & 8.0 & 1032.6 & 10.0 & 1033.7 & 26.2 & 1033.7 & 26.2 & 99.8 \\
\hline SYN12-5-27 & 1029.3 & 18.4 & 1031.0 & 23.5 & 1034.7 & 62.1 & 1034.7 & 62.1 & 99.5 \\
\hline SYN12-5-2 & 1045.3 & 30.3 & 1042.3 & 37.5 & 1035.9 & 97.4 & 1035.9 & 97.4 & 100.9 \\
\hline SYN12-5-94 & 1043.9 & 15.1 & 1042.0 & 20.3 & 1037.9 & 54.4 & 1037.9 & 54.4 & 100.6 \\
\hline SYN12-5-73 & 997.2 & 8.8 & 1010.9 & 16.7 & 1040.7 & 49.0 & 1040.7 & 49.0 & 95.8 \\
\hline SYN12-5-89 & 1032.6 & 11.8 & 1036.6 & 11.2 & 1045.2 & 24.3 & 1045.2 & 24.3 & 98.8 \\
\hline SYN12-5-67 & 997.9 & 12.0 & 1014.3 & 25.2 & 1049.9 & 74.8 & 1049.9 & 74.8 & 95.0 \\
\hline SYN12-5-47 & 1021.3 & 23.9 & 1031.4 & 57.5 & 1052.9 & 171.6 & 1052.9 & 171.6 & 97.0 \\
\hline SYN12-5-33 & 1050.1 & 6.3 & 1054.7 & 6.9 & 1064.2 & 16.8 & 1064.2 & 16.8 & 98.7 \\
\hline SYN12-5-13 & 1064.8 & 8.4 & 1067.3 & 12.0 & 1072.4 & 32.2 & 1072.4 & 32.2 & 99.3 \\
\hline SYN12-5-31 & 1025.0 & 19.5 & 1044.6 & 17.8 & 1085.8 & 35.7 & 1085.8 & 35.7 & 94.4 \\
\hline SYN12-5-78 & 1077.8 & 8.9 & 1083.1 & 27.3 & 1093.7 & 80.0 & 1093.7 & 80.0 & 98.6 \\
\hline SYN12-5-42 & 1047.3 & 28.1 & 1073.1 & 63.8 & 1125.9 & 183.2 & 1125.9 & 183.2 & 93.0 \\
\hline SYN12-5-41 & 1134.2 & 82.7 & 1134.1 & 55.2 & 1134.0 & 28.8 & 1134.0 & 28.8 & 100.0 \\
\hline SYN12-5-19 & 1200.6 & 17.7 & 1181.5 & 24.2 & 1146.7 & 60.9 & 1146.7 & 60.9 & 104.7 \\
\hline SYN12-5-64 & 1187.1 & 9.7 & 1173.4 & 16.3 & 1148.2 & 43.1 & 1148.2 & 43.1 & 103.4 \\
\hline SYN12-5-103 & 1112.1 & 29.2 & 1125.1 & 19.9 & 1150.1 & 12.3 & 1150.1 & 12.3 & 96.7 \\
\hline SYN12-5-86 & 1172.4 & 47.8 & 1171.3 & 74.6 & 1169.2 & 193.3 & 1169.2 & 193.3 & 100.3 \\
\hline SYN12-5-100 & 1255.9 & 12.2 & 1249.1 & 8.9 & 1237.4 & 12.5 & 1237.4 & 12.5 & 101.5 \\
\hline SYN12-5-44 & 1346.7 & 15.0 & 1335.3 & 10.6 & 1317.2 & 13.9 & 1317.2 & 13.9 & 102.2 \\
\hline SYN12-5-24 & 1348.1 & 14.7 & 1341.2 & 12.6 & 1330.1 & 22.8 & 1330.1 & 22.8 & 101.4 \\
\hline SYN12-5-51 & 1444.7 & 24.2 & 1433.8 & 16.4 & 1417.7 & 19.9 & 1417.7 & 19.9 & 101.9 \\
\hline SYN12-5-15 & 1450.9 & 12.3 & 1443.6 & 11.9 & 1432.8 & 23.4 & 1432.8 & 23.4 & 101.3 \\
\hline SYN12-5-54 & 1440.0 & 21.8 & 1439.5 & 14.0 & 1438.6 & 12.9 & 1438.6 & 12.9 & 100.1 \\
\hline
\end{tabular}




\begin{tabular}{|l|l|l|l|l|l|l|l|l|l|} 
SYN12-5-48 & 1461.9 & 7.9 & 1455.5 & 8.5 & 1446.2 & 17.5 & 1446.2 & 17.5 & 101.1 \\
\hline SYN12-5-102 & 1656.4 & 19.5 & 1655.6 & 12.2 & 1654.5 & 12.2 & 1654.5 & 12.2 & 100.1 \\
\hline SYN12-5-71 & 1657.5 & 17.2 & 1662.7 & 17.8 & 1669.2 & 33.8 & 1669.2 & 33.8 & 99.3 \\
\hline SYN12-5-97 & 1716.3 & 15.0 & 1717.6 & 11.2 & 1719.3 & 16.7 & 1719.3 & 16.7 & 99.8 \\
\hline SYN12-5-6 & 1701.5 & 13.6 & 1713.7 & 10.7 & 1728.5 & 16.8 & 1728.5 & 16.8 & 98.4 \\
\hline SYN12-5-69 & 1712.6 & 23.9 & 1725.4 & 13.8 & 1741.1 & 8.9 & 1741.1 & 8.9 & 98.4 \\
\hline SYN12-5-61 & 1739.9 & 33.0 & 1742.6 & 18.4 & 1745.9 & 8.0 & 1745.9 & 8.0 & 99.7 \\
\hline SYN12-5-5 & 1813.7 & 15.1 & 1801.4 & 13.6 & 1787.1 & 23.6 & 1787.1 & 23.6 & 101.5 \\
\hline SYN12-5-38 & 1842.5 & 11.2 & 1840.6 & 10.3 & 1838.4 & 18.0 & 1838.4 & 18.0 & 100.2 \\
\hline SYN12-5-60 & 1996.6 & 13.9 & 1992.0 & 12.7 & 1987.3 & 21.5 & 1987.3 & 21.5 & 100.5 \\
\hline SYN12-5-53 & 2087.7 & 46.5 & 2080.3 & 24.0 & 2072.9 & 13.4 & 2072.9 & 13.4 & 100.7 \\
\hline SYN12-5-29 & 2113.3 & 12.4 & 2104.3 & 8.7 & 2095.6 & 12.1 & 2095.6 & 12.1 & 100.8 \\
\hline SYN12-5-88 & 2523.1 & 18.4 & 2504.3 & 10.1 & 2489.1 & 10.7 & 2489.1 & 10.7 & 101.4 \\
\hline SYN12-5-59 & 2657.8 & 27.9 & 2669.1 & 13.4 & 2677.7 & 10.2 & 2677.7 & 10.2 & 99.3 \\
\hline SYN12-5-56 & 2939.9 & 51.1 & 3029.2 & 21.0 & 3089.0 & 3.3 & 3089.0 & 3.3 & 95.2 \\
\hline
\end{tabular}

\begin{tabular}{|l|l|l|l|l|l|l|l|l|l|}
\hline Analysis & $206 \mathrm{~Pb}^{*}$ & \pm & $207 \mathrm{~Pb}^{*}$ & \pm & $206 \mathrm{~Pb}^{*}$ & \pm & Best age & \pm & Conc \\
\hline & $238 \mathrm{U}^{*}$ & $(\mathrm{Ma})$ & $235 \mathrm{U}$ & $(\mathrm{Ma})$ & $207 \mathrm{~Pb}$ & $(\mathrm{Ma})$ & $(\mathrm{Ma})$ & $(\mathrm{Ma})$ & $(\%)$ \\
\hline & & & & & & & & & \\
\hline & & & & & & & & & \\
\hline & & & & & & & & & \\
\hline SYN12-6-21 & 99.5 & 1.4 & 93.3 & 9.1 & -63.8 & 246.9 & 99.5 & 1.4 & NA \\
\hline SYN12-6-101 & 151.4 & 2.6 & 157.5 & 4.4 & 250.2 & 56.1 & 151.4 & 2.6 & NA \\
\hline SYN12-6-103 & 155.4 & 2.7 & 156.8 & 6.5 & 178.5 & 95.5 & 155.4 & 2.7 & NA \\
\hline SYN12-6-68 & 157.9 & 3.5 & 156.8 & 4.1 & 140.2 & 40.5 & 157.9 & 3.5 & NA \\
\hline SYN12-6-60 & 159.2 & 3.3 & 162.2 & 18.1 & 205.5 & 277.3 & 159.2 & 3.3 & NA \\
\hline SYN12-6-69 & 159.2 & 3.0 & 157.5 & 4.4 & 131.6 & 55.5 & 159.2 & 3.0 & NA \\
\hline SYN12-6-40 & 160.7 & 3.6 & 161.3 & 7.7 & 169.1 & 107.8 & 160.7 & 3.6 & NA \\
\hline SYN12-6-88 & 162.1 & 3.5 & 160.2 & 7.1 & 131.6 & 100.3 & 162.1 & 3.5 & NA \\
\hline SYN12-6-83 & 162.2 & 4.3 & 164.2 & 4.6 & 193.8 & 34.7 & 162.2 & 4.3 & NA \\
\hline SYN12-6-100 & 228.6 & 3.8 & 224.5 & 11.9 & 181.7 & 131.7 & 228.6 & 3.8 & NA \\
\hline SYN12-6-23 & 228.8 & 17.8 & 286.1 & 75.9 & 785.0 & 629.2 & 228.8 & 17.8 & NA \\
\hline SYN12-6-29 & 238.3 & 10.7 & 224.6 & 50.5 & 83.3 & 592.1 & 238.3 & 10.7 & NA \\
\hline SYN12-6-37 & 240.8 & 5.0 & 238.2 & 14.9 & 212.8 & 154.8 & 240.8 & 5.0 & NA \\
\hline SYN12-6-05 & 249.0 & 8.2 & 243.5 & 12.5 & 190.8 & 108.4 & 249.0 & 8.2 & NA \\
\hline SYN12-6-66 & 281.1 & 8.2 & 290.5 & 18.5 & 366.9 & 151.1 & 281.1 & 8.2 & NA \\
\hline SYN12-6-81 & 287.1 & 6.7 & 283.9 & 10.8 & 257.8 & 83.9 & 287.1 & 6.7 & NA \\
\hline SYN12-6-65 & 312.4 & 8.9 & 307.5 & 14.8 & 271.2 & 109.3 & 312.4 & 8.9 & NA \\
\hline SYN12-6-50 & 422.0 & 9.8 & 414.2 & 16.6 & 370.8 & 96.0 & 422.0 & 9.8 & 113.8 \\
\hline & & & & & & & & & \\
\hline & & & & & & & \\
\hline
\end{tabular}




\begin{tabular}{|c|c|c|c|c|c|c|c|c|c|}
\hline SYN12-6-63 & 427.8 & 11.0 & 431.0 & 29.8 & 448.1 & 179.1 & 427.8 & 11.0 & 95.5 \\
\hline SYN12-6-11 & 436.0 & 9.4 & 442.0 & 28.9 & 473.2 & 171.9 & 436.0 & 9.4 & 92.1 \\
\hline SYN12-6-101 & 454.0 & 11.1 & 546.6 & 69.5 & 953.8 & 333.8 & 454.0 & 11.1 & 47.6 \\
\hline SYN12-6-75 & 472.1 & 13.4 & 465.5 & 13.9 & 433.0 & 51.5 & 472.1 & 13.4 & 109.0 \\
\hline SYN12-6-04 & 482.6 & 4.0 & 487.3 & 15.7 & 509.6 & 87.3 & 482.6 & 4.0 & 94.7 \\
\hline SYN12-6-30 & 490.0 & 10.3 & 484.5 & 15.7 & 458.6 & 76.4 & 490.0 & 10.3 & 106.8 \\
\hline SYN12-6-104 & 535.7 & 7.5 & 526.2 & 10.6 & 485.2 & 47.3 & 535.7 & 7.5 & 110.4 \\
\hline SYN12-6-91 & 538.5 & 19.1 & 537.4 & 32.1 & 533.1 & 147.8 & 538.5 & 19.1 & 101.0 \\
\hline SYN12-6-34 & 587.0 & 7.8 & 585.6 & 17.2 & 580.0 & 78.2 & 587.0 & 7.8 & 101.2 \\
\hline SYN12-6-31 & 600.3 & 10.2 & 603.2 & 11.6 & 614.0 & 39.5 & 600.3 & 10.2 & 97.8 \\
\hline SYN12-6-43 & 605.7 & 7.8 & 609.8 & 11.3 & 625.3 & 44.5 & 605.7 & 7.8 & 96.9 \\
\hline SYN12-6-06 & 607.3 & 13.8 & 611.7 & 22.2 & 627.9 & 90.6 & 607.3 & 13.8 & 96.7 \\
\hline SYN12-6-78 & 619.3 & 10.6 & 617.5 & 13.0 & 611.1 & 47.0 & 619.3 & 10.6 & 101.3 \\
\hline SYN12-6-73 & 629.0 & 13.1 & 623.9 & 15.3 & 605.4 & 52.8 & 629.0 & 13.1 & 103.9 \\
\hline SYN12-6-92 & 934.8 & 15.7 & 923.9 & 14.8 & 898.1 & 34.0 & 898.1 & 34.0 & 104.1 \\
\hline SYN12-6-94 & 953.1 & 18.2 & 947.3 & 40.2 & 934.0 & 127.0 & 934.0 & 127.0 & 102.1 \\
\hline SYN12-6-93 & 1019.8 & 28.1 & 1008.6 & 52.7 & 984.2 & 156.6 & 984.2 & 156.6 & 103.6 \\
\hline SYN12-6-13 & 997.3 & 11.2 & 1002.0 & 9.4 & 1012.3 & 16.9 & 1012.3 & 16.9 & 98.5 \\
\hline SYN12-6-14 & 988.5 & 25.6 & 997.6 & 18.2 & 1017.6 & 12.9 & 1017.6 & 12.9 & 97.1 \\
\hline SYN12-6-57 & 1027.9 & 19.9 & 1026.4 & 25.3 & 1023.1 & 66.9 & 1023.1 & 66.9 & 100.5 \\
\hline SYN12-6-56 & 1029.4 & 15.6 & 1030.4 & 21.8 & 1032.6 & 59.5 & 1032.6 & 59.5 & 99.7 \\
\hline SYN12-6-76 & 1069.0 & 19.2 & 1057.6 & 32.8 & 1034.0 & 92.7 & 1034.0 & 92.7 & 103.4 \\
\hline SYN12-6-44 & 1018.2 & 18.3 & 1024.0 & 15.2 & 1036.3 & 27.0 & 1036.3 & 27.0 & 98.3 \\
\hline SYN12-6-67 & 1036.0 & 16.0 & 1041.7 & 36.1 & 1053.6 & 106.3 & 1053.6 & 106.3 & 98.3 \\
\hline SYN12-6-55 & 1053.3 & 13.6 & 1054.1 & 10.6 & 1055.5 & 16.1 & 1055.5 & 16.1 & 99.8 \\
\hline SYN12-6-87 & 1070.2 & 10.8 & 1065.9 & 8.7 & 1057.1 & 14.9 & 1057.1 & 14.9 & 101.2 \\
\hline SYN12-6-22 & 1021.1 & 20.2 & 1032.7 & 18.7 & 1057.5 & 38.9 & 1057.5 & 38.9 & 96.6 \\
\hline SYN12-6-70 & 1075.8 & 27.5 & 1073.4 & 21.7 & 1068.8 & 35.2 & 1068.8 & 35.2 & 100.7 \\
\hline SYN12-6-18 & 1069.5 & 24.1 & 1069.5 & 19.5 & 1069.5 & 32.8 & 1069.5 & 32.8 & 100.0 \\
\hline SYN12-6-38 & 1046.5 & 22.1 & 1054.0 & 27.1 & 1069.6 & 69.2 & 1069.6 & 69.2 & 97.8 \\
\hline SYN12-6-51 & 1103.1 & 24.8 & 1096.4 & 23.7 & 1083.0 & 51.3 & 1083.0 & 51.3 & 101.9 \\
\hline SYN12-6-15 & 1085.5 & 10.2 & 1087.8 & 14.6 & 1092.6 & 38.8 & 1092.6 & 38.8 & 99.3 \\
\hline SYN12-6-01 & 1068.6 & 8.5 & 1078.9 & 8.7 & 1099.8 & 19.5 & 1099.8 & 19.5 & 97.2 \\
\hline SYN12-6-52 & 1092.6 & 24.2 & 1097.1 & 31.9 & 1106.0 & 82.1 & 1106.0 & 82.1 & 98.8 \\
\hline SYN12-6-96 & 1120.4 & 17.7 & 1115.5 & 12.0 & 1106.0 & 7.7 & 1106.0 & 7.7 & 101.3 \\
\hline SYN12-6-20 & 1092.3 & 19.2 & 1098.0 & 17.0 & 1109.2 & 33.2 & 1109.2 & 33.2 & 98.5 \\
\hline SYN12-6-89 & 1130.2 & 16.8 & 1125.8 & 11.8 & 1117.4 & 12.0 & 1117.4 & 12.0 & 101.1 \\
\hline SYN12-6-09 & 1069.2 & 23.7 & 1087.4 & 34.3 & 1123.9 & 90.5 & 1123.9 & 90.5 & 95.1 \\
\hline SYN12-6-98 & 1156.5 & 15.8 & 1149.6 & 11.6 & 1136.6 & 15.9 & 1136.6 & 15.9 & 101.8 \\
\hline
\end{tabular}




\begin{tabular}{|c|c|c|c|c|c|c|c|c|c|}
\hline SYN12-6-54 & 1119.7 & 19.2 & 1126.2 & 21.5 & 1138.7 & 50.5 & 1138.7 & 50.5 & 98.3 \\
\hline SYN12-6-48 & 1163.6 & 15.2 & 1156.5 & 15.9 & 1143.1 & 35.9 & 1143.1 & 35.9 & 101.8 \\
\hline SYN12-6-79 & 1156.4 & 17.7 & 1160.5 & 16.3 & 1168.1 & 33.1 & 1168.1 & 33.1 & 99.0 \\
\hline SYN12-6-32 & 1062.1 & 36.5 & 1097.6 & 27.8 & 1168.8 & 36.0 & 1168.8 & 36.0 & 90.9 \\
\hline SYN12-6-62 & 1137.0 & 17.8 & 1148.4 & 17.7 & 1170.0 & 38.1 & 1170.0 & 38.1 & 97.2 \\
\hline SYN12-6-99R & 1196.6 & 19.8 & 1193.2 & 13.2 & 1187.1 & 9.5 & 1187.1 & 9.5 & 100.8 \\
\hline SYN12-6-90 & 1212.8 & 22.5 & 1204.4 & 51.5 & 1189.4 & 138.4 & 1189.4 & 138.4 & 102.0 \\
\hline SYN12-6-42 & 1157.2 & 31.5 & 1168.6 & 29.3 & 1189.8 & 58.9 & 1189.8 & 58.9 & 97.3 \\
\hline SYN12-6-99C & 1154.2 & 47.0 & 1167.7 & 32.7 & 1192.9 & 30.5 & 1192.9 & 30.5 & 96.8 \\
\hline SYN12-6-46 & 1271.9 & 62.4 & 1284.9 & 39.8 & 1306.8 & 15.3 & 1306.8 & 15.3 & 97.3 \\
\hline SYN12-6-36 & 1283.5 & 12.5 & 1294.7 & 20.1 & 1313.3 & 49.0 & 1313.3 & 49.0 & 97.7 \\
\hline SYN12-6-27 & 1305.6 & 18.6 & 1309.2 & 25.0 & 1314.9 & 58.4 & 1314.9 & 58.4 & 99.3 \\
\hline SYN12-6-82 & 1316.3 & 40.0 & 1330.4 & 25.8 & 1353.1 & 17.5 & 1353.1 & 17.5 & 97.3 \\
\hline SYN12-6-49 & 1360.4 & 30.8 & 1364.5 & 23.5 & 1371.0 & 36.1 & 1371.0 & 36.1 & 99.2 \\
\hline SYN12-6-59 & 1385.3 & 24.0 & 1390.2 & 14.8 & 1397.7 & 5.7 & 1397.7 & 5.7 & 99.1 \\
\hline SYN12-6-02 & 1376.0 & 28.6 & 1415.0 & 19.2 & 1474.2 & 18.7 & 1474.2 & 18.7 & 93.3 \\
\hline SYN12-6-82 & 1511.3 & 44.6 & 1497.0 & 27.9 & 1476.8 & 24.8 & 1476.8 & 24.8 & 102.3 \\
\hline SYN12-6-71 & 1498.9 & 21.6 & 1503.9 & 20.9 & 1510.9 & 40.0 & 1510.9 & 40.0 & 99.2 \\
\hline SYN12-6-61 & 1615.3 & 51.1 & 1615.9 & 29.2 & 1616.8 & 8.6 & 1616.8 & 8.6 & 99.9 \\
\hline SYN12-6-35 & 1627.1 & 50.7 & 16 & 30.2 & 3.0 & 22.0 & 3.0 & 22.0 & 99.9 \\
\hline SYN12-6-47 & 1621.0 & 26.9 & 1627.3 & 16.3 & 1635.5 & 13.4 & 1635.5 & 13.4 & 99.1 \\
\hline SYN12-6-39 & 1627.5 & 43.6 & 1635.6 & 25.6 & 1646.0 & 15.6 & 1646.0 & 15.6 & 98.9 \\
\hline SYN12-6-58 & 1659.2 & 42.9 & 1653.4 & 24.6 & 1646.1 & 13.3 & 1646.1 & 13.3 & 100.8 \\
\hline SYN12-6-72 & 1655.4 & 20.7 & 1653.5 & 12.8 & 1651.0 & 12.6 & 1651.0 & 12.6 & 100.3 \\
\hline SYN12-6-03 & 1645.2 & 21.3 & 1649.1 & 12.5 & 1654.0 & 8.1 & 1654.0 & 8.1 & 99.5 \\
\hline SYN12-6-97 & 1673.9 & 17.5 & 1667.4 & 10.3 & 1659.1 & 7.4 & 1659.1 & 7.4 & 100.9 \\
\hline SYN12-6-33 & 1649.5 & 50.9 & 1669.2 & 29.5 & 1693.9 & 15.9 & 1693.9 & 15.9 & 97.4 \\
\hline SYN12-6-74 & 1676.5 & 15.7 & 1701.2 & 11.5 & 1731.8 & 16.7 & 1731.8 & 16.7 & 96.8 \\
\hline SYN12-6-77 & 1729.7 & 30.0 & 1732.0 & 16.6 & 1734.9 & 5.4 & 1734.9 & 5.4 & 99.7 \\
\hline SYN12-6-16 & 1734.3 & 23.5 & 1741.8 & 13.3 & 1750.8 & 7.5 & 1750.8 & 7.5 & 99.1 \\
\hline SYN12-6-07 & 1800.6 & 16.6 & 1801.2 & 9.1 & 1801.8 & 4.2 & 1801.8 & 4.2 & 99.9 \\
\hline SYN12-6-64 & 1681.1 & 28.2 & 1747.4 & 16.1 & 1827.7 & 5.6 & 1827.7 & 5.6 & 92.0 \\
\hline SYN12-6-95 & 1544.8 & 64.2 & 1679.2 & 39.4 & 1851.3 & 19.8 & 1851.3 & 19.8 & 83.4 \\
\hline SYN12-6-26 & 1671.3 & 48.3 & 1764.0 & 27.7 & 1875.6 & 7.3 & 1875.6 & 7.3 & 89.1 \\
\hline SYN12-6-24 & 1851.8 & 15.8 & 1864.6 & 9.6 & 1878.8 & 10.0 & 1878.8 & 10.0 & 98.6 \\
\hline SYN12-6-10 & 1857.4 & 23.9 & 1871.3 & 13.0 & 1886.7 & 6.0 & 1886.7 & 6.0 & 98.4 \\
\hline SYN12-6-41 & 1907.2 & 37.4 & 1904.4 & 19.8 & 1901.4 & 7.5 & 1901.4 & 7.5 & 100.3 \\
\hline SYN12-6-08 & 2113.0 & 30.6 & 2121.7 & 16.6 & 2130.2 & 13.6 & 2130.2 & 13.6 & 99.2 \\
\hline SYN12-6-45 & 2747.6 & 89.3 & 2700.5 & 51.9 & 2665.5 & 62.4 & 2665.5 & 62.4 & 103.1 \\
\hline
\end{tabular}




\begin{tabular}{|l|l|l|l|l|l|l|l|l|l|} 
SYN12-6-80 & 2721.5 & 28.4 & 2730.9 & 12.2 & 2737.8 & 3.0 & 2737.8 & 3.0 & 99.4 \\
\hline SYN12-6-28 & 2821.3 & 31.1 & 2817.3 & 14.2 & 2814.4 & 10.0 & 2814.4 & 10.0 & 100.2 \\
\hline SYN12-6-25 & 3116.7 & 37.2 & 3091.3 & 15.2 & 3074.9 & 7.3 & 3074.9 & 7.3 & 101.4 \\
\hline SYN12-6-12 & 3826.2 & 61.6 & 3816.9 & 21.4 & 3812.0 & 4.3 & 3812.0 & 4.3 & 100.4 \\
\hline
\end{tabular}

\begin{tabular}{|c|c|c|c|c|c|c|c|c|c|}
\hline Analysis & $206 \mathrm{~Pb}^{*}$ & \pm & 207Pb* & \pm & $206 \mathrm{~Pb}^{*}$ & \pm & Best age & \pm & Conc \\
\hline & $238 U^{*}$ & (Ma) & $235 U$ & (Ma) & $207 \mathrm{~Pb}^{*}$ & (Ma) & (Ma) & (Ma) & $(\%)$ \\
\hline & & & & & & & & & \\
\hline & & & & & & & & & \\
\hline SYN12-8B-82 & 99.8 & 5.1 & 116.7 & 18.1 & 477.8 & 345.4 & 99.8 & 5.1 & NA \\
\hline SYN12-8B-55 & 104.2 & 6.7 & 111.4 & 22.7 & 267.7 & 474.4 & 104.2 & 6.7 & NA \\
\hline SYN12-8B-85 & 109.0 & 2.4 & 111.5 & 5.0 & 163.8 & 98.1 & 109.0 & 2.4 & NA \\
\hline SYN12-8B-59 & 110.7 & 4.4 & 113.8 & 10.9 & 180.9 & 216.3 & 110.7 & 4.4 & NA \\
\hline SYN12-8B-76 & 112.0 & 4.4 & 108.6 & 10.7 & 35.2 & 231.2 & 112.0 & 4.4 & NA \\
\hline SYN12-8B-78 & 233.0 & 3.5 & 234.0 & 10.0 & 244.0 & 104.0 & 233.0 & 3.5 & NA \\
\hline SYN12-8B-66 & 237.1 & 3.5 & 237.0 & 5.1 & 236.1 & 43.3 & 237.1 & 3.5 & NA \\
\hline SYN12-8B-89 & 240.7 & 4.1 & 247.5 & 14.9 & 312.5 & 149.5 & 240.7 & 4.1 & NA \\
\hline SYN12-8B-62 & 245.3 & 8.4 & 242.1 & 7.9 & 210.9 & 25.7 & 245.3 & 8.4 & NA \\
\hline SYN12-8B-31 & 246.3 & 3.7 & 248.5 & 10.5 & 269.5 & 103.0 & 246.3 & 3.7 & NA \\
\hline SYN12-8B-81 & 250.3 & 4.2 & 253.1 & 9.9 & 279.0 & 93.8 & 250.3 & 4.2 & NA \\
\hline SYN12-8B-33 & 268.5 & 12.2 & 311.2 & 15.0 & 645.2 & 68.0 & 268.5 & 12.2 & NA \\
\hline SYN12-8B-65 & 333.0 & 6.7 & 334.4 & 9.9 & 344.1 & 63.5 & 333.0 & 6.7 & NA \\
\hline SYN12-8B-83 & 376.0 & 6.1 & 379.9 & 6.1 & 403.9 & 21.0 & 376.0 & 6.1 & NA \\
\hline SYN12-8B-60 & 383.2 & 5.9 & 390.0 & 14.4 & 430.6 & 92.8 & 383.2 & 5.9 & NA \\
\hline SYN12-8B-92 & 394.8 & 5.0 & 392.8 & 6.5 & 380.5 & 34.0 & 394.8 & 5.0 & NA \\
\hline SYN12-8B-49 & 416.8 & 14.6 & 420.3 & 22.7 & 439.5 & 122.5 & 416.8 & 14.6 & 94.8 \\
\hline SYN12-8B-3 & 416.9 & 8.2 & 416.8 & 23.0 & 416.3 & 143.4 & 416.9 & 8.2 & 100.2 \\
\hline SYN12-8B-61 & 419.4 & 35.0 & 286.5 & 82.3 & -694.7 & 898.3 & 419.4 & 35.0 & NA \\
\hline SYN12-8B-15 & 422.3 & 5.6 & 420.4 & 7.5 & 409.7 & 37.9 & 422.3 & 5.6 & 103.1 \\
\hline SYN12-8B-17 & 423.6 & 7.6 & 421.0 & 8.4 & 406.4 & 35.5 & 423.6 & 7.6 & 104.3 \\
\hline SYN12-8B-35 & 425.1 & 9.1 & 433.2 & 22.2 & 476.7 & 130.3 & 425.1 & 9.1 & 89.2 \\
\hline SYN12-8B-25 & 430.1 & 11.6 & 425.9 & 14.2 & 403.2 & 67.3 & 430.1 & 11.6 & 106.7 \\
\hline SYN12-8B-52 & 431.0 & 11.2 & 431.7 & 14.7 & 435.3 & 71.3 & 431.0 & 11.2 & 99.0 \\
\hline SYN12-8B-70 & 433.6 & 6.7 & 433.7 & 6.8 & 434.3 & 24.4 & 433.6 & 6.7 & 99.8 \\
\hline SYN12-8B-48 & 435.8 & 4.9 & 440.4 & 20.7 & 464.3 & 125.9 & 435.8 & 4.9 & 93.9 \\
\hline SYN12-8B-4 & 448.5 & 18.9 & 448.9 & 16.2 & 450.7 & 20.2 & 448.5 & 18.9 & 99.5 \\
\hline SYN12-8B-46 & 518.1 & 7.3 & 511.0 & 24.7 & 479.3 & 132.0 & 518.1 & 7.3 & 108.1 \\
\hline SYN12-8B-16 & 542.1 & 10.8 & 568.9 & 28.5 & 677.7 & 132.5 & 542.1 & 10.8 & 80.0 \\
\hline
\end{tabular}




\begin{tabular}{|c|c|c|c|c|c|c|c|c|c|}
\hline SYN12-8B-86 & 558.3 & 10.6 & 561.0 & 10.5 & 572.0 & 31.1 & 558.3 & 10.6 & 97.6 \\
\hline SYN12-8B-91 & 574.0 & 6.8 & 572.4 & 6.0 & 566.0 & 12.6 & 574.0 & 6.8 & 101.4 \\
\hline SYN12-8B-77 & 582.9 & 6.0 & 577.6 & 9.5 & 556.5 & 40.6 & 582.9 & 6.0 & 104.7 \\
\hline SYN12-8B-1 & 593.9 & 11.9 & 601.5 & 20.9 & 630.3 & 88.3 & 593.9 & 11.9 & 94.2 \\
\hline SYN12-8B-38 & 600.3 & 8.2 & 596.8 & 9.3 & 583.5 & 32.2 & 600.3 & 8.2 & 102.9 \\
\hline SYN12-8B-13 & 603.9 & 7.7 & 609.4 & 7.6 & 629.9 & 21.0 & 603.9 & 7.7 & 95.9 \\
\hline SYN12-8B-8 & 606.6 & 10.8 & 608.4 & 11.7 & 614.8 & 37.7 & 606.6 & 10.8 & 98.7 \\
\hline SYN12-8B-99 & 616.1 & 42.7 & 623.2 & 34.2 & 648.9 & 21.2 & 616.1 & 42.7 & 94.9 \\
\hline SYN12-8B-41 & 640.2 & 11.4 & 643.7 & 10.1 & 656.2 & 20.8 & 640.2 & 11.4 & 97.6 \\
\hline SYN12-8B-11 & 653.8 & 18.9 & 649.1 & 27.0 & 633.0 & 102.0 & 653.8 & 18.9 & 103.3 \\
\hline SYN12-8B-73 & 1041.1 & 21.8 & 1028.9 & 30.5 & 1003.0 & 84.0 & 1003.0 & 84.0 & 103.8 \\
\hline SYN12-8B-96 & 1023.8 & 11.6 & 1021.4 & 9.2 & 1016.2 & 14.9 & 1016.2 & 14.9 & 100.8 \\
\hline SYN12-8B-29 & 1023.4 & 10.1 & 1026.3 & 12.2 & 1032.5 & 31.6 & 1032.5 & 31.6 & 99.1 \\
\hline SYN12-8B-88 & 1079.0 & 23.8 & 1074.5 & 56.6 & 1065.3 & 164.8 & 1065.3 & 164.8 & 101.3 \\
\hline SYN12-8B-34 & 1073.9 & 21.0 & 1072.2 & 16.7 & 1068.6 & 27.1 & 1068.6 & 27.1 & 100.5 \\
\hline SYN12-8B-72 & 1063.6 & 14.3 & 1066.3 & 10.2 & 1071.9 & 10.0 & 1071.9 & 10.0 & 99.2 \\
\hline SYN12-8B-30 & 1082.3 & 19.9 & 1079.7 & 16.9 & 1074.6 & 31.5 & 1074.6 & 31.5 & 100.7 \\
\hline SYN12-8B-68 & 1071.8 & 12.6 & 1073.9 & 14.0 & 1078.1 & 33.8 & 1078.1 & 33.8 & 99.4 \\
\hline SYN12-8B-100 & 1048.7 & 20.3 & 1060.4 & 14.1 & 1084.6 & 8.1 & 1084.6 & 8.1 & 96.7 \\
\hline SYN12-8B-20 & 1051.4 & 8.6 & 1065.6 & 8.9 & 1094.8 & 20.2 & 1094.8 & 20.2 & 96.0 \\
\hline SYN12-8B-57 & 1082.0 & 27.9 & 1087.4 & 25.4 & 1098.2 & 51.5 & 1098.2 & 51.5 & 98.5 \\
\hline SYN12-8B-94 & 1200.9 & 17.7 & 1182.1 & 13.5 & 1147.8 & 21.1 & 1147.8 & 21.1 & 104.6 \\
\hline SYN12-8B-50 & 1188.4 & 28.0 & 1191.0 & 18.3 & 1195.7 & 8.7 & 1195.7 & 8.7 & 99.4 \\
\hline SYN12-8B-69 & 1090.2 & 27.4 & 1132.5 & 25.1 & 1214.4 & 48.4 & 1214.4 & 48.4 & 89.8 \\
\hline SYN12-8B-32 & 1168.6 & 15.1 & 1184.9 & 15.8 & 1214.7 & 34.7 & 1214.7 & 34.7 & 96.2 \\
\hline SYN12-8B-43 & 1205.1 & 21.3 & 1211.4 & 20.8 & 1222.7 & 43.4 & 1222.7 & 43.4 & 98.6 \\
\hline SYN12-8B-26 & 1140.1 & 25.3 & 1172.7 & 35.1 & 1233.4 & 87.0 & 1233.4 & 87.0 & 92.4 \\
\hline SYN12-8B-63 & 1251.1 & 14.8 & 1247.8 & 14.2 & 1241.9 & 29.1 & 1241.9 & 29.1 & 100.7 \\
\hline SYN12-8B-95 & 1270.3 & 26.0 & 1270.7 & 19.5 & 1271.4 & 28.4 & 1271.4 & 28.4 & 99.9 \\
\hline SYN12-8B-87 & 1328.0 & 14.8 & 1321.6 & 11.2 & 1311.2 & 16.9 & 1311.2 & 16.9 & 101.3 \\
\hline SYN12-8B-51 & 1305.2 & 39.4 & 1317.7 & 28.8 & 1338.0 & 39.4 & 1338.0 & 39.4 & 97.5 \\
\hline SYN12-8B-47 & 1312.7 & 25.8 & 1324.8 & 17.2 & 1344.5 & 15.8 & 1344.5 & 15.8 & 97.6 \\
\hline SYN12-8B-9 & 1378.4 & 14.9 & 1384.0 & 10.2 & 1392.5 & 11.8 & 1392.5 & 11.8 & 99.0 \\
\hline SYN12-8B-21 & 1237.7 & 21.2 & 1309.7 & 35.0 & 1429.7 & 83.3 & 1429.7 & 83.3 & 86.6 \\
\hline SYN12-8B-67 & 1440.2 & 18.4 & 1436.9 & 12.8 & 1432.1 & 16.4 & 1432.1 & 16.4 & 100.6 \\
\hline SYN12-8B-7 & 1380.7 & 41.8 & 1401.1 & 26.6 & 1432.2 & 18.5 & 1432.2 & 18.5 & 96.4 \\
\hline SYN12-8B-91 & 1502.0 & 21.0 & 1500.5 & 13.2 & 1498.2 & 11.9 & 1498.2 & 11.9 & 100.3 \\
\hline SYN12-8B-19 & 1534.4 & 123.1 & 1522.7 & 71.3 & 1506.5 & 8.2 & 1506.5 & 8.2 & 101.9 \\
\hline SYN12-8B-14 & 1605.3 & 12.6 & 1606.8 & 8.5 & 1608.9 & 10.5 & 1608.9 & 10.5 & 99.8 \\
\hline
\end{tabular}




\begin{tabular}{|l|l|l|l|l|l|l|l|l|l|}
\hline SYN12-8B-90 & 1664.0 & 61.3 & 1641.6 & 46.7 & 1613.0 & 73.2 & 1613.0 & 73.2 & 103.2 \\
\hline SYN12-8B-22 & 1629.0 & 46.1 & 1631.2 & 26.3 & 1634.1 & 8.5 & 1634.1 & 8.5 & 99.7 \\
\hline SYN12-8B-2 & 1670.1 & 16.0 & 1654.6 & 10.8 & 1635.0 & 14.2 & 1635.0 & 14.2 & 102.1 \\
\hline SYN12-8B-28 & 1613.2 & 43.6 & 1624.6 & 26.4 & 1639.5 & 21.3 & 1639.5 & 21.3 & 98.4 \\
\hline SYN12-8B-36 & 1663.4 & 13.4 & 1654.1 & 8.1 & 1642.3 & 7.2 & 1642.3 & 7.2 & 101.3 \\
\hline SYN12-8B-93 & 1653.0 & 19.3 & 1652.3 & 13.8 & 1651.3 & 19.3 & 1651.3 & 19.3 & 100.1 \\
\hline SYN12-8B-27 & 1624.3 & 79.2 & 1642.4 & 46.3 & 1665.8 & 25.9 & 1665.8 & 25.9 & 97.5 \\
\hline SYN12-8B-44 & 1707.0 & 57.2 & 1692.8 & 31.6 & 1675.2 & 8.4 & 1675.2 & 8.4 & 101.9 \\
\hline SYN12-8B-12 & 1614.1 & 24.1 & 1641.8 & 19.0 & 1677.4 & 29.7 & 1677.4 & 29.7 & 96.2 \\
\hline SYN12-8B-97 & 1653.1 & 24.9 & 1688.1 & 14.1 & 1731.7 & 2.3 & 1731.7 & 2.3 & 95.5 \\
\hline SYN12-8B-64 & 1753.3 & 23.6 & 1747.7 & 14.0 & 1741.0 & 12.7 & 1741.0 & 12.7 & 100.7 \\
\hline SYN12-8B-74 & 1789.9 & 21.0 & 1773.1 & 11.4 & 1753.3 & 3.1 & 1753.3 & 3.1 & 102.1 \\
\hline SYN12-8B-6 & 1758.4 & 19.9 & 1770.9 & 11.0 & 1785.7 & 3.8 & 1785.7 & 3.8 & 98.5 \\
\hline SYN12-8B-58 & 1838.5 & 39.7 & 1823.7 & 21.9 & 1806.7 & 13.6 & 1806.7 & 13.6 & 101.8 \\
\hline SYN12-8B-10 & 1853.1 & 25.6 & 1883.3 & 13.6 & 1916.7 & 1.9 & 1916.7 & 1.9 & 96.7 \\
\hline SYN12-8B-75 & 2019.8 & 27.3 & 2024.0 & 14.0 & 2028.2 & 5.1 & 2028.2 & 5.1 & 99.6 \\
\hline SYN12-8B-54 & 2389.1 & 48.5 & 2436.5 & 22.5 & 2476.3 & 3.5 & 2476.3 & 3.5 & 96.5 \\
\hline SYN12-8B-5 & 2569.3 & 46.8 & 2605.2 & 21.8 & 2633.3 & 12.1 & 2633.3 & 12.1 & 97.6 \\
\hline SYN12-8B-71 & 2693.0 & 38.7 & 2688.1 & 17.0 & 2684.4 & 6.9 & 2684.4 & 6.9 & 100.3 \\
\hline SYN12-8B-37 & 2602.1 & 47.6 & 2650.2 & 28.1 & 2687.1 & 33.0 & 2687.1 & 33.0 & 96.8 \\
\hline SYN12-8B-53 & 2708.7 & 89.6 & 2703.8 & 38.5 & 2700.1 & 8.0 & 2700.1 & 8.0 & 100.3 \\
\hline SYN12-8B-98 & 2804.6 & 39.1 & 2745.8 & 17.1 & 2702.9 & 9.2 & 2702.9 & 9.2 & 103.8 \\
\hline SYN12-8B-18 & 2695.3 & 35.2 & 2722.2 & 15.3 & 2742.2 & 4.0 & 2742.2 & 4.0 & 98.3 \\
\hline SYN12-8B-45 & 2886.0 & 140.5 & 2827.7 & 58.0 & 2786.3 & 12.1 & 2786.3 & 12.1 & 103.6 \\
\hline
\end{tabular}

\begin{tabular}{|l|l|l|l|l|l|l|l|l|l|}
\hline Analysis & $206 \mathrm{~Pb}^{*}$ & \pm & $207 \mathrm{~Pb}^{*}$ & \pm & $206 \mathrm{~Pb}^{*}$ & \pm & Best age & \pm & Conc \\
\hline & $238 \mathrm{U}^{*}$ & $(\mathrm{Ma})$ & $235 \mathrm{U}$ & $(\mathrm{Ma})$ & $207 \mathrm{~Pb}^{*}$ & $(\mathrm{Ma})$ & $(\mathrm{Ma})$ & $(\mathrm{Ma})$ & $(\%)$ \\
\hline & & & & & & & & & \\
\hline & & & & & & & & & \\
\hline & & & & & & & & & \\
\hline SYN12-10-11 & 98.8 & 7.3 & 90.7 & 43.0 & -118.6 & 1279.0 & 98.8 & 7.3 & NA \\
\hline SYN12-10-4 & 99.5 & 0.9 & 103.4 & 5.9 & 194.2 & 137.8 & 99.5 & 0.9 & NA \\
\hline SYN12-10-109 & 99.6 & 1.3 & 102.2 & 6.9 & 163.4 & 164.4 & 99.6 & 1.3 & NA \\
\hline SYN12-10-56 & 99.7 & 2.4 & 104.3 & 6.8 & 210.1 & 148.2 & 99.7 & 2.4 & NA \\
\hline SYN12-10-3 & 100.1 & 3.5 & 94.1 & 10.0 & -54.4 & 257.2 & 100.1 & 3.5 & NA \\
\hline SYN12-10-23 & 100.2 & 2.2 & 98.8 & 4.7 & 64.1 & 107.6 & 100.2 & 2.2 & NA \\
\hline SYN12-10-70 & 100.3 & 3.0 & 84.6 & 28.2 & -339.1 & 914.7 & 100.3 & 3.0 & NA \\
\hline SYN12-10-41 & 100.4 & 4.2 & 111.1 & 10.7 & 346.1 & 210.2 & 100.4 & 4.2 & NA \\
\hline SYN12-10-24 & 100.6 & 3.1 & 100.8 & 4.2 & 105.9 & 73.6 & 100.6 & 3.1 & NA \\
\hline
\end{tabular}




\begin{tabular}{|c|c|c|c|c|c|c|c|c|c|}
\hline SYN12-10-83 & 100.8 & 1.8 & 90.2 & 6.5 & -182.8 & 181.3 & 100.8 & 1.8 & NA \\
\hline SYN12-10-66 & 101.1 & 1.7 & 99.0 & 4.6 & 49.2 & 109.5 & 101.1 & 1.7 & NA \\
\hline SYN12-10-80 & 101.1 & 2.3 & 104.4 & 6.4 & 179.2 & 140.4 & 101.1 & 2.3 & NA \\
\hline SYN12-10-103 & 101.1 & 1.1 & 99.9 & 5.6 & 69.8 & 137.2 & 101.1 & 1.1 & NA \\
\hline SYN12-10-64 & 101.3 & 2.3 & 103.7 & 8.3 & 158.3 & 188.9 & 101.3 & 2.3 & NA \\
\hline SYN12-10-71 & 101.4 & 2.0 & 95.4 & 7.6 & -51.0 & 198.9 & 101.4 & 2.0 & NA \\
\hline SYN12-10-89 & 101.5 & 2.0 & 99.6 & 12.1 & 53.8 & 302.0 & 101.5 & 2.0 & NA \\
\hline SYN12-10-25 & 101.6 & 2.5 & 101.2 & 9.9 & 92.4 & 235.5 & 101.6 & 2.5 & NA \\
\hline SYN12-10-58 & 101.7 & 2.4 & 93.0 & 11.3 & -123.0 & 308.3 & 101.7 & 2.4 & NA \\
\hline SYN12-10-119 & 101.8 & 2.2 & 101.4 & 8.2 & 91.7 & 193.8 & 101.8 & 2.2 & NA \\
\hline SYN12-10-63 & 101.9 & 3.5 & 95.9 & 28.0 & -51.8 & 756.0 & 101.9 & 3.5 & NA \\
\hline SYN12-10-34 & 102.2 & 3.2 & 100.4 & 5.6 & 59.1 & 118.0 & 102.2 & 3.2 & NA \\
\hline SYN12-10-117 & 102.4 & 4.2 & 95.7 & 21.8 & -66.8 & 581.0 & 102.4 & 4.2 & NA \\
\hline SYN12-10-14 & 102.4 & 2.2 & 103.0 & 7.5 & 117.8 & 174.0 & 102.4 & 2.2 & NA \\
\hline SYN12-10-40 & 102.6 & 5.1 & 119.4 & 18.9 & 469.6 & 355.3 & 102.6 & 5.1 & NA \\
\hline SYN12-10-73 & 102.7 & 2.2 & 97.0 & 16.3 & -42.5 & 427.0 & 102.7 & 2.2 & $\mathrm{NA}$ \\
\hline SYN12-10-55 & 102.9 & 2.9 & 100.6 & 6.1 & 46.5 & 135.2 & 102.9 & 2.9 & NA \\
\hline SYN12-10-85 & 103.3 & 1.3 & 104.1 & 2.7 & 123.8 & 56.9 & 103.3 & 1.3 & NA \\
\hline SYN12-10-2 & 103.4 & 2.3 & 95.3 & 15.5 & -102.0 & 417.1 & 103.4 & 2.3 & NA \\
\hline SYN12-10-07 & 103.4 & 1.3 & 104.6 & 3.0 & 131.7 & 64.0 & 103.4 & 1.3 & NA \\
\hline SYN12-10-18 & 103.7 & 2.5 & 100.7 & 5.6 & 29.2 & 126.9 & 103.7 & 2.5 & NA \\
\hline SYN12-10-95 & 104.0 & 3.7 & 106.1 & 8.4 & 153.8 & 176.7 & 104.0 & 3.7 & NA \\
\hline SYN12-10-76 & 104.1 & 5.7 & 111.9 & 30.9 & 280.1 & 666.7 & 104.1 & 5.7 & NA \\
\hline SYN12-10-44 & 105.5 & 2.7 & 109.7 & 6.4 & 202.2 & 129.6 & 105.5 & 2.7 & NA \\
\hline SYN12-10-75 & 113.9 & 4.0 & 101.0 & 33.1 & -194.5 & 878.1 & 113.9 & 4.0 & NA \\
\hline SYN12-10-61 & 135.0 & 5.2 & 138.1 & 16.6 & 191.3 & 286.3 & 135.0 & 5.2 & NA \\
\hline SYN12-10-28 & 156.1 & 5.7 & 148.0 & 21.9 & 20.3 & 373.7 & 156.1 & 5.7 & NA \\
\hline SYN12-10-13 & 157.7 & 5.6 & 149.4 & 13.5 & 19.2 & 217.2 & 157.7 & 5.6 & NA \\
\hline SYN12-10-47 & 159.5 & 2.4 & 162.1 & 7.0 & 200.9 & 102.8 & 159.5 & 2.4 & NA \\
\hline SYN12-10-97 & 262.3 & 5.2 & 263.6 & 10.9 & 275.0 & 97.7 & 262.3 & 5.2 & NA \\
\hline SYN12-10-50 & 307.4 & 5.8 & 298.9 & 9.9 & 233.1 & 76.9 & 307.4 & 5.8 & NA \\
\hline SYN12-10-93 & 327.2 & 4.2 & 323.9 & 9.0 & 300.3 & 67.7 & 327.2 & 4.2 & NA \\
\hline SYN12-10-36 & 363.2 & 6.0 & 365.1 & 11.0 & 376.7 & 71.2 & 363.2 & 6.0 & NA \\
\hline SYN12-10-33 & 393.9 & 20.3 & 381.7 & 28.6 & 308.2 & 164.6 & 393.9 & 20.3 & NA \\
\hline SYN12-10-91 & 497.7 & 13.6 & 492.3 & 76.3 & 467.5 & 431.1 & 497.7 & 13.6 & 106.5 \\
\hline SYN12-10-116 & 556.4 & 11.1 & 540.5 & 41.5 & 474.2 & 214.7 & 556.4 & 11.1 & 117.3 \\
\hline SYN12-10-98 & 597.5 & 7.9 & 598.7 & 8.5 & 603.3 & 27.5 & 597.5 & 7.9 & 99.0 \\
\hline SYN12-10-78 & 609.7 & 6.6 & 615.4 & 7.7 & 636.4 & 26.2 & 609.7 & 6.6 & 95.8 \\
\hline SYN12-10-52 & 609.7 & 8.8 & 607.8 & 12.7 & 600.7 & 50.6 & 609.7 & 8.8 & 101.5 \\
\hline
\end{tabular}




\begin{tabular}{|c|c|c|c|c|c|c|c|c|c|}
\hline SYN12-10-86 & 641.0 & 28.4 & 635.7 & 24.8 & 616.9 & 52.7 & 641.0 & 28.4 & 103.9 \\
\hline SYN12-10-99 & 653.5 & 9.7 & 663.4 & 22.3 & 697.2 & 91.8 & 653.5 & 9.7 & 93.7 \\
\hline SYN12-10-60 & 702.9 & 9.6 & 702.1 & 12.6 & 699.6 & 43.0 & 702.9 & 9.6 & 100.5 \\
\hline SYN12-10-102 & 953.7 & 17.9 & 944.8 & 18.3 & 924.2 & 44.7 & 924.2 & 44.7 & 103.2 \\
\hline SYN12-10-107 & 944.6 & 11.1 & 948.8 & 10.7 & 958.4 & 24.3 & 958.4 & 24.3 & 98.6 \\
\hline SYN12-10-96 & 976.1 & 8.8 & 981.4 & 13.5 & 993.3 & 39.0 & 993.3 & 39.0 & 98.3 \\
\hline SYN12-10-31 & 1031.6 & 17.1 & 1021.4 & 16.5 & 999.5 & 37.2 & 999.5 & 37.2 & 103.2 \\
\hline SYN12-10-17 & 1037.5 & 22.7 & 1028.2 & 23.9 & 1008.4 & 57.8 & 1008.4 & 57.8 & 102.9 \\
\hline SYN12-10-49 & 1007.6 & 8.3 & 1009.2 & 12.3 & 1012.8 & 34.5 & 1012.8 & 34.5 & 99.5 \\
\hline SYN12-10-118 & 1046.7 & 15.2 & 1040.1 & 13.5 & 1026.1 & 27.4 & 1026.1 & 27.4 & 102.0 \\
\hline SYN12-10-29 & 1017.3 & 21.0 & 1023.5 & 15.6 & 1036.9 & 18.7 & 1036.9 & 18.7 & 98.1 \\
\hline SYN12-10-32 & 1013.5 & 15.7 & 1026.2 & 11.4 & 1053.4 & 11.5 & 1053.4 & 11.5 & 96.2 \\
\hline SYN12-10-1 & 1058.7 & 27.8 & 1062.0 & 25.1 & 1068.9 & 50.9 & 1068.9 & 50.9 & 99.1 \\
\hline SYN12-10-105 & 1075.4 & 11.8 & 1074.3 & 11.0 & 1072.1 & 23.1 & 1072.1 & 23.1 & 100.3 \\
\hline SYN12-10-38 & 1073.8 & 17.8 & 1073.7 & 13.0 & 1073.5 & 15.9 & 1073.5 & 15.9 & 100.0 \\
\hline SYN12-10-112 & 1085.0 & 16.6 & 1084.7 & 13.3 & 1084.1 & 22.4 & 1084.1 & 22.4 & 100.1 \\
\hline SYN12-10-15 & 1096.7 & 15.0 & 1097.2 & 12.1 & 1098.2 & 20.0 & 1098.2 & 20.0 & 99.9 \\
\hline SYN12-10-35 & 1132.9 & 16.5 & 1135.0 & 11.3 & 1139.2 & 8.9 & 1139.2 & 8.9 & 99.4 \\
\hline SYN12-10-69 & 1183.8 & 42.8 & 1196.8 & 29.5 & 1220.3 & 27.2 & 1220.3 & 27.2 & 97.0 \\
\hline SYN12-10-30 & 1214.1 & 17.0 & 1216.4 & 13.7 & 1220.5 & 23.0 & 1220.5 & 23.0 & 99.5 \\
\hline SYN12-10-57 & 1270.3 & 38.6 & 1258.9 & 41.1 & 1239.5 & 90.3 & 1239.5 & 90.3 & 102.5 \\
\hline SYN12-10-90 & 1303.7 & 19.2 & 1298.9 & 12.4 & 1290.9 & 9.4 & 1290.9 & 9.4 & 101.0 \\
\hline SYN12-10-39 & 1442.0 & 46.2 & 1417.3 & 29.2 & 1380.5 & 26.0 & 1380.5 & 26.0 & 104.5 \\
\hline SYN12-10-88 & 1448.7 & 22.6 & 1454.6 & 13.6 & 1463.3 & 4.7 & 1463.3 & 4.7 & 99.0 \\
\hline SYN12-10-79 & 1493.1 & 19.6 & 1493.3 & 17.1 & 1493.6 & 30.6 & 1493.6 & 30.6 & 100.0 \\
\hline SYN12-10-19 & 1489.2 & 21.2 & 1491.2 & 14.1 & 1494.0 & 16.2 & 1494.0 & 16.2 & 99.7 \\
\hline SYN12-10-59 & 1501.7 & 20.1 & 1502.7 & 12.2 & 1504.1 & 7.6 & 1504.1 & 7.6 & 99.8 \\
\hline SYN12-10-72 & 1603.9 & 35.6 & 1612.3 & 20.9 & 1623.3 & 11.2 & 1623.3 & 11.2 & 98.8 \\
\hline SYN12-10-51 & 1673.0 & 41.2 & 1655.2 & 31.6 & 1632.7 & 49.8 & 1632.7 & 49.8 & 102.5 \\
\hline SYN12-10-48 & 1651.6 & 25.6 & 1649.7 & 16.1 & 1647.1 & 16.8 & 1647.1 & 16.8 & 100.3 \\
\hline SYN12-10-65 & 1660.6 & 15.9 & 1655.1 & 9.0 & 1648.2 & 2.7 & 1648.2 & 2.7 & 100.8 \\
\hline SYN12-10-87 & 1620.1 & 29.9 & 1632.8 & 17.6 & 1649.2 & 10.8 & 1649.2 & 10.8 & 98.2 \\
\hline SYN12-10-06 & 1665.0 & 22.8 & 1659.4 & 15.2 & 1652.4 & 18.9 & 1652.4 & 18.9 & 100.8 \\
\hline SYN12-10-84 & 1689.4 & 34.5 & 1690.2 & 21.2 & 1691.2 & 20.5 & 1691.2 & 20.5 & 99.9 \\
\hline SYN12-10-12 & 1675.4 & 23.1 & 1682.8 & 14.2 & 1692.1 & 13.6 & 1692.1 & 13.6 & 99.0 \\
\hline SYN12-10-111 & 1675.8 & 23.9 & 1684.4 & 23.1 & 1695.3 & 42.3 & 1695.3 & 42.3 & 98.9 \\
\hline SYN12-10-101 & 1715.5 & 20.6 & 1711.5 & 13.8 & 1706.6 & 17.7 & 1706.6 & 17.7 & 100.5 \\
\hline SYN12-10-114 & 1728.1 & 22.8 & 1721.2 & 14.2 & 1712.9 & 15.1 & 1712.9 & 15.1 & 100.9 \\
\hline SYN12-10-94 & 1775.4 & 33.0 & 1770.1 & 29.5 & 1763.8 & 51.2 & 1763.8 & 51.2 & 100.7 \\
\hline
\end{tabular}




\begin{tabular}{|l|l|l|l|l|l|l|l|l|l|}
\hline SYN12-10-45 & 1534.2 & 31.7 & 1639.1 & 19.1 & 1776.4 & 6.1 & 1776.4 & 6.1 & 86.4 \\
\hline SYN12-10-54 & 1776.7 & 14.3 & 1780.0 & 8.0 & 1783.8 & 4.5 & 1783.8 & 4.5 & 99.6 \\
\hline SYN12-10-62 & 1795.0 & 21.7 & 1791.1 & 12.1 & 1786.6 & 6.6 & 1786.6 & 6.6 & 100.5 \\
\hline SYN12-10-26 & 1765.4 & 22.6 & 1776.3 & 13.8 & 1789.0 & 13.7 & 1789.0 & 13.7 & 98.7 \\
\hline SYN12-10-77 & 1843.4 & 19.6 & 1843.7 & 10.9 & 1844.1 & 7.2 & 1844.1 & 7.2 & 100.0 \\
\hline SYN12-10-68 & 1838.5 & 14.4 & 1845.1 & 11.0 & 1852.6 & 16.8 & 1852.6 & 16.8 & 99.2 \\
\hline SYN12-10-82 & 1810.0 & 38.7 & 1830.4 & 21.3 & 1853.6 & 10.0 & 1853.6 & 10.0 & 97.6 \\
\hline SYN12-10-37 & 1831.0 & 35.3 & 1843.1 & 23.2 & 1856.8 & 28.6 & 1856.8 & 28.6 & 98.6 \\
\hline SYN12-10-92 & 1866.3 & 31.5 & 1863.1 & 17.4 & 1859.6 & 10.8 & 1859.6 & 10.8 & 100.4 \\
\hline SYN12-10-08 & 1887.4 & 48.7 & 1881.7 & 26.1 & 1875.4 & 11.9 & 1875.4 & 11.9 & 100.6 \\
\hline SYN12-10-67 & 1797.3 & 95.5 & 1841.4 & 54.3 & 1891.6 & 34.6 & 1891.6 & 34.6 & 95.0 \\
\hline SYN12-10-43 & 1842.7 & 27.2 & 1877.3 & 14.6 & 1915.7 & 2.7 & 1915.7 & 2.7 & 96.2 \\
\hline SYN12-10-74 & 1927.4 & 28.0 & 1929.8 & 15.7 & 1932.4 & 12.6 & 1932.4 & 12.6 & 99.7 \\
\hline SYN12-10-115 & 2010.4 & 19.8 & 2023.1 & 10.6 & 2036.1 & 6.7 & 2036.1 & 6.7 & 98.7 \\
\hline SYN12-10-27 & 2108.4 & 26.1 & 2111.7 & 15.9 & 2114.9 & 18.5 & 2114.9 & 18.5 & 99.7 \\
\hline SYN12-10-106 & 2305.5 & 58.6 & 2288.3 & 55.0 & 2273.0 & 90.2 & 2273.0 & 90.2 & 101.4 \\
\hline SYN12-10-09 & 2347.9 & 26.6 & 2334.4 & 12.5 & 2322.7 & 3.5 & 2322.7 & 3.5 & 101.1 \\
\hline SYN12-10-5 & 2547.3 & 22.9 & 2534.0 & 11.2 & 2523.3 & 8.4 & 2523.3 & 8.4 & 101.0 \\
\hline SYN12-10-53 & 2495.5 & 29.2 & 2594.9 & 13.3 & 2673.4 & 3.3 & 2673.4 & 3.3 & 93.3 \\
\hline SYN12-10-20 & 2712.5 & 77.1 & 2703.2 & 33.1 & 2696.3 & 6.3 & 2696.3 & 6.3 & 100.6 \\
\hline SYN12-10-113 & 2715.4 & 43.7 & 2714.6 & 18.8 & 2713.9 & 4.0 & 2713.9 & 4.0 & 100.1 \\
\hline SYN12-10-21 & 2729.7 & 39.5 & 2743.0 & 17.0 & 2752.7 & 4.8 & 2752.7 & 4.8 & 99.2 \\
\hline SYN12-10-120 & 2824.0 & 30.9 & 2786.8 & 13.0 & 2760.0 & 3.4 & 2760.0 & 3.4 & 102.3 \\
\hline SYN12-10-108 & 3009.0 & 21.2 & 2997.2 & 8.7 & 2989.4 & 3.6 & 2989.4 & 3.6 & 100.7 \\
\hline
\end{tabular}

\begin{tabular}{|l|l|l|l|l|l|l|l|l|l|}
\hline Analysis & $206 \mathrm{~Pb}^{*}$ & \pm & $207 \mathrm{~Pb}^{*}$ & \pm & $206 \mathrm{~Pb}^{*}$ & \pm & Best age & \pm & Conc \\
\hline & $238 \mathrm{U}^{*}$ & $(\mathrm{Ma})$ & $235 \mathrm{U}$ & $(\mathrm{Ma})$ & $207 \mathrm{~Pb}^{*}$ & $(\mathrm{Ma})$ & $(\mathrm{Ma})$ & $(\mathrm{Ma})$ & $(\%)$ \\
\hline & & & & & & & & & \\
\hline & & & & & & & & & \\
\hline & & & & & & & & & \\
\hline SYN11A-93 & 94.9 & 4.8 & 118.6 & 11.5 & 623.4 & 193.3 & 94.9 & 4.8 & NA \\
\hline SYN11A-87 & 95.5 & 6.5 & 103.1 & 21.7 & 282.9 & 484.6 & 95.5 & 6.5 & NA \\
\hline SYN11A-86 & 97.8 & 2.5 & 86.9 & 14.1 & -201.3 & 422.9 & 97.8 & 2.5 & NA \\
\hline unknown SYN11a-56 & 98.1 & 1.4 & 103.1 & 4.6 & 220.7 & 102.1 & 98.1 & 1.4 & NA \\
\hline SYN11A-90 & 98.4 & 2.2 & 109.5 & 12.9 & 359.1 & 275.5 & 98.4 & 2.2 & NA \\
\hline SYN11A-46 & 98.4 & 2.0 & 100.5 & 10.1 & 151.6 & 242.7 & 98.4 & 2.0 & NA \\
\hline SYN11A-98 & 98.7 & 4.1 & 116.6 & 27.3 & 499.3 & 544.8 & 98.7 & 4.1 & NA \\
\hline SYN11A-40 & 99.2 & 2.0 & 99.1 & 6.0 & 96.9 & 144.2 & 99.2 & 2.0 & NA \\
\hline SYN11A-41 & 99.2 & 2.0 & 100.8 & 2.8 & 139.4 & 50.9 & 99.2 & 2.0 & NA \\
\hline
\end{tabular}




\begin{tabular}{|c|c|c|c|c|c|c|c|c|c|}
\hline SYN11A-14 & 99.3 & 2.2 & 96.4 & 6.8 & 26.6 & 169.8 & 99.3 & 2.2 & NA \\
\hline SYN11A-15 & 99.3 & 2.0 & 94.6 & 6.2 & -21.7 & 158.2 & 99.3 & 2.0 & NA \\
\hline SYN11A-85 & 99.6 & 1.9 & 101.9 & 15.0 & 155.1 & 361.5 & 99.6 & 1.9 & NA \\
\hline SYN11A-84 & 99.7 & 3.6 & 94.2 & 7.0 & -42.1 & 166.3 & 99.7 & 3.6 & NA \\
\hline SYN11A-39 & 99.7 & 2.8 & 96.1 & 7.5 & 8.6 & 185.9 & 99.7 & 2.8 & NA \\
\hline SYN11A-9 & 99.9 & 3.0 & 95.2 & 7.0 & -21.9 & 171.2 & 99.9 & 3.0 & NA \\
\hline SYN11A-89 & 100.0 & 2.7 & 90.1 & 14.6 & -164.1 & 418.2 & 100.0 & 2.7 & NA \\
\hline SYN11A-57 & 100.0 & 3.2 & 100.3 & 9.0 & 106.1 & 208.7 & 100.0 & 3.2 & NA \\
\hline SYN11A-82 & 100.1 & 4.5 & 104.2 & 20.7 & 198.6 & 478.1 & 100.1 & 4.5 & NA \\
\hline SYN11A-83 & 100.1 & 2.7 & 91.8 & 9.3 & -119.5 & 252.1 & 100.1 & 2.7 & NA \\
\hline SYN11A-36 & 100.2 & 2.2 & 98.7 & 5.0 & 63.7 & 115.1 & 100.2 & 2.2 & NA \\
\hline SYN11A-30 & 100.2 & 2.4 & 98.1 & 9.4 & 46.5 & 234.9 & 100.2 & 2.4 & NA \\
\hline SYN11A-78 & 100.2 & 0.8 & 100.0 & 6.0 & 95.0 & 148.6 & 100.2 & 0.8 & NA \\
\hline SYN11A-76 & 100.6 & 3.1 & 99.6 & 7.1 & 75.8 & 161.3 & 100.6 & 3.1 & NA \\
\hline SYN11A-71 & 100.6 & 1.8 & 97.1 & 6.6 & 11.9 & 167.3 & 100.6 & 1.8 & NA \\
\hline SYN11A-35 & 100.9 & 1.3 & 100.5 & 1.7 & 91.4 & 26.7 & 100.9 & 1.3 & NA \\
\hline SYN11A-11 & 100.9 & 2.7 & 102.7 & 6.6 & 144.1 & 144.4 & 100.9 & 2.7 & NA \\
\hline SYN11A-68 & 101.1 & 2.7 & 106.4 & 4.6 & 226.2 & 85.9 & 101.1 & 2.7 & NA \\
\hline SYN11A-60 & 101.1 & 2.4 & 95.6 & 7.4 & -41.6 & 188.4 & 101.1 & 2.4 & NA \\
\hline SYN11A-79 & 101.2 & 1.9 & 99.6 & 4.0 & 63.0 & 90.8 & 101.2 & 1.9 & NA \\
\hline SYN11A-18 & 101.2 & 4.4 & 105.4 & 18.6 & 201.0 & 422.3 & 101.2 & 4.4 & NA \\
\hline SYN11A-16 & 101.3 & 1.1 & 111.4 & 18.2 & 332.7 & 393.2 & 101.3 & 1.1 & NA \\
\hline SYN11A-59 & 101.4 & 2.3 & 100.1 & 2.8 & 68.0 & 44.6 & 101.4 & 2.3 & NA \\
\hline SYN11A-53 & 101.5 & 2.1 & 98.2 & 8.9 & 20.4 & 223.9 & 101.5 & 2.1 & NA \\
\hline SYN11A-20 & 101.5 & 3.9 & 91.6 & 12.0 & -159.8 & 329.2 & 101.5 & 3.9 & NA \\
\hline SYN11A-80 & 101.6 & 1.6 & 89.1 & 9.7 & -233.6 & 284.6 & 101.6 & 1.6 & NA \\
\hline SYN11A-19 & 101.6 & 2.6 & 104.9 & 5.5 & 179.5 & 114.9 & 101.6 & 2.6 & NA \\
\hline SYN11A-38 & 101.7 & 2.6 & 95.9 & 12.9 & -44.6 & 337.6 & 101.7 & 2.6 & NA \\
\hline SYN11A-31 & 101.7 & 3.0 & 99.8 & 12.1 & 55.2 & 296.0 & 101.7 & 3.0 & NA \\
\hline SYN11A-23 & 101.9 & 2.8 & 78.6 & 13.0 & -585.2 & 463.0 & 101.9 & 2.8 & NA \\
\hline SYN11A-26 & 102.0 & 2.1 & 99.6 & 9.4 & 43.5 & 233.0 & 102.0 & 2.1 & NA \\
\hline SYN11A-94 & 102.0 & 1.8 & 97.9 & 8.7 & -1.2 & 221.7 & 102.0 & 1.8 & NA \\
\hline SYN11A-37 & 102.2 & 2.4 & 103.5 & 3.7 & 133.4 & 66.6 & 102.2 & 2.4 & NA \\
\hline SYN11A-48 & 102.2 & 3.6 & 101.3 & 14.8 & 80.9 & 355.2 & 102.2 & 3.6 & NA \\
\hline SYN11A-99 & 102.2 & 3.2 & 101.4 & 5.6 & 81.7 & 114.3 & 102.2 & 3.2 & NA \\
\hline SYN11A-28 & 102.5 & 1.9 & 108.3 & 4.2 & 237.9 & 84.1 & 102.5 & 1.9 & NA \\
\hline SYN11A-52 & 102.5 & 1.5 & 102.1 & 3.8 & 91.6 & 86.3 & 102.5 & 1.5 & NA \\
\hline SYN11A-44 & 102.9 & 2.1 & 100.9 & 4.7 & 54.3 & 105.8 & 102.9 & 2.1 & NA \\
\hline SYN11A-51 & 102.9 & 2.1 & 104.6 & 4.1 & 143.0 & 84.4 & 102.9 & 2.1 & NA \\
\hline
\end{tabular}




\begin{tabular}{|c|c|c|c|c|c|c|c|c|c|}
\hline SYN11A-24 & 103.0 & 3.9 & 95.7 & 20.8 & -81.6 & 556.8 & 103.0 & 3.9 & NA \\
\hline SYN11A-63 & 103.2 & 4.9 & 99.7 & 20.9 & 17.7 & 521.3 & 103.2 & 4.9 & NA \\
\hline SYN11A-47 & 103.2 & 2.1 & 103.4 & 5.7 & 108.7 & 126.6 & 103.2 & 2.1 & NA \\
\hline SYN11A-42 & 103.2 & 1.2 & 104.3 & 5.3 & 129.5 & 123.5 & 103.2 & 1.2 & NA \\
\hline SYN11A-92 & 103.3 & 3.7 & 95.7 & 17.8 & -90.4 & 472.4 & 103.3 & 3.7 & NA \\
\hline SYN11A-100 & 103.4 & 2.7 & 99.2 & 7.5 & -0.9 & 180.1 & 103.4 & 2.7 & NA \\
\hline SYN11A-3 & 103.5 & 2.4 & 103.7 & 4.4 & 108.6 & 90.8 & 103.5 & 2.4 & NA \\
\hline SYN11A-54 & 103.5 & 8.5 & 124.2 & 19.9 & 540.9 & 326.1 & 103.5 & 8.5 & NA \\
\hline SYN11A-33 & 103.6 & 2.3 & 104.5 & 4.4 & 125.6 & 90.8 & 103.6 & 2.3 & NA \\
\hline SYN11A-75 & 103.6 & 3.5 & 109.2 & 8.5 & 231.7 & 172.3 & 103.6 & 3.5 & NA \\
\hline SYN11A-1 & 103.7 & 2.4 & 96.1 & 15.7 & -88.2 & 417.4 & 103.7 & 2.4 & NA \\
\hline SYN11A-66 & 103.8 & 2.0 & 104.3 & 6.5 & 117.1 & 148.7 & 103.8 & 2.0 & NA \\
\hline SYN11A-10 & 103.9 & 1.8 & 111.1 & 14.9 & 269.3 & 323.8 & 103.9 & 1.8 & NA \\
\hline SYN11A-12 & 103.9 & 3.1 & 103.1 & 8.1 & 85.3 & 182.3 & 103.9 & 3.1 & NA \\
\hline SYN11A-97 & 103.9 & 3.1 & 104.2 & 4.9 & 110.8 & 93.8 & 103.9 & 3.1 & NA \\
\hline SYN11A-50 & 104.0 & 2.6 & 106.2 & 7.1 & 156.7 & 154.4 & 104.0 & 2.6 & NA \\
\hline SYN11A-55 & 104.2 & 3.3 & 103.6 & 4.9 & 89.5 & 90.0 & 104.2 & 3.3 & NA \\
\hline SYN11A-34 & 104.6 & 2.9 & 104.0 & 7.5 & 90.0 & 167.0 & 104.6 & 2.9 & NA \\
\hline SYN11A-65 & 105.3 & 1.7 & 105.2 & 4.9 & 102.0 & 107.9 & 105.3 & 1.7 & NA \\
\hline SYN11A-7 & 106.0 & 3.5 & 108.8 & 9.5 & 171.7 & 202.1 & 106.0 & 3.5 & NA \\
\hline SYN11A-4 & 106.1 & 3.6 & 107.8 & 16.5 & 146.7 & 372.7 & 106.1 & 3.6 & NA \\
\hline SYN11A-96 & 106.7 & 2.2 & 109.3 & 4.0 & 166.3 & 75.7 & 106.7 & 2.2 & NA \\
\hline SYN11A-22 & 109.2 & 3.4 & 105.4 & 21.8 & 20.6 & 522.3 & 109.2 & 3.4 & NA \\
\hline SYN11A-27 & 111.5 & 5.0 & 121.8 & 17.5 & 328.1 & 332.4 & 111.5 & 5.0 & NA \\
\hline SYN11A-73 & 114.8 & 2.5 & 110.4 & 15.7 & 17.0 & 359.3 & 114.8 & 2.5 & NA \\
\hline SYN11A-6 & 158.8 & 4.9 & 153.0 & 11.8 & 64.7 & 183.1 & 158.8 & 4.9 & NA \\
\hline SYN11A-81 & 159.6 & 2.9 & 165.2 & 10.6 & 245.9 & 155.1 & 159.6 & 2.9 & NA \\
\hline SYN11A-43 & 168.4 & 2.3 & 171.8 & 8.4 & 217.9 & 119.1 & 168.4 & 2.3 & NA \\
\hline SYN11A-5 & 170.5 & 1.7 & 168.9 & 4.4 & 147.6 & 62.3 & 170.5 & 1.7 & NA \\
\hline SYN11A-8 & 250.4 & 7.2 & 232.2 & 35.9 & 52.5 & 408.8 & 250.4 & 7.2 & NA \\
\hline SYN11A-49 & 333.7 & 8.4 & 333.8 & 13.8 & 334.1 & 93.4 & 333.7 & 8.4 & NA \\
\hline SYN11A-70 & 443.1 & 10.8 & 444.0 & 10.0 & 448.6 & 26.2 & 443.1 & 10.8 & 98.8 \\
\hline SYN11A-13 & 595.6 & 8.8 & 590.7 & 13.6 & 572.2 & 56.6 & 595.6 & 8.8 & 104.1 \\
\hline SYN11A-69 & 1019.6 & 33.6 & 1028.2 & 44.7 & 1046.5 & 119.5 & 1046.5 & 119.5 & 97.4 \\
\hline SYN11A-77 & 1145.9 & 12.2 & 1147.6 & 18.9 & 1150.9 & 49.4 & 1150.9 & 49.4 & 99.6 \\
\hline SYN11A-25 & 1464.3 & 24.0 & 1453.9 & 17.1 & 1438.8 & 23.8 & 1438.8 & 23.8 & 101.8 \\
\hline SYN11A-62 & 1626.8 & 23.5 & 1637.3 & 13.6 & 1650.8 & 6.9 & 1650.8 & 6.9 & 98.5 \\
\hline SYN11A-2 & 1794.9 & 8.4 & 1770.4 & 5.0 & 1741.5 & 4.8 & 1741.5 & 4.8 & 103.1 \\
\hline SYN11A-17 & 1785.1 & 32.2 & 1782.1 & 18.5 & 1778.7 & 14.2 & 1778.7 & 14.2 & 100.4 \\
\hline
\end{tabular}




\begin{tabular}{|l|l|l|l|l|l|l|l|l|l|} 
SYN11A-88 & 1834.8 & 17.2 & 1851.4 & 28.7 & 1870.1 & 57.6 & 1870.1 & 57.6 & 98.1 \\
\hline SYN11A-21 & 1896.2 & 29.6 & 1889.2 & 15.8 & 1881.5 & 6.7 & 1881.5 & 6.7 & 100.8 \\
\hline SYN11A-61 & 1975.7 & 17.5 & 1950.0 & 9.5 & 1922.8 & 7.0 & 1922.8 & 7.0 & 102.7 \\
\hline
\end{tabular}

\begin{tabular}{|c|c|c|c|c|c|c|c|c|c|}
\hline Analysis & $206 \mathrm{~Pb}^{*}$ & \pm & 207Pb* & \pm & $206 \mathrm{~Pb} *$ & \pm & Best age & \pm & Conc \\
\hline & $238 U^{*}$ & (Ma) & $235 U$ & (Ma) & 207Pb* & (Ma) & (Ma) & (Ma) & $(\%)$ \\
\hline & & & & & & & & & \\
\hline & & & & & & & & & \\
\hline SYN12-12-04 & 97.8 & 3.5 & 93.2 & 23.3 & -23.8 & 637.3 & 97.8 & 3.5 & NA \\
\hline SYN12-12-90 & 98.5 & 9.4 & 70.5 & 24.1 & -801.2 & 991.0 & 98.5 & 9.4 & NA \\
\hline SYN12-12-03 & 98.6 & 2.7 & 102.0 & 30.3 & 182.0 & 740.9 & 98.6 & 2.7 & NA \\
\hline SYN12-12-64 & 99.4 & 2.6 & 98.5 & 4.5 & 77.0 & 96.1 & 99.4 & 2.6 & NA \\
\hline SYN12-12-36 & 99.5 & 2.2 & 76.4 & 21.3 & -598.7 & 798.4 & 99.5 & 2.2 & NA \\
\hline SYN12-12-23 & 100.1 & 1.9 & 95.6 & 14.3 & -13.3 & 378.1 & 100.1 & 1.9 & NA \\
\hline SYN12-12-02 & 100.1 & 4.4 & 106.3 & 10.3 & 245.8 & 212.1 & 100.1 & 4.4 & NA \\
\hline SYN12-12-20 & 100.3 & 1.4 & 101.5 & 12.7 & 130.7 & 307.9 & 100.3 & 1.4 & NA \\
\hline SYN12-12-31 & 100.3 & 2.0 & 102.6 & 4.1 & 154.9 & 85.9 & 100.3 & 2.0 & NA \\
\hline SYN12-12-68 & 100.6 & 2.5 & 96.3 & 9.6 & -8.1 & 244.5 & 100.6 & 2.5 & NA \\
\hline SYN12-12-47 & 100.7 & 3.4 & 96.6 & 14.4 & -1.9 & 369.7 & 100.7 & 3.4 & NA \\
\hline SYN12-12-113 & 100.9 & 5.8 & 99.3 & 16.2 & 62.0 & 386.1 & 100.9 & 5.8 & NA \\
\hline SYN12-12-53 & 100.9 & 2.0 & 100.2 & 15.7 & 82.9 & 390.4 & 100.9 & 2.0 & NA \\
\hline SYN12-12-62 & 100.9 & 3.1 & 98.1 & 15.2 & 30.6 & 383.5 & 100.9 & 3.1 & NA \\
\hline SYN12 1224 & 101.0 & 6.2 & 90.3 & 65.4 & 182.4 & 2219.7 & 101.0 & 6.2 & AA \\
\hline SYN12-12-72 & 101.3 & 2.2 & 99.6 & 9.3 & 57.5 & 228.0 & 101.3 & 2.2 & NA \\
\hline SYN12-12-58 & 101.5 & 3.3 & 91.0 & 21.3 & -174.7 & 611.6 & 101.5 & 3.3 & NA \\
\hline SYN12-12-11 & 101.6 & 1.3 & 86.5 & 10.7 & -312.1 & 328.7 & 101.6 & 1.3 & NA \\
\hline SYN12-12-27 & 101.7 & 1.9 & 98.2 & 7.7 & 15.5 & 192.8 & 101.7 & 1.9 & NA \\
\hline SYN12-12-16 & 101.9 & 3.7 & 96.0 & 13.4 & -47.8 & 345.2 & 101.9 & 3.7 & NA \\
\hline SYN12-12-13 & 101.9 & 3.2 & 97.9 & 11.3 & 2.4 & 281.6 & 101.9 & 3.2 & NA \\
\hline SYN12-12-43 & 102.0 & 1.9 & 96.2 & 8.3 & -44.5 & 215.2 & 102.0 & 1.9 & NA \\
\hline SYN12-12-37 & 102.1 & 1.6 & 101.5 & 11.6 & 87.2 & 282.5 & 102.1 & 1.6 & NA \\
\hline SYN12-12-115 & 102.5 & 1.7 & 102.4 & 2.3 & 100.0 & 38.8 & 102.5 & 1.7 & NA \\
\hline SYN12-12-71 & 102.6 & 3.2 & 103.7 & 6.2 & 129.2 & 128.9 & 102.6 & 3.2 & NA \\
\hline SYN12-12-59 & 103.2 & 2.0 & 108.2 & 11.1 & 218.2 & 247.7 & 103.2 & 2.0 & NA \\
\hline SYN12-12-51 & 103.8 & 4.6 & 99.4 & 14.9 & -3.4 & 366.2 & 103.8 & 4.6 & NA \\
\hline SYN12-12-09 & 104.3 & 3.5 & 99.6 & 12.4 & -10.5 & 306.4 & 104.3 & 3.5 & NA \\
\hline SYN12-12-80 & 104.3 & 2.1 & 97.7 & 10.5 & -59.9 & 271.2 & 104.3 & 2.1 & NA \\
\hline SYN12-12-26 & 104.4 & 6.1 & 105.4 & 10.8 & 129.5 & 211.5 & 104.4 & 6.1 & NA \\
\hline
\end{tabular}




\begin{tabular}{|c|c|c|c|c|c|c|c|c|c|}
\hline SYN12-12-69 & 163.1 & 12.1 & 257.4 & 21.9 & 1236.9 & 117.4 & 163.1 & 12.1 & NA \\
\hline SYN12-12-81 & 403.6 & 2.5 & 401.9 & 3.9 & 391.7 & 21.9 & 403.6 & 2.5 & 103.0 \\
\hline SYN12-12-01 & 405.5 & 4.9 & 397.8 & 21.3 & 353.2 & 143.7 & 405.5 & 4.9 & 114.8 \\
\hline SYN12-12-08 & 421.4 & 5.3 & 423.7 & 13.4 & 436.2 & 80.8 & 421.4 & 5.3 & 96.6 \\
\hline SYN12-12-15 & 454.0 & 7.1 & 460.1 & 28.6 & 490.8 & 166.7 & 454.0 & 7.1 & 92.5 \\
\hline SYN12-12-119 & 466.8 & 7.8 & 467.6 & 17.8 & 471.4 & 97.6 & 466.8 & 7.8 & 99.0 \\
\hline SYN12-12-70 & 552.8 & 4.9 & 537.6 & 16.8 & 473.4 & 86.7 & 552.8 & 4.9 & 116.8 \\
\hline SYN12-12-98 & 575.3 & 13.4 & 569.6 & 28.2 & 547.0 & 131.0 & 575.3 & 13.4 & 105.2 \\
\hline SYN12-12-118 & 610.2 & 9.6 & 605.1 & 19.1 & 586.1 & 83.7 & 610.2 & 9.6 & 104.1 \\
\hline SYN12-12-21 & 854.4 & 48.7 & 858.6 & 36.0 & 869.3 & 24.1 & 854.4 & 48.7 & 98.3 \\
\hline SYN12-12-82 & 1001.8 & 14.7 & 1000.7 & 11.9 & 998.4 & 20.0 & 998.4 & 20.0 & 100.3 \\
\hline SYN12-12-83 & 1033.5 & 10.0 & 1024.5 & 9.5 & 1005.3 & 21.1 & 1005.3 & 21.1 & 102.8 \\
\hline SYN12-12-114 & 1021.6 & 10.0 & 1025.4 & 10.1 & 1033.7 & 23.4 & 1033.7 & 23.4 & 98.8 \\
\hline SYN12-12-75 & 1073.6 & 5.0 & 1072.5 & 6.8 & 1070.4 & 17.8 & 1070.4 & 17.8 & 100.3 \\
\hline SYN12-12-74 & 1074.1 & 34.6 & 1074.0 & 58.5 & 1073.7 & 162.7 & 1073.7 & 162.7 & 100.0 \\
\hline SYN12-12-78 & 1085.3 & 10.5 & 1082.6 & 12.2 & 1077.0 & 30.3 & 1077.0 & 30.3 & 100.8 \\
\hline SYN12-12-34 & 1075.6 & 7.8 & 1078.5 & 6.1 & 1084.3 & 9.3 & 1084.3 & 9.3 & 99.2 \\
\hline SYN12-12-95 & 1059.6 & 19.9 & 1070.1 & 30.3 & 1091.4 & 82.2 & 1091.4 & 82.2 & 97.1 \\
\hline SYN12-12-101 & 1076.8 & 17.9 & 1084.0 & 33.8 & 1098.4 & 95.0 & 1098.4 & 95.0 & 98.0 \\
\hline SYN12-12-117 & 1107.5 & 24.3 & 1105.8 & 26.4 & 1102.4 & 62.1 & 1102.4 & 62.1 & 100.5 \\
\hline SYN12-12-40 & 1126.0 & 10.9 & 1128.8 & 8.9 & 1134.1 & 15.2 & 1134.1 & 15.2 & 99.3 \\
\hline SYN12-12-97 & 1115.8 & 16.2 & 1126.7 & 16.2 & 1147.9 & 35.6 & 1147.9 & 35.6 & 97.2 \\
\hline SYN12-12-105 & 1277.7 & 15.8 & 1268.1 & 20.5 & 1251.7 & 48.6 & 1251.7 & 48.6 & 102.1 \\
\hline SYN12-12-29 & 1332.9 & 9.8 & 1328.5 & 8.2 & 1321.4 & 14.4 & 1321.4 & 14.4 & 100.9 \\
\hline SYN12-12-55 & 1331.7 & 16.1 & 1328.4 & 10.7 & 1323.0 & 10.2 & 1323.0 & 10.2 & 100.7 \\
\hline SYN12-12-88 & 1411.2 & 23.9 & 1406.1 & 41.3 & 1398.5 & 97.6 & 1398.5 & 97.6 & 100.9 \\
\hline SYN12-12-60 & 1427.7 & 15.1 & 1427.6 & 18.0 & 1427.3 & 38.9 & 1427.3 & 38.9 & 100.0 \\
\hline SYN12-12-57 & 1442.8 & 28.2 & 1440.8 & 18.0 & 1437.9 & 16.2 & 1437.9 & 16.2 & 100.3 \\
\hline SYN12-12-41 & 1422.9 & 11.4 & 1434.2 & 12.3 & 1450.9 & 25.3 & 1450.9 & 25.3 & 98.1 \\
\hline SYN12-12-25 & 1479.5 & 21.3 & 1470.7 & 12.9 & 1457.9 & 7.4 & 1457.9 & 7.4 & 101.5 \\
\hline SYN12-12-107 & 1577.6 & 43.8 & 1555.4 & 36.2 & 1525.5 & 62.2 & 1525.5 & 62.2 & 103.4 \\
\hline SYN12-12-116 & 1557.1 & 29.7 & 1565.2 & 20.2 & 1576.1 & 25.1 & 1576.1 & 25.1 & 98.8 \\
\hline SYN12-12-87 & 1648.8 & 26.4 & 1638.6 & 19.1 & 1625.6 & 27.5 & 1625.6 & 27.5 & 101.4 \\
\hline SYN12-12-05 & 1591.8 & 33.7 & 1615.0 & 20.0 & 1645.3 & 11.7 & 1645.3 & 11.7 & 96.7 \\
\hline SYN12-12-85 & 1683.3 & 17.8 & 1668.2 & 10.6 & 1649.2 & 9.0 & 1649.2 & 9.0 & 102.1 \\
\hline SYN12-12-99 & 1669.1 & 11.6 & 1661.1 & 14.5 & 1650.9 & 29.4 & 1650.9 & 29.4 & 101.1 \\
\hline SYN12-12-110 & 1700.5 & 19.9 & 1689.2 & 15.2 & 1675.2 & 23.6 & 1675.2 & 23.6 & 101.5 \\
\hline SYN12-12-63 & 1717.3 & 42.8 & 1715.0 & 23.8 & 1712.3 & 8.1 & 1712.3 & 8.1 & 100.3 \\
\hline SYN12-12-22 & 1681.2 & 18.6 & 1698.4 & 13.0 & 1719.7 & 17.4 & 1719.7 & 17.4 & 97.8 \\
\hline
\end{tabular}




\begin{tabular}{|c|c|c|c|c|c|c|c|c|c|}
\hline SYN12-12-77 & 1741.6 & 33.5 & 1742.4 & 20.9 & 1743.3 & 22.2 & \begin{tabular}{|l}
1743.3 \\
\end{tabular} & 22.2 & 99.9 \\
\hline SYN12-12-32 & 1798.0 & 31.1 & 1775.0 & 17.2 & 1748.0 & 9.7 & 1748.0 & 9.7 & 102.9 \\
\hline SYN12-12-89 & 1786.5 & 26.1 & 1778.1 & 14.3 & 1768.2 & 6.0 & 1768.2 & 6.0 & 101.0 \\
\hline SYN12-12-65 & 1775.6 & 8.9 & 1775.2 & 5.1 & 1774.7 & 3.8 & 1774.7 & 3.8 & 100.0 \\
\hline SYN12-12-44 & 1802.0 & 28.5 & 1795.2 & 15.3 & 1787.4 & 3.4 & 1787.4 & 3.4 & 100.8 \\
\hline SYN12-12-108 & 1799.4 & 12.7 & 1794.2 & 7.6 & 1788.2 & 7.4 & 1788.2 & 7.4 & 100.6 \\
\hline SYN12-12-96 & 1830.1 & 16.9 & 1813.3 & 9.5 & 1794.0 & 6.9 & 1794.0 & 6.9 & 102.0 \\
\hline SYN12-12-49 & 1821.4 & 15.2 & 1811.7 & 10.2 & 1800.5 & 13.5 & 1800.5 & 13.5 & 101.2 \\
\hline SYN12-12-33 & 1810.6 & 12.5 & 1808.8 & 6.8 & 1806.7 & 3.2 & 1806.7 & 3.2 & 100.2 \\
\hline SYN12-12-67 & 1847.5 & 17.8 & 1838.7 & 12.3 & 1828.7 & 16.9 & 1828.7 & 16.9 & 101.0 \\
\hline SYN12-12-17 & 1833.9 & 23.5 & 1831.7 & 13.5 & 1829.2 & 11.2 & 1829.2 & 11.2 & 100.3 \\
\hline SYN12-12-46 & 1837.6 & 16.1 & 1836.2 & 10.3 & 1834.6 & 12.2 & 1834.6 & 12.2 & 100.2 \\
\hline SYN12-12-100 & 1866.5 & 24.8 & 1854.9 & 14.1 & 1841.9 & 11.5 & 1841.9 & 11.5 & 101.3 \\
\hline SYN12-12-12 & 1825.2 & 12.1 & 1834.2 & 7.1 & 1844.4 & 6.1 & 1844.4 & 6.1 & 99.0 \\
\hline SYN12-12-103 & 1870.4 & 24.0 & 1859.5 & 15.8 & 1847.4 & 20.2 & 1847.4 & 20.2 & 101.2 \\
\hline SYN12-12-10 & 1854.8 & 22.6 & 1852.2 & 12.1 & 1849.3 & 4.1 & 1849.3 & 4.1 & 100.3 \\
\hline SYN12-12-61 & 1869.2 & 15.7 & 1860.3 & 8.6 & 1850.3 & 5.1 & 1850.3 & 5.1 & 101.0 \\
\hline SYN12-12-50 & 1850.6 & 17.9 & 1850.8 & 11.3 & 1851.1 & 13.1 & 1851.1 & 13.1 & 100.0 \\
\hline SYN12-12-93 & 1871.3 & 34.5 & 1864.4 & 20.9 & 1856.8 & 22.2 & 1856.8 & 22.2 & 100.8 \\
\hline SYN12-12-07 & 1898.9 & 36.5 & 1885.3 & 19.3 & 1870.3 & 7.4 & 1870.3 & 7.4 & 101.5 \\
\hline SYN12-12-86 & 1908.4 & 29.2 & 1890.8 & 17.9 & 1871.4 & 20.2 & 1871.4 & 20.2 & 102.0 \\
\hline SYN12-12-112 & 1856.7 & 18.6 & 1865.6 & 20.6 & 1875.5 & 38.2 & 1875.5 & 38.2 & 99.0 \\
\hline SYN12-12-35 & 1706.9 & 68.7 & 1784.7 & 38.6 & 1876.9 & 6.0 & 1876.9 & 6.0 & 90.9 \\
\hline SYN12-12-54 & 1908.6 & 10.1 & 1900.3 & 6.0 & 1891.2 & 6.0 & 1891.2 & 6.0 & 100.9 \\
\hline SYN12-12-18 & 1908.6 & 17.7 & 1922.0 & 11.9 & 1936.5 & 15.6 & 1936.5 & 15.6 & 98.6 \\
\hline SYN12-12-56 & 1961.7 & 22.4 & 1949.8 & 15.2 & 1937.3 & 20.6 & 1937.3 & 20.6 & 101.3 \\
\hline SYN12-12-39 & 1940.9 & 28.3 & 1953.7 & 15.1 & 1967.3 & 7.3 & 1967.3 & 7.3 & 98.7 \\
\hline SYN12-12-104 & 2050.8 & 32.0 & 2053.7 & 16.3 & 2056.6 & 5.9 & 2056.6 & 5.9 & 99.7 \\
\hline SYN12-12-94 & 2078.5 & 36.5 & 2077.1 & 18.6 & 2075.7 & 8.4 & 2075.7 & 8.4 & 100.1 \\
\hline SYN12-12-14 & 2155.9 & 43.3 & 2213.1 & 22.3 & 2266.5 & 12.4 & 2266.5 & 12.4 & 95.1 \\
\hline SYN12-12-109 & 2534.1 & 31.9 & 2520.7 & 14.2 & 2510.0 & 2.4 & 2510.0 & 2.4 & 101.0 \\
\hline SYN12-12-111 & 2487.5 & 67.2 & 2571.2 & 39.5 & 2637.9 & 44.6 & 2637.9 & 44.6 & 94.3 \\
\hline SYN12-12-45 & 2682.7 & 25.1 & 2683.3 & 10.9 & 2683.7 & 2.9 & 2683.7 & 2.9 & 100.0 \\
\hline SYN12-12-06 & 2575.5 & 44.1 & 2646.5 & 19.8 & 2701.2 & 5.7 & 2701.2 & 5.7 & 95.3 \\
\hline SYN12-12-91 & 2765.6 & 20.4 & 2734.4 & 9.3 & 2711.4 & 6.0 & 2711.4 & 6.0 & 102.0 \\
\hline SYN12-12-84 & 2755.1 & 24.8 & 2738.8 & 14.7 & 2726.8 & 17.9 & 2726.8 & 17.9 & 101.0 \\
\hline SYN12-12-73 & 2725.2 & 13.1 & 2726.5 & 6.2 & 2727.4 & 4.8 & 2727.4 & 4.8 & 99.9 \\
\hline SYN12-12-48 & 2759.5 & 21.1 & 2744.7 & 9.5 & 2733.8 & 5.7 & 2733.8 & 5.7 & 100.9 \\
\hline SYN12-12-42 & 2578.2 & 28.2 & 2670.4 & 12.7 & 2740.9 & 4.1 & 2740.9 & 4.1 & 94.1 \\
\hline
\end{tabular}




\begin{tabular}{|l|l|l|l|l|l|l|l|l|l|} 
SYN12-12-52 & 2735.2 & 45.6 & 2744.4 & 21.5 & 2751.1 & 16.1 & 2751.1 & 16.1 & 99.4 \\
\hline SYN12-12-120 & 3105.1 & 19.8 & 3164.7 & 14.6 & 3202.7 & 20.1 & 3202.7 & 20.1 & 97.0 \\
\hline
\end{tabular}

\begin{tabular}{|c|c|c|c|c|c|c|c|c|c|}
\hline Analysis & 206Pb* & \pm & 207Pb* & \pm & 206Pb* & \pm & Best age & \pm & Conc \\
\hline & $238 U^{*}$ & (Ma) & $235 \mathrm{U}$ & (Ma) & 207Pb* & (Ma) & (Ma) & (Ma) & $(\%)$ \\
\hline & & & & & & & & & \\
\hline & & & & & & & & & \\
\hline SYN12-13R-39 & 94.1 & 8.6 & 117.4 & 43.6 & 619.9 & 854.2 & 94.1 & 8.6 & NA \\
\hline SYN12-13R-19 & 95.2 & 5.1 & -129.6 & $\begin{array}{l}- \\
479.5 \\
\end{array}$ & NA & NA & 95.2 & 5.1 & NA \\
\hline SYN12-13R-55 & 95.4 & 3.4 & 78.5 & 29.0 & -410.2 & 1031.4 & 95.4 & 3.4 & NA \\
\hline SYN12-13R-111 & 96.0 & 1.8 & 95.2 & 7.6 & 75.2 & 193.4 & 96.0 & 1.8 & NA \\
\hline SYN12-13R-114 & 96.2 & 6.6 & 51.4 & 26.7 & NA & NA & 96.2 & 6.6 & NA \\
\hline SYN12-13R-109 & 96.4 & 0.9 & 96.8 & 6.6 & 105.6 & 167.9 & 96.4 & 0.9 & NA \\
\hline SYN12-13R-69 & 96.9 & 3.4 & 89.1 & 18.1 & -115.1 & 521.2 & 96.9 & 3.4 & NA \\
\hline SYN12-13R-30 & 97.2 & 3.1 & 90.7 & 23.7 & -74.6 & 672.1 & 97.2 & 3.1 & NA \\
\hline SYN12-13R-118 & 97.2 & 4.9 & 95.3 & 19.0 & 46.5 & 487.2 & 97.2 & 4.9 & NA \\
\hline SYN12-13R-98 & 97.3 & 0.9 & 97.8 & 5.7 & 111.7 & 143.1 & 97.3 & 0.9 & NA \\
\hline SYN12-13R-63 & 97.3 & 3.9 & 98.5 & 6.2 & 127.9 & 121.1 & 97.3 & 3.9 & NA \\
\hline SYN12-13R-47 & 97.3 & 5.3 & 90.2 & 20.2 & -93.9 & 565.4 & 97.3 & 5.3 & NA \\
\hline SYN12-13R-34 & 97.6 & 2.6 & 97.2 & 16.3 & 87.8 & 413.7 & 97.6 & 2.6 & NA \\
\hline SYN12-13R-74 & 97.8 & 1.7 & 94.6 & 7.3 & 15.7 & 190.1 & 97.8 & 1.7 & NA \\
\hline SYN12-13R-32 & 97.9 & 2.0 & 92.4 & 23.4 & -47.0 & 652.9 & 97.9 & 2.0 & NA \\
\hline SYN12-13R-65 & 97.9 & 4.9 & 98.9 & 7.7 & 122.6 & 151.5 & 97.9 & 4.9 & NA \\
\hline SYN12-13R-102 & 98.2 & 7.8 & 120.1 & 16.6 & 577.4 & 268.7 & 98.2 & 7.8 & NA \\
\hline SYN12-13R-35 & 98.3 & 1.7 & 98.4 & 4.8 & 99.8 & 114.4 & 98.3 & 1.7 & NA \\
\hline SYN12-13R-25 & 98.3 & 3.9 & 93.7 & 25.8 & -23.5 & 704.5 & 98.3 & 3.9 & NA \\
\hline SYN12-13R-5 & 98.4 & 2.8 & 93.7 & 10.4 & -24.2 & 273.5 & 98.4 & 2.8 & NA \\
\hline SYN12-13R-67 & 98.4 & 1.6 & 101.4 & 10.6 & 172.7 & 255.1 & 98.4 & 1.6 & NA \\
\hline SYN12-13R-85 & 98.4 & 3.3 & 94.3 & 15.1 & -8.8 & 398.6 & 98.4 & 3.3 & NA \\
\hline SYN12-13R-108 & 98.4 & 4.1 & 107.6 & 15.5 & 316.6 & 333.0 & 98.4 & 4.1 & NA \\
\hline SYN12-13R-14 & 98.5 & 1.8 & 95.1 & 9.3 & 12.9 & 242.5 & 98.5 & 1.8 & NA \\
\hline SYN12-13R-93 & 98.5 & 1.2 & 96.9 & 7.6 & 58.4 & 193.2 & 98.5 & 1.2 & NA \\
\hline SYN12-13R-64 & 98.5 & 1.4 & 97.5 & 4.3 & 72.0 & 105.4 & 98.5 & 1.4 & NA \\
\hline SYN12-13R-57 & 98.6 & 3.7 & 85.0 & 9.6 & -281.3 & 284.6 & 98.6 & 3.7 & NA \\
\hline SYN12-13R-36 & 98.8 & 2.0 & 90.7 & 9.4 & -117.0 & 264.5 & 98.8 & 2.0 & NA \\
\hline SYN12-13R-2 & 98.8 & 2.6 & 99.2 & 23.1 & 107.9 & 581.8 & 98.8 & 2.6 & NA \\
\hline SYN12-13R-38 & 98.8 & 2.8 & 95.0 & 7.7 & -1.0 & 194.7 & 98.8 & 2.8 & NA \\
\hline SYN12-13R-117 & 98.9 & 2.5 & 92.6 & 11.7 & -65.0 & 317.7 & 98.9 & 2.5 & NA \\
\hline
\end{tabular}




\begin{tabular}{|c|c|c|c|c|c|c|c|c|c|}
\hline SYN12-13R-27 & 98.9 & 1.4 & 101.5 & 3.5 & 162.5 & 77.9 & 98.9 & 1.4 & NA \\
\hline SYN12-13R-88 & 98.9 & 0.8 & 100.4 & 7.7 & 135.5 & 188.8 & 98.9 & 0.8 & NA \\
\hline SYN12-13R-66 & 98.9 & 7.1 & 99.6 & 31.4 & 116.3 & 778.1 & 98.9 & 7.1 & NA \\
\hline SYN12-13R-94 & 98.9 & 2.1 & 100.7 & 7.8 & 142.9 & 184.9 & 98.9 & 2.1 & NA \\
\hline SYN12-13R-84 & 99.2 & 3.2 & 107.5 & 14.8 & 295.1 & 325.1 & 99.2 & 3.2 & NA \\
\hline SYN12-13R-48 & 99.3 & 2.2 & 95.8 & 9.0 & 11.3 & 230.8 & 99.3 & 2.2 & NA \\
\hline SYN12-13R-96 & 99.5 & 1.5 & 106.9 & 12.6 & 277.1 & 283.8 & 99.5 & 1.5 & NA \\
\hline SYN12-13R-16 & 99.5 & 1.4 & 99.0 & 4.1 & 85.8 & 97.2 & 99.5 & 1.4 & NA \\
\hline SYN12-13R-1 & 99.5 & 1.8 & 101.6 & 16.4 & 150.5 & 398.3 & 99.5 & 1.8 & NA \\
\hline SYN12-13R-24 & 99.7 & 2.0 & 93.0 & 9.0 & -76.0 & 242.5 & 99.7 & 2.0 & NA \\
\hline SYN12-13R-11 & 99.7 & 2.3 & 102.1 & 8.4 & 157.1 & 196.0 & 99.7 & 2.3 & NA \\
\hline SYN12-13R-77 & 99.7 & 3.1 & 95.4 & 20.8 & -10.3 & 552.6 & 99.7 & 3.1 & NA \\
\hline SYN12-13R-72 & 99.7 & 2.1 & 101.1 & 10.2 & 133.1 & 245.0 & 99.7 & 2.1 & NA \\
\hline SYN12-13R-83 & 99.8 & 6.6 & 88.0 & 21.4 & -220.9 & 625.1 & 99.8 & 6.6 & NA \\
\hline SYN12-13R-13 & 99.9 & 2.1 & 95.7 & 4.0 & -7.8 & 91.2 & 99.9 & 2.1 & NA \\
\hline SYN12-13R-40 & 99.9 & 2.7 & 94.5 & 15.6 & -40.3 & 416.6 & 99.9 & 2.7 & NA \\
\hline SYN12-13R-9 & 100.1 & 1.8 & 93.3 & 17.5 & -78.1 & 483.1 & 100.1 & 1.8 & NA \\
\hline SYN12-13R-3 & 100.1 & 1.2 & 95.8 & 7.4 & -9.4 & 193.0 & 100.1 & 1.2 & NA \\
\hline SYN12-13R-92 & 100.1 & 9.1 & 97.1 & 24.9 & 22.8 & 615.7 & 100.1 & 9.1 & NA \\
\hline SYN12-13R-49 & 100.2 & 1.6 & 101.1 & 5.2 & 123.8 & 120.8 & 100.2 & 1.6 & NA \\
\hline SYN12-13R-87 & 100.7 & 3.1 & 123.2 & 19.3 & 584.1 & 356.5 & 100.7 & 3.1 & NA \\
\hline SYN12-13R-8 & 101.0 & 1.5 & 96.7 & 8.5 & -8.1 & 219.3 & 101.0 & 1.5 & NA \\
\hline SYN12-13R-86 & 101.0 & 1.6 & 100.7 & 5.4 & 93.6 & 128.1 & 101.0 & 1.6 & NA \\
\hline SYN12-13R-4 & 101.4 & 5.1 & 80.3 & 43.1 & -509.1 & 1592.9 & 101.4 & 5.1 & NA \\
\hline SYN12-13R-120 & 101.7 & 2.2 & 108.7 & 11.5 & 264.1 & 251.3 & 101.7 & 2.2 & NA \\
\hline SYN12-13R-76 & 103.0 & 9.6 & 105.8 & 42.6 & 168.4 & 1003.0 & 103.0 & 9.6 & NA \\
\hline SYN12-13R-22 & 104.5 & 1.9 & 94.5 & 22.3 & -151.7 & 620.4 & 104.5 & 1.9 & NA \\
\hline SYN12-13R-60 & 104.6 & 9.0 & 135.8 & 38.7 & 722.5 & 631.5 & 104.6 & 9.0 & NA \\
\hline SYN12-13R-41 & 117.8 & 4.7 & 122.4 & 13.2 & 212.4 & 248.8 & 117.8 & 4.7 & NA \\
\hline SYN12-13R-90 & 121.0 & 3.8 & 94.9 & 14.3 & -521.5 & 414.4 & 121.0 & 3.8 & NA \\
\hline SYN12-13R-103 & 154.6 & 7.3 & 180.1 & 56.1 & 529.6 & 756.2 & 154.6 & 7.3 & NA \\
\hline SYN12-13R-113 & 154.8 & 4.0 & 143.8 & 18.4 & -33.5 & 327.5 & 154.8 & 4.0 & NA \\
\hline SYN12-13R-112 & 270.5 & 1.9 & 278.1 & 9.3 & 342.7 & 84.5 & 270.5 & 1.9 & NA \\
\hline SYN12-13R-17 & 328.6 & 9.0 & 249.4 & 76.9 & -442.5 & 933.8 & 328.6 & 9.0 & NA \\
\hline SYN12-13R-71 & 330.5 & 8.6 & 326.8 & 14.2 & 300.2 & 98.2 & 330.5 & 8.6 & NA \\
\hline SYN12-13R-29 & 419.1 & 6.9 & 400.8 & 20.2 & 296.4 & 133.9 & 419.1 & 6.9 & 141.4 \\
\hline SYN12-13R-45 & 425.2 & 7.9 & 430.1 & 16.4 & 456.5 & 94.6 & 425.2 & 7.9 & 93.2 \\
\hline SYN12-13R-50 & 442.9 & 9.0 & 445.5 & 13.0 & 459.3 & 65.2 & 442.9 & 9.0 & 96.4 \\
\hline SYN12-13R-105 & 619.4 & 5.3 & 624.2 & 15.8 & 641.7 & 70.1 & 619.4 & 5.3 & 96.5 \\
\hline
\end{tabular}




\begin{tabular}{|c|c|c|c|c|c|c|c|c|c|}
\hline SYN12-13R-115 & 679.2 & 8.8 & 696.2 & 10.8 & 751.8 & 34.9 & 679.2 & 8.8 & 90.3 \\
\hline SYN12-13R-58 & 991.6 & 35.7 & 995.1 & 38.6 & 1002.8 & 95.0 & 1002.8 & 95.0 & 98.9 \\
\hline SYN12-13R-97 & 996.8 & 14.3 & 1003.4 & 13.9 & 1017.7 & 31.3 & 1017.7 & 31.3 & 97.9 \\
\hline SYN12-13R-33 & 1035.0 & 6.2 & 1031.6 & 11.6 & 1024.3 & 33.7 & 1024.3 & 33.7 & 101.0 \\
\hline SYN12-13R-82 & 993.9 & 15.3 & 1008.2 & 46.6 & 1039.3 & 143.5 & 1039.3 & 143.5 & 95.6 \\
\hline SYN12-13R-20 & 998.8 & 31.3 & 1011.6 & 22.6 & 1039.6 & 20.4 & 1039.6 & 20.4 & 96.1 \\
\hline SYN12-13R-99 & 1037.8 & 10.5 & 1042.6 & 21.5 & 1052.6 & 62.8 & 1052.6 & 62.8 & 98.6 \\
\hline SYN12-13R-106 & 1074.9 & 12.4 & 1076.9 & 12.4 & 1081.1 & 28.0 & 1081.1 & 28.0 & 99.4 \\
\hline SYN12-13R-107 & 1040.8 & 9.5 & 1065.9 & 23.6 & 1117.8 & 68.6 & 1117.8 & 68.6 & 93.1 \\
\hline SYN12-13R-42 & 1195.1 & 16.2 & 1175.6 & 19.9 & 1139.8 & 48.5 & 1139.8 & 48.5 & 104.9 \\
\hline SYN12-13R-68 & 1171.1 & 7.8 & 1166.2 & 9.6 & 1157.2 & 23.3 & 1157.2 & 23.3 & 101.2 \\
\hline SYN12-13R-46 & 1195.4 & 17.4 & 1194.4 & 34.8 & 1192.6 & 92.6 & 1192.6 & 92.6 & 100.2 \\
\hline SYN12-13R-26 & 1175.6 & 34.3 & 1194.4 & 61.4 & 1228.5 & 159.8 & 1228.5 & 159.8 & 95.7 \\
\hline SYN12-13R-78 & 1220.0 & 9.7 & 1226.6 & 9.8 & 1238.1 & 20.8 & 1238.1 & 20.8 & 98.5 \\
\hline SYN12-13R-80 & 1324.9 & 42.2 & 1324.0 & 31.6 & 1322.6 & 47.1 & 1322.6 & 47.1 & 100.2 \\
\hline SYN12-13R-15 & 1361.2 & 18.5 & 1355.9 & 20.4 & 1347.6 & 43.8 & 1347.6 & 43.8 & 101.0 \\
\hline SYN12-13R-79 & 1341.8 & 10.6 & 1360.9 & 18.6 & 1391.0 & 44.6 & 1391.0 & 44.6 & 96.5 \\
\hline SYN12-13R-51 & 1445.2 & 12.2 & 1441.0 & 17.9 & 1434.7 & 40.4 & 1434.7 & 40.4 & 100.7 \\
\hline SYN12-13R-21 & 1453.0 & 15.8 & 1448.4 & 10.0 & 1441.6 & 8.7 & 1441.6 & 8.7 & 100.8 \\
\hline SYN12-13R-101 & 1420.2 & 14.2 & 1429.3 & 16.0 & 1442.8 & 33.6 & 1442.8 & 33.6 & 98.4 \\
\hline SYN12-13R-73 & 1310.0 & 7.8 & 1361.8 & 6.7 & 1444.0 & 11.7 & 1444.0 & 11.7 & 90.7 \\
\hline SYN12-13R-56 & 1458.2 & 24.7 & 1456.6 & 22.7 & 1454.2 & 42.7 & 1454.2 & 42.7 & 100.3 \\
\hline SYN12-13R-89 & 1477.2 & 16.3 & 1468.8 & 15.4 & 1456.5 & 29.4 & 1456.5 & 29.4 & 101.4 \\
\hline SYN12-13R-12 & 1476.1 & 13.0 & 1482.2 & 10.7 & 1491.0 & 18.1 & 1491.0 & 18.1 & 99.0 \\
\hline SYN12-13R-81 & 1516.6 & 9.6 & 1515.9 & 14.4 & 1514.8 & 31.8 & 1514.8 & 31.8 & 100.1 \\
\hline SYN12-13R-59 & 1528.8 & 13.5 & 1526.5 & 9.8 & 1523.3 & 13.9 & 1523.3 & 13.9 & 100.4 \\
\hline SYN12-13R-95 & 1626.3 & 16.4 & 1611.7 & 11.2 & 1592.8 & 14.7 & 1592.8 & 14.7 & 102.1 \\
\hline SYN12-13R-28 & 1539.2 & 24.3 & 1565.9 & 18.7 & 1602.0 & 28.7 & 1602.0 & 28.7 & 96.1 \\
\hline SYN12-13R-53 & 1617.1 & 15.5 & 1618.9 & 12.6 & 1621.4 & 20.7 & 1621.4 & 20.7 & 99.7 \\
\hline SYN12-13R-31 & 1638.1 & 31.6 & 1637.2 & 18.4 & 1636.0 & 10.3 & 1636.0 & 10.3 & 100.1 \\
\hline SYN12-13R-7 & 1666.8 & 33.7 & 1683.2 & 20.2 & 1703.8 & 16.3 & 1703.8 & 16.3 & 97.8 \\
\hline SYN12-13R-91 & 1715.5 & 27.3 & 1714.2 & 15.1 & 1712.6 & 4.8 & 1712.6 & 4.8 & 100.2 \\
\hline SYN12-13R-70 & 1812.4 & 33.4 & 1821.8 & 20.1 & 1832.5 & 19.4 & 1832.5 & 19.4 & 98.9 \\
\hline SYN12-13R-116 & 1860.9 & 14.0 & 1852.1 & 18.9 & 1842.2 & 36.9 & 1842.2 & 36.9 & 101.0 \\
\hline SYN12-13R-62 & 1892.4 & 29.5 & 1886.0 & 16.1 & 1878.9 & 9.7 & 1878.9 & 9.7 & 100.7 \\
\hline SYN12-13R-110 & 1934.3 & 18.1 & 1914.6 & 17.5 & 1893.3 & 31.0 & 1893.3 & 31.0 & 102.2 \\
\hline SYN12-13R-54 & 1931.2 & 14.5 & 1924.5 & 10.7 & 1917.3 & 16.0 & 1917.3 & 16.0 & 100.7 \\
\hline SYN12-13R-43 & 2180.0 & 16.7 & 2165.4 & 8.7 & 2151.6 & 6.1 & 2151.6 & 6.1 & 101.3 \\
\hline SYN12-13R-10 & 2326.6 & 34.4 & 2344.8 & 17.3 & 2360.7 & 11.9 & 2360.7 & 11.9 & 98.6 \\
\hline
\end{tabular}




\begin{tabular}{|l|l|l|l|l|l|l|l|l|l|} 
SYN12-13R-104 & 2579.2 & 45.3 & 2542.2 & 34.6 & 2512.8 & 51.0 & 2512.8 & 51.0 & 102.6 \\
\hline SYN12-13R-23 & 2445.5 & 60.4 & 2548.7 & 28.9 & 2631.9 & 15.0 & 2631.9 & 15.0 & 92.9 \\
\hline SYN12-13R-61 & 2705.5 & 67.6 & 2741.4 & 29.3 & 2768.0 & 7.5 & 2768.0 & 7.5 & 97.7 \\
\hline SYN12-13R-6 & 2786.4 & 20.1 & 2789.3 & 8.7 & 2791.4 & 3.9 & 2791.4 & 3.9 & 99.8 \\
\hline
\end{tabular}

\begin{tabular}{|c|c|c|c|c|c|c|c|c|c|}
\hline Analysis & 206Pb* & \pm & 207Pb* & \pm & $206 \mathrm{~Pb}^{*}$ & \pm & Best age & \pm & Conc \\
\hline & $238 U^{*}$ & (Ma) & $235 U$ & (Ma) & 207Pb* & (Ma) & (Ma) & (Ma) & (\%) \\
\hline & & & & & & & & & \\
\hline & & & & & & & & & \\
\hline SYN12-14-8 & 92.3 & 1.9 & 103.7 & 6.8 & 373.6 & 148.9 & 92.3 & 1.9 & NA \\
\hline SYN12-14-22 & 98.1 & 4.3 & 85.4 & 12.8 & -256.7 & 381.5 & 98.1 & 4.3 & NA \\
\hline SYN12-14-17 & 98.2 & 2.6 & 105.0 & 11.3 & 262.4 & 253.8 & 98.2 & 2.6 & NA \\
\hline SYN12-14-2 & 98.4 & 7.1 & 79.7 & 25.1 & -452.3 & 858.6 & 98.4 & 7.1 & NA \\
\hline SYN12-14-6 & 99.0 & 1.9 & 95.1 & 5.4 & -0.5 & 136.3 & 99.0 & 1.9 & NA \\
\hline SYN12-14-3 & 99.1 & 1.8 & 100.6 & 2.8 & 134.5 & 54.6 & 99.1 & 1.8 & NA \\
\hline SYN12-14-14 & 99.2 & 1.2 & 100.8 & 1.9 & 140.6 & 36.5 & 99.2 & 1.2 & NA \\
\hline SYN12-14-19 & 99.5 & 1.0 & 101.9 & 4.4 & 158.0 & 104.3 & 99.5 & 1.0 & NA \\
\hline SYN12-14-13 & 99.5 & 2.4 & 103.2 & 9.6 & 189.5 & 220.9 & 99.5 & 2.4 & NA \\
\hline SYN12-14-20 & 99.6 & 3.4 & 99.6 & 5.6 & 100.0 & 112.7 & 99.6 & 3.4 & NA \\
\hline SYN12-14-26 & 99.8 & 2.7 & 97.3 & 7.4 & 36.8 & 178.7 & 99.8 & 2.7 & NA \\
\hline SYN12-14-33 & 99.9 & 3.3 & 99.8 & 5.4 & 96.1 & 111.2 & 99.9 & 3.3 & NA \\
\hline SYN12-14-4 & 100.0 & 1.3 & 99.9 & 3.6 & 97.3 & 83.3 & 100.0 & 1.3 & NA \\
\hline SYN12-14-38 & 100.0 & 2.2 & 100.2 & 7.6 & 104.2 & 181.9 & 100.0 & 2.2 & NA \\
\hline SYN12-14-39 & 100.2 & 1.4 & 93.6 & 23.5 & -70.9 & 651.1 & 100.2 & 1.4 & NA \\
\hline SYN12-14-7 & 100.3 & 4.5 & 103.6 & 14.8 & 180.4 & 336.9 & 100.3 & 4.5 & NA \\
\hline SYN12-14-29 & 100.7 & 1.5 & 97.3 & 4.6 & 16.6 & 113.0 & 100.7 & 1.5 & NA \\
\hline SYN12-14-27 & 100.9 & 3.3 & 105.3 & 10.3 & 205.5 & 226.8 & 100.9 & 3.3 & NA \\
\hline SYN12-14-23 & 101.0 & 1.9 & 101.0 & 3.9 & 101.2 & 85.1 & 101.0 & 1.9 & NA \\
\hline SYN12-14-40 & 101.6 & 1.7 & 101.1 & 2.6 & 90.0 & 49.2 & 101.6 & 1.7 & NA \\
\hline SYN12-14-9 & 101.6 & 1.7 & 98.0 & 9.5 & 11.2 & 241.4 & 101.6 & 1.7 & NA \\
\hline SYN12-14-41 & 102.4 & 3.6 & 102.7 & 11.2 & 110.1 & 257.0 & 102.4 & 3.6 & NA \\
\hline SYN12-14-1 & 155.7 & 3.9 & 156.9 & 5.4 & 175.5 & 61.7 & 155.7 & 3.9 & NA \\
\hline SYN12-14-12 & 157.9 & 6.0 & 174.0 & 8.9 & 399.3 & 90.3 & 157.9 & 6.0 & NA \\
\hline SYN12-14-15 & 1025.8 & 14.8 & 1025.9 & 14.6 & 1026.1 & 33.2 & 1026.1 & 33.2 & 100.0 \\
\hline SYN12-14-24 & 1031.4 & 38.9 & 1048.2 & 40.8 & 1083.4 & 94.8 & 1083.4 & 94.8 & 95.2 \\
\hline SYN12-14-31 & 1087.2 & 22.2 & 1107.4 & 32.8 & 1147.3 & 86.0 & 1147.3 & 86.0 & 94.8 \\
\hline SYN12-14-11 & 1441.5 & 9.3 & 1432.8 & 16.5 & 1419.8 & 38.6 & 1419.8 & 38.6 & 101.5 \\
\hline SYN12-14-36 & 1451.1 & 30.4 & 1445.2 & 19.3 & 1436.6 & 17.2 & 1436.6 & 17.2 & 101.0 \\
\hline
\end{tabular}




\begin{tabular}{|l|l|l|l|l|l|l|l|l|l|} 
SYN12-14-30 & 1709.3 & 28.8 & 1680.8 & 17.9 & 1645.5 & 19.3 & 1645.5 & 19.3 & 103.9 \\
\hline SYN12-14-35 & 1512.3 & 100.3 & 1571.6 & 59.8 & 1652.2 & 13.5 & 1652.2 & 13.5 & 91.5 \\
\hline SYN12-14-10 & 1756.9 & 16.9 & 1758.3 & 10.6 & 1759.9 & 11.8 & 1759.9 & 11.8 & 99.8 \\
\hline SYN12-14-32 & 1839.3 & 15.6 & 1836.0 & 10.2 & 1832.2 & 12.8 & 1832.2 & 12.8 & 100.4 \\
\hline SYN12-14-21 & 1867.7 & 18.1 & 1853.2 & 13.0 & 1836.9 & 18.9 & 1836.9 & 18.9 & 101.7 \\
\hline SYN12-14-34 & 1917.3 & 14.0 & 1906.5 & 10.5 & 1894.7 & 16.0 & 1894.7 & 16.0 & 101.2 \\
\hline SYN12-14-16 & 2018.6 & 29.5 & 2003.7 & 15.4 & 1988.4 & 8.2 & 1988.4 & 8.2 & 101.5 \\
\hline SYN12-14-37 & 2097.3 & 17.7 & 2097.1 & 12.8 & 2096.9 & 18.4 & 2096.9 & 18.4 & 100.0 \\
\hline SYN12-14-25 & 2326.6 & 32.5 & 2315.7 & 15.6 & 2306.0 & 6.4 & 2306.0 & 6.4 & 100.9 \\
\hline SYN12-14-28 & 2757.5 & 120.9 & 2885.1 & 51.9 & 2975.5 & 10.7 & 2975.5 & 10.7 & 92.7 \\
\hline
\end{tabular}

\begin{tabular}{|c|c|c|c|c|c|c|c|c|c|}
\hline Analysis & $206 \mathrm{~Pb}^{*}$ & \pm & $207 \mathrm{~Pb}^{*}$ & \pm & $206 \mathrm{~Pb}^{*}$ & \pm & Best age & \pm & Conc \\
\hline & $238 U^{*}$ & (Ma) & $235 U$ & (Ma) & $207 \mathrm{~Pb}^{*}$ & (Ma) & (Ma) & (Ma) & $(\%)$ \\
\hline SYN12-15-6 & 87.7 & 6.9 & 248.1 & 673.2 & 2307.4 & 178.6 & 87.7 & 6.9 & NA \\
\hline SYN12-15-11 & 89.2 & 5.2 & 77.3 & 20.2 & -275.5 & 684.7 & 89.2 & 5.2 & NA \\
\hline SYN12-15-47 & 90.9 & 4.2 & 84.4 & 17.2 & -95.6 & 513.6 & 90.9 & 4.2 & NA \\
\hline SYN12-15-8 & 91.2 & 2.9 & 92.6 & 6.7 & 129.0 & 160.0 & 91.2 & 2.9 & NA \\
\hline SYN12-15-98 & 91.2 & 6.9 & 92.3 & 15.3 & 119.0 & 370.2 & 91.2 & 6.9 & NA \\
\hline SYN12-15-86 & 91.5 & 2.1 & 98.3 & 12.4 & 265.5 & 300.4 & 91.5 & 2.1 & NA \\
\hline SYN12-15-23 & 91.7 & 4.4 & 90.8 & 9.6 & 65.6 & 236.7 & 91.7 & 4.4 & NA \\
\hline SYN12-15-60 & 92.4 & 2.6 & 92.8 & 15.0 & 103.9 & 396.3 & 92.4 & 2.6 & NA \\
\hline SYN12-15-90 & 92.6 & 2.6 & 95.3 & 11.7 & 163.4 & 294.8 & 92.6 & 2.6 & NA \\
\hline SYN12-15-38 & 92.9 & 1.4 & 95.3 & 7.1 & 155.6 & 180.4 & 92.9 & 1.4 & NA \\
\hline SYN12-15-19 & 93.2 & 3.8 & 93.7 & 7.2 & 106.0 & 165.1 & 93.2 & 3.8 & NA \\
\hline SYN12-15-96 & 93.3 & 8.5 & 92.1 & 57.0 & 59.9 & 1701.1 & 93.3 & 8.5 & NA \\
\hline SYN12-15-80 & 93.8 & 2.3 & 97.6 & 6.4 & 193.4 & 150.4 & 93.8 & 2.3 & NA \\
\hline SYN12-15-51 & 94.3 & 1.8 & 90.6 & 4.8 & -6.0 & 125.2 & 94.3 & 1.8 & NA \\
\hline SYN12-15-15 & 94.7 & 3.7 & 78.5 & 20.1 & -391.2 & 693.7 & 94.7 & 3.7 & NA \\
\hline SYN12-15-50 & 94.9 & 1.1 & 92.1 & 10.5 & 20.5 & 286.3 & 94.9 & 1.1 & NA \\
\hline SYN12-15-65 & 94.9 & 5.7 & 78.6 & 33.8 & -392.1 & 1202.3 & 94.9 & 5.7 & NA \\
\hline SYN12-15-34 & 95.1 & 4.4 & 93.5 & 9.2 & 52.8 & 218.3 & 95.1 & 4.4 & NA \\
\hline SYN12-15-30 & 95.3 & 2.2 & 88.1 & 12.7 & -101.1 & 367.1 & 95.3 & 2.2 & NA \\
\hline SYN12-15-40 & 95.4 & 5.6 & 84.7 & 95.3 & -208.4 & 1373.4 & 95.4 & 5.6 & NA \\
\hline SYN12-15-44 & 96.0 & 2.6 & 102.5 & 24.5 & 257.0 & 583.0 & 96.0 & 2.6 & NA \\
\hline SYN12-15-53 & 96.0 & 1.2 & 98.0 & 7.1 & 145.1 & 175.0 & 96.0 & 1.2 & NA \\
\hline SYN12-15-75 & 96.1 & 3.9 & 93.3 & 7.2 & 20.7 & 165.8 & 96.1 & 3.9 & NA \\
\hline SYN12-15-72 & 96.2 & 1.8 & 83.7 & 8.7 & -258.3 & 269.8 & 96.2 & 1.8 & NA \\
\hline
\end{tabular}




\begin{tabular}{|c|c|c|c|c|c|c|c|c|c|}
\hline SYN12-15-95 & 96.7 & 5.5 & 94.2 & 23.0 & 31.9 & 604.3 & 96.7 & 5.5 & NA \\
\hline SYN12-15-18 & 96.9 & 6.1 & 81.1 & 65.9 & -360.1 & 2749.8 & 96.9 & 6.1 & NA \\
\hline SYN12-15-66 & 97.1 & 2.3 & 92.2 & 10.7 & -32.9 & 290.4 & 97.1 & 2.3 & NA \\
\hline SYN12-15-4 & 97.4 & 1.1 & 99.3 & 4.1 & 145.8 & 98.8 & 97.4 & 1.1 & NA \\
\hline SYN12-15-57 & 97.4 & 2.2 & 82.5 & 16.1 & -329.7 & 522.8 & 97.4 & 2.2 & NA \\
\hline SYN12-15-9 & 97.6 & 0.7 & 102.3 & 3.7 & 212.9 & 87.2 & 97.6 & 0.7 & NA \\
\hline SYN12-15-83 & 97.8 & 3.0 & 99.4 & 14.3 & 140.1 & 348.9 & 97.8 & 3.0 & NA \\
\hline SYN12-15-26 & 97.8 & 3.1 & 82.8 & 21.7 & -331.7 & 706.6 & 97.8 & 3.1 & NA \\
\hline SYN12-15-27 & 98.8 & 3.3 & 105.4 & 5.5 & 257.4 & 99.5 & 98.8 & 3.3 & NA \\
\hline SYN12-15-1 & 98.9 & 2.8 & 92.6 & 19.6 & -67.1 & 542.3 & 98.9 & 2.8 & NA \\
\hline SYN12-15-62 & 99.1 & 5.4 & 110.4 & 17.4 & 362.1 & 355.1 & 99.1 & 5.4 & NA \\
\hline SYN12-15-39 & 99.8 & 4.5 & 104.9 & 52.1 & 221.9 & 1287.2 & 99.8 & 4.5 & NA \\
\hline SYN12-15-71 & 100.0 & 1.5 & 99.2 & 7.5 & 78.7 & 185.6 & 100.0 & 1.5 & NA \\
\hline SYN12-15-12 & 101.0 & 3.2 & 106.1 & 7.4 & 223.4 & 152.3 & 101.0 & 3.2 & NA \\
\hline SYN12-15-64 & 101.5 & 1.9 & 101.0 & 13.1 & 89.9 & 321.9 & 101.5 & 1.9 & NA \\
\hline SYN12-15-49 & 101.7 & 3.1 & 114.0 & 8.4 & 377.5 & 162.0 & 101.7 & 3.1 & NA \\
\hline SYN12-15-29 & 106.0 & 7.7 & 125.2 & 40.8 & 507.7 & 765.2 & 106.0 & 7.7 & NA \\
\hline SYN12-15-10 & 111.0 & 4.9 & 106.7 & 25.3 & 11.6 & 598.5 & 111.0 & 4.9 & NA \\
\hline SYN12-15-93 & 164.0 & 8.4 & 175.0 & 13.2 & 326.3 & 145.8 & 164.0 & 8.4 & NA \\
\hline SYN12-15-61 & 251.1 & 3.1 & 246.8 & 9.4 & 206.4 & 95.0 & 251.1 & 3.1 & NA \\
\hline SYN12-15-84 & 331.4 & 4.4 & 321.6 & 14.9 & 251.3 & 120.3 & 331.4 & 4.4 & NA \\
\hline SYN12-15-86 & 395.2 & 11.5 & 388.2 & 28.3 & 346.8 & 186.6 & 395.2 & 11.5 & NA \\
\hline SYN12-15-21 & 475.2 & 20.4 & 489.3 & 34.9 & 555.8 & 170.7 & 475.2 & 20.4 & 85.5 \\
\hline SYN12-15-52 & 492.9 & 10.3 & 513.1 & 21.5 & 604.3 & 105.4 & 492.9 & 10.3 & 81.6 \\
\hline SYN12-15-94 & 547.6 & 3.5 & 539.6 & 11.5 & 505.8 & 58.9 & 547.6 & 3.5 & 108.3 \\
\hline SYN12-15-25 & 615.5 & 10.9 & 619.8 & 26.8 & 635.7 & 117.6 & 615.5 & 10.9 & 96.8 \\
\hline SYN12-15-46 & 648.2 & 12.4 & 674.2 & 15.2 & 761.9 & 49.0 & 648.2 & 12.4 & 85.1 \\
\hline SYN12-15-42 & 994.3 & 13.4 & 992.9 & 15.0 & 989.8 & 37.9 & 989.8 & 37.9 & 100.5 \\
\hline SYN12-15-45 & 998.7 & 8.5 & 996.2 & 17.2 & 990.8 & 51.7 & 990.8 & 51.7 & 100.8 \\
\hline SYN12-15-85 & 1045.5 & 18.8 & 1042.7 & 13.5 & 1036.8 & 14.0 & 1036.8 & 14.0 & 100.8 \\
\hline SYN12-15-82 & 1024.9 & 29.2 & 1029.3 & 51.3 & 1038.5 & 147.5 & 1038.5 & 147.5 & 98.7 \\
\hline SYN12-15-24 & 1034.5 & 15.8 & 1038.3 & 20.3 & 1046.3 & 53.3 & 1046.3 & 53.3 & 98.9 \\
\hline SYN12-15-20 & 1080.4 & 28.1 & 1071.3 & 38.0 & 1052.7 & 100.7 & 1052.7 & 100.7 & 102.6 \\
\hline SYN12-15-81 & 1041.3 & 12.5 & 1053.7 & 23.1 & 1079.4 & 65.7 & 1079.4 & 65.7 & 96.5 \\
\hline SYN12-15-67 & 1081.6 & 13.1 & 1082.1 & 17.5 & 1083.0 & 45.6 & 1083.0 & 45.6 & 99.9 \\
\hline SYN12-15-77 & 1040.9 & 28.5 & 1061.2 & 27.3 & 1103.1 & 58.1 & 1103.1 & 58.1 & 94.4 \\
\hline SYN12-15-2 & 1153.8 & 15.7 & 1139.5 & 39.0 & 1112.3 & 109.8 & 1112.3 & 109.8 & 103.7 \\
\hline SYN12-15-73 & 1164.1 & 31.1 & 1159.6 & 68.2 & 1151.3 & 187.2 & 1151.3 & 187.2 & 101.1 \\
\hline SYN12-15-16 & 1129.4 & 7.2 & 1139.3 & 6.5 & 1158.4 & 12.9 & 1158.4 & 12.9 & 97.5 \\
\hline
\end{tabular}




\begin{tabular}{|c|c|c|c|c|c|c|c|c|c|}
\hline SYN12-15-97 & 1169.7 & 21.7 & 1168.0 & 30.0 & 1164.9 & 75.6 & 1164.9 & 75.6 & 100.4 \\
\hline SYN12-15-17 & 1117.3 & 8.5 & 1133.6 & 7.4 & 1165.0 & 14.2 & 1165.0 & 14.2 & 95.9 \\
\hline SYN12-15-5 & 1113.8 & 36.4 & 1134.2 & 24.5 & 1173.4 & 7.7 & 1173.4 & 7.7 & 94.9 \\
\hline SYN12-15-32 & 1387.7 & 11.2 & 1384.6 & 14.5 & 1379.9 & 32.5 & 1379.9 & 32.5 & 100.6 \\
\hline SYN12-15-58 & 1409.3 & 24.3 & 1399.6 & 18.5 & 1384.9 & 28.8 & 1384.9 & 28.8 & 101.8 \\
\hline SYN12-15-14 & 1428.8 & 9.8 & 1430.0 & 18.0 & 1431.8 & 42.4 & 1431.8 & 42.4 & 99.8 \\
\hline SYN12-15-43 & 1457.6 & 7.7 & 1451.0 & 6.3 & 1441.3 & 10.5 & 1441.3 & 10.5 & 101.1 \\
\hline SYN12-15-91 & 1475.2 & 19.9 & 1473.8 & 16.0 & 1471.7 & 26.5 & 1471.7 & 26.5 & 100.2 \\
\hline SYN12-15-41 & 1579.1 & 7.4 & 1593.5 & 5.9 & 1612.6 & 9.5 & 1612.6 & 9.5 & 97.9 \\
\hline SYN12-15-100 & 1655.8 & 10.2 & 1642.7 & 11.0 & 1626.0 & 21.5 & 1626.0 & 21.5 & 101.8 \\
\hline SYN12-15-89 & 1646.2 & 18.2 & 1650.5 & 14.0 & 1655.9 & 21.7 & 1655.9 & 21.7 & 99.4 \\
\hline SYN12-15-3 & 1671.3 & 19.5 & 1665.1 & 14.1 & 1657.2 & 20.6 & 1657.2 & 20.6 & 100.8 \\
\hline SYN12-15-63 & 1652.9 & 38.0 & 1669.5 & 34.4 & 1690.5 & 60.7 & 1690.5 & 60.7 & 97.8 \\
\hline SYN12-15-69 & 1457.3 & 116.5 & 1571.9 & 71.8 & 1729.2 & 14.4 & 1729.2 & 14.4 & 84.3 \\
\hline SYN12-15-37 & 1647.2 & 41.0 & 1695.5 & 24.5 & 1755.7 & 17.0 & 1755.7 & 17.0 & 93.8 \\
\hline SYN12-15-92 & 1752.7 & 9.8 & 1755.0 & 11.3 & 1757.7 & 21.9 & 1757.7 & 21.9 & 99.7 \\
\hline SYN12-15-59 & 1689.5 & 80.7 & 1726.9 & 45.3 & 1772.5 & 9.4 & 1772.5 & 9.4 & 95.3 \\
\hline SYN12-15-22 & 1779.2 & 21.8 & 1779.1 & 13.9 & 1778.9 & 15.9 & 1778.9 & 15.9 & 100.0 \\
\hline SYN12-15-35 & 1783.9 & 34.0 & 1788.7 & 28.2 & 1794.3 & 46.4 & 1794.3 & 46.4 & 99.4 \\
\hline SYN12-15-78 & 1838.1 & 15.3 & 1823.6 & 22.6 & 1807.2 & 45.2 & 1807.2 & 45.2 & 101.7 \\
\hline SYN12-15-36 & 1828.3 & 24.2 & 1825.5 & 14.1 & 1822.2 & 12.3 & 1822.2 & 12.3 & 100.3 \\
\hline SYN12-15-79 & 1857.0 & 13.4 & 1846.1 & 22.0 & 1833.9 & 44.3 & 1833.9 & 44.3 & 101.3 \\
\hline SYN12-15-48 & 1856.4 & 7.4 & 1846.8 & 5.4 & 1835.9 & 8.0 & 1835.9 & 8.0 & 101.1 \\
\hline SYN12-15-31 & 1873.7 & 11.4 & 1861.5 & 6.4 & 1847.9 & 5.0 & 1847.9 & 5.0 & 101.4 \\
\hline SYN12-15-56 & 1956.2 & 22.6 & 1941.2 & 13.8 & 1925.3 & 15.6 & 1925.3 & 15.6 & 101.6 \\
\hline SYN12-15-70 & 2039.5 & 42.0 & 2034.8 & 31.0 & 2029.9 & 45.6 & 2029.9 & 45.6 & 100.5 \\
\hline SYN12-15-74 & 2727.9 & 17.1 & 2721.9 & 7.6 & 2717.4 & 3.9 & 2717.4 & 3.9 & 100.4 \\
\hline SYN12-15-33 & 2728.8 & 80.4 & 2732.7 & 34.9 & 2735.6 & 12.0 & 2735.6 & 12.0 & 99.8 \\
\hline SYN12-15-13 & 2983.9 & 26.8 & 2975.0 & 11.0 & 2969.0 & 3.1 & 2969.0 & 3.1 & 100.5 \\
\hline
\end{tabular}

\begin{tabular}{|l|l|l|l|l|l|l|l|l|l|}
\hline Analysis & $206 \mathrm{~Pb}^{*}$ & \pm & $207 \mathrm{~Pb}^{*}$ & \pm & $206 \mathrm{~Pb}^{*}$ & \pm & Best age & \pm & Conc \\
\hline & $238 \mathrm{U}^{*}$ & $(\mathrm{Ma})$ & $235 \mathrm{U}$ & $(\mathrm{Ma})$ & $207 \mathrm{~Pb}^{*}$ & $(\mathrm{Ma})$ & $(\mathrm{Ma})$ & $(\mathrm{Ma})$ & $(\%)$ \\
\hline & & & & & & & & & \\
\hline & & & & & & & & & \\
\hline & & & & & & & & & \\
\hline SYN12-16-41 & 90.2 & 1.3 & 89.1 & 4.3 & 57.7 & 116.3 & 90.2 & 1.3 & NA \\
\hline SYN12-16-52 & 93.4 & 2.0 & 95.6 & 5.1 & 150.8 & 120.2 & 93.4 & 2.0 & NA \\
\hline SYN12-16-95 & 95.3 & 1.8 & 95.8 & 8.9 & 108.1 & 227.4 & 95.3 & 1.8 & NA \\
\hline SYN12-16-97 & 97.8 & 4.1 & 92.4 & 17.0 & -42.3 & 459.4 & 97.8 & 4.1 & NA \\
\hline
\end{tabular}




\begin{tabular}{|c|c|c|c|c|c|c|c|c|c|}
\hline SYN12-16-98 & 103.3 & 3.1 & 99.2 & 9.8 & 2.7 & 238.0 & 103.3 & 3.1 & NA \\
\hline SYN12-16-50 & 201.4 & 3.9 & 191.3 & 11.0 & 68.3 & 143.0 & 201.4 & 3.9 & NA \\
\hline SYN12-16-72 & 270.1 & 5.4 & 298.6 & 11.0 & 527.4 & 81.9 & 270.1 & 5.4 & NA \\
\hline SYN12-16-75 & 328.0 & 6.5 & 339.3 & 15.5 & 417.6 & 111.1 & 328.0 & 6.5 & NA \\
\hline SYN12-16-70 & 341.1 & 2.7 & 346.5 & 7.4 & 382.3 & 53.5 & 341.1 & 2.7 & NA \\
\hline SYN12-16-3 & 379.8 & 7.9 & 374.5 & 26.7 & 342.3 & 187.0 & 379.8 & 7.9 & NA \\
\hline SYN12-16-56 & 415.9 & 4.8 & 402.8 & 15.3 & 328.7 & 100.6 & 415.9 & 4.8 & 126.5 \\
\hline SYN12-16-92 & 418.3 & 9.8 & 415.0 & 20.3 & 396.8 & 122.2 & 418.3 & 9.8 & 105.4 \\
\hline SYN12-16-69 & 434.0 & 5.5 & 437.8 & 10.4 & 457.6 & 58.1 & 434.0 & 5.5 & 94.8 \\
\hline SYN12-16-1 & 435.3 & 2.8 & 437.4 & 7.5 & 448.4 & 44.5 & 435.3 & 2.8 & 97.1 \\
\hline SYN12-16-38 & 454.5 & 22.0 & 465.3 & 19.3 & 519.1 & 27.9 & 454.5 & 22.0 & 87.5 \\
\hline SYN12-16-78 & 455.0 & 4.3 & 450.3 & 13.9 & 426.2 & 82.7 & 455.0 & 4.3 & 106.7 \\
\hline SYN12-16-9 & 458.7 & 6.0 & 462.4 & 23.9 & 481.1 & 138.8 & 458.7 & 6.0 & 95.3 \\
\hline SYN12-16-24 & 552.1 & 7.1 & 561.9 & 8.4 & 601.8 & 30.8 & 552.1 & 7.1 & 91.7 \\
\hline SYN12-16-99 & 557.9 & 37.2 & 573.8 & 31.1 & 637.2 & 28.2 & 557.9 & 37.2 & 87.6 \\
\hline SYN12-16-46 & 565.3 & 45.8 & 583.5 & 39.4 & 655.4 & 57.5 & 565.3 & 45.8 & 86.2 \\
\hline SYN12-16-7 & 581.0 & 20.4 & 624.3 & 61.2 & 784.4 & 265.5 & 581.0 & 20.4 & 74.1 \\
\hline SYN12-16-58 & 594.0 & 8.6 & 593.8 & 11.1 & 593.3 & 42.3 & 594.0 & 8.6 & 100.1 \\
\hline SYN12-16-54 & 600.9 & 11.7 & 604.9 & 16.2 & 619.8 & 62.5 & 600.9 & 11.7 & 97.0 \\
\hline SYN12-16-4 & 621.1 & 14.0 & 621.8 & 15.2 & 624.6 & 48.9 & 621.1 & 14.0 & 99.4 \\
\hline SYN12-16-71 & 632.6 & 22.5 & 663.1 & 28.0 & 768.2 & 92.2 & 632.6 & 22.5 & 82.4 \\
\hline SYN12-16-81 & 637.5 & 13.3 & 647.0 & 14.6 & 680.3 & 45.7 & 637.5 & 13.3 & 93.7 \\
\hline SYN12-16-84 & 639.5 & 13.3 & 670.9 & 35.7 & 777.6 & 145.9 & 639.5 & 13.3 & 82.2 \\
\hline SYN12-16-73 & 670.3 & 12.9 & 692.9 & 11.7 & 767.1 & 24.4 & 670.3 & 12.9 & 87.4 \\
\hline SYN12-16-87 & 677.0 & 4.9 & 675.6 & 4.7 & 671.2 & 12.5 & 677.0 & 4.9 & 100.9 \\
\hline SYN12-16-66 & 852.6 & 56.8 & 841.6 & 50.1 & 812.8 & 106.9 & 852.6 & 56.8 & 104.9 \\
\hline SYN12-16-35 & 954.5 & 13.7 & 957.5 & 21.0 & 964.2 & 61.7 & 964.2 & 61.7 & 99.0 \\
\hline SYN12-16-74 & 881.2 & 25.2 & 924.6 & 26.1 & 1029.6 & 61.1 & 1029.6 & 61.1 & 85.6 \\
\hline SYN12-16-10 & 1037.8 & 42.6 & 1036.4 & 43.3 & 1033.4 & 100.5 & 1033.4 & 100.5 & 100.4 \\
\hline SYN12-16-62 & 1041.2 & 17.1 & 1041.1 & 13.0 & 1040.9 & 18.5 & 1040.9 & 18.5 & 100.0 \\
\hline SYN12-16-2 & 1070.2 & 22.4 & 1063.5 & 22.7 & 1049.8 & 52.2 & 1049.8 & 52.2 & 101.9 \\
\hline SYN12-16-28 & 1047.5 & 10.7 & 1049.6 & 8.8 & 1054.1 & 15.3 & 1054.1 & 15.3 & 99.4 \\
\hline SYN12-16-60 & 1065.0 & 7.0 & 1063.4 & 9.8 & 1060.2 & 26.4 & 1060.2 & 26.4 & 100.5 \\
\hline SYN12-16-47 & 1055.5 & 14.1 & 1061.9 & 12.4 & 1075.1 & 24.0 & 1075.1 & 24.0 & 98.2 \\
\hline SYN12-16-40 & 1129.3 & 58.4 & 1133.6 & 38.7 & 1141.7 & 11.2 & 1141.7 & 11.2 & 98.9 \\
\hline SYN12-16-14 & 1168.0 & 24.2 & 1166.4 & 27.5 & 1163.6 & 64.5 & 1163.6 & 64.5 & 100.4 \\
\hline SYN12-16-61 & 1193.2 & 50.0 & 1196.8 & 34.2 & 1203.4 & 31.3 & 1203.4 & 31.3 & 99.1 \\
\hline SYN12-16-51 & 1133.9 & 37.8 & 1185.2 & 26.0 & 1280.1 & 15.5 & 1280.1 & 15.5 & 88.6 \\
\hline SYN12-16-22 & 1330.4 & 25.1 & 1320.5 & 19.2 & 1304.6 & 30.1 & 1304.6 & 30.1 & 102.0 \\
\hline
\end{tabular}




\begin{tabular}{|c|c|c|c|c|c|c|c|c|c|}
\hline SYN12-16-88 & 1282.5 & 7.4 & 1292.4 & 9.1 & 1308.8 & 20.6 & 1308.8 & 20.6 & 98.0 \\
\hline SYN12-16-86 & 1379.3 & 10.7 & 1371.8 & 7.7 & 1360.2 & 10.6 & 1360.2 & 10.6 & 101.4 \\
\hline SYN12-16-23 & 1368.3 & 7.3 & 1371.4 & 11.5 & 1376.0 & 27.1 & 1376.0 & 27.1 & 99.4 \\
\hline SYN12-16-6 & 1460.3 & 14.8 & 1437.0 & 12.9 & 1402.5 & 23.9 & 1402.5 & 23.9 & 104.1 \\
\hline SYN12-16-33 & 1464.3 & 19.5 & 1451.8 & 13.8 & 1433.5 & 19.1 & 1433.5 & 19.1 & 102.1 \\
\hline SYN12-16-67 & 1294.9 & 13.8 & 1352.4 & 11.6 & 1444.4 & 19.2 & 1444.4 & 19.2 & 89.7 \\
\hline SYN12-16-94 & 1497.4 & 90.5 & 1477.0 & 53.1 & 1447.8 & 14.8 & 1447.8 & 14.8 & 103.4 \\
\hline SYN12-16-36 & 1395.7 & 9.6 & 1416.7 & 6.9 & 1448.2 & 9.0 & 1448.2 & 9.0 & 96.4 \\
\hline SYN12-16-12 & 1490.1 & 22.1 & 1492.8 & 15.7 & 1496.6 & 21.3 & 1496.6 & 21.3 & 99.6 \\
\hline SYN12-16-68 & 1515.3 & 19.0 & 1509.0 & 13.7 & 1500.2 & 19.6 & 1500.2 & 19.6 & 101.0 \\
\hline SYN12-16-26 & 1402.7 & 24.5 & 1462.2 & 17.6 & 1549.8 & 21.9 & 1549.8 & 21.9 & 90.5 \\
\hline SYN12-16-79 & 1577.1 & 10.0 & 1582.2 & 6.1 & 1589.0 & 5.0 & 1589.0 & 5.0 & 99.3 \\
\hline SYN12-16-16 & 1587.1 & 34.6 & 1596.6 & 20.5 & 1609.2 & 12.4 & 1609.2 & 12.4 & 98.6 \\
\hline SYN12-16-21 & 1634.0 & 25.7 & 1627.8 & 31.4 & 1619.9 & 64.0 & 1619.9 & 64.0 & 100.9 \\
\hline SYN12-16-15 & 1656.6 & 32.0 & 1651.7 & 18.1 & 1645.5 & 6.3 & 1645.5 & 6.3 & 100.7 \\
\hline SYN12-16-93 & 1649.6 & 34.9 & 1647.8 & 21.7 & 1645.5 & 21.4 & 1645.5 & 21.4 & 100.3 \\
\hline SYN12-16-13 & 1643.0 & 9.0 & 1644.6 & 10.8 & 1646.7 & 21.8 & 1646.7 & 21.8 & 99.8 \\
\hline SYN12-16-57 & 1671.1 & 9.6 & 1667.8 & 7.5 & 1663.8 & 11.9 & 1663.8 & 11.9 & 100.4 \\
\hline SYN12-16-76 & 1668.5 & 21.9 & 1672.8 & 12.7 & 1678.3 & 7.8 & 1678.3 & 7.8 & 99.4 \\
\hline SYN12-16-18 & 1703.1 & 11.7 & 1697.7 & 7.3 & 1691.0 & 7.6 & 1691.0 & 7.6 & 100.7 \\
\hline SYN12-16-31 & 1634.9 & 25.1 & 1681.9 & 14.9 & 1741.0 & 9.4 & 1741.0 & 9.4 & 93.9 \\
\hline SYN12-16-64 & 1489.8 & 20.0 & 1597.6 & 12.6 & 1742.9 & 8.2 & 1742.9 & 8.2 & 85.5 \\
\hline SYN12-16-80 & 1786.6 & 4.5 & 1767.5 & 3.1 & 1744.9 & 4.1 & 1744.9 & 4.1 & 102.4 \\
\hline SYN12-16-77 & 1767.9 & 18.8 & 1762.5 & 14.7 & 1756.2 & 23.4 & 1756.2 & 23.4 & 100.7 \\
\hline SYN12-16-8 & 1810.6 & 16.2 & 1814.4 & 9.1 & 1818.8 & 5.6 & 1818.8 & 5.6 & 99.5 \\
\hline SYN12-16-20 & 1785.1 & 55.0 & 1808.1 & 31.0 & 1834.6 & 18.0 & 1834.6 & 18.0 & 97.3 \\
\hline SYN12-16-89 & 1844.9 & 13.5 & 1841.5 & 17.4 & 1837.6 & 33.9 & 1837.6 & 33.9 & 100.4 \\
\hline SYN12-16-34 & 1767.1 & 31.4 & 1803.7 & 18.0 & 1846.3 & 12.1 & 1846.3 & 12.1 & 95.7 \\
\hline SYN12-16-63 & 1854.1 & 10.3 & 1852.4 & 7.0 & 1850.4 & 9.5 & 1850.4 & 9.5 & 100.2 \\
\hline SYN12-16-48 & 1903.4 & 12.3 & 1904.2 & 8.6 & 1905.1 & 12.1 & 1905.1 & 12.1 & 99.9 \\
\hline SYN12-16-83 & 1844.6 & 10.9 & 1875.2 & 6.2 & 1909.3 & 4.2 & 1909.3 & 4.2 & 96.6 \\
\hline SYN12-16-5 & 2008.7 & 13.1 & 2011.6 & 17.9 & 2014.6 & 33.7 & 2014.6 & 33.7 & 99.7 \\
\hline SYN12-16-43 & 2081.0 & 59.5 & 2078.9 & 30.2 & 2076.9 & 12.3 & 2076.9 & 12.3 & 100.2 \\
\hline SYN12-16-30 & 2060.4 & 24.1 & 2114.2 & 21.1 & 2166.9 & 33.8 & 2166.9 & 33.8 & 95.1 \\
\hline SYN12-16-29 & 2252.6 & 40.0 & 2378.7 & 19.9 & 2488.7 & 8.5 & 2488.7 & 8.5 & 90.5 \\
\hline SYN12-16-42 & 2535.6 & 19.5 & 2522.1 & 9.0 & 2511.3 & 4.5 & 2511.3 & 4.5 & 101.0 \\
\hline SYN12-16-45 & 2661.7 & 18.6 & 2664.9 & 18.4 & 2667.2 & 29.2 & 2667.2 & 29.2 & 99.8 \\
\hline SYN12-16-59 & 2717.0 & 33.0 & 2716.3 & 14.7 & 2715.8 & 7.3 & 2715.8 & 7.3 & 100.0 \\
\hline SYN12-16-65 & 2744.3 & 43.1 & 2737.4 & 18.7 & 2732.2 & 6.9 & 2732.2 & 6.9 & 100.4 \\
\hline
\end{tabular}




\begin{tabular}{|c|c|c|c|c|c|c|c|c|c|}
\hline SYN12-16-11 & 2694.0 & 23.8 & 2725.5 & 11.3 & 2749.0 & 8.4 & 2749.0 & 8.4 & 98.0 \\
\hline SYN12-16-19 & 2937.9 & 122.6 & 2908.0 & 49.8 & 2887.3 & 3.1 & 2887.3 & 3.1 & 101.8 \\
\hline \multirow[t]{2}{*}{ Analysis } & $206 \mathrm{~Pb} *$ & \pm & 207Pb* & \pm & $206 \mathrm{~Pb}^{*}$ & \pm & Best age & \pm & Conc \\
\hline & $238 U^{*}$ & (Ma) & $235 \mathrm{U}$ & (Ma) & $207 \mathrm{~Pb}^{*}$ & (Ma) & (Ma) & (Ma) & $(\%)$ \\
\hline SYN12-17-1 & 92.1 & 3.6 & 94.0 & 11.0 & 143.3 & 274.2 & 92.1 & 3.6 & NA \\
\hline SYN12-17-87 & 92.2 & 4.8 & 86.9 & 15.3 & -57.3 & 431.0 & 92.2 & 4.8 & NA \\
\hline SYN12-17-16 & 93.9 & 3.5 & 106.2 & 15.3 & 390.7 & 331.1 & 93.9 & 3.5 & NA \\
\hline SYN12-17-96 & 94.3 & 1.7 & 95.4 & 7.2 & 120.9 & 183.1 & 94.3 & 1.7 & NA \\
\hline SYN12-17-43 & 94.4 & 2.4 & 106.1 & 15.1 & 378.6 & 334.1 & 94.4 & 2.4 & NA \\
\hline SYN12-17-74 & 94.7 & 1.8 & 93.9 & 9.0 & 74.6 & 234.8 & 94.7 & 1.8 & NA \\
\hline SYN12-17-97 & 95.0 & 2.0 & 99.3 & 15.9 & 204.6 & 390.1 & 95.0 & 2.0 & NA \\
\hline SYN12-17-36 & 96.1 & 5.8 & 80.4 & 46.3 & -364.9 & 1682.5 & 96.1 & 5.8 & NA \\
\hline SYN12-17-81 & 97.2 & 4.3 & 77.1 & 35.2 & -509.9 & 1323.4 & 97.2 & 4.3 & NA \\
\hline SYN12-17-11 & 98.4 & 0.4 & 99.1 & 1.6 & 114.6 & 38.0 & 98.4 & 0.4 & NA \\
\hline SYN12-17-59 & 99.9 & 2.9 & 97.0 & 10.0 & 25.1 & 249.6 & 99.9 & 2.9 & NA \\
\hline SYN12-17-28 & 100.4 & 2.4 & 95.8 & 13.0 & -16.8 & 340.5 & 100.4 & 2.4 & NA \\
\hline SYN12-17-92 & 117.0 & 4.3 & 123.9 & 32.8 & 259.5 & 650.8 & 117.0 & 4.3 & NA \\
\hline SYN12-17-18 & 159.6 & 1.0 & 160.1 & 1.6 & 167.1 & 20.7 & 159.6 & 1.0 & NA \\
\hline SYN12-17-95 & 224.1 & 2.2 & 225.6 & 6.2 & 241.1 & 67.0 & 224.1 & 2.2 & NA \\
\hline SYN12-17-32 & 267.8 & 8.8 & 288.6 & 13.8 & 461.0 & 96.5 & 267.8 & 8.8 & NA \\
\hline SYN12-17-70 & 387.6 & 5.6 & 376.3 & 20.9 & 307.8 & 147.9 & 387.6 & 5.6 & NA \\
\hline SYN12-17-8 & 423.3 & 4.5 & 418.0 & 10.6 & 389.0 & 64.8 & 423.3 & 4.5 & 108.8 \\
\hline SYN12-17-101 & 468.5 & 3.6 & 470.6 & 10.8 & 480.9 & 60.6 & 468.5 & 3.6 & 97.4 \\
\hline SYN12-17-34 & 500.8 & 19.7 & 503.8 & 44.4 & 517.2 & 229.0 & 500.8 & 19.7 & 96.8 \\
\hline SYN12-17-73 & 577.1 & 13.0 & 570.5 & 76.2 & 544.0 & 379.9 & 577.1 & 13.0 & 106.1 \\
\hline SYN12-17-4 & 608.5 & 5.9 & 614.6 & 31.0 & 637.4 & 142.9 & 608.5 & 5.9 & 95.5 \\
\hline SYN12-17-46 & 908.8 & 7.9 & 904.6 & 6.7 & 894.3 & 12.6 & 894.3 & 12.6 & 101.6 \\
\hline SYN12-17-26 & 1031.7 & 18.1 & 1018.0 & 20.9 & 988.5 & 53.5 & 988.5 & 53.5 & 104.4 \\
\hline SYN12-17-85 & 1007.1 & 12.5 & 1006.1 & 17.6 & 1003.9 & 49.0 & 1003.9 & 49.0 & 100.3 \\
\hline SYN12-17-61 & 1039.7 & 10.2 & 1030.1 & 10.7 & 1009.8 & 25.8 & 1009.8 & 25.8 & 103.0 \\
\hline SYN12-17-58 & 1033.2 & 9.8 & 1026.4 & 11.7 & 1011.7 & 30.1 & 1011.7 & 30.1 & 102.1 \\
\hline SYN12-17-56 & 1036.0 & 9.1 & 1028.3 & 10.5 & 1012.0 & 26.8 & 1012.0 & 26.8 & 102.4 \\
\hline SYN12-17-84 & 1034.3 & 16.7 & 1028.4 & 42.8 & 1015.9 & 129.4 & 1015.9 & 129.4 & 101.8 \\
\hline SYN12-17-9 & 1038.1 & 5.4 & 1036.1 & 7.4 & 1032.0 & 20.0 & 1032.0 & 20.0 & 100.6 \\
\hline SYN12-17-40 & 1036.3 & 5.8 & 1035.0 & 15.0 & 1032.2 & 45.2 & 1032.2 & 45.2 & 100.4 \\
\hline SYN12-17-55 & 1078.5 & 9.1 & 1063.4 & 11.6 & 1032.4 & 30.6 & 1032.4 & 30.6 & 104.5 \\
\hline
\end{tabular}




\begin{tabular}{|c|c|c|c|c|c|c|c|c|c|}
\hline SYN12-17-10 & 1026.2 & 10.3 & 1029.0 & 11.0 & 1035.0 & 26.2 & 1035.0 & 26.2 & 99.1 \\
\hline SYN12-17-23 & 1041.3 & 9.9 & 1040.4 & 6.9 & 1038.5 & 5.7 & 1038.5 & 5.7 & 100.3 \\
\hline SYN12-17-68 & 1036.6 & 8.9 & 1038.8 & 16.0 & 1043.3 & 45.9 & 1043.3 & 45.9 & 99.4 \\
\hline SYN12-17-63 & 1060.0 & 6.7 & 1058.2 & 19.5 & 1054.5 & 58.1 & 1054.5 & 58.1 & 100.5 \\
\hline SYN12-17-27 & 1087.1 & 10.4 & 1077.4 & 17.5 & 1057.8 & 48.7 & 1057.8 & 48.7 & 102.8 \\
\hline SYN12-17-78 & 1052.2 & 8.8 & 1056.0 & 7.6 & 1063.8 & 14.6 & 1063.8 & 14.6 & 98.9 \\
\hline SYN12-17-21 & 1011.2 & 21.3 & 1030.3 & 53.9 & 1071.2 & 161.0 & 1071.2 & 161.0 & 94.4 \\
\hline SYN12-17-69 & 1077.5 & 15.7 & 1076.8 & 17.7 & 1075.3 & 43.2 & 1075.3 & 43.2 & 100.2 \\
\hline SYN12-17-31 & 1077.5 & 6.5 & 1079.2 & 17.0 & 1082.7 & 49.7 & 1082.7 & 49.7 & 99.5 \\
\hline SYN12-17-25 & 1053.5 & 23.1 & 1068.8 & 16.6 & 1100.0 & 15.8 & 1100.0 & 15.8 & 95.8 \\
\hline SYN12-17-2 & 1117.4 & 6.4 & 1112.2 & 5.2 & 1102.2 & 9.1 & 1102.2 & 9.1 & 101.4 \\
\hline SYN12-17-41 & 1134.0 & 6.6 & 1130.2 & 6.3 & 1122.9 & 13.5 & 1122.9 & 13.5 & 101.0 \\
\hline SYN12-17-57 & 1142.6 & 16.8 & 1137.1 & 13.2 & 1126.8 & 21.5 & 1126.8 & 21.5 & 101.4 \\
\hline SYN12-17-64 & 1062.2 & 20.5 & 1086.1 & 15.5 & 1134.3 & 20.1 & 1134.3 & 20.1 & 93.6 \\
\hline SYN12-17-33 & 1113.0 & 9.3 & 1123.4 & 11.5 & 1143.6 & 28.4 & 1143.6 & 28.4 & 97.3 \\
\hline SYN12-17-77 & 1140.5 & 6.0 & 1142.1 & 9.2 & 1145.3 & 24.1 & 1145.3 & 24.1 & 99.6 \\
\hline SYN12-17-51 & 1141.8 & 10.8 & 1144.5 & 21.6 & 1149.8 & 59.1 & 1149.8 & 59.1 & 99.3 \\
\hline SYN12-17-13 & 1171.5 & 13.0 & 1165.7 & 15.3 & 1155.0 & 36.6 & 1155.0 & 36.6 & 101.4 \\
\hline SYN12-17-29 & 1104.4 & 7.6 & 1126.5 & 13.6 & 1169.3 & 36.5 & 1169.3 & 36.5 & 94.5 \\
\hline SYN12-17-67 & 1178.6 & 8.3 & 1176.7 & 6.4 & 1173.2 & 10.2 & 1173.2 & 10.2 & 100.5 \\
\hline SYN12-17-30 & 1137.7 & 4.7 & 1157.5 & 13.8 & 1194.8 & 38.3 & 1194.8 & 38.3 & 95.2 \\
\hline SYN12-17-75 & 1193.6 & 10.1 & 1199.1 & 10.3 & 1209.1 & 22.3 & 1209.1 & 22.3 & 98.7 \\
\hline SYN12-17-17 & 1182.2 & 15.1 & 1196.4 & 50.1 & 1222.1 & 137.4 & 1222.1 & \begin{tabular}{|l|}
137.4 \\
\end{tabular} & 96.7 \\
\hline SYN12-17-5 & 1170.0 & 46.6 & 1202.5 & 32.5 & 1261.5 & 29.9 & 1261.5 & 29.9 & 92.7 \\
\hline SYN12-17-80 & 1312.6 & 11.4 & 1306.4 & 7.9 & 1296.3 & 9.7 & 1296.3 & 9.7 & 101.3 \\
\hline SYN12-17-53 & 1331.4 & 19.3 & 1332.6 & 32.5 & 1334.7 & 78.8 & 1334.7 & 78.8 & 99.8 \\
\hline SYN12-17-54 & 1399.5 & 17.0 & 1391.7 & 12.8 & 1379.9 & 19.5 & 1379.9 & 19.5 & 101.4 \\
\hline SYN12-17-82 & 1395.4 & 13.6 & 1396.8 & 14.9 & 1398.8 & 31.3 & 1398.8 & 31.3 & 99.8 \\
\hline SYN12-17-39 & 1467.7 & 10.3 & 1458.9 & 7.3 & 1446.1 & 9.8 & 1446.1 & 9.8 & 101.5 \\
\hline SYN12-17-50 & 1463.7 & 12.8 & 1458.4 & 12.2 & 1450.7 & 23.7 & 1450.7 & 23.7 & 100.9 \\
\hline SYN12-17-98 & 1455.3 & 10.1 & 1461.0 & 12.1 & 1469.4 & 25.7 & 1469.4 & 25.7 & 99.0 \\
\hline SYN12-17-12 & 1522.4 & 6.8 & 1510.4 & 4.5 & 1493.5 & 5.1 & 1493.5 & 5.1 & 101.9 \\
\hline SYN12-17-99 & 1475.3 & 15.1 & 1493.7 & 21.6 & 1519.7 & 47.4 & 1519.7 & 47.4 & 97.1 \\
\hline SYN12-17-79 & 1517.1 & 25.0 & 1522.1 & 25.2 & 1529.0 & 49.0 & 1529.0 & 49.0 & 99.2 \\
\hline SYN12-17-14 & 1567.2 & 4.6 & 1566.8 & 7.9 & 1566.3 & 17.6 & 1566.3 & 17.6 & 100.1 \\
\hline SYN12-17-93 & 1563.5 & 13.0 & 1574.5 & 21.0 & 1589.3 & 45.9 & 1589.3 & 45.9 & 98.4 \\
\hline SYN12-17-88 & 1568.3 & 14.7 & 1578.9 & 21.8 & 1593.0 & 46.8 & 1593.0 & 46.8 & 98.4 \\
\hline SYN12-17-48 & 1562.1 & 22.4 & 1580.0 & 14.8 & 1603.9 & 17.0 & 1603.9 & 17.0 & 97.4 \\
\hline SYN12-17-44 & 1655.3 & 16.3 & 1644.9 & 21.2 & 1631.5 & 43.7 & 1631.5 & 43.7 & 101.5 \\
\hline
\end{tabular}




\begin{tabular}{|l|l|l|l|l|l|l|l|l|l|} 
SYN12-17-86 & 1651.6 & 7.4 & 1644.9 & 7.7 & 1636.2 & 14.9 & 1636.2 & 14.9 & 100.9 \\
\hline SYN12-17-104 & 1656.9 & 16.5 & 1650.1 & 12.7 & 1641.4 & 19.9 & 1641.4 & 19.9 & 100.9 \\
\hline SYN12-17-38 & 1644.6 & 10.1 & 1643.2 & 7.8 & 1641.5 & 12.3 & 1641.5 & 12.3 & 100.2 \\
\hline SYN12-17-76 & 1672.4 & 16.9 & 1661.1 & 12.5 & 1646.8 & 18.8 & 1646.8 & 18.8 & 101.6 \\
\hline SYN12-17-65 & 1642.8 & 10.3 & 1644.8 & 8.1 & 1647.4 & 12.9 & 1647.4 & 12.9 & 99.7 \\
\hline SYN12-17-89 & 1664.9 & 13.4 & 1659.6 & 10.3 & 1652.9 & 16.2 & 1652.9 & 16.2 & 100.7 \\
\hline SYN12-17-35 & 1649.1 & 14.8 & 1653.0 & 10.7 & 1658.0 & 15.1 & 1658.0 & 15.1 & 99.5 \\
\hline SYN12-17-62 & 1702.6 & 12.6 & 1696.9 & 7.4 & 1689.9 & 5.7 & 1689.9 & 5.7 & 100.7 \\
\hline SYN12-17-60 & 1756.8 & 9.6 & 1747.1 & 6.7 & 1735.5 & 9.4 & 1735.5 & 9.4 & 101.2 \\
\hline SYN12-17-7 & 1747.9 & 14.0 & 1751.7 & 8.4 & 1756.2 & 7.9 & 1756.2 & 7.9 & 99.5 \\
\hline SYN12-17-24 & 1772.4 & 12.9 & 1768.3 & 11.5 & 1763.4 & 19.8 & 1763.4 & 19.8 & 100.5 \\
\hline SYN12-17-6 & 1850.3 & 14.4 & 1838.6 & 16.6 & 1825.3 & 31.5 & 1825.3 & 31.5 & 101.4 \\
\hline SYN12-17-3 & 1902.9 & 19.8 & 1881.7 & 12.6 & 1858.3 & 15.3 & 1858.3 & 15.3 & 102.4 \\
\hline SYN12-17-71 & 1888.3 & 14.1 & 1896.2 & 12.6 & 1904.7 & 21.5 & 1904.7 & 21.5 & 99.1 \\
\hline SYN12-17-100 & 1939.9 & 10.2 & 1924.3 & 14.2 & 1907.5 & 27.4 & 1907.5 & 27.4 & 101.7 \\
\hline SYN12-17-15 & 1964.7 & 13.7 & 1954.6 & 9.8 & 1944.0 & 14.2 & 1944.0 & 14.2 & 101.1 \\
\hline SYN12-17-42 & 1900.0 & 19.9 & 1925.2 & 13.5 & 1952.4 & 17.8 & 1952.4 & 17.8 & 97.3 \\
\hline SYN12-17-90 & 2010.5 & 12.0 & 2018.1 & 7.1 & 2025.8 & 7.6 & 2025.8 & 7.6 & 99.2 \\
\hline SYN12-17-52 & 2016.7 & 24.1 & 2024.2 & 19.0 & 2031.9 & 29.5 & 2031.9 & 29.5 & 99.2 \\
\hline SYN12-17-22 & 2102.1 & 16.7 & 2097.3 & 8.8 & 2092.5 & 5.8 & 2092.5 & 5.8 & 100.5 \\
\hline SYN12-17-49 & 498.3 & 21.9 & 1058.0 & 31.4 & 2513.4 & 23.6 & 2513.4 & 23.6 & 19.8 \\
\hline SYN12-17-47 & 2472.2 & 38.0 & 2577.2 & 17.7 & 2660.9 & 6.2 & 2660.9 & 6.2 & 92.9 \\
\hline SYN12-17-94 & 2746.8 & 18.1 & 2764.9 & 8.8 & 2778.1 & 7.4 & 2778.1 & 7.4 & 98.9 \\
\hline SYN12-17-102 & 3360.3 & 78.9 & 3508.5 & 29.7 & 3594.2 & 1.9 & 3594.2 & 1.9 & 93.5 \\
\hline
\end{tabular}

\begin{tabular}{|l|l|l|l|l|l|l|l|l|l|}
\hline Analysis & $206 \mathrm{~Pb}^{*}$ & \pm & $207 \mathrm{~Pb}^{*}$ & \pm & $206 \mathrm{~Pb}^{*}$ & \pm & Best age & \pm & Conc \\
\hline & $238 \mathrm{U}^{*}$ & $(\mathrm{Ma})$ & $235 \mathrm{U}$ & $(\mathrm{Ma})$ & $207 \mathrm{~Pb} *$ & $(\mathrm{Ma})$ & $(\mathrm{Ma})$ & $(\mathrm{Ma})$ & $(\%)$ \\
\hline & & & & & & & & & \\
\hline & & & & & & & & & \\
\hline SYN12-18-64 & 311.2 & 3.0 & 315.7 & 4.9 & 349.0 & 34.1 & 311.2 & 3.0 & NA \\
\hline SYN12-18-19 & 314.4 & 5.2 & 343.4 & 61.3 & 544.8 & 462.1 & 314.4 & 5.2 & NA \\
\hline SYN12-18-23 & 320.4 & 4.4 & 325.5 & 6.5 & 361.9 & 41.4 & 320.4 & 4.4 & NA \\
\hline SYN12-18-74 & 346.2 & 3.8 & 345.9 & 6.1 & 344.1 & 40.0 & 346.2 & 3.8 & NA \\
\hline SYN12-18-97 & 346.9 & 4.3 & 346.8 & 11.9 & 346.1 & 86.7 & 346.9 & 4.3 & NA \\
\hline SYN12-18-41 & 348.1 & 2.2 & 344.7 & 8.1 & 322.1 & 61.6 & 348.1 & 2.2 & NA \\
\hline SYN12-18-8 & 370.6 & 5.1 & 379.9 & 11.2 & 437.2 & 72.1 & 370.6 & 5.1 & NA \\
\hline SYN12-18-59 & 371.5 & 4.9 & 374.6 & 8.6 & 393.9 & 53.3 & 371.5 & 4.9 & NA \\
\hline SYN12-18-67 & 378.2 & 2.8 & 384.3 & 13.3 & 420.8 & 90.9 & 378.2 & 2.8 & NA \\
\hline SYN12-18-62 & 378.5 & 8.3 & 379.8 & 16.5 & 387.6 & 105.3 & 378.5 & 8.3 & NA \\
\hline
\end{tabular}




\begin{tabular}{|c|c|c|c|c|c|c|c|c|c|}
\hline SYN12-18-43 & 385.7 & 4.3 & 392.8 & 19.1 & 434.6 & 128.0 & 385.7 & 4.3 & NA \\
\hline SYN12-18-76 & 387.1 & 12.8 & 393.4 & 16.9 & 430.9 & 87.6 & 387.1 & 12.8 & NA \\
\hline SYN12-18-18 & 394.4 & 11.3 & 413.8 & 13.3 & 523.5 & 56.6 & 394.4 & 11.3 & NA \\
\hline SYN12-18-90 & 404.5 & 5.3 & 407.5 & 14.7 & 424.4 & 92.7 & 404.5 & 5.3 & 95.3 \\
\hline SYN12-18-39 & 404.7 & 5.8 & 411.2 & 13.1 & 447.8 & 79.7 & 404.7 & 5.8 & 90.4 \\
\hline SYN12-18-68 & 406.4 & 17.2 & 410.9 & 15.4 & 436.6 & 28.3 & 406.4 & 17.2 & 93.1 \\
\hline SYN12-18-86 & 410.1 & 4.9 & 407.6 & 9.7 & 393.8 & 58.7 & 410.1 & 4.9 & 104.1 \\
\hline SYN12-18-38 & 412.1 & 5.9 & 408.1 & 16.4 & 385.2 & 104.7 & 412.1 & 5.9 & 107.0 \\
\hline SYN12-18-88 & 414.5 & 4.4 & 409.2 & 8.1 & 379.0 & 47.7 & 414.5 & 4.4 & 109.4 \\
\hline SYN12-18-94 & 419.7 & 4.2 & 419.9 & 13.2 & 420.9 & 82.4 & 419.7 & 4.2 & 99.7 \\
\hline SYN12-18-7 & 431.6 & 24.2 & 432.7 & 21.6 & 438.3 & 43.6 & 431.6 & 24.2 & 98.5 \\
\hline SYN12-18-50 & 432.3 & 5.9 & 437.3 & 11.7 & 463.4 & 65.9 & 432.3 & 5.9 & 93.3 \\
\hline SYN12-18-65 & 436.2 & 4.2 & 433.2 & 9.8 & 417.5 & 58.2 & 436.2 & 4.2 & 104.5 \\
\hline SYN12-18-29 & 436.4 & 4.0 & 438.6 & 5.3 & 450.1 & 25.7 & 436.4 & 4.0 & 97.0 \\
\hline SYN12-18-28 & 445.6 & 7.5 & 443.0 & 7.9 & 429.3 & 29.8 & 445.6 & 7.5 & 103.8 \\
\hline SYN12-18-15 & 459.9 & 7.7 & 459.1 & 10.0 & 454.8 & 46.4 & 459.9 & 7.7 & 101.1 \\
\hline SYN12-18-4 & 469.1 & 7.3 & 464.3 & 17.2 & 440.8 & 96.1 & 469.1 & 7.3 & 106.4 \\
\hline SYN12-18-73 & 535.3 & 48.3 & 550.7 & 40.4 & 615.0 & 30.1 & 535.3 & 48.3 & 87.0 \\
\hline SYN12-18-44 & 554.0 & 11.5 & 546.5 & 46.6 & 515.1 & 237.9 & 554.0 & 11.5 & 107.6 \\
\hline SYN12-18-99 & 558.2 & 5.7 & 564.6 & 16.0 & 590.4 & 76.5 & 558.2 & 5.7 & 94.6 \\
\hline SYN12-18-45 & 588.8 & 4.1 & 591.9 & 4.6 & 603.7 & 15.7 & 588.8 & 4.1 & 97.5 \\
\hline SYN12-18-1 & 588.9 & 14.5 & 609.5 & 26.8 & 686.8 & 112.1 & 588.9 & 14.5 & 85.7 \\
\hline SYN12-18-34 & 596.7 & 10.9 & 610.8 & 13.6 & 663.3 & 48.8 & 596.7 & 10.9 & 90.0 \\
\hline SYN12-18-82 & 597.9 & 16.4 & 617.6 & 55.8 & 690.3 & 250.3 & 597.9 & 16.4 & 86.6 \\
\hline SYN12-18-84 & 609.2 & 3.5 & 611.6 & 8.3 & 620.6 & 36.6 & 609.2 & 3.5 & 98.2 \\
\hline SYN12-18-35 & 617.7 & 9.9 & 617.3 & 10.6 & 616.0 & 33.4 & 617.7 & 9.9 & 100.3 \\
\hline SYN12-18-36 & 623.3 & 12.1 & 624.5 & 14.8 & 628.9 & 52.6 & 623.3 & 12.1 & 99.1 \\
\hline SYN12-18-6 & 623.4 & 7.3 & 618.8 & 44.7 & 602.0 & 207.6 & 623.4 & 7.3 & 103.6 \\
\hline SYN12-18-78 & 636.0 & 3.8 & 630.5 & 13.2 & 611.1 & 59.4 & 636.0 & 3.8 & 104.1 \\
\hline SYN12-18-5 & 661.6 & 32.7 & 656.1 & 33.3 & 637.1 & 96.9 & 661.6 & 32.7 & 103.9 \\
\hline SYN12-18-60 & 684.5 & 17.3 & 679.7 & 40.8 & 663.8 & 167.1 & 684.5 & 17.3 & 103.1 \\
\hline SYN12-18-48 & 942.3 & 5.6 & 938.7 & 10.4 & 930.1 & 32.5 & 930.1 & 32.5 & 101.3 \\
\hline SYN12-18-77 & 913.4 & 29.7 & 926.7 & 22.3 & 958.6 & 23.5 & 958.6 & 23.5 & 95.3 \\
\hline SYN12-18-33 & 1044.2 & 11.4 & 1033.6 & 22.1 & 1011.1 & 65.0 & 1011.1 & 65.0 & 103.3 \\
\hline SYN12-18-37 & 1038.5 & 9.8 & 1032.2 & 12.0 & 1018.8 & 31.3 & 1018.8 & 31.3 & 101.9 \\
\hline SYN12-18-47 & 1047.9 & 14.3 & 1045.4 & 23.8 & 1040.2 & 67.3 & 1040.2 & 67.3 & 100.7 \\
\hline SYN12-18-87 & 1039.1 & 8.9 & 1040.6 & 10.7 & 1043.6 & 27.5 & 1043.6 & 27.5 & 99.6 \\
\hline SYN12-18-92 & 1049.1 & 5.7 & 1047.4 & 7.1 & 1043.6 & 18.6 & 1043.6 & 18.6 & 100.5 \\
\hline SYN12-18-42 & 1071.9 & 13.3 & 1063.0 & 15.3 & 1044.7 & 38.0 & 1044.7 & 38.0 & 102.6 \\
\hline
\end{tabular}




\begin{tabular}{|c|c|c|c|c|c|c|c|c|c|}
\hline SYN12-18-56 & 1056.9 & 7.8 & 1053.5 & 6.2 & 1046.3 & 10.1 & 1046.3 & 10.1 & 101.0 \\
\hline SYN12-18-80 & 1035.7 & 6.9 & 1041.5 & 11.2 & 1053.7 & 31.6 & 1053.7 & 31.6 & 98.3 \\
\hline SYN12-18-85 & 1078.6 & 16.0 & 1074.4 & 20.4 & 1066.1 & 52.5 & 1066.1 & 52.5 & 101.2 \\
\hline SYN12-18-81 & 1076.3 & 32.1 & 1074.4 & 62.0 & 1070.6 & 176.4 & 1070.6 & 176.4 & 100.5 \\
\hline SYN12-18-24 & 1074.3 & 5.7 & 1073.7 & 17.7 & 1072.3 & 52.4 & 1072.3 & 52.4 & 100.2 \\
\hline SYN12-18-75 & 1070.9 & 26.4 & 1072.3 & 19.4 & 1075.4 & 23.9 & 1075.4 & 23.9 & 99.6 \\
\hline SYN12-18-21 & 1079.7 & 14.7 & 1079.3 & 25.5 & 1078.3 & 71.0 & 1078.3 & 71.0 & 100.1 \\
\hline SYN12-18-91 & 1076.5 & 9.1 & 1078.3 & 6.5 & 1081.9 & 6.9 & 1081.9 & 6.9 & 99.5 \\
\hline SYN12-18-52 & 1105.6 & 17.0 & 1106.8 & 15.4 & 1109.2 & 31.1 & 1109.2 & 31.1 & 99.7 \\
\hline SYN12-18-13 & 1173.5 & 18.6 & 1156.3 & 40.0 & 1124.0 & 110.2 & 1124.0 & 110.2 & 104.4 \\
\hline SYN12-18-69 & 1168.1 & 27.1 & 1155.1 & 22.8 & 1130.7 & 42.3 & 1130.7 & 42.3 & 103.3 \\
\hline SYN12-18-61 & 1131.9 & 14.5 & 1133.2 & 17.1 & 1135.7 & 41.4 & 1135.7 & 41.4 & 99.7 \\
\hline SYN12-18-63 & 1156.4 & 9.9 & 1154.9 & 11.2 & 1152.1 & 26.5 & 1152.1 & 26.5 & 100.4 \\
\hline SYN12-18-66 & 1168.5 & 8.0 & 1163.8 & 6.9 & 1155.1 & 13.1 & 1155.1 & 13.1 & 101.2 \\
\hline SYN12-18-20 & 1164.0 & 42.9 & 1161.5 & 30.7 & 1156.7 & 36.8 & 1156.7 & 36.8 & 100.6 \\
\hline SYN12-18-96 & 1183.8 & 5.0 & 1174.5 & 8.7 & 1157.4 & 23.2 & 1157.4 & 23.2 & 102.3 \\
\hline SYN12-18-53 & 1131.8 & 9.1 & 1143.6 & 6.8 & 1166.0 & 9.0 & 1166.0 & 9.0 & 97.1 \\
\hline SYN12-18-16 & 1194.0 & 12.4 & 1187.1 & 8.7 & 1174.4 & 10.3 & 1174.4 & 10.3 & 101.7 \\
\hline SYN12-18-31 & 1171.9 & 24.1 & 1179.6 & 26.5 & 1193.8 & 60.3 & 1193.8 & 60.3 & 98.2 \\
\hline SYN12-18-72 & 1180.8 & 10.9 & 1186.1 & 16.3 & 1195.7 & 41.4 & 1195.7 & 41.4 & 98.7 \\
\hline SYN12-18-55 & 1219.4 & 25.1 & 1217.6 & 16.8 & 1214.5 & 14.3 & 1214.5 & 14.3 & 100.4 \\
\hline SYN12-18-10 & 1200.2 & 15.5 & 1206.2 & 15.4 & 1217.1 & 32.6 & 1217.1 & 32.6 & 98.6 \\
\hline SYN12-18-71 & 1277.9 & 8.9 & 1274.4 & 22.4 & 1268.4 & 58.2 & 1268.4 & 58.2 & 100.8 \\
\hline SYN12-18-2 & 1252.9 & 25.3 & 1262.8 & 33.3 & 1279.6 & 78.6 & 1279.6 & 78.6 & 97.9 \\
\hline SYN12-18-14 & 1281.1 & 17.4 & 1281.7 & 16.8 & 1282.6 & 34.2 & 1282.6 & 34.2 & 99.9 \\
\hline SYN12-18-9 & 1321.6 & 12.6 & 1326.0 & 8.1 & 1333.0 & 5.3 & 1333.0 & 5.3 & 99.1 \\
\hline SYN12-18-79 & 1382.8 & 16.2 & 1380.9 & 24.4 & 1377.9 & 56.7 & 1377.9 & 56.7 & 100.4 \\
\hline SYN12-18-40 & 1387.6 & 12.2 & 1401.8 & 7.8 & 1423.4 & 6.1 & 1423.4 & 6.1 & 97.5 \\
\hline SYN12-18-26 & 1464.6 & 18.6 & 1457.4 & 22.2 & 1446.9 & 47.5 & 1446.9 & 47.5 & 101.2 \\
\hline SYN12-18-98 & 1397.7 & 12.9 & 1431.2 & 9.4 & 1481.3 & 12.7 & 1481.3 & 12.7 & 94.4 \\
\hline SYN12-18-22 & 1618.6 & 17.9 & 1605.8 & 14.4 & 1589.1 & 23.8 & 1589.1 & 23.8 & 101.9 \\
\hline SYN12-18-3 & 1598.2 & 17.6 & 1607.4 & 15.5 & 1619.4 & 27.3 & 1619.4 & 27.3 & 98.7 \\
\hline SYN12-18-11 & 1678.7 & 26.4 & 1681.8 & 18.4 & 1685.8 & 24.9 & 1685.8 & 24.9 & 99.6 \\
\hline SYN12-18-27 & 1754.4 & 24.1 & 1732.1 & 13.3 & 1705.3 & 6.1 & 1705.3 & 6.1 & 102.9 \\
\hline SYN12-18-93 & 2144.9 & 16.9 & 2129.0 & 8.4 & 2113.8 & 3.4 & 2113.8 & 3.4 & 101.5 \\
\hline SYN12-18-89 & 2341.3 & 34.8 & 2505.0 & 17.6 & 2640.5 & 11.1 & 2640.5 & 11.1 & 88.7 \\
\hline SYN12-18-25 & 2565.4 & 25.1 & 2638.8 & 11.8 & 2695.6 & 6.7 & 2695.6 & 6.7 & 95.2 \\
\hline SYN12-18-30 & 2331.3 & 56.1 & 2545.0 & 27.3 & 2720.0 & 10.0 & 2720.0 & 10.0 & 85.7 \\
\hline SYN12-18-54 & 2754.4 & 27.6 & 2748.5 & 12.9 & 2744.2 & 9.5 & 2744.2 & 9.5 & 100.4 \\
\hline
\end{tabular}




\begin{tabular}{|c|c|c|c|c|c|c|c|c|c|}
\hline Analysis & $206 \mathrm{~Pb}^{*}$ & \pm & 207Pb* & \pm & 206Pb* & \pm & Best age & \pm & Conc \\
\hline & $238 U^{*}$ & (Ma) & $235 \mathrm{U}$ & (Ma) & 207Pb* & (Ma) & (Ma) & (Ma) & $(\%)$ \\
\hline SYN12-19-87 & 293.9 & 6.0 & 294.7 & 6.2 & 300.5 & 28.4 & 293.9 & 6.0 & NA \\
\hline SYN12-19-20 & 304.7 & 2.6 & 304.8 & 4.8 & 305.4 & 36.7 & 304.7 & 2.6 & NA \\
\hline SYN12-19-23 & 305.6 & 2.4 & 305.4 & 7.2 & 303.6 & 59.6 & 305.6 & 2.4 & NA \\
\hline SYN12-19-58 & 312.7 & 9.4 & 303.0 & 29.8 & 228.7 & 253.3 & 312.7 & 9.4 & NA \\
\hline SYN12-19-52 & 329.0 & 2.0 & 325.4 & 5.5 & 300.0 & 42.4 & 329.0 & 2.0 & NA \\
\hline SYN12-19-81 & 367.8 & 5.1 & 356.8 & 19.8 & 285.9 & 146.9 & 367.8 & 5.1 & NA \\
\hline SYN12-19-7 & 376.0 & 1.6 & 378.5 & 6.8 & 393.9 & 47.4 & 376.0 & 1.6 & NA \\
\hline SYN12-19-16 & 376.5 & 3.6 & 376.9 & 8.1 & 379.2 & 53.6 & 376.5 & 3.6 & NA \\
\hline SYN12-19-85 & 398.7 & 23.3 & 413.7 & 23.3 & 498.6 & 72.0 & 398.7 & 23.3 & NA \\
\hline SYN12-19-36 & 411.8 & 15.9 & 428.0 & 44.0 & 515.8 & 262.9 & 411.8 & 15.9 & 79.8 \\
\hline SYN12-19-67 & 421.6 & 5.5 & 420.2 & 39.0 & 412.8 & 252.3 & 421.6 & 5.5 & 102.1 \\
\hline SYN12-19-42 & 422.8 & 2.7 & 423.4 & 5.8 & 426.6 & 34.8 & 422.8 & 2.7 & 99.1 \\
\hline SYN12-19-68 & 427.5 & 4.0 & 430.8 & 7.7 & 448.0 & 43.6 & 427.5 & 4.0 & 95.4 \\
\hline SYN12-19-83 & 429.7 & 12.2 & 430.4 & 34.7 & 434.2 & 211.0 & 429.7 & 12.2 & 99.0 \\
\hline SYN12-19-9 & 441.8 & 7.9 & 456.8 & 10.5 & 533.2 & 47.4 & 441.8 & 7.9 & 82.9 \\
\hline SYN12-19-5 & 445.5 & 12.5 & 431.5 & 76.3 & 357.4 & 489.3 & 445.5 & 12.5 & 124.7 \\
\hline SYN12-19-38 & 447.4 & 23.4 & 442.2 & 28.7 & 415.3 & 132.0 & 447.4 & 23.4 & 107.7 \\
\hline SYN12-19-37 & 470.5 & 22.7 & 481.8 & 30.5 & 535.9 & 135.0 & 470.5 & 22.7 & 87.8 \\
\hline SYN12-19-13 & 471.2 & 9.4 & 445.1 & 24.5 & 312.6 & 147.4 & 471.2 & 9.4 & 150.7 \\
\hline SYN12-19-24 & 525.8 & 3.7 & 530.4 & 7.4 & 550.2 & 35.9 & 525.8 & 3.7 & 95.6 \\
\hline SYN12-19-80 & 538.4 & 8.0 & 535.4 & 10.1 & 522.5 & 40.9 & 538.4 & 8.0 & 103.0 \\
\hline SYN12-19-41 & 540.5 & 3.7 & 537.3 & 14.3 & 523.6 & 73.7 & 540.5 & 3.7 & 103.2 \\
\hline SYN12-19-27 & 600.5 & 6.8 & 602.5 & 8.6 & 610.1 & 31.9 & 600.5 & 6.8 & 98.4 \\
\hline SYN12-19-29 & 609.7 & 7.4 & 611.9 & 14.1 & 620.0 & 60.3 & 609.7 & 7.4 & 98.3 \\
\hline SYN12-19-100 & 612.8 & 12.6 & 606.6 & 22.1 & 583.6 & 94.1 & 612.8 & 12.6 & 105.0 \\
\hline SYN12-19-66 & 620.2 & 6.2 & 621.5 & 16.8 & 626.4 & 74.7 & 620.2 & 6.2 & 99.0 \\
\hline SYN12-19-51 & 623.7 & 6.2 & 622.4 & 9.9 & 617.7 & 40.0 & 623.7 & 6.2 & 101.0 \\
\hline SYN12-19-53 & 628.1 & 9.8 & 631.9 & 17.9 & 645.6 & 73.9 & 628.1 & 9.8 & 97.3 \\
\hline SYN12-19-82 & 632.3 & 7.5 & 630.5 & 8.9 & 623.8 & 30.5 & 632.3 & 7.5 & 101.4 \\
\hline SYN12-19-97 & 633.9 & 7.6 & 628.7 & 26.1 & 609.9 & 117.3 & 633.9 & 7.6 & 103.9 \\
\hline SYN12-19-59 & 637.0 & 6.3 & 637.5 & 44.4 & 639.5 & 200.6 & 637.0 & 6.3 & 99.6 \\
\hline SYN12-19-65 & 644.6 & 4.9 & 651.3 & 21.8 & 674.8 & 95.5 & 644.6 & 4.9 & 95.5 \\
\hline SYN12-19-61 & 647.5 & 14.1 & 672.4 & 43.6 & 756.5 & 181.1 & 647.5 & 14.1 & 85.6 \\
\hline SYN12-19-3 & 729.7 & 4.8 & 726.9 & 6.8 & 718.5 & 23.4 & 729.7 & 4.8 & 101.5 \\
\hline
\end{tabular}




\begin{tabular}{|c|c|c|c|c|c|c|c|c|c|}
\hline SYN12-19-74 & 797.8 & 8.9 & 794.7 & 13.5 & 786.0 & 45.1 & 797.8 & 8.9 & 101.5 \\
\hline SYN12-19-46 & 1003.1 & 54.5 & 998.4 & 40.6 & 988.2 & 51.3 & 988.2 & 51.3 & 101.5 \\
\hline SYN12-19-17 & 963.4 & 6.7 & 971.0 & 20.5 & 988.4 & 65.0 & 988.4 & 65.0 & 97.5 \\
\hline SYN12-19-91 & 974.1 & 5.8 & 980.4 & 7.4 & 994.5 & 20.0 & 994.5 & 20.0 & 97.9 \\
\hline SYN12-19-73 & 1023.4 & 9.0 & 1022.7 & 10.4 & 1021.3 & 26.4 & 1021.3 & 26.4 & 100.2 \\
\hline SYN12-19-2 & 1033.9 & 4.8 & 1030.0 & 4.8 & 1021.8 & 11.1 & 1021.8 & 11.1 & 101.2 \\
\hline SYN12-19-39 & 1030.2 & 8.6 & 1029.0 & 8.3 & 1026.4 & 18.5 & 1026.4 & 18.5 & 100.4 \\
\hline SYN12-19-32 & 1050.6 & 7.9 & 1043.2 & 7.1 & 1027.6 & 14.7 & 1027.6 & 14.7 & 102.2 \\
\hline SYN12-19-25 & 1061.0 & 7.5 & 1054.3 & 43.3 & 1040.4 & 132.5 & 1040.4 & 132.5 & 102.0 \\
\hline SYN12-19-88 & 1033.4 & 4.9 & 1035.7 & 7.7 & 1040.5 & 21.4 & 1040.5 & 21.4 & 99.3 \\
\hline SYN12-19-34 & 1040.0 & 11.6 & 1042.9 & 13.1 & 1049.0 & 32.2 & 1049.0 & 32.2 & 99.1 \\
\hline SYN12-19-49 & 1075.1 & 10.0 & 1067.7 & 8.2 & 1052.6 & 14.5 & 1052.6 & 14.5 & 102.1 \\
\hline SYN12-19-55 & 1087.4 & 9.9 & 1080.5 & 29.7 & 1066.4 & 87.5 & 1066.4 & 87.5 & 102.0 \\
\hline SYN12-19-44 & 1118.3 & 10.7 & 1106.3 & 12.5 & 1082.8 & 30.7 & 1082.8 & 30.7 & 103.3 \\
\hline SYN12-19-43 & 1086.5 & 7.2 & 1086.2 & 8.6 & 1085.6 & 21.6 & 1085.6 & 21.6 & 100.1 \\
\hline SYN12-19-22 & 1096.1 & 9.2 & 1098.0 & 11.5 & 1101.9 & 29.2 & 1101.9 & 29.2 & 99.5 \\
\hline SYN12-19-33 & 1047.5 & 10.7 & 1074.6 & 42.3 & 1130.0 & 125.2 & 1130.0 & 125.2 & 92.7 \\
\hline SYN12-19-35 & 1107.1 & 13.4 & 1114.9 & 10.0 & 1130.0 & 13.0 & 1130.0 & 13.0 & 98.0 \\
\hline SYN12-19-8 & 1151.4 & 12.7 & 1144.2 & 18.9 & 1130.6 & 49.3 & 1130.6 & 49.3 & 101.8 \\
\hline SYN12-19-1 & 1173.3 & 4.6 & 1166.2 & 3.8 & 1153.0 & 6.9 & 1153.0 & 6.9 & 101.8 \\
\hline SYN12-19-57 & 1150.3 & 9.1 & 1151.9 & 7.0 & 1154.9 & 10.5 & 1154.9 & 10.5 & 99.6 \\
\hline SYN12-19-63 & 1193.9 & 45.4 & 1183.8 & 37.2 & 1165.4 & 65.5 & 1165.4 & 65.5 & 102.4 \\
\hline SYN12-19-95 & 1147.6 & 17.4 & 1154.0 & 24.6 & 1166.1 & 62.6 & 1166.1 & 62.6 & 98.4 \\
\hline SYN12-19-14 & 1094.1 & 32.3 & 1123.6 & 23.4 & 1181.1 & 24.5 & 1181.1 & 24.5 & 92.6 \\
\hline SYN12-19-72 & 1192.5 & 12.3 & 1188.7 & 13.3 & 1181.8 & 30.1 & 1181.8 & 30.1 & 100.9 \\
\hline SYN12-19-86 & 1188.2 & 9.9 & 1191.7 & 13.1 & 1198.1 & 32.2 & 1198.1 & 32.2 & 99.2 \\
\hline SYN12-19-98 & 1198.2 & 8.6 & 1199.1 & 6.6 & 1200.6 & 10.3 & 1200.6 & 10.3 & 99.8 \\
\hline SYN12-19-50 & 1251.3 & 7.5 & 1248.8 & 9.3 & 1244.3 & 21.8 & 1244.3 & 21.8 & 100.6 \\
\hline SYN12-19-62 & 1286.5 & 5.6 & 1290.0 & 5.5 & 1295.7 & 11.3 & 1295.7 & 11.3 & 99.3 \\
\hline SYN12-19-10 & 1345.4 & 8.3 & 1340.0 & 7.3 & 1331.4 & 13.5 & 1331.4 & 13.5 & 101.0 \\
\hline SYN12-19-60 & 1385.7 & 18.8 & 1384.4 & 13.4 & 1382.4 & 17.8 & 1382.4 & 17.8 & 100.2 \\
\hline SYN12-19-21 & 1388.2 & 11.3 & 1393.5 & 7.8 & 1401.6 & 9.1 & 1401.6 & 9.1 & 99.0 \\
\hline SYN12-19-89 & 1384.8 & 21.4 & 1421.1 & 15.9 & 1475.9 & 22.5 & 1475.9 & 22.5 & 93.8 \\
\hline SYN12-19-77 & 1483.7 & 11.7 & 1482.2 & 7.9 & 1479.9 & 9.4 & 1479.9 & 9.4 & 100.3 \\
\hline SYN12-19-31 & 1569.6 & 23.9 & 1601.8 & 30.2 & 1644.2 & 61.9 & 1644.2 & 61.9 & 95.5 \\
\hline SYN12-19-56 & 1700.6 & 12.2 & 1678.6 & 10.4 & 1651.1 & 17.9 & 1651.1 & 17.9 & 103.0 \\
\hline SYN12-19-47 & 1724.3 & 21.1 & 1699.6 & 19.3 & 1669.2 & 34.7 & 1669.2 & 34.7 & 103.3 \\
\hline SYN12-19-69 & 1669.3 & 22.0 & 1698.2 & 12.6 & 1733.9 & 5.9 & 1733.9 & 5.9 & 96.3 \\
\hline SYN12-19-92 & 1738.4 & 8.3 & 1748.3 & 6.2 & 1760.2 & 9.2 & 1760.2 & 9.2 & 98.8 \\
\hline
\end{tabular}




\begin{tabular}{|l|l|l|l|l|l|l|l|l|l|} 
SYN12-19-28 & 1767.8 & 24.7 & 1876.2 & 14.9 & 1998.4 & 12.0 & 1998.4 & 12.0 & 88.5 \\
\hline SYN12-19-30 & 2003.7 & 13.5 & 2001.3 & 8.3 & 1998.8 & 9.6 & 1998.8 & 9.6 & 100.2 \\
\hline SYN12-19-18 & 1981.9 & 19.2 & 1998.0 & 10.3 & 2014.6 & 6.4 & 2014.6 & 6.4 & 98.4 \\
\hline SYN12-19-15 & 1931.8 & 24.4 & 1987.6 & 13.0 & 2046.1 & 5.0 & 2046.1 & 5.0 & 94.4 \\
\hline SYN12-19-26 & 1932.2 & 29.6 & 1994.9 & 16.4 & 2060.4 & 11.2 & 2060.4 & 11.2 & 93.8 \\
\hline SYN12-19-99 & 2500.4 & 21.2 & 2489.5 & 10.7 & 2480.6 & 8.9 & 2480.6 & 8.9 & 100.8 \\
\hline SYN12-19-6 & 2365.4 & 69.7 & 2528.7 & 34.1 & 2662.4 & 16.8 & 2662.4 & 16.8 & 88.8 \\
\hline SYN12-19-84 & 2742.6 & 14.0 & 2752.1 & 6.1 & 2759.1 & 1.9 & 2759.1 & 1.9 & 99.4 \\
\hline
\end{tabular}

\begin{tabular}{|c|c|c|c|c|c|c|c|c|c|}
\hline Analysis & $206 \mathrm{~Pb} *$ & \pm & $207 \mathrm{~Pb}^{*}$ & \pm & $206 \mathrm{~Pb}^{*}$ & \pm & Best age & \pm & Conc \\
\hline & $238 U^{*}$ & (Ma) & $235 U$ & (Ma) & $207 \mathrm{~Pb}^{*}$ & (Ma) & (Ma) & (Ma) & $(\%)$ \\
\hline & & & & & & & & & \\
\hline SYN14-1_Run2- 187 & 145.8 & 3.0 & 151.8 & 6.8 & 247.9 & 100.7 & 145.8 & 3.0 & NA \\
\hline SYN14-1_Run1- 62 & 147.7 & 2.5 & 146.1 & 7.9 & 120.7 & 130.0 & 147.7 & 2.5 & NA \\
\hline SYN14-1_Run1- 13 & 148.8 & 2.8 & 145.8 & 6.4 & 97.3 & 102.7 & 148.8 & 2.8 & NA \\
\hline SYN14-1_Run2- 211 & 149.1 & 5.2 & 153.0 & 13.0 & 213.8 & 195.6 & 149.1 & 5.2 & NA \\
\hline SYN14-1_Run2- 118 & 149.4 & 3.2 & 150.5 & 6.5 & 167.9 & 95.6 & 149.4 & 3.2 & NA \\
\hline SYN14-1_Run1- 7 & 151.2 & 3.8 & 199.8 & 11.9 & 820.5 & 125.9 & 151.2 & 3.8 & NA \\
\hline SYN14-1_Run1- 59 & 152.7 & 2.6 & 181.9 & 9.5 & 580.6 & 117.6 & 152.7 & 2.6 & NA \\
\hline SYN14-1_Run2- 189 & 152.8 & 3.7 & 146.2 & 12.6 & 40.3 & 214.2 & 152.8 & 3.7 & NA \\
\hline SYN14-1_Run1-87 & 153.4 & 4.0 & 150.8 & 9.7 & 110.4 & 150.8 & 153.4 & 4.0 & NA \\
\hline SYN14-1_Run1- 3 & 154.8 & 3.1 & 149.4 & 5.8 & 64.3 & 87.3 & 154.8 & 3.1 & NA \\
\hline SYN14-1_Run1- 102 & 157.6 & 3.1 & 158.3 & 4.1 & 167.8 & 45.0 & 157.6 & 3.1 & NA \\
\hline SYN14-1_Run1- 48 & 160.3 & 3.6 & 197.5 & 13.1 & 668.8 & 149.1 & 160.3 & 3.6 & NA \\
\hline SYN14-1_Run2- 181 & 174.1 & 2.9 & 175.3 & 3.5 & 191.2 & 31.3 & 174.1 & 2.9 & NA \\
\hline SYN14-1_Run1- 73 & 234.6 & 4.3 & 239.9 & 6.2 & 292.2 & 51.6 & 234.6 & 4.3 & NA \\
\hline SYN14-1_Run1-80 & 244.9 & 3.8 & 250.3 & 7.3 & 301.5 & 66.1 & 244.9 & 3.8 & NA \\
\hline SYN14-1_Run2- 195 & 251.3 & 5.9 & 254.9 & 7.7 & 288.4 & 55.2 & 251.3 & 5.9 & NA \\
\hline SYN14-1_Run1- 76 & 253.3 & 6.9 & 256.3 & 11.9 & 283.9 & 102.5 & 253.3 & 6.9 & NA \\
\hline SYN14-1_Run1-49 & 289.1 & 5.8 & 286.3 & 9.7 & 263.8 & 76.4 & 289.1 & 5.8 & NA \\
\hline SYN14-1_Run1-83 & 344.9 & 4.5 & 353.9 & 6.7 & 413.6 & 40.6 & 344.9 & 4.5 & NA \\
\hline SYN14-1_Run1- 69 & 380.3 & 5.1 & 377.0 & 8.3 & 356.6 & 50.9 & 380.3 & 5.1 & NA \\
\hline SYN14-1_Run1- 9 & 396.9 & 6.6 & 395.1 & 10.4 & 384.5 & 60.2 & 396.9 & 6.6 & NA \\
\hline SYN14-1_Run1- 37 & 399.6 & 8.0 & 382.1 & 16.0 & 277.5 & 105.1 & 399.6 & 8.0 & NA \\
\hline SYN14-1_Run2- 173 & 400.5 & 9.7 & 398.4 & 12.6 & 385.8 & 65.0 & 400.5 & 9.7 & 103.8 \\
\hline SYN14-1_Run1- 36 & 413.9 & 6.4 & 411.7 & 9.1 & 399.1 & 48.3 & 413.9 & 6.4 & 103.7 \\
\hline SYN14-1_Run2- 190 & 422.4 & 4.8 & 427.8 & 6.2 & 456.5 & 29.7 & 422.4 & 4.8 & 92.5 \\
\hline SYN14-1_Run2- 216 & 425.4 & 6.2 & 432.7 & 9.7 & 471.6 & 51.5 & 425.4 & 6.2 & 90.2 \\
\hline
\end{tabular}




\begin{tabular}{|c|c|c|c|c|c|c|c|c|c|}
\hline SYN14-1_Run2- 119 & 428.6 & 6.9 & 436.1 & 11.6 & 476.3 & 62.1 & 428.6 & 6.9 & 90.0 \\
\hline SYN14-1_Run2- 120 & 438.2 & 9.2 & 436.9 & 9.6 & 429.9 & 35.8 & 438.2 & 9.2 & 101.9 \\
\hline SYN14-1_Run1- 106 & 446.0 & 6.6 & 449.5 & 8.6 & 467.2 & 39.8 & 446.0 & 6.6 & 95.5 \\
\hline SYN14-1_Run1- 90 & 454.0 & 11.7 & 454.7 & 12.7 & 458.5 & 48.8 & 454.0 & 11.7 & 99.0 \\
\hline SYN14-1_Run1- 24 & 459.7 & 9.6 & 477.7 & 12.8 & 565.0 & 55.8 & 459.7 & 9.6 & 81.4 \\
\hline SYN14-1_Run1- 12 & 461.2 & 15.2 & 458.9 & 13.9 & 447.7 & 36.3 & 461.2 & 15.2 & 103.0 \\
\hline SYN14-1_Run2- 147 & 544.6 & 13.3 & 564.4 & 15.1 & 645.0 & 50.9 & 544.6 & 13.3 & 84.4 \\
\hline SYN14-1_Run1- 99 & 546.0 & 9.6 & 547.5 & 13.1 & 553.6 & 54.6 & 546.0 & 9.6 & 98.6 \\
\hline SYN14-1_Run1- 46 & 547.9 & 18.7 & 552.1 & 18.1 & 569.7 & 50.8 & 547.9 & 18.7 & 96.2 \\
\hline SYN14-1_Run1- 47 & 553.9 & 19.4 & 554.2 & 21.7 & 555.4 & 77.2 & 553.9 & 19.4 & 99.7 \\
\hline SYN14-1_Run1- 16 & 577.4 & 10.9 & 578.9 & 15.8 & 584.8 & 64.9 & 577.4 & 10.9 & 98.7 \\
\hline SYN14-1_Run2- 111 & 579.8 & 13.9 & 590.3 & 14.1 & 630.9 & 40.6 & 579.8 & 13.9 & 91.9 \\
\hline SYN14-1_Run2- 177 & 595.6 & 10.0 & 611.3 & 12.7 & 669.7 & 45.4 & 595.6 & 10.0 & 88.9 \\
\hline SYN14-1_Run1- 20 & 599.1 & 11.0 & 590.9 & 12.6 & 559.6 & 45.0 & 599.1 & 11.0 & 107.1 \\
\hline SYN14-1_Run1- 108 & 601.7 & 13.1 & 606.1 & 13.2 & 622.5 & 38.1 & 601.7 & 13.1 & 96.7 \\
\hline SYN14-1_Run2- 212 & 603.7 & 11.7 & 607.7 & 12.9 & 622.2 & 42.1 & 603.7 & 11.7 & 97.0 \\
\hline SYN14-1_Run2- 186 & 626.0 & 9.3 & 641.8 & 16.6 & 697.7 & 66.5 & 626.0 & 9.3 & 89.7 \\
\hline SYN14-1_Run2- 170 & 714.6 & 13.3 & 718.3 & 15.2 & 729.9 & 46.7 & 714.6 & 13.3 & 97.9 \\
\hline SYN14-1_Run1- 97 & 1005.6 & 34.9 & 979.3 & 31.8 & 920.6 & 70.0 & 920.6 & 70.0 & 109.2 \\
\hline SYN14-1_Run1- 68 & 897.4 & 16.4 & 909.6 & 14.2 & 939.4 & 27.2 & 939.4 & 27.2 & 95.5 \\
\hline SYN14-1_Run1- 6 & 985.2 & 20.0 & 977.3 & 19.5 & 959.6 & 45.0 & 959.6 & 45.0 & 102.7 \\
\hline SYN14-1_Run2- 191 & 945.6 & 22.0 & 951.1 & 27.6 & 963.9 & 75.5 & 963.9 & 75.5 & 98.1 \\
\hline SYN14-1_Run2- 124 & 906.9 & 16.4 & 924.8 & 13.0 & 967.7 & 18.6 & 967.7 & 18.6 & 93.7 \\
\hline SYN14-1_Run2- 209 & 920.1 & 13.7 & 934.4 & 16.4 & 968.4 & 44.1 & 968.4 & 44.1 & 95.0 \\
\hline SYN14-1_Run2- 200 & 854.1 & 18.1 & 887.4 & 17.4 & 971.3 & 38.1 & 971.3 & 38.1 & 87.9 \\
\hline SYN14-1_Run1- 79 & 957.2 & 16.5 & 963.3 & 17.4 & 977.3 & 42.6 & 977.3 & 42.6 & 97.9 \\
\hline SYN14-1_Run1- 74 & 821.1 & 20.1 & 866.6 & 19.5 & 985.1 & 42.5 & 985.1 & 42.5 & 83.3 \\
\hline SYN14-1_Run1- 89 & 1047.4 & 13.2 & 1033.9 & 18.9 & 1005.6 & 52.2 & 1005.6 & 52.2 & 104.2 \\
\hline SYN14-1_Run2- 179 & 929.8 & 16.9 & 952.9 & 16.9 & 1006.4 & 38.8 & 1006.4 & 38.8 & 92.4 \\
\hline SYN14-1_Run2- 204 & 995.6 & 19.5 & 1000.9 & 21.4 & 1012.4 & 52.9 & 1012.4 & 52.9 & 98.3 \\
\hline SYN14-1_Run2- 157 & 856.4 & 24.0 & 902.6 & 19.4 & 1017.5 & 25.4 & 1017.5 & 25.4 & 84.2 \\
\hline SYN14-1_Run1- 29 & 1064.9 & 19.8 & 1049.9 & 22.0 & 1018.7 & 54.6 & 1018.7 & 54.6 & 104.5 \\
\hline SYN14-1_Run1- 51 & 978.3 & 14.9 & 991.7 & 13.4 & 1021.6 & 27.2 & 1021.6 & 27.2 & 95.8 \\
\hline SYN14-1_Run1- 18 & 997.3 & 40.8 & 1005.8 & 29.9 & 1024.4 & 32.0 & 1024.4 & 32.0 & 97.4 \\
\hline SYN14-1_Run2- 116 & 1028.9 & 13.8 & 1027.5 & 13.2 & 1024.5 & 29.1 & 1024.5 & 29.1 & 100.4 \\
\hline SYN14-1_Run2- 112 & 1023.2 & 12.4 & 1025.7 & 12.2 & 1031.2 & 27.6 & 1031.2 & 27.6 & 99.2 \\
\hline SYN14-1_Run2- 199 & 1011.8 & 32.1 & 1018.9 & 32.6 & 1034.2 & 75.2 & 1034.2 & 75.2 & 97.8 \\
\hline SYN14-1_Run1- 65 & 1024.9 & 12.1 & 1028.0 & 10.7 & 1034.5 & 21.1 & 1034.5 & 21.1 & 99.1 \\
\hline SYN14-1_Run2- 214 & 1016.1 & 20.0 & 1022.9 & 20.7 & 1037.5 & 48.6 & 1037.5 & 48.6 & 97.9 \\
\hline
\end{tabular}




\begin{tabular}{|c|c|c|c|c|c|c|c|c|c|}
\hline SYN14-1_Run1- 25 & 1080.2 & 17.3 & 1067.9 & 15.0 & 1042.6 & 29.3 & 1042.6 & 29.3 & 103.6 \\
\hline SYN14-1_Run1- 50 & 962.9 & 22.5 & 988.8 & 18.0 & 1047.0 & 27.0 & 1047.0 & 27.0 & 92.0 \\
\hline SYN14-1_Run1- 105 & 1029.9 & 12.9 & 1035.6 & 12.4 & 1047.7 & 27.1 & 1047.7 & 27.1 & 98.3 \\
\hline SYN14-1_Run2- 217 & 1020.1 & 14.8 & 1029.0 & 11.9 & 1047.9 & 19.4 & 1047.9 & 19.4 & 97.3 \\
\hline SYN14-1_Run1- 15 & 1073.2 & 15.7 & 1066.0 & 15.2 & 1051.3 & 33.6 & 1051.3 & 33.6 & 102.1 \\
\hline SYN14-1_Run2- 178 & 1066.7 & 13.9 & 1063.9 & 14.8 & 1058.0 & 35.2 & 1058.0 & 35.2 & 100.8 \\
\hline SYN14-1_Run2- 145 & 1011.3 & 14.6 & 1026.4 & 12.9 & 1058.6 & 25.1 & 1058.6 & 25.1 & 95.5 \\
\hline SYN14-1_Run2- 176 & 1022.6 & 16.6 & 1034.8 & 16.3 & 1060.8 & 36.0 & 1060.8 & 36.0 & 96.4 \\
\hline SYN14-1_Run2- 165 & 992.0 & 21.0 & 1014.2 & 23.1 & 1062.5 & 56.0 & 1062.5 & 56.0 & 93.4 \\
\hline SYN14-1_Run1- 72 & 1025.9 & 17.0 & 1037.7 & 16.9 & 1062.6 & 37.6 & 1062.6 & 37.6 & 96.6 \\
\hline SYN14-1_Run2- 163 & 1013.0 & 17.9 & 1029.9 & 16.4 & 1065.8 & 33.3 & 1065.8 & 33.3 & 95.1 \\
\hline SYN14-1_Run2- 218 & 973.5 & 21.0 & 1002.5 & 17.5 & 1066.4 & 29.5 & 1066.4 & 29.5 & 91.3 \\
\hline SYN14-1_Run1- 94 & 1006.8 & 12.7 & 1025.8 & 12.8 & 1066.6 & 28.9 & 1066.6 & 28.9 & 94.4 \\
\hline SYN14-1_Run2- 175 & 1035.1 & 13.7 & 1050.9 & 14.0 & 1083.8 & 32.0 & 1083.8 & 32.0 & 95.5 \\
\hline SYN14-1_Run1- 101 & 997.2 & 18.4 & 1024.9 & 17.6 & 1084.4 & 37.3 & 1084.4 & 37.3 & 92.0 \\
\hline SYN14-1_Run2- 194 & 1052.9 & 31.9 & 1064.2 & 30.4 & 1087.5 & 65.0 & 1087.5 & 65.0 & 96.8 \\
\hline SYN14-1_Run1- 30 & 1057.8 & 24.7 & 1069.0 & 24.5 & 1091.9 & 54.4 & 1091.9 & 54.4 & 96.9 \\
\hline SYN14-1_Run1- 32 & 1051.1 & 21.7 & 1065.2 & 21.6 & 1094.3 & 47.9 & 1094.3 & 47.9 & 96.1 \\
\hline SYN14-1_Run2- 166 & 1067.9 & 9.6 & 1077.7 & 10.8 & 1097.5 & 26.1 & 1097.5 & 26.1 & 97.3 \\
\hline SYN14-1_Run2- 151 & 1104.3 & 18.2 & 1106.4 & 22.6 & 1110.5 & 56.4 & 1110.5 & 56.4 & 99.4 \\
\hline SYN14-1_Run2- 210 & 1088.8 & 30.6 & 1097.2 & 21.8 & 1113.8 & 21.8 & 1113.8 & 21.8 & 97.8 \\
\hline SYN14-1_Run2- 161 & 1004.4 & 29.2 & 1041.2 & 25.0 & 1119.3 & 43.8 & 1119.3 & 43.8 & 89.7 \\
\hline SYN14-1_Run1- 103 & 1157.7 & 20.8 & 1144.6 & 16.2 & 1119.8 & 26.3 & 1119.8 & 26.3 & 103.4 \\
\hline SYN14-1_Run1- 17 & 1149.0 & 17.8 & 1139.2 & 14.3 & 1120.4 & 24.5 & 1120.4 & 24.5 & 102.6 \\
\hline SYN14-1_Run1- 78 & 1138.0 & 23.3 & 1134.8 & 18.3 & 1128.6 & 29.3 & 1128.6 & 29.3 & 100.8 \\
\hline SYN14-1_Run1- 21 & 1056.2 & 14.4 & 1080.6 & 13.0 & 1130.2 & 25.6 & 1130.2 & 25.6 & 93.5 \\
\hline SYN14-1_Run2- 183 & 1162.6 & 18.5 & 1152.7 & 14.7 & 1134.2 & 24.5 & 1134.2 & 24.5 & 102.5 \\
\hline SYN14-1_Run1- 64 & 1144.3 & 13.4 & 1142.4 & 12.2 & 1138.7 & 24.8 & 1138.7 & 24.8 & 100.5 \\
\hline SYN14-1_Run2- 132 & 1121.5 & 18.8 & 1127.4 & 17.2 & 1138.8 & 34.6 & 1138.8 & 34.6 & 98.5 \\
\hline SYN14-1_Run2- 193 & 1145.6 & 18.5 & 1143.4 & 25.2 & 1139.1 & 64.2 & 1139.1 & 64.2 & 100.6 \\
\hline SYN14-1_Run2- 158 & 1067.2 & 28.0 & 1098.3 & 23.7 & 1160.5 & 41.2 & 1160.5 & 41.2 & 92.0 \\
\hline SYN14-1_Run1- 82 & 1173.0 & 32.6 & 1168.9 & 23.2 & 1161.4 & 27.5 & 1161.4 & 27.5 & 101.0 \\
\hline SYN14-1_Run1- 43 & 1163.3 & 20.8 & 1164.4 & 19.3 & 1166.4 & 39.3 & 1166.4 & 39.3 & 99.7 \\
\hline SYN14-1_Run2- 169 & 1134.3 & 15.6 & 1146.3 & 14.3 & 1169.1 & 28.5 & 1169.1 & 28.5 & 97.0 \\
\hline SYN14-1_Run2- 154 & 1076.9 & 28.6 & 1109.8 & 21.9 & 1174.7 & 29.5 & 1174.7 & 29.5 & 91.7 \\
\hline SYN14-1_Run1- 67 & 1148.0 & 19.4 & 1157.4 & 22.1 & 1175.0 & 51.7 & 1175.0 & 51.7 & 97.7 \\
\hline SYN14-1_Run1- 91 & 1113.2 & 17.1 & 1135.9 & 18.2 & 1179.6 & 40.7 & 1179.6 & 40.7 & 94.4 \\
\hline SYN14-1_Run1- 60 & 1123.8 & 19.8 & 1144.2 & 17.7 & 1183.0 & 34.1 & 1183.0 & 34.1 & 95.0 \\
\hline SYN14-1_Run2- 168 & 1143.7 & 23.2 & 1158.6 & 23.8 & 1186.6 & 52.2 & 1186.6 & 52.2 & 96.4 \\
\hline
\end{tabular}




\begin{tabular}{|c|c|c|c|c|c|c|c|c|c|}
\hline SYN14-1_Run1- 28 & 1247.9 & 15.2 & 1239.3 & 20.7 & 1224.5 & 50.3 & 1224.5 & 50.3 & 101.9 \\
\hline SYN14-1_Run1- 53 & 1201.4 & 17.5 & 1212.8 & 13.7 & 1233.2 & 21.6 & 1233.2 & 21.6 & 97.4 \\
\hline SYN14-1_Run2- 201 & 1233.9 & 15.4 & 1238.9 & 16.7 & 1247.5 & 37.1 & 1247.5 & 37.1 & 98.9 \\
\hline SYN14-1_Run2- 159 & 1211.4 & 22.8 & 1226.0 & 20.7 & 1251.8 & 39.9 & 1251.8 & 39.9 & 96.8 \\
\hline SYN14-1_Run1- 96 & 1261.1 & 20.2 & 1257.9 & 17.0 & 1252.2 & 30.8 & 1252.2 & 30.8 & 100.7 \\
\hline SYN14-1_Run1- 1 & 1190.7 & 20.6 & 1214.4 & 21.0 & 1256.8 & 44.5 & 1256.8 & 44.5 & 94.7 \\
\hline SYN14-1_Run1- 70 & 1303.2 & 32.2 & 1286.1 & 27.8 & 1257.7 & 51.9 & 1257.7 & 51.9 & 103.6 \\
\hline SYN14-1_Run2- 174 & 1287.8 & 19.2 & 1303.4 & 15.1 & 1329.2 & 23.7 & 1329.2 & 23.7 & 96.9 \\
\hline SYN14-1_Run2- 143 & 1260.4 & 23.1 & 1287.0 & 19.9 & 1331.6 & 35.3 & 1331.6 & 35.3 & 94.6 \\
\hline SYN14-1_Run1- 33 & 1272.9 & 19.8 & 1300.3 & 15.3 & 1345.7 & 22.8 & 1345.7 & 22.8 & 94.6 \\
\hline SYN14-1_Run1- 107 & 1358.3 & 23.3 & 1362.3 & 19.1 & 1368.6 & 32.5 & 1368.6 & 32.5 & 99.3 \\
\hline SYN14-1_Run2- 121 & 1377.0 & 23.4 & 1376.0 & 18.9 & 1374.3 & 31.7 & 1374.3 & 31.7 & 100.2 \\
\hline SYN14-1_Run1- 55 & 1380.3 & 19.5 & 1379.5 & 16.3 & 1378.1 & 28.3 & 1378.1 & 28.3 & 100.2 \\
\hline SYN14-1_Run1- 54 & 1326.7 & 18.1 & 1348.8 & 16.1 & 1384.0 & 29.8 & 1384.0 & 29.8 & 95.9 \\
\hline SYN14-1_Run2- 162 & 1341.3 & 23.9 & 1359.8 & 19.7 & 1388.8 & 33.2 & 1388.8 & 33.2 & 96.6 \\
\hline SYN14-1_Run1- 44 & 1371.1 & 35.4 & 1378.2 & 24.5 & 1389.3 & 29.5 & 1389.3 & 29.5 & 98.7 \\
\hline SYN14-1_Run1- 84 & 1348.2 & 18.2 & 1368.4 & 17.4 & 1400.1 & 34.0 & 1400.1 & 34.0 & 96.3 \\
\hline SYN14-1_Run1- 26 & 1275.2 & 36.6 & 1333.1 & 25.1 & 1427.4 & 22.9 & 1427.4 & 22.9 & 89.3 \\
\hline SYN14-1_Run1- 39 & 1435.1 & 21.7 & 1432.2 & 16.3 & 1427.9 & 24.8 & 1427.9 & 24.8 & 100.5 \\
\hline SYN14-1_Run2- 182 & 1402.3 & 14.1 & 1413.8 & 13.0 & 1431.2 & 24.5 & 1431.2 & 24.5 & 98.0 \\
\hline SYN14-1_Run1- 34 & 1433.9 & 43.1 & 1442.2 & 28.7 & 1454.5 & 30.6 & 1454.5 & 30.6 & 98.6 \\
\hline SYN14-1_Run2- 133 & 1363.8 & 22.9 & 1406.0 & 23.7 & 1470.6 & 47.4 & 1470.6 & 47.4 & 92.7 \\
\hline SYN14-1_Run1- 100 & 1486.0 & 22.8 & 1488.8 & 17.6 & 1492.6 & 27.7 & 1492.6 & 27.7 & 99.6 \\
\hline SYN14-1_Run1- 8 & 1478.1 & 41.1 & 1488.3 & 27.5 & 1502.8 & 30.8 & 1502.8 & 30.8 & 98.4 \\
\hline SYN14-1_Run1- 61 & 1452.8 & 25.9 & 1474.0 & 20.5 & 1504.6 & 32.6 & 1504.6 & 32.6 & 96.6 \\
\hline SYN14-1_Run2- 205 & 1421.0 & 48.9 & 1455.3 & 32.3 & 1505.7 & 31.1 & 1505.7 & 31.1 & 94.4 \\
\hline SYN14-1_Run2- 126 & 1517.3 & 61.9 & 1512.9 & 38.2 & 1506.8 & 30.7 & 1506.8 & 30.7 & 100.7 \\
\hline SYN14-1_Run2- 203 & 1461.1 & 22.5 & 1480.4 & 19.2 & 1508.1 & 33.3 & 1508.1 & 33.3 & 96.9 \\
\hline SYN14-1_Run2- 196 & 1501.1 & 27.2 & 1504.4 & 19.0 & 1509.1 & 24.9 & 1509.1 & 24.9 & 99.5 \\
\hline SYN14-1_Run1- 10 & 1513.8 & 31.1 & 1545.1 & 22.1 & 1588.1 & 29.3 & 1588.1 & 29.3 & 95.3 \\
\hline SYN14-1_Run1- 45 & 1428.0 & 37.0 & 1497.5 & 26.4 & 1597.2 & 32.4 & 1597.2 & 32.4 & 89.4 \\
\hline SYN14-1_Run2- 153 & 1628.0 & 18.3 & 1624.0 & 14.3 & 1618.7 & 22.8 & 1618.7 & 22.8 & 100.6 \\
\hline SYN14-1_Run1- 81 & 1620.2 & 27.2 & 1623.6 & 20.2 & 1627.9 & 30.2 & 1627.9 & 30.2 & 99.5 \\
\hline SYN14-1_Run1- 14 & 1585.8 & 28.6 & 1604.9 & 21.5 & 1630.1 & 32.1 & 1630.1 & 32.1 & 97.3 \\
\hline SYN14-1_Run1- 52 & 1621.3 & 27.0 & 1628.2 & 18.7 & 1637.2 & 24.8 & 1637.2 & 24.8 & 99.0 \\
\hline SYN14-1_Run2- 113 & 1585.3 & 19.7 & 1609.1 & 15.5 & 1640.4 & 24.4 & 1640.4 & 24.4 & 96.6 \\
\hline SYN14-1_Run1- 98 & 1633.7 & 76.1 & 1637.6 & 43.7 & 1642.5 & 19.7 & 1642.5 & 19.7 & 99.5 \\
\hline SYN14-1_Run1- 104 & 1640.6 & 18.9 & 1641.4 & 15.9 & 1642.6 & 26.9 & 1642.6 & 26.9 & 99.9 \\
\hline SYN14-1_Run2- 188 & 1606.5 & 23.2 & 1625.2 & 21.5 & 1649.6 & 38.9 & 1649.6 & 38.9 & 97.4 \\
\hline
\end{tabular}




\begin{tabular}{|c|c|c|c|c|c|c|c|c|c|}
\hline SYN14-1_Run2- 180 & 1735.9 & 40.9 & 1697.7 & 24.4 & 1650.7 & 22.9 & 1650.7 & 22.9 & 105.2 \\
\hline SYN14-1_Run2- 127 & 1593.7 & 21.9 & 1621.2 & 19.8 & 1657.2 & 35.0 & 1657.2 & 35.0 & 96.2 \\
\hline SYN14-1_Run1- 35 & 1584.1 & 18.4 & 1616.1 & 15.2 & 1658.1 & 25.1 & 1658.1 & 25.1 & 95.5 \\
\hline SYN14-1_Run2- 122 & 1609.5 & 49.9 & 1632.6 & 30.2 & 1662.4 & 23.3 & 1662.4 & 23.3 & 96.8 \\
\hline SYN14-1_Run2- 220 & 1581.3 & 41.4 & 1617.6 & 25.7 & 1665.1 & 21.4 & 1665.1 & 21.4 & 95.0 \\
\hline SYN14-1_Run2- 146 & 1654.5 & 24.5 & 1659.5 & 17.1 & 1666.0 & 23.1 & 1666.0 & 23.1 & 99.3 \\
\hline SYN14-1_Run2- 184 & 1615.3 & 54.7 & 1637.7 & 33.6 & 1666.5 & 28.9 & 1666.5 & 28.9 & 96.9 \\
\hline SYN14-1_Run2- 215 & 1587.1 & 23.1 & 1628.4 & 21.7 & 1682.0 & 39.1 & 1682.0 & 39.1 & 94.4 \\
\hline SYN14-1_Run1-95 & 1360.4 & 20.5 & 1498.2 & 15.8 & 1699.0 & 20.7 & 1699.0 & 20.7 & 80.1 \\
\hline SYN14-1_Run2- 208 & 1677.4 & 18.5 & 1690.0 & 17.0 & 1705.8 & 30.4 & 1705.8 & 30.4 & 98.3 \\
\hline SYN14-1_Run2- 160 & 1732.1 & 31.4 & 1721.7 & 20.7 & 1709.1 & 25.8 & 1709.1 & 25.8 & 101.3 \\
\hline SYN14-1_Run2- 123 & 1522.9 & 23.0 & 1607.2 & 20.0 & 1719.4 & 33.3 & 1719.4 & 33.3 & 88.6 \\
\hline SYN14-1_Run2- 125 & 1739.0 & 46.5 & 1731.0 & 26.8 & 1721.4 & 19.0 & 1721.4 & 19.0 & 101.0 \\
\hline SYN14-1_Run2- 185 & 1755.2 & 24.0 & 1740.3 & 16.7 & 1722.4 & 23.4 & 1722.4 & 23.4 & 101.9 \\
\hline SYN14-1_Run2- 156 & 1715.1 & 26.9 & 1722.4 & 17.7 & 1731.3 & 21.2 & 1731.3 & 21.2 & 99.1 \\
\hline SYN14-1_Run1- 57 & 1708.1 & 22.4 & 1720.6 & 15.5 & 1735.9 & 20.8 & 1735.9 & 20.8 & 98.4 \\
\hline SYN14-1_Run1- 88 & 1684.7 & 16.3 & 1707.8 & 13.4 & 1736.1 & 21.9 & 1736.1 & 21.9 & 97.0 \\
\hline SYN14-1_Run1- 11 & 1729.0 & 20.4 & 1735.7 & 18.0 & 1743.8 & 31.0 & 1743.8 & 31.0 & 99.1 \\
\hline SYN14-1_Run1- 5 & 1661.1 & 25.0 & 1700.6 & 21.1 & 1749.7 & 34.8 & 1749.7 & 34.8 & 94.9 \\
\hline SYN14-1_Run2- 130 & 1704.8 & 24.8 & 1732.6 & 18.4 & 1766.4 & 26.9 & 1766.4 & 26.9 & 96.5 \\
\hline SYN14-1_Run2- 197 & 1764.8 & 19.7 & 1772.1 & 14.3 & 1780.8 & 20.6 & 1780.8 & 20.6 & 99.1 \\
\hline SYN14-1_Run1- 110 & 1782.2 & 39.7 & 1788.1 & 23.6 & 1795.0 & 21.3 & 1795.0 & 21.3 & 99.3 \\
\hline SYN14-1_Run1- 2 & 1748.0 & 37.8 & 1771.8 & 22.5 & 1799.9 & 19.1 & 1799.9 & 19.1 & 97.1 \\
\hline SYN14-1_Run1- 77 & 1773.3 & 28.4 & 1786.0 & 18.8 & 1800.9 & 23.3 & 1800.9 & 23.3 & 98.5 \\
\hline SYN14-1_Run1- 38 & 1777.1 & 22.7 & 1791.5 & 15.5 & 1808.3 & 20.3 & 1808.3 & 20.3 & 98.3 \\
\hline SYN14-1_Run1- 85 & 1773.5 & 28.5 & 1792.6 & 18.6 & 1814.8 & 22.4 & 1814.8 & 22.4 & 97.7 \\
\hline SYN14-1_Run2- 167 & 1813.7 & 32.6 & 1816.5 & 25.6 & 1819.8 & 40.1 & 1819.8 & 40.1 & 99.7 \\
\hline SYN14-1_Run2- 198 & 1772.5 & 34.5 & 1798.5 & 21.4 & 1828.8 & 22.1 & 1828.8 & 22.1 & 96.9 \\
\hline SYN14-1_Run2- 171 & 1815.8 & 30.3 & 1822.1 & 21.6 & 1829.3 & 30.7 & 1829.3 & 30.7 & 99.3 \\
\hline SYN14-1_Run1- 92 & 1836.3 & 24.4 & 1836.2 & 15.8 & 1836.0 & 19.2 & 1836.0 & 19.2 & 100.0 \\
\hline SYN14-1_Run1- 71 & 1589.3 & 25.5 & 1699.2 & 17.4 & 1837.5 & 19.8 & 1837.5 & 19.8 & 86.5 \\
\hline SYN14-1_Run1- 63 & 1827.8 & 39.0 & 1832.4 & 24.8 & 1837.6 & 29.0 & 1837.6 & 29.0 & 99.5 \\
\hline SYN14-1_Run2- 152 & 1819.7 & 25.1 & 1838.1 & 15.7 & 1859.0 & 17.0 & 1859.0 & 17.0 & 97.9 \\
\hline SYN14-1_Run2- 155 & 1873.3 & 25.9 & 1878.6 & 17.7 & 1884.6 & 23.7 & 1884.6 & 23.7 & 99.4 \\
\hline SYN14-1_Run1- 27 & 1807.7 & 41.2 & 1846.3 & 28.6 & 1890.1 & 38.2 & 1890.1 & 38.2 & 95.6 \\
\hline SYN14-1_Run2- 150 & 1643.7 & 24.2 & 1760.8 & 17.4 & 1902.6 & 22.6 & 1902.6 & 22.6 & 86.4 \\
\hline SYN14-1_Run2- 129 & 1925.9 & 37.6 & 1937.2 & 22.3 & 1949.3 & 22.1 & 1949.3 & 22.1 & 98.8 \\
\hline SYN14-1_Run2- 144 & 1929.3 & 28.5 & 1952.7 & 19.1 & 1977.6 & 24.8 & 1977.6 & 24.8 & 97.6 \\
\hline SYN14-1_Run2- 213 & 1896.2 & 29.7 & 1938.9 & 20.4 & 1984.8 & 27.0 & 1984.8 & 27.0 & 95.5 \\
\hline
\end{tabular}




\begin{tabular}{|l|l|l|l|l|l|l|l|l|l|} 
SYN14-1_Run1- 23 & 1932.7 & 25.4 & 1961.1 & 16.9 & 1991.2 & 21.6 & 1991.2 & 21.6 & 97.1 \\
\hline SYN14-1_Run2-172 & 2470.1 & 31.1 & 2453.2 & 17.1 & 2439.3 & 18.0 & 2439.3 & 18.0 & 101.3 \\
\hline SYN14-1_Run1- 40 & 2092.8 & 54.5 & 2324.7 & 29.9 & 2535.0 & 20.2 & 2535.0 & 20.2 & 82.6 \\
\hline SYN14-1_Run2-149 & 2571.7 & 42.3 & 2627.3 & 22.1 & 2670.3 & 20.7 & 2670.3 & 20.7 & 96.3 \\
\hline SYN14-1_Run1- 66 & 2676.8 & 43.6 & 2707.0 & 22.5 & 2729.5 & 21.7 & 2729.5 & 21.7 & 98.1 \\
\hline SYN14-1_Run2-114 & 2720.8 & 32.1 & 2742.0 & 18.7 & 2757.6 & 22.1 & 2757.6 & 22.1 & 98.7 \\
\hline SYN14-1_Run2-219 & 2637.3 & 45.2 & 2708.0 & 24.4 & 2761.3 & 25.1 & 2761.3 & 25.1 & 95.5 \\
\hline SYN14-1_Run1-109 & 2696.4 & 50.8 & 2742.3 & 24.3 & 2776.3 & 18.3 & 2776.3 & 18.3 & 97.1 \\
\hline SYN14-1_Run2-192 & 2804.8 & 38.0 & 2812.2 & 18.5 & 2817.5 & 16.2 & 2817.5 & 16.2 & 99.5 \\
\hline
\end{tabular}

\begin{tabular}{|c|c|c|c|c|c|c|c|c|c|}
\hline Analysis & $206 \mathrm{~Pb}^{*}$ & \pm & 207Pb* & \pm & $206 \mathrm{~Pb} *$ & \pm & Best age & \pm & Conc \\
\hline & $238 U^{*}$ & (Ma) & $235 \mathrm{U}$ & (Ma) & $207 \mathrm{~Pb}^{*}$ & (Ma) & (Ma) & (Ma) & $(\%)$ \\
\hline & & & & & & & & & \\
\hline SYN14-3_Run1- 10 & 108.8 & 2.2 & 131.6 & 8.0 & 567.1 & 134.6 & 108.8 & 2.2 & NA \\
\hline SYN14-3_Run1- 83 & 112.9 & 2.9 & 105.6 & 7.6 & 56.2 & 172.7 & 112.9 & 2.9 & NA \\
\hline SYN14-3_Run2- 199 & 123.0 & 2.2 & 122.9 & 3.9 & 119.6 & 66.4 & 123.0 & 2.2 & NA \\
\hline SYN14-3_Run2- 217 & 164.0 & 6.5 & 169.3 & 7.9 & 243.9 & 71.6 & 164.0 & 6.5 & NA \\
\hline SYN14-3_Run2- 171 & 235.1 & 4.3 & 235.1 & 7.3 & 234.6 & 67.0 & 235.1 & 4.3 & NA \\
\hline SYN14-3_Run2- 170 & 290.7 & 4.9 & 298.6 & 8.2 & 360.8 & 59.9 & 290.7 & 4.9 & NA \\
\hline SYN14-3_Run1- 43 & 319.1 & 6.7 & 331.9 & 10.6 & 422.6 & 68.6 & 319.1 & 6.7 & NA \\
\hline SYN14-3_Run1- 27 & 334.7 & 6.4 & 343.7 & 9.0 & 405.3 & 53.1 & 334.7 & 6.4 & NA \\
\hline SYN14-3_Run2- 202 & 347.1 & 8.3 & 357.3 & 10.2 & 424.1 & 52.3 & 347.1 & 8.3 & NA \\
\hline SYN14-3_Run1- 13 & 350.4 & 7.2 & 358.3 & 8.9 & 410.1 & 46.6 & 350.4 & 7.2 & NA \\
\hline SYN14-3_Run1- 46 & 368.0 & 6.0 & 370.5 & 8.5 & 386.0 & 48.4 & 368.0 & 6.0 & NA \\
\hline SYN14-3_Run1- 91 & 379.9 & 7.2 & 443.1 & 9.0 & 786.2 & 33.0 & 379.9 & 7.2 & NA \\
\hline SYN14-3_Run2- 210 & 394.8 & 7.9 & 404.7 & 10.5 & 461.6 & 52.0 & 394.8 & 7.9 & NA \\
\hline SYN14-3_Run1- 55 & 401.3 & 10.6 & 406.4 & 11.8 & 435.6 & 49.5 & 401.3 & 10.6 & 92.1 \\
\hline SYN14-3_Run1- 78 & 402.6 & 9.1 & 397.4 & 9.9 & 367.4 & 43.0 & 402.6 & 9.1 & 109.6 \\
\hline SYN14-3_Run1-89 & 403.3 & 5.7 & 392.9 & 10.5 & 332.2 & 65.2 & 403.3 & 5.7 & 121.4 \\
\hline SYN14-3_Run2- 183 & 419.0 & 7.6 & 433.5 & 10.4 & 511.2 & 50.5 & 419.0 & 7.6 & 82.0 \\
\hline SYN14-3_Run2- 215 & 423.2 & 9.6 & 436.7 & 13.4 & 508.3 & 64.9 & 423.2 & 9.6 & 83.3 \\
\hline SYN14-3_Run2- 219 & 424.3 & 7.1 & 430.3 & 8.8 & 462.3 & 40.2 & 424.3 & 7.1 & 91.8 \\
\hline SYN14-3_Run1- 26 & 425.6 & 7.3 & 424.7 & 11.6 & 419.9 & 62.8 & 425.6 & 7.3 & 101.3 \\
\hline SYN14-3_Run1- 47 & 425.9 & 6.3 & 427.9 & 11.5 & 438.9 & 64.8 & 425.9 & 6.3 & 97.0 \\
\hline SYN14-3_Run2- 180 & 429.3 & 9.3 & 426.4 & 11.4 & 410.7 & 53.3 & 429.3 & 9.3 & 104.5 \\
\hline SYN14-3_Run1- 80 & 431.2 & 5.8 & 428.6 & 9.7 & 414.9 & 54.0 & 431.2 & 5.8 & 103.9 \\
\hline SYN14-3_Run2- 120 & 435.2 & 6.5 & 434.9 & 9.1 & 433.5 & 45.6 & 435.2 & 6.5 & 100.4 \\
\hline SYN14-3_Run2- 173 & 435.2 & 9.0 & 435.4 & 10.4 & 436.5 & 44.5 & 435.2 & 9.0 & 99.7 \\
\hline
\end{tabular}




\begin{tabular}{|c|c|c|c|c|c|c|c|c|c|}
\hline SYN14-3_Run1- 82 & 435.7 & 8.5 & 438.5 & 11.4 & 453.0 & 55.3 & 435.7 & 8.5 & 96.2 \\
\hline SYN14-3_Run1- 99 & 462.1 & 10.2 & 457.1 & 13.7 & 432.1 & 65.3 & 462.1 & 10.2 & 106.9 \\
\hline SYN14-3_Run1- 51 & 463.1 & 8.1 & 471.9 & 10.6 & 515.0 & 47.4 & 463.1 & 8.1 & 89.9 \\
\hline SYN14-3_Run1- 66 & 466.3 & 26.3 & 466.3 & 24.2 & 466.2 & 61.9 & 466.3 & 26.3 & 100.0 \\
\hline SYN14-3_Run1- 40 & 474.5 & 9.5 & 469.3 & 12.0 & 444.2 & 53.9 & 474.5 & 9.5 & 106.8 \\
\hline SYN14-3_Run1- 56 & 474.5 & 7.8 & 478.3 & 10.5 & 496.5 & 47.4 & 474.5 & 7.8 & 95.6 \\
\hline SYN14-3_Run1- 62 & 475.3 & 7.9 & 489.6 & 9.1 & 557.2 & 34.5 & 475.3 & 7.9 & 85.3 \\
\hline SYN14-3_Run1- 50 & 489.9 & 6.2 & 492.1 & 7.1 & 502.0 & 27.3 & 489.9 & 6.2 & 97.6 \\
\hline SYN14-3_Run2- 161 & 531.3 & 8.9 & 546.0 & 12.0 & 607.6 & 48.4 & 531.3 & 8.9 & 87.4 \\
\hline SYN14-3_Run2- 205 & 533.8 & 19.9 & 539.5 & 20.5 & 563.6 & 65.3 & 533.8 & 19.9 & 94.7 \\
\hline SYN14-3_Run1- 59 & 535.9 & 10.2 & 539.6 & 11.4 & 555.6 & 40.4 & 535.9 & 10.2 & 96.4 \\
\hline SYN14-3_Run1- 14 & 555.3 & 7.2 & 582.3 & 9.5 & 689.0 & 35.8 & 555.3 & 7.2 & 80.6 \\
\hline SYN14-3_Run2- 184 & 569.4 & 7.4 & 583.9 & 8.4 & 640.6 & 28.1 & 569.4 & 7.4 & 88.9 \\
\hline SYN14-3_Run2- 185 & 570.4 & 9.7 & 567.8 & 9.5 & 557.4 & 27.6 & 570.4 & 9.7 & 102.3 \\
\hline SYN14-3_Run1- 9 & 578.2 & 8.4 & 583.6 & 9.6 & 604.5 & 33.3 & 578.2 & 8.4 & 95.6 \\
\hline SYN14-3_Run2- 194 & 579.3 & 9.2 & 595.0 & 9.1 & 655.4 & 24.8 & 579.3 & 9.2 & 88.4 \\
\hline SYN14-3_Run1- 61 & 581.3 & 10.9 & 593.0 & 12.8 & 638.1 & 44.2 & 581.3 & 10.9 & 91.1 \\
\hline SYN14-3_Run2- 212 & 599.9 & 12.4 & 609.8 & 13.2 & 646.5 & 41.2 & 599.9 & 12.4 & 92.8 \\
\hline SYN14-3_Run2- 116 & 605.6 & 7.9 & 610.9 & 12.1 & 630.5 & 48.4 & 605.6 & 7.9 & 96.0 \\
\hline SYN14-3_Run2- 190 & 609.9 & 21.1 & 627.6 & 19.3 & 691.8 & 42.2 & 609.9 & 21.1 & 88.2 \\
\hline SYN14-3_Run1- 15 & 612.7 & 13.8 & 618.6 & 14.7 & 640.2 & 45.2 & 612.7 & 13.8 & 95.7 \\
\hline SYN14-3_Run2- 168 & 613.2 & 20.8 & 639.6 & 19.5 & 734.1 & 43.5 & 613.2 & 20.8 & 83.5 \\
\hline SYN14-3_Run1- 86 & 615.8 & 11.2 & 617.7 & 11.0 & 624.4 & 30.4 & 615.8 & 11.2 & 98.6 \\
\hline SYN14-3_Run1- 74 & 624.1 & 22.2 & 615.8 & 17.9 & 585.7 & 22.8 & 624.1 & 22.2 & 106.6 \\
\hline SYN14-3_Run2- 137 & 624.2 & 9.7 & 648.6 & 10.4 & 734.8 & 29.9 & 624.2 & 9.7 & 84.9 \\
\hline SYN14-3_Run2- 149 & 630.7 & 19.9 & 645.0 & 19.1 & 695.3 & 48.2 & 630.7 & 19.9 & 90.7 \\
\hline SYN14-3_Run2- 142 & 780.6 & 14.0 & 809.1 & 13.4 & 888.4 & 30.0 & 780.6 & 14.0 & 87.9 \\
\hline SYN14-3_Run2- 122 & 840.9 & 19.0 & 839.0 & 15.6 & 834.1 & 26.9 & 840.9 & 19.0 & 100.8 \\
\hline SYN14-3_Run2- 198 & 948.7 & 11.7 & 941.5 & 12.0 & 925.0 & 29.7 & 925.0 & 29.7 & 102.6 \\
\hline SYN14-3_Run1- 104 & 926.3 & 33.8 & 908.2 & 28.2 & 864.4 & 53.5 & 926.3 & 33.8 & 107.2 \\
\hline SYN14-3_Run2- 204 & 953.3 & 19.5 & 958.2 & 17.5 & 969.4 & 35.8 & 969.4 & 35.8 & 98.3 \\
\hline SYN14-3_Run1- 54 & 970.8 & 17.8 & 970.9 & 16.0 & 971.3 & 33.0 & 971.3 & 33.0 & 99.9 \\
\hline SYN14-3_Run2- 118 & 983.5 & 15.3 & 983.7 & 12.2 & 984.2 & 19.7 & 984.2 & 19.7 & 99.9 \\
\hline SYN14-3_Run1- 57 & 1010.0 & 12.5 & 1005.0 & 13.9 & 994.1 & 34.7 & 994.1 & 34.7 & 101.6 \\
\hline SYN14-3_Run1- 109 & 994.3 & 14.3 & 994.5 & 16.5 & 994.9 & 42.4 & 994.9 & 42.4 & 99.9 \\
\hline SYN14-3_Run2- 164 & 899.0 & 19.4 & 931.7 & 18.4 & 1009.9 & 39.1 & 1009.9 & 39.1 & 89.0 \\
\hline SYN14-3_Run2- 211 & 1042.8 & 25.6 & 1040.4 & 19.7 & 1035.3 & 28.9 & 1035.3 & 28.9 & 100.7 \\
\hline SYN14-3_Run2- 206 & 987.8 & 18.3 & 1003.5 & 14.2 & 1038.0 & 20.0 & 1038.0 & 20.0 & 95.2 \\
\hline SYN14-3_Run2- 165 & 962.4 & 14.8 & 985.9 & 15.3 & 1038.5 & 35.9 & 1038.5 & 35.9 & 92.7 \\
\hline
\end{tabular}




\begin{tabular}{|c|c|c|c|c|c|c|c|c|c|}
\hline SYN14-3_Run1- 19 & 909.2 & 16.0 & 956.5 & 14.2 & 1066.8 & 26.0 & 1066.8 & 26.0 & 85.2 \\
\hline SYN14-3_Run1- 106 & 1045.8 & 17.3 & 1055.8 & 15.6 & 1076.5 & 31.1 & 1076.5 & 31.1 & 97.1 \\
\hline SYN14-3_Run2- 134 & 1000.0 & 16.1 & 1024.7 & 13.3 & 1077.9 & 22.5 & 1077.9 & 22.5 & 92.8 \\
\hline SYN14-3_Run1- 1 & 887.1 & 15.1 & 944.2 & 23.7 & 1079.9 & 68.2 & 1079.9 & 68.2 & 82.1 \\
\hline SYN14-3_Run2- 125 & 997.6 & 31.1 & 1024.3 & 24.7 & 1081.8 & 36.8 & 1081.8 & 36.8 & 92.2 \\
\hline SYN14-3_Run2- 157 & 1057.0 & 30.7 & 1066.1 & 22.4 & 1084.7 & 25.4 & 1084.7 & 25.4 & 97.5 \\
\hline SYN14-3_Run2- 214 & 1006.7 & 20.9 & 1031.7 & 18.1 & 1085.3 & 33.3 & 1085.3 & 33.3 & 92.8 \\
\hline SYN14-3_Run1- 24 & 1099.4 & 21.0 & 1095.3 & 17.6 & 1087.0 & 32.1 & 1087.0 & 32.1 & 101.1 \\
\hline SYN14-3_Run2- 196 & 1049.7 & 22.1 & 1063.2 & 18.0 & 1090.9 & 30.2 & 1090.9 & 30.2 & 96.2 \\
\hline SYN14-3_Run2- 143 & 1066.1 & 13.1 & 1074.3 & 11.6 & 1091.1 & 22.7 & 1091.1 & 22.7 & 97.7 \\
\hline SYN14-3_Run1- 42 & 1102.8 & 19.0 & 1102.2 & 17.5 & 1101.0 & 36.1 & 1101.0 & 36.1 & 100.2 \\
\hline SYN14-3_Run2- 160 & 1057.6 & 16.4 & 1072.3 & 16.8 & 1102.4 & 38.0 & 1102.4 & 38.0 & 95.9 \\
\hline SYN14-3_Run1- 23 & 1002.3 & 16.4 & 1041.6 & 14.4 & 1124.8 & 26.3 & 1124.8 & 26.3 & 89.1 \\
\hline SYN14-3_Run2- 197 & 1132.2 & 21.8 & 1134.9 & 21.0 & 1140.2 & 44.6 & 1140.2 & 44.6 & 99.3 \\
\hline SYN14-3_Run2- 169 & 1032.3 & 12.9 & 1067.8 & 11.6 & 1141.1 & 22.3 & 1141.1 & 22.3 & 90.5 \\
\hline SYN14-3_Run2- 220 & 1140.4 & 18.8 & 1145.7 & 15.8 & 1155.6 & 28.3 & 1155.6 & 28.3 & 98.7 \\
\hline SYN14-3_Run2- 153 & 971.1 & 28.1 & 1031.3 & 25.9 & 1161.3 & 49.5 & 1161.3 & 49.5 & 83.6 \\
\hline SYN14-3_Run2- 145 & 1150.8 & 23.3 & 1156.7 & 17.5 & 1167.7 & 24.7 & 1167.7 & 24.7 & 98.6 \\
\hline SYN14-3_Run1- 11 & 998.5 & 35.2 & 1055.1 & 27.2 & 1174.3 & 32.8 & 1174.3 & 32.8 & 85.0 \\
\hline SYN14-3_Run2- 155 & 1045.9 & 18.1 & 1088.6 & 16.2 & 1175.0 & 30.5 & 1175.0 & 30.5 & 89.0 \\
\hline SYN14-3_Run1- 77 & 1066.1 & 22.5 & 1104.4 & 23.6 & 1180.7 & 52.7 & 1180.7 & 52.7 & 90.3 \\
\hline SYN14-3_Run2- 129 & 1039.2 & 15.8 & 1086.7 & 13.2 & 1183.0 & 21.8 & 1183.0 & 21.8 & 87.8 \\
\hline SYN14-3_Run1- 49 & 1152.1 & 20.6 & 1171.1 & 17.8 & 1206.5 & 32.9 & 1206.5 & 32.9 & 95.5 \\
\hline SYN14-3_Run2- 113 & 1183.4 & 26.3 & 1194.4 & 20.8 & 1214.4 & 32.9 & 1214.4 & 32.9 & 97.5 \\
\hline SYN14-3_Run2- 213 & 1221.1 & 23.4 & 1219.8 & 18.7 & 1217.4 & 30.9 & 1217.4 & 30.9 & 100.3 \\
\hline SYN14-3_Run1- 72 & 1237.9 & 23.8 & 1241.1 & 18.9 & 1246.6 & 31.2 & 1246.6 & 31.2 & 99.3 \\
\hline SYN14-3_Run2- 172 & 1297.9 & 23.1 & 1295.7 & 17.5 & 1292.1 & 26.3 & 1292.1 & 26.3 & 100.5 \\
\hline SYN14-3_Run2- 119 & 1071.6 & 29.9 & 1153.2 & 24.3 & 1310.2 & 35.1 & 1310.2 & 35.1 & 81.8 \\
\hline SYN14-3_Run1- 93 & 1321.0 & 23.1 & 1320.6 & 18.3 & 1320.0 & 30.1 & 1320.0 & 30.1 & 100.1 \\
\hline SYN14-3_Run1- 53 & 1262.5 & 25.1 & 1292.9 & 19.4 & 1343.8 & 29.2 & 1343.8 & 29.2 & 94.0 \\
\hline SYN14-3_Run2- 163 & 1214.4 & 19.9 & 1273.0 & 18.7 & 1373.3 & 35.7 & 1373.3 & 35.7 & 88.4 \\
\hline SYN14-3_Run2- 182 & 1184.6 & 21.8 & 1255.5 & 16.4 & 1379.1 & 20.3 & 1379.1 & 20.3 & 85.9 \\
\hline SYN14-3_Run2- 121 & 1364.3 & 18.8 & 1375.6 & 16.8 & 1393.2 & 31.3 & 1393.2 & 31.3 & 97.9 \\
\hline SYN14-3_Run1- 48 & 1365.1 & 23.9 & 1379.5 & 19.7 & 1401.8 & 33.4 & 1401.8 & 33.4 & 97.4 \\
\hline SYN14-3_Run2- 200 & 1359.4 & 20.5 & 1377.2 & 18.3 & 1404.9 & 33.7 & 1404.9 & 33.7 & 96.8 \\
\hline SYN14-3_Run2- 195 & 1384.5 & 28.6 & 1397.2 & 21.0 & 1416.7 & 29.8 & 1416.7 & 29.8 & 97.7 \\
\hline SYN14-3_Run2- 141 & 1344.9 & 19.9 & 1382.8 & 15.3 & 1441.9 & 22.8 & 1441.9 & 22.8 & 93.3 \\
\hline SYN14-3_Run2- 166 & 1421.0 & 17.0 & 1431.3 & 14.7 & 1446.7 & 26.1 & 1446.7 & 26.1 & 98.2 \\
\hline SYN14-3_Run2- 188 & 1350.0 & 22.7 & 1388.1 & 18.3 & 1447.3 & 29.5 & 1447.3 & 29.5 & 93.3 \\
\hline
\end{tabular}




\begin{tabular}{|c|c|c|c|c|c|c|c|c|c|}
\hline SYN14-3_Run1- 92 & 1413.1 & 24.9 & 1427.3 & 17.1 & 1448.5 & 20.4 & 1448.5 & 20.4 & 97.6 \\
\hline SYN14-3_Run1- 16 & 1364.2 & 30.6 & 1411.4 & 21.6 & 1483.3 & 25.4 & 1483.3 & 25.4 & 92.0 \\
\hline SYN14-3_Run1- 100 & 1493.5 & 24.2 & 1489.4 & 17.5 & 1483.6 & 25.1 & 1483.6 & 25.1 & 100.7 \\
\hline SYN14-3_Run1- 107 & 1468.4 & 21.5 & 1477.7 & 15.1 & 1491.1 & 19.7 & 1491.1 & 19.7 & 98.5 \\
\hline SYN14-3_Run2- 209 & 1372.2 & 25.1 & 1420.2 & 17.9 & 1492.9 & 22.0 & 1492.9 & 22.0 & 91.9 \\
\hline SYN14-3_Run2- 115 & 1460.5 & 26.0 & 1478.8 & 20.5 & 1505.0 & 32.4 & 1505.0 & 32.4 & 97.0 \\
\hline SYN14-3_Run2- 147 & 1495.7 & 25.0 & 1500.1 & 18.0 & 1506.3 & 25.1 & 1506.3 & 25.1 & 99.3 \\
\hline SYN14-3_Run2- 175 & 1424.4 & 25.3 & 1458.1 & 20.3 & 1507.6 & 32.5 & 1507.6 & 32.5 & 94.5 \\
\hline SYN14-3_Run1- 87 & 1455.6 & 23.6 & 1478.0 & 18.6 & 1510.3 & 29.5 & 1510.3 & 29.5 & 96.4 \\
\hline SYN14-3_Run2- 179 & 1437.9 & 33.3 & 1467.9 & 22.7 & 1511.5 & 25.9 & 1511.5 & 25.9 & 95.1 \\
\hline SYN14-3_Run1- 102 & 1449.6 & 23.4 & 1477.4 & 16.0 & 1517.6 & 18.8 & 1517.6 & 18.8 & 95.5 \\
\hline SYN14-3_Run2- 124 & 1270.2 & 19.2 & 1367.0 & 15.8 & 1521.6 & 24.1 & 1521.6 & 24.1 & 83.5 \\
\hline SYN14-3_Run2- 191 & 1460.7 & 21.1 & 1485.8 & 17.5 & 1521.9 & 29.3 & 1521.9 & 29.3 & 96.0 \\
\hline SYN14-3_Run1- 17 & 1505.9 & 28.7 & 1512.6 & 21.6 & 1522.0 & 32.7 & 1522.0 & 32.7 & 98.9 \\
\hline SYN14-3_Run1- 69 & 1417.7 & 19.6 & 1460.5 & 17.5 & 1523.2 & 31.0 & 1523.2 & 31.0 & 93.1 \\
\hline SYN14-3_Run2- 152 & 1426.4 & 23.9 & 1466.3 & 23.1 & 1524.6 & 43.8 & 1524.6 & 43.8 & 93.6 \\
\hline SYN14-3_Run1- 96 & 1488.2 & 27.3 & 1503.3 & 18.2 & 1524.7 & 20.5 & 1524.7 & 20.5 & 97.6 \\
\hline SYN14-3_Run2- 133 & 1441.1 & 38.5 & 1478.0 & 26.2 & 1531.3 & 29.1 & 1531.3 & 29.1 & 94.1 \\
\hline SYN14-3_Run2- 201 & 1326.6 & 23.2 & 1416.7 & 20.4 & 1554.7 & 34.4 & 1554.7 & 34.4 & 85.3 \\
\hline SYN14-3_Run2- 144 & 1503.2 & 16.8 & 1526.4 & 24.0 & 1558.8 & 51.9 & 1558.8 & 51.9 & 96.4 \\
\hline SYN14-3_Run2- 131 & 1592.9 & 29.4 & 1580.8 & 19.6 & 1564.6 & 23.9 & 1564.6 & 23.9 & 101.8 \\
\hline SYN14-3_Run1- 79 & 1513.7 & 22.9 & 1539.0 & 18.7 & 1574.0 & 30.7 & 1574.0 & 30.7 & 96.2 \\
\hline SYN14-3_Run1- 12 & 1394.7 & 15.3 & 1468.0 & 15.0 & 1575.6 & 28.0 & 1575.6 & 28.0 & 88.5 \\
\hline SYN14-3_Run1- 18 & 1512.9 & 25.1 & 1544.4 & 19.9 & 1587.8 & 31.3 & 1587.8 & 31.3 & 95.3 \\
\hline SYN14-3_Run2- 207 & 1279.1 & 33.0 & 1401.8 & 23.5 & 1593.6 & 22.7 & 1593.6 & 22.7 & 80.3 \\
\hline SYN14-3_Run2- 159 & 1563.1 & 26.6 & 1576.4 & 17.8 & 1594.1 & 20.9 & 1594.1 & 20.9 & 98.1 \\
\hline SYN14-3_Run1- 67 & 1464.3 & 37.2 & 1521.0 & 24.5 & 1600.8 & 23.6 & 1600.8 & 23.6 & 91.5 \\
\hline SYN14-3_Run2- 192 & 1511.5 & 25.3 & 1551.6 & 18.6 & 1606.7 & 26.0 & 1606.7 & 26.0 & 94.1 \\
\hline SYN14-3_Run1- 103 & 1601.2 & 25.6 & 1607.3 & 17.3 & 1615.2 & 21.3 & 1615.2 & 21.3 & 99.1 \\
\hline SYN14-3_Run2- 174 & 1595.1 & 20.1 & 1605.3 & 15.9 & 1618.7 & 25.3 & 1618.7 & 25.3 & 98.5 \\
\hline SYN14-3_Run1- 76 & 1573.6 & 28.4 & 1594.4 & 19.5 & 1621.9 & 24.6 & 1621.9 & 24.6 & 97.0 \\
\hline SYN14-3_Run1- 90 & 1589.7 & 26.7 & 1606.1 & 18.6 & 1627.6 & 24.6 & 1627.6 & 24.6 & 97.7 \\
\hline SYN14-3_Run2- 154 & 1596.7 & 31.3 & 1610.2 & 24.5 & 1627.8 & 38.6 & 1627.8 & 38.6 & 98.1 \\
\hline SYN14-3_Run1- 98 & 1537.1 & 27.2 & 1575.8 & 22.0 & 1627.8 & 35.5 & 1627.8 & 35.5 & 94.4 \\
\hline SYN14-3_Run2- 156 & 1666.4 & 33.7 & 1650.5 & 20.2 & 1630.4 & 17.1 & 1630.4 & 17.1 & 102.2 \\
\hline SYN14-3_Run2- 128 & 1627.5 & 19.4 & 1630.4 & 12.2 & 1634.0 & 12.1 & 1634.0 & 12.1 & 99.6 \\
\hline SYN14-3_Run2- 146 & 1585.6 & 22.7 & 1607.0 & 18.2 & 1635.1 & 29.4 & 1635.1 & 29.4 & 97.0 \\
\hline SYN14-3_Run2- 178 & 1629.5 & 38.7 & 1633.4 & 25.6 & 1638.4 & 30.6 & 1638.4 & 30.6 & 99.5 \\
\hline SYN14-3_Run1- 75 & 1611.7 & 25.7 & 1624.5 & 17.8 & 1641.1 & 23.1 & 1641.1 & 23.1 & 98.2 \\
\hline
\end{tabular}




\begin{tabular}{|c|c|c|c|c|c|c|c|c|c|}
\hline SYN14-3_Run1- 29 & 1574.0 & 25.2 & 1606.5 & 17.3 & 1649.4 & 21.3 & 1649.4 & 21.3 & 95.4 \\
\hline SYN14-3_Run2- 218 & 1641.1 & 26.0 & 1644.9 & 16.9 & 1649.8 & 19.6 & 1649.8 & 19.6 & 99.5 \\
\hline SYN14-3_Run2- 150 & 1676.2 & 29.5 & 1666.1 & 18.0 & 1653.4 & 16.9 & 1653.4 & 16.9 & 101.4 \\
\hline SYN14-3_Run1- 73 & 1604.7 & 34.7 & 1634.3 & 22.2 & 1672.5 & 22.7 & 1672.5 & 22.7 & 95.9 \\
\hline SYN14-3_Run2- 208 & 1410.7 & 58.1 & 1519.1 & 38.8 & 1673.6 & 32.9 & 1673.6 & 32.9 & 84.3 \\
\hline SYN14-3_Run1- 5 & 1681.2 & 24.7 & 1684.9 & 17.1 & 1689.6 & 22.9 & 1689.6 & 22.9 & 99.5 \\
\hline SYN14-3_Run2- 167 & 1597.6 & 30.6 & 1645.0 & 20.7 & 1706.0 & 24.3 & 1706.0 & 24.3 & 93.6 \\
\hline SYN14-3_Run1- 41 & 1657.4 & 30.1 & 1680.6 & 21.0 & 1709.6 & 28.0 & 1709.6 & 28.0 & 96.9 \\
\hline SYN14-3_Run2- 187 & 1665.0 & 21.3 & 1686.9 & 18.1 & 1714.2 & 30.3 & 1714.2 & 30.3 & 97.1 \\
\hline SYN14-3_Run1- 70 & 1463.1 & 54.5 & 1571.8 & 39.7 & 1720.9 & 49.6 & 1720.9 & 49.6 & 85.0 \\
\hline SYN14-3_Run1- 8 & 1693.8 & 27.3 & 1712.1 & 18.0 & 1734.7 & 21.6 & 1734.7 & 21.6 & 97.6 \\
\hline SYN14-3_Run1- 2 & 1807.5 & 27.8 & 1776.9 & 18.1 & 1741.1 & 22.8 & 1741.1 & 22.8 & 103.8 \\
\hline SYN14-3_Run1- 64 & 1750.4 & 20.5 & 1752.2 & 13.7 & 1754.2 & 17.5 & 1754.2 & 17.5 & 99.8 \\
\hline SYN14-3_Run1- 84 & 1747.6 & 28.0 & 1751.2 & 18.6 & 1755.5 & 23.3 & 1755.5 & 23.3 & 99.5 \\
\hline SYN14-3_Run1- 60 & 1720.2 & 26.0 & 1743.0 & 17.1 & 1770.4 & 20.5 & 1770.4 & 20.5 & 97.2 \\
\hline SYN14-3_Run2- 123 & 1722.3 & 29.2 & 1757.0 & 18.8 & 1798.4 & 21.2 & 1798.4 & 21.2 & 95.8 \\
\hline SYN14-3_Run2- 193 & 1555.1 & 30.2 & 1662.4 & 20.5 & 1800.8 & 22.1 & 1800.8 & 22.1 & 86.4 \\
\hline SYN14-3_Run1- 65 & 1775.5 & 31.3 & 1790.5 & 19.5 & 1808.1 & 20.9 & 1808.1 & 20.9 & 98.2 \\
\hline SYN14-3_Run1- 58 & 1866.0 & 59.3 & 1861.3 & 33.1 & 1855.9 & 23.3 & 1855.9 & 23.3 & 100.5 \\
\hline SYN14-3_Run2- 189 & 1640.1 & 26.0 & 1760.2 & 19.3 & 1906.0 & 26.2 & 1906.0 & 26.2 & 86.0 \\
\hline SYN14-3_Run1- 110 & 1816.7 & 31.9 & 1870.4 & 21.6 & 1930.5 & 27.5 & 1930.5 & 27.5 & 94.1 \\
\hline SYN14-3_Run2- 203 & 1842.6 & 29.9 & 1898.1 & 20.5 & 1959.4 & 26.4 & 1959.4 & 26.4 & 94.0 \\
\hline SYN14-3_Run1- 20 & 1936.0 & 29.9 & 1948.2 & 21.5 & 1961.2 & 30.8 & 1961.2 & 30.8 & 98.7 \\
\hline SYN14-3_Run1- 28 & 1757.3 & 27.9 & 1856.0 & 17.8 & 1968.5 & 18.6 & 1968.5 & 18.6 & 89.3 \\
\hline SYN14-3_Run1- 97 & 1950.7 & 30.3 & 1960.8 & 17.4 & 1971.4 & 15.8 & 1971.4 & 15.8 & 99.0 \\
\hline SYN14-3_Run2- 140 & 1900.7 & 25.2 & 1938.1 & 17.0 & 1978.4 & 21.9 & 1978.4 & 21.9 & 96.1 \\
\hline SYN14-3_Run2- 162 & 1666.2 & 52.5 & 1821.4 & 32.7 & 2003.8 & 26.0 & 2003.8 & 26.0 & 83.1 \\
\hline SYN14-3_Run1- 95 & 1737.1 & 60.4 & 1866.3 & 36.1 & 2013.3 & 26.2 & 2013.3 & 26.2 & 86.3 \\
\hline SYN14-3_Run2- 186 & 1928.7 & 47.3 & 2008.4 & 26.8 & 2091.5 & 20.5 & 2091.5 & 20.5 & 92.2 \\
\hline SYN14-3_Run2- 176 & 2364.7 & 33.1 & 2495.2 & 19.5 & 2603.2 & 21.1 & 2603.2 & 21.1 & 90.8 \\
\hline SYN14-3_Run1- 94 & 2426.0 & 52.3 & 2532.8 & 28.6 & 2619.5 & 27.4 & 2619.5 & 27.4 & 92.6 \\
\hline SYN14-3_Run2- 136 & 2610.0 & 36.6 & 2628.2 & 19.1 & 2642.3 & 18.6 & 2642.3 & 18.6 & 98.8 \\
\hline SYN14-3_Run1- 88 & 2472.4 & 41.3 & 2567.0 & 22.2 & 2642.5 & 21.1 & 2642.5 & 21.1 & 93.6 \\
\hline SYN14-3_Run2- 112 & 2404.5 & 35.0 & 2554.2 & 18.1 & 2675.4 & 14.2 & 2675.4 & 14.2 & 89.9 \\
\hline SYN14-3_Run1- 4 & 2665.5 & 29.7 & 2688.5 & 15.3 & 2705.8 & 14.4 & 2705.8 & 14.4 & 98.5 \\
\hline SYN14-3_Run2- 111 & 2647.0 & 44.7 & 2681.8 & 22.8 & 2708.1 & 20.9 & 2708.1 & 20.9 & 97.7 \\
\hline SYN14-3_Run1- 3 & 2627.1 & 38.0 & 2676.9 & 21.9 & 2714.7 & 24.9 & 2714.7 & 24.9 & 96.8 \\
\hline SYN14-3_Run2- 177 & 2765.1 & 41.4 & 2789.3 & 19.5 & 2806.9 & 14.9 & 2806.9 & 14.9 & 98.5 \\
\hline SYN14-3_Run2- 181 & 2714.0 & 37.3 & 2786.0 & 17.9 & 2838.6 & 13.7 & 2838.6 & 13.7 & 95.6 \\
\hline
\end{tabular}




\begin{tabular}{|c|c|c|c|c|c|c|c|c|c|}
\hline Analysis & 206Pb* & \pm & 207Pb* & \pm & 206Pb* & \pm & Best age & \pm & Conc \\
\hline & $238 U^{*}$ & (Ma) & $235 \mathrm{U}$ & (Ma) & 207Pb* & (Ma) & (Ma) & (Ma) & $(\%)$ \\
\hline SYN14-4 run 1-- 1 & 409.1 & 10.2 & 417.7 & 17.8 & 465.5 & 100.0 & 409.1 & 10.2 & 87.9 \\
\hline SYN14-4 run 1-- 2 & 1540.3 & 31.2 & 1613.8 & 22.5 & 1711.1 & 29.6 & 1711.1 & 29.6 & 90.0 \\
\hline SYN14-4 run 1-- 3 & 1071.9 & 25.5 & 1080.3 & 23.4 & 1097.5 & 47.8 & 1097.5 & 47.8 & 97.7 \\
\hline SYN14-4 run 1-- 4 & 1499.1 & 53.3 & 1496.6 & 33.7 & 1493.0 & 30.9 & 1493.0 & 30.9 & 100.4 \\
\hline SYN14-4 run 1-- 5 & 1745.2 & 49.1 & 1785.6 & 32.1 & 1833.2 & 37.6 & 1833.2 & 37.6 & 95.2 \\
\hline SYN14-4 run 1-- 6 & 1580.4 & 29.6 & 1616.7 & 24.5 & 1664.2 & 40.3 & 1664.2 & 40.3 & 95.0 \\
\hline SYN14-4 run 1-- 7 & 1306.3 & 26.0 & 1319.5 & 21.7 & 1341.0 & 37.6 & 1341.0 & 37.6 & 97.4 \\
\hline SYN14-4 run 1-- 8 & 1059.6 & 35.7 & 1079.5 & 28.4 & 1119.9 & 43.9 & 1119.9 & 43.9 & 94.6 \\
\hline SYN14-4 run 1-- 9 & 1819.8 & 71.8 & 1843.0 & 41.9 & 1869.3 & 35.2 & 1869.3 & 35.2 & 97.4 \\
\hline SYN14-4 run 1-- 10 & 1146.0 & 28.4 & 1145.8 & 23.5 & 1145.3 & 41.4 & 1145.3 & 41.4 & 100.1 \\
\hline SYN14-4 run 1-- 11 & 972.3 & 25.1 & 989.3 & 21.3 & 1027.3 & 38.7 & 1027.3 & 38.7 & 94.6 \\
\hline SYN14-4 run 1-- 12 & 1045.7 & 33.6 & 1068.0 & 29.0 & 1113.7 & 53.5 & 1113.7 & 53.5 & 93.9 \\
\hline SYN14-4 run 1-- 13 & 1582.4 & 37.4 & 1603.4 & 28.0 & 1631.0 & 41.2 & 1631.0 & 41.2 & 97.0 \\
\hline SYN14-4 run 1-- 14 & 1225.1 & 28.0 & 1274.5 & 26.4 & 1358.7 & 50.8 & 1358.7 & 50.8 & 90.2 \\
\hline SYN14-4 run 1-- 15 & 1244.2 & 46.9 & 1271.8 & 33.1 & 1318.7 & 36.6 & 1318.7 & 36.6 & 94.3 \\
\hline SYN14-4 run 1-- 16 & 1014.7 & 17.0 & 1037.2 & 16.4 & 1085.1 & 35.1 & 1085.1 & 35.1 & 93.5 \\
\hline SYN14-4 run 1-- 17 & 1390.1 & 35.2 & 1421.5 & 25.7 & 1468.8 & 34.9 & 1468.8 & 34.9 & 94.6 \\
\hline SYN14-4 run 1-- 18 & 2611.7 & 68.5 & 2680.0 & 34.3 & 2732.0 & 28.7 & 2732.0 & 28.7 & 95.6 \\
\hline SYN14-4 run 1-- 19 & 2418.6 & 38.4 & 2700.8 & 24.3 & 2919.3 & 28.0 & 2919.3 & 28.0 & 82.8 \\
\hline SYN14-4 run 1-- 20 & 1031.8 & 21.8 & 1041.5 & 18.4 & 1062.1 & 33.2 & 1062.1 & 33.2 & 97.1 \\
\hline SYN14-4 run 1-- 21 & 1105.1 & 39.3 & 1118.6 & 29.2 & 1144.9 & 37.7 & 1144.9 & 37.7 & 96.5 \\
\hline SYN14-4 run 1-- 22 & 1647.9 & 45.4 & 1676.9 & 31.6 & 1713.3 & 41.4 & 1713.3 & 41.4 & 96.2 \\
\hline SYN14-4 run 1-- 23 & 1425.0 & 34.4 & 1448.3 & 25.0 & 1482.7 & 34.1 & 1482.7 & 34.1 & 96.1 \\
\hline SYN14-4 run 1-- 25 & 1077.8 & 34.4 & 1116.2 & 28.3 & 1191.6 & 45.8 & 1191.6 & 45.8 & 90.5 \\
\hline SYN14-4 run 1-- 26 & 962.3 & 34.7 & 967.2 & 28.4 & 978.4 & 48.5 & 978.4 & 48.5 & 98.4 \\
\hline SYN14-4 run 1-- 27 & 1774.6 & 35.8 & 1736.5 & 24.1 & 1691.0 & 32.3 & 1691.0 & 32.3 & 104.9 \\
\hline SYN14-4 run 1-- 28 & 1086.7 & 27.9 & 1097.7 & 23.7 & 1119.7 & 43.0 & 1119.7 & 43.0 & 97.0 \\
\hline SYN14-4 run 1-- 29 & 1914.5 & 74.1 & 1956.3 & 40.9 & 2000.9 & 26.3 & 2000.9 & 26.3 & 95.7 \\
\hline SYN14-4 run 1-- 30 & 1795.8 & 71.8 & 1850.2 & 41.3 & 1911.9 & 28.3 & 1911.9 & 28.3 & 93.9 \\
\hline SYN14-4 run 1-- 31 & 996.8 & 25.2 & 1005.7 & 22.8 & 1025.3 & 46.7 & 1025.3 & 46.7 & 97.2 \\
\hline SYN14-4 run 1-- 32 & 1155.5 & 41.8 & 1168.3 & 32.5 & 1192.3 & 49.9 & 1192.3 & 49.9 & 96.9 \\
\hline SYN14-4 run 1-- 34 & 1619.4 & 32.2 & 1623.4 & 24.5 & 1628.6 & 37.8 & 1628.6 & 37.8 & 99.4 \\
\hline SYN14-4 run 1-- 35 & 2664.1 & 119.4 & 2688.0 & 54.4 & 2706.0 & 29.8 & 2706.0 & 29.8 & 98.5 \\
\hline SYN14-4 run 1-- 36 & 1101.6 & 34.1 & 1125.7 & 28.2 & 1172.4 & 47.6 & 1172.4 & 47.6 & 94.0 \\
\hline
\end{tabular}




\begin{tabular}{|c|c|c|c|c|c|c|c|c|c|}
\hline SYN14-4 run 1-- 37 & 1540.9 & 52.1 & 1565.4 & 41.5 & 1598.7 & 66.3 & 1598.7 & 66.3 & 96.4 \\
\hline SYN14-4 run 1-- 38 & 532.5 & 12.0 & 542.1 & 15.8 & 582.3 & 63.5 & 532.5 & 12.0 & 91.5 \\
\hline SYN14-4 run 1-- 39 & 993.6 & 31.1 & 1020.7 & 28.1 & 1079.4 & 55.1 & 1079.4 & 55.1 & 92.1 \\
\hline SYN14-4 run 1-- 40 & 1203.3 & 47.5 & 1232.3 & 37.2 & 1283.5 & 56.4 & 1283.5 & 56.4 & 93.7 \\
\hline SYN14-4 run 1-- 41 & 1250.7 & 43.4 & 1309.1 & 32.2 & 1406.3 & 41.0 & 1406.3 & 41.0 & 88.9 \\
\hline SYN14-4 run 1-- 42 & 424.7 & 12.5 & 434.9 & 19.4 & 489.6 & 100.5 & 424.7 & 12.5 & 86.7 \\
\hline SYN14-4 run 1-- 43 & 912.5 & 27.6 & 929.9 & 26.8 & 971.2 & 60.6 & 971.2 & 60.6 & 94.0 \\
\hline SYN14-4 run 1-- 44 & 1800.7 & 62.1 & 1797.2 & 37.9 & 1793.2 & 39.3 & 1793.2 & 39.3 & 100.4 \\
\hline SYN14-4 run 1-- 45 & 393.0 & 13.5 & 408.8 & 16.9 & 498.5 & 78.7 & 393.0 & 13.5 & NA \\
\hline SYN14-4 run 1-- 46 & 1132.3 & 25.8 & 1139.3 & 20.2 & 1152.7 & 31.3 & 1152.7 & 31.3 & 98.2 \\
\hline SYN14-4 run 1-- 47 & 1008.3 & 32.0 & 1010.8 & 31.3 & 1016.2 & 70.7 & 1016.2 & 70.7 & 99.2 \\
\hline SYN14-4 run 1-- 49 & 1335.3 & 26.7 & 1363.9 & 21.8 & 1408.8 & 35.9 & 1408.8 & 35.9 & 94.8 \\
\hline SYN14-4 run 1-- 50 & 2582.0 & 53.1 & 2635.5 & 31.1 & 2676.8 & 35.9 & 2676.8 & 35.9 & 96.5 \\
\hline SYN14-4 run 1-- 51 & 1100.8 & 28.1 & 1089.9 & 27.3 & 1068.4 & 60.1 & 1068.4 & 60.1 & 103.0 \\
\hline SYN14-4 run 1-- 52 & 967.6 & 50.5 & 1012.1 & 37.3 & 1109.9 & 30.0 & 1109.9 & 30.0 & 87.2 \\
\hline SYN14-4 run 1-- 53 & 407.3 & 10.2 & 414.2 & 11.9 & 452.7 & 52.1 & 407.3 & 10.2 & 90.0 \\
\hline SYN14-4 run 1-- 56 & 1274.0 & 30.3 & 1285.0 & 24.6 & 1303.4 & 41.2 & 1303.4 & 41.2 & 97.7 \\
\hline SYN14-4 run 1-- 57 & 2327.9 & 57.6 & 2423.9 & 32.0 & 2505.6 & 31.0 & 2505.6 & 31.0 & 92.9 \\
\hline SYN14-4 run 1-- 58 & 1054.9 & 48.8 & 1045.3 & 35.0 & 1025.3 & 38.5 & 1025.3 & 38.5 & 102.9 \\
\hline SYN14-4 run 1-- 59 & 1721.7 & 52.3 & 1754.7 & 35.2 & 1794.2 & 43.8 & 1794.2 & 43.8 & 96.0 \\
\hline SYN14-4 run 1-- 60 & 457.2 & 13.7 & 471.7 & 14.3 & 542.8 & 47.5 & 457.2 & 13.7 & 84.2 \\
\hline SYN14-4 run 1-- 61 & 584.6 & 33.0 & 599.4 & 29.0 & 656.1 & 53.5 & 584.6 & 33.0 & 89.1 \\
\hline SYN14-4 run 1-- 62 & 983.5 & 25.5 & 1007.4 & 25.9 & 1060.0 & 59.1 & 1060.0 & 59.1 & 92.8 \\
\hline SYN14-4 run 1-- 63 & 934.9 & 26.4 & 952.7 & 24.2 & 994.3 & 50.0 & 994.3 & 50.0 & 94.0 \\
\hline SYN14-4 run 1-- 64 & 1431.9 & 34.5 & 1445.1 & 23.2 & 1464.5 & 25.7 & 1464.5 & 25.7 & 97.8 \\
\hline SYN14-4 run 1-- 65 & 1437.0 & 39.0 & 1451.6 & 28.1 & 1473.1 & 38.2 & 1473.1 & 38.2 & 97.6 \\
\hline SYN14-4 run 1-- 66 & 572.4 & 14.6 & 578.6 & 15.3 & 603.1 & 48.3 & 572.4 & 14.6 & 94.9 \\
\hline SYN14-4 run 1-- 67 & 1686.5 & 65.9 & 1708.2 & 41.5 & 1734.8 & 42.9 & 1734.8 & 42.9 & 97.2 \\
\hline SYN14-4 run 1-- 68 & 1280.1 & 36.7 & 1305.4 & 24.6 & 1347.1 & 21.8 & 1347.1 & 21.8 & 95.0 \\
\hline SYN14-4 run 1-- 69 & 919.1 & 19.6 & 906.7 & 22.3 & 876.5 & 60.5 & 919.1 & 19.6 & 104.9 \\
\hline SYN14-4 run 1-- 70 & 1443.6 & 33.6 & 1454.4 & 22.8 & 1470.1 & 26.7 & 1470.1 & 26.7 & 98.2 \\
\hline SYN14-4 run 1-- 72 & 583.0 & 28.8 & 587.2 & 30.0 & 603.5 & 92.9 & 583.0 & 28.8 & 96.6 \\
\hline SYN14-4 run 1-- 73 & 1049.4 & 24.0 & 1076.3 & 21.3 & 1131.3 & 40.5 & 1131.3 & 40.5 & 92.8 \\
\hline SYN14-4 run 1-- 74 & 1341.9 & 27.0 & 1333.1 & 20.5 & 1319.0 & 31.5 & 1319.0 & 31.5 & 101.7 \\
\hline SYN14-4 run 1-- 75 & 1708.2 & 49.8 & 1717.9 & 30.4 & 1729.7 & 29.0 & 1729.7 & 29.0 & 98.8 \\
\hline SYN14-4 run 1-- 76 & 1882.6 & 58.8 & 1895.0 & 35.2 & 1908.6 & 35.2 & 1908.6 & 35.2 & 98.6 \\
\hline SYN14-4 run 1-- 77 & 1601.4 & 60.2 & 1637.6 & 35.9 & 1684.4 & 21.8 & 1684.4 & 21.8 & 95.1 \\
\hline SYN14-4 run 1-- 78 & 2176.1 & 94.2 & 2220.3 & 48.8 & 2261.4 & 31.2 & 2261.4 & 31.2 & 96.2 \\
\hline SYN14-4 run 1-- 79 & 2629.3 & 59.9 & 2683.6 & 33.6 & 2724.7 & 36.8 & 2724.7 & 36.8 & 96.5 \\
\hline
\end{tabular}




\begin{tabular}{|c|c|c|c|c|c|c|c|c|c|}
\hline SYN14-4 run 1-- 80 & 1583.9 & 48.8 & 1615.8 & 30.7 & 1657.5 & 27.9 & 1657.5 & 27.9 & 95.6 \\
\hline SYN14-4 run 1-- 81 & 1797.3 & 71.2 & 1795.7 & 43.8 & 1793.8 & 46.3 & 1793.8 & 46.3 & 100.2 \\
\hline SYN14-4 run 1-- 82 & 1815.0 & 52.5 & 1782.5 & 30.7 & 1744.7 & 27.9 & 1744.7 & 27.9 & 104.0 \\
\hline SYN14-4 run 1-- 83 & 981.7 & 41.1 & 994.8 & 30.9 & 1023.7 & 36.8 & 1023.7 & 36.8 & 95.9 \\
\hline SYN14-4 run 1-- 85 & 388.4 & 12.0 & 386.3 & 13.0 & 373.8 & 55.9 & 388.4 & 12.0 & NA \\
\hline SYN14-4 run 1-- 86 & 1002.2 & 24.3 & 1013.7 & 20.3 & 1038.6 & 35.9 & 1038.6 & 35.9 & 96.5 \\
\hline SYN14-4 run 1-- 87 & 1268.2 & 31.5 & 1298.6 & 22.8 & 1349.1 & 28.7 & 1349.1 & 28.7 & 94.0 \\
\hline SYN14-4 run 1-- 88 & 1391.9 & 44.5 & 1436.8 & 33.3 & 1504.1 & 46.7 & 1504.1 & 46.7 & 92.5 \\
\hline SYN14-4 run 1-- 89 & 1640.3 & 43.8 & 1654.8 & 29.1 & 1673.2 & 34.6 & 1673.2 & 34.6 & 98.0 \\
\hline SYN14-4 run 1-- 90 & 380.2 & 7.5 & 381.1 & 9.2 & 386.9 & 46.9 & 380.2 & 7.5 & NA \\
\hline SYN14-4 run 1-- 91 & 1276.4 & 50.3 & 1273.2 & 34.8 & 1267.8 & 39.4 & 1267.8 & 39.4 & 100.7 \\
\hline SYN14-4 run 1-- 92 & 1902.5 & 41.9 & 1872.1 & 26.6 & 1838.4 & 32.6 & 1838.4 & 32.6 & 103.5 \\
\hline SYN14-4 run 1-- 93 & 979.7 & 36.3 & 986.1 & 30.7 & 1000.2 & 56.2 & 1000.2 & 56.2 & 97.9 \\
\hline SYN14-4 run 1-- 94 & 1229.2 & 25.7 & 1236.1 & 22.7 & 1248.2 & 42.8 & 1248.2 & 42.8 & 98.5 \\
\hline SYN14-4 run 1-- 95 & 327.0 & 12.0 & 322.5 & 14.6 & 290.4 & 84.2 & 327.0 & 12.0 & NA \\
\hline SYN14-4 run 1-- 96 & 1561.1 & 36.6 & 1597.3 & 34.1 & 1645.2 & 61.6 & 1645.2 & 61.6 & 94.9 \\
\hline SYN14-4 run 1-- 97 & 2137.2 & 57.7 & 2156.7 & 34.3 & 2175.4 & 37.6 & 2175.4 & 37.6 & 98.2 \\
\hline SYN14-4 run 1-- 98 & 1444.8 & 54.8 & 1472.9 & 42.2 & 3.6 & 64.1 & 3.6 & 64.1 & 95.5 \\
\hline SYN14-4 run 1-- 99 & 1010.8 & 24.7 & 101 & 22.8 & 2.4 & 48.2 & 1022.4 & 48.2 & 98.9 \\
\hline SYN14-4 run 1-- 100 & 1493.1 & 34.0 & 1524.8 & 24.0 & 1569.1 & 31.1 & 1569.1 & 31.1 & 95.2 \\
\hline SYN14-4 run 1-- 101 & 1116.0 & 34.6 & 1140.5 & 25.4 & 1187.5 & 30.0 & 1187.5 & 30.0 & 94.0 \\
\hline SYN14-4 run 1-- 102 & 1498.0 & 32.2 & 1485.0 & 23.7 & 1466.5 & 35.0 & 1466.5 & 35.0 & 102.1 \\
\hline SYN14-4 run 1-- 103 & 1622.9 & 41.9 & 1611.5 & 30.5 & 1596.7 & 44.6 & 1596.7 & 44.6 & 101.6 \\
\hline SYN14-4 run 1-- 104 & 1468.7 & 33.8 & 1478.7 & 24.2 & 1493.0 & 33.1 & 1493.0 & 33.1 & 98.4 \\
\hline SYN14-4 run 1-- 105 & 1049.8 & 24.5 & 1045.0 & 19.6 & 1035.1 & 32.6 & 1035.1 & 32.6 & 101.4 \\
\hline SYN14-4 run 1-- 106 & 1285.1 & 38.2 & 1302.4 & 31.0 & 1331.0 & 51.5 & 1331.0 & 51.5 & 96.5 \\
\hline SYN14-4 run 1-- 107 & 1480.1 & 41.4 & 1484.5 & 27.8 & 1490.8 & 32.0 & 1490.8 & 32.0 & 99.3 \\
\hline SYN14-4 run 1-- 108 & 1304.5 & 29.9 & 1300.5 & 22.5 & 1293.9 & 33.7 & 1293.9 & 33.7 & 100.8 \\
\hline SYN14-4 run 1-- 109 & 2681.6 & 78.5 & 2701.1 & 37.7 & 2715.8 & 29.1 & 2715.8 & 29.1 & 98.7 \\
\hline SYN14-4 run 1-- 110 & 1238.1 & 26.6 & 1222.8 & 20.3 & 1196.0 & 31.6 & 1196.0 & 31.6 & 103.5 \\
\hline SYN14-4_Run2- 163 & 301.3 & 9.1 & 327.6 & 18.1 & 519.1 & 125.2 & 301.3 & 9.1 & NA \\
\hline SYN14-4_Run2- 205 & 341.5 & 9.6 & 340.4 & 11.9 & 333.1 & 67.0 & 341.5 & 9.6 & NA \\
\hline SYN14-4_Run2- 218 & 372.7 & 15.7 & 374.7 & 20.9 & 387.2 & 114.1 & 372.7 & 15.7 & NA \\
\hline SYN14-4_Run2- 144 & 393.1 & 9.8 & 395.5 & 10.6 & 410.0 & 43.6 & 393.1 & 9.8 & NA \\
\hline SYN14-4_Run2- 116 & 405.0 & 11.0 & 405.7 & 12.2 & 409.5 & 52.5 & 405.0 & 11.0 & 98.9 \\
\hline SYN14-4_Run2- 213 & 409.9 & 9.2 & 415.1 & 12.3 & 444.3 & 61.9 & 409.9 & 9.2 & 92.2 \\
\hline SYN14-4_Run2- 200 & 413.5 & 11.5 & 411.0 & 13.4 & 397.1 & 61.6 & 413.5 & 11.5 & 104.1 \\
\hline SYN14-4_Run2- 191 & 414.8 & 10.4 & 420.7 & 13.6 & 453.2 & 66.6 & 414.8 & 10.4 & 91.5 \\
\hline SYN14-4_Run2- 139 & 420.2 & 11.2 & 429.4 & 14.3 & 479.2 & 67.0 & 420.2 & \begin{tabular}{|l|}
11.2 \\
\end{tabular} & 87.7 \\
\hline
\end{tabular}




\begin{tabular}{|c|c|c|c|c|c|c|c|c|c|}
\hline SYN14-4_Run2- 182 & 424.5 & 10.9 & 429.3 & 11.4 & 455.3 & 41.2 & 424.5 & 10.9 & 93.2 \\
\hline SYN14-4_Run2- 153 & 426.4 & 7.2 & 429.2 & 11.0 & 444.4 & 57.9 & 426.4 & 7.2 & 96.0 \\
\hline SYN14-4_Run2- 141 & 463.4 & 13.4 & 468.0 & 16.8 & 490.7 & 73.4 & 463.4 & 13.4 & 94.4 \\
\hline SYN14-4_Run2- 186 & 520.2 & 27.5 & 521.3 & 26.2 & 526.1 & 72.6 & 520.2 & 27.5 & 98.9 \\
\hline SYN14-4_Run2- 199 & 554.8 & 22.5 & 561.1 & 23.5 & 587.1 & 74.6 & 554.8 & 22.5 & 94.5 \\
\hline SYN14-4_Run2- 190 & 570.8 & 16.3 & 586.5 & 19.6 & 648.0 & 69.5 & 570.8 & 16.3 & 88.1 \\
\hline SYN14-4_Run2- 133 & 580.6 & 11.9 & 581.5 & 18.3 & 585.0 & 76.8 & 580.6 & 11.9 & 99.2 \\
\hline SYN14-4_Run2- 193 & 599.4 & 17.3 & 606.4 & 17.5 & 632.8 & 50.9 & 599.4 & 17.3 & 94.7 \\
\hline SYN14-4_Run2- 118 & 610.9 & 13.8 & 621.5 & 16.1 & 660.3 & 54.5 & 610.9 & 13.8 & 92.5 \\
\hline SYN14-4_Run2- 155 & 615.5 & 15.1 & 624.3 & 16.0 & 656.5 & 48.4 & 615.5 & 15.1 & 93.8 \\
\hline SYN14-4_Run2- 208 & 762.9 & 28.4 & 763.4 & 29.0 & 764.9 & 77.8 & 762.9 & 28.4 & 99.7 \\
\hline SYN14-4_Run2- 220 & 958.4 & 27.4 & 955.7 & 25.5 & 949.5 & 56.1 & 949.5 & 56.1 & 100.9 \\
\hline SYN14-4_Run2- 185 & 968.7 & 19.7 & 967.8 & 19.3 & 965.8 & 44.7 & 965.8 & 44.7 & 100.3 \\
\hline SYN14-4_Run2- 215 & 989.8 & 27.1 & 982.4 & 25.0 & 966.1 & 54.3 & 966.1 & 54.3 & 102.5 \\
\hline SYN14-4_Run2- 131 & 955.2 & 25.8 & 958.7 & 22.6 & 966.8 & 44.7 & 966.8 & 44.7 & 98.8 \\
\hline SYN14-4_Run2- 166 & 1019.8 & 26.6 & 1007.8 & 26.4 & 981.6 & 61.7 & 981.6 & 61.7 & 103.9 \\
\hline SYN14-4_Run2- 201 & 1033.4 & 34.8 & 1020.2 & 27.7 & 991.9 & 46.8 & 991.9 & 46.8 & 104.2 \\
\hline SYN14-4_Run2- 152 & 931.8 & 24.0 & 950.9 & 21.5 & 995.5 & 43.1 & 995.5 & 43.1 & 93.6 \\
\hline SYN14-4_Run2- 198 & 977.0 & 22.2 & 983.3 & 24.0 & 997.5 & 59.2 & 997.5 & 59.2 & 97.9 \\
\hline SYN14-4_Run2- 113 & 942.1 & 20.9 & 958.9 & 18.7 & 997.6 & 37.2 & 997.6 & 37.2 & 94.4 \\
\hline SYN14-4_Run2- 192 & 949.6 & 23.8 & 968.8 & 30.4 & 1012.7 & 82.1 & 1012.7 & 82.1 & 93.8 \\
\hline SYN14-4_Run2- 135 & 1027.1 & 25.7 & 1026.4 & 24.3 & 1024.8 & 53.0 & 1024.8 & 53.0 & 100.2 \\
\hline SYN14-4_Run2- 196 & 1001.1 & 27.7 & 1013.1 & 22.7 & 1039.0 & 38.1 & 1039.0 & 38.1 & 96.4 \\
\hline SYN14-4_Run2- 158 & 961.4 & 21.2 & 985.7 & 21.5 & 1040.0 & 49.5 & 1040.0 & 49.5 & 92.4 \\
\hline SYN14-4_Run2- 194 & 1020.8 & 43.0 & 1027.2 & 33.4 & 1040.9 & 49.1 & 1040.9 & 49.1 & 98.1 \\
\hline SYN14-4_Run2- 128 & 1008.5 & 25.4 & 1019.6 & 21.3 & 1043.3 & 38.1 & 1043.3 & 38.1 & 96.7 \\
\hline SYN14-4_Run2- 127 & 1008.2 & 21.3 & 1019.7 & 27.1 & 1044.4 & 71.5 & 1044.4 & 71.5 & 96.5 \\
\hline SYN14-4_Run2- 170 & 1065.3 & 18.8 & 1065.5 & 17.5 & 1066.1 & 37.0 & 1066.1 & 37.0 & 99.9 \\
\hline SYN14-4_Run2- 160 & 1054.0 & 27.3 & 1058.6 & 24.4 & 1068.2 & 48.7 & 1068.2 & 48.7 & 98.7 \\
\hline SYN14-4_Run2- 212 & 1027.4 & 32.2 & 1043.1 & 26.3 & 1075.9 & 43.8 & 1075.9 & 43.8 & 95.5 \\
\hline SYN14-4_Run2- 171 & 1062.4 & 27.5 & 1069.4 & 21.1 & 1083.7 & 30.3 & 1083.7 & 30.3 & 98.0 \\
\hline SYN14-4_Run2- 216 & 1042.6 & 29.4 & 1056.0 & 22.5 & 1083.9 & 31.3 & 1083.9 & 31.3 & 96.2 \\
\hline SYN14-4_Run2- 112 & 1048.9 & 41.3 & 1061.2 & 40.8 & 1086.6 & 90.0 & 1086.6 & 90.0 & 96.5 \\
\hline SYN14-4_Run2- 111 & 1023.9 & 25.9 & 1044.5 & 23.5 & 1088.1 & 47.1 & 1088.1 & 47.1 & 94.1 \\
\hline SYN14-4_Run2- 156 & 1114.0 & 28.5 & 1105.4 & 22.6 & 1088.7 & 37.3 & 1088.7 & 37.3 & 102.3 \\
\hline SYN14-4_Run2- 169 & 1081.1 & 24.1 & 1083.8 & 19.7 & 1089.2 & 33.9 & 1089.2 & 33.9 & 99.3 \\
\hline SYN14-4_Run2- 165 & 1082.3 & 24.8 & 1085.2 & 22.9 & 1090.9 & 47.5 & 1090.9 & 47.5 & 99.2 \\
\hline SYN14-4_Run2- 137 & 1018.8 & 25.9 & 1042.5 & 20.9 & 1092.5 & 32.9 & 1092.5 & 32.9 & 93.3 \\
\hline SYN14-4_Run2- 161 & 1060.1 & 26.1 & 1071.5 & 25.7 & 1094.7 & 56.5 & 1094.7 & 56.5 & 96.8 \\
\hline
\end{tabular}




\begin{tabular}{|c|c|c|c|c|c|c|c|c|c|}
\hline SYN14-4_Run2- 168 & 1005.7 & 31.6 & 1034.1 & 25.9 & 1094.7 & 41.8 & 1094.7 & 41.8 & 91.9 \\
\hline SYN14-4_Run2- 188 & 1123.5 & 27.0 & 1116.3 & 25.4 & 1102.2 & 53.7 & 1102.2 & 53.7 & 101.9 \\
\hline SYN14-4_Run2- 146 & 1057.4 & 27.7 & 1073.0 & 21.2 & 1104.9 & 29.6 & 1104.9 & 29.6 & 95.7 \\
\hline SYN14-4_Run2- 164 & 1161.0 & 26.3 & 1144.5 & 21.1 & 1113.4 & 36.3 & 1113.4 & 36.3 & 104.3 \\
\hline SYN14-4_Run2- 209 & 1122.5 & 23.0 & 1120.1 & 21.4 & 1115.5 & 44.4 & 1115.5 & 44.4 & 100.6 \\
\hline SYN14-4_Run2- 175 & 1183.6 & 33.4 & 1164.2 & 26.8 & 1128.4 & 46.2 & 1128.4 & 46.2 & 104.9 \\
\hline SYN14-4_Run2- 121 & 1054.2 & 44.4 & 1081.0 & 33.5 & 1135.5 & 42.1 & 1135.5 & 42.1 & 92.8 \\
\hline SYN14-4_Run2- 181 & 1069.8 & 21.0 & 1094.7 & 18.8 & 1144.5 & 36.3 & 1144.5 & 36.3 & 93.5 \\
\hline SYN14-4_Run2- 195 & 1127.9 & 40.0 & 1136.0 & 29.7 & 1151.5 & 39.1 & 1151.5 & 39.1 & 97.9 \\
\hline SYN14-4_Run2- 147 & 1158.0 & 38.9 & 1159.8 & 27.4 & 1163.2 & 30.0 & 1163.2 & 30.0 & 99.5 \\
\hline SYN14-4_Run2- 206 & 1131.1 & 48.8 & 1153.2 & 37.3 & 1194.9 & 52.6 & 1194.9 & 52.6 & 94.7 \\
\hline SYN14-4_Run2- 150 & 1122.5 & 36.5 & 1148.3 & 28.0 & 1197.6 & 39.5 & 1197.6 & 39.5 & 93.7 \\
\hline SYN14-4_Run2- 177 & 1222.5 & 30.1 & 1228.3 & 24.8 & 1238.6 & 43.3 & 1238.6 & 43.3 & 98.7 \\
\hline SYN14-4_Run2- 129 & 1220.4 & 17.7 & 1233.2 & 16.4 & 1255.7 & 32.3 & 1255.7 & 32.3 & 97.2 \\
\hline SYN14-4_Run2- 149 & 1283.0 & 27.7 & 1288.2 & 19.5 & 1296.7 & 23.4 & 1296.7 & 23.4 & 98.9 \\
\hline SYN14-4_Run2- 172 & 1216.6 & 39.8 & 1259.1 & 32.7 & 1332.5 & 53.8 & 1332.5 & 53.8 & 91.3 \\
\hline SYN14-4_Run2- 140 & 1356.3 & 31.5 & 1352.2 & 23.7 & 1345.7 & 35.5 & 45.7 & 35.5 & 100.8 \\
\hline SYN14-4_Run2- 167 & 1414.9 & 39.2 & 1396.5 & 26.8 & 1368.4 & 33.3 & 1368.4 & 33.3 & 103.4 \\
\hline SYN14-4_Run2- 148 & 1430.3 & 41.9 & 1408.8 & 28.1 & 6.4 & 33.0 & 6.4 & 33.0 & 103.9 \\
\hline SYN14-4_Run2-174 & 14 & 3 & & 30.6 & & & & .8 & 1.1 \\
\hline SYN14-4_Run2- 132 & 1334.0 & 3 & & 28.0 & 7.2 & 41.9 & 7.2 & .9 & 92.8 \\
\hline SYN14-4_Run2- 126 & 140 & 3 & 14 & 25.0 & 1.4 & 25.8 & 1.4 & .8 & 97.6 \\
\hline SYN14-4_Run2- 123 & 1416.6 & 34.3 & 1439.8 & 27.3 & 2 & 43.7 & 74.2 & 43.7 & 96.1 \\
\hline SYN14-4_Run2- 143 & 1294.5 & 46.0 & 1365.0 & 32.2 & 1477.2 & 32.7 & 1477.2 & 32.7 & 87.6 \\
\hline SYN14-4_Run2- 145 & 1439.2 & 40.6 & 1468.4 & 28.4 & 1510.9 & 34.8 & 1510.9 & 34.8 & 95.3 \\
\hline SYN14-4_Run2- 122 & 1502.9 & 92.9 & 1527.4 & 56.2 & 1561.4 & 29.8 & 1561.4 & 29.8 & 96.3 \\
\hline SYN14-4_Run2- 197 & 1605.6 & 49.6 & 1626.5 & 34.2 & 1653.5 & 43.7 & 1653.5 & 43.7 & 97.1 \\
\hline SYN14-4_Run2- 204 & 1601.9 & 38.5 & 1630.4 & 25.8 & 1667.4 & 30.8 & 1667.4 & 30.8 & 96.1 \\
\hline SYN14-4_Run2- 154 & 1598.2 & 27.8 & 1645.1 & 24.0 & 1705.6 & 40.4 & 1705.6 & 40.4 & 93.7 \\
\hline SYN14-4_Run2- 173 & 1750.7 & 46.6 & 1737.5 & 31.2 & 1721.8 & 40.3 & 1721.8 & 40.3 & 101.7 \\
\hline SYN14-4_Run2- 114 & 1609.7 & 29.3 & 1661.8 & 21.2 & 1728.3 & 28.9 & 1728.3 & 28.9 & 93.1 \\
\hline SYN14-4_Run2- 117 & 1638.7 & 36.1 & 1695.2 & 25.6 & 1765.8 & 33.9 & 1765.8 & 33.9 & 92.8 \\
\hline SYN14-4_Run2- 187 & 1672.4 & 36.0 & 1714.3 & 23.6 & 1766.0 & 26.6 & 1766.0 & 26.6 & 94.7 \\
\hline SYN14-4_Run2- 138 & 1769.3 & 46.0 & 1791.0 & 29.6 & 1816.3 & 34.3 & 1816.3 & 34.3 & 97.4 \\
\hline SYN14-4_Run2- 151 & 1621.7 & 37.7 & 1719.2 & 25.9 & 1840.2 & 30.5 & 1840.2 & 30.5 & 88.1 \\
\hline SYN14-4_Run2- 176 & 1814.1 & 69.3 & 1838.5 & 42.0 & 1866.2 & 41.4 & 1866.2 & 41.4 & 97.2 \\
\hline SYN14-4_Run2- 184 & 1840.3 & 41.7 & 1868.8 & 28.5 & 1900.7 & 37.6 & 1900.7 & 37.6 & 96.8 \\
\hline SYN14-4_Run2- 179 & 1852.5 & 46.0 & 1888.7 & 28.4 & 1928.8 & 29.8 & 1928.8 & 29.8 & 96.0 \\
\hline SYN14-4_Run2- 134 & 1732.9 & 45.1 & 1831.2 & 28.1 & 1944.8 & 26.4 & 1944.8 & 26.4 & 89.1 \\
\hline
\end{tabular}




\begin{tabular}{|c|c|c|c|c|c|c|c|c|c|}
\hline SYN14-4_Run2- 159 & 1457.4 & 44.9 & 1686.3 & 35.9 & 1984.1 & 47.7 & 1984.1 & 47.7 & 73.5 \\
\hline SYN14-4_Run2- 142 & 1975.8 & 58.8 & 1985.3 & 33.4 & 1995.2 & 29.4 & 1995.2 & 29.4 & 99.0 \\
\hline SYN14-4_Run2- 211 & 2054.6 & 77.7 & 2028.6 & 41.8 & 2002.3 & 31.6 & 2002.3 & 31.6 & 102.6 \\
\hline SYN14-4_Run2- 115 & 1951.9 & 34.6 & 2004.8 & 23.1 & 2059.6 & 29.2 & 2059.6 & 29.2 & 94.8 \\
\hline SYN14-4_Run2- 162 & 2440.1 & 68.6 & 2484.5 & 36.6 & 2521.0 & 34.3 & 2521.0 & 34.3 & 96.8 \\
\hline SYN14-4_Run2- 219 & 2573.6 & 45.8 & 2587.9 & 25.6 & 2599.0 & 27.9 & 2599.0 & 27.9 & 99.0 \\
\hline SYN14-4_Run2- 130 & 2716.4 & 64.2 & 2688.3 & 32.6 & 2667.2 & 31.0 & 2667.2 & 31.0 & 101.8 \\
\hline SYN14-4_Run2- 119 & 2343.5 & 59.9 & 2524.8 & 32.0 & 2674.0 & 26.3 & 2674.0 & 26.3 & 87.6 \\
\hline SYN14-4_Run2- 207 & 2580.7 & 81.6 & 2641.4 & 42.2 & 2688.2 & 38.6 & 2688.2 & 38.6 & 96.0 \\
\hline SYN14-4_Run2- 214 & 3141.7 & 81.2 & 3225.1 & 38.7 & 3277.4 & 35.7 & 3277.4 & 35.7 & 95.9 \\
\hline - SYN14-4 run 3-- 307 & 220.3 & 7.2 & 219.0 & 8.8 & 205.0 & 68.3 & 220.3 & 7.2 & NA \\
\hline - SYN14-4 run 3-- 243 & 245.9 & 8.9 & 246.6 & 9.4 & 253.0 & 51.6 & 245.9 & 8.9 & NA \\
\hline - SYN14-4 run 3-- 315 & 304.5 & 13.9 & 311.1 & 14.2 & 360.7 & 57.8 & 304.5 & 13.9 & NA \\
\hline - SYN14-4 run 3-- 311 & 341.0 & 7.9 & 341.4 & 9.6 & 344.3 & 52.6 & 341.0 & 7.9 & NA \\
\hline - SYN14-4 run 3-- 225 & 349.2 & 10.5 & 349.2 & 12.9 & 349.0 & 70.4 & 349.2 & 10.5 & NA \\
\hline - SYN14-4 run 3-- 222 & 379.0 & 11.6 & 380.8 & 12.1 & 391.7 & 48.2 & 379.0 & 11.6 & NA \\
\hline - SYN14-4 run 3-- 229 & 396.5 & 11.7 & 394.9 & 12.3 & 385.8 & 49.4 & 396.5 & 11.7 & NA \\
\hline - SYN14-4 run 3-- 319 & 398.1 & 8.5 & 391.0 & 11.0 & 349.3 & 57.8 & 398.1 & 8.5 & NA \\
\hline - SYN14-4 run 3-- 238 & 399.3 & 11.6 & 396.5 & 11.6 & 379.9 & 41.8 & 399.3 & 11.6 & NA \\
\hline - SYN14-4 run 3-- 269 & 400.0 & 16.0 & 391.7 & 15.6 & 342.9 & 55.9 & 400.0 & 16.0 & NA \\
\hline - SYN14-4 run 3-- 265 & 419.1 & 11.8 & 432.1 & 12.5 & 501.8 & 44.3 & 419.1 & 11.8 & 83.5 \\
\hline - SYN14-4 run 3-- 257 & 426.3 & 12.1 & 446.6 & 22.2 & 552.7 & 118.1 & 426.3 & 12.1 & 77.1 \\
\hline - SYN14-4 run 3-- 282 & 512.1 & 18.5 & 537.3 & 21.1 & 646.0 & 73.0 & 512.1 & 18.5 & 79.3 \\
\hline - SYN14-4 run 3-- 250 & 513.3 & 13.7 & 536.9 & 18.3 & 638.4 & 73.2 & 513.3 & 13.7 & 80.4 \\
\hline - SYN14-4 run 3-- 293 & 513.4 & 11.0 & 586.8 & 15.7 & 881.8 & 56.8 & 513.4 & 11.0 & 58.2 \\
\hline - SYN14-4 run 3-- 286 & 527.8 & 17.3 & 534.3 & 21.4 & 562.2 & 84.5 & 527.8 & 17.3 & 93.9 \\
\hline - SYN14-4 run 3-- 318 & 536.8 & 14.0 & 542.6 & 14.6 & 566.9 & 47.3 & 536.8 & 14.0 & 94.7 \\
\hline - SYN14-4 run 3-- 288 & 540.9 & 12.3 & 540.2 & 16.5 & 537.3 & 68.4 & 540.9 & 12.3 & 100.7 \\
\hline - SYN14-4 run 3-- 274 & 547.8 & 22.9 & 543.6 & 27.7 & 526.1 & 108.2 & 547.8 & 22.9 & 104.1 \\
\hline - SYN14-4 run 3-- 308 & 564.7 & 13.2 & 588.3 & 18.2 & 680.0 & 69.8 & 564.7 & 13.2 & 83.0 \\
\hline - SYN14-4 run 3-- 252 & 584.7 & 13.3 & 588.4 & 14.6 & 602.9 & 48.7 & 584.7 & 13.3 & 97.0 \\
\hline - SYN14-4 run 3-- 234 & 591.2 & 16.5 & 607.9 & 20.4 & 671.0 & 72.1 & 591.2 & 16.5 & 88.1 \\
\hline - SYN14-4 run 3-- 228 & 607.7 & 17.6 & 606.5 & 17.8 & 602.3 & 52.7 & 607.7 & 17.6 & 100.9 \\
\hline - SYN14-4 run 3-- 268 & 702.0 & 24.0 & 729.4 & 29.0 & 814.7 & 88.9 & 702.0 & 24.0 & 86.2 \\
\hline - SYN14-4 run 3-- 305 & 960.1 & 26.5 & 934.6 & 27.7 & 875.0 & 70.9 & 875.0 & 70.9 & 109.7 \\
\hline - SYN14-4 run 3-- 289 & 964.2 & 25.5 & 943.9 & 26.6 & 896.9 & 67.2 & 896.9 & 67.2 & 107.5 \\
\hline - SYN14-4 run 3-- 325 & 922.6 & 24.8 & 901.0 & 22.9 & 848.5 & 52.2 & 922.6 & 24.8 & 108.7 \\
\hline - SYN14-4 run 3-- 326 & 922.5 & 27.6 & 928.9 & 22.4 & 944.3 & 37.0 & 944.3 & 37.0 & 97.7 \\
\hline - SYN14-4 run 3-- 323 & 967.2 & 26.4 & 963.3 & 21.9 & 954.4 & 39.2 & 954.4 & 39.2 & 101.3 \\
\hline
\end{tabular}




\begin{tabular}{|c|c|c|c|c|c|c|c|c|c|}
\hline - SYN14-4 run 3-- 270 & 905.7 & 26.7 & 921.0 & 22.9 & 957.8 & 42.5 & 957.8 & 42.5 & 94.6 \\
\hline - SYN14-4 run 3-- 248 & 1018.8 & 33.6 & 1009.6 & 27.5 & 989.8 & 48.7 & 989.8 & 48.7 & 102.9 \\
\hline - SYN14-4 run 3-- 328 & 1034.9 & 36.5 & 1021.9 & 27.5 & 994.4 & 38.5 & 994.4 & 38.5 & 104.1 \\
\hline - SYN14-4 run 3-- 300 & 897.1 & 30.9 & 927.4 & 28.3 & 1000.1 & 58.0 & 1000.1 & 58.0 & 89.7 \\
\hline - SYN14-4 run 3-- 267 & 988.5 & 29.4 & 996.1 & 30.5 & 1013.1 & 72.4 & 1013.1 & 72.4 & 97.6 \\
\hline - SYN14-4 run 3-- 237 & 1024.6 & 26.4 & 1022.2 & 22.9 & 1016.8 & 44.7 & 1016.8 & 44.7 & 100.8 \\
\hline - SYN14-4 run 3-- 322 & 1057.6 & 49.5 & 1049.1 & 41.6 & 1031.4 & 77.6 & 1031.4 & 77.6 & 102.5 \\
\hline - SYN14-4 run 3-- 279 & 1031.7 & 28.8 & 1035.3 & 25.6 & 1042.8 & 50.9 & 1042.8 & 50.9 & 98.9 \\
\hline - SYN14-4 run 3-- 253 & 987.9 & 27.1 & 1007.2 & 21.7 & 1049.5 & 33.3 & 1049.5 & 33.3 & 94.1 \\
\hline - SYN14-4 run 3-- 313 & 1059.3 & 22.2 & 1056.4 & 17.7 & 1050.4 & 29.3 & 1050.4 & 29.3 & 100.8 \\
\hline - SYN14-4 run 3-- 276 & 983.2 & 20.0 & 1006.4 & 21.4 & 1057.5 & 50.9 & 1057.5 & 50.9 & 93.0 \\
\hline - SYN14-4 run 3-- 224 & 1033.5 & 30.3 & 1043.5 & 28.7 & 1064.5 & 61.4 & 1064.5 & 61.4 & 97.1 \\
\hline - SYN14-4 run 3-- 242 & 1075.5 & 20.4 & 1073.4 & 15.9 & 1069.1 & 24.9 & 1069.1 & 24.9 & 100.6 \\
\hline - SYN14-4 run 3-- 275 & 1002.5 & 23.7 & 1024.4 & 19.0 & 1071.4 & 29.6 & 1071.4 & 29.6 & 93.6 \\
\hline - SYN14-4 run 3-- 260 & 976.9 & 26.4 & 1006.8 & 26.4 & 1072.2 & 58.9 & 1072.2 & 58.9 & 91.1 \\
\hline - SYN14-4 run 3-- 310 & 1039.2 & 30.1 & 1054.4 & 24.2 & 1086.0 & 38.8 & 1086.0 & 38.8 & 95.7 \\
\hline - SYN14-4 run 3-- 240 & 990.5 & 28.8 & 1023.3 & 27.7 & 94.0 & 58.6 & 109 & 58.6 & 90.5 \\
\hline - SYN14-4 run 3-- 290 & 1088.9 & 48.6 & 1092.7 & 37.3 & 1100.5 & 55.0 & 1100.5 & 55.0 & 98.9 \\
\hline - SYN14-4 run 3-- 302 & 1151.5 & 23.3 & 1135.6 & 19.5 & 1105.2 & 36.0 & 1105.2 & 36.0 & 104.2 \\
\hline - SYN14-4 run 3-- 277 & 1033.3 & 32.0 & 1058.1 & 23.5 & 1109.4 & 25.2 & 1109.4 & 25.2 & 93.1 \\
\hline - SYN14-4 run 3-- 245 & 1121.4 & 28.9 & 1130.0 & 22.9 & 1146.6 & 36.9 & 1146.6 & 36.9 & 97.8 \\
\hline - SYN14-4 run 3-- 317 & 1152.8 & 22.5 & 1151.6 & 20.4 & 1149.5 & 40.9 & 1149.5 & 40.9 & 100.3 \\
\hline - SYN14-4 run 3-- 298 & 1128.4 & 25.8 & 1144.2 & 20.8 & 1174.3 & 34.5 & 1174.3 & 34.5 & 96.1 \\
\hline - SYN14-4 run 3-- 292 & 1114.4 & 29.1 & 1136.5 & 22.1 & 1179.0 & 30.3 & 1179.0 & 30.3 & 94.5 \\
\hline - SYN14-4 run 3-- 281 & 1060.2 & 19.3 & 1109.7 & 22.4 & 1208.0 & 52.4 & 1208.0 & 52.4 & 87.8 \\
\hline - SYN14-4 run 3-- 223 & 1144.7 & 28.1 & 1167.7 & 29.1 & 1210.6 & 63.3 & 1210.6 & 63.3 & 94.6 \\
\hline - SYN14-4 run 3-- 261 & 1130.2 & 25.8 & 1161.9 & 21.0 & 1221.4 & 34.2 & 1221.4 & 34.2 & 92.5 \\
\hline - SYN14-4 run 3-- 324 & 1215.1 & 54.8 & 1224.4 & 37.0 & 1240.8 & 31.4 & 1240.8 & 31.4 & 97.9 \\
\hline - SYN14-4 run 3-- 299 & 1185.6 & 52.3 & 1206.5 & 37.9 & 1244.2 & 45.7 & 1244.2 & 45.7 & 95.3 \\
\hline - SYN14-4 run 3-- 284 & 1184.7 & 31.3 & 1211.0 & 23.0 & 1258.1 & 29.2 & 1258.1 & 29.2 & 94.2 \\
\hline - SYN14-4 run 3-- 259 & 1355.9 & 39.1 & 1362.8 & 28.2 & 1373.6 & 38.0 & 1373.6 & 38.0 & 98.7 \\
\hline - SYN14-4 run 3-- 232 & 1282.4 & 22.9 & 1328.0 & 19.9 & 1402.4 & 35.0 & 1402.4 & 35.0 & 91.4 \\
\hline - SYN14-4 run 3-- 291 & 1349.2 & 33.3 & 1386.3 & 28.0 & 1443.8 & 47.6 & 1443.8 & 47.6 & 93.4 \\
\hline - SYN14-4 run 3-- 321 & 1463.5 & 32.0 & 1455.9 & 22.0 & 1444.8 & 27.9 & 1444.8 & 27.9 & 101.3 \\
\hline - SYN14-4 run 3-- 297 & 1342.1 & 32.8 & 1385.3 & 27.0 & 1452.6 & 44.2 & 1452.6 & 44.2 & 92.4 \\
\hline - SYN14-4 run 3-- 246 & 1369.4 & 29.7 & 1409.1 & 26.9 & 1469.7 & 49.1 & 1469.7 & 49.1 & 93.2 \\
\hline - SYN14-4 run 3-- 312 & 1349.0 & 35.1 & 1400.9 & 26.2 & 1480.8 & 35.9 & 1480.8 & 35.9 & 91.1 \\
\hline - SYN14-4 run 3-- 273 & 1452.4 & 46.4 & 1472.6 & 30.9 & 1501.8 & 33.1 & 1501.8 & 33.1 & 96.7 \\
\hline - SYN14-4 run 3-- 329 & 1434.5 & 41.6 & 1463.1 & 32.4 & 1504.9 & 49.8 & 1504.9 & 49.8 & 95.3 \\
\hline
\end{tabular}




\begin{tabular}{|c|c|c|c|c|c|c|c|c|c|}
\hline - SYN14-4 run 3-- 221 & 1473.6 & 48.2 & 1487.3 & 31.6 & 1506.7 & 32.5 & 1506.7 & 32.5 & 97.8 \\
\hline - SYN14-4 run 3-- 306 & 1443.1 & 47.5 & 1471.1 & 34.8 & 1511.6 & 48.4 & 1511.6 & 48.4 & 95.5 \\
\hline - SYN14-4 run 3-- 330 & 1638.6 & 49.6 & 1606.8 & 35.0 & 1565.3 & 49.9 & 1565.3 & 49.9 & 104.7 \\
\hline - SYN14-4 run 3-- 251 & 1625.9 & 32.3 & 1623.6 & 23.7 & 1620.6 & 34.9 & 1620.6 & 34.9 & 100.3 \\
\hline - SYN14-4 run 3-- 258 & 1526.0 & 51.1 & 56.1 & 34.4 & 1620.6 & 39.0 & 0.6 & 39.0 & 94.2 \\
\hline - SYN14-4 run 3-- 287 & 1613.0 & 33.4 & 6.9 & 23.8 & 1621.8 & 33.3 & 1.8 & 33.3 & 99.5 \\
\hline - SYN14-4 run 3-- 231 & 1668.6 & 29.4 & 1649.6 & 20.6 & 1625.6 & 28.6 & 1625.6 & 28.6 & 102.6 \\
\hline - SYN14-4 run 3-- 241 & 1622.6 & 39.8 & 1627.9 & 27.3 & 1634.8 & 35.4 & ט.דטעו & 35.4 & 99.3 \\
\hline - SYN14-4 rur & 1143.4 & 28.1 & 1326.7 & 22.7 & 1636.3 & 27.6 & 10 & 27.6 & 69.9 \\
\hline - SYN14-4 run 3-- 301 & 1541.7 & 36.0 & 1588.5 & 26.3 & 1651.2 & 36.2 & 1651.2 & 36.2 & 93.4 \\
\hline - SYN14-4 run 3-- 271 & 1626.0 & 37.3 & 1638.2 & 27.7 & 1653.8 & 41.0 & 1653.8 & 41.0 & 98.3 \\
\hline - SYN14-4 run 3-- 230 & 1599.1 & 41.5 & 1624.0 & 28.8 & 1656.4 & 37.3 & 1656.4 & 37.3 & 96.5 \\
\hline - SYN14-4 run 3-- 235 & 1609.7 & 51.8 & 1647.1 & 39.1 & 1695.1 & 57.8 & 1695.1 & 57.8 & 95.0 \\
\hline - SYN14-4 run 3-- 303 & 1615.0 & 36.8 & 1660.1 & 27.0 & 1717.5 & 37.9 & 1717.5 & 37.9 & 94.0 \\
\hline - SYN14-4 run 3-- 239 & 1685.9 & 39.8 & 1703.0 & 28.2 & 1724.2 & 38.7 & 1724.2 & 38.7 & 97.8 \\
\hline - SYN14-4 run 3-- 278 & 1768.3 & 43.3 & 2.2 & 25.9 & 33.0 & 24.4 & 3.0 & 24.4 & 102.0 \\
\hline--233 & 1840.1 & 41.3 & 1.1 & 26.9 & 4.6 & 34.8 & 6 & 34.8 & 06.1 \\
\hline - SYN14-4 run 3-- 264 & .7 & $2+2$ & 0 & 20.1 & 3.2 & 28.0 & 3.2 & 28.0 & 96.5 \\
\hline - SYN14-4 run 3-- 266 & 0 & 7 & 3 & 27.4 & .6 & 37 & .6 & 37.7 & 79.1 \\
\hline - SYN14-4 run 3-- 304 & 1.6 & 32.3 & 3.7 & 24.2 & 34.0 & 30.1 & 34.0 & 30.1 & 79.7 \\
\hline - SYN14-4 r & 1849.3 & 37.8 & 1822.9 & 24.4 & 1792.8 & 30.2 & 1792.8 & 30.2 & 103.2 \\
\hline - SYN14-4 run 3-- 272 & 1764.5 & 38.9 & 1815.4 & 26.9 & 1874.4 & 34.9 & 1874.4 & 34.9 & 94.1 \\
\hline - SYN14-4 run 3-- 226 & 1872.7 & 41.1 & 1874.9 & 26.4 & 1877.2 & 31.7 & 1877.2 & 31.7 & 99.8 \\
\hline - SYN14-4 run 3-- 236 & 1760.9 & 31.7 & 1847.0 & 25.4 & 1945.3 & 38.7 & 1945.3 & 38.7 & 90.5 \\
\hline - SYN14-4 run 3-- 262 & 1829.0 & 56.9 & 1897.5 & 36.9 & 1973.3 & 42.4 & 1973.3 & 42.4 & 92.7 \\
\hline - SYN14-4 run 3-- 244 & 1852.3 & 61.7 & 1912.1 & 39.6 & 1977.5 & 45.1 & 1977.5 & 45.1 & 93.7 \\
\hline - SYN14-4 run 3-- 254 & 1907.6 & 39.8 & 3.3 & 29.3 & 1.5 & 42.1 & 1.5 & 42.1 & 96.3 \\
\hline - SYN14-4 run 3-- 255 & 1919.4 & 60.6 & 1982.1 & 36.2 & 2048.2 & 35.0 & 2048.2 & 35.0 & 93.7 \\
\hline - SYN14-4 run 3-- 296 & 2510.1 & 42.6 & 2522.0 & 24.7 & 2531.5 & 28.3 & 2531.5 & 28.3 & 99.2 \\
\hline - SYN14-4 run 3-- 314 & 2614.7 & 63.2 & 2609.9 & 30.0 & 2606.1 & 21.2 & 2606.1 & 21.2 & 100.3 \\
\hline - SYN14-4 run 3-- 263 & 2573.2 & 56.3 & 2634.0 & 31.4 & 2681.0 & 33.6 & 2681.0 & 33.6 & 96.0 \\
\hline - SYN14-4 run 3-- 316 & 2585.1 & 46.4 & 2643.0 & 24.5 & 2687.6 & 23.5 & 2687.6 & 23.5 & 96.2 \\
\hline - SYN14-4 run 3-- 327 & 2615.8 & 62.8 & 2660.2 & 30.8 & 2694.1 & 24.5 & 2694.1 & 24.5 & 97.1 \\
\hline - SYN14-4 run 3-- 294 & 2584.3 & 78.2 & 2650.8 & 38.5 & 2702.0 & 29.5 & 2702.0 & 29.5 & 95.6 \\
\hline - SYN14-4 run 3-- 249 & 2717.7 & 119.0 & 2745.3 & 54.3 & 2765.6 & 32.6 & 2765.6 & 32.6 & 98.3 \\
\hline - SYN14-4 run 3-- 295 & 2733.5 & 56.5 & 2756.2 & 29.9 & 2772.8 & 30.6 & 2772.8 & 30.6 & 98.6 \\
\hline - SYN14-4 run 3-- 283 & 5.5 & 65.2 & 5.4 & 29.2 & 5.7 & 26.0 & 55.7 & 26.0 & 109.5 \\
\hline - SYN14-4 run 3-- 320 & 3067.1 & 62.7 & 3057.1 & 30.7 & 3050.4 & 30.0 & 3050.4 & 30.0 & 100.5 \\
\hline
\end{tabular}




\begin{tabular}{|c|c|c|c|c|c|c|c|c|c|}
\hline Analysis & 206Pb* & \pm & $207 \mathrm{~Pb}^{*}$ & \pm & $206 \mathrm{~Pb}^{*}$ & \pm & Best age & \pm & Conc \\
\hline & $238 U^{*}$ & (Ma) & $235 \mathrm{U}$ & $(\mathrm{Ma})$ & $207 \mathrm{~Pb}^{*}$ & (Ma) & (Ma) & (Ma) & $(\%)$ \\
\hline SYN14-5_Run2- 149 & 107.3 & 3.3 & 111.6 & 4.4 & 203.6 & 62.2 & 107.3 & 3.3 & NA \\
\hline SYN14-5_Run2- 196 & 219.2 & 4.6 & 219.0 & 8.3 & 216.8 & 84.3 & 219.2 & 4.6 & NA \\
\hline SYN14-5_Run2- 120 & 249.9 & 7.6 & 252.2 & 9.1 & 273.9 & 60.6 & 249.9 & 7.6 & NA \\
\hline SYN14-5_Run1- 6 & 260.0 & 4.4 & 253.2 & 5.8 & 190.8 & 45.0 & 260.0 & 4.4 & NA \\
\hline SYN14-5_Run3- 317 & 270.1 & 5.1 & 272.1 & 5.8 & 290.0 & 33.6 & 270.1 & 5.1 & NA \\
\hline SYN14-5_Run2- 155 & 290.8 & 5.8 & 302.5 & 10.0 & 393.8 & 72.7 & 290.8 & 5.8 & NA \\
\hline SYN14-5_Run2- 152 & 299.9 & 5.7 & 304.8 & 8.9 & 343.1 & 62.9 & 299.9 & 5.7 & NA \\
\hline SYN14-5_Run2- 124 & 300.5 & 5.9 & 301.4 & 9.2 & 308.2 & 65.7 & 300.5 & 5.9 & NA \\
\hline SYN14-5_Run3- 242 & 312.2 & 6.2 & 314.8 & 13.6 & 334.3 & 104.4 & 312.2 & 6.2 & NA \\
\hline SYN14-5_Run2- 180 & 321.7 & 4.6 & 326.3 & 6.6 & 359.5 & 41.7 & 321.7 & 4.6 & NA \\
\hline SYN14-5_Run1- 93 & 336.4 & 5.2 & 341.3 & 7.5 & 375.2 & 45.7 & 336.4 & 5.2 & NA \\
\hline SYN14-5_Run2- 197 & 337.2 & 4.8 & 335.6 & 5.4 & 323.9 & 27.1 & 337.2 & 4.8 & NA \\
\hline SYN14-5_Run3- 308 & 340.2 & 4.9 & 337.4 & 7.3 & 318.4 & 47.0 & 340.2 & 4.9 & NA \\
\hline SYN14-5_Run2- 199 & 344.6 & 8.5 & 337.6 & 8.2 & 290.1 & 29.8 & 344.6 & 8.5 & NA \\
\hline SYN14-5_Run1- 18 & 346.2 & 5.4 & 343.7 & 6.6 & 326.5 & 36.7 & 346.2 & 5.4 & $\mathrm{NA}$ \\
\hline SYN14-5_Run1- 74 & 362.8 & 6.4 & 367.1 & 8.2 & 394.5 & 43.8 & 362.8 & 6.4 & NA \\
\hline SYN14-5_Run3- 258 & 365.3 & 5.5 & 368.3 & 6.8 & 387.3 & 34.5 & 365.3 & 5.5 & NA \\
\hline SYN14-5_Run1- 27 & 373.2 & 4.5 & 371.0 & 6.7 & 357.5 & 39.9 & 373.2 & 4.5 & NA \\
\hline SYN14-5_Run3- 225 & 380.9 & 6.1 & 386.8 & 11.1 & 422.2 & 67.4 & 380.9 & 6.1 & NA \\
\hline SYN14-5_Run3- 221 & 384.8 & 5.8 & 389.2 & 8.0 & 415.3 & 43.4 & 384.8 & 5.8 & NA \\
\hline SYN14-5_Run2- 181 & 391.9 & 7.0 & 388.8 & 12.3 & 370.6 & 74.7 & 391.9 & 7.0 & NA \\
\hline SYN14-5_Run3- 252 & 397.2 & 7.1 & 395.9 & 9.1 & 388.2 & 46.9 & 397.2 & 7.1 & NA \\
\hline SYN14-5_Run3- 307 & 398.9 & 10.1 & 410.6 & 12.2 & 476.6 & 55.8 & 398.9 & 10.1 & NA \\
\hline SYN14-5_Run1- 2 & 405.0 & 6.9 & 403.0 & 7.3 & 391.7 & 29.9 & 405.0 & 6.9 & 103.4 \\
\hline SYN14-5_Run1- 103 & 406.7 & 5.7 & 406.0 & 8.1 & 402.1 & 43.6 & 406.7 & 5.7 & 101.1 \\
\hline SYN14-5_Run1- 52 & 409.9 & 6.8 & 410.6 & 8.0 & 414.2 & 36.4 & 409.9 & 6.8 & 99.0 \\
\hline SYN14-5_Run2- 142 & 411.5 & 8.7 & 410.1 & 10.9 & 402.2 & 53.4 & 411.5 & 8.7 & 102.3 \\
\hline SYN14-5_Run2- 177 & 413.5 & 6.3 & 413.7 & 9.3 & 415.0 & 50.3 & 413.5 & 6.3 & 99.6 \\
\hline SYN14-5_Run1- 56 & 413.8 & 16.7 & 418.2 & 16.0 & 442.6 & 47.8 & 413.8 & 16.7 & 93.5 \\
\hline SYN14-5_Run1-83 & 416.3 & 7.1 & 420.9 & 11.1 & 445.8 & 59.9 & 416.3 & 7.1 & 93.4 \\
\hline SYN14-5_Run1- 78 & 419.5 & 10.1 & 420.7 & 11.0 & 427.5 & 44.2 & 419.5 & 10.1 & 98.1 \\
\hline SYN14-5_Run3- 263 & 426.1 & 6.9 & 432.8 & 8.5 & 468.5 & 38.6 & 426.1 & 6.9 & 91.0 \\
\hline SYN14-5_Run1- 39 & 427.4 & 6.0 & 434.8 & 11.7 & 474.6 & 65.8 & 427.4 & 6.0 & 90.0 \\
\hline SYN14-5_Run3- 303 & 432.5 & 11.2 & 430.3 & 10.2 & 418.2 & 25.9 & 432.5 & 11.2 & 103.4 \\
\hline SYN14-5_Run2- 209 & 435.4 & 5.0 & 437.4 & 7.7 & 447.7 & 40.5 & 435.4 & 5.0 & 97.3 \\
\hline
\end{tabular}




\begin{tabular}{|c|c|c|c|c|c|c|c|c|c|}
\hline SYN14-5_Run2- 111 & 438.3 & 7.7 & 436.3 & 8.6 & 426.0 & 35.7 & 438.3 & 7.7 & 102.9 \\
\hline SYN14-5_Run3- 233 & 438.8 & 6.0 & 435.6 & 7.0 & 419.0 & 30.5 & 438.8 & 6.0 & 104.7 \\
\hline SYN14-5_Run1- 49 & 438.9 & 7.6 & 445.0 & 9.7 & 476.4 & 44.4 & 438.9 & 7.6 & 92.1 \\
\hline SYN14-5_Run2- 146 & 451.6 & 10.9 & 453.0 & 10.5 & 460.3 & 31.3 & 451.6 & 10.9 & 98.1 \\
\hline SYN14-5_Run3- 329 & 453.5 & 6.0 & 463.9 & 6.3 & 515.5 & 21.9 & 453.5 & 6.0 & 88.0 \\
\hline SYN14-5_Run2- 200 & 459.5 & 8.6 & 460.7 & 13.6 & 466.5 & 69.0 & 459.5 & 8.6 & 98.5 \\
\hline SYN14-5_Run3- 246 & 461.0 & 7.3 & 468.4 & 17.6 & 504.8 & 96.8 & 461.0 & 7.3 & 91.3 \\
\hline SYN14-5_Run3- 248 & 462.1 & 9.5 & 463.2 & 10.2 & 468.6 & 37.7 & 462.1 & 9.5 & 98.6 \\
\hline SYN14-5_Run1- 38 & 469.9 & 5.0 & 472.3 & 7.4 & 484.1 & 35.5 & 469.9 & 5.0 & 97.1 \\
\hline SYN14-5_Run2- 169 & 472.9 & 6.2 & 473.6 & 9.9 & 476.9 & 49.6 & 472.9 & 6.2 & 99.1 \\
\hline SYN14-5_Run2- 114 & 521.1 & 8.7 & 524.5 & 9.4 & 539.3 & 33.0 & 521.1 & 8.7 & 96.6 \\
\hline SYN14-5_Run2- 204 & 524.8 & 9.5 & 528.2 & 20.3 & 542.6 & 99.3 & 524.8 & 9.5 & 96.7 \\
\hline SYN14-5_Run3- 247 & 539.5 & 9.3 & 541.1 & 11.0 & 547.7 & 41.6 & 539.5 & 9.3 & 98.5 \\
\hline SYN14-5_Run3- 319 & 540.6 & 8.1 & 543.5 & 19.4 & 555.6 & 94.9 & 540.6 & 8.1 & 97.3 \\
\hline SYN14-5_Run3- 323 & 546.6 & 10.6 & 551.7 & 13.5 & 572.9 & 53.5 & 546.6 & 10.6 & 95.4 \\
\hline SYN14-5_Run3- 253 & 562.2 & 11.4 & 561.1 & 19.5 & 556.4 & 87.3 & 562.2 & 11.4 & 101.1 \\
\hline SYN14-5_Run1- 29 & 567.5 & 12.1 & 575.1 & 13.0 & 604.9 & 42.3 & 567.5 & 12.1 & 93.8 \\
\hline SYN14-5_Run1- 96 & 568.9 & 7.6 & 575.7 & 12.5 & 602.6 & 53.8 & 568.9 & 7.6 & 94.4 \\
\hline SYN14-5_Run2- 137 & 577.7 & 8.1 & 577.0 & 9.2 & 574.1 & 32.6 & 577.7 & 8.1 & 100.6 \\
\hline SYN14-5_Run2- 194 & 578.4 & 8.0 & 600.7 & 11.4 & 685.7 & 43.9 & 578.4 & 8.0 & 84.4 \\
\hline SYN14-5_Run2- 208 & 582.9 & 7.5 & 594.3 & 9.1 & 638.3 & 32.7 & 582.9 & 7.5 & 91.3 \\
\hline SYN14-5_Run1- 10 & 586.2 & 13.9 & 590.7 & 18.8 & 608.3 & 73.4 & 586.2 & 13.9 & 96.4 \\
\hline SYN14-5_Run1- 51 & 594.6 & 9.0 & 607.3 & 12.6 & 654.8 & 48.7 & 594.6 & 9.0 & 90.8 \\
\hline SYN14-5_Run3- 298 & 599.0 & 13.0 & 605.7 & 15.9 & 630.8 & 57.2 & 599.0 & 13.0 & 95.0 \\
\hline SYN14-5_Run2- 160 & 599.6 & 12.0 & 603.7 & 22.2 & 619.1 & 94.9 & 599.6 & 12.0 & 96.8 \\
\hline SYN14-5_Run1- 72 & 603.7 & 9.9 & 604.5 & 12.1 & 607.6 & 44.1 & 603.7 & 9.9 & 99.3 \\
\hline SYN14-5_Run3- 288 & 604.0 & 22.0 & 618.1 & 20.8 & 670.1 & 50.4 & 604.0 & 22.0 & 90.1 \\
\hline SYN14-5_Run2- 182 & 609.2 & 13.0 & 606.0 & 15.7 & 593.9 & 56.9 & 609.2 & 13.0 & 102.6 \\
\hline SYN14-5_Run2- 121 & 609.7 & 12.1 & 619.5 & 13.5 & 655.6 & 43.9 & 609.7 & 12.1 & 93.0 \\
\hline SYN14-5_Run3- 293 & 611.3 & 7.5 & 611.6 & 10.0 & 613.0 & 37.7 & 611.3 & 7.5 & 99.7 \\
\hline SYN14-5_Run1- 22 & 615.0 & 8.6 & 616.7 & 10.3 & 622.7 & 36.3 & 615.0 & 8.6 & 98.8 \\
\hline SYN14-5_Run2- 143 & 615.1 & 12.8 & 636.9 & 15.2 & 715.2 & 50.3 & 615.1 & 12.8 & 86.0 \\
\hline SYN14-5_Run3- 243 & 617.7 & 12.1 & 614.7 & 18.9 & 603.8 & 76.4 & 617.7 & 12.1 & 102.3 \\
\hline SYN14-5_Run3- 268 & 620.0 & 9.3 & 624.9 & 9.8 & 642.5 & 29.9 & 620.0 & 9.3 & 96.5 \\
\hline SYN14-5_Run3- 229 & 626.9 & 10.6 & 622.2 & 11.2 & 605.4 & 34.8 & 626.9 & 10.6 & 103.5 \\
\hline SYN14-5_Run3- 235 & 627.5 & 12.8 & 643.1 & 20.8 & 698.3 & 81.1 & 627.5 & 12.8 & 89.9 \\
\hline SYN14-5_Run3- 227 & 629.6 & 14.5 & 638.9 & 14.2 & 671.9 & 37.6 & 629.6 & 14.5 & 93.7 \\
\hline SYN14-5_Run2- 186 & 633.2 & 11.2 & 641.8 & 12.9 & 672.2 & 42.0 & 633.2 & 11.2 & 94.2 \\
\hline SYN14-5_Run2- 165 & 646.7 & 9.5 & 643.6 & 12.4 & 633.1 & 44.9 & 646.7 & 9.5 & 102.1 \\
\hline
\end{tabular}




\begin{tabular}{|c|c|c|c|c|c|c|c|c|c|}
\hline SYN14-5_Run1- 8 & 924.0 & 15.5 & 921.1 & 19.6 & 914.2 & 55.6 & 914.2 & 55.6 & 101.1 \\
\hline SYN14-5_Run3- 286 & 925.7 & 16.6 & 925.3 & 15.5 & 924.5 & 34.3 & 924.5 & 34.3 & 100.1 \\
\hline SYN14-5_Run1- 107 & 884.3 & 14.1 & 896.8 & 14.9 & 928.0 & 37.8 & 928.0 & 37.8 & 95.3 \\
\hline SYN14-5_Run1- 86 & 912.1 & 12.4 & 918.5 & 14.8 & 933.9 & 40.2 & 933.9 & 40.2 & 97.7 \\
\hline SYN14-5_Run1- 101 & 910.8 & 15.2 & 918.8 & 18.7 & 938.1 & 51.7 & 938.1 & 51.7 & 97.1 \\
\hline SYN14-5_Run3- 321 & 952.6 & 18.8 & 954.1 & 20.9 & 957.6 & 53.8 & 957.6 & 53.8 & 99.5 \\
\hline SYN14-5_Run2- 207 & 947.8 & 19.9 & 953.4 & 19.8 & 966.3 & 46.6 & 966.3 & 46.6 & 98.1 \\
\hline SYN14-5_Run3- 278 & 966.0 & 9.8 & 967.2 & 16.2 & 970.0 & 48.1 & 970.0 & 48.1 & 99.6 \\
\hline SYN14-5_Run3- 249 & 958.5 & 12.9 & 962.2 & 11.9 & 970.7 & 25.7 & 970.7 & 25.7 & 98.7 \\
\hline SYN14-5_Run2- 214 & 1001.6 & 18.1 & 998.7 & 18.5 & 992.3 & 43.9 & 992.3 & 43.9 & 100.9 \\
\hline SYN14-5_Run1- 94 & 1013.8 & 20.1 & 1008.8 & 27.3 & 998.1 & 75.0 & 998.1 & 75.0 & 101.6 \\
\hline SYN14-5_Run1- 45 & 1028.6 & 16.9 & 1019.1 & 16.9 & 998.7 & 39.3 & 998.7 & 39.3 & 103.0 \\
\hline SYN14-5_Run2- 192 & 937.0 & 13.6 & 956.0 & 18.1 & 999.9 & 50.3 & 999.9 & 50.3 & 93.7 \\
\hline SYN14-5_Run1- 110 & 970.1 & 12.3 & 979.3 & 14.5 & 999.9 & 37.8 & 999.9 & 37.8 & 97.0 \\
\hline SYN14-5_Run2- 151 & 1026.6 & 11.6 & 1019.1 & 14.4 & 1002.9 & 38.0 & 1002.9 & 38.0 & 102.4 \\
\hline SYN14-5_Run2- 113 & 993.1 & 15.0 & 997.3 & 15.2 & 1006.3 & 35.5 & 1006.3 & 35.5 & 98.7 \\
\hline SYN14-5_Run3- 271 & 1014.2 & 16.0 & 1011.9 & 14.6 & 1006.9 & 30.8 & 1006.9 & 30.8 & 100.7 \\
\hline SYN14-5_Run1- 41 & 999.2 & 13.7 & 1001.7 & 12.0 & 1007.0 & 23.7 & 1007.0 & 23.7 & 99.2 \\
\hline SYN14-5_Run2- 156 & 967.3 & 13.6 & 979.8 & 17.5 & 1007.8 & 47.3 & 1007.8 & 47.3 & 96.0 \\
\hline SYN14-5_Run3- 230 & 1008.5 & 15.1 & 1008.9 & 18.1 & 1009.7 & 47.3 & 1009.7 & 47.3 & 99.9 \\
\hline SYN14-5_Run3- 257 & 970.5 & 15.3 & 982.7 & 13.5 & 1009.9 & 26.4 & 1009.9 & 26.4 & 96.1 \\
\hline SYN14-5_Run3- 295 & 1031.2 & 16.5 & 1026.2 & 15.1 & 1015.4 & 31.9 & 1015.4 & 31.9 & 101.6 \\
\hline SYN14-5_Run1-98 & 997.2 & 15.6 & 1003.4 & 14.7 & 1016.7 & 31.8 & 1016.7 & 31.8 & 98.1 \\
\hline SYN14-5_Run2- 154 & 1030.0 & 14.2 & 1025.9 & 17.0 & 1017.2 & 43.9 & 1017.2 & 43.9 & 101.3 \\
\hline SYN14-5_Run1- 28 & 1017.0 & 22.1 & 1017.2 & 20.0 & 1017.6 & 41.5 & 1017.6 & 41.5 & 99.9 \\
\hline SYN14-5_Run1- 109 & 967.7 & 12.6 & 983.2 & 15.9 & 1018.0 & 42.5 & 1018.0 & 42.5 & 95.1 \\
\hline SYN14-5_Run1- 36 & 1018.8 & 18.5 & 1018.6 & 14.9 & 1018.1 & 25.0 & 1018.1 & 25.0 & 100.1 \\
\hline SYN14-5_Run3- 239 & 1024.4 & 15.7 & 1022.7 & 13.1 & 1019.1 & 23.7 & 1019.1 & 23.7 & 100.5 \\
\hline SYN14-5_Run1- 88 & 1000.8 & 17.5 & 1006.8 & 15.8 & 1019.8 & 32.1 & 1019.8 & 32.1 & 98.1 \\
\hline SYN14-5_Run1- 61 & 939.9 & 18.5 & 964.4 & 18.5 & 1020.7 & 42.2 & 1020.7 & 42.2 & 92.1 \\
\hline SYN14-5_Run1- 55 & 894.8 & 21.3 & 932.1 & 24.0 & 1021.4 & 61.0 & 1021.4 & 61.0 & 87.6 \\
\hline SYN14-5_Run2- 117 & 914.9 & 28.8 & 946.8 & 23.6 & 1021.7 & 36.6 & 1021.7 & 36.6 & 89.6 \\
\hline SYN14-5_Run3- 305 & 1003.4 & 11.7 & 1009.2 & 12.7 & 1021.7 & 30.9 & 1021.7 & 30.9 & 98.2 \\
\hline SYN14-5_Run1-80 & 1019.9 & 16.5 & 1021.2 & 13.6 & 1024.0 & 24.4 & 1024.0 & 24.4 & 99.6 \\
\hline SYN14-5_Run1- 104 & 1027.7 & 14.6 & 1026.8 & 11.1 & 1024.8 & 15.5 & 1024.8 & 15.5 & 100.3 \\
\hline SYN14-5_Run2- 112 & 936.9 & 15.2 & 963.5 & 13.8 & 1024.8 & 27.9 & 1024.8 & 27.9 & 91.4 \\
\hline SYN14-5_Run2- 157 & 1025.4 & 12.1 & 1025.6 & 13.4 & 1025.9 & 33.0 & 1025.9 & 33.0 & 99.9 \\
\hline SYN14-5_Run1- 63 & 1048.8 & 20.8 & 1041.5 & 16.5 & 1026.1 & 27.2 & 1026.1 & 27.2 & 102.2 \\
\hline SYN14-5_Run3- 292 & 1046.0 & 21.2 & 1039.7 & 19.6 & 1026.4 & 41.7 & 1026.4 & 41.7 & 101.9 \\
\hline
\end{tabular}




\begin{tabular}{|c|c|c|c|c|c|c|c|c|c|}
\hline SYN14-5_Run2- 139 & 1021.5 & 14.9 & 1024.1 & 15.2 & 1029.8 & 35.3 & 1029.8 & 35.3 & 99.2 \\
\hline SYN14-5_Run3- 316 & 1050.1 & 15.8 & 1043.9 & 16.8 & 1030.7 & 40.3 & 1030.7 & 40.3 & 101.9 \\
\hline SYN14-5_Run3- 232 & 1076.8 & 17.9 & 1062.4 & 15.5 & 1032.8 & 30.7 & 1032.8 & 30.7 & 104.3 \\
\hline SYN14-5_Run1- 9 & 1006.3 & 15.8 & 1015.7 & 15.1 & 1036.2 & 33.1 & 1036.2 & 33.1 & 97.1 \\
\hline SYN14-5_Run1- 7 & 985.3 & 16.1 & 1001.5 & 15.2 & 1037.1 & 32.7 & 1037.1 & 32.7 & 95.0 \\
\hline SYN14-5_Run3- 313 & 1023.0 & 18.3 & 1027.9 & 14.4 & 1038.3 & 22.0 & 1038.3 & 22.0 & 98.5 \\
\hline SYN14-5_Run3- 277 & 1047.9 & 15.3 & 1045.0 & 12.2 & 1039.0 & 20.1 & 1039.0 & 20.1 & 100.9 \\
\hline SYN14-5_Run1- 48 & 1031.3 & 11.2 & 1034.5 & 12.8 & 1041.3 & 32.0 & 1041.3 & 32.0 & 99.0 \\
\hline SYN14-5_Run1- 75 & 1073.3 & 19.0 & 1063.0 & 21.1 & 1042.0 & 51.5 & 1042.0 & 51.5 & 103.0 \\
\hline SYN14-5_Run2- 144 & 990.8 & 11.2 & 1007.0 & 13.9 & 1042.6 & 36.2 & 1042.6 & 36.2 & 95.0 \\
\hline SYN14-5_Run1- 17 & 1054.9 & 29.8 & 1051.0 & 22.8 & 1043.0 & 33.8 & 1043.0 & 33.8 & 101.1 \\
\hline SYN14-5_Run2- 170 & 1017.4 & 16.0 & 1026.0 & 14.9 & 1044.3 & 31.4 & 1044.3 & 31.4 & 97.4 \\
\hline SYN14-5_Run2- 135 & 1008.8 & 13.2 & 1020.3 & 13.6 & 1045.0 & 31.8 & 1045.0 & 31.8 & 96.5 \\
\hline SYN14-5_Run1- 59 & 984.4 & 12.1 & 1003.4 & 11.3 & 1045.1 & 23.6 & 1045.1 & 23.6 & 94.2 \\
\hline SYN14-5_Run2- 134 & 1004.3 & 30.8 & 1017.4 & 22.6 & 1045.6 & 24.2 & 1045.6 & 24.2 & 96.0 \\
\hline SYN14-5_Run1-13 & 1038.1 & 18.9 & 1040.6 & 20.2 & 1045.9 & 48.4 & 1045.9 & 48.4 & 99.2 \\
\hline SYN14-5_Run2- 187 & 1032.0 & 16.1 & 1036.9 & 20.7 & 1047.2 & 54.6 & 1047.2 & 54.6 & 98.6 \\
\hline SYN14-5_Run3- 241 & 982.0 & 15.9 & 1002.4 & 15.0 & 1047.3 & 32.1 & 1047.3 & 32.1 & 93.8 \\
\hline SYN14-5_Run1- 69 & 1037.9 & 19.3 & 1041.2 & 16.1 & 1048.1 & 28.7 & 1048.1 & 28.7 & 99.0 \\
\hline SYN14-5_Run3- 228 & 1014.4 & 19.1 & 1028.1 & 16.2 & 1057.4 & 29.5 & 1057.4 & 29.5 & 95.9 \\
\hline SYN14-5_Run3- 275 & 1076.0 & 14.1 & 1069.9 & 13.4 & 1057.5 & 28.9 & 1057.5 & 28.9 & 101.7 \\
\hline SYN14-5_Run3- 245 & 1022.5 & 17.8 & 1033.7 & 15.7 & 1057.5 & 30.7 & 1057.5 & 30.7 & 96.7 \\
\hline SYN14-5_Run1- 92 & 1022.2 & 38.6 & 1033.7 & 27.9 & 1058.2 & 27.3 & 1058.2 & 27.3 & 96.6 \\
\hline SYN14-5_Run2- 211 & 1049.0 & 19.2 & 1052.2 & 18.6 & 1058.8 & 40.7 & 1058.8 & 40.7 & 99.1 \\
\hline SYN14-5_Run2- 179 & 995.3 & 14.5 & 1015.6 & 18.6 & 1059.6 & 49.0 & 1059.6 & 49.0 & 93.9 \\
\hline SYN14-5_Run1- 1 & 1100.0 & 13.1 & 1086.9 & 11.1 & 1060.8 & 21.2 & 1060.8 & 21.2 & 103.7 \\
\hline SYN14-5_Run1- 47 & 1056.3 & 16.2 & 1057.8 & 19.1 & 1061.0 & 48.1 & 1061.0 & 48.1 & 99.6 \\
\hline SYN14-5_Run3- 315 & 1020.5 & 21.4 & 1033.5 & 19.3 & 1061.2 & 39.1 & 1061.2 & 39.1 & 96.2 \\
\hline SYN14-5_Run1- 85 & 1054.3 & 15.1 & 1056.9 & 13.2 & 1062.3 & 25.7 & 1062.3 & 25.7 & 99.2 \\
\hline SYN14-5_Run3- 238 & 1054.3 & 15.7 & 1057.7 & 12.7 & 1064.8 & 21.3 & 1064.8 & 21.3 & 99.0 \\
\hline SYN14-5_Run3- 310 & 993.8 & 22.1 & 1016.8 & 20.6 & 1066.7 & 42.7 & 1066.7 & 42.7 & 93.2 \\
\hline SYN14-5_Run1- 91 & 1040.7 & 13.5 & 1050.0 & 13.4 & 1069.3 & 29.8 & 1069.3 & 29.8 & 97.3 \\
\hline SYN14-5_Run1- 33 & 1045.0 & 16.8 & 1054.8 & 13.6 & 1075.2 & 22.3 & 1075.2 & 22.3 & 97.2 \\
\hline SYN14-5_Run2- 163 & 1021.3 & 22.1 & 1038.7 & 25.5 & 1075.6 & 63.4 & 1075.6 & 63.4 & 95.0 \\
\hline SYN14-5_Run1- 20 & 1030.3 & 17.5 & 1045.1 & 17.1 & 1076.2 & 37.4 & 1076.2 & 37.4 & 95.7 \\
\hline SYN14-5_Run3- 260 & 1039.9 & 19.1 & 1053.1 & 17.0 & 1080.7 & 33.5 & 1080.7 & 33.5 & 96.2 \\
\hline SYN14-5_Run1- 35 & 1014.0 & 14.6 & 1036.2 & 14.1 & 1083.5 & 30.3 & 1083.5 & 30.3 & 93.6 \\
\hline SYN14-5_Run2- 220 & 1021.3 & 34.1 & 1041.4 & 28.2 & 1083.9 & 48.0 & 1083.9 & 48.0 & 94.2 \\
\hline SYN14-5_Run2- 171 & 1078.6 & 21.1 & 1080.5 & 16.0 & 1084.3 & 22.8 & 1084.3 & 22.8 & 99.5 \\
\hline
\end{tabular}




\begin{tabular}{|c|c|c|c|c|c|c|c|c|c|}
\hline SYN14-5_Run2- 203 & 1040.3 & 40.5 & 1054.6 & 32.1 & 1084.3 & 49.7 & 1084.3 & 49.7 & 95.9 \\
\hline SYN14-5_Run1- 60 & 1043.5 & 18.5 & 1058.5 & 21.0 & 1089.7 & 51.2 & 1089.7 & 51.2 & 95.8 \\
\hline SYN14-5_Run3- 291 & 1051.3 & 15.2 & 1063.9 & 14.8 & 1089.9 & 32.1 & 1089.9 & 32.1 & 96.5 \\
\hline SYN14-5_Run2- 115 & 1077.9 & 22.7 & 1082.7 & 16.5 & 1092.4 & 19.1 & 1092.4 & 19.1 & 98.7 \\
\hline SYN14-5_Run3- 266 & 1041.7 & 14.1 & 1058.7 & 11.9 & 1093.8 & 20.9 & 1093.8 & 20.9 & 95.2 \\
\hline SYN14-5_Run2- 132 & 1090.5 & 14.5 & 1092.3 & 13.6 & 1095.8 & 28.6 & 1095.8 & 28.6 & 99.5 \\
\hline SYN14-5_Run2- 150 & 1059.4 & 15.0 & 1071.9 & 13.5 & 1097.4 & 26.7 & 1097.4 & 26.7 & 96.5 \\
\hline SYN14-5_Run2- 162 & 1040.5 & 13.8 & 1059.1 & 20.2 & 1097.7 & 54.3 & 1097.7 & 54.3 & 94.8 \\
\hline SYN14-5_Run3- 322 & 1037.4 & 22.1 & 1057.2 & 21.3 & 1098.5 & 45.6 & 1098.5 & 45.6 & 94.4 \\
\hline SYN14-5_Run3- 234 & 1055.2 & 17.6 & 1070.5 & 19.3 & 1101.8 & 45.6 & 1101.8 & 45.6 & 95.8 \\
\hline SYN14-5_Run2- 145 & 1057.5 & 22.2 & 1072.5 & 21.2 & 1103.0 & 45.1 & 1103.0 & 45.1 & 95.9 \\
\hline SYN14-5_Run3- 222 & 1076.2 & 15.7 & 1086.4 & 13.7 & 1106.8 & 26.0 & 1106.8 & 26.0 & 97.2 \\
\hline SYN14-5_Run1- 50 & 1043.1 & 33.2 & 1064.0 & 26.3 & 1107.0 & 40.1 & 1107.0 & 40.1 & 94.2 \\
\hline SYN14-5_Run3- 240 & 1098.0 & 19.2 & 1101.4 & 16.6 & 1108.0 & 31.7 & 1108.0 & 31.7 & 99.1 \\
\hline SYN14-5_Run3- 296 & 1077.0 & 20.4 & 1088.5 & 22.6 & 1111.6 & 53.7 & 1111.6 & 53.7 & 96.9 \\
\hline SYN14-5_Run2- 198 & 1125.5 & 12.0 & 1122.4 & 18.8 & 1116.5 & 49.9 & 1116.5 & 49.9 & 100.8 \\
\hline SYN14-5_Run3- 284 & 1116.2 & 18.5 & 1116.9 & 18.4 & 1118.2 & 40.4 & 1118.2 & 40.4 & 99.8 \\
\hline SYN14-5_Run3- 281 & 11 & 22.1 & 1135.2 & 19.9 & 1121.5 & 40.0 & 1121.5 & 40.0 & 101.9 \\
\hline SYN14-5_Run2- 188 & 1100.5 & 20.1 & 11 & 18.3 & 2.5 & 36.9 & .5 & 36.9 & 98.0 \\
\hline SYN14-5_R & 10 & 2 & 3 & 21.7 & 1 & 4 & .1 & 8 & .6 \\
\hline SYN14-5_Run1- 53 & 10 & 15 & 7 & 15.0 & .0 & 31.6 & 1127.0 & 31.6 & 94.0 \\
\hline SYN14-5_Run3- 267 & 10 & 13.0 & 11 & 12.6 & 7.5 & 26.9 & 1127.5 & 26.9 & 97.0 \\
\hline SYN14-5_Run1- 57 & 998.7 & 12.7 & 1041.0 & 11.9 & 1131.1 & 23.8 & 1131.1 & 23.8 & 88.3 \\
\hline SYN14-5_Run1- 102 & 1094.4 & 16.5 & 1108.1 & 13.8 & 1135.2 & 24.3 & 1135.2 & 24.3 & 96.4 \\
\hline SYN14-5_Run2- 158 & 1051.5 & 13.6 & 1079.8 & 17.4 & 1137.4 & 44.1 & 1137.4 & 44.1 & 92.5 \\
\hline SYN14-5_Run2- 118 & 1150.7 & 21.0 & 1147.0 & 15.6 & 1140.2 & 21.5 & 1140.2 & 21.5 & 100.9 \\
\hline SYN14-5_Run1- 67 & 1088.2 & 18.5 & 1106.4 & 17.5 & 1142.3 & 36.1 & 1142.3 & 36.1 & 95.3 \\
\hline SYN14-5_Run1- 64 & 1155.9 & 19.9 & 1151.3 & 15.7 & 1142.6 & 25.5 & 1142.6 & 25.5 & 101.2 \\
\hline SYN14-5_Run2- 216 & 1016.5 & 16.1 & 1058.2 & 23.6 & 1145.0 & 62.8 & 1145.0 & 62.8 & 88.8 \\
\hline SYN14-5_Run1- 82 & 1158.6 & 12.8 & 1157.0 & 12.1 & 1154.0 & 25.0 & 1154.0 & 25.0 & 100.4 \\
\hline SYN14-5_Run3- 289 & 1105.4 & 18.7 & 1122.8 & 15.6 & 1156.6 & 27.3 & 1156.6 & 27.3 & 95.6 \\
\hline SYN14-5_Run2- 184 & 944.4 & 12.5 & 1011.4 & 11.8 & 1159.3 & 23.2 & 1159.3 & 23.2 & 81.5 \\
\hline SYN14-5_Run3- 326 & 1181.2 & 15.8 & 1173.8 & 12.1 & 1160.1 & 18.6 & 1160.1 & 18.6 & 101.8 \\
\hline SYN14-5_Run2- 215 & 1155.5 & 12.2 & 1158.6 & 10.9 & 1164.3 & 21.1 & 1164.3 & 21.1 & 99.2 \\
\hline SYN14-5_Run1- 16 & 1116.6 & 16.2 & 1134.0 & 13.9 & 1167.6 & 25.3 & 1167.6 & 25.3 & 95.6 \\
\hline SYN14-5_Run2- 189 & 1118.6 & 27.2 & 1135.8 & 19.8 & 1168.7 & 22.9 & 1168.7 & 22.9 & 95.7 \\
\hline SYN14-5_Run3- 330 & 1103.6 & 41.2 & 1126.5 & 32.6 & 1170.8 & 49.8 & 1170.8 & 49.8 & 94.3 \\
\hline SYN14-5_Run2- 147 & 1127.9 & 13.4 & 1142.7 & 15.5 & 1171.0 & 36.8 & 1171.0 & 36.8 & 96.3 \\
\hline SYN14-5_Run1- 24 & 1155.5 & 19.8 & 1161.4 & 25.0 & 1172.6 & 61.1 & 1172.6 & 61.1 & 98.5 \\
\hline
\end{tabular}




\begin{tabular}{|c|c|c|c|c|c|c|c|c|c|}
\hline SYN14-5_Run2- 178 & 1157.4 & 14.5 & 1163.4 & 15.2 & 1174.6 & 34.1 & 1174.6 & 34.1 & 98.5 \\
\hline SYN14-5_Run1- 5 & 1154.8 & 18.5 & 1164.9 & 24.2 & 1183.9 & 59.7 & 1183.9 & 59.7 & 97.5 \\
\hline SYN14-5_Run3- 306 & 1128.1 & 41.8 & 1149.5 & 31.2 & 1190.2 & 40.6 & 1190.2 & 40.6 & 94.8 \\
\hline SYN14-5_Run1- 81 & 1146.2 & 22.4 & 1162.3 & 20.5 & 1192.3 & 40.6 & 1192.3 & 40.6 & 96.1 \\
\hline SYN14-5_Run1- 77 & 1147.0 & 14.4 & 1164.3 & 13.3 & 1196.8 & 26.4 & 1196.8 & 26.4 & 95.8 \\
\hline SYN14-5_Run1- 79 & 1119.2 & 24.2 & 1146.2 & 20.5 & 1197.9 & 35.9 & 1197.9 & 35.9 & 93.4 \\
\hline SYN14-5_Run2- 168 & 1166.7 & 27.0 & 1178.1 & 24.9 & 1199.2 & 49.6 & 1199.2 & 49.6 & 97.3 \\
\hline SYN14-5_Run3- 259 & 1209.0 & 13.2 & 1205.8 & 11.0 & 1199.9 & 19.9 & 1199.9 & 19.9 & 100.8 \\
\hline SYN14-5_Run1- 70 & 1169.1 & 27.8 & 1181.8 & 21.9 & 1205.2 & 34.3 & 1205.2 & 34.3 & 97.0 \\
\hline SYN14-5_Run3- 251 & 1239.2 & 20.6 & 1227.6 & 17.4 & 1207.4 & 32.1 & 1207.4 & 32.1 & 102.6 \\
\hline SYN14-5_Run1- 84 & 1059.8 & 39.1 & 1113.0 & 29.6 & 1218.4 & 35.2 & 1218.4 & 35.2 & 87.0 \\
\hline SYN14-5_Run3- 324 & 1126.1 & 26.8 & 1159.8 & 26.0 & 1223.5 & 53.3 & 1223.5 & 53.3 & 92.0 \\
\hline SYN14-5_Run1- 30 & 1189.4 & 18.3 & 1207.3 & 16.5 & 1239.4 & 31.7 & 1239.4 & 31.7 & 96.0 \\
\hline SYN14-5_Run3- 309 & 1207.5 & 18.5 & 1222.0 & 21.4 & 1247.8 & 49.0 & 1247.8 & 49.0 & 96.8 \\
\hline SYN14-5_Run1- 46 & 1220.5 & 15.9 & 1231.3 & 14.7 & 1250.2 & 29.0 & 1250.2 & 29.0 & 97.6 \\
\hline SYN14-5_Run3- 285 & 1190.0 & 25.7 & 1213.2 & 21.9 & 1254.8 & 39.0 & 1254.8 & 39.0 & 94.8 \\
\hline SYN14-5_Run3- 265 & 1245.6 & 14.7 & 1251.5 & 12.7 & 1261.7 & 23.4 & 1261.7 & 23.4 & 98.7 \\
\hline SYN14-5_Run3- 302 & 1254.4 & 19.2 & 1260.1 & 15.0 & 1270.0 & 23.5 & 1270.0 & 23.5 & 98.8 \\
\hline SYN14-5_Run2- 173 & 1256.6 & 26.4 & 1263.2 & 19.3 & 1274.5 & 26.1 & 1274.5 & 26.1 & 98.6 \\
\hline SYN14-5_Run2- 183 & 1235.5 & 16.5 & 1269.9 & 14.5 & 1328.8 & 26.2 & 1328.8 & 26.2 & 93.0 \\
\hline SYN14-5_Run1- 12 & 1362.5 & 15.6 & 1350.8 & 17.2 & 1332.3 & 37.3 & 1332.3 & 37.3 & 102.3 \\
\hline SYN14-5_Run3- 256 & 1314.3 & 24.2 & 1322.3 & 17.9 & 1335.2 & 25.2 & 1335.2 & 25.2 & 98.4 \\
\hline SYN14-5_Run1- 71 & 1274.3 & 16.3 & 1297.3 & 13.7 & 1335.7 & 23.8 & 1335.7 & 23.8 & 95.4 \\
\hline SYN14-5_Run1- 62 & 1306.8 & 32.6 & 1318.5 & 23.3 & 1337.6 & 29.7 & 1337.6 & 29.7 & 97.7 \\
\hline SYN14-5_Run1- 26 & 1231.7 & 20.6 & 1272.1 & 22.1 & 1341.2 & 46.9 & 1341.2 & 46.9 & 91.8 \\
\hline SYN14-5_Run1- 19 & 1384.2 & 25.3 & 1370.5 & 17.3 & 1349.3 & 20.9 & 1349.3 & 20.9 & 102.6 \\
\hline SYN14-5_Run2- 172 & 1313.3 & 19.0 & 1327.2 & 18.3 & 1349.8 & 36.3 & 1349.8 & 36.3 & 97.3 \\
\hline SYN14-5_Run2- 140 & 1372.8 & 21.0 & 1364.9 & 16.1 & 1352.5 & 25.2 & 1352.5 & 25.2 & 101.5 \\
\hline SYN14-5_Run3- 255 & 1385.8 & 19.6 & 1379.1 & 15.7 & 1368.7 & 26.1 & 1368.7 & 26.1 & 101.2 \\
\hline SYN14-5_Run1- 106 & 1358.1 & 31.4 & 1367.9 & 23.3 & 1383.4 & 33.3 & 1383.4 & 33.3 & 98.2 \\
\hline SYN14-5_Run2- 128 & 1362.1 & 16.4 & 1370.8 & 12.0 & 1384.4 & 16.6 & 1384.4 & 16.6 & 98.4 \\
\hline SYN14-5_Run1- 87 & 1317.0 & 32.1 & 1343.6 & 22.3 & 1386.1 & 25.3 & 1386.1 & 25.3 & 95.0 \\
\hline SYN14-5_Run1- 99 & 1323.2 & 20.7 & 1348.7 & 18.5 & 1389.2 & 34.1 & 1389.2 & 34.1 & 95.2 \\
\hline SYN14-5_Run3- 237 & 1363.4 & 18.3 & 1382.7 & 13.1 & 1412.6 & 17.2 & 1412.6 & 17.2 & 96.5 \\
\hline SYN14-5_Run3- 287 & 1397.6 & 25.6 & 1410.1 & 18.2 & 1428.9 & 23.8 & 1428.9 & 23.8 & 97.8 \\
\hline SYN14-5_Run3- 328 & 1408.5 & 17.3 & 1419.9 & 15.5 & 1437.0 & 28.6 & 1437.0 & 28.6 & 98.0 \\
\hline SYN14-5_Run2- 148 & 1322.6 & 29.9 & 1368.6 & 23.4 & 1441.2 & 35.1 & 1441.2 & 35.1 & 91.8 \\
\hline SYN14-5_Run2- 206 & 1428.0 & 19.7 & 1433.3 & 17.4 & 1441.2 & 31.7 & 1441.2 & 31.7 & 99.1 \\
\hline SYN14-5_Run2- 133 & 1352.0 & 20.1 & 1388.5 & 15.2 & 1444.9 & 21.8 & 1444.9 & 21.8 & 93.6 \\
\hline
\end{tabular}




\begin{tabular}{|c|c|c|c|c|c|c|c|c|c|}
\hline SYN14-5_Run2- 153 & 1407.6 & 28.0 & 1422.7 & 19.9 & 1445.3 & 26.1 & 1445.3 & 26.1 & 97.4 \\
\hline SYN14-5_Run3- 312 & 1524.4 & 27.3 & 1496.5 & 17.7 & 1457.0 & 19.8 & 1457.0 & 19.8 & 104.6 \\
\hline SYN14-5_Run1- 25 & 1431.4 & 17.2 & 1443.6 & 15.0 & 1461.6 & 27.0 & 1461.6 & 27.0 & 97.9 \\
\hline SYN14-5_Run3- 269 & 1428.3 & 31.2 & 1442.6 & 23.4 & 1463.7 & 34.8 & 1463.7 & 34.8 & 97.6 \\
\hline SYN14-5_Run3- 274 & 1453.5 & 25.3 & 1459.0 & 21.8 & 1467.1 & 38.5 & 1467.1 & 38.5 & 99.1 \\
\hline SYN14-5_Run2- 116 & 1449.7 & 35.9 & 1460.8 & 24.2 & 1476.9 & 27.7 & 1476.9 & 27.7 & 98.2 \\
\hline SYN14-5_Run1- 54 & 1451.1 & 24.1 & 1463.0 & 16.8 & 1480.2 & 21.4 & 1480.2 & 21.4 & 98.0 \\
\hline SYN14-5_Run3- 314 & 1424.0 & 23.7 & 1447.1 & 19.6 & 1481.2 & 32.9 & 1481.2 & 32.9 & 96.1 \\
\hline SYN14-5_Run1- 44 & 1471.4 & 25.0 & 1475.6 & 16.4 & 1481.7 & 17.5 & 1481.7 & 17.5 & 99.3 \\
\hline SYN14-5_Run3- 261 & 1500.4 & 23.2 & 1498.5 & 16.9 & 1495.8 & 24.4 & 1495.8 & 24.4 & 100.3 \\
\hline SYN14-5_Run1- 42 & 1477.0 & 20.8 & 1485.1 & 15.9 & 1496.8 & 24.5 & 1496.8 & 24.5 & 98.7 \\
\hline SYN14-5_Run2- 213 & 1468.7 & 20.7 & 1487.8 & 16.3 & 1515.1 & 25.8 & 1515.1 & 25.8 & 96.9 \\
\hline SYN14-5_Run1- 11 & 1395.4 & 23.2 & 1454.9 & 20.6 & 1542.9 & 36.1 & 1542.9 & 36.1 & 90.4 \\
\hline SYN14-5_Run2- 212 & 1499.7 & 29.1 & 1520.5 & 18.6 & 1549.6 & .3 & 49.6 & 17.3 & 96.8 \\
\hline SYN14-5_Run1- 90 & 1520.7 & 15.1 & 1535.4 & 13.6 & 1555.7 & 24.6 & 1555.7 & 24.6 & 97.8 \\
\hline SYN14-5_Run2- 131 & 1534.6 & 36.1 & 1544.3 & 22.9 & 1557.6 & 21.6 & 557.6 & 21.6 & 98.5 \\
\hline SYN14-5_Run3- 290 & 1499.2 & 18.4 & 1527.7 & 13.6 & 1567.3 & 19.3 & 67.3 & 19.3 & 95.7 \\
\hline SYN14-5_Run1- 73 & 1546.9 & 31.7 & 1570.2 & 22.2 & 1601.7 & 29.1 & 01.7 & 29.1 & 96.6 \\
\hline SYN14-5_Run1- 32 & 1533.0 & 25.4 & 1570.3 & 19.6 & 1620.7 & 29.9 & 20.7 & 29.9 & 94.6 \\
\hline SYN14-5_Run2- 201 & 1592.6 & 44.0 & 1604.9 & 28.6 & 1621.1 & 31.3 & 21.1 & 31.3 & 98.2 \\
\hline SYN14-5_Run1- 37 & 1614.6 & 22.0 & 1621.4 & 16.6 & 1630.3 & 25.2 & 1630.3 & 25.2 & 99.0 \\
\hline SYN14-5_Run2- 164 & 1620.3 & 22.0 & 1626.9 & 15.5 & 1635.5 & 21.3 & 1635.5 & 21.3 & 99.1 \\
\hline SYN14-5_Run3- 304 & 1629.1 & 31.0 & 1633.7 & 19.3 & 1639.7 & 18.4 & 1639.7 & 18.4 & 99.4 \\
\hline SYN14-5_Run1-89 & 1587.1 & 24.8 & 1610.0 & 18.3 & 1640.1 & 26.4 & 1640.1 & 26.4 & 96.8 \\
\hline SYN14-5_Run3- 318 & 1683.9 & 20.1 & 1665.9 & 14.4 & 1643.3 & 20.6 & 1643.3 & 20.6 & 102.5 \\
\hline SYN14-5_Run2- 174 & 1648.8 & 28.0 & 1646.8 & 19.6 & 1644.1 & 26.5 & 1644.1 & 26.5 & 100.3 \\
\hline SYN14-5_Run2- 129 & 1617.8 & 30.5 & 1631.2 & 20.8 & 1648.4 & 26.6 & 1648.4 & 26.6 & 98.1 \\
\hline SYN14-5_Run3- 270 & 1620.5 & 21.6 & 1632.8 & 17.4 & 1648.6 & 28.3 & 1648.6 & 28.3 & 98.3 \\
\hline SYN14-5_Run3- 279 & 1679.5 & 35.8 & 1665.8 & 21.5 & 1648.6 & 18.9 & 1648.6 & 18.9 & 101.9 \\
\hline SYN14-5_Run2- 217 & 1622.9 & 22.4 & 1636.3 & 18.3 & 1653.6 & 30.2 & 1653.6 & 30.2 & 98.1 \\
\hline SYN14-5_Run3- 311 & 1651.1 & 23.9 & 1652.6 & 16.6 & 1654.5 & 22.4 & 1654.5 & 22.4 & 99.8 \\
\hline SYN14-5_Run2- 202 & 1617.7 & 16.5 & 1635.7 & 15.2 & 1658.9 & 27.4 & 1658.9 & 27.4 & 97.5 \\
\hline SYN14-5_Run3- 254 & 1625.9 & 25.1 & 1645.1 & 18.2 & 1669.7 & 25.7 & 1669.7 & 25.7 & 97.4 \\
\hline SYN14-5_Run3- 236 & 1718.8 & 31.3 & 1701.6 & 20.3 & 1680.4 & 24.6 & 1680.4 & 24.6 & 102.3 \\
\hline SYN14-5_Run3- 280 & 1696.0 & 28.4 & 1701.7 & 20.9 & 1708.7 & 30.8 & 1708.7 & 30.8 & 99.3 \\
\hline SYN14-5_Run2- 190 & 1674.7 & 30.6 & 1691.8 & 24.7 & 1713.1 & 39.9 & 1713.1 & 39.9 & 97.8 \\
\hline SYN14-5_Run2- 166 & 1725.8 & 23.4 & 1724.6 & 18.8 & 1723.1 & 30.3 & 1723.1 & 30.3 & 100.2 \\
\hline SYN14-5_Run3- 223 & 1686.0 & 22.2 & 1702.9 & 14.9 & 1723.8 & 18.3 & 1723.8 & 18.3 & 97.8 \\
\hline SYN14-5_Run2- 123 & 1680.9 & 23.9 & 1701.2 & 16.2 & 1726.3 & 20.6 & 1726.3 & 20.6 & 97.4 \\
\hline
\end{tabular}




\begin{tabular}{|c|c|c|c|c|c|c|c|c|c|}
\hline SYN14-5_Run1- 31 & 1733.8 & 19.5 & 1731.2 & 16.0 & 1728.1 & 26.5 & 1728.1 & 26.5 & 100.3 \\
\hline SYN14-5_Run3- 320 & 1729.1 & 23.8 & 1729.4 & 15.9 & 1729.7 & 20.0 & 1729.7 & 20.0 & 100.0 \\
\hline SYN14-5_Run1- 76 & 1720.3 & 20.4 & 1725.1 & 15.7 & 1730.9 & 24.3 & 1730.9 & 24.3 & 99.4 \\
\hline SYN14-5_Run3- 327 & 1735.5 & 28.6 & 1734.7 & 18.7 & 1733.7 & 22.5 & 1733.7 & 22.5 & 100.1 \\
\hline SYN14-5_Run1- 66 & 1732.9 & 25.6 & 1736.1 & 16.1 & 1740.1 & 17.6 & 1740.1 & 17.6 & 99.6 \\
\hline SYN14-5_Run3- 231 & 1745.0 & 24.0 & 1744.8 & 20.7 & 1744.6 & 35.2 & 1744.6 & 35.2 & 100.0 \\
\hline SYN14-5_Run2- 122 & 1714.2 & 24.1 & 1729.2 & 18.2 & 1747.2 & 27.4 & 1747.2 & 27.4 & 98.1 \\
\hline SYN14-5_Run2- 219 & 1727.8 & 22.4 & 1742.2 & 15.4 & 1759.5 & 20.3 & 1759.5 & 20.3 & 98.2 \\
\hline SYN14-5_Run1- 3 & 1706.2 & 17.4 & 1734.7 & 13.9 & 1769.2 & 22.1 & 1769.2 & 22.1 & 96.4 \\
\hline SYN14-5_Run2- 141 & 1710.2 & 35.7 & 1739.8 & 21.0 & 1775.4 & 15.7 & 1775.4 & 15.7 & 96.3 \\
\hline SYN14-5_Run1- 40 & 1648.9 & 22.2 & 1717.0 & 21.4 & 1801.1 & 38.0 & 1801.1 & 38.0 & 91.5 \\
\hline SYN14-5_Run3- 325 & 1867.4 & 30.3 & 1845.9 & 19.8 & 1821.9 & 25.2 & 1821.9 & 25.2 & 102.5 \\
\hline SYN14-5_Run3- 262 & 1814.3 & 22.6 & 1824.4 & 15.1 & 1835.9 & 19.3 & 1835.9 & 19.3 & 98.8 \\
\hline SYN14-5_Run1- 21 & 1816.4 & 26.2 & 1825.8 & 17.7 & 1836.4 & 23.1 & 1836.4 & 23.1 & 98.9 \\
\hline SYN14-5_Run1- 34 & 1811.2 & 24.8 & 1824.7 & 19.2 & 1840.2 & 29.6 & 1840.2 & 29.6 & 98.4 \\
\hline 5_Run1- 4 & 1803.7 & 29.2 & 1838.4 & 17.7 & 1877.9 & 17.3 & 77.9 & 17.3 & 96.0 \\
\hline SYN14-5_Run2- 126 & 1898.3 & 26.3 & 1896.2 & 17.0 & 1893.8 & 21.2 & 1893.8 & 21.2 & 100.2 \\
\hline SYN14-5_Run2- 185 & 1855.9 & 24.5 & 1890.6 & 21.6 & 1928.9 & 35.8 & 1928.9 & 35.8 & 96.2 \\
\hline SYN14-5_Run3- 301 & 1958.2 & 22.0 & 1945.8 & 15.6 & 1932.7 & 22.3 & 1932.7 & 22.3 & 101.3 \\
\hline SYN14-5_Run3- 250 & 1974.8 & 22.0 & 1977.8 & 14.4 & 1980.9 & 18.3 & 1980.9 & 18.3 & 99.7 \\
\hline SYN14-5_Run1- 105 & 1934.6 & 21.5 & 1964.0 & 16.1 & 1995.2 & 23.8 & 1995.2 & 23.8 & 97.0 \\
\hline SYN14-5_Run3- 294 & 1980.0 & 24.7 & 1991.7 & 14.7 & 2003.9 & 15.3 & 2003.9 & 15.3 & 98.8 \\
\hline SYN14-5_Run2- 159 & 1994.2 & 24.6 & 2002.5 & 16.3 & 2011.1 & 21.1 & 2011.1 & 21.1 & 99.2 \\
\hline SYN14-5_Run2- 218 & 2037.2 & 24.5 & 2032.6 & 16.2 & 2028.0 & 21.2 & 2028.0 & 21.2 & 100.5 \\
\hline SYN14-5_Run3- 299 & 2056.1 & 39.0 & 2059.3 & 24.3 & 2062.6 & 28.9 & 2062.6 & 28.9 & 99.7 \\
\hline SYN14-5_Run2- 119 & 2051.7 & 40.4 & 2069.0 & 22.6 & 2086.1 & 19.7 & 2086.1 & 19.7 & 98.4 \\
\hline SYN14-5_Run1- 43 & 2064.5 & 28.9 & 2076.1 & 16.1 & 2087.7 & 13.9 & 2087.7 & 13.9 & 98.9 \\
\hline SYN14-5_Run3- 244 & 2042.3 & 22.8 & 2067.2 & 15.3 & 2092.1 & 20.0 & 2092.1 & 20.0 & 97.6 \\
\hline SYN14-5_Run1- 23 & 2057.7 & 29.6 & 2099.4 & 20.4 & 2140.5 & 27.6 & 2140.5 & 27.6 & 96.1 \\
\hline SYN14-5_Run1- 108 & 2358.5 & 41.2 & 2399.3 & 22.6 & 2434.0 & 22.0 & 2434.0 & 22.0 & 96.9 \\
\hline SYN14-5_Run2- 176 & 2177.5 & 64.4 & 2325.3 & 32.8 & 2457.7 & 14.6 & 2457.7 & 14.6 & 88.6 \\
\hline SYN14-5_Run1- 68 & 2548.2 & 32.2 & 2560.9 & 19.4 & 2571.0 & 23.5 & 2571.0 & 23.5 & 99.1 \\
\hline SYN14-5_Run3- 297 & 2523.3 & 47.9 & 2620.3 & 22.9 & 2696.0 & 13.9 & 2696.0 & 13.9 & 93.6 \\
\hline SYN14-5_Run1- 100 & 2634.3 & 46.8 & 2671.4 & 24.0 & 2699.6 & 22.1 & 2699.6 & 22.1 & 97.6 \\
\hline SYN14-5_Run2- 136 & 2550.9 & 36.3 & 2638.7 & 19.0 & 2706.7 & 17.3 & 2706.7 & 17.3 & 94.2 \\
\hline SYN14-5_Run1- 95 & 2624.1 & 34.3 & 2674.8 & 20.8 & 2713.3 & 25.3 & 2713.3 & 25.3 & 96.7 \\
\hline SYN14-5_Run2- 161 & 2716.9 & 31.4 & 2722.3 & 14.5 & 2726.2 & 9.6 & 2726.2 & 9.6 & 99.7 \\
\hline SYN14-5_Run2- 138 & 2747.1 & 43.6 & 2756.9 & 20.5 & 2764.0 & 15.3 & 2764.0 & 15.3 & 99.4 \\
\hline SYN14-5_Run3- 273 & 2855.5 & 55.4 & 2894.3 & 31.7 & 2921.4 & 37.0 & 2921.4 & 37.0 & 97.7 \\
\hline
\end{tabular}




\begin{tabular}{|c|c|c|c|c|c|c|c|c|c|}
\hline Analysis & 206Pb* & \pm & 207Pb* & \pm & $206 \mathrm{~Pb}^{*}$ & \pm & Best age & \pm & Conc \\
\hline & $238 U^{*}$ & (Ma) & $235 \mathrm{U}$ & (Ma) & 207Pb* & (Ma) & (Ma) & (Ma) & $(\%)$ \\
\hline SYN14_9_Run 1-- 40 & 166.1 & 3.7 & 164.4 & 6.1 & 140.8 & 77.7 & 166.1 & 3.7 & NA \\
\hline SYN14_9_Run 2-- 188 & 170.4 & 3.0 & 168.1 & 4.5 & 135.6 & 53.9 & 170.4 & 3.0 & NA \\
\hline SYN14_9_Run 3-- 292 & 173.1 & 3.0 & 178.1 & 5.6 & 245.9 & 67.5 & 173.1 & 3.0 & NA \\
\hline SYN14_9_Run 1-- 41 & 178.0 & 3.2 & 173.9 & 9.7 & 119.3 & 137.0 & 178.0 & 3.2 & NA \\
\hline SYN14_9_Run 1-- 46 & 222.8 & 4.7 & 230.1 & 7.0 & 304.7 & 59.6 & 222.8 & 4.7 & NA \\
\hline SYN14_9_Run 2-- 151 & 231.7 & 5.0 & 237.9 & 9.7 & 299.9 & 91.6 & 231.7 & 5.0 & NA \\
\hline SYN14_9_Run 1-- 76 & 244.2 & 6.7 & 247.8 & 9.8 & 281.8 & 79.2 & 244.2 & 6.7 & NA \\
\hline SYN14_9_Run 3-- 227 & 256.5 & 4.0 & 258.0 & 7.5 & 271.6 & 65.4 & 256.5 & 4.0 & NA \\
\hline SYN14_9_Run 1-- 72 & 258.1 & 7.5 & 258.2 & 11.8 & 258.9 & 97.2 & 258.1 & 7.5 & NA \\
\hline SYN14_9_Run 3-- 231 & 259.1 & 5.1 & 261.6 & 7.3 & 283.6 & 55.9 & 259.1 & 5.1 & NA \\
\hline SYN14_9_Run 2-- 215 & 334.6 & 5.6 & 331.9 & 12.9 & 313.2 & 96.1 & 334.6 & 5.6 & NA \\
\hline SYN14_9_Run 1-- 70 & 335.7 & 6.3 & 340.1 & 9.2 & 370.0 & 57.4 & 335.7 & 6.3 & NA \\
\hline SYN14_9_Run 3-- 326 & 340.2 & 4.2 & 336.4 & 6.1 & 309.8 & 39.3 & 340.2 & 4.2 & NA \\
\hline SYN14_9_Run 3-- 225 & 349.0 & 6.2 & 360.0 & 11.8 & 431.2 & 76.6 & 349.0 & 6.2 & NA \\
\hline SYN14_9_Run 1-- 91 & 352.3 & 6.1 & 366.3 & 16.2 & 456.1 & 109.8 & 352.3 & 6.1 & NA \\
\hline SYN14_9_Run 1-- 26 & 369.4 & 13.3 & 361.5 & 17.6 & 311.6 & 101.1 & 369.4 & 13.3 & NA \\
\hline SYN14_9_Run 1-- 94 & 371.1 & 7.7 & 367.6 & 8.9 & 345.4 & 43.4 & 371.1 & 7.7 & NA \\
\hline SYN14_9_Run 2-- 125 & 371.6 & 11.5 & 370.1 & 11.2 & 360.3 & 37.8 & 371.6 & 11.5 & NA \\
\hline SYN14_9_Run 2-- 172 & 373.0 & 5.9 & 386.5 & 6.8 & 468.4 & 30.4 & 373.0 & 5.9 & NA \\
\hline SYN14_9_Run 3-- 316 & 375.9 & 8.2 & 375.5 & 9.8 & 372.8 & 48.5 & 375.9 & 8.2 & NA \\
\hline SYN14_9_Run 2-- 154 & 379.7 & 8.2 & 380.7 & 9.3 & 387.3 & 43.1 & 379.7 & 8.2 & NA \\
\hline SYN14_9_Run 1-- 101 & 387.9 & 7.9 & 397.1 & 10.6 & 450.4 & 55.0 & 387.9 & 7.9 & NA \\
\hline SYN14_9_Run 1-- 73 & 392.0 & 6.7 & 397.2 & 8.6 & 427.9 & 42.8 & 392.0 & 6.7 & NA \\
\hline SYN14_9_Run 1-- 81 & 402.1 & 10.4 & 407.6 & 12.1 & 438.9 & 54.4 & 402.1 & 10.4 & 91.6 \\
\hline SYN14_9_Run 3-- 291 & 402.7 & 9.6 & 399.9 & 12.2 & 383.6 & 61.7 & 402.7 & 9.6 & 105.0 \\
\hline SYN14_9_Run 3-- 293 & 402.9 & 9.6 & 417.3 & 19.4 & 497.6 & 112.4 & 402.9 & 9.6 & 81.0 \\
\hline SYN14_9_Run 1-- 56 & 409.6 & 7.9 & 423.2 & 21.8 & 498.3 & 131.6 & 409.6 & 7.9 & 82.2 \\
\hline SYN14_9_Run 3-- 249 & 419.4 & 8.6 & 417.3 & 10.1 & 405.6 & 46.2 & 419.4 & 8.6 & 103.4 \\
\hline SYN14_9_Run 2-- 147 & 419.7 & 8.3 & 422.8 & 17.4 & 439.8 & 101.9 & 419.7 & 8.3 & 95.4 \\
\hline SYN14_9_Run 1-- 103 & 422.7 & 8.3 & 429.1 & 11.3 & 463.8 & 55.4 & 422.7 & 8.3 & 91.1 \\
\hline SYN14_9_Run 2-- 169 & 424.5 & 7.9 & 426.7 & 8.5 & 438.9 & 33.2 & 424.5 & 7.9 & 96.7 \\
\hline SYN14_9_Run 2-- 212 & 427.4 & 8.2 & 421.4 & 15.5 & 388.8 & 90.2 & 427.4 & 8.2 & 109.9 \\
\hline SYN14_9_Run 1-- 43 & 428.0 & 11.3 & 427.6 & 13.1 & 425.2 & 57.4 & 428.0 & 11.3 & 100.7 \\
\hline SYN14_9_Run 1-- 2 & 428.1 & 6.5 & 430.3 & 7.2 & 442.5 & 30.0 & 428.1 & 6.5 & 96.7 \\
\hline
\end{tabular}




\begin{tabular}{|c|c|c|c|c|c|c|c|c|c|}
\hline SYN14_9_Run 2-- 122 & 434.1 & 7.3 & 437.0 & 13.1 & 452.7 & 72.7 & 434.1 & 7.3 & 95.9 \\
\hline SYN14_9_Run 3-- 235 & 448.4 & 7.9 & 434.8 & 11.9 & 363.7 & 63.9 & 448.4 & 7.9 & 123.3 \\
\hline SYN14_9_Run 2-- 183 & 457.5 & 7.6 & 451.6 & 12.2 & 421.8 & 64.1 & 457.5 & 7.6 & 108.5 \\
\hline SYN14_9_Run 3-- 247 & 457.7 & 7.1 & 460.6 & 11.7 & 475.6 & 60.0 & 457.7 & 7.1 & 96.2 \\
\hline SYN14_9_Run 2-- 132 & 468.8 & 13.6 & 478.4 & 16.0 & 524.6 & 63.5 & 468.8 & 13.6 & 89.4 \\
\hline SYN14_9_Run 1-- 27 & 476.7 & 10.5 & 473.5 & 12.3 & 457.8 & 51.3 & 476.7 & 10.5 & 104.1 \\
\hline SYN14_9_Run 2-- 196 & 511.6 & 13.3 & 532.8 & 18.0 & 624.7 & 73.2 & 511.6 & 13.3 & 81.9 \\
\hline SYN14_9_Run 1-- 25 & 533.1 & 12.3 & 535.0 & 13.1 & 542.9 & 44.7 & 533.1 & 12.3 & 98.2 \\
\hline SYN14_9_Run 3-- 290 & 553.4 & 13.7 & 549.0 & 14.8 & 530.7 & 51.0 & 553.4 & 13.7 & 104.3 \\
\hline SYN14_9_Run 2-- 195 & 560.0 & 12.1 & 555.5 & 19.9 & 537.2 & 88.7 & 560.0 & 12.1 & 104.2 \\
\hline SYN14_9_Run 3-- 313 & 561.7 & 13.0 & 589.2 & 15.3 & 696.8 & 51.6 & 561.7 & 13.0 & 80.6 \\
\hline SYN14_9_Run 3-- 314 & 562.2 & 10.4 & 560.8 & 17.0 & 555.4 & 75.0 & 562.2 & 10.4 & 101.2 \\
\hline SYN14_9_Run 1-- 53 & 577.8 & 18.8 & 573.6 & 18.4 & 557.2 & 53.6 & 577.8 & 18.8 & 103.7 \\
\hline SYN14_9_Run 3-- 245 & 595.9 & 10.7 & 605.4 & 12.8 & 641.0 & 44.8 & 595.9 & 10.7 & 93.0 \\
\hline SYN14_9_Run 1-- 9 & 608.8 & 15.7 & 612.6 & 14.6 & 626.7 & 35.4 & 608.8 & 15.7 & 97.1 \\
\hline SYN14_9_Run 1-- 37 & 611.5 & 10.4 & 619.9 & 14.2 & 650.7 & 53.7 & 611.5 & 10.4 & 94.0 \\
\hline SYN14_9_Run 2-- 201 & 614.0 & 10.2 & 619.3 & 15.8 & 638.9 & 63.1 & 614.0 & 10.2 & 96.1 \\
\hline SYN14_9_Run 3-- 233 & 615.7 & 11.3 & 616.6 & 11.9 & 619.6 & 36.6 & 615.7 & 11.3 & 99.4 \\
\hline SYN14_9_Run 2-- 153 & 617.8 & 12.7 & 623.7 & 15.3 & 645.2 & 53.0 & 617.8 & 12.7 & 95.8 \\
\hline SYN14_9_Run 2-- 163 & 620.6 & 9.7 & 620.1 & 12.5 & 618.1 & 46.4 & 620.6 & 9.7 & 100.4 \\
\hline SYN14_9_Run 3-- 224 & 632.9 & 8.6 & 630.1 & 12.9 & 620.1 & 50.9 & 632.9 & 8.6 & 102.1 \\
\hline SYN14_9_Run 1-- 47 & 633.0 & 10.9 & 636.0 & 13.8 & 646.9 & 49.3 & 633.0 & 10.9 & 97.9 \\
\hline SYN14_9_Run 2-- 184 & 636.1 & 11.0 & 634.0 & 11.0 & 626.5 & 31.6 & 636.1 & 11.0 & 101.5 \\
\hline SYN14_9_Run 1-- 48 & 638.8 & 12.2 & 637.6 & 12.8 & 633.4 & 39.0 & 638.8 & 12.2 & 100.8 \\
\hline SYN14_9_Run 3-- 328 & 677.6 & 14.4 & 699.1 & 19.2 & 768.6 & 64.5 & 677.6 & 14.4 & 88.2 \\
\hline SYN14_9_Run 2-- 185 & 934.7 & 14.7 & 934.9 & 17.1 & 935.3 & 45.9 & 935.3 & 45.9 & 99.9 \\
\hline SYN14_9_Run 2-- 162 & 938.0 & 17.3 & 946.6 & 18.3 & 966.8 & 45.1 & 966.8 & 45.1 & 97.0 \\
\hline SYN14_9_Run 1-- 98 & 905.8 & 16.7 & 927.6 & 16.8 & 979.5 & 39.4 & 979.5 & 39.4 & 92.5 \\
\hline SYN14_9_Run 2-- 171 & 950.4 & 20.0 & 961.0 & 25.7 & 985.1 & 70.3 & 985.1 & 70.3 & 96.5 \\
\hline SYN14_9_Run 3-- 311 & 1006.4 & 15.4 & 1002.7 & 13.2 & 994.7 & 25.3 & 994.7 & 25.3 & 101.2 \\
\hline SYN14_9_Run 2-- 177 & 996.0 & 15.5 & 997.7 & 15.2 & 1001.2 & 34.7 & 1001.2 & 34.7 & 99.5 \\
\hline SYN14_9_Run 1-- 30 & 1016.6 & 19.5 & 1013.2 & 19.7 & 1005.8 & 45.9 & 1005.8 & 45.9 & 101.1 \\
\hline SYN14_9_Run 2-- 123 & 1023.5 & 13.2 & 1018.3 & 15.0 & 1007.0 & 38.0 & 1007.0 & 38.0 & 101.6 \\
\hline SYN14_9_Run 1-- 87 & 1008.0 & 13.1 & 1008.2 & 11.3 & 1008.8 & 21.8 & 1008.8 & 21.8 & 99.9 \\
\hline SYN14_9_Run 3-- 318 & 983.3 & 17.2 & 991.5 & 20.3 & 1009.7 & 52.4 & 1009.7 & 52.4 & 97.4 \\
\hline SYN14_9_Run 2-- 131 & 968.5 & 13.1 & 981.3 & 17.5 & 1010.1 & 48.0 & 1010.1 & 48.0 & 95.9 \\
\hline SYN14_9_Run 3-- 223 & 987.6 & 9.6 & 994.7 & 10.5 & 1010.4 & 25.8 & 1010.4 & 25.8 & 97.7 \\
\hline SYN14_9_Run 2-- 114 & 1016.0 & 18.9 & 1016.0 & 26.7 & 1016.0 & 73.8 & 1016.0 & 73.8 & 100.0 \\
\hline SYN14_9_Run 3-- 229 & 1022.7 & 22.5 & 1020.9 & 18.8 & 1017.0 & 34.4 & 1017.0 & 34.4 & 100.6 \\
\hline
\end{tabular}




\begin{tabular}{|c|c|c|c|c|c|c|c|c|c|}
\hline SYN14_9_Run 1-- 59 & 1030.3 & 20.2 & 1026.3 & 22.8 & 1018.0 & 57.1 & 1018.0 & 57.1 & 101.2 \\
\hline SYN14_9_Run 2-- 148 & 1020.4 & 17.0 & 1020.0 & 18.1 & 1019.2 & 43.8 & 1019.2 & 43.8 & 100.1 \\
\hline SYN14_9_Run 1-- 69 & 1030.3 & 20.0 & 1028.3 & 16.4 & 1024.0 & 29.0 & 1024.0 & 29.0 & 100.6 \\
\hline SYN14_9_Run 2-- 129 & 1027.8 & 18.7 & 1026.6 & 15.7 & 1024.0 & 28.7 & 1024.0 & 28.7 & 100.4 \\
\hline SYN14_9_Run 2-- 130 & 990.6 & 20.1 & 1001.4 & 30.2 & 1025.2 & 85.2 & 1025.2 & 85.2 & 96.6 \\
\hline SYN14_9_Run 3-- 294 & 1044.7 & 11.6 & 1039.2 & 10.4 & 1027.6 & 21.4 & 1027.6 & 21.4 & 101.7 \\
\hline SYN14_9_Run 2-- 146 & 1013.1 & 17.4 & 1019.7 & 14.3 & 1033.9 & 25.0 & 1033.9 & 25.0 & 98.0 \\
\hline SYN14_9_Run 3-- 285 & 923.1 & 16.6 & 956.7 & 13.5 & 1034.6 & 19.9 & 1034.6 & 19.9 & 89.2 \\
\hline SYN14_9_Run 2-- 180 & 1025.5 & 24.6 & 1028.7 & 18.7 & 1035.6 & 25.7 & 1035.6 & 25.7 & 99.0 \\
\hline SYN14_9_Run 2-- 216 & 1033.8 & 15.4 & 1034.6 & 12.7 & 1036.4 & 22.4 & 1036.4 & 22.4 & 99.8 \\
\hline SYN14_9_Run 3-- 246 & 998.7 & 16.4 & 1010.7 & 19.3 & 1036.7 & 49.0 & 1036.7 & 49.0 & 96.3 \\
\hline SYN14_9_Run 1-- 24 & 1106.1 & 21.5 & 1084.0 & 18.7 & 1039.8 & 37.1 & 1039.8 & 37.1 & 106.4 \\
\hline SYN14_9_Run 2-- 137 & 1015.5 & 15.0 & 1023.8 & 12.1 & 1041.6 & 20.0 & 1041.6 & 20.0 & 97.5 \\
\hline SYN14_9_Run 2-- 187 & 1056.6 & 22.9 & 1051.8 & 17.1 & 1041.7 & 22.8 & 1041.7 & 22.8 & 101.4 \\
\hline SYN14_9_Run 1-- 57 & 1031.2 & 16.7 & 1035.3 & 15.4 & 1043.9 & 32.1 & 1043.9 & 32.1 & 98.8 \\
\hline SYN14_9_Run 1-- 71 & 1005.1 & 30.4 & 1017.8 & 22.6 & 1045.1 & 26.1 & 1045.1 & 26.1 & 96.2 \\
\hline SYN14_9_Run 1-- 36 & 1015.5 & 16.8 & 1025.1 & 15.9 & 1045.6 & 34.1 & 1045.6 & 34.1 & 97.1 \\
\hline SYN14_9_Run 2-- 211 & 993.2 & 16.8 & 1009.9 & 18.8 & 1046.2 & 46.6 & 1046.2 & 46.6 & 94.9 \\
\hline SYN14_9_Run 1-- 38 & 98 & 12.6 & 1008.0 & 13.3 & 7.8 & 31.4 & 1047.8 & 31.4 & 94.5 \\
\hline SYN14_9_Run 1-- 79 & & 1 & & 13.5 & & 31.5 & .9 & .5 & 1 \\
\hline SYN14_9_Run 2-- 197 & 10 & 1 & 1 & 3 & 9 & 31.7 & .9 & .7 & .7 \\
\hline SYN14_9_Run 1-- 39 & 990.5 & 1 & 1 & .7 & 5 & 42.2 & 6.5 & .2 & 93.7 \\
\hline SYN14_9_Run 2-- 149 & 1079.8 & 26.2 & 1072.2 & 22.9 & 8 & 45.0 & 56.8 & 45.0 & 102.2 \\
\hline SYN14_9_Run 2-- 193 & 994.5 & 18.2 & 1014.6 & 17.8 & 1058.3 & 39.3 & 1058.3 & 39.3 & 94.0 \\
\hline SYN14_9_Run 3-- 320 & 1024.3 & 18.0 & 1035.2 & 16.9 & 1058.3 & 35.7 & 1058.3 & 35.7 & 96.8 \\
\hline SYN14_9_Run 2-- 143 & 1063.1 & 13.4 & 1062.9 & 12.8 & 1062.4 & 27.7 & 1062.4 & 27.7 & 100.1 \\
\hline SYN14_9_Run 2-- 145 & 1014.8 & 24.4 & 1030.4 & 19.2 & 1063.8 & 28.4 & 1063.8 & 28.4 & 95.4 \\
\hline SYN14_9_Run 2-- 111 & 1009.3 & 19.2 & 1027.1 & 16.1 & 1065.3 & 28.5 & 1065.3 & 28.5 & 94.7 \\
\hline SYN14_9_Run 3-- 282 & 1037.4 & 12.3 & 1047.0 & 13.3 & 1067.2 & 31.7 & 1067.2 & 31.7 & 97.2 \\
\hline SYN14_9_Run 1-- 6 & 983.5 & 23.8 & 1011.4 & 18.6 & 1072.2 & 25.5 & 1072.2 & 25.5 & 91.7 \\
\hline SYN14_9_Run 3-- 310 & 1045.9 & 13.9 & 1055.2 & 14.9 & 1074.4 & 35.3 & 1074.4 & 35.3 & 97.3 \\
\hline SYN14_9_Run 3-- 240 & 1047.4 & 17.1 & 1056.3 & 18.6 & 1074.7 & 44.5 & 1074.7 & 44.5 & 97.5 \\
\hline SYN14_9_Run 1-- 54 & 1053.1 & 17.3 & 1060.3 & 15.6 & 1075.3 & 31.5 & 1075.3 & 31.5 & 97.9 \\
\hline SYN14_9_Run 3-- 248 & 1024.7 & 19.7 & 1041.1 & 19.2 & 1075.6 & 42.0 & 1075.6 & 42.0 & 95.3 \\
\hline SYN14_9_Run 2-- 179 & 1071.0 & 14.3 & 1072.8 & 11.2 & 1076.4 & 17.5 & 1076.4 & 17.5 & 99.5 \\
\hline SYN14_9_Run 3-- 232 & 1002.2 & 15.0 & 1026.4 & 21.8 & 1078.5 & 59.5 & 1078.5 & 59.5 & 92.9 \\
\hline SYN14_9_Run 1-- 50 & 1037.0 & 17.4 & 1050.7 & 18.1 & 1079.3 & 42.0 & 1079.3 & 42.0 & 96.1 \\
\hline SYN14_9_Run 1-- 51 & 1086.5 & 23.0 & 1084.4 & 19.6 & 1080.1 & 37.0 & 1080.1 & 37.0 & 100.6 \\
\hline SYN14_9_Run 2-- 186 & 1039.4 & 18.2 & 1052.7 & 16.1 & 1080.2 & 31.5 & 1080.2 & 31.5 & 96.2 \\
\hline
\end{tabular}




\begin{tabular}{|c|c|c|c|c|c|c|c|c|c|}
\hline SYN14_9_Run 2-- 165 & 1013.1 & 15.8 & 1035.0 & 16.2 & 1081.6 & 37.0 & 1081.6 & 37.0 & 93.7 \\
\hline SYN14_9_Run 3-- 252 & 1073.6 & 23.5 & 1076.9 & 18.7 & 1083.4 & 30.1 & 1083.4 & 30.1 & 99.1 \\
\hline SYN14_9_Run 1-- 92 & 1014.7 & 16.9 & 1037.6 & 18.2 & 1086.3 & 43.2 & 1086.3 & 43.2 & 93.4 \\
\hline SYN14_9_Run 3-- 288 & 1052.4 & 14.0 & 1064.1 & 16.7 & 1088.3 & 41.8 & 1088.3 & 41.8 & 96.7 \\
\hline SYN14_9_Run 2-- 167 & 996.2 & 18.9 & 1025.6 & 26.2 & 1089.0 & 70.1 & 1089.0 & 70.1 & 91.5 \\
\hline SYN14_9_Run 1-- 82 & 1088.0 & 24.5 & 1088.5 & 18.5 & 1089.5 & 26.0 & 1089.5 & 26.0 & 99.9 \\
\hline SYN14_9_Run 2-- 217 & 1002.9 & 22.3 & 1030.9 & 22.4 & 1090.9 & 49.8 & 1090.9 & 49.8 & 91.9 \\
\hline SYN14_9_Run 2-- 176 & 1065.6 & 27.4 & 1074.0 & 25.5 & 1091.1 & 53.2 & 1091.1 & 53.2 & 97.7 \\
\hline SYN14_9_Run 2-- 206 & 1033.6 & 22.4 & 1054.3 & 24.7 & 1097.5 & 58.9 & 1097.5 & 58.9 & 94.2 \\
\hline SYN14_9_Run 2-- 181 & 1050.1 & 15.5 & 1065.7 & 12.7 & 1097.8 & 21.5 & 1097.8 & 21.5 & 95.7 \\
\hline SYN14_9_Run 1-- 52 & 1044.1 & 22.4 & 1063.6 & 18.7 & 1103.7 & 32.5 & 1103.7 & 32.5 & 94.6 \\
\hline SYN14_9_Run 1-- 18 & 1068.8 & 19.2 & 1081.7 & 20.5 & 1107.8 & 47.7 & 1107.8 & 47.7 & 96.5 \\
\hline SYN14_9_Run 1-- 58 & 1011.5 & 13.6 & 1043.1 & 15.1 & 1109.8 & 36.1 & 1109.8 & 36.1 & 91.1 \\
\hline SYN14_9_Run 2-- 150 & 1064.3 & 20.0 & 1082.4 & 17.8 & 1118.8 & 34.7 & 1118.8 & 34.7 & 95.1 \\
\hline SYN14_9_Run 3-- 309 & 1089.5 & 17.2 & 1099.3 & 16.9 & 1118.9 & 36.9 & 1118.9 & 36.9 & 97.4 \\
\hline SYN14_9_Run 3-- 238 & 1111.5 & 15.5 & 1114.2 & 15.8 & 1119.6 & 35.5 & 1119.6 & 35.5 & 99.3 \\
\hline SYN14_9_Run 1-- 35 & 1073.2 & 22.0 & 1089.5 & 17.0 & 1122.1 & 24.9 & 1122.1 & 24.9 & 95.6 \\
\hline SYN14_9_Run 3-- 236 & 1020.0 & 19.2 & 1054.1 & 17.1 & 1125.4 & 32.4 & 1125.4 & 32.4 & 90.6 \\
\hline SYN14_9_Run 1-- 33 & 1091.8 & 19.8 & 1103.9 & 18.6 & 1127.9 & 38.7 & 1127.9 & 38.7 & 96.8 \\
\hline SYN14_9_Run 1-- 65 & 1045.6 & 14.0 & 1073.1 & 19.3 & 1129.3 & 50.4 & 1129.3 & 50.4 & 92.6 \\
\hline SYN14_9_Run 3-- 228 & 1121.7 & 19.3 & 1125.2 & 17.5 & 1131.8 & 34.9 & 1131.8 & 34.9 & 99.1 \\
\hline SYN14_9_Run 2-- 164 & 1165.3 & 12.1 & 1155.4 & 11.8 & 1136.8 & 25.4 & 1136.8 & 25.4 & 102.5 \\
\hline SYN14_9_Run 2-- 204 & 1089.3 & 21.9 & 1105.6 & 19.7 & 1137.8 & 38.9 & 1137.8 & 38.9 & 95.7 \\
\hline SYN14_9_Run 2-- 189 & 1149.5 & 21.6 & 1146.0 & 20.9 & 1139.4 & 44.5 & 1139.4 & 44.5 & 100.9 \\
\hline SYN14_9_Run 3-- 241 & 1098.9 & 19.3 & 1112.7 & 18.5 & 1139.9 & 38.8 & 1139.9 & 38.8 & 96.4 \\
\hline SYN14_9_Run 2-- 170 & 1148.6 & 20.2 & 1146.8 & 16.2 & 1143.3 & 27.4 & 1143.3 & 27.4 & 100.5 \\
\hline SYN14_9_Run 1-- 77 & 1124.8 & 19.1 & 1133.5 & 18.7 & 1150.1 & 40.1 & 1150.1 & 40.1 & 97.8 \\
\hline SYN14_9_Run 3-- 289 & 1094.3 & 22.7 & 1114.2 & 21.7 & 1153.3 & 45.1 & 1153.3 & 45.1 & 94.9 \\
\hline SYN14_9_Run 3-- 330 & 1121.7 & 16.1 & 1134.5 & 13.7 & 1159.3 & 25.1 & 1159.3 & 25.1 & 96.8 \\
\hline SYN14_9_Run 3-- 273 & 1182.4 & 21.1 & 1174.7 & 16.1 & 1160.5 & 24.8 & 1160.5 & 24.8 & 101.9 \\
\hline SYN14_9_Run 1-- 64 & 1152.3 & 13.1 & 1155.3 & 14.9 & 1160.9 & 35.0 & 1160.9 & 35.0 & 99.3 \\
\hline SYN14_9_Run 3-- 283 & 1155.1 & 19.5 & 1157.8 & 17.2 & 1163.0 & 33.3 & 1163.0 & 33.3 & 99.3 \\
\hline SYN14_9_Run 3-- 304 & 1103.0 & 10.4 & 1124.4 & 12.7 & 1165.9 & 31.0 & 1165.9 & 31.0 & 94.6 \\
\hline SYN14_9_Run 1-- 7 & 1163.7 & 13.0 & 1164.5 & 11.5 & 1165.9 & 22.5 & 1165.9 & 22.5 & 99.8 \\
\hline SYN14_9_Run 1-- 21 & 1069.9 & 20.3 & 1103.5 & 16.4 & 1170.5 & 26.1 & 1170.5 & 26.1 & 91.4 \\
\hline SYN14_9_Run 2-- 138 & 1152.5 & 21.6 & 1161.2 & 17.5 & 1177.5 & 29.4 & 1177.5 & 29.4 & 97.9 \\
\hline SYN14_9_Run 3-- 321 & 1132.1 & 21.6 & 1148.3 & 19.7 & 1179.1 & 39.1 & 1179.1 & 39.1 & 96.0 \\
\hline SYN14_9_Run 1-- 97 & 1154.8 & 17.3 & 1163.5 & 18.4 & 1179.6 & 41.3 & 1179.6 & 41.3 & 97.9 \\
\hline SYN14_9_Run 3-- 243 & 1147.4 & 28.6 & 1159.1 & 21.7 & 1181.1 & 30.8 & 1181.1 & 30.8 & 97.1 \\
\hline
\end{tabular}




\begin{tabular}{|c|c|c|c|c|c|c|c|c|c|}
\hline SYN14_9_Run 2-- 191 & 1131.6 & 23.5 & 1149.0 & 16.6 & 1181.9 & 16.5 & 1181.9 & 16.5 & 95.7 \\
\hline SYN14_9_Run 3-- 237 & 1143.6 & 13.2 & 1157.2 & 11.8 & 1182.6 & 22.8 & 1182.6 & 22.8 & 96.7 \\
\hline SYN14_9_Run 2-- 178 & 1082.5 & 16.4 & 1117.1 & 16.1 & 1185.1 & 33.7 & 1185.1 & 33.7 & 91.3 \\
\hline SYN14_9_Run 1-- 19 & 1183.1 & 24.6 & 1184.5 & 21.7 & 1187.2 & 41.4 & 1187.2 & 41.4 & 99.6 \\
\hline SYN14_9_Run 1-- 80 & 1141.2 & 18.5 & 1159.2 & 16.2 & 1193.0 & 30.2 & 1193.0 & 30.2 & 95.7 \\
\hline SYN14_9_Run 1-- 61 & 1159.2 & 20.3 & 1171.0 & 26.6 & 1193.0 & 65.6 & 1193.0 & 65.6 & 97.2 \\
\hline SYN14_9_Run 2-- 152 & 1160.8 & 17.6 & 1174.9 & 14.3 & 1200.8 & 24.2 & 1200.8 & 24.2 & 96.7 \\
\hline SYN14_9_Run 3-- 305 & 1213.1 & 17.9 & 1209.5 & 13.6 & 1202.9 & 20.2 & 1202.9 & 20.2 & 100.8 \\
\hline SYN14_9_Run 2-- 200 & 1121.0 & 16.5 & 1150.0 & 14.8 & 1205.3 & 28.1 & 1205.3 & 28.1 & 93.0 \\
\hline SYN14_9_Run 2-- 121 & 1137.2 & 17.7 & 1161.6 & 19.0 & 1207.4 & 42.6 & 1207.4 & 42.6 & 94.2 \\
\hline SYN14_9_Run 2-- 173 & 1194.7 & 16.8 & 1211.2 & 15.7 & 1240.6 & 31.4 & 1240.6 & 31.4 & 96.3 \\
\hline SYN14_9_Run 2-- 133 & 1176.4 & 23.5 & 1199.3 & 19.0 & 1240.7 & 31.1 & 1240.7 & 31.1 & 94.8 \\
\hline SYN14_9_Run 1-- 62 & 1082.3 & 14.2 & 1137.3 & 13.4 & 1244.0 & 26.1 & 1244.0 & 26.1 & 87.0 \\
\hline SYN14_9_Run 3-- 306 & 1186.8 & 27.6 & 1207.8 & 25.7 & 1245.4 & 50.9 & 1245.4 & 50.9 & 95.3 \\
\hline SYN14_9_Run 2-- 116 & 1263.5 & 27.4 & 1260.3 & 23.2 & 1254.8 & 42.1 & 1254.8 & 42.1 & 100.7 \\
\hline SYN14_9_Run 2-- 119 & 1218.5 & 37.2 & 1247.4 & 30.4 & 1297.5 & 50.2 & 1297.5 & 50.2 & 93.9 \\
\hline SYN14_9_Run 1-- 86 & 1295.8 & 22.0 & 1300.7 & 18.8 & 1308.8 & 33.8 & 1308.8 & 33.8 & 99.0 \\
\hline SYN14_9_Run 1-- 100 & 1287.1 & 22.7 & 1298.9 & 20.8 & 1318.4 & 40.0 & 1318.4 & 40.0 & 97.6 \\
\hline SYN14_9_Run 1-- 83 & 1305.7 & 21.7 & 1311.4 & 16.6 & 1320.7 & 25.5 & 1320.7 & 25.5 & 98.9 \\
\hline SYN14_9_Run 1-- 23 & 1328.5 & 32.0 & 1333.1 & 22.9 & 1340.6 & 30.0 & 1340.6 & 30.0 & 99.1 \\
\hline SYN14_9_Run 2-- 140 & 1389.8 & 21.9 & 1371.4 & 16.6 & 1342.8 & 26.0 & 1342.8 & 26.0 & 103.5 \\
\hline SYN14_9_Run 1-- 32 & 1386.6 & 21.0 & 1376.5 & 17.7 & 1360.8 & 31.7 & 1360.8 & 31.7 & 101.9 \\
\hline SYN14_9_Run 2-- 207 & 1290.9 & 24.6 & 1326.8 & 20.2 & 1385.3 & 33.4 & 1385.3 & 33.4 & 93.2 \\
\hline SYN14_9_Run 1-- 89 & 1361.1 & 23.4 & 1372.1 & 17.3 & 1389.3 & 24.7 & 1389.3 & 24.7 & 98.0 \\
\hline SYN14_9_Run 1-- 67 & 1353.1 & 25.7 & 1377.5 & 20.6 & 1415.5 & 33.3 & 1415.5 & 33.3 & 95.6 \\
\hline SYN14_9_Run 2-- 220 & 1451.5 & 26.2 & 1442.4 & 18.3 & 1429.0 & 24.2 & 1429.0 & 24.2 & 101.6 \\
\hline SYN14_9_Run 2-- 128 & 1377.6 & 16.6 & 1401.2 & 12.7 & 1437.3 & 19.2 & 1437.3 & 19.2 & 95.8 \\
\hline SYN14_9_Run 3-- 308 & 1435.1 & 15.7 & 1436.1 & 11.2 & 1437.6 & 15.2 & 1437.6 & 15.2 & 99.8 \\
\hline SYN14_9_Run 2-- 139 & 1388.4 & 30.0 & 1408.6 & 22.9 & 1439.2 & 34.3 & 1439.2 & 34.3 & 96.5 \\
\hline SYN14_9_Run 2-- 210 & 1470.9 & 25.2 & 1458.2 & 18.2 & 1439.9 & 26.1 & 1439.9 & 26.1 & 102.2 \\
\hline SYN14_9_Run 3-- 323 & 1357.9 & 29.7 & 1391.0 & 31.1 & 1442.0 & 63.1 & 1442.0 & 63.1 & 94.2 \\
\hline SYN14_9_Run 3-- 325 & 1420.0 & 23.4 & 1433.3 & 17.8 & 1453.3 & 26.9 & 1453.3 & 26.9 & 97.7 \\
\hline SYN14_9_Run 1-- 45 & 1479.9 & 22.5 & 1473.0 & 18.3 & 1463.1 & 30.9 & 1463.1 & 30.9 & 101.1 \\
\hline SYN14_9_Run 1-- 1 & 1470.1 & 22.0 & 1469.6 & 17.6 & 1468.8 & 29.1 & 1468.8 & 29.1 & 100.1 \\
\hline SYN14_9_Run 3-- 244 & 1479.4 & 22.1 & 1475.1 & 21.3 & 1468.9 & 41.1 & 1468.9 & 41.1 & 100.7 \\
\hline SYN14_9_Run 2-- 208 & 1265.0 & 21.4 & 1348.2 & 22.7 & 1482.9 & 45.5 & 1482.9 & 45.5 & 85.3 \\
\hline SYN14_9_Run 2-- 194 & 1457.0 & 23.5 & 1469.7 & 21.8 & 1488.1 & 40.8 & 1488.1 & 40.8 & 97.9 \\
\hline SYN14_9_Run 2-- 209 & 1515.3 & 23.2 & 1508.4 & 15.8 & 1498.9 & 19.5 & 1498.9 & 19.5 & 101.1 \\
\hline SYN14_9_Run 1-- 60 & 1426.9 & 27.9 & 1462.6 & 23.1 & 1514.8 & 38.6 & 1514.8 & 38.6 & 94.2 \\
\hline
\end{tabular}




\begin{tabular}{|c|c|c|c|c|c|c|c|c|c|}
\hline SYN14_9_Run 2-- 205 & 1441.5 & 21.6 & 1474.2 & 17.9 & 1521.5 & 29.7 & 1521.5 & 29.7 & 94.7 \\
\hline SYN14_9_Run 2-- 127 & 1554.0 & 25.6 & 1553.7 & 20.0 & 1553.3 & 31.7 & 1553.3 & 31.7 & 100.0 \\
\hline SYN14_9_Run 2-- 134 & 1484.4 & 47.7 & 1519.8 & 30.8 & 1569.5 & 28.8 & 1569.5 & 28.8 & 94.6 \\
\hline SYN14_9_Run 1-- 102 & 1353.1 & 39.0 & 1449.5 & 28.0 & 1594.0 & 32.1 & 1594.0 & 32.1 & 84.9 \\
\hline SYN14_9_Run 2-- 144 & 1576.0 & 18.2 & 1583.8 & 15.7 & 1594.1 & 27.4 & 1594.1 & 27.4 & 98.9 \\
\hline SYN14_9_Run 1-- 31 & 1438.8 & 21.8 & 1510.5 & 17.1 & 1612.5 & 25.5 & 1612.5 & 25.5 & 89.2 \\
\hline SYN14_9_Run 3-- 324 & 1589.4 & 25.5 & 1604.3 & 22.0 & 1623.9 & 38.0 & 1623.9 & 38.0 & 97.9 \\
\hline SYN14_9_Run 3-- 329 & 1630.6 & 24.1 & 1628.1 & 17.1 & 1624.8 & 23.6 & 1624.8 & 23.6 & 100.4 \\
\hline SYN14_9_Run 3-- 250 & 1634.1 & 19.4 & 1633.3 & 14.0 & 1632.3 & 20.0 & 1632.3 & 20.0 & 100.1 \\
\hline SYN14_9_Run 2-- 199 & 1584.3 & 27.2 & 1609.6 & 18.0 & 1642.9 & 20.6 & 1642.9 & 20.6 & 96.4 \\
\hline SYN14_9_Run 2-- 182 & 1631.2 & 25.8 & 1639.1 & 19.2 & 1649.1 & 28.5 & 1649.1 & 28.5 & 98.9 \\
\hline SYN14_9_Run 3-- 286 & 1582.5 & 19.6 & 1612.2 & 16.3 & 1651.1 & 26.8 & 1651.1 & 26.8 & 95.8 \\
\hline SYN14_9_Run 2-- 192 & 1617.5 & 19.4 & 1632.9 & 14.3 & 1652.9 & 20.9 & 1652.9 & 20.9 & 97.9 \\
\hline SYN14_9_Run 1-- 29 & 1660.7 & 21.3 & 1657.4 & 15.9 & 1653.3 & 23.7 & 1653.3 & 23.7 & 100.4 \\
\hline SYN14_9_Run 3-- 307 & 1672.6 & 26.9 & 1676.8 & 17.4 & 1682.0 & 19.8 & 1682.0 & 19.8 & 99.4 \\
\hline SYN14_9_Run 1-- 22 & 1638.6 & 26.5 & 1660.5 & 19.6 & 1688.3 & 28.6 & 1688.3 & 28.6 & 97.1 \\
\hline SYN14_9_Run 1-- 96 & 1550.0 & 34.2 & 1614.5 & 25.6 & 1699.7 & 36.2 & 1699.7 & 36.2 & 91.2 \\
\hline SYN14_9_Run 2-- 174 & 1636.8 & 38.8 & 1667.0 & 24.0 & 1705.2 & 21.6 & 1705.2 & 21.6 & 96.0 \\
\hline SYN14_9_Run 1-- 88 & 1603.2 & 24.9 & 1650.7 & 20.9 & 1711.7 & 34.2 & 1711.7 & 34.2 & 93.7 \\
\hline SYN14_9_Run 3-- 327 & 1772.0 & 31.9 & 1746.5 & 18.3 & 1716.0 & 13.7 & 1716.0 & 13.7 & 103.3 \\
\hline SYN14_9_Run 3-- 221 & 1742.0 & 20.2 & 1734.7 & 12.9 & 1725.9 & 14.9 & 1725.9 & 14.9 & 100.9 \\
\hline SYN14_9_Run 3-- 284 & 1739.6 & 32.5 & 1733.5 & 22.0 & 1726.2 & 28.9 & 1726.2 & 28.9 & 100.8 \\
\hline SYN14_9_Run 2-- 202 & 1676.1 & 26.2 & 1698.7 & 16.7 & 1726.6 & 17.8 & 1726.6 & 17.8 & 97.1 \\
\hline SYN14_9_Run 3-- 319 & 1718.7 & 30.8 & 1723.9 & 28.7 & 1730.2 & 51.2 & 1730.2 & 51.2 & 99.3 \\
\hline SYN14_9_Run 1-- 68 & 1768.8 & 30.0 & 1760.4 & 18.9 & 1750.5 & 21.2 & 1750.5 & 21.2 & 101.0 \\
\hline SYN14_9_Run 3-- 315 & 1710.8 & 28.7 & 1729.5 & 18.8 & 1752.2 & 22.3 & 1752.2 & 22.3 & 97.6 \\
\hline SYN14_9_Run 2-- 135 & 1719.8 & 29.6 & 1740.5 & 19.1 & 1765.3 & 21.9 & 1765.3 & 21.9 & 97.4 \\
\hline SYN14_9_Run 1-- 99 & 1743.4 & 29.0 & 1763.9 & 19.5 & 1788.1 & 24.6 & 1788.1 & 24.6 & 97.5 \\
\hline SYN14_9_Run 1-- 84 & 1813.2 & 36.1 & 1806.9 & 20.3 & 1799.6 & 13.9 & 1799.6 & 13.9 & 100.8 \\
\hline SYN14_9_Run 1-- 28 & 1772.4 & 23.4 & 1785.8 & 15.5 & 1801.6 & 19.5 & 1801.6 & 19.5 & 98.4 \\
\hline SYN14_9_Run 2-- 166 & 1844.2 & 27.1 & 1826.7 & 19.3 & 1806.8 & 27.7 & 1806.8 & 27.7 & 102.1 \\
\hline SYN14_9_Run 2-- 198 & 1513.4 & 39.2 & 1646.5 & 27.2 & 1820.9 & 29.6 & 1820.9 & 29.6 & 83.1 \\
\hline SYN14_9_Run 3-- 265 & 1687.5 & 25.5 & 1748.3 & 15.8 & 1821.8 & 14.8 & 1821.8 & 14.8 & 92.6 \\
\hline SYN14_9_Run 1-- 8 & 1641.6 & 24.9 & 1732.9 & 17.6 & 1844.8 & 22.2 & 1844.8 & 22.2 & 89.0 \\
\hline SYN14_9_Run 1-- 66 & 1880.3 & 29.7 & 1866.8 & 19.8 & 1851.7 & 25.8 & 1851.7 & 25.8 & 101.5 \\
\hline SYN14_9_Run 2-- 218 & 1829.1 & 24.6 & 1840.5 & 15.8 & 1853.4 & 18.6 & 1853.4 & 18.6 & 98.7 \\
\hline SYN14_9_Run 1-- 20 & 1665.1 & 28.2 & 1753.8 & 19.8 & 1861.2 & 25.2 & 1861.2 & 25.2 & 89.5 \\
\hline SYN14_9_Run 3-- 317 & 1820.6 & 23.9 & 1842.6 & 17.0 & 1867.4 & 23.6 & 1867.4 & 23.6 & 97.5 \\
\hline SYN14_9_Run 2-- 118 & 1982.5 & 28.1 & 1938.1 & 18.0 & 1891.1 & 22.7 & 1891.1 & 22.7 & 104.8 \\
\hline
\end{tabular}




\begin{tabular}{|l|l|l|l|l|l|l|l|l|l|} 
SYN14_9_Run 3-- 222 & 1864.0 & 32.6 & 1885.6 & 21.0 & 1909.5 & 25.1 & 1909.5 & 25.1 & 97.6 \\
\hline SYN14_9_Run 3-- 322 & 1910.5 & 27.1 & 1918.3 & 16.3 & 1926.9 & 16.7 & 1926.9 & 16.7 & 99.1 \\
\hline SYN14_9_Run 1-- 17 & 1961.9 & 37.3 & 1956.5 & 20.2 & 1950.7 & 13.8 & 1950.7 & 13.8 & 100.6 \\
\hline SYN14_9_Run 2-- 190 & 1888.4 & 23.6 & 1935.7 & 16.2 & 1986.7 & 21.4 & 1986.7 & 21.4 & 95.0 \\
\hline SYN14_9_Run 2-- 175 & 1946.1 & 27.3 & 1968.8 & 16.9 & 1992.7 & 19.1 & 1992.7 & 19.1 & 97.7 \\
\hline SYN14_9_Run 2-- 142 & 2007.3 & 25.5 & 2021.7 & 15.5 & 2036.4 & 17.3 & 2036.4 & 17.3 & 98.6 \\
\hline SYN14_9_Run 1-- 55 & 2440.1 & 33.6 & 2455.0 & 17.4 & 2467.3 & 15.0 & 2467.3 & 15.0 & 98.9 \\
\hline SYN14_9_Run 1-- 95 & 2592.9 & 43.1 & 2585.4 & 21.2 & 2579.5 & 17.0 & 2579.5 & 17.0 & 100.5 \\
\hline SYN14_9_Run 2-- 113 & 2590.5 & 39.7 & 2611.0 & 24.8 & 2626.9 & 31.2 & 2626.9 & 31.2 & 98.6 \\
\hline SYN14_9_Run 1-- 10 & 2518.6 & 47.9 & 2596.0 & 23.2 & 2657.0 & 15.4 & 2657.0 & 15.4 & 94.8 \\
\hline SYN14_9_Run 1-- 74 & 2693.2 & 29.4 & 2686.9 & 16.7 & 2682.2 & 19.2 & 2682.2 & 19.2 & 100.4 \\
\hline SYN14_9_Run 2-- 124 & 2422.9 & 34.6 & 2567.6 & 21.3 & 2683.9 & 24.9 & 2683.9 & 24.9 & 90.3 \\
\hline SYN14_9_Run 3-- 287 & 2655.7 & 34.7 & 2684.3 & 20.1 & 2705.9 & 23.3 & 2705.9 & 23.3 & 98.1 \\
\hline SYN14_9_Run 2-- 120 & 2619.4 & 48.7 & 2678.5 & 25.4 & 2723.4 & 24.0 & 2723.4 & 24.0 & 96.2 \\
\hline SYN14_9_Run 1-- 34 & 2666.1 & 43.7 & 2700.0 & 20.9 & 2725.5 & 15.7 & 2725.5 & 15.7 & 97.8 \\
\hline SYN14_9_Run 1-- 78 & 2637.6 & 48.6 & 2689.8 & 24.4 & 2729.2 & 21.0 & 2729.2 & 21.0 & 96.6 \\
\hline SYN14_9_Run 3-- 312 & 2739.2 & 56.8 & 2792.3 & 26.4 & 2830.8 & 18.1 & 2830.8 & 18.1 & 96.8 \\
\hline SYN14_9_Run 2-- 141 & 2841.8 & 42.5 & 2846.9 & 20.3 & 2850.5 & 17.3 & 2850.5 & 17.3 & 99.7 \\
\hline SYN14_9_Run 3-- 253 & 3121.5 & 73.0 & 3196.8 & 30.9 & 3244.3 & 18.5 & 3244.3 & 18.5 & 96.2 \\
\hline
\end{tabular}

\begin{tabular}{|l|l|l|l|l|l|l|l|l|l|}
\hline Analysis & $206 \mathrm{~Pb}^{*}$ & \pm & $207 \mathrm{~Pb}^{*}$ & \pm & $206 \mathrm{~Pb}^{*}$ & \pm & Best age & \pm & Conc \\
\hline & $238 \mathrm{U}^{*}$ & $(\mathrm{Ma})$ & $235 \mathrm{U}$ & $(\mathrm{Ma})$ & $207 \mathrm{~Pb}^{*}$ & $(\mathrm{Ma})$ & $(\mathrm{Ma})$ & $(\mathrm{Ma})$ & $(\%)$ \\
\hline & & & & & & & & & \\
\hline SYN14-10 run 1-- 53 & 110.1 & 2.5 & 111.8 & 9.4 & 147.5 & 202.4 & 110.1 & 2.5 & NA \\
\hline SYN14-10 run 1-- 89 & 112.7 & 3.6 & 115.8 & 10.9 & 179.7 & 220.0 & 112.7 & 3.6 & NA \\
\hline SYN14-10 run 1-- 39 & 114.2 & 3.1 & 108.9 & 7.2 & 5.0 & 153.9 & 114.2 & 3.1 & NA \\
\hline SYN14-10 run 1-- 40 & 115.2 & 4.2 & 115.4 & 10.2 & 120.4 & 203.2 & 115.2 & 4.2 & NA \\
\hline SYN14-10 run 1-- 47 & 115.3 & 4.3 & 128.0 & 9.6 & 371.1 & 158.5 & 115.3 & 4.3 & NA \\
\hline SYN14-10 run 1-- 96 & 119.1 & 4.4 & 113.7 & 9.1 & 1.5 & 182.4 & 119.1 & 4.4 & NA \\
\hline SYN14-10 run 1-- 103 & 119.9 & 4.8 & 115.1 & 8.4 & 18.6 & 156.6 & 119.9 & 4.8 & NA \\
\hline SYN14-10 run 1-- 87 & 188.5 & 5.4 & 311.2 & 8.8 & 1375.1 & $z 8.9$ & 188.5 & 5.4 & NA \\
\hline SYN14-10 run 1-- 102 & 237.2 & 5.0 & 245.0 & 7.1 & 320.5 & 55.0 & 237.2 & 5.0 & NA \\
\hline SYN14-10 run 1-- 23 & 246.1 & 4.1 & 246.6 & 6.0 & 251.1 & 48.9 & 246.1 & 4.1 & NA \\
\hline SYN14-10 run 1-- 110 & 246.6 & 6.6 & 246.0 & 8.4 & 240.3 & 62.2 & 246.6 & 6.6 & NA \\
\hline SYN14-10 run 1-- 49 & 268.0 & 6.7 & 270.0 & 16.6 & 286.8 & 149.0 & 268.0 & 6.7 & NA \\
\hline SYN14-10 run 1-- 97 & 287.9 & 10.2 & 311.0 & 17.4 & 488.3 & 118.7 & 287.9 & 10.2 & NA \\
\hline SYN14-10 run 1-- 99 & 322.8 & 5.6 & 325.0 & 9.0 & 341.4 & 61.3 & 322.8 & 5.6 & NA \\
\hline SYN14-10 run 1-- 9 & 353.8 & 7.2 & 361.3 & 8.3 & 409.9 & 39.7 & 353.8 & 7.2 & NA \\
\hline
\end{tabular}




\begin{tabular}{|c|c|c|c|c|c|c|c|c|c|}
\hline SYN14-10 run 1-- 84 & 409.0 & 8.1 & 411.0 & 9.4 & 422.6 & 42.4 & 409.0 & 8.1 & 96.8 \\
\hline SYN14-10 run 1-- 13 & 412.8 & 8.5 & 411.2 & 11.0 & 402.4 & 55.2 & 412.8 & 8.5 & 102.6 \\
\hline SYN14-10 run 1-- 26 & 426.8 & 10.7 & 500.0 & 34.4 & 851.0 & 173.1 & 426.8 & 10.7 & 50.1 \\
\hline SYN14-10 run 1-- 57 & 434.1 & 10.8 & 426.8 & 12.5 & 387.5 & 56.4 & 434.1 & 10.8 & 112.0 \\
\hline SYN14-10 run 1-- 73 & 435.8 & 11.9 & 440.9 & 12.2 & 467.5 & 42.2 & 435.8 & 11.9 & 93.2 \\
\hline SYN14-10 run 1-- 29 & 438.8 & 14.8 & 417.7 & 19.6 & 302.3 & 103.7 & 438.8 & 14.8 & 145.1 \\
\hline SYN14-10 run 1-- 65 & 482.6 & 10.1 & 484.6 & 12.3 & 494.2 & 51.2 & 482.6 & 10.1 & 97.7 \\
\hline SYN14-10 run 1-- 12 & 510.7 & 15.3 & 518.2 & 20.6 & 551.6 & 87.8 & 510.7 & 15.3 & 92.6 \\
\hline SYN14-10 run 1-- 36 & 528.2 & 14.4 & 533.1 & 15.2 & 554.2 & 50.6 & 528.2 & 14.4 & 95.3 \\
\hline SYN14-10 run 1-- 59 & 545.8 & 11.8 & 539.6 & 13.1 & 513.3 & 48.1 & 545.8 & 11.8 & 106.3 \\
\hline SYN14-10 run 1-- 64 & 551.6 & 14.4 & 553.2 & 13.4 & 559.9 & 34.6 & 551.6 & 14.4 & 98.5 \\
\hline SYN14-10 run 1-- 104 & 558.0 & 9.3 & 571.3 & 11.6 & 624.9 & 43.3 & 558.0 & 9.3 & 89.3 \\
\hline SYN14-10 run 1-- 83 & 576.0 & 10.8 & 582.7 & 13.7 & 609.2 & 51.5 & 576.0 & 10.8 & 94.6 \\
\hline SYN14-10 run 1-- 37 & 580.4 & 10.8 & 592.4 & 11.6 & 638.9 & 36.9 & 580.4 & 10.8 & 90.8 \\
\hline SYN14-10 run 1-- 54 & 582.3 & 15.8 & 600.7 & 25.7 & 670.8 & 104.8 & 582.3 & 15.8 & 86.8 \\
\hline SYN14-10 run 1-- 45 & 584.4 & 12.3 & 587.2 & 18.7 & 598.4 & 77.0 & 584.4 & 12.3 & 97.7 \\
\hline SYN14-10 run 1-- 56 & 589.6 & 22.7 & 610.6 & 23.1 & 689.2 & 64.2 & 589.6 & 22.7 & 85.6 \\
\hline SYN14-10 run 1-- 106 & 589.7 & 9.4 & 592.1 & 16.9 & 601.2 & 72.9 & 589.7 & 9.4 & 98.1 \\
\hline SYN14-10 run 1-- 92 & 599.4 & 12.8 & 607.7 & 11.9 & 638.5 & 28.1 & 599.4 & 12.8 & 93.9 \\
\hline SYN14-10 run 1-- 5 & 606.0 & 16.1 & 611.0 & 17.3 & 629.7 & 54.7 & 606.0 & 16.1 & 96.2 \\
\hline SYN14-10 run 1-- 51 & 610.3 & 26.2 & 613.4 & 23.6 & 624.9 & 52.8 & 610.3 & 26.2 & 97.7 \\
\hline SYN14-10 run 1-- 81 & 613.3 & 16.1 & 614.4 & 14.2 & 618.4 & 30.5 & 613.3 & 16.1 & 99.2 \\
\hline SYN14-10 run 1-- 78 & 618.6 & 15.1 & 625.5 & 16.3 & 650.7 & 51.0 & 618.6 & 15.1 & 95.1 \\
\hline SYN14-10 run 1-- 77 & 1015.7 & 20.5 & 995.9 & 20.2 & 952.6 & 47.5 & 952.6 & 47.5 & 106.6 \\
\hline SYN14-10 run 1-- 7 & 938.2 & 14.3 & 943.9 & 16.1 & 957.1 & 41.6 & 957.1 & 41.6 & 98.0 \\
\hline SYN14-10 run 1-- 86 & 990.1 & 13.2 & 984.6 & 16.2 & 972.4 & 43.5 & 972.4 & 43.5 & 101.8 \\
\hline SYN14-10 run 1-- 85 & 1003.7 & 20.0 & 995.5 & 17.5 & 977.4 & 35.5 & 977.4 & 35.5 & 102.7 \\
\hline SYN14-10 run 1-- 93 & 1055.9 & 12.3 & 1033.2 & 13.5 & 985.3 & 33.4 & 985.3 & 33.4 & 107.2 \\
\hline SYN14-10 run 1-- 88 & 1023.2 & 18.3 & 1013.5 & 15.9 & 992.5 & 31.6 & 992.5 & 31.6 & 103.1 \\
\hline SYN14-10 run 1-- 44 & 987.3 & 21.7 & 992.0 & 19.9 & 1002.3 & 41.7 & 1002.3 & 41.7 & 98.5 \\
\hline SYN14-10 run 1-- 79 & 1002.0 & 16.4 & 1002.8 & 12.9 & 1004.4 & 19.8 & 1004.4 & 19.8 & 99.8 \\
\hline SYN14-10 run 1-- 75 & 1029.7 & 23.5 & 1022.7 & 19.2 & 1007.9 & 34.0 & 1007.9 & 34.0 & 102.2 \\
\hline SYN14-10 run 1-- 63 & 1071.4 & 20.7 & 1052.8 & 17.7 & 1014.6 & 34.6 & 1014.6 & 34.6 & 105.6 \\
\hline SYN14-10 run 1-- 52 & 972.4 & 20.9 & 985.8 & 17.0 & 1015.6 & 28.1 & 1015.6 & 28.1 & 95.7 \\
\hline SYN14-10 run 1-- 35 & 1058.6 & 22.6 & 1046.5 & 24.6 & 1021.2 & 60.3 & 1021.2 & 60.3 & 103.7 \\
\hline SYN14-10 run 1-- 60 & 1011.3 & 20.5 & 1016.3 & 22.2 & 1027.3 & 54.1 & 1027.3 & 54.1 & 98.4 \\
\hline SYN14-10 run 1-- 18 & 1028.9 & 14.5 & 1029.6 & 13.9 & 1031.0 & 30.5 & 1031.0 & 30.5 & 99.8 \\
\hline SYN14-10 run 1-- 91 & 1030.2 & 36.9 & 1031.0 & 27.5 & 1032.7 & 35.5 & 1032.7 & 35.5 & 99.8 \\
\hline SYN14-10 run 1-- 38 & 1054.3 & 17.1 & 1048.4 & 15.7 & 1036.2 & 33.2 & 1036.2 & 33.2 & 101.7 \\
\hline
\end{tabular}




\begin{tabular}{|c|c|c|c|c|c|c|c|c|c|}
\hline SYN14-10 run 1-- 19 & 999.6 & 20.0 & 1013.0 & 19.4 & 1042.1 & 42.9 & 1042.1 & 42.9 & 95.9 \\
\hline SYN14-10 run 1-- 2 & 1038.2 & 14.6 & 1042.6 & 12.7 & 1051.7 & 24.6 & 1051.7 & 24.6 & 98.7 \\
\hline SYN14-10 run 1-- 95 & 1041.9 & 23.8 & 1045.3 & 17.8 & 1052.3 & 23.3 & 1052.3 & 23.3 & 99.0 \\
\hline SYN14-10 run 1-- 33 & 1006.9 & 23.8 & 1024.2 & 21.4 & 1061.5 & 42.7 & 1061.5 & 42.7 & 94.8 \\
\hline SYN14-10 run 1-- 62 & 1077.1 & 16.6 & 1078.0 & 15.7 & 1080.0 & 33.7 & 1080.0 & 33.7 & 99.7 \\
\hline SYN14-10 run 1-- 1 & 1019.7 & 17.5 & 1039.6 & 16.6 & 1081.8 & 34.9 & 1081.8 & 34.9 & 94.3 \\
\hline SYN14-10 run 1-- 4 & 1102.0 & 60.1 & 1096.6 & 41.7 & 1085.9 & 36.7 & 1085.9 & 36.7 & 101.5 \\
\hline SYN14-10 run 1-- 14 & 1041.0 & 23.4 & 1056.6 & 20.2 & 1089.0 & 37.8 & 1089.0 & 37.8 & 95.6 \\
\hline SYN14-10 run 1-- 42 & 1118.9 & 38.5 & 1111.3 & 31.4 & 1096.4 & 55.1 & 1096.4 & 55.1 & 102.1 \\
\hline SYN14-10 run 1-- 69 & 1050.1 & 32.4 & 1065.5 & 26.0 & 1097.0 & 41.6 & 1097.0 & 41.6 & 95.7 \\
\hline SYN14-10 run 1-- 70 & 1032.1 & 21.1 & 1054.3 & 20.0 & 1100.6 & 41.8 & 1100.6 & 41.8 & 93.8 \\
\hline SYN14-10 run 1-- 17 & 1042.7 & 16.6 & 1063.6 & 14.9 & 1106.6 & 29.4 & 1106.6 & 29.4 & 94.2 \\
\hline SYN14-10 ru & 1014.7 & 20.0 & 1046.0 & 20.6 & 1111.9 & 46.3 & 1111.9 & 46.3 & 91.3 \\
\hline SYN14-10 run 1-- 74 & 967.0 & 19.0 & 1013.5 & 23.1 & 1115.3 & 58.1 & 1115.3 & 58.1 & 86.7 \\
\hline SYN14-10 run 1-- 68 & 1114.4 & 27.7 & 1119.7 & 24.6 & 1130.1 & 48.3 & 1130.1 & 48.3 & 98.6 \\
\hline SYN14-10 run 1-- 67 & 1154.5 & 20.3 & 1150.5 & 18.8 & 1143.0 & 38.5 & 1143.0 & 38.5 & 101.0 \\
\hline SYN14-10 run 1-- 82 & 1111.5 & 21.3 & 1122.2 & 19.4 & 1143.1 & 38.6 & 1143.1 & 38.6 & 97.2 \\
\hline SYN14-10 run 1-- 109 & 1139.8 & 16.7 & 1142.8 & 13.8 & 1148.6 & 24.5 & 1148.6 & 24.5 & 99.2 \\
\hline SYN14-10 run 1-- 3 & 1186.9 & 17.0 & 1187.8 & 13.5 & 1189.3 & 22.2 & 1189.3 & 22.2 & 99.8 \\
\hline SYN14-10 run 1-- 41 & 1238.4 & 22.1 & 1231.6 & 16.3 & 1219.8 & 22.8 & 1219.8 & 22.8 & 101.5 \\
\hline SYN14-10 run 1-- 20 & 1038.0 & 18.9 & 1101.3 & 15.7 & 1228.5 & 24.7 & 1228.5 & 24.7 & 84.5 \\
\hline SYN14-10 run 1-- 21 & 1143.0 & 23.7 & 1180.1 & 23.6 & 1248.6 & 49.2 & 1248.6 & 49.2 & 91.5 \\
\hline SYN14-10 run 1-- 8 & 1318.1 & 44.0 & 1307.1 & 28.7 & 1289.2 & 24.3 & 1289.2 & 24.3 & 102.2 \\
\hline SYN14-10 run 1-- 11 & 1081.3 & 15.5 & 1165.2 & 17.9 & 1324.8 & 40.0 & 1324.8 & 40.0 & 81.6 \\
\hline SYN14-10 run 1-- 46 & 1259.3 & 17.0 & 1. & 13.1 & 1338.9 & 19.4 & 1338.9 & 19.4 & 94.1 \\
\hline SYN14-10 run 1-- 107 & 1431.3 & 21.7 & 1429.4 & 16.0 & 1426.6 & 23.3 & 1426.6 & 23.3 & 100.3 \\
\hline SYN14-10 run 1-- 27 & 1368.9 & 18.4 & 1393.9 & 16.7 & 1432.4 & 31.1 & 1432.4 & 31.1 & 95.6 \\
\hline SYN14-10 run 1-- 98 & 1432.1 & 31.9 & 1436.5 & 21.9 & 1442.9 & 26.8 & 1442.9 & 26.8 & 99.3 \\
\hline SYN14-10 run 1-- 22 & 1433.9 & 23.4 & 1440.6 & 19.5 & 1450.4 & 33.5 & 1450.4 & 33.5 & 98.9 \\
\hline SYN14-10 run 1-- 10 & 1420.9 & 64.8 & 1435.2 & 39.7 & 1456.3 & 18.0 & 1456.3 & 18.0 & 97.6 \\
\hline SYN14-10 run 1-- 16 & 1442.2 & 48.8 & 1454.6 & 31.7 & 1472.7 & 30.2 & 1472.7 & 30.2 & 97.9 \\
\hline SYN14-10 run 1-- 105 & 1127.9 & 30.1 & 1253.0 & 23.5 & 1474.9 & 28.5 & 1474.9 & 28.5 & 76.5 \\
\hline SYN14-10 run 1-- 90 & 1487.1 & 27.5 & 1495.3 & 26.3 & 1506.9 & 50.1 & 1506.9 & 50.1 & 98.7 \\
\hline SYN14-10 run 1-- 66 & 1565.2 & 19.8 & 1560.6 & 14.9 & 1554.4 & 22.8 & 1554.4 & 22.8 & 100.7 \\
\hline SYN14-10 run 1-- 61 & 1605.0 & 40.6 & 1622.3 & 25.4 & 1644.8 & 24.0 & 1644.8 & 24.0 & 97.6 \\
\hline SYN14-10 run 1-- 24 & 1649.3 & 29.3 & 1653.6 & 18.8 & 1659.2 & 20.6 & 1659.2 & 20.6 & 99.4 \\
\hline SYN14-10 run 1-- 94 & 1708.0 & 32.4 & 1714.7 & 20.2 & 1722.8 & 20.8 & 1722.8 & 20.8 & 99.1 \\
\hline SYN14-10 run 1-- 108 & 1711.4 & 54.6 & 1720.1 & 31.8 & 1730.7 & 22.8 & 1730.7 & 22.8 & 98.9 \\
\hline SYN14-10 run 1-- 72 & 1719.1 & 26.3 & 1724.7 & 16.4 & 1731.4 & 17.2 & 1731.4 & 17.2 & 99.3 \\
\hline
\end{tabular}




\begin{tabular}{|c|c|c|c|c|c|c|c|c|c|}
\hline SYN14-10 run 1-- 80 & 1693.3 & 31.2 & 1723.0 & 20.8 & 1759.3 & 25.2 & 1759.3 & 25.2 & 96.2 \\
\hline SYN14-10 run 1-- 15 & 1759.6 & 25.1 & 1760.0 & 16.8 & 1760.4 & 21.5 & 1760.4 & 21.5 & 100.0 \\
\hline SYN14-10 run 1-- 50 & 1738.0 & 38.2 & 1748.3 & 24.8 & 1760.7 & 29.0 & 1760.7 & 29.0 & 98.7 \\
\hline SYN14-10 run 1-- 101 & 1781.6 & 31.6 & 1782.5 & 20.2 & 1783.6 & 23.7 & 1783.6 & 23.7 & 99.9 \\
\hline SYN14-10 run 1-- 28 & 1748.4 & 26.7 & 1782.1 & 21.9 & 1821.7 & 35.3 & 1821.7 & 35.3 & 96.0 \\
\hline SYN14-10 run 1-- 100 & 1817.7 & 57.2 & 1842.1 & 33.1 & 1869.8 & 26.2 & 1869.8 & 26.2 & 97.2 \\
\hline SYN14-10 run 1-- 30 & 1833.1 & 27.2 & 1852.8 & 22.8 & 1875.0 & 37.1 & 1875.0 & 37.1 & 97.8 \\
\hline SYN14-10 run 1-- 71 & 1904.4 & 20.4 & 1894.4 & 14.4 & 1883.3 & 20.5 & 1883.3 & 20.5 & 101.1 \\
\hline SYN14-10 run 1-- 48 & 1939.2 & 31.4 & 1960.5 & 19.6 & 1983.0 & 22.4 & 1983.0 & 22.4 & 97.8 \\
\hline SYN14-10 run 1-- 31 & 2008.9 & 49.2 & 2062.3 & 26.8 & 2116.0 & 18.3 & 2116.0 & 18.3 & 94.9 \\
\hline SYN14-10 run 1-- 34 & 2100.4 & 35.0 & 2143.2 & 22.0 & 2184.5 & 26.0 & 2184.5 & 26.0 & 96.2 \\
\hline SYN14-10 run 1-- 58 & 2473.9 & 50.8 & 2499.3 & 26.5 & 2520.1 & 24.0 & 2520.1 & 24.0 & 98.2 \\
\hline SYN14-10 run 1-- 25 & 2512.1 & 47.8 & 2541.3 & 23.3 & 2564.6 & 16.4 & 2564.6 & 16.4 & 98.0 \\
\hline SYN14-10 run 1-- 43 & 2553.7 & 39.0 & 2628.9 & 20.4 & 2687.2 & 18.8 & 2687.2 & 18.8 & 95.0 \\
\hline SYN14-10 run 1-- 55 & 657.9 & 236.7 & 1362.8 & 346.2 & 2749.5 & 389.3 & 2749.5 & 389.3 & 23.9 \\
\hline SYN14-10 run 1-- 76 & 2911.5 & 45.8 & 2882.2 & 26.8 & 2861.8 & 32.8 & 2861.8 & 32.8 & 101.7 \\
\hline SYN14-10 Run2- 126 & 113.2 & 2.7 & 107.3 & 6.8 & 20.9 & 150.7 & 113.2 & 2.7 & NA \\
\hline SYN14-10 Run2- 192 & 114.6 & 6.9 & 118.0 & 12.5 & 186.5 & 220.6 & 4.6 & 6.9 & NA \\
\hline SYN14-10 Run2- 147 & 115.9 & 4.1 & 123.8 & 7.1 & 277.6 & 112.3 & .9 & 4.1 & NA \\
\hline SYN14-10 Run2- 178 & 11 & 3.3 & 106.5 & 7.3 & 8 & .6 & 1 & 3.3 & NA \\
\hline SYN14-10 Run2- 215 & 167.0 & 2.5 & 170.9 & 8.5 & 2 & 9.9 & .0 & 2.5 & NA \\
\hline SYN14-10 Run2- 163 & 227.1 & 8.9 & 229.6 & 12.4 & 2 & 4.5 & .1 & 8.9 & NA \\
\hline SYN14-10 Run2- 145 & 231.1 & 6.3 & 230.4 & 15.0 & 223.0 & 155.2 & 231.1 & 6.3 & NA \\
\hline SYN14-10 Run2- 116 & 247.2 & 7.4 & 251.3 & 8.6 & 290.0 & 53.8 & 247.2 & 7.4 & NA \\
\hline SYN14-10 Run2- 144 & 248.3 & 4.5 & 254.7 & 8.1 & 314.1 & 70.1 & 248.3 & 4.5 & NA \\
\hline SYN14-10 Run2- 121 & 262.7 & 5.4 & 268.1 & 7.2 & 315.0 & 51.1 & 262.7 & 5.4 & NA \\
\hline SYN14-10 Run2- 142 & 272.0 & 5.1 & 274.8 & 10.6 & 298.9 & 90.9 & 272.0 & 5.1 & NA \\
\hline SYN14-10 Run2- 216 & 315.2 & 5.7 & 319.4 & 11.5 & 349.5 & 84.9 & 315.2 & 5.7 & NA \\
\hline SYN14-10 Run2- 187 & 331.0 & 5.4 & 328.4 & 7.3 & 309.4 & 45.3 & 331.0 & 5.4 & NA \\
\hline SYN14-10 Run2- 119 & 363.4 & 5.4 & 365.4 & 5.9 & 377.6 & 26.5 & 363.4 & 5.4 & NA \\
\hline SYN14-10 Run2- 206 & 368.5 & 7.3 & 379.2 & 8.8 & 444.9 & 41.6 & 368.5 & 7.3 & NA \\
\hline SYN14-10 Run2- 166 & 372.2 & 5.2 & 370.8 & 11.1 & 361.8 & 73.6 & 372.2 & 5.2 & NA \\
\hline SYN14-10 Run2- 203 & 375.9 & 4.9 & 376.9 & 10.1 & 383.0 & 65.5 & 375.9 & 4.9 & NA \\
\hline SYN14-10 Run2- 172 & 390.1 & 6.3 & 389.9 & 7.4 & 388.4 & 35.4 & 390.1 & 6.3 & NA \\
\hline SYN14-10 Run2- 164 & 391.2 & 15.5 & 399.1 & 15.1 & 445.1 & 46.0 & 391.2 & 15.5 & NA \\
\hline SYN14-10 Run2- 129 & 402.2 & 10.9 & 403.7 & 10.5 & 412.0 & 32.0 & 402.2 & 10.9 & 97.6 \\
\hline SYN14-10 Run2- 208 & 420.4 & 7.7 & 429.6 & 9.6 & 478.7 & 44.2 & 420.4 & 7.7 & 87.8 \\
\hline SYN14-10 Run2- 139 & 425.1 & 9.6 & 421.8 & 10.3 & 404.0 & 41.8 & 425.1 & 9.6 & 105.2 \\
\hline SYN14-10 Run2- 134 & 426.0 & 10.4 & 431.9 & 15.2 & 463.5 & 78.1 & 426.0 & 10.4 & 91.9 \\
\hline
\end{tabular}




\begin{tabular}{|c|c|c|c|c|c|c|c|c|c|}
\hline SYN14-10 Run2- 220 & 426.8 & 7.8 & 427.6 & 11.5 & 431.8 & 59.7 & 426.8 & 7.8 & 98.8 \\
\hline SYN14-10 Run2- 168 & 429.0 & 11.2 & 426.6 & 11.4 & 413.8 & 41.6 & 429.0 & 11.2 & 103.7 \\
\hline SYN14-10 Run2- 205 & 437.3 & 10.3 & 436.5 & 11.1 & 431.9 & 43.3 & 437.3 & 10.3 & 101.3 \\
\hline SYN14-10 Run2- 217 & 470.3 & 40.8 & 572.7 & 55.5 & 1003.1 & 181.2 & 470.3 & 40.8 & 46.9 \\
\hline SYN14-10 Run2- 167 & 473.2 & 9.8 & 476.6 & 15.3 & 492.9 & 74.6 & 473.2 & 9.8 & 96.0 \\
\hline SYN14-10 Run2- 194 & 534.9 & 9.0 & 558.1 & 14.0 & 654.3 & 58.7 & 534.9 & 9.0 & 81.7 \\
\hline SYN14-10 Run2- 151 & 548.3 & 9.1 & 554.0 & 12.0 & 577.8 & 48.1 & 548.3 & 9.1 & 94.9 \\
\hline SYN14-10 Run2- 189 & 566.7 & 12.1 & 570.8 & 12.5 & 587.5 & 38.7 & 566.7 & 12.1 & 96.5 \\
\hline SYN14-10 Run2- 112 & 573.5 & 24.8 & 583.3 & 26.8 & 621.5 & 86.7 & 573.5 & 24.8 & 92.3 \\
\hline SYN14-10 Run2- 211 & 600.0 & 9.2 & 607.3 & 10.2 & 634.7 & 33.1 & 600.0 & 9.2 & 94.5 \\
\hline SYN14-10 Run2- 169 & 610.1 & 13.2 & 606.8 & 16.2 & 594.6 & 59.4 & 610.1 & 13.2 & 102.6 \\
\hline SYN14-10 Run2- 165 & 622.0 & 10.9 & 616.6 & 11.3 & 597.2 & 34.8 & 622.0 & 10.9 & 104.2 \\
\hline SYN14-10 Run2- 197 & 625.8 & 7.9 & 630.6 & 10.0 & 648.2 & 36.1 & 625.8 & 7.9 & 96.5 \\
\hline SYN14-10 Run2- 152 & 628.1 & 12.7 & 634.9 & 12.5 & 659.2 & 34.3 & 628.1 & 12.7 & 95.3 \\
\hline SYN14-10 Run2- 118 & 635.3 & 10.8 & 635.3 & 11.2 & 635.4 & 34.1 & 635.3 & 10.8 & 100.0 \\
\hline SYN14-10 Run2- 159 & 673.3 & 18.7 & 680.3 & 16.3 & 703.6 & 31.9 & 673.3 & 18.7 & 95.7 \\
\hline SYN14-10 Run2- 155 & 836.9 & 22.7 & 827.5 & 18.1 & 802.4 & 28.9 & 836.9 & 22.7 & 104.3 \\
\hline SYN14-10 Run2- 150 & 1015.9 & 12.7 & 996.0 & 16.0 & 952.4 & 43.5 & 952.4 & 43.5 & 106.7 \\
\hline SYN14-10 Run2- 174 & 976.6 & 15.5 & 970.0 & 17.0 & 955.1 & 43.4 & 955.1 & 43.4 & 102.2 \\
\hline SYN14-10 Run2- 213 & 870.6 & 19.4 & 900.9 & 18.7 & 975.9 & 41.4 & 975.9 & 41.4 & 89.2 \\
\hline SYN14-10 Run2- 128 & 1011.6 & 30.7 & 1006.1 & 24.2 & 994.2 & 38.5 & 994.2 & 38.5 & 101.7 \\
\hline SYN14-10 Run2- 190 & 957.7 & 20.8 & 973.3 & 17.6 & 1008.8 & 31.9 & 1008.8 & 31.9 & 94.9 \\
\hline SYN14-10 Run2- 195 & 999.0 & 19.6 & 1003.2 & 18.4 & 1012.4 & 39.9 & 1012.4 & 39.9 & 98.7 \\
\hline SYN14-10 Run2- 120 & 1014.7 & 20.9 & 1015.1 & 19.2 & 1015.9 & 40.3 & 1015.9 & 40.3 & 99.9 \\
\hline SYN14-10 Run2- 113 & 1012.2 & 21.0 & 1015.7 & 18.4 & 1023.3 & 36.1 & 1023.3 & 36.1 & 98.9 \\
\hline SYN14-10 Run2- 125 & 1010.6 & 18.2 & 1015.1 & 19.6 & 1024.8 & 47.8 & 1024.8 & 47.8 & 98.6 \\
\hline SYN14-10 Run2- 153 & 1006.5 & 23.0 & 1012.5 & 18.9 & 1025.4 & 32.6 & 1025.4 & 32.6 & 98.2 \\
\hline SYN14-10 Run2- 133 & 999.5 & 29.1 & 1008.2 & 21.5 & 1027.0 & 24.0 & 1027.0 & 24.0 & 97.3 \\
\hline SYN14-10 Run2- 179 & 990.5 & 18.7 & 1003.3 & 24.0 & 1031.2 & 64.0 & 1031.2 & 64.0 & 96.1 \\
\hline SYN14-10 Run2- 156 & 1038.5 & 13.2 & 1037.1 & 12.4 & 1034.1 & 26.5 & 1034.1 & 26.5 & 100.4 \\
\hline SYN14-10 Run2- 141 & 1021.6 & 19.0 & 1026.7 & 23.4 & 1037.6 & 61.0 & 1037.6 & 61.0 & 98.5 \\
\hline SYN14-10 Run2- 132 & 1006.2 & 22.6 & 1019.2 & 22.8 & 1047.1 & 52.2 & 1047.1 & 52.2 & 96.1 \\
\hline SYN14-10 Run2- 199 & 1035.9 & 19.5 & 1039.6 & 16.4 & 1047.5 & 30.1 & 1047.5 & 30.1 & 98.9 \\
\hline SYN14-10 Run2- 188 & 1056.0 & 17.9 & 1053.2 & 18.0 & 1047.6 & 41.2 & 1047.6 & 41.2 & 100.8 \\
\hline SYN14-10 Run2- 136 & 985.9 & 16.2 & 1005.7 & 15.2 & 1049.0 & 32.3 & 1049.0 & 32.3 & 94.0 \\
\hline SYN14-10 Run2- 182 & 1005.3 & 14.1 & 1020.2 & 14.4 & 1052.4 & 33.2 & 1052.4 & 33.2 & 95.5 \\
\hline SYN14-10 Run2- 124 & 964.5 & 23.6 & 992.6 & 23.2 & 1055.3 & 51.1 & 1055.3 & 51.1 & 91.4 \\
\hline SYN14-10 Run2- 210 & 1038.0 & 18.6 & 1046.2 & 19.5 & 1063.4 & 45.8 & 1063.4 & 45.8 & 97.6 \\
\hline SYN14-10 Run2- 170 & 1005.7 & 21.2 & 1024.7 & 19.8 & 1065.6 & 41.1 & 1065.6 & 41.1 & 94.4 \\
\hline
\end{tabular}




\begin{tabular}{|c|c|c|c|c|c|c|c|c|c|}
\hline SYN14-10 Run2- 140 & 1006.0 & 20.9 & 1031.1 & 22.9 & 1084.8 & 54.9 & 1084.8 & 54.9 & 92.7 \\
\hline SYN14-10 Run2- 209 & 1020.1 & 13.3 & 1041.4 & 16.8 & 1086.6 & 43.5 & 1086.6 & 43.5 & 93.9 \\
\hline SYN14-10 Run2- 214 & 1005.8 & 24.6 & 1034.5 & 22.4 & 1095.8 & 44.4 & 1095.8 & 44.4 & 91.8 \\
\hline SYN14-10 Run2- 180 & 1073.9 & 20.7 & 1082.2 & 17.1 & 1098.8 & 29.9 & 1098.8 & 29.9 & 97.7 \\
\hline SYN14-10 Run2- 175 & 1089.4 & 22.8 & 1093.1 & 16.7 & 1100.5 & 20.2 & 1100.5 & 20.2 & 99.0 \\
\hline SYN14-10 Run2- 204 & 1108.8 & 19.4 & 1108.4 & 18.2 & 1107.6 & 38.2 & 1107.6 & 38.2 & 100.1 \\
\hline SYN14-10 Run2- 200 & 1050.6 & 35.8 & 1073.5 & 28.9 & 1120.5 & 46.1 & 1120.5 & 46.1 & 93.8 \\
\hline SYN14-10 Run2- 160 & 1182.2 & 21.5 & 1176.8 & 16.9 & 1166.9 & 27.4 & 1166.9 & 27.4 & 101.3 \\
\hline SYN14-10 Run2- 177 & 1142.9 & 20.1 & 1152.4 & 18.0 & 1170.3 & 35.1 & 1170.3 & 35.1 & 97.7 \\
\hline SYN14-10 Run2- 212 & 1153.5 & 25.8 & 1163.0 & 20.2 & 1180.8 & 31.6 & 1180.8 & 31.6 & 97.7 \\
\hline SYN14-10 Run2- 171 & 1169.6 & 23.5 & 1180.8 & 21.7 & 1201.3 & 43.4 & 1201.3 & 43.4 & 97.4 \\
\hline SYN14-10 Run2- 193 & 1151.7 & 34.8 & 1177.3 & 24.8 & 1224.7 & 26.3 & 1224.7 & 26.3 & 94.0 \\
\hline SYN14-10 Run2- 181 & 1133.0 & 21.9 & 1167.5 & 20.4 & 1232.2 & 40.1 & 1232.2 & 40.1 & 91.9 \\
\hline SYN14-10 Run2- 202 & 1164.1 & 17.8 & 1194.8 & 14.2 & 1250.7 & 22.6 & 1250.7 & 22.6 & 93.1 \\
\hline SYN14-10 Run2- 185 & 1225.1 & 24.7 & 1239.9 & 18.8 & 1265.7 & 27.9 & 1265.7 & 27.9 & 96.8 \\
\hline SYN14-10 Run2- 198 & 1314.8 & 16.7 & 1319.2 & 14.3 & 1326.3 & 25.8 & 1326.3 & 25.8 & 99.1 \\
\hline SYN14-10 Run2- 191 & 1349.0 & 21.7 & 1344.2 & 15.7 & 1336.4 & 21.6 & 133 & 21.6 & 100.9 \\
\hline SYN14-10 Run2- 161 & 1369.0 & 20.9 & 1356.7 & 15.4 & 1337.3 & 22.6 & 1337.3 & 22.6 & 102.4 \\
\hline SYN14-10 Run2- 219 & 1381.2 & 23.6 & 1398.3 & 18.6 & 1424.5 & 29.6 & 1424.5 & 29.6 & 97.0 \\
\hline SYN14-10 Run2- 143 & 1406.6 & 23.5 & 1417.3 & 18.3 & 1433.5 & 28.7 & 1433.5 & 28.7 & 98.1 \\
\hline SYN14-10 Run2- 122 & 1412.0 & 20.4 & 1424.9 & 16.0 & 1444.2 & 25.4 & 1444.2 & 25.4 & 97.8 \\
\hline SYN14-10 Run2- 149 & 1253.2 & 24.5 & 1329.6 & 20.2 & 1454.9 & 31.7 & 1454.9 & 31.7 & 86.1 \\
\hline SYN14-10 Run2- 186 & 1427.5 & 19.0 & 1446.6 & 15.7 & 1474.7 & 26.5 & 1474.7 & 26.5 & 96.8 \\
\hline SYN14-10 Run2- 138 & 1448.9 & 32.4 & 1460.6 & 21.1 & 1477.8 & 20.6 & 1477.8 & 20.6 & 98.0 \\
\hline SYN14-10 Run2- 218 & 1425.2 & 31.8 & 1453.6 & 25.5 & 1495.5 & 41.1 & 1495.5 & 41.1 & 95.3 \\
\hline SYN14-10 Run2- 130 & 1576.3 & 28.6 & 1595.9 & 27.5 & 1621.9 & 50.8 & 1621.9 & 50.8 & 97.2 \\
\hline SYN14-10 Run2- 184 & 1685.1 & 29.1 & 1660.3 & 20.4 & 1629.0 & 28.7 & 1629.0 & 28.7 & 103.4 \\
\hline SYN14-10 Run2- 137 & 1463.8 & 32.0 & 1538.3 & 23.9 & 1642.1 & 32.8 & 1642.1 & 32.8 & 89.1 \\
\hline SYN14-10 Run2- 176 & 1566.9 & 32.1 & 1599.9 & 22.5 & 1643.6 & 29.3 & 1643.6 & 29.3 & 95.3 \\
\hline SYN14-10 Run2- 196 & 1612.5 & 50.8 & 1641.5 & 31.3 & 1678.7 & 26.7 & 1678.7 & 26.7 & 96.1 \\
\hline SYN14-10 Run2- 123 & 1709.0 & 22.7 & 1708.0 & 17.7 & 1706.9 & 28.0 & 1706.9 & 28.0 & 100.1 \\
\hline SYN14-10 Run2- 131 & 1688.7 & 25.4 & 1703.1 & 17.4 & 1720.8 & 22.8 & 1720.8 & 22.8 & 98.1 \\
\hline SYN14-10 Run2- 114 & 1730.6 & 26.5 & 1745.0 & 17.5 & 1762.1 & 21.5 & 1762.1 & 21.5 & 98.2 \\
\hline SYN14-10 Run2- 201 & 1857.3 & 32.7 & 1854.6 & 22.5 & 1851.5 & 30.8 & 1851.5 & 30.8 & 100.3 \\
\hline SYN14-10 Run2- 207 & 1655.7 & 42.6 & 1752.8 & 29.0 & 1870.7 & 34.0 & 1870.7 & 34.0 & 88.5 \\
\hline SYN14-10 Run2- 115 & 1851.5 & 28.4 & 1913.8 & 19.3 & 1981.9 & 24.6 & 1981.9 & 24.6 & 93.4 \\
\hline SYN14-10 Run2- 111 & 1929.1 & 27.6 & 1975.3 & 19.2 & 2024.0 & 25.7 & 2024.0 & 25.7 & 95.3 \\
\hline SYN14-10 Run2- 148 & 2044.1 & 33.6 & 2052.2 & 20.8 & 2060.3 & 24.2 & 2060.3 & 24.2 & 99.2 \\
\hline SYN14-10 Run2- 127 & 1642.4 & 62.7 & 1846.7 & 39.5 & 2085.4 & 29.6 & 2085.4 & 29.6 & 78.8 \\
\hline
\end{tabular}




\begin{tabular}{|c|c|c|c|c|c|c|c|c|c|}
\hline SYN14-10 Run2- 117 & 1995.6 & 27.3 & 2066.8 & 17.3 & 2138.5 & 20.0 & 2138.5 & 20.0 & 93.3 \\
\hline SYN14-10 Run2- 135 & 2120.9 & 38.3 & 2246.7 & 21.0 & 2363.3 & 16.3 & 2363.3 & 16.3 & 89.7 \\
\hline SYN14-10 Run2- 162 & 2418.7 & 34.0 & 2450.2 & 21.2 & 2476.4 & 26.3 & 2476.4 & 26.3 & 97.7 \\
\hline SYN14-10 Run2- 183 & 2464.5 & 42.3 & 2491.7 & 21.7 & 2513.9 & 18.4 & 2513.9 & 18.4 & 98.0 \\
\hline SYN14-10 Run2- 157 & 2576.9 & 54.7 & 2656.1 & 26.7 & 2717.0 & 19.3 & 2717.0 & 19.3 & 94.8 \\
\hline SYN14-10 Run2- 173 & 2673.7 & 79.4 & 2699.6 & 36.1 & 2718.9 & 19.7 & 2718.9 & 19.7 & 98.3 \\
\hline SYN14-10 Run2- 158 & 2730.0 & 37.9 & 2746.0 & 20.0 & 2757.7 & 20.4 & 2757.7 & 20.4 & 99.0 \\
\hline SYN14-10 Run3- 267 & 111.0 & 3.5 & 102.1 & 7.6 & 100.8 & 176.2 & 111.0 & 3.5 & NA \\
\hline SYN14-10 Run3- 255 & 116.3 & 2.9 & 122.5 & 10.3 & 244.1 & 197.7 & 116.3 & 2.9 & NA \\
\hline SYN14-10 Run3- 264 & 222.9 & 5.9 & 226.4 & 7.2 & 263.0 & 51.6 & 222.9 & 5.9 & NA \\
\hline SYN14-10 Run3- 318 & 231.9 & 3.0 & 229.9 & 5.4 & 209.4 & 52.8 & 231.9 & 3.0 & NA \\
\hline SYN14-10 Run3- 270 & 235.0 & 3.7 & 233.8 & 8.3 & 221.9 & 84.7 & 235.0 & 3.7 & NA \\
\hline SYN14-10 Run3- 238 & 255.7 & 5.6 & 253.5 & 6.5 & 232.9 & 42.5 & 255.7 & 5.6 & NA \\
\hline SYN14-10 Run3- 322 & 259.6 & 3.5 & 252.6 & 7.4 & 188.2 & 69.7 & 259.6 & 3.5 & NA \\
\hline SYN14-10 Run3- 277 & 264.6 & 5.3 & 265.1 & 6.0 & 269.4 & 35.4 & 264.6 & 5.3 & NA \\
\hline SYN14-10 Run3- 275 & 289.2 & 5.2 & 288.4 & 8.3 & 282.1 & 62.5 & 289.2 & 5.2 & NA \\
\hline SYN14-10 Run3- 258 & 309.2 & 6.6 & 317.3 & 12.6 & 376.8 & 92.1 & 309.2 & 6.6 & NA \\
\hline SYN14-10 Run3- 320 & 354.0 & 8.4 & 368.4 & 13.2 & 460.2 & 77.5 & 354.0 & 8.4 & NA \\
\hline SYN14-10 Run3- 235 & 364.6 & 8.8 & 359.9 & 10.2 & 329.5 & 52.0 & 364.6 & 8.8 & NA \\
\hline SYN14-10 Run3- 292 & 373.8 & 10.8 & 378.1 & 13.1 & 404.2 & 64.1 & 373.8 & 10.8 & NA \\
\hline SYN14-10 Run3- 245 & 381.5 & 9.2 & 385.5 & 19.5 & 409.6 & 124.0 & 381.5 & 9.2 & NA \\
\hline SYN14-10 Run3- 288 & 396.3 & 13.6 & 827.0 & 144.4 & 2274.5 & 440.3 & 396.3 & 13.6 & AA \\
\hline SYN14-10 Run3- 252 & 400.8 & 13.6 & 413.4 & 15.4 & 484.9 & 63.7 & 400.8 & 13.6 & 82.7 \\
\hline SYN14-10 Run3- 306 & 408.0 & 8.0 & 398.5 & 10.0 & 343.5 & 51.3 & 408.0 & 8.0 & 118.8 \\
\hline SYN14-10 Run3- 268 & 415.8 & 9.0 & 425.7 & 9.3 & 479.9 & 32.5 & 415.8 & 9.0 & 86.6 \\
\hline SYN14-10 Run3- 253 & 418.6 & 13.2 & 428.3 & 13.3 & 480.5 & 44.3 & 418.6 & 13.2 & 87.1 \\
\hline SYN14-10 Run3- 274 & 421.3 & 7.8 & 425.4 & 10.7 & 447.5 & 53.5 & 421.3 & 7.8 & 94.1 \\
\hline SYN14-10 Run3- 305 & 430.8 & 8.3 & 436.8 & 11.5 & 468.9 & 56.3 & 430.8 & 8.3 & 91.9 \\
\hline SYN14-10 Run3- 231 & 533.5 & 8.5 & 532.2 & 11.1 & 526.7 & 45.9 & 533.5 & 8.5 & 101.3 \\
\hline SYN14-10 Run3- 327 & 539.0 & 17.1 & 658.0 & 26.3 & 1090.7 & 86.2 & 539.0 & 17.1 & 49.4 \\
\hline SYN14-10 Run3- 289 & 543.0 & 8.7 & 547.1 & 12.4 & 564.1 & 52.4 & 543.0 & 8.7 & 96.2 \\
\hline SYN14-10 Run3- 328 & 568.2 & 11.8 & 569.3 & 15.1 & 573.9 & 58.9 & 568.2 & 11.8 & 99.0 \\
\hline SYN14-10 Run3- 250 & 572.4 & 14.9 & 575.3 & 14.4 & 587.1 & 40.1 & 572.4 & 14.9 & 97.5 \\
\hline SYN14-10 Run3- 291 & 615.0 & 10.3 & 618.2 & 10.6 & 630.0 & 32.1 & 615.0 & 10.3 & 97.6 \\
\hline SYN14-10 Run3- 279 & 626.3 & 10.7 & 632.1 & 12.2 & 653.2 & 40.4 & 626.3 & 10.7 & 95.9 \\
\hline SYN14-10 Run3- 282 & 720.1 & 16.9 & 714.1 & 17.4 & 695.3 & 49.2 & 720.1 & 16.9 & 103.6 \\
\hline SYN14-10 Run3- 247 & 758.3 & 35.4 & 764.0 & 27.5 & 780.7 & 28.5 & 758.3 & 35.4 & 97.1 \\
\hline SYN14-10 Run3- 237 & 992.9 & 21.2 & 987.8 & 17.9 & 976.4 & 33.5 & 976.4 & 33.5 & 101.7 \\
\hline SYN14-10 Run3- 273 & 1006.6 & 21.6 & 998.0 & 16.9 & 978.9 & 26.9 & 978.9 & 26.9 & 102.8 \\
\hline
\end{tabular}




\begin{tabular}{|c|c|c|c|c|c|c|c|c|c|}
\hline SYN14-10 Run3- 299 & 958.1 & 14.4 & 964.7 & 15.6 & 979.8 & 39.0 & 979.8 & 39.0 & 97.8 \\
\hline SYN14-10 Run3- 243 & 901.5 & 31.3 & 927.0 & 27.7 & 988.0 & 53.3 & 988.0 & 53.3 & 91.2 \\
\hline SYN14-10 Run3- 229 & 998.6 & 18.7 & 996.3 & 19.9 & 991.3 & 48.9 & 991.3 & 48.9 & 100.7 \\
\hline SYN14-10 Run3- 272 & 989.0 & 18.2 & 990.3 & 21.1 & 993.1 & 54.7 & 993.1 & 54.7 & 99.6 \\
\hline SYN14-10 Run3- 314 & 998.3 & 19.3 & 998.2 & 15.3 & 998.0 & 24.5 & 998.0 & 24.5 & 100.0 \\
\hline SYN14-10 Run3- 244 & 951.0 & 23.0 & 965.3 & 19.3 & 998.2 & 34.5 & 998.2 & 34.5 & 95.3 \\
\hline SYN14-10 Run3- 300 & 1027.0 & 23.2 & 1018.0 & 18.5 & 998.7 & 30.7 & 998.7 & 30.7 & 102.8 \\
\hline SYN14-10 Run3- 321 & 931.0 & 21.3 & 951.5 & 21.4 & 999.3 & 50.0 & 999.3 & 50.0 & 93.2 \\
\hline SYN14-10 Run3- 298 & 978.8 & 15.3 & 986.0 & 17.9 & 1001.9 & 46.3 & 1001.9 & 46.3 & 97.7 \\
\hline SYN14-10 Run3- 240 & 973.9 & 32.0 & 984.0 & 24.8 & 1006.5 & 34.6 & 1006.5 & 34.6 & 96.8 \\
\hline SYN14-10 Run3- 302 & 961.0 & 18.3 & 976.2 & 19.1 & 1010.4 & 45.5 & 1010.4 & 45.5 & 95.1 \\
\hline SYN14-10 Run3- 257 & 953.3 & 19.4 & 971.2 & 16.1 & 1012.0 & 27.5 & 1012.0 & 27.5 & 94.2 \\
\hline SYN14-10 Run3- 234 & 1057.7 & 16.2 & 1043.0 & 13.4 & 1012.3 & 24.5 & 1012.3 & 24.5 & 104.5 \\
\hline SYN14-10 Run3- 297 & 1054.7 & 96.0 & 1041.0 & 64.7 & 1012.5 & 20.8 & 1012.5 & 20.8 & 104.2 \\
\hline SYN14-10 Run3- 256 & 991.1 & 20.1 & 998.3 & 18.4 & 1014.1 & 38.2 & 1014.1 & 38.2 & 97.7 \\
\hline SYN14-10 Run3- 224 & 989.8 & 15.8 & 997.7 & 14.5 & 1015.2 & 30.4 & 1015.2 & 30.4 & 97.5 \\
\hline SYN14-10 Ru & 1043.0 & 13.7 & 1034.3 & 14.2 & 1016.0 & 33.7 & 1016.0 & 33.7 & 102.7 \\
\hline SYN14-10 Run3- 325 & 1060.1 & 16.9 & 1046.0 & 17.2 & 1016.6 & 40.2 & 1016.6 & 40.2 & 104.3 \\
\hline SYN14-10 Run3- 223 & 1016.6 & 15.0 & 1017.0 & 12.8 & 1017.9 & 24.0 & 1017.9 & 24.0 & 99.9 \\
\hline SYN14-10 Run3- 330 & 1002.3 & 16.5 & 1008.1 & 16.0 & 1020.7 & 35.5 & 1020.7 & 35.5 & 98.2 \\
\hline SYN14-10 Run3- 230 & 1014.5 & 18.3 & 1017.7 & 16.0 & 1024.5 & 31.3 & 1024.5 & 31.3 & 99.0 \\
\hline SYN14-10 Run3- 259 & 1008.6 & 18.3 & 1014.3 & 15.9 & 1026.7 & 30.7 & 1026.7 & 30.7 & 98.2 \\
\hline SYN14-10 Run3- 251 & 1032.9 & 22.7 & 1031.9 & 17.7 & 1030.0 & 27.5 & 1030.0 & 27.5 & 100.3 \\
\hline SYN14-10 Run3- 248 & 1023.7 & 23.2 & 1026.2 & 23.6 & 1031.5 & 54.7 & 1031.5 & 54.7 & 99.2 \\
\hline SYN14-10 Run3- 261 & 1037.9 & 35.7 & 1038.0 & 25.8 & 1038.3 & 27.4 & 1038.3 & 27.4 & 100.0 \\
\hline SYN14-10 Run3- 319 & 998.8 & 25.5 & 1011.5 & 21.0 & 1039.0 & 35.9 & 1039.0 & 35.9 & 96.1 \\
\hline SYN14-10 Run3- 242 & 997.1 & 20.7 & 1011.9 & 17.7 & 44.1 & 32.5 & 1044.1 & 32.5 & 95.5 \\
\hline SYN14-10 Run3- 310 & 1016.4 & 25.8 & 1025.4 & 21.2 & 1044.5 & 36.3 & 1044.5 & 36.3 & 97.3 \\
\hline SYN14-10 Run3- 303 & 1102.4 & 29.6 & 1085.1 & 23.4 & 1050.4 & 39.3 & 1050.4 & 39.3 & 104.9 \\
\hline SYN14-10 Run3- 266 & 1030.9 & 38.5 & 1044.7 & 28.8 & 1073.8 & 35.7 & 1073.8 & 35.7 & 96.0 \\
\hline SYN14-10 Run3- 227 & 1148.8 & 58.5 & 1126.0 & 42.0 & 1082.1 & 53.9 & 1082.1 & 53.9 & 106.2 \\
\hline SYN14-10 Run3- 286 & 1055.0 & 15.0 & 1065.1 & 13.6 & 1085.8 & 27.6 & 1085.8 & 27.6 & 97.2 \\
\hline SYN14-10 Run3- 254 & 1032.9 & 18.1 & 1050.2 & 18.0 & 1086.2 & 40.0 & 1086.2 & 40.0 & 95.1 \\
\hline SYN14-10 Run3- 232 & 1048.9 & 17.6 & 1062.0 & 20.7 & 1089.2 & 51.3 & 1089.2 & 51.3 & 96.3 \\
\hline SYN14-10 Run3- 246 & 1065.5 & 17.1 & 1075.9 & 16.6 & 1097.1 & 36.0 & 1097.1 & 36.0 & 97.1 \\
\hline SYN14-10 Run3- 280 & 1071.8 & 20.3 & 1081.6 & 15.3 & 1101.4 & 20.5 & 1101.4 & 20.5 & 97.3 \\
\hline SYN14-10 Run3- 260 & 970.5 & 14.0 & 1012.8 & 16.0 & 1105.3 & 38.9 & 1105.3 & 38.9 & 87.8 \\
\hline SYN14-10 Run3- 278 & 1092.4 & 24.5 & 1104.8 & 21.0 & 1129.4 & 38.6 & 1129.4 & 38.6 & 96.7 \\
\hline SYN14-10 Run3- 293 & 1142.7 & 26.2 & 1139.6 & 20.7 & 1133.9 & 34.1 & 1133.9 & 34.1 & 100.8 \\
\hline
\end{tabular}




\begin{tabular}{|c|c|c|c|c|c|c|c|c|c|}
\hline SYN14-10 Run3- 323 & 1155.5 & 21.0 & 1148.5 & 16.8 & 1135.3 & 28.1 & 1135.3 & 28.1 & 101.8 \\
\hline SYN14-10 Run3- 239 & 1155.8 & 19.0 & 1154.5 & 17.6 & 1152.0 & 36.1 & 1152.0 & 36.1 & 100.3 \\
\hline SYN14-10 Run3- 222 & 1164.2 & 27.3 & 1160.3 & 23.3 & 1153.2 & 43.6 & 1153.2 & 43.6 & 101.0 \\
\hline SYN14-10 Run3- 296 & 1149.0 & 29.1 & 1150.9 & 21.3 & 1154.6 & 27.3 & 1154.6 & 27.3 & 99.5 \\
\hline SYN14-10 Run3- 290 & 1146.8 & 22.7 & 1151.3 & 17.0 & 1159.9 & 23.5 & 1159.9 & 23.5 & 98.9 \\
\hline SYN14-10 Run3- 225 & 1154.3 & 17.1 & 1156.8 & 13.3 & 1161.5 & 20.4 & 1161.5 & 20.4 & 99.4 \\
\hline SYN14-10 Run3- 241 & 1129.0 & 19.8 & 1145.5 & 18.5 & 1176.8 & 37.5 & 1176.8 & 37.5 & 95.9 \\
\hline SYN14-10 Run3- 284 & 1127.8 & 18.2 & 1145.7 & 19.3 & 1179.8 & 43.4 & 1179.8 & 43.4 & 95.6 \\
\hline SYN14-10 Run3- 294 & 1106.2 & 22.8 & 1134.7 & 25.6 & 1189.5 & 59.2 & 1189.5 & 59.2 & 93.0 \\
\hline SYN14-10 Run3- 309 & 1161.0 & 15.9 & 1175.7 & 21.2 & 1202.8 & 52.3 & 1202.8 & 52.3 & 96.5 \\
\hline SYN14-10 Run3- 308 & 1224.0 & 31.0 & 1232.9 & 21.5 & 1248.5 & 22.3 & 1248.5 & 22.3 & 98.0 \\
\hline SYN14-10 Run3- 301 & 1262.6 & 34.8 & 1266.9 & 25.1 & 1274.2 & 32.9 & 1274.2 & 32.9 & 99.1 \\
\hline SYN14-10 Run3- 228 & 1257.7 & 24.5 & 1268.7 & 19.3 & 1287.5 & 30.7 & 1287.5 & 30.7 & 97.7 \\
\hline SYN14-10 Run3- 326 & 1320.5 & 24.6 & 1309.2 & 19.9 & 1290.7 & 34.1 & 1290.7 & 34.1 & 102.3 \\
\hline SYN14-10 Run3- 316 & 1333.4 & 28.9 & 1344.7 & 20.5 & 1362.7 & 26.2 & 1362.7 & 26.2 & 97.8 \\
\hline SYN14-10 Run3- 307 & 1416.6 & 23.4 & 1406.8 & 17.8 & 1392.1 & 27.6 & 1392.1 & 27.6 & 101.8 \\
\hline SYN14-10 Run3- 233 & 1353.7 & 22.1 & 1380.0 & 16.7 & 1420.8 & 24.2 & 20.8 & 24.2 & 95.3 \\
\hline SYN14-10 Run3- 324 & 1435.8 & 13.0 & 1432.9 & 13.5 & 1428.7 & 27.6 & 8.7 & 27.6 & 100.5 \\
\hline SYN14-10 Run3- 269 & 1399.4 & 37.0 & 1413.2 & 25.2 & 1433.9 & 28.9 & 3.9 & 28.9 & 97.6 \\
\hline SYN14-10 Run3- 226 & & 40.2 & & 27.2 & .0 & 5 & & .5 & 0 \\
\hline SYN14-10 Run3- 283 & $14 C$ & 22.3 & & 19.8 & .5 & 1 & .5 & 35.1 & 93.4 \\
\hline SYN14-10 Run3- 329 & 15 & 35.9 & 154 & 23.3 & .7 & 23.9 & .7 & 23.9 & 97.5 \\
\hline SYN14-10 Run3- 236 & 1602.4 & 30.0 & 1590.1 & 19.4 & 1573.8 & 21.7 & 73.8 & 21.7 & 101.8 \\
\hline SYN14-10 Run3- 315 & 1615.3 & 76.0 & 1622.3 & 44.5 & 1631.3 & 25.4 & 1631.3 & 25.4 & 99.0 \\
\hline SYN14-10 Run3- 311 & 1622.7 & 21.4 & 1627.7 & 14.6 & 1634.3 & 18.9 & 1634.3 & 18.9 & 99.3 \\
\hline SYN14-10 Run3- 317 & 1615.2 & 29.2 & 1628.7 & 21.9 & 1646.1 & 32.9 & 1646.1 & 32.9 & 98.1 \\
\hline SYN14-10 Run3- 249 & 1590.0 & 56.3 & 1616.2 & 35.2 & 1650.4 & 31.7 & 1650.4 & 31.7 & 96.3 \\
\hline SYN14-10 Run3- 276 & 1711.6 & 24.9 & 1701.7 & 17.2 & 1689.6 & 23.3 & 1689.6 & 23.3 & 101.3 \\
\hline SYN14-10 Run3- 265 & 1685.4 & 41.4 & 1687.3 & 26.6 & 1689.7 & 30.1 & 1689.7 & 30.1 & 99.7 \\
\hline SYN14-10 Run3- 313 & 1804.2 & 22.9 & 1825.5 & 14.8 & 1849.8 & 17.6 & 1849.8 & 17.6 & 97.5 \\
\hline SYN14-10 Run3- 281 & 2098.9 & 29.2 & 2107.9 & 17.4 & 2116.7 & 19.1 & 2116.7 & 19.1 & 99.2 \\
\hline SYN14-10 Run3- 285 & 2088.7 & 33.0 & 2133.3 & 19.0 & 2176.4 & 18.5 & 2176.4 & 18.5 & 96.0 \\
\hline SYN14-10 Run3- 304 & 2597.5 & 36.9 & 2616.1 & 21.2 & 2630.4 & 24.2 & 2630.4 & 24.2 & 98.8 \\
\hline SYN14-10 Run3- 263 & 2682.1 & 115.1 & 2707.1 & 51.3 & 2725.8 & 23.0 & 2725.8 & 23.0 & 98.4 \\
\hline SYN14-10 Run3- 271 & 2740.5 & 44.3 & 2776.0 & 22.3 & 2801.9 & 20.4 & 2801.9 & 20.4 & 97.8 \\
\hline SYN14-10 Run3- 262 & 2812.3 & 45.6 & 2809.8 & 24.3 & 2808.0 & 25.9 & 2808.0 & 25.9 & 100.2 \\
\hline SYN14-10 Run3- 221 & 3354.1 & 91.1 & 3407.7 & 36.4 & 3439.3 & 20.0 & 3439.3 & 20.0 & 97.5 \\
\hline Analysis & $206 \mathrm{~Pb}^{*}$ & \pm & 207Pb* & \pm & $206 \mathrm{~Pb}^{*}$ & \pm & Best age & \pm & Conc \\
\hline
\end{tabular}




\begin{tabular}{|c|c|c|c|c|c|c|c|c|c|}
\hline & $238 U^{*}$ & (Ma) & $235 \mathrm{U}$ & (Ma) & $207 \mathrm{~Pb}^{*}$ & (Ma) & (Ma) & (Ma) & $(\%)$ \\
\hline & & & & & & & & & \\
\hline SYN14-11_Run1- 10 & 1707.3 & 20.5 & 1705.3 & 16.8 & 1702.9 & 27.8 & 1702.9 & 27.8 & 100.3 \\
\hline SYN14-11_Run1- 79 & 106.0 & 4.0 & 114.4 & 14.1 & 293.0 & 286.2 & 106.0 & 4.0 & NA \\
\hline SYN14-11_Run2- 201 & 106.5 & 2.5 & 106.7 & 4.6 & 110.5 & 92.5 & 106.5 & 2.5 & NA \\
\hline SYN14-11_Run1- 110 & 111.6 & 2.8 & 116.6 & 5.2 & 220.1 & 91.5 & 111.6 & 2.8 & NA \\
\hline SYN14-11_Run3- 278 & 163.2 & 4.0 & 166.3 & 12.2 & 211.2 & 176.2 & 163.2 & 4.0 & NA \\
\hline SYN14-11_Run3- 297 & 164.8 & 5.8 & 161.6 & 7.1 & 114.5 & 73.9 & 164.8 & 5.8 & NA \\
\hline SYN14-11_Run2- 204 & 166.3 & 4.7 & 165.5 & 8.0 & 155.5 & 102.3 & 166.3 & 4.7 & NA \\
\hline SYN14-11_Run1- 21 & 170.2 & 3.4 & 171.7 & 6.6 & 191.9 & 85.5 & 170.2 & 3.4 & NA \\
\hline SYN14-11_Run2- 118 & 232.6 & 4.5 & 237.2 & 8.7 & 282.5 & 82.0 & 232.6 & 4.5 & NA \\
\hline SYN14-11_Run1- 52 & 234.2 & 5.6 & 234.2 & 6.4 & 233.8 & 42.6 & 234.2 & 5.6 & NA \\
\hline SYN14-11_Run2- 133 & 234.4 & 5.7 & 235.4 & 7.6 & 245.4 & 61.1 & 234.4 & 5.7 & NA \\
\hline SYN14-11_Run2- 168 & 238.6 & 5.4 & 256.6 & 9.5 & 423.8 & 77.8 & 238.6 & 5.4 & NA \\
\hline SYN14-11_Run1- 47 & 244.8 & 4.8 & 243.8 & 7.2 & 234.0 & 61.7 & 244.8 & 4.8 & NA \\
\hline SYN14-11_Run2- 187 & 246.4 & 6.2 & 247.0 & 7.1 & 252.8 & 44.6 & 246.4 & 6.2 & NA \\
\hline SYN14-11_Run2- 115 & 252.6 & 7.2 & 265.1 & 9.9 & 377.1 & 69.1 & 252.6 & 7.2 & NA \\
\hline SYN14-11_Run1- 53 & 254.2 & 4.6 & 294.5 & 7.6 & 627.2 & 50.6 & 254.2 & 4.6 & NA \\
\hline SYN14-11_Run1- 100 & 256.2 & 3.9 & 255.2 & 5.3 & 245.5 & 40.2 & 256.2 & 3.9 & NA \\
\hline SYN14-11_Run2- 144 & 266.1 & 6.4 & 260.5 & 11.7 & 211.1 & 103.0 & 266.1 & 6.4 & NA \\
\hline SYN14-11_Run3- 250 & 267.7 & 4.8 & 268.2 & 6.4 & 272.7 & 45.9 & 267.7 & 4.8 & NA \\
\hline SYN14-11_Run1- 92 & 278.6 & 5.5 & 275.2 & 9.1 & 246.2 & 73.2 & 278.6 & 5.5 & NA \\
\hline SYN14-11_Run2- 218 & 286.6 & 5.6 & 290.1 & 6.2 & 318.0 & 32.3 & 286.6 & 5.6 & NA \\
\hline SYN14-11_Run1- 57 & 314.7 & 7.1 & 321.0 & 9.8 & 367.2 & 60.3 & 314.7 & 7.1 & NA \\
\hline SYN14-11_Run1- 108 & 314.9 & 9.1 & 314.8 & 11.9 & 314.5 & 74.3 & 314.9 & 9.1 & NA \\
\hline SYN14-11_Run1- 91 & 315.0 & 7.9 & 314.0 & 9.5 & 306.2 & 54.6 & 315.0 & 7.9 & NA \\
\hline SYN14-11_Run2- 153 & 317.3 & 4.9 & 323.5 & 9.7 & 368.2 & 70.6 & 317.3 & 4.9 & NA \\
\hline SYN14-11_Run1- 66 & 321.3 & 4.8 & 308.4 & 10.4 & 212.1 & 83.3 & 321.3 & 4.8 & NA \\
\hline SYN14-11_Run1- 24 & 346.1 & 5.8 & 338.4 & 8.0 & 286.0 & 49.5 & 346.1 & 5.8 & NA \\
\hline SYN14-11_Run2- 163 & 350.3 & 5.3 & 369.2 & 5.6 & 489.5 & 21.0 & 350.3 & 5.3 & NA \\
\hline SYN14-11_Run3- 314 & 369.5 & 6.3 & 375.2 & 8.7 & 410.9 & 48.4 & 369.5 & 6.3 & NA \\
\hline SYN14-11_Run1- 56 & 373.9 & 7.0 & 367.5 & 12.4 & 327.2 & 80.0 & 373.9 & 7.0 & NA \\
\hline SYN14-11_Run2- 114 & 377.6 & 7.4 & 376.5 & 7.9 & 369.4 & 33.7 & 377.6 & 7.4 & NA \\
\hline SYN14-11_Run2- 155 & 385.1 & 8.8 & 382.1 & 13.3 & 363.9 & 77.5 & 385.1 & 8.8 & NA \\
\hline SYN14-11_Run3- 311 & 392.3 & 5.7 & 396.1 & 15.8 & 418.1 & 102.1 & 392.3 & 5.7 & NA \\
\hline SYN14-11_Run1- 63 & 392.9 & 6.7 & 396.4 & 8.1 & 417.3 & 39.0 & 392.9 & 6.7 & NA \\
\hline SYN14-11_Run1- 3 & 394.0 & 5.8 & 392.6 & 7.6 & 384.4 & 39.2 & 394.0 & 5.8 & NA \\
\hline SYN14-11_Run3- 221 & 397.2 & 5.6 & 414.2 & 10.3 & 510.1 & 58.3 & 397.2 & 5.6 & NA \\
\hline
\end{tabular}




\begin{tabular}{|c|c|c|c|c|c|c|c|c|c|}
\hline SYN14-11_Run1- 40 & 399.3 & 6.6 & 404.9 & 10.4 & 437.3 & 57.9 & 399.3 & 6.6 & NA \\
\hline SYN14-11_Run3- 246 & 399.9 & 7.8 & 409.2 & 11.8 & 462.4 & 63.6 & 399.9 & 7.8 & NA \\
\hline SYN14-11_Run3- 299 & 411.4 & 10.0 & 420.9 & 9.5 & 473.2 & 25.8 & 411.4 & 10.0 & 86.9 \\
\hline SYN14-11_Run2- 127 & 411.7 & 6.8 & 417.2 & 8.9 & 448.0 & 43.4 & 411.7 & 6.8 & 91.9 \\
\hline SYN14-11_Run3- 301 & 415.1 & 7.3 & 418.4 & 9.9 & 436.5 & 49.6 & 415.1 & 7.3 & 95.1 \\
\hline SYN14-11_Run3- 307 & 417.9 & 6.7 & 419.1 & 9.2 & 426.1 & 46.7 & 417.9 & 6.7 & 98.1 \\
\hline SYN14-11_Run2- 161 & 418.5 & 6.5 & 432.4 & 10.5 & 507.2 & 55.1 & 418.5 & 6.5 & 82.5 \\
\hline SYN14-11_Run1- 86 & 418.9 & 6.0 & 409.2 & 9.2 & 355.0 & 52.2 & 418.9 & 6.0 & 118.0 \\
\hline SYN14-11_Run2- 172 & 419.1 & 6.4 & 413.4 & 9.0 & 381.9 & 48.1 & 419.1 & 6.4 & 109.7 \\
\hline SYN14-11_Run1- 81 & 429.0 & 7.5 & 429.2 & 10.8 & 430.1 & 55.4 & 429.0 & 7.5 & 99.7 \\
\hline SYN14-11_Run1- 102 & 434.6 & 7.4 & 434.3 & 9.8 & 432.6 & 48.0 & 434.6 & 7.4 & 100.5 \\
\hline SYN14-11_Run3- 258 & 438.0 & 9.1 & 439.6 & 11.6 & 447.8 & 54.0 & 438.0 & 9.1 & 97.8 \\
\hline SYN14-11_Run1- 32 & 438.8 & 8.6 & 438.7 & 8.9 & 438.4 & 32.6 & 438.8 & 8.6 & 100.1 \\
\hline SYN14-11_Run2- 205 & 438.9 & 7.9 & 437.1 & 9.6 & 427.2 & 43.6 & 438.9 & 7.9 & 102.8 \\
\hline SYN14-11_Run3- 296 & 443.1 & 8.6 & 440.6 & 10.1 & 427.4 & 44.7 & 443.1 & 8.6 & 103.7 \\
\hline SYN14-11_Run3- 257 & 454.7 & 7.1 & 454.7 & 12.7 & 454.9 & 67.9 & 454.7 & 7.1 & 99.9 \\
\hline SYN14-11_Run3- 236 & 456.9 & 9.1 & 456.9 & 9.4 & 456.8 & 33.5 & 456.9 & 9.1 & 100.0 \\
\hline SYN14-11_Run2- 210 & 462.4 & 10.3 & 477.6 & 11.5 & 551.3 & 42.3 & 462.4 & 10.3 & 83.9 \\
\hline SYN14-11_Run3- 275 & 466.2 & 12.4 & 464.5 & 12.3 & 455.9 & 40.1 & 466.2 & 12.4 & 102.3 \\
\hline SYN14-11_Run2- 200 & 514.1 & 9.9 & 520.8 & 13.2 & 550.3 & 55.3 & 514.1 & 9.9 & 93.4 \\
\hline SYN14-11_Run1- 33 & 547.5 & 9.2 & 549.5 & 10.5 & 557.7 & 38.1 & 547.5 & 9.2 & 98.2 \\
\hline SYN14-11_Run1- 46 & 552.5 & 8.1 & 562.7 & 9.3 & 603.9 & 32.7 & 552.5 & 8.1 & 91.5 \\
\hline SYN14-11_Run1- 27 & 556.5 & 7.6 & 564.2 & 7.9 & 595.7 & 24.4 & 556.5 & 7.6 & 93.4 \\
\hline SYN14-11_Run2- 219 & 580.1 & 7.8 & 583.4 & 9.4 & 596.3 & 34.1 & 580.1 & 7.8 & 97.3 \\
\hline SYN14-11_Run3- 312 & 584.2 & 14.6 & 604.8 & 14.9 & 682.7 & 41.8 & 584.2 & 14.6 & 85.6 \\
\hline SYN14-11_Run2- 214 & 594.5 & 11.3 & 600.6 & 11.5 & 623.6 & 34.1 & 594.5 & 11.3 & 95.3 \\
\hline SYN14-11_Run2- 175 & 596.8 & 9.5 & 598.6 & 11.3 & 605.5 & 40.6 & 596.8 & 9.5 & 98.6 \\
\hline SYN14-11_Run3- 313 & 598.8 & 14.5 & 586.6 & 12.7 & 539.8 & 28.5 & 598.8 & 14.5 & 110.9 \\
\hline SYN14-11_Run1- 19 & 600.1 & 12.8 & 616.1 & 16.4 & 675.7 & 58.9 & 600.1 & 12.8 & 88.8 \\
\hline SYN14-11_Run1- 45 & 600.3 & 9.9 & 614.7 & 10.4 & 668.1 & 31.0 & 600.3 & 9.9 & 89.8 \\
\hline SYN14-11_Run1- 42 & 601.8 & 21.7 & 599.0 & 21.6 & 588.1 & 63.1 & 601.8 & 21.7 & 102.3 \\
\hline SYN14-11_Run1- 69 & 607.1 & 10.7 & 609.0 & 11.7 & 616.1 & 38.2 & 607.1 & 10.7 & 98.5 \\
\hline SYN14-11_Run2- 174 & 612.1 & 9.1 & 619.2 & 14.2 & 645.4 & 56.4 & 612.1 & 9.1 & 94.8 \\
\hline SYN14-11_Run1- 62 & 615.7 & 9.5 & 622.3 & 10.7 & 646.6 & 34.9 & 615.7 & 9.5 & 95.2 \\
\hline SYN14-11_Run2- 166 & 625.7 & 7.0 & 629.3 & 7.5 & 642.5 & 23.4 & 625.7 & 7.0 & 97.4 \\
\hline SYN14-11_Run1- 29 & 629.2 & 6.1 & 627.8 & 8.7 & 622.7 & 33.7 & 629.2 & 6.1 & 101.0 \\
\hline SYN14-11_Run1- 39 & 641.1 & 12.1 & 651.0 & 11.0 & 685.5 & 24.5 & 641.1 & 12.1 & 93.5 \\
\hline SYN14-11_Run1- 85 & 641.4 & 14.7 & 641.6 & 15.2 & 642.4 & 45.2 & 641.4 & 14.7 & 99.8 \\
\hline SYN14-11_Run2- 119 & 661.8 & 7.5 & 659.7 & 9.1 & 652.7 & 31.4 & 661.8 & 7.5 & 101.4 \\
\hline
\end{tabular}




\begin{tabular}{|c|c|c|c|c|c|c|c|c|c|}
\hline SYN14-11_Run2- 117 & 666.7 & 13.1 & 663.0 & 20.5 & 650.3 & 78.7 & 666.7 & 13.1 & 102.5 \\
\hline SYN14-11_Run3- 284 & 683.0 & 13.1 & 677.3 & 13.3 & 658.3 & 38.1 & 683.0 & 13.1 & 103.8 \\
\hline SYN14-11_Run2- 134 & 686.0 & 10.7 & 694.5 & 10.9 & 722.2 & 30.3 & 686.0 & 10.7 & 95.0 \\
\hline SYN14-11_Run3- 281 & 934.5 & 16.9 & 932.4 & 18.1 & 927.6 & 46.1 & 927.6 & 46.1 & 100.7 \\
\hline SYN14-11_Run3- 233 & 924.6 & 17.2 & 927.7 & 16.5 & 935.1 & 37.9 & 935.1 & 37.9 & 98.9 \\
\hline SYN14-11_Run3- 326 & 946.2 & 15.4 & 948.4 & 14.2 & 953.6 & 30.4 & 953.6 & 30.4 & 99.2 \\
\hline SYN14-11_Run1- 90 & 971.9 & 13.2 & 967.0 & 14.1 & 955.8 & 35.4 & 955.8 & 35.4 & 101.7 \\
\hline SYN14-11_Run2- 196 & 960.5 & 19.4 & 959.4 & 20.4 & 956.7 & 50.6 & 956.7 & 50.6 & 100.4 \\
\hline SYN14-11_Run3- 308 & 977.6 & 15.4 & 972.0 & 14.2 & 959.4 & 31.0 & 959.4 & 31.0 & 101.9 \\
\hline SYN14-11_Run3- 249 & 933.7 & 18.4 & 941.5 & 18.6 & 959.6 & 44.4 & 959.6 & 44.4 & 97.3 \\
\hline SYN14-11_Run1- 77 & 917.6 & 19.2 & 930.3 & 15.2 & 960.5 & 22.3 & 960.5 & 22.3 & 95.5 \\
\hline SYN14-11_Run2- 180 & 969.9 & 14.6 & 968.2 & 12.3 & 964.3 & 22.6 & 964.3 & 22.6 & 100.6 \\
\hline SYN14-11_Run1- 12 & 1001.3 & 23.1 & 990.9 & 20.0 & 968.1 & 39.7 & 968.1 & 39.7 & 103.4 \\
\hline SYN14-11_Run2- 137 & 955.6 & 19.0 & 959.5 & 16.7 & 968.4 & 33.3 & 968.4 & 33.3 & 98.7 \\
\hline SYN14-11_Run3- 283 & 989.4 & 17.4 & 983.6 & 16.8 & 970.6 & 38.1 & 970.6 & 38.1 & 101.9 \\
\hline SYN14-11_Run1- 61 & 938.9 & 24.2 & 948.7 & 18.7 & 971.5 & 25.1 & 971.5 & 25.1 & 96.6 \\
\hline SYN14-11_Run3- 230 & 946.8 & 18.0 & 955.7 & 18.5 & 976.4 & 44.3 & 976.4 & 44.3 & 97.0 \\
\hline SYN14-11_Run1- 70 & 1024.7 & 21.1 & 1012.4 & 18.3 & 986.0 & 36.7 & 986.0 & 36.7 & 103.9 \\
\hline SYN14-11_Run1- 103 & 976.5 & 12.6 & 980.5 & 14.9 & 989.4 & 39.2 & 989.4 & 39.2 & 98.7 \\
\hline SYN14-11_Run1- 104 & 1048.6 & 16.1 & 1030.4 & 12.8 & 991.9 & 21.8 & 991.9 & 21.8 & 105.7 \\
\hline SYN14-11_Run1- 64 & 1036.8 & 18.0 & 1022.8 & 15.7 & 992.9 & 31.7 & 992.9 & 31.7 & 104.4 \\
\hline SYN14-11_Run3- 287 & 1017.3 & 15.1 & 1010.0 & 13.1 & 994.1 & 26.0 & 994.1 & 26.0 & 102.3 \\
\hline SYN14-11_Run3- 264 & 944.2 & 16.2 & 959.9 & 16.6 & 996.1 & 39.3 & 996.1 & 39.3 & 94.8 \\
\hline SYN14-11_Run1- 28 & 983.1 & 14.1 & 987.2 & 11.9 & 996.3 & 21.7 & 996.3 & 21.7 & 98.7 \\
\hline SYN14-11_Run2- 191 & 1020.4 & 17.2 & 1012.8 & 15.4 & 996.5 & 31.9 & 996.5 & 31.9 & 102.4 \\
\hline SYN14-11_Run1- 7 & 1053.6 & 13.3 & 1035.5 & 14.0 & 997.6 & 33.9 & 997.6 & 33.9 & 105.6 \\
\hline SYN14-11_Run2- 136 & 864.0 & 14.8 & 902.6 & 12.8 & 998.2 & 22.4 & 998.2 & 22.4 & 86.6 \\
\hline SYN14-11_Run1- 17 & 1029.6 & 13.7 & 1019.9 & 12.0 & 999.2 & 23.9 & 999.2 & 23.9 & 103.0 \\
\hline SYN14-11_Run2- 215 & 1021.1 & 18.6 & 1014.3 & 15.1 & 999.6 & 26.1 & 999.6 & 26.1 & 102.2 \\
\hline SYN14-11_Run3- 289 & 983.4 & 20.1 & 988.5 & 18.0 & 999.7 & 36.7 & 999.7 & 36.7 & 98.4 \\
\hline SYN14-11_Run3- 259 & 1006.1 & 13.5 & 1005.2 & 11.6 & 1003.1 & 22.1 & 1003.1 & 22.1 & 100.3 \\
\hline SYN14-11_Run2- 111 & 1054.2 & 12.5 & 1039.1 & 17.8 & 1007.3 & 48.9 & 1007.3 & 48.9 & 104.7 \\
\hline SYN14-11_Run1- 98 & 1013.8 & 18.7 & 1012.3 & 15.2 & 1009.0 & 25.9 & 1009.0 & 25.9 & 100.5 \\
\hline SYN14-11_Run3- 272 & 957.1 & 17.4 & 974.0 & 16.0 & 1012.3 & 33.1 & 1012.3 & 33.1 & 94.6 \\
\hline SYN14-11_Run2- 124 & 1078.3 & 24.9 & 1057.0 & 25.7 & 1013.1 & 60.7 & 1013.1 & 60.7 & 106.4 \\
\hline SYN14-11_Run3- 309 & 1019.0 & 20.9 & 1017.3 & 16.5 & 1013.7 & 26.1 & 1013.7 & 26.1 & 100.5 \\
\hline SYN14-11_Run1- 83 & 1026.8 & 18.4 & 1023.7 & 14.6 & 1017.1 & 23.8 & 1017.1 & 23.8 & 100.9 \\
\hline SYN14-11_Run2- 129 & 956.5 & 19.4 & 975.4 & 20.8 & 1018.0 & 50.6 & 1018.0 & 50.6 & 94.0 \\
\hline SYN14-11_Run1- 95 & 1024.1 & 13.3 & 1022.4 & 18.1 & 1018.7 & 49.3 & 1018.7 & 49.3 & 100.5 \\
\hline
\end{tabular}




\begin{tabular}{|c|c|c|c|c|c|c|c|c|c|}
\hline SYN14-11_Run3- 304 & 1017.0 & 16.3 & 1017.7 & 14.4 & 1019.1 & 28.6 & 1019.1 & 28.6 & 99.8 \\
\hline SYN14-11_Run3- 330 & 943.0 & 16.1 & 966.2 & 13.4 & 1019.3 & 22.3 & 1019.3 & 22.3 & 92.5 \\
\hline SYN14-11_Run3- 241 & 972.4 & 12.2 & 987.3 & 16.2 & 1020.5 & 44.2 & 1020.5 & 44.2 & 95.3 \\
\hline SYN14-11_Run2- 198 & 1003.7 & 13.8 & 1009.1 & 12.8 & 1020.9 & 27.2 & 1020.9 & 27.2 & 98.3 \\
\hline SYN14-11_Run2- 208 & 999.4 & 19.1 & 1006.3 & 22.0 & 1021.3 & 55.9 & 1021.3 & 55.9 & 97.8 \\
\hline SYN14-11_Run1- 23 & 1043.0 & 11.8 & 1036.1 & 17.4 & 1021.7 & 48.1 & 1021.7 & 48.1 & 102.1 \\
\hline SYN14-11_Run1- 5 & 1061.7 & 14.1 & 1049.4 & 11.9 & 1023.9 & 22.7 & 1023.9 & 22.7 & 103.7 \\
\hline SYN14-11_Run3- 243 & 825.7 & 21.0 & 881.5 & 24.0 & 1024.3 & 61.2 & 1024.3 & 61.2 & 80.6 \\
\hline SYN14-11_Run3- 320 & 1025.5 & 15.1 & 1025.3 & 14.1 & 1025.0 & 30.1 & 1025.0 & 30.1 & 100.1 \\
\hline SYN14-11_Run3- 295 & 1053.2 & 18.7 & 1044.7 & 14.9 & 1026.8 & 25.0 & 1026.8 & 25.0 & 102.6 \\
\hline SYN14-11_Run2- 190 & 990.7 & 17.7 & 1002.1 & 15.4 & 1027.3 & 29.7 & 1027.3 & 29.7 & 96.4 \\
\hline SYN14-11_Run2- 116 & 1069.5 & 21.6 & 1057.2 & 21.4 & 1031.9 & 48.8 & 1031.9 & 48.8 & 103.6 \\
\hline SYN14-11_Run1- 107 & 1037.8 & 20.9 & 1036.5 & 17.2 & 1033.8 & 30.3 & 1033.8 & 30.3 & 100.4 \\
\hline SYN14-11_Run1- 51 & 1035.0 & 18.3 & 1034.8 & 18.2 & 1034.3 & 41.7 & 1034.3 & 41.7 & 100.1 \\
\hline SYN14-11_Run2- 179 & 975.6 & 17.2 & 994.0 & 15.7 & 1034.9 & 31.9 & 1034.9 & 31.9 & 94.3 \\
\hline SYN14-11_Run2- 132 & 1019.3 & 12.9 & 1024.3 & 11.0 & 1035.0 & 20.6 & 1035.0 & 20.6 & 98.5 \\
\hline SYN14-11_Run3- 225 & 1017.3 & 15.3 & 1023.5 & 12.4 & 36.6 & 20.7 & 36.6 & 20.7 & 98.1 \\
\hline SYN14-11_Run3- 280 & 1004.6 & 24.5 & 1016.2 & 19.5 & 1041.2 & 30.5 & 1041.2 & 30.5 & 96.5 \\
\hline SYN14-11_Run2- 181 & 1079.3 & 21.0 & 1067.0 & 16.8 & 1042.0 & 28.3 & 1042.0 & 28.3 & 103.6 \\
\hline SYN14-11_Run2- 185 & 993.7 & 14.0 & 1009.4 & 16.8 & 1043.6 & 43.2 & 1043.6 & 43.2 & 95.2 \\
\hline SYN14-11_Run3- 292 & 1095.4 & 20.3 & 1078.3 & 14.9 & 1043.8 & 19.9 & 1043.8 & 19.9 & 104.9 \\
\hline SYN14-11_Run1- 74 & 1090.6 & 14.2 & 1075.4 & 15.4 & 1044.7 & 37.3 & 1044.7 & 37.3 & 104.4 \\
\hline SYN14-11_Run3- 306 & 1023.5 & 20.7 & 1030.4 & 17.2 & 1045.1 & 30.7 & 1045.1 & 30.7 & 97.9 \\
\hline SYN14-11_Run3- 262 & 1053.5 & 20.2 & 1051.2 & 18.5 & 1046.5 & 38.5 & 1046.5 & 38.5 & 100.7 \\
\hline SYN14-11_Run2- 183 & 1053.2 & 17.6 & 1051.9 & 15.6 & 1049.2 & 31.0 & 1049.2 & 31.0 & 100.4 \\
\hline SYN14-11_Run3- 265 & 996.2 & 20.6 & 1013.1 & 18.7 & 1049.9 & 38.0 & 1049.9 & 38.0 & 94.9 \\
\hline SYN14-11_Run1- 72 & 1007.1 & 15.4 & 1020.7 & 12.9 & 1050.1 & 22.7 & 1050.1 & 22.7 & 95.9 \\
\hline SYN14-11_Run2- 207 & 997.5 & 26.4 & 1014.3 & 21.5 & 1050.8 & 35.5 & 1050.8 & 35.5 & 94.9 \\
\hline SYN14-11_Run2- 220 & 1018.8 & 19.4 & 1029.2 & 14.7 & 1051.3 & 19.2 & 1051.3 & 19.2 & 96.9 \\
\hline SYN14-11_Run3- 226 & 1057.8 & 17.7 & 1056.4 & 15.0 & 1053.6 & 28.0 & 1053.6 & 28.0 & 100.4 \\
\hline SYN14-11_Run2- 171 & 991.9 & 16.9 & 1011.7 & 14.6 & 1054.8 & 27.3 & 1054.8 & 27.3 & 94.0 \\
\hline SYN14-11_Run3- 316 & 1056.2 & 16.8 & 1056.6 & 15.2 & 1057.6 & 31.1 & 1057.6 & 31.1 & 99.9 \\
\hline SYN14-11_Run3- 256 & 1071.8 & 20.6 & 1067.7 & 16.2 & 1059.4 & 25.9 & 1059.4 & 25.9 & 101.2 \\
\hline SYN14-11_Run3- 327 & 1063.0 & 15.1 & 1062.3 & 13.1 & 1060.8 & 25.3 & 1060.8 & 25.3 & 100.2 \\
\hline SYN14-11_Run2- 151 & 1007.4 & 23.2 & 1025.0 & 23.3 & 1062.6 & 52.8 & 1062.6 & 52.8 & 94.8 \\
\hline SYN14-11_Run1- 11 & 1041.2 & 15.3 & 1048.1 & 18.7 & 1062.7 & 47.8 & 1062.7 & 47.8 & 98.0 \\
\hline SYN14-11_Run1- 94 & 1033.2 & 21.5 & 1042.9 & 19.0 & 1063.3 & 37.3 & 1063.3 & 37.3 & 97.2 \\
\hline SYN14-11_Run2- 209 & 1069.9 & 19.7 & 1068.3 & 15.3 & 1065.0 & 23.5 & 1065.0 & 23.5 & 100.5 \\
\hline SYN14-11_Run1- 60 & 1028.1 & 15.9 & 1040.6 & 13.5 & 1066.8 & 24.5 & 1066.8 & 24.5 & 96.4 \\
\hline
\end{tabular}




\begin{tabular}{|c|c|c|c|c|c|c|c|c|c|}
\hline SYN14-11_Run2- 162 & 949.4 & 33.0 & 986.5 & 26.2 & 1070.0 & 36.1 & 1070.0 & 36.1 & 88.7 \\
\hline SYN14-11_Run1- 35 & 1074.6 & 17.6 & 1073.2 & 14.1 & 1070.4 & 23.4 & 1070.4 & 23.4 & 100.4 \\
\hline SYN14-11_Run2- 217 & 1028.9 & 16.5 & 1042.5 & 17.0 & 1071.2 & 39.3 & 1071.2 & 39.3 & 96.1 \\
\hline SYN14-11_Run1- 82 & 1054.7 & 27.6 & 1060.1 & 22.4 & 1071.2 & 37.6 & 1071.2 & 37.6 & 98.5 \\
\hline SYN14-11_Run3- 315 & 934.9 & 12.9 & 976.5 & 12.9 & 1071.4 & 28.6 & 1071.4 & 28.6 & 87.3 \\
\hline SYN14-11_Run3- 251 & 1048.9 & 18.8 & 1056.6 & 14.7 & 1072.4 & 22.4 & 1072.4 & 22.4 & 97.8 \\
\hline SYN14-11_Run2- 184 & 1070.1 & 18.2 & 1071.1 & 16.2 & 1073.2 & 32.1 & 1073.2 & 32.1 & 99.7 \\
\hline SYN14-11_Run2- 216 & 1061.2 & 26.1 & 1065.3 & 19.3 & 1073.6 & 23.8 & 1073.6 & 23.8 & 98.8 \\
\hline SYN14-11_Run2- 120 & 976.9 & 19.4 & 1007.7 & 16.8 & 1075.2 & 30.6 & 1075.2 & 30.6 & 90.9 \\
\hline SYN14-11_Run2- 152 & 1049.0 & 25.2 & 1058.5 & 21.6 & 1078.2 & 40.4 & 1078.2 & 40.4 & 97.3 \\
\hline SYN14-11_Run3- 248 & 1080.7 & 19.7 & 1080.0 & 18.2 & 1078.5 & 37.8 & 1078.5 & 37.8 & 100.2 \\
\hline SYN14-11_Run3- 234 & 1066.2 & 16.0 & 1071.2 & 14.9 & 1081.4 & 31.1 & 1081.4 & 31.1 & 98.6 \\
\hline SYN14-11_Run1- 8 & 1082.0 & 20.3 & 1081.9 & 17.8 & 1081.5 & 34.5 & 1081.5 & 34.5 & 100.0 \\
\hline SYN14-11_Run2- 203 & 1054.2 & 18.0 & 1063.5 & 14.4 & 1082.5 & 23.4 & 1082.5 & 23.4 & 97.4 \\
\hline SYN14-11_Run2- 158 & 1069.1 & 18.5 & 1073.6 & 14.8 & 1082.7 & 24.4 & 1082.7 & 24.4 & 98.7 \\
\hline SYN14-11_Run2- 199 & 1064.4 & 17.5 & 1071.7 & 15.4 & 1086.7 & 30.0 & 1086.7 & 30.0 & 97.9 \\
\hline SYN14-11_Run1- 30 & 1050.7 & 20.2 & 1063.1 & 17.1 & 1088.6 & 31.0 & 1088.6 & 31.0 & 96.5 \\
\hline SYN14-11_Run3- 318 & 1103.5 & 15.4 & 1101.0 & 13.2 & 1096.2 & 24.8 & 1096.2 & 24.8 & 100.7 \\
\hline SYN14-11_Run3- 323 & 1074.0 & 17.4 & 1084.5 & 16.1 & 1105.5 & 32.9 & 1105.5 & 32.9 & 97.1 \\
\hline SYN14-11_Run3- 244 & 1059.0 & 16.6 & 1074.9 & 14.2 & 1107.3 & 26.1 & 1107.3 & 26.1 & 95.6 \\
\hline SYN14-11_Run2- 141 & 1047.4 & 16.0 & 1067.2 & 25.0 & 1108.0 & 68.0 & 1108.0 & 68.0 & 94.5 \\
\hline SYN14-11_Run2- 138 & 1063.5 & 14.5 & 1079.1 & 14.1 & 1110.6 & 30.3 & 1110.6 & 30.3 & 95.8 \\
\hline SYN14-11_Run3- 328 & 946.4 & 26.5 & 997.7 & 21.2 & 1111.9 & 29.1 & 1111.9 & 29.1 & 85.1 \\
\hline SYN14-11_Run2- 186 & 1130.4 & 18.1 & 1127.3 & 15.9 & 1121.4 & 31.0 & 1121.4 & 31.0 & 100.8 \\
\hline SYN14-11_Run3- 239 & 1072.7 & 19.2 & 1089.1 & 17.7 & 1122.0 & 36.1 & 1122.0 & 36.1 & 95.6 \\
\hline SYN14-11_Run1- 34 & 1091.6 & 21.0 & 1101.9 & 19.1 & 1122.1 & 38.5 & 1122.1 & 38.5 & 97.3 \\
\hline SYN14-11_Run2- 112 & 1137.6 & 19.2 & 1133.9 & 21.0 & 1126.7 & 48.9 & 1126.7 & 48.9 & 101.0 \\
\hline SYN14-11_Run1- 105 & 1141.8 & 18.4 & 1136.9 & 14.2 & 1127.6 & 22.0 & 1127.6 & 22.0 & 101.3 \\
\hline SYN14-11_Run1- 88 & 1117.4 & 14.5 & 1122.1 & 13.2 & 1131.1 & 26.5 & 1131.1 & 26.5 & 98.8 \\
\hline SYN14-11_Run3- 291 & 1168.1 & 22.4 & 1155.3 & 19.1 & 1131.5 & 36.0 & 1131.5 & 36.0 & 103.2 \\
\hline SYN14-11_Run2- 178 & 1138.0 & 16.7 & 1135.8 & 15.6 & 1131.6 & 32.5 & 1131.6 & 32.5 & 100.6 \\
\hline SYN14-11_Run2- 128 & 1137.3 & 18.0 & 1136.8 & 16.4 & 1136.0 & 33.0 & 1136.0 & 33.0 & 100.1 \\
\hline SYN14-11_Run2- 189 & 1186.4 & 17.2 & 1171.0 & 12.9 & 1142.5 & 18.9 & 1142.5 & 18.9 & 103.8 \\
\hline SYN14-11_Run3- 269 & 1135.7 & 15.0 & 1139.7 & 15.9 & 1147.4 & 36.3 & 1147.4 & 36.3 & 99.0 \\
\hline SYN14-11_Run2- 194 & 1053.7 & 15.6 & 1086.1 & 15.4 & 1151.8 & 33.1 & 1151.8 & 33.1 & 91.5 \\
\hline SYN14-11_Run3- 229 & 1149.3 & 19.9 & 1150.5 & 18.7 & 1152.8 & 38.9 & 1152.8 & 38.9 & 99.7 \\
\hline SYN14-11_Run3- 271 & 1185.1 & 15.9 & 1174.0 & 12.4 & 1153.5 & 19.9 & 1153.5 & 19.9 & 102.7 \\
\hline SYN14-11_Run2- 182 & 1147.7 & 17.3 & 1149.8 & 17.5 & 1153.7 & 38.4 & 1153.7 & 38.4 & 99.5 \\
\hline SYN14-11_Run3- 222 & 1117.0 & 18.4 & 1133.7 & 16.5 & 1165.8 & 32.2 & 1165.8 & 32.2 & 95.8 \\
\hline
\end{tabular}




\begin{tabular}{|c|c|c|c|c|c|c|c|c|c|}
\hline SYN14-11_Run3- 329 & 1196.3 & 28.3 & 1186.5 & 22.6 & 1168.7 & 38.2 & 1168.7 & 38.2 & 102.4 \\
\hline SYN14-11_Run2- 121 & 1146.5 & 18.9 & 1155.0 & 13.9 & 1170.9 & 17.7 & 1170.9 & 17.7 & 97.9 \\
\hline SYN14-11_Run1- 99 & 1154.6 & 22.1 & 161.5 & 17.6 & 1174.5 & 28.6 & 1174.5 & 28.6 & 98.3 \\
\hline SYN14-11_Run2- 211 & 1140.9 & 20.2 & 52.6 & 15.5 & 1174.6 & 22.9 & 174.6 & 22.9 & 97.1 \\
\hline SYN14-11_Run1- 96 & 1118.7 & 32.5 & 138.2 & 24.7 & 1175.5 & 34.2 & 1175.5 & 34.2 & 95.2 \\
\hline SYN14-11_Run1- 50 & 1150.5 & 22.9 & 59.5 & 19.7 & 1176.4 & 36.4 & 176.4 & 36.4 & 97.8 \\
\hline SYN14-11_Run1- 9 & 1190.6 & 14.5 & 1186.0 & 11.5 & 1177.7 & 18.8 & 1177.7 & 18.8 & 101.1 \\
\hline SYN14-11_Run3- 266 & 1233.0 & 14.5 & 1213.5 & 12.7 & 1179.0 & 24.4 & 1179.0 & 24.4 & 104.6 \\
\hline SYN14-11_Run1- 59 & 1148.1 & 14.0 & 1159.2 & 15.8 & 1180.0 & 36.8 & 1180.0 & 36.8 & 97.3 \\
\hline SYN14-11_Run2- 164 & 1162.2 & 19.5 & 1169.4 & 15.9 & 1183.0 & 27.3 & 1183.0 & 27.3 & 98.2 \\
\hline SYN14-11_Run2- 177 & 1146.0 & 20.3 & 1159.2 & 18.0 & 1183.9 & 34.7 & 1183.9 & 34.7 & 96.8 \\
\hline SYN14-11_Run3- 282 & 1246.4 & 24.1 & 1227.5 & 19.2 & 1194.2 & 32.7 & 1194.2 & 32.7 & 104.4 \\
\hline SYN14-11_Run2- 139 & 1192.8 & 18.5 & 1194.8 & 17.7 & 1198.3 & 8 & 1198.3 & 36.8 & 99.5 \\
\hline SYN14-11_Run2- 159 & 1166.7 & 24.3 & 1179.0 & 21.1 & 1201.7 & 39.2 & 1201.7 & 39.2 & 97.1 \\
\hline SYN14-11_Run2- 156 & 1190.5 & 18.3 & 1199.5 & 19.1 & 1215.7 & 41.7 & 1215.7 & 41.7 & 97.9 \\
\hline SYN14-11_Run3- 288 & 1220.8 & 23.6 & 20.3 & 18.9 & 9.5 & 31.7 & 9.5 & 31.7 & 100.1 \\
\hline SYN14-11_Run2- 130 & 1169.5 & 15.6 & 7.5 & 12.7 & 0.3 & 21.0 & 0.3 & 21.0 & 95.8 \\
\hline SYN14-11_Run2- 157 & 1136.9 & 19.7 & 1166.3 & 27.9 & 1221.3 & 69.9 & 1221.3 & 69.9 & 93.1 \\
\hline SYN14-11_Run2- 123 & 1232.1 & 27.6 & 9.2 & 22.1 & 24.2 & 37.2 & 24.2 & 37.2 & 100.6 \\
\hline SYN14-11_Run3- 277 & 1274.0 & 23.1 & 1257.0 & 17.5 & 1228.0 & 27.2 & 8.0 & 27.2 & 103.7 \\
\hline SYN14-11_Run2- 149 & 1259.5 & 19.5 & 1250.4 & 16.9 & 1234.8 & 31.7 & 1234.8 & 31.7 & 102.0 \\
\hline SYN14-11_Run2- 197 & 1243.5 & 15.5 & 1242.5 & 12.0 & 1240.8 & 19.1 & 1240.8 & 19.1 & 100.2 \\
\hline SYN14-11_Run3- 285 & 1065.1 & 19.1 & 1124.6 & 17.0 & 1241.4 & 30.5 & 1241.4 & 30.5 & 85.8 \\
\hline SYN14-11_Run1- 26 & 1232.2 & 13.0 & 1238.2 & 15.6 & 1248.6 & 36.1 & 1248.6 & 36.1 & 98.7 \\
\hline SYN14-11_Run2- 113 & 1176.3 & 16.9 & 1208.1 & 13.6 & 1265.3 & 21.5 & 1265.3 & 21.5 & 93.0 \\
\hline SYN14-11_Run3- 274 & 1260.3 & 20.2 & 1267.8 & 15.7 & 1280.5 & 24.5 & 1280.5 & 24.5 & 98.4 \\
\hline SYN14-11_Run3- 255 & 1294.5 & 25.2 & 1305.0 & 26.3 & 1322.3 & 55.6 & 1322.3 & 55.6 & 97.9 \\
\hline SYN14-11_Run3- 300 & 1325.5 & 21.2 & 1324.8 & 16.8 & 1323.9 & 27.5 & 1323.9 & 27.5 & 100.1 \\
\hline SYN14-11_Run3- 245 & 1251.1 & 18.1 & 1279.5 & 27.6 & 1327.6 & 66.7 & 1327.6 & 66.7 & 94.2 \\
\hline SYN14-11_Run2- 146 & 1276.9 & 18.2 & 1296.8 & 15.7 & 1330.0 & 28.2 & 1330.0 & 28.2 & 96.0 \\
\hline SYN14-11_Run3- 232 & 1325.6 & 22.8 & 1337.2 & 21.3 & 1355.8 & 41.1 & 1355.8 & 41.1 & 97.8 \\
\hline SYN14-11_Run3- 253 & 1312.9 & 44.6 & 1330.9 & 29.2 & 1359.9 & 23.1 & 1359.9 & 23.1 & 96.5 \\
\hline SYN14-11_Run2- 193 & 1429.5 & 16.0 & 1404.4 & 13.7 & 1366.6 & 24.8 & 1366.6 & 24.8 & 104.6 \\
\hline SYN14-11_Run3- 242 & 1142.0 & 22.0 & 1222.3 & 20.0 & 1366.8 & 36.0 & 1366.8 & 36.0 & 83.6 \\
\hline SYN14-11_Run2- 202 & 1403.8 & 27.3 & 1392.7 & 22.6 & 1375.7 & 39.6 & 1375.7 & 39.6 & 102.0 \\
\hline SYN14-11_Run1- 80 & 1367.1 & 26.0 & 1370.6 & 20.5 & 1375.9 & 33.3 & 1375.9 & 33.3 & 99.4 \\
\hline SYN14-11_Run3- 227 & 1390.3 & 16.2 & 1385.5 & 11.8 & 1378.2 & 16.9 & 1378.2 & 16.9 & 100.9 \\
\hline SYN14-11_Run3- 247 & 1354.3 & 23.9 & 1371.4 & 18.6 & 1398.2 & 28.9 & 1398.2 & 28.9 & 96.9 \\
\hline SYN14-11_Run2- 165 & 1423.8 & 23.0 & 1418.2 & 15.7 & 1409.9 & 19.0 & 1409.9 & 19.0 & 101.0 \\
\hline
\end{tabular}




\begin{tabular}{|c|c|c|c|c|c|c|c|c|c|}
\hline SYN14-11_Run1- 84 & 1441.9 & 24.6 & 1430.5 & 17.6 & 1413.5 & 24.7 & 1413.5 & 24.7 & 102.0 \\
\hline SYN14-11_Run3- 322 & 1485.5 & 23.0 & 1461.1 & 16.1 & 1425.8 & 21.8 & 1425.8 & 21.8 & 104.2 \\
\hline SYN14-11_Run2- 143 & 1392.5 & 22.2 & 1406.2 & 19.3 & 1427.0 & 34.6 & 1427.0 & 34.6 & 97.6 \\
\hline SYN14-11_Run1- 2 & 1423.9 & 25.4 & 1425.5 & 18.2 & 1428.0 & 24.9 & 1428.0 & 24.9 & 99.7 \\
\hline SYN14-11_Run1- 44 & 1428.0 & 24.0 & 1432.5 & 16.7 & 1439.2 & 21.1 & 1439.2 & 21.1 & 99.2 \\
\hline SYN14-11_Run3- 263 & 1430.7 & 17.4 & 1444.8 & 20.9 & 1465.6 & 44.6 & 1465.6 & 44.6 & 97.6 \\
\hline SYN14-11_Run3- 276 & 1437.6 & 32.8 & 1451.6 & 22.9 & 1472.2 & 28.7 & 1472.2 & 28.7 & 97.6 \\
\hline SYN14-11_Run3- 321 & 1463.4 & 25.8 & 1468.6 & 19.4 & 1476.2 & 29.2 & 1476.2 & 29.2 & 99.1 \\
\hline SYN14-11_Run3- 260 & 1509.4 & 22.6 & 1500.7 & 18.0 & 1488.4 & 29.8 & 1488.4 & 29.8 & 101.4 \\
\hline SYN14-11_Run1- 15 & 1475.3 & 25.9 & 1484.0 & 18.3 & 1496.5 & 24.1 & 1496.5 & 24.1 & 98.6 \\
\hline SYN14-11_Run3- 290 & 1267.8 & 19.7 & 1359.9 & 14.4 & 1507.6 & 16.2 & 1507.6 & 16.2 & 84.1 \\
\hline SYN14-11_Run3- 237 & 1521.2 & 26.9 & 1519.5 & 20.3 & 1517.0 & 31.1 & 1517.0 & 31.1 & 100.3 \\
\hline SYN14-11_Run2- 150 & 1524.8 & 28.1 & 1532.5 & 21.3 & 1543.1 & 32.3 & 1543.1 & 32.3 & 98.8 \\
\hline SYN14-11_Run2- 125 & 1373.2 & 27.0 & 1447.1 & 19.6 & 1557.3 & 24.4 & 1557.3 & 24.4 & 88.2 \\
\hline SYN14-11_Run3- 224 & 1528.3 & 21.7 & 1549.7 & 25.9 & 1579.0 & 53.2 & 1579.0 & 53.2 & 96.8 \\
\hline SYN14-11_Run1- 20 & 1629.5 & 21.5 & 1616.9 & 14.3 & 1600.4 & 17.7 & 1600.4 & 17.7 & 101.8 \\
\hline SYN14-11_Run2- 173 & 1495.8 & 39.6 & 1548.2 & 26.2 & 20.5 & 27.1 & 0.5 & 27.1 & 92.3 \\
\hline SYN14-11_Run3- 261 & 1632.8 & 27.5 & 1630.4 & 20.8 & 1627.4 & 32.0 & 1627.4 & 32.0 & 100.3 \\
\hline SYN14-11_Run2- 212 & 1552.8 & 20.6 & 1585.3 & 18.9 & 1628.9 & 33.7 & 1628.9 & 33.7 & 95.3 \\
\hline SYN14-11_Run2- 148 & 1604.1 & 21.2 & 1616.1 & 16.3 & 1631.8 & 25.2 & 1631.8 & 25.2 & 98.3 \\
\hline SYN14-11_Run2- 170 & 1646.8 & 19.3 & 1644.0 & 15.1 & 1640.5 & 23.9 & 1640.5 & 23.9 & 100.4 \\
\hline SYN14-11_Run1- 71 & 1625.6 & 16.6 & 1635.8 & 12.6 & 1649.0 & 19.2 & 1649.0 & 19.2 & 98.6 \\
\hline SYN14-11_Run3- 273 & 1657.8 & 27.0 & 1654.2 & 19.6 & 1649.7 & 28.6 & 1649.7 & 28.6 & 100.5 \\
\hline SYN14-11_Run1- 109 & 1604.5 & 39.5 & 1626.7 & 24.4 & 1655.5 & 21.2 & 1655.5 & 21.2 & 96.9 \\
\hline SYN14-11_Run3- 223 & 1614.1 & 24.0 & 1632.2 & 17.5 & 1655.7 & 25.1 & 1655.7 & 25.1 & 97.5 \\
\hline SYN14-11_Run1- 89 & 1649.8 & 24.8 & 1653.9 & 19.1 & 1659.1 & 29.7 & 1659.1 & 29.7 & 99.4 \\
\hline SYN14-11_Run1- 73 & 1611.3 & 21.6 & 1633.9 & 14.4 & 1663.1 & 17.1 & 1663.1 & 17.1 & 96.9 \\
\hline SYN14-11_Run1- 38 & 1639.3 & 44.0 & 1662.4 & 28.8 & 1691.7 & 32.7 & 1691.7 & 32.7 & 96.9 \\
\hline SYN14-11_Run2- 147 & 1658.4 & 37.5 & 1674.1 & 23.5 & 1693.9 & 23.5 & 1693.9 & 23.5 & 97.9 \\
\hline SYN14-11_Run2- 188 & 1677.3 & 25.1 & 1685.8 & 17.9 & 1696.4 & 25.0 & 1696.4 & 25.0 & 98.9 \\
\hline SYN14-11_Run2- 192 & 1642.5 & 29.2 & 1668.1 & 19.4 & 1700.4 & 22.9 & 1700.4 & 22.9 & 96.6 \\
\hline SYN14-11_Run3- 238 & 1669.9 & 30.3 & 1684.3 & 20.3 & 1702.4 & 25.0 & 1702.4 & 25.0 & 98.1 \\
\hline SYN14-11_Run3- 240 & 1675.7 & 27.2 & 1691.9 & 18.7 & 1712.1 & 24.4 & 1712.1 & 24.4 & 97.9 \\
\hline SYN14-11_Run1- 48 & 1672.1 & 27.7 & 1691.6 & 19.5 & 1715.8 & 26.6 & 1715.8 & 26.6 & 97.5 \\
\hline SYN14-11_Run1- 16 & 1640.1 & 34.5 & 1674.4 & 23.0 & 1717.6 & 27.1 & 1717.6 & 27.1 & 95.5 \\
\hline SYN14-11_Run2- 206 & 1706.0 & 24.5 & 1712.0 & 16.5 & 1719.3 & 21.0 & 1719.3 & 21.0 & 99.2 \\
\hline SYN14-11_Run2- 140 & 1740.3 & 24.9 & 1730.9 & 15.4 & 1719.5 & 16.4 & 1719.5 & 16.4 & 101.2 \\
\hline SYN14-11_Run3- 319 & 1700.2 & 26.9 & 1716.9 & 18.8 & 1737.2 & 25.5 & 1737.2 & 25.5 & 97.9 \\
\hline SYN14-11_Run2- 135 & 1739.6 & 24.9 & 1741.3 & 17.4 & 1743.2 & 24.0 & 1743.2 & 24.0 & 99.8 \\
\hline
\end{tabular}




\begin{tabular}{|c|c|c|c|c|c|c|c|c|c|}
\hline SYN14-11_Run2- 154 & 1711.0 & 32.1 & 1727.4 & 21.7 & 1747.3 & 27.6 & 1747.3 & 27.6 & 97.9 \\
\hline SYN14-11_Run3- 294 & 1767.4 & 29.5 & 1759.1 & 21.2 & 1749.3 & 30.5 & 1749.3 & 30.5 & 101.0 \\
\hline SYN14-11_Run1- 43 & 1724.8 & 25.8 & 1738.5 & 16.2 & 1755.1 & 17.4 & 1755.1 & 17.4 & 98.3 \\
\hline SYN14-11_Run3- 228 & 1666.9 & 28.7 & 1706.4 & 19.2 & 1755.3 & 23.2 & 1755.3 & 23.2 & 95.0 \\
\hline SYN14-11_Run3- 310 & 1732.9 & 30.5 & 1743.8 & 19.4 & 1756.9 & 21.7 & 1756.9 & 21.7 & 98.6 \\
\hline SYN14-11_Run3- 252 & 1706.4 & 36.3 & 1739.1 & 22.9 & 1778.5 & 23.8 & 1778.5 & 23.8 & 95.9 \\
\hline SYN14-11_Run1- 14 & 1767.9 & 40.1 & 1774.4 & 23.9 & 1782.1 & 21.5 & 1782.1 & 21.5 & 99.2 \\
\hline SYN14-11_Run2- 126 & 1733.6 & 16.8 & 1759.7 & 16.2 & 1790.7 & 29.0 & 1790.7 & 29.0 & 96.8 \\
\hline SYN14-11_Run2- 160 & 1683.5 & 27.8 & 1732.8 & 20.3 & 1792.9 & 28.5 & 1792.9 & 28.5 & 93.9 \\
\hline SYN14-11_Run1- 54 & 1762.0 & 20.2 & 1779.0 & 13.8 & 1798.9 & 18.1 & 1798.9 & 18.1 & 97.9 \\
\hline SYN14-11_Run3- 324 & 1757.9 & 41.6 & 1776.9 & 25.5 & 1799.2 & 25.2 & 1799.2 & 25.2 & 97.7 \\
\hline SYN14-11_Run3- 279 & 1795.8 & 34.6 & 1799.4 & 20.5 & 1803.4 & 18.6 & 1803.4 & 18.6 & 99.6 \\
\hline SYN14-11_Run1- 49 & 1814.9 & 24.3 & 1826.8 & 17.6 & 1840.4 & 25.3 & 1840.4 & 25.3 & 98.6 \\
\hline SYN14-11_Run3- 317 & 1800.4 & 31.4 & 1831.2 & 21.2 & 1866.4 & 27.0 & 1866.4 & 27.0 & 96.5 \\
\hline SYN14-11_Run3- 267 & 1881.5 & 21.9 & 1886.5 & 13.9 & 1891.9 & 16.5 & 1891.9 & 16.5 & 99.5 \\
\hline SYN14-11_Run1- 25 & 1951.4 & 22.0 & 1930.7 & 14.9 & 1908.6 & 20.2 & 1908.6 & 20.2 & 102.2 \\
\hline SYN14-11_Run2- 176 & 1947.8 & 31.4 & 1948.3 & 17.8 & 1948.9 & 15.2 & 948.9 & 15.2 & 99.9 \\
\hline SYN14-11_Run2- 169 & 2028.1 & 33.2 & 2023.3 & 19.8 & 2018.3 & 21.6 & 2018.3 & 21.6 & 100.5 \\
\hline SYN14-11_Run2- 195 & 2045.5 & 29.3 & 2032.1 & 17.8 & 2018.6 & 20.2 & 2018.6 & 20.2 & 101.3 \\
\hline SYN14-11_Run1- 65 & 2026.1 & 33.0 & 2046.1 & 18.0 & 2066.2 & 13.5 & 2066.2 & 13.5 & 98.1 \\
\hline SYN14-11_Run3- 235 & 2088.8 & 32.5 & 2091.9 & 21.7 & 2095.0 & 28.9 & 2095.0 & 28.9 & 99.7 \\
\hline SYN14-11_Run2- 131 & 2006.5 & 32.1 & 2055.8 & 20.6 & 2105.6 & 24.8 & 2105.6 & 24.8 & 95.3 \\
\hline SYN14-11_Run3- 293 & 1951.9 & 39.7 & 2078.7 & 25.2 & 2206.8 & 27.7 & 2206.8 & 27.7 & 88.4 \\
\hline SYN14-11_Run2- 167 & 2120.3 & 29.7 & 2212.1 & 19.7 & 2298.3 & 24.8 & 2298.3 & 24.8 & 92.3 \\
\hline SYN14-11_Run3- 302 & 2387.8 & 40.7 & 2399.6 & 21.6 & 2409.6 & 19.7 & 2409.6 & 19.7 & 99.1 \\
\hline SYN14-11_Run1- 67 & 2518.2 & 26.3 & 2516.0 & 19.1 & 2514.3 & 27.3 & 2514.3 & 27.3 & 100.2 \\
\hline SYN14-11_Run2- 145 & 2457.6 & 36.1 & 2504.7 & 19.3 & 2543.0 & 18.4 & 2543.0 & 18.4 & 96.6 \\
\hline SYN14-11_Run1- 4 & 2547.4 & 23.9 & 2545.4 & 16.1 & 2543.8 & 21.8 & 2543.8 & 21.8 & 100.1 \\
\hline SYN14-11_Run1- 93 & 2514.0 & 39.6 & 2535.8 & 20.7 & 2553.4 & 19.1 & 2553.4 & 19.1 & 98.5 \\
\hline SYN14-11_Run1- 58 & 2591.4 & 26.6 & 2598.5 & 16.2 & 2603.9 & 20.0 & 2603.9 & 20.0 & 99.5 \\
\hline SYN14-11_Run1- 87 & 2622.3 & 36.3 & 2644.8 & 19.5 & 2662.1 & 20.1 & 2662.1 & 20.1 & 98.5 \\
\hline SYN14-11_Run1- 55 & 2622.4 & 32.4 & 2647.5 & 18.9 & 2666.7 & 22.1 & 2666.7 & 22.1 & 98.3 \\
\hline SYN14-11_Run1- 6 & 2723.6 & 42.8 & 2696.8 & 23.1 & 2676.9 & 24.9 & 2676.9 & 24.9 & 101.7 \\
\hline SYN14-11_Run2- 142 & 2625.8 & 35.7 & 2656.5 & 18.7 & 2679.9 & 18.3 & 2679.9 & 18.3 & 98.0 \\
\hline SYN14-11_Run1- 36 & 2528.4 & 35.8 & 2615.1 & 21.4 & 2682.9 & 24.9 & 2682.9 & 24.9 & 94.2 \\
\hline SYN14-11_Run1- 75 & 2564.4 & 42.6 & 2633.2 & 22.4 & 2686.4 & 20.9 & 2686.4 & 20.9 & 95.5 \\
\hline SYN14-11_Run1- 22 & 2679.3 & 31.6 & 2692.7 & 16.1 & 2702.7 & 15.0 & 2702.7 & 15.0 & 99.1 \\
\hline SYN14-11_Run3- 268 & 2690.7 & 27.8 & 2698.0 & 16.4 & 2703.5 & 19.6 & 2703.5 & 19.6 & 99.5 \\
\hline SYN14-11_Run3- 298 & 2672.7 & 35.2 & 2695.6 & 18.9 & 2712.8 & 19.7 & 2712.8 & 19.7 & 98.5 \\
\hline
\end{tabular}




\begin{tabular}{|l|l|l|l|l|l|l|l|l|l|} 
SYN14-11_Run3- 254 & 2687.4 & 59.2 & 2706.4 & 28.6 & 2720.5 & 22.6 & 2720.5 & 22.6 & 98.8 \\
\hline SYN14-11_Run1- 31 & 2735.6 & 38.6 & 2727.5 & 20.2 & 2721.4 & 20.5 & 2721.4 & 20.5 & 100.5 \\
\hline SYN14-11_Run1- 106 & 2651.0 & 33.0 & 2702.4 & 17.0 & 2741.0 & 16.1 & 2741.0 & 16.1 & 96.7 \\
\hline SYN14-11_Run3- 305 & 2792.2 & 41.7 & 2764.3 & 23.1 & 2744.0 & 26.3 & 2744.0 & 26.3 & 101.8 \\
\hline SYN14-11_Run3- 303 & 2721.0 & 47.3 & 2755.7 & 23.0 & 2781.2 & 19.0 & 2781.2 & 19.0 & 97.8 \\
\hline SYN14-11_Run1-97 & 2679.7 & 34.2 & 2752.5 & 17.9 & 2806.3 & 17.3 & 2806.3 & 17.3 & 95.5 \\
\hline
\end{tabular}

\begin{tabular}{|c|c|c|c|c|c|c|c|c|c|}
\hline Analysis & $206 \mathrm{~Pb}^{*}$ & \pm & $207 \mathrm{~Pb} *$ & \pm & $206 \mathrm{~Pb}^{*}$ & \pm & Best age & \pm & Conc \\
\hline & $238 U^{*}$ & (Ma) & $235 \mathrm{U}$ & (Ma) & $207 \mathrm{~Pb}^{*}$ & (Ma) & (Ma) & (Ma) & $(\%)$ \\
\hline & & & & & & & & & \\
\hline SYN14-12_Run1- 1 & 856.3 & 27.9 & 907.2 & 25.8 & 1033.2 & 51.2 & 1033.2 & 51.2 & 82.9 \\
\hline SYN14-12_Run1- 10 & 1024.0 & 20.5 & 1017.6 & 16.7 & 1003.9 & 29.3 & 1003.9 & 29.3 & 102.0 \\
\hline SYN14-12_Run1- 100 & 988.7 & 20.7 & 1010.0 & 17.5 & 1056.3 & 31.0 & 1056.3 & 31.0 & 93.6 \\
\hline SYN14-12_Run1- 101 & 300.3 & 6.8 & 310.9 & 10.7 & 391.7 & 72.8 & 300.3 & 6.8 & NA \\
\hline SYN14-12_Run1- 102 & 963.6 & 27.5 & 941.7 & 24.8 & 890.9 & 54.2 & 890.9 & 54.2 & 108.2 \\
\hline SYN14-12_Run1- 103 & 2619.4 & 83.7 & 2676.0 & 38.8 & 2719.1 & 22.0 & 2719.1 & 22.0 & 96.3 \\
\hline SYN14-12_Run1- 104 & 1871.1 & 27.9 & 1858.7 & 18.9 & 1844.9 & 25.4 & 1844.9 & 25.4 & 101.4 \\
\hline SYN14-12_Run1- 105 & 1129.7 & 32.0 & 1143.8 & 22.5 & 1170.5 & 22.3 & 1170.5 & 22.3 & 96.5 \\
\hline SYN14-12_Run1- 106 & 418.5 & 10.1 & 421.6 & 10.3 & 438.9 & 36.9 & 418.5 & 10.1 & 95.3 \\
\hline SYN14-12_Run1- 107 & 1064.2 & 20.1 & 1064.3 & 21.3 & 1064.7 & 50.1 & 1064.7 & 50.1 & 99.9 \\
\hline SYN14-12_Run1- 108 & 933.7 & 26.1 & 971.0 & 25.0 & 1056.6 & 53.3 & 1056.6 & 53.3 & 88.4 \\
\hline SYN14-12_Run1- 109 & 1045.1 & 22.2 & 1049.0 & 22.1 & 1057.2 & 49.6 & 1057.2 & 49.6 & 98.9 \\
\hline SYN14-12_Run1- 110 & 1159.1 & 30.3 & 1168.9 & 24.4 & 1187.0 & 40.7 & 1187.0 & 40.7 & 97.7 \\
\hline SYN14-12_Run1- 12 & 362.3 & 7.6 & 370.4 & 10.1 & 421.3 & 54.0 & 362.3 & 7.6 & NA \\
\hline SYN14-12_Run1- 13 & 1193.5 & 23.3 & 1181.6 & 19.3 & 1159.9 & 34.8 & 1159.9 & 34.8 & 102.9 \\
\hline SYN14-12_Run1- 14 & 2438.7 & 53.5 & 2471.2 & 27.6 & 2498.0 & 23.2 & 2498.0 & 23.2 & 97.6 \\
\hline SYN14-12_Run1- 15 & 560.5 & 16.7 & 561.5 & 18.9 & 565.7 & 67.1 & 560.5 & 16.7 & 99.1 \\
\hline SYN14-12_Run1- 16 & 1623.4 & 28.1 & 1617.8 & 20.9 & 1610.4 & 31.4 & 1610.4 & 31.4 & 100.8 \\
\hline SYN14-12_Run1- 17 & 1046.0 & 17.0 & 1047.2 & 15.8 & 1049.6 & 33.3 & 1049.6 & 33.3 & 99.7 \\
\hline SYN14-12_Run1- 18 & 1148.1 & 36.2 & 1154.6 & 27.6 & 1166.8 & 40.3 & 1166.8 & 40.3 & 98.4 \\
\hline SYN14-12_Run1- 19 & 1900.1 & 30.7 & 1911.0 & 20.4 & 1922.8 & 26.2 & 1922.8 & 26.2 & 98.8 \\
\hline SYN14-12_Run1- 2 & 1070.9 & 24.5 & 1073.9 & 19.8 & 1080.0 & 33.1 & 1080.0 & 33.1 & 99.1 \\
\hline SYN14-12_Run1- 20 & 2628.8 & 46.2 & 2677.4 & 27.4 & 2714.4 & 32.5 & 2714.4 & 32.5 & 96.8 \\
\hline SYN14-12_Run1- 21 & 1088.8 & 43.3 & 1089.4 & 31.3 & 1090.5 & 36.1 & 1090.5 & 36.1 & 99.8 \\
\hline SYN14-12_Run1- 22 & 1909.8 & 66.3 & 1921.6 & 37.9 & 1934.4 & 32.0 & 1934.4 & 32.0 & 98.7 \\
\hline SYN14-12_Run1- 23 & 1037.2 & 18.6 & 1047.5 & 16.1 & 1069.2 & 30.5 & 1069.2 & 30.5 & 97.0 \\
\hline SYN14-12_Run1- 24 & 1022.7 & 23.3 & 1031.6 & 17.7 & 1050.5 & 23.9 & 1050.5 & 23.9 & 97.4 \\
\hline SYN14-12_Run1- 25 & 311.8 & 8.5 & 313.9 & 10.2 & 329.8 & 57.1 & 311.8 & 8.5 & NA \\
\hline
\end{tabular}




\begin{tabular}{|c|c|c|c|c|c|c|c|c|c|}
\hline SYN14-12_Run1- 26 & 1627.7 & 34.3 & 1641.4 & 24.2 & 1658.9 & 33.1 & 1658.9 & 33.1 & 98.1 \\
\hline SYN14-12_Run1- 27 & 1033.3 & 28.8 & 1036.8 & 22.0 & 1044.3 & 31.4 & 1044.3 & 31.4 & 98.9 \\
\hline SYN14-12_Run1- 28 & 1130.2 & 28.1 & 1147.6 & 23.9 & 1180.6 & 43.2 & 1180.6 & 43.2 & 95.7 \\
\hline SYN14-12_Run1- 29 & 1605.3 & 22.2 & 1626.2 & 19.2 & 1653.4 & 33.0 & 1653.4 & 33.0 & 97.1 \\
\hline SYN14-12_Run1- 3 & 854.1 & 22.3 & 884.5 & 18.8 & 961.3 & 31.1 & 961.3 & 31.1 & 88.8 \\
\hline SYN14-12_Run1- 30 & 1015.9 & 25.8 & 1032.5 & 23.3 & 1068.0 & 46.4 & 1068.0 & 46.4 & 95.1 \\
\hline SYN14-12_Run1- 31 & 800.0 & 19.3 & 830.6 & 19.1 & 913.3 & 45.0 & 800.0 & 19.3 & 87.6 \\
\hline SYN14-12_Run1- 32 & 1529.9 & 30.6 & 1558.3 & 20.5 & 1596.8 & 23.6 & 1596.8 & 23.6 & 95.8 \\
\hline SYN14-12_Run1- 33 & 1034.4 & 18.7 & 1046.2 & 20.1 & 1071.1 & 47.9 & 1071.1 & 47.9 & 96.6 \\
\hline SYN14-12_Run1- 34 & 1231.0 & 32.2 & 1227.5 & 22.0 & 1221.5 & 22.5 & 1221.5 & 22.5 & 100.8 \\
\hline SYN14-12_Run1- 35 & 1338.0 & 48.0 & 1343.6 & 32.8 & 1352.6 & 36.4 & 1352.6 & 36.4 & 98.9 \\
\hline SYN14-12_Run1- 36 & 2472.8 & 60.3 & 2506.7 & 30.3 & 2534.3 & 23.7 & 2534.3 & 23.7 & 97.6 \\
\hline SYN14-12_Run1- 37 & 1009.5 & 21.3 & 1020.7 & 18.3 & 1044.8 & 34.4 & 1044.8 & 34.4 & 96.6 \\
\hline SYN14-12_Run1- 38 & 2037.7 & 39.6 & 2071.6 & 23.0 & 2105.5 & 22.5 & 2105.5 & 22.5 & 96.8 \\
\hline SYN14-12_Run1- 39 & 2634.4 & 44.8 & 2670.6 & 25.4 & 2698.0 & 28.5 & 2698.0 & 28.5 & 97.6 \\
\hline SYN14-12_Run1- 4 & 1043.0 & 25.3 & 1041.6 & 22.2 & 1038.7 & 43.8 & 1038.7 & 43.8 & 100.4 \\
\hline SYN14-12_Run1- 40 & 1452.4 & 34.1 & 1436.9 & 22.6 & 1414.0 & 25.7 & 1414.0 & 25.7 & 102.7 \\
\hline SYN14-12_Run1- 41 & 534.3 & 21.5 & 511.0 & 19.0 & 408.2 & 49.8 & 534.3 & 21.5 & 130.9 \\
\hline SYN14-12_Run1- 42 & 1943.3 & 32.5 & 1947.3 & 20.6 & 1951.6 & 24.5 & 1951.6 & 24.5 & 99.6 \\
\hline SYN14-12_Run1- 43 & 1030.3 & 29.4 & 1061.5 & 31.0 & 1126.1 & 70.7 & 1126.1 & 70.7 & 91.5 \\
\hline SYN14-12_Run1- 44 & 1007.5 & 21.5 & 1020.5 & 20.1 & 1048.7 & 42.5 & 1048.7 & 42.5 & 96.1 \\
\hline SYN14-12_Run1- 45 & 612.0 & 14.5 & 610.4 & 16.6 & 604.3 & 57.0 & 612.0 & 14.5 & 101.3 \\
\hline SYN14-12_Run1- 46 & 1668.2 & 37.0 & 1682.1 & 23.0 & 1699.4 & 22.5 & 1699.4 & 22.5 & 98.2 \\
\hline SYN14-12_Run1- 47 & 1669.2 & 63.5 & 1685.0 & 37.6 & 1704.7 & 27.6 & 1704.7 & 27.6 & 97.9 \\
\hline SYN14-12_Run1- 48 & 1034.2 & 20.6 & 1038.9 & 19.3 & 1048.8 & 41.2 & 1048.8 & 41.2 & 98.6 \\
\hline SYN14-12_Run1- 49 & 2534.0 & 40.0 & 2591.6 & 20.2 & 2637.0 & 16.7 & 2637.0 & 16.7 & 96.1 \\
\hline SYN14-12_Run1- 50 & 1697.1 & 28.8 & 1684.7 & 23.0 & 1669.2 & 37.5 & 1669.2 & 37.5 & 101.7 \\
\hline SYN14-12_Run1- 51 & 1056.3 & 29.5 & 1065.7 & 24.5 & 1085.1 & 43.2 & 1085.1 & 43.2 & 97.3 \\
\hline SYN14-12_Run1- 53 & 230.3 & 7.9 & 228.6 & 9.5 & 212.1 & 71.1 & 230.3 & 7.9 & NA \\
\hline SYN14-12_Run1- 54 & 1792.7 & 29.0 & 1780.1 & 20.3 & 1765.4 & 28.5 & 1765.4 & 28.5 & 101.5 \\
\hline SYN14-12_Run1- 55 & 1214.8 & 39.4 & 1221.4 & 29.8 & 1233.1 & 43.5 & 1233.1 & 43.5 & 98.5 \\
\hline SYN14-12_Run1- 56 & 956.9 & 22.6 & 950.6 & 20.4 & 936.0 & 43.3 & 936.0 & 43.3 & 102.2 \\
\hline SYN14-12_Run1- 57 & 1003.7 & 27.5 & 1008.0 & 21.8 & 1017.4 & 34.4 & 1017.4 & 34.4 & 98.7 \\
\hline SYN14-12_Run1- 58 & 1005.9 & 22.2 & 1019.3 & 21.8 & 1048.3 & 48.4 & 1048.3 & 48.4 & 96.0 \\
\hline SYN14-12_Run1- 59 & 1041.4 & 31.1 & 1032.4 & 23.9 & 1013.2 & 36.2 & 1013.2 & 36.2 & 102.8 \\
\hline SYN14-12_Run1- 6 & 1089.2 & 19.8 & 1071.1 & 17.8 & 1034.5 & 36.6 & 1034.5 & 36.6 & 105.3 \\
\hline SYN14-12_Run1- 60 & 1049.3 & 22.8 & 1058.1 & 19.3 & 1076.5 & 35.3 & 1076.5 & 35.3 & 97.5 \\
\hline SYN14-12_Run1- 61 & 1031.0 & 21.1 & 1016.3 & 20.1 & 984.8 & 45.2 & 984.8 & 45.2 & 104.7 \\
\hline SYN14-12_Run1- 62 & 2700.4 & 61.3 & 2703.7 & 31.0 & 2706.1 & 28.8 & 2706.1 & 28.8 & 99.8 \\
\hline
\end{tabular}




\begin{tabular}{|c|c|c|c|c|c|c|c|c|c|}
\hline SYN14-12_Run1- 63 & 1653.1 & 28.0 & 1652.1 & 19.2 & 1650.8 & 25.1 & 1650.8 & 25.1 & 100.1 \\
\hline SYN14-12_Run1- 64 & 1039.5 & 22.9 & 1051.4 & 23.9 & 1076.2 & 55.3 & 1076.2 & 55.3 & 96.6 \\
\hline SYN14-12_Run1- 65 & 2597.9 & 65.8 & 2669.1 & 33.3 & 2723.4 & 28.5 & 2723.4 & 28.5 & 95.4 \\
\hline SYN14-12_Run1- 66 & 437.2 & 11.4 & 452.1 & 17.3 & 528.6 & 85.8 & 437.2 & 11.4 & 82.7 \\
\hline SYN14-12_Run1- 67 & 1037.7 & 39.7 & 1051.1 & 28.7 & 1079.0 & 29.0 & 1079.0 & 29.0 & 96.2 \\
\hline SYN14-12_Run1- 68 & 1225.9 & 44.9 & 1243.1 & 30.7 & 1272.9 & 28.3 & 1272.9 & 28.3 & 96.3 \\
\hline SYN14-12_Run1- 69 & 1005.6 & 17.7 & 1001.7 & 21.1 & 993.0 & 55.2 & 993.0 & 55.2 & 101.3 \\
\hline SYN14-12_Run1- 7 & 994.0 & 23.0 & 1004.4 & 19.7 & 1027.2 & 37.0 & 1027.2 & 37.0 & 96.8 \\
\hline SYN14-12_Run1- 70 & 2742.8 & 45.8 & 2744.3 & 23.8 & 2745.4 & 23.9 & 2745.4 & 23.9 & 99.9 \\
\hline SYN14-12_Run1- 71 & 1401.3 & 24.4 & 1410.8 & 17.7 & 1425.1 & 24.2 & 1425.1 & 24.2 & 98.3 \\
\hline SYN14-12_Run1- 72 & 233.7 & 5.2 & 221.3 & 11.3 & 91.2 & 123.1 & 233.7 & 5.2 & NA \\
\hline SYN14-12_Run1- 73 & 1150.2 & 33.8 & 1138.0 & 27.9 & 1114.8 & 50.0 & 1114.8 & 50.0 & 103.2 \\
\hline SYN14-12_Run1- 74 & 1665.2 & 32.7 & 1714.8 & 24.4 & 1776.0 & 35.3 & 1776.0 & 35.3 & 93.8 \\
\hline SYN14-12_Run1- 75 & 1079.6 & 16.0 & 1061.0 & 15.2 & 1022.9 & 33.7 & 1022.9 & 33.7 & 105.5 \\
\hline SYN14-12_Run1- 76 & 1348.3 & 36.8 & 1377.9 & 26.6 & 1424.0 & 34.8 & 1424.0 & 34.8 & 94.7 \\
\hline SYN14-12_Run1- 77 & 1043.8 & 35.9 & 1034.4 & 25.4 & 1014.8 & 24.2 & 1014.8 & 24.2 & 102.9 \\
\hline SYN14-12_Run1- 78 & 1483.6 & 36.1 & 1457.3 & 23.7 & 1419.2 & 27.1 & 1419.2 & 27.1 & 104.5 \\
\hline SYN14-12_Run1- 79 & 1038.6 & 17.8 & 1045.1 & 17.1 & 1058.7 & 37.2 & 1058.7 & 37.2 & 98.1 \\
\hline SYN14-12_Run1- 8 & 239.0 & 7.7 & 240.7 & 8.5 & 258.1 & 52.5 & 239.0 & 7.7 & NA \\
\hline SYN14-12_Run1- 80 & 1296.9 & 25.7 & 1318.9 & 22.0 & 1354.8 & 38.9 & 1354.8 & 38.9 & 95.7 \\
\hline SYN14-12_Run1- 81 & 592.6 & 15.8 & 602.8 & 18.5 & 641.5 & 63.9 & 592.6 & 15.8 & 92.4 \\
\hline SYN14-12_Run1- 82 & 236.4 & 6.7 & 233.9 & 8.8 & 208.3 & 71.4 & 236.4 & 6.7 & NA \\
\hline SYN14-12_Run1- 83 & 975.0 & 16.5 & 989.8 & 19.6 & 1022.7 & 50.9 & 1022.7 & 50.9 & 95.3 \\
\hline SYN14-12_Run1- 84 & 770.3 & 20.3 & 763.6 & 19.1 & 744.0 & 46.3 & 770.3 & 20.3 & 103.5 \\
\hline SYN14-12_Run1- 85 & 585.8 & 15.6 & 584.6 & 16.4 & 580.0 & 52.7 & 585.8 & 15.6 & 101.0 \\
\hline SYN14-12_Run1- 86 & 433.8 & 8.1 & 434.3 & 9.6 & 436.7 & 42.8 & 433.8 & 8.1 & 99.3 \\
\hline SYN14-12_Run1- 87 & 2691.7 & 60.8 & 2722.4 & 29.6 & 2745.3 & 24.3 & 2745.3 & 24.3 & 98.0 \\
\hline SYN14-12_Run1- 88 & 1485.0 & 34.8 & 1481.4 & 22.5 & 1476.3 & 22.8 & 1476.3 & 22.8 & 100.6 \\
\hline SYN14-12_Run1- 89 & 1730.0 & 34.3 & 1727.9 & 24.7 & 1725.4 & 35.5 & 1725.4 & 35.5 & 100.3 \\
\hline SYN14-12_Run1- 9 & 1099.9 & 29.0 & 1088.3 & 28.5 & 1065.1 & 63.8 & 1065.1 & 63.8 & 103.3 \\
\hline SYN14-12_Run1- 90 & 379.9 & 6.0 & 376.1 & 6.9 & 352.4 & 32.9 & 379.9 & 6.0 & NA \\
\hline SYN14-12_Run1- 91 & 905.0 & 22.3 & 927.9 & 19.9 & 982.7 & 39.4 & 982.7 & 39.4 & 92.1 \\
\hline SYN14-12_Run1- 92 & 1045.9 & 25.9 & 1024.4 & 19.1 & 978.8 & 25.6 & 978.8 & 25.6 & 106.9 \\
\hline SYN14-12_Run1- 93 & 1126.7 & 26.8 & 1140.5 & 21.4 & 1166.8 & 34.3 & 1166.8 & 34.3 & 96.6 \\
\hline SYN14-12_Run1- 94 & 429.7 & 8.8 & 440.8 & 12.2 & 499.2 & 58.4 & 429.7 & 8.8 & 86.1 \\
\hline SYN14-12_Run1- 95 & 1578.3 & 31.5 & 1569.0 & 21.7 & 1556.5 & 28.6 & 1556.5 & 28.6 & 101.4 \\
\hline SYN14-12_Run1- 96 & 1128.7 & 26.8 & 1133.6 & 23.7 & 1143.0 & 46.2 & 1143.0 & 46.2 & 98.7 \\
\hline SYN14-12_Run1- 97 & 1089.6 & 30.6 & 1110.0 & 28.4 & 1150.3 & 57.3 & 1150.3 & 57.3 & 94.7 \\
\hline SYN14-12_Run1- 98 & 1126.8 & 22.4 & 1163.8 & 22.2 & 1233.3 & 46.4 & 1233.3 & 46.4 & 91.4 \\
\hline
\end{tabular}




\begin{tabular}{|c|c|c|c|c|c|c|c|c|c|}
\hline SYN14-12_Run1- 99 & 1131.4 & 21.0 & 1133.9 & 17.1 & 1138.5 & 29.1 & 1138.5 & 29.1 & 99.4 \\
\hline SYN14-12_Run2- 111 & 980.7 & 28.9 & 986.1 & 21.4 & 997.9 & 24.3 & 997.9 & 24.3 & 98.3 \\
\hline SYN14-12_Run2- 112 & 419.2 & 17.4 & 420.5 & 19.2 & 427.8 & 79.5 & 419.2 & 17.4 & 98.0 \\
\hline SYN14-12_Run2- 113 & 808.2 & 17.8 & 838.9 & 18.4 & 921.2 & 45.3 & 808.2 & 17.8 & 87.7 \\
\hline SYN14-12_Run2- 114 & 1003.2 & 16.9 & 1007.6 & 14.7 & 1017.3 & 28.6 & 1017.3 & 28.6 & 98.6 \\
\hline SYN14-12_Run2- 115 & 1000.8 & 30.0 & 988.4 & 23.0 & 961.0 & 34.4 & 961.0 & 34.4 & 104.1 \\
\hline SYN14-12_Run2- 116 & 403.7 & 7.4 & 398.6 & 8.9 & 369.0 & 43.0 & 403.7 & 7.4 & 109.4 \\
\hline SYN14-12_Run2- 117 & 996.9 & 21.2 & 1018.3 & 20.1 & 1064.7 & 42.8 & 1064.7 & 42.8 & 93.6 \\
\hline SYN14-12_Run2- 118 & 1021.2 & 23.3 & 1025.6 & 20.0 & 1034.9 & 38.2 & 1034.9 & 38.2 & 98.7 \\
\hline SYN14-12_Run2- 119 & 1906.3 & 30.1 & 1929.5 & 20.7 & 1954.5 & 27.8 & 1954.5 & 27.8 & 97.5 \\
\hline SYN14-12_Run2- 120 & 1187.3 & 50.9 & 1212.5 & 36.7 & 1257.6 & 43.1 & 1257.6 & 43.1 & 94.4 \\
\hline SYN14-12_Run2- 121 & 1101.4 & 23.6 & 1093.8 & 18.2 & 1078.6 & 28.0 & 1078.6 & 28.0 & 102.1 \\
\hline SYN14-12_Run2- 123 & 1005.4 & 16.3 & 1015.4 & 15.9 & 1037.0 & 35.1 & 1037.0 & 35.1 & 96.9 \\
\hline SYN14-12_Run2- 126 & 1123.4 & 21.8 & 1133.2 & 20.2 & 1152.2 & 40.9 & 1152.2 & 40.9 & 97.5 \\
\hline SYN14-12_Run2- 127 & 1415.6 & 52.4 & 1439.1 & 32.8 & 1473.9 & 20.6 & 1473.9 & 20.6 & 96.0 \\
\hline SYN14-12_Run2- 128 & 989.2 & 20.9 & 1009.4 & 16.6 & 1053.6 & 25.2 & 1053.6 & 25.2 & 93.9 \\
\hline SYN14-12_Run2- 129 & 2296.2 & 48.3 & 2350.0 & 28.6 & 2397.0 & 31.7 & 2397.0 & 31.7 & 95.8 \\
\hline SYN14-12_Run2- 130 & 322.9 & 6.9 & 305.8 & 9.5 & 177.0 & 66.6 & 322.9 & 6.9 & NA \\
\hline SYN14-12_Run2- 131 & 2717.9 & 46.4 & 2739.0 & 25.5 & 2754.5 & 27.7 & 2754.5 & 27.7 & 98.7 \\
\hline SYN14-12_Run2- 132 & 1083.7 & 22.1 & 1092.6 & 17.7 & 1110.3 & 29.2 & 1110.3 & 29.2 & 97.6 \\
\hline SYN14-12_Run2- 133 & 1618.3 & 32.7 & 1622.6 & 21.1 & 1628.2 & 23.1 & 1628.2 & 23.1 & 99.4 \\
\hline SYN14-12_Run2- 134 & 415.1 & 10.4 & 416.0 & 13.6 & 421.0 & 67.8 & 415.1 & 10.4 & 98.6 \\
\hline SYN14-12_Run2- 135 & 989.6 & 16.0 & 990.4 & 16.5 & 992.2 & 39.5 & 992.2 & 39.5 & 99.7 \\
\hline SYN14-12_Run2- 137 & 981.4 & 29.4 & 999.6 & 24.7 & 1039.7 & 43.8 & 1039.7 & 43.8 & 94.4 \\
\hline SYN14-12_Run2- 138 & 561.6 & 18.1 & 570.1 & 21.1 & 604.3 & 75.1 & 561.6 & 18.1 & 92.9 \\
\hline SYN14-12_Run2- 139 & 367.7 & 8.6 & 368.2 & 10.4 & 371.2 & 53.2 & 367.7 & 8.6 & NA \\
\hline SYN14-12_Run2- 140 & 1208.2 & 26.8 & 1237.4 & 20.8 & 1288.6 & 31.0 & 1288.6 & 31.0 & 93.8 \\
\hline SYN14-12_Run2- 141 & 1677.4 & 61.9 & 1694.4 & 36.9 & 1715.5 & 29.1 & 1715.5 & 29.1 & 97.8 \\
\hline SYN14-12_Run2- 142 & 1070.0 & 32.5 & 1089.2 & 25.1 & 1128.0 & 35.6 & 1128.0 & 35.6 & 94.9 \\
\hline SYN14-12_Run2- 144 & 1405.6 & 48.6 & 1438.7 & 32.2 & 1488.0 & 31.0 & 1488.0 & 31.0 & 94.5 \\
\hline SYN14-12_Run2- 145 & 1041.4 & 24.1 & 1036.2 & 18.7 & 1025.2 & 29.0 & 1025.2 & 29.0 & 101.6 \\
\hline SYN14-12_Run2- 146 & 298.1 & 6.1 & 304.4 & 8.5 & 352.4 & 55.4 & 298.1 & 6.1 & NA \\
\hline SYN14-12_Run2- 147 & 1036.7 & 25.2 & 1055.5 & 21.1 & 1094.6 & 37.1 & 1094.6 & 37.1 & 94.7 \\
\hline SYN14-12_Run2- 148 & 1110.8 & 32.1 & 1133.7 & 22.7 & 1177.8 & 20.7 & 1177.8 & 20.7 & 94.3 \\
\hline SYN14-12_Run2- 149 & 2627.9 & 37.4 & 2660.9 & 19.5 & 2686.1 & 18.8 & 2686.1 & 18.8 & 97.8 \\
\hline SYN14-12_Run2- 150 & 590.3 & 14.6 & 601.7 & 18.6 & 644.8 & 68.6 & 590.3 & 14.6 & 91.5 \\
\hline SYN14-12_Run2- 151 & 408.4 & 5.6 & 411.0 & 7.3 & 425.8 & 36.0 & 408.4 & 5.6 & 95.9 \\
\hline SYN14-12_Run2- 152 & 1009.2 & 21.4 & 1022.1 & 19.7 & 1050.0 & 40.8 & 1050.0 & 40.8 & 96.1 \\
\hline SYN14-12_Run2- 153 & 1795.8 & 27.7 & 1791.9 & 21.8 & 1787.3 & 34.6 & 1787.3 & 34.6 & 100.5 \\
\hline
\end{tabular}




\begin{tabular}{|c|c|c|c|c|c|c|c|c|c|}
\hline SYN14-12_Run2- 155 & 397.9 & 7.6 & 401.0 & 8.3 & 419.2 & 33.8 & 397.9 & 7.6 & NA \\
\hline SYN14-12_Run2- 156 & 1004.1 & 23.4 & 1013.0 & 19.9 & 1032.3 & 36.7 & 1032.3 & 36.7 & 97.3 \\
\hline SYN14-12_Run2- 157 & 977.9 & 12.9 & 986.4 & 13.2 & 1005.2 & 31.0 & 1005.2 & 31.0 & 97.3 \\
\hline SYN14-12_Run2- 158 & 1129.1 & 34.6 & 1143.3 & 30.5 & 1170.2 & 57.9 & 1170.2 & 57.9 & 96.5 \\
\hline SYN14-12_Run2- 159 & 2669.2 & 37.7 & 2696.9 & 20.2 & 2717.6 & 20.7 & 2717.6 & 20.7 & 98.2 \\
\hline SYN14-12_Run2- 160 & 1138.5 & 31.3 & 1142.8 & 22.8 & 1150.9 & 28.7 & 1150.9 & 28.7 & 98.9 \\
\hline SYN14-12_Run2- 161 & 1050.4 & 16.0 & 1054.8 & 16.3 & 1064.0 & 37.2 & 1064.0 & 37.2 & 98.7 \\
\hline SYN14-12_Run2- 162 & 250.7 & 7.1 & 248.9 & 10.2 & 231.8 & 82.5 & 250.7 & 7.1 & NA \\
\hline SYN14-12_Run2- 163 & 1938.7 & 36.4 & 1943.8 & 21.1 & 1949.3 & 19.6 & 1949.3 & 19.6 & 99.5 \\
\hline SYN14-12_Run2- 164 & 1036.8 & 20.4 & 1028.7 & 17.2 & 1011.3 & 32.1 & 1011.3 & 32.1 & 102.5 \\
\hline SYN14-12_Run2- 177 & 862.4 & 27.7 & 911.8 & 25.5 & 1033.5 & 50.0 & 1033.5 & 50.0 & 83.4 \\
\hline SYN14-12_Run2- 178 & 446.3 & 10.3 & 452.1 & 14.0 & 482.1 & 66.0 & 446.3 & 10.3 & 92.6 \\
\hline SYN14-12_Run2- 179 & 2672.7 & 75.8 & 2731.5 & 35.8 & 2775.2 & 24.8 & 2775.2 & 24.8 & 96.3 \\
\hline SYN14-12_Run2- 180 & 1703.1 & 49.7 & 1702.3 & 31.3 & 01.2 & 33.8 & 1.2 & 33.8 & 100.1 \\
\hline SYN14-12_Run2- 181 & 1481.2 & 33.4 & 1617.4 & 23.6 & 1799.5 & 26.5 & 1799.5 & 26.5 & 82.3 \\
\hline SYN14-12_Run2- 182 & 2590.4 & 80.4 & 2672.0 & 40.0 & 2734.3 & 32.1 & 2 & 32.1 & 94.7 \\
\hline SYN14-12_Run2- 183 & 1170.1 & 24.8 & 1189.3 & 19.8 & 1224.3 & 31.6 & 1 & 31.6 & 95.6 \\
\hline SYN14-12_Run2- 184 & 441.1 & 12.5 & 435.9 & 13.1 & 3.6 & 50.8 & 4 & 12.5 & 107.9 \\
\hline SYN14-12_Run2- 185 & 664.4 & 13.6 & 721.4 & 14.7 & 902.8 & 38.7 & 664.4 & 13.6 & 73.6 \\
\hline SYN14-12_Run2- 186 & 1009.8 & 32.8 & 1007.2 & 27.0 & 1001.5 & 47.9 & 10 & 47.9 & 100.8 \\
\hline SYN14-12_Run2- 187 & 1830.6 & 52.3 & 1873.3 & 31.0 & 1921.0 & 27.8 & 1921.0 & 27.8 & 95.3 \\
\hline SYN14-12_Run2- 188 & 1782.0 & 32.0 & 1767.9 & 20.6 & 1751.1 & 24.7 & 1751.1 & 24.7 & 101.8 \\
\hline SYN14-12_Run2- 189 & 1719.8 & 37.4 & 1719.9 & 25.3 & 1719.9 & 32.8 & 1719.9 & 32.8 & 100.0 \\
\hline SYN14-12_Run2- 190 & 1347.8 & 39.6 & 1351.1 & 27.3 & 1356.4 & 31.8 & 1356.4 & 31.8 & 99.4 \\
\hline SYN14-12_Run2- 191 & 1051.7 & 21.0 & 1055.3 & 17.1 & 1062.8 & 29.0 & 1062.8 & 29.0 & 99.0 \\
\hline SYN14-12_Run2- 192 & 1685.5 & 29.7 & 1706.6 & 18.9 & 1732.5 & 20.5 & 1732.5 & 20.5 & 97.3 \\
\hline SYN14-12_Run2- 193 & 1250.0 & 26.0 & 1224.4 & 21.9 & 1179.7 & 40.5 & 1179.7 & 40.5 & 106.0 \\
\hline SYN14-12_Run2- 194 & 1674.9 & 39.6 & 1709.2 & 28.4 & 1751.5 & 39.4 & 1751.5 & 39.4 & 95.6 \\
\hline SYN14-12_Run2- 196 & 905.8 & 21.6 & 928.1 & 19.6 & 981.4 & 40.0 & 981.4 & 40.0 & 92.3 \\
\hline SYN14-12_Run2- 197 & 1633.3 & 29.7 & 1639.4 & 22.6 & 1647.2 & 34.6 & 1647.2 & 34.6 & 99.2 \\
\hline SYN14-12_Run2- 198 & 617.4 & 10.4 & 609.6 & 10.9 & 580.7 & 34.2 & 617.4 & 10.4 & 106.3 \\
\hline SYN14-12_Run2- 200 & 1675.7 & 40.0 & 1679.2 & 25.1 & 1683.6 & 26.4 & 1683.6 & 26.4 & 99.5 \\
\hline SYN14-12_Run2- 201 & 1563.4 & 31.0 & 1572.3 & 20.3 & 1584.2 & 22.7 & 1584.2 & 22.7 & 98.7 \\
\hline SYN14-12_Run2- 202 & 272.1 & 6.3 & 277.7 & 7.1 & 324.5 & 39.4 & 272.1 & 6.3 & NA \\
\hline SYN14-12_Run2- 203 & 1525.4 & 27.6 & 1538.6 & 22.2 & 1556.7 & 36.3 & 1556.7 & 36.3 & 98.0 \\
\hline SYN14-12_Run2- 204 & 1034.5 & 28.2 & 1047.5 & 24.1 & 1074.7 & 44.3 & 1074.7 & 44.3 & 96.3 \\
\hline SYN14-12_Run2- 205 & 1379.6 & 46.1 & 1405.6 & 31.6 & 1445.3 & 35.5 & 1445.3 & 35.5 & 95.5 \\
\hline SYN14-12_Run2- 206 & 1830.5 & 57.9 & 1854.1 & 32.5 & 1880.7 & 20.9 & 1880.7 & 20.9 & 97.3 \\
\hline SYN14-12_Run2- 207 & 1461.3 & 38.8 & 1446.0 & 26.7 & 1423.4 & 34.0 & 1423.4 & 34.0 & 102.7 \\
\hline
\end{tabular}




\begin{tabular}{|c|c|c|c|c|c|c|c|c|c|}
\hline SYN14-12_Run2- 208 & 168.7 & 4.5 & 174.8 & 6.0 & 258.9 & 59.5 & 168.7 & 4.5 & NA \\
\hline SYN14-12_Run2- 209 & 1362.2 & 17.1 & 1380.5 & 14.3 & 1408.8 & 24.6 & 1408.8 & 24.6 & 96.7 \\
\hline SYN14-12_Run2- 210 & 348.1 & 8.4 & 348.1 & 9.3 & 348.2 & 44.0 & 348.1 & 8.4 & NA \\
\hline SYN14-12_Run2- 211 & 2505.6 & 71.1 & 2551.8 & 36.2 & 2588.7 & 30.4 & 2588.7 & 30.4 & 96.8 \\
\hline SYN14-12_Run2- 212 & 566.0 & 11.7 & 568.4 & 12.4 & 578.2 & 40.3 & 566.0 & 11.7 & 97.9 \\
\hline SYN14-12_Run2- 213 & 1854.2 & 83.7 & 1863.0 & 46.5 & 1872.9 & 29.4 & 1872.9 & 29.4 & 99.0 \\
\hline SYN14-12_Run2- 214 & 589.1 & 14.9 & 600.1 & 17.7 & 642.2 & 61.9 & 589.1 & 14.9 & 91.7 \\
\hline SYN14-12_Run2- 215 & 998.8 & 30.9 & 1003.2 & 22.8 & 1012.8 & 25.8 & 1012.8 & 25.8 & 98.6 \\
\hline SYN14-12_Run2- 216 & 1756.2 & 58.5 & 1742.8 & 34.0 & 1726.6 & 27.1 & 1726.6 & 27.1 & 101.7 \\
\hline SYN14-12_Run2- 217 & 1074.7 & 18.1 & 1081.0 & 18.9 & 1093.7 & 43.6 & 1093.7 & 43.6 & 98.3 \\
\hline SYN14-12_Run2- 218 & 960.9 & 15.8 & 974.5 & 14.0 & 1005.3 & 27.6 & 1005.3 & 27.6 & 95.6 \\
\hline SYN14-12_Run2- 219 & 1095.2 & 32.6 & 1105.4 & 24.4 & 1125.4 & 32.5 & 1125.4 & 32.5 & 97.3 \\
\hline SYN14-12_Run2- 220 & 1014.8 & 26.6 & 1030.8 & 20.8 & 1065.0 & 30.7 & 1065.0 & 30.7 & 95.3 \\
\hline SYN14-12_Run3- 221 & 2727.6 & 56.6 & 2788.0 & 27.7 & 2832.1 & 23.3 & 2832.1 & 23.3 & 96.3 \\
\hline SYN14-12_Run3- 222 & 1343.3 & 26.2 & 1349.0 & 18.7 & 1358.0 & 24.2 & 1358.0 & 24.2 & 98.9 \\
\hline SYN14-12_Run3- 223 & 1069.5 & 35.3 & 1066.6 & 25.7 & 1060.7 & 30.9 & 1060.7 & 30.9 & 100.8 \\
\hline SYN14-12_Run3- 224 & 2620.6 & 47.3 & 2718.8 & 23.2 & 2792.5 & 17.9 & 92.5 & 17.9 & 93.8 \\
\hline SYN14-12_Run3- 225 & 525.0 & 10.6 & 524.8 & 10.0 & 524.0 & 27.0 & 525.0 & 10.6 & 100.2 \\
\hline SYN14-12_Run3- 226 & 1315.6 & 44.2 & 1314.3 & 30.3 & 1312.2 & 34.0 & 1312.2 & 34.0 & 100.3 \\
\hline SYN14-12_Run3- 227 & 1155.1 & 21.9 & 1183.9 & 18.6 & 1236.9 & 32.7 & 1236.9 & 32.7 & 93.4 \\
\hline SYN14-12_Run3- 228 & 311.1 & 12.0 & 300.7 & 17.4 & 220.5 & 124.6 & 311.1 & 12.0 & NA \\
\hline SYN14-12_Run3- 229 & 460.4 & 6.9 & 450.5 & 10.2 & 400.8 & 52.3 & 460.4 & 6.9 & 114.9 \\
\hline SYN14-12_Run3- 230 & 1005.6 & 20.1 & 1026.6 & 21.9 & 1071.8 & 52.4 & 1071.8 & 52.4 & 93.8 \\
\hline SYN14-12_Run3- 231 & 410.6 & 10.6 & 418.5 & 10.9 & 462.4 & 38.0 & 410.6 & 10.6 & 88.8 \\
\hline SYN14-12_Run3- 232 & 1141.7 & 17.9 & 1176.4 & 15.9 & 1240.8 & 29.7 & 1240.8 & 29.7 & 92.0 \\
\hline SYN14-12_Run3- 233 & 612.8 & 10.9 & 600.2 & 10.7 & 552.6 & 31.7 & 612.8 & 10.9 & 110.9 \\
\hline SYN14-12_Run3- 234 & 1305.0 & 64.2 & 1335.6 & 45.8 & 1384.9 & 55.6 & 1384.9 & 55.6 & 94.2 \\
\hline SYN14-12_Run3- 235 & 1133.8 & 29.8 & 1134.4 & 23.6 & 1135.4 & 38.4 & 1135.4 & 38.4 & 99.9 \\
\hline SYN14-12_Run3- 236 & 342.6 & 7.4 & 340.9 & 12.8 & 329.5 & 86.5 & 342.6 & 7.4 & NA \\
\hline SYN14-12_Run3- 237 & 1878.4 & 35.7 & 1876.1 & 21.9 & 1873.5 & 23.9 & 1873.5 & 23.9 & 100.3 \\
\hline SYN14-12_Run3- 238 & 1351.5 & 26.8 & 1356.2 & 21.3 & 1363.5 & 34.8 & 1363.5 & 34.8 & 99.1 \\
\hline SYN14-12_Run3- 239 & 1038.9 & 31.9 & 1043.7 & 22.9 & 1053.8 & 22.3 & 1053.8 & 22.3 & 98.6 \\
\hline SYN14-12_Run3- 240 & 1435.3 & 33.7 & 1432.5 & 23.4 & 1428.5 & 29.5 & 1428.5 & 29.5 & 100.5 \\
\hline SYN14-12_Run3- 241 & 1455.7 & 24.9 & 1479.4 & 18.3 & 1513.6 & 25.7 & 1513.6 & 25.7 & 96.2 \\
\hline SYN14-12_Run3- 242 & 1015.7 & 22.4 & 1025.3 & 18.5 & 1046.0 & 32.0 & 1046.0 & 32.0 & 97.1 \\
\hline SYN14-12_Run3- 243 & 998.8 & 29.9 & 993.6 & 26.1 & 982.2 & 52.1 & 982.2 & 52.1 & 101.7 \\
\hline SYN14-12_Run3- 244 & 312.7 & 12.7 & 308.0 & 12.8 & 272.5 & 55.7 & 312.7 & 12.7 & NA \\
\hline SYN14-12_Run3- 245 & 1068.5 & 34.1 & 1080.1 & 25.0 & 1103.6 & 29.4 & 1103.6 & 29.4 & 96.8 \\
\hline SYN14-12_Run3- 246 & 1430.2 & 39.1 & 1437.3 & 28.2 & 1447.8 & 38.8 & 1447.8 & 38.8 & 98.8 \\
\hline
\end{tabular}




\begin{tabular}{|c|c|c|c|c|c|c|c|c|c|}
\hline SYN14-12_Run3- 247 & 1362.1 & 25.8 & 1381.0 & 19.9 & 1410.3 & 30.6 & 1410.3 & 30.6 & 96.6 \\
\hline SYN14-12_Run3- 248 & 514.6 & 14.1 & 578.0 & 16.3 & 835.6 & 48.8 & 514.6 & 14.1 & 61.6 \\
\hline SYN14-12_Run3- 249 & 981.4 & 29.5 & 1002.8 & 37.1 & 1050.0 & 97.6 & 1050.0 & 97.6 & 93.5 \\
\hline SYN14-12_Run3- 250 & 1217.0 & 23.4 & 1226.6 & 21.3 & 1243.4 & 41.4 & 1243.4 & 41.4 & 97.9 \\
\hline SYN14-12_Run3- 251 & 459.9 & 8.7 & 460.0 & 9.3 & 460.7 & 35.1 & 459.9 & 8.7 & 99.8 \\
\hline SYN14-12_Run3- 252 & 2608.4 & 75.3 & 2609.6 & 35.7 & 2610.6 & 24.4 & 2610.6 & 24.4 & 99.9 \\
\hline SYN14-12_Run3- 253 & 1012.8 & 22.1 & 1009.3 & 19.3 & 1001.7 & 37.9 & 1001.7 & 37.9 & 101.1 \\
\hline SYN14-12_Run3- 254 & 1009.6 & 21.2 & 1019.9 & 16.7 & 1042.0 & 25.0 & 1042.0 & 25.0 & 96.9 \\
\hline SYN14-12_Run3- 255 & 1114.0 & 32.5 & 1082.6 & 23.7 & 1019.9 & 32.5 & 1019.9 & 32.5 & 109.2 \\
\hline SYN14-12_Run3- 256 & 1175.7 & 27.7 & 1129.0 & 20.7 & 1040.2 & 32.2 & 1040.2 & 32.2 & 113.0 \\
\hline SYN14-12_Run3- 266 & 1347.8 & 39.3 & 1338.8 & 27.0 & 1324.3 & 32.3 & 1324.3 & 32.3 & 101.8 \\
\hline SYN14-12_Run3- 267 & 1758.7 & 29.3 & 1774.2 & 20.2 & 1792.5 & 26.8 & 1792.5 & 26.8 & 98.1 \\
\hline SYN14-12_Run3- 268 & 949.8 & 19.5 & 939.3 & 16.3 & 914.8 & 30.5 & 914.8 & 30.5 & 103.8 \\
\hline SYN14-12_Run3- 269 & 1051.5 & 19.6 & 1065.8 & 16.3 & 1095.1 & 28.7 & 1095.1 & 28.7 & 96.0 \\
\hline SYN14-12_Run3- 270 & 2616.3 & 41.8 & 2646.8 & 23.5 & 2670.2 & 25.9 & 2670.2 & 25.9 & 98.0 \\
\hline SYN14-12_Run3- 271 & 351.0 & 14.6 & 344.8 & 14.5 & 303.1 & 58.1 & 351.0 & 14.6 & NA \\
\hline SYN14-12_Run3- 272 & 438.7 & 10.1 & 455.7 & 18.8 & 2.5 & 99.3 & 7 & 10.1 & 80.9 \\
\hline SYN14-12_Run3- 273 & 750.2 & 14.2 & 808.2 & 13.8 & 971.3 & 29.9 & 750.2 & 14.2 & 77.2 \\
\hline SYN14-12_Run3- 274 & 1386.2 & 21.4 & 1412.9 & 16.1 & 1453.4 & 23.4 & 1453.4 & 23.4 & 95.4 \\
\hline SYN14-12_Run3- 275 & 1005.7 & 15.6 & 998.7 & 14.4 & 983.4 & 31.2 & 983.4 & 31.2 & 102.3 \\
\hline SYN14-12_Run3- 276 & 1187.2 & 17.4 & 1185.5 & 15.2 & 1182.4 & 29.0 & 1182.4 & 29.0 & 100.4 \\
\hline SYN14-12_Run3- 277 & 1329.2 & 60.2 & 1334.8 & 39.7 & 1343.7 & 35.8 & 1343.7 & 35.8 & 98.9 \\
\hline SYN14-12_Run3- 278 & 1694.8 & 54.3 & 1689.1 & 31.9 & 1682.0 & 24.3 & 1682.0 & 24.3 & 100.8 \\
\hline SYN14-12_Run3- 279 & 997.6 & 24.1 & 997.9 & 20.0 & 998.5 & 35.5 & 998.5 & 35.5 & 99.9 \\
\hline SYN14-12_Run3- 280 & 1437.2 & 28.9 & 1446.5 & 23.1 & 1460.1 & 37.7 & 1460.1 & 37.7 & 98.4 \\
\hline SYN14-12_Run3- 281 & 429.7 & 8.3 & 427.7 & 11.8 & 417.1 & 60.8 & 429.7 & 8.3 & 103.0 \\
\hline SYN14-12_Run3- 282 & 2713.6 & 47.7 & 2720.4 & 24.1 & 2725.5 & 22.3 & 2725.5 & 22.3 & 99.6 \\
\hline SYN14-12_Run3- 283 & 1028.4 & 25.0 & 1049.0 & 22.6 & 1092.3 & 44.9 & 1092.3 & 44.9 & 94.1 \\
\hline SYN14-12_Run3- 284 & 458.8 & 12.6 & 453.7 & 11.6 & 428.0 & 31.5 & 458.8 & 12.6 & 107.2 \\
\hline SYN14-12_Run3- 285 & 1416.3 & 29.3 & 1430.7 & 20.7 & 1452.0 & 26.7 & 1452.0 & 26.7 & 97.5 \\
\hline SYN14-12_Run3- 286 & 593.2 & 9.9 & 582.0 & 9.8 & 538.5 & 29.9 & 593.2 & 9.9 & 110.2 \\
\hline SYN14-12_Run3- 287 & 1612.8 & 33.1 & 1617.2 & 23.7 & 1622.9 & 33.2 & 1622.9 & 33.2 & 99.4 \\
\hline SYN14-12_Run3- 288 & 1062.6 & 26.3 & 1038.5 & 23.6 & 988.3 & 49.6 & 988.3 & 49.6 & 107.5 \\
\hline SYN14-12_Run3- 289 & 974.2 & 20.3 & 1004.2 & 16.9 & 1070.2 & 28.5 & 1070.2 & 28.5 & 91.0 \\
\hline SYN14-12_Run3- 290 & 1867.7 & 37.3 & 1884.2 & 21.9 & 1902.4 & 20.0 & 1902.4 & 20.0 & 98.2 \\
\hline SYN14-12_Run3- 291 & 945.6 & 35.8 & 958.7 & 26.4 & 988.8 & 25.2 & 988.8 & 25.2 & 95.6 \\
\hline SYN14-12_Run3- 292 & 1052.4 & 23.0 & 1024.7 & 27.7 & 966.1 & 73.2 & 966.1 & 73.2 & 108.9 \\
\hline SYN14-12_Run3- 293 & 1131.8 & 23.4 & 1139.4 & 17.6 & 1153.9 & 24.1 & 1153.9 & 24.1 & 98.1 \\
\hline SYN14-12_Run3- 294 & 1094.9 & 40.7 & 1097.3 & 29.3 & 1101.9 & 33.2 & 1101.9 & 33.2 & 99.4 \\
\hline
\end{tabular}




\begin{tabular}{|c|c|c|c|c|c|c|c|c|c|}
\hline SYN14-12_Run3- 295 & 1020.1 & 21.1 & 1043.8 & 19.8 & 1093.7 & 41.3 & 1093.7 & 41.3 & 93.3 \\
\hline SYN14-12_Run3- 296 & 1425.1 & 52.3 & 1423.3 & 33.2 & 1420.5 & 27.9 & 1420.5 & 27.9 & 100.3 \\
\hline SYN14-12_Run3- 297 & 1050.3 & 22.4 & 1050.9 & 19.8 & 1052.3 & 39.4 & 1052.3 & 39.4 & 99.8 \\
\hline SYN14-12_Run3- 299 & 1118.1 & 29.4 & 1132.9 & 21.4 & 1161.2 & 25.3 & 1161.2 & 25.3 & 96.3 \\
\hline SYN14-12_Run3- 300 & 1036.5 & 17.7 & 1031.0 & 16.0 & 1019.2 & 33.3 & 019.2 & 33.3 & 101.7 \\
\hline SYN14-12_Run3- 301 & 1051.5 & 27.3 & 1046.9 & 19.8 & 1037.3 & 22.4 & 1037.3 & 22.4 & 101.4 \\
\hline SYN14-12_Run3- 302 & 2301.8 & 41.0 & 2409.8 & 23.1 & 2502.3 & 22.7 & 2502.3 & 22.7 & 92.0 \\
\hline SYN14-12_Run3- 303 & 436.0 & 8.0 & 444.1 & 13.2 & 486.4 & 69.3 & 436.0 & 8.0 & 89.6 \\
\hline SYN14-12_Run3- 304 & 2391.0 & 73.0 & 2478.6 & 36.0 & 2551.2 & 21.8 & 2551.2 & 21.8 & 93.7 \\
\hline SYN14-12_Run3- 305 & 1445.6 & 32.4 & 1563.7 & 27.1 & 1726.9 & 42.3 & 1726.9 & 42.3 & 83.7 \\
\hline SYN14-12_Run3- 306 & 411.9 & 8.1 & 411.9 & 9.5 & 2.2 & 43.1 & 1.9 & 8.1 & 99.9 \\
\hline SYN14-12_Run3- 307 & 10 & 20.5 & 1019.7 & 18.0 & 1041.1 & 35.0 & 1041.1 & 35.0 & 97.0 \\
\hline SYN14-12_Run3- 308 & 993.5 & 21.6 & 984.2 & 23.2 & 963.7 & 58.1 & 963.7 & 58.1 & 103.1 \\
\hline SYN14-12_Run3- 309 & 1951.1 & 32.7 & 1967.6 & 24.4 & 1984.9 & 36.2 & 1984.9 & 36.2 & 98.3 \\
\hline SYN14-12_Run3- 310 & 966.0 & 21.8 & 989.0 & 23.3 & 1040.3 & 56.1 & 1040.3 & 56.1 & 92.9 \\
\hline SYN14-12_Run3- 311 & 1444.9 & 25.5 & 1476.5 & 18.5 & 1522.1 & 25.1 & 1522.1 & 25.1 & 94.9 \\
\hline SYN14-12_Run3- 312 & 1304.3 & 23.6 & 1318.7 & 21.1 & 1342.3 & 39.6 & 1342.3 & 39.6 & 97.2 \\
\hline SYN14-12_Run3- 313 & 983.4 & 22.8 & 986.2 & 19.5 & 992.5 & 37.0 & 992.5 & 37.0 & 99.1 \\
\hline SYN14-12_Run3- 314 & 1196.5 & 25.4 & 1191.9 & 19.3 & 1183.4 & 29.3 & 1183.4 & 29.3 & 101.1 \\
\hline SYN14-12_Run3- 315 & 1033.5 & 24.6 & 1066.9 & 25.8 & 1135.9 & 58.5 & 1135.9 & 58.5 & 91.0 \\
\hline SYN14-12_Run3- 316 & 994.2 & 20.6 & 1022.4 & 18.9 & 1083.3 & 38.2 & 1083.3 & 38.2 & 91.8 \\
\hline SYN14-12_Run3- 317 & 1882.3 & 37.6 & 1867.1 & 24.5 & 1850.2 & 30.9 & 1850.2 & 30.9 & 101.7 \\
\hline SYN14-12_Run3- 318 & 422.0 & 5.9 & 426.3 & 10.2 & 449.8 & 57.0 & 422.0 & 5.9 & 93.8 \\
\hline SYN14-12_Run3- 319 & 343.3 & 9.2 & 340.6 & 9.7 & 322.3 & 42.9 & 343.3 & 9.2 & NA \\
\hline SYN14-12_Run3- 320 & 1806.0 & 41.5 & 1819.7 & 25.4 & 1835.4 & 26.2 & 1835.4 & 26.2 & 98.4 \\
\hline SYN14-12_Run3- 321 & 308.2 & 8.0 & 313.8 & 10.4 & 355.4 & 62.5 & 308.2 & 8.0 & NA \\
\hline SYN14-12_Run3- 322 & 961.2 & 16.3 & 953.1 & 14.9 & 934.6 & 32.3 & 934.6 & 32.3 & 102.8 \\
\hline SYN14-12_Run3- 323 & 1048.1 & 38.7 & 1054.9 & 29.5 & 1069.0 & 40.9 & 1069.0 & 40.9 & 98.0 \\
\hline SYN14-12_Run3- 324 & 1408.3 & 43.3 & 1430.7 & 31.3 & 1464.2 & 42.2 & 1464.2 & 42.2 & 96.2 \\
\hline SYN14-12_Run3- 326 & 1166.1 & 17.9 & 1164.1 & 17.3 & 1160.5 & 36.7 & 1160.5 & 36.7 & 100.5 \\
\hline SYN14-12_Run3- 327 & 994.6 & 17.8 & 1010.2 & 16.1 & 1044.4 & 32.7 & 1044.4 & 32.7 & 95.2 \\
\hline SYN14-12_Run3- 328 & 1864.7 & 70.9 & 1888.9 & 40.8 & 1915.6 & 33.2 & 1915.6 & 33.2 & 97.3 \\
\hline SYN14-12_Run3- 329 & 2659.6 & 48.2 & 2685.9 & 24.7 & 2705.7 & 23.2 & 2705.7 & 23.2 & 98.3 \\
\hline SYN14-12_Run3- 330 & 1452.7 & 28.5 & 1446.7 & 20.1 & 1437.8 & 27.2 & 1437.8 & 27.2 & 101.0 \\
\hline
\end{tabular}

\begin{tabular}{|l|l|l|l|l|l|l|l|l|l|}
\hline Analysis & $206 \mathrm{~Pb}^{*}$ & \pm & $207 \mathrm{~Pb}^{*}$ & \pm & $206 \mathrm{~Pb}^{*}$ & \pm & Best age & \pm & Conc \\
\hline & $238 \mathrm{U}^{*}$ & $(\mathrm{Ma})$ & $235 \mathrm{U}$ & $(\mathrm{Ma})$ & $207 \mathrm{~Pb}^{*}$ & $(\mathrm{Ma})$ & $(\mathrm{Ma})$ & $(\mathrm{Ma})$ & $(\%)$ \\
\hline & & & & & & & & & \\
\hline & & & & & & & & & \\
\hline
\end{tabular}




\begin{tabular}{|c|c|c|c|c|c|c|c|c|c|}
\hline SYN14-13_Run1- 1 & 230.2 & 4.6 & 238.4 & 6.2 & 320.1 & 48.0 & 230.2 & 4.6 & NA \\
\hline SYN14-13_Run1- 73 & 113.4 & 2.2 & 115.8 & 5.4 & 166.1 & 106.3 & 113.4 & 2.2 & NA \\
\hline SYN14-13_Run2- 215 & 113.9 & 3.1 & 114.0 & 4.9 & 114.8 & 86.4 & 113.9 & 3.1 & NA \\
\hline SYN14-13_Run2- 212 & 114.4 & 3.8 & 122.2 & 7.6 & 276.9 & 129.3 & 114.4 & 3.8 & NA \\
\hline SYN14-13_Run2- 116 & 115.9 & 1.9 & 117.9 & 3.0 & 158.2 & 50.3 & 115.9 & 1.9 & NA \\
\hline SYN14-13_Run1- 76 & 116.2 & 3.1 & 122.7 & 7.4 & 250.6 & 133.4 & 116.2 & 3.1 & NA \\
\hline SYN14-13_Run1- 64 & 116.5 & 3.1 & 116.9 & 5.3 & 125.2 & 94.2 & 116.5 & 3.1 & NA \\
\hline SYN14-13_Run2- 131 & 117.0 & 2.4 & 117.4 & 3.9 & 125.5 & 67.3 & 117.0 & 2.4 & NA \\
\hline SYN14-13_Run2- 132 & 118.6 & 2.6 & 116.6 & 5.2 & 75.5 & 99.0 & 118.6 & 2.6 & NA \\
\hline SYN14-13_Run2- 119 & 119.9 & 1.9 & 117.8 & 4.1 & 75.7 & 80.0 & 119.9 & 1.9 & NA \\
\hline SYN14-13_Run1- 6 & 164.2 & 4.2 & 205.2 & 17.8 & 706.9 & 196.6 & 164.2 & 4.2 & NA \\
\hline SYN14-13_Run1- 68 & 166.2 & 4.9 & 167.4 & 6.0 & 184.8 & 57.3 & 166.2 & 4.9 & NA \\
\hline SYN14-13_Run1- 86 & 176.4 & 4.3 & 213.1 & 8.4 & 642.1 & 76.5 & 176.4 & 4.3 & NA \\
\hline SYN14-13_Run1- 96 & 217.3 & 5.0 & 216.4 & 6.8 & 206.2 & 59.8 & 217.3 & 5.0 & NA \\
\hline SYN14-13_Run1- 43 & 291.8 & 5.3 & 301.2 & 10.1 & 374.6 & 76.8 & 291.8 & 5.3 & NA \\
\hline SYN14-13_Run1- 79 & 328.5 & 6.3 & 333.4 & 7.4 & 368.2 & 38.8 & 328.5 & 6.3 & NA \\
\hline SYN14-13_Run1- 71 & 370.3 & 13.7 & 435.9 & 15.5 & 799.4 & 45.3 & 370.3 & 13.7 & NA \\
\hline SYN14-13_Run1- 74 & 371.5 & 27.8 & 700.0 & 42.6 & 1976.2 & 59.6 & 371.5 & 27.8 & NA \\
\hline SYN14-13_Run2- 169 & 384.2 & 10.4 & 407.1 & 14.1 & 539.2 & 68.9 & 384.2 & 10.4 & NA \\
\hline SYN14-13_Run2- 111 & 395.2 & 8.6 & 393.5 & 10.7 & 383.8 & 54.1 & 395.2 & 8.6 & NA \\
\hline SYN14-13_Run2- 170 & 398.7 & 9.2 & 401.7 & 11.1 & 419.0 & 52.8 & 398.7 & 9.2 & NA \\
\hline SYN14-13_Run1- 3 & 417.5 & 12.4 & 424.6 & 12.4 & 463.0 & 40.7 & 417.5 & 12.4 & 90.2 \\
\hline SYN14-13_Run1- 53 & 421.8 & 25.4 & 425.6 & 22.7 & 445.9 & 45.2 & 421.8 & 25.4 & 94.6 \\
\hline SYN14-13_Run1-84 & 428.2 & 9.2 & 425.1 & 10.9 & 408.2 & 49.5 & 428.2 & 9.2 & 104.9 \\
\hline SYN14-13_Run1- 7 & 428.7 & 7.7 & 427.7 & 9.6 & 422.5 & 44.8 & 428.7 & 7.7 & 101.5 \\
\hline SYN14-13_Run1- 91 & 466.3 & 9.0 & 466.6 & 11.7 & 468.0 & 53.0 & 466.3 & 9.0 & 99.6 \\
\hline SYN14-13_Run1- 18 & 487.0 & 10.2 & 491.3 & 10.5 & 511.4 & 34.9 & 487.0 & 10.2 & 95.2 \\
\hline SYN14-13_Run2- 204 & 496.0 & 7.4 & 512.3 & 9.5 & 585.7 & 38.4 & 496.0 & 7.4 & 84.7 \\
\hline SYN14-13_Run1- 98 & 505.7 & 9.4 & 521.1 & 10.5 & 589.3 & 37.2 & 505.7 & 9.4 & 85.8 \\
\hline SYN14-13_Run1- 21 & 513.8 & 11.5 & 528.5 & 15.7 & 592.4 & 65.6 & 513.8 & 11.5 & 86.7 \\
\hline SYN14-13_Run1- 34 & 518.3 & 9.5 & 540.2 & 19.2 & 633.7 & 90.0 & 518.3 & 9.5 & 81.8 \\
\hline SYN14-13_Run2- 123 & 528.4 & 11.0 & 530.3 & 12.2 & 538.3 & 44.2 & 528.4 & 11.0 & 98.2 \\
\hline SYN14-13_Run1- 82 & 545.7 & 9.0 & 550.4 & 10.3 & 570.1 & 37.3 & 545.7 & 9.0 & 95.7 \\
\hline SYN14-13_Run1- 101 & 556.1 & 9.3 & 563.2 & 10.6 & 591.8 & 37.4 & 556.1 & 9.3 & 94.0 \\
\hline SYN14-13_Run2- 202 & 561.6 & 8.0 & 560.0 & 12.7 & 553.3 & 55.8 & 561.6 & 8.0 & 101.5 \\
\hline SYN14-13_Run2- 220 & 581.0 & 11.7 & 606.8 & 13.8 & 704.4 & 46.3 & 581.0 & 11.7 & 82.5 \\
\hline SYN14-13_Run2- 113 & 582.0 & 10.8 & 586.3 & 12.5 & 602.9 & 43.6 & 582.0 & 10.8 & 96.5 \\
\hline SYN14-13_Run1- 63 & 589.7 & 8.8 & 605.3 & 11.7 & 664.4 & 43.9 & 589.7 & 8.8 & 88.8 \\
\hline SYN14-13_Run1- 41 & 593.8 & 13.5 & 598.4 & 12.8 & 616.0 & 33.1 & 593.8 & 13.5 & 96.4 \\
\hline
\end{tabular}




\begin{tabular}{|c|c|c|c|c|c|c|c|c|c|}
\hline SYN14-13_Run2- 213 & 600.3 & 11.4 & 605.9 & 13.0 & 626.6 & 44.1 & 600.3 & 11.4 & 95.8 \\
\hline SYN14-13_Run2- 128 & 603.1 & 10.6 & 597.1 & 13.5 & 574.5 & 51.1 & 603.1 & 10.6 & 105.0 \\
\hline SYN14-13_Run2- 196 & 611.6 & 15.2 & 609.1 & 17.5 & 599.7 & 60.9 & 611.6 & 15.2 & 102.0 \\
\hline SYN14-13_Run1- 100 & 646.3 & 10.6 & 659.9 & 12.5 & 706.6 & 40.4 & 646.3 & 10.6 & 91.5 \\
\hline SYN14-13_Run2- 199 & 834.5 & 16.1 & 842.9 & 16.8 & 865.1 & 43.3 & 834.5 & 16.1 & 96.5 \\
\hline SYN14-13_Run1- 17 & 920.8 & 16.9 & 916.7 & 15.0 & 906.6 & 31.5 & 906.6 & 31.5 & 101.6 \\
\hline SYN14-13_Run2- 146 & 928.6 & 16.5 & 939.1 & 15.9 & 963.8 & 36.0 & 963.8 & 36.0 & 96.4 \\
\hline SYN14-13_Run2- 124 & 953.8 & 20.4 & 963.7 & 20.4 & 986.5 & 47.8 & 986.5 & 47.8 & 96.7 \\
\hline SYN14-13_Run1- 36 & 1020.3 & 15.8 & 1010.6 & 18.0 & 989.5 & 46.1 & 989.5 & 46.1 & 103.1 \\
\hline SYN14-13_Run1- 48 & 983.3 & 22.8 & 991.3 & 19.3 & 1009.1 & 35.1 & 1009.1 & 35.1 & 97.4 \\
\hline SYN14-13_Run2- 141 & 1066.0 & 27.6 & 1058.7 & 26.0 & 1043.6 & 56.1 & 1043.6 & 56.1 & 102.2 \\
\hline SYN14-13_Run2- 179 & 1058.3 & 28.9 & 1055.4 & 23.1 & 1049.4 & 38.6 & 1049.4 & 38.6 & 100.8 \\
\hline SYN14-13_Run2- 158 & 1064.5 & 20.3 & 1060.3 & 17.8 & 1051.8 & 35.2 & 1051.8 & 35.2 & 101.2 \\
\hline SYN14-13_Run2- 125 & 1029.7 & 22.2 & 1043.7 & 19.7 & 1073.1 & 38.6 & 1073.1 & 38.6 & 96.0 \\
\hline SYN14-13_Run1- 95 & 1070.3 & 10.0 & 1078.7 & 12.3 & 1095.7 & 30.8 & 1095.7 & 30.8 & 97.7 \\
\hline SYN14-13_Run1- 67 & 1059.7 & 24.6 & 1074.5 & 26.2 & 1104.8 & 61.0 & 1104.8 & 61.0 & 95.9 \\
\hline SYN14-13_Run1- 75 & 1086.0 & 31.0 & 1096.2 & 23.3 & 1116.3 & 31.0 & 1116.3 & 31.0 & 97.3 \\
\hline SYN14-13_Run1- 54 & 952.3 & 19.6 & 1003.3 & 17.4 & 1116.6 & 32.0 & 1116.6 & 32.0 & 85.3 \\
\hline SYN14-13_Run2- 164 & 1123.7 & 27.5 & 1122.8 & 21.6 & 1121.1 & 34.2 & 1121.1 & 34.2 & 100.2 \\
\hline SYN14-13_Run2- 218 & 1063.7 & 22.5 & 1083.6 & 17.7 & 1123.9 & 26.5 & 1123.9 & 26.5 & 94.6 \\
\hline SYN14-13_Run1- 107 & 1066.1 & 17.3 & 1089.3 & 15.2 & 1136.1 & 28.8 & 1136.1 & 28.8 & 93.8 \\
\hline SYN14-13_Run1- 49 & 1054.1 & 20.6 & 1083.3 & 17.6 & 1142.6 & 31.5 & 1142.6 & 31.5 & 92.3 \\
\hline SYN14-13_Run1- 27 & 1099.5 & 40.1 & 1114.2 & 28.5 & 1143.1 & 28.0 & 1143.1 & 28.0 & 96.2 \\
\hline SYN14-13_Run1- 42 & 1123.5 & 19.1 & 1130.7 & 15.4 & 1144.5 & 25.5 & 1144.5 & 25.5 & 98.2 \\
\hline SYN14-13_Run1- 14 & 1023.7 & 25.5 & 1064.2 & 20.6 & 1148.3 & 31.5 & 1148.3 & 31.5 & 89.1 \\
\hline SYN14-13_Run2- 150 & 1117.4 & 19.1 & 1128.8 & 21.3 & 1150.7 & 49.7 & 1150.7 & 49.7 & 97.1 \\
\hline SYN14-13_Run2- 217 & 1164.9 & 20.4 & 1164.0 & 16.6 & 1162.3 & 28.5 & 1162.3 & 28.5 & 100.2 \\
\hline SYN14-13_Run2- 154 & 1117.3 & 33.9 & 1133.8 & 24.6 & 1165.6 & 28.3 & 1165.6 & 28.3 & 95.9 \\
\hline SYN14-13_Run2- 167 & 1108.2 & 19.1 & 1130.6 & 16.7 & 1173.9 & 30.9 & 1173.9 & 30.9 & 94.4 \\
\hline SYN14-13_Run1- 97 & 1180.3 & 20.7 & 1186.5 & 17.7 & 1197.8 & 32.7 & 1197.8 & 32.7 & 98.5 \\
\hline SYN14-13_Run2- 208 & 1156.0 & 28.9 & 1172.3 & 24.2 & 1202.5 & 42.7 & 1202.5 & 42.7 & 96.1 \\
\hline SYN14-13_Run1- 105 & 1218.9 & 24.4 & 1214.5 & 20.8 & 1206.5 & 38.4 & 1206.5 & 38.4 & 101.0 \\
\hline SYN14-13_Run1- 16 & 1224.0 & 27.0 & 1218.7 & 20.8 & 1209.3 & 32.6 & 1209.3 & 32.6 & 101.2 \\
\hline SYN14-13_Run1- 77 & 1222.3 & 20.0 & 1218.5 & 14.3 & 1211.7 & 18.0 & 1211.7 & 18.0 & 100.9 \\
\hline SYN14-13_Run2- 183 & 1012.5 & 33.9 & 1083.0 & 27.3 & 1227.7 & 37.7 & 1227.7 & 37.7 & 82.5 \\
\hline SYN14-13_Run1- 104 & 1241.9 & 27.1 & 1244.5 & 19.4 & 1248.9 & 24.4 & 1248.9 & 24.4 & 99.4 \\
\hline SYN14-13_Run1- 30 & 1273.5 & 31.8 & 1266.3 & 23.4 & 1254.1 & 32.9 & 1254.1 & 32.9 & 101.5 \\
\hline SYN14-13_Run2- 174 & 1204.7 & 39.2 & 1225.6 & 28.1 & 1262.5 & 33.2 & 1262.5 & 33.2 & 95.4 \\
\hline SYN14-13_Run1- 59 & 1092.7 & 43.7 & 1155.5 & 30.9 & 1275.2 & 21.0 & 1275.2 & 21.0 & 85.7 \\
\hline
\end{tabular}




\begin{tabular}{|c|c|c|c|c|c|c|c|c|c|}
\hline SYN14-13_Run1- 45 & 1240.6 & 29.7 & 1257.3 & 20.4 & 1286.0 & 20.5 & 1286.0 & 20.5 & 96.5 \\
\hline SYN14-13_Run1- 35 & 1211.0 & 19.9 & 1239.6 & 17.7 & 1289.5 & 33.2 & 1289.5 & 33.2 & 93.9 \\
\hline SYN14-13_Run2- 191 & 1153.2 & 24.1 & 1206.0 & 18.9 & 1301.7 & 27.2 & 1301.7 & 27.2 & 88.6 \\
\hline SYN14-13_Run1- 66 & 1307.6 & 23.9 & 1307.5 & 20.4 & 1307.3 & 37.0 & 1307.3 & 37.0 & 100.0 \\
\hline SYN14-13_Run2- 161 & 1309.5 & 42.5 & 1309.3 & 33.9 & 1309.0 & 56.1 & 1309.0 & 56.1 & 100.0 \\
\hline SYN14-13_Run1- 69 & 1309.3 & 28.8 & 1312.6 & 22.4 & 1318.1 & 35.3 & 1318.1 & 35.3 & 99.3 \\
\hline SYN14-13_Run2- 149 & 1291.0 & 22.7 & 1303.6 & 16.7 & 1324.4 & 23.2 & 1324.4 & 23.2 & 97.5 \\
\hline SYN14-13_Run1- 37 & 1211.9 & 28.5 & 1261.3 & 25.2 & 1346.6 & 45.5 & 1346.6 & 45.5 & 90.0 \\
\hline SYN14-13_Run2- 209 & 1267.4 & 27.6 & 1298.5 & 21.4 & 1350.1 & 32.5 & 1350.1 & 32.5 & 93.9 \\
\hline SYN14-13_Run2- 151 & 1394.2 & 20.0 & 1377.4 & 15.8 & 1351.4 & 26.3 & 1351.4 & 26.3 & 103.2 \\
\hline SYN14-13_Run2- 112 & 1391.6 & 43.8 & 1381.9 & 31.9 & 1366.9 & 45.4 & 1366.9 & 45.4 & 101.8 \\
\hline SYN14-13_Run1- 13 & 1406.9 & 24.6 & 1402.5 & 17.9 & 1395.8 & 25.4 & 1395.8 & 25.4 & 100.8 \\
\hline SYN14-13_Run2-133 & 1358.8 & 39.6 & 1376.2 & 26.0 & 1403.3 & 22.8 & 1403.3 & 22.8 & 96.8 \\
\hline SYN14-13_Run1- 89 & 1396.7 & 33.7 & 1401.4 & 24.6 & 1408.4 & 34.7 & 1408.4 & 34.7 & 99.2 \\
\hline SYN14-13_Run1- 20 & 1366.7 & 25.2 & 1384.4 & 18.5 & 1411.6 & 25.8 & 1411.6 & 25.8 & 96.8 \\
\hline SYN14-13_Run2- 152 & 1349.1 & 33.3 & 1379.2 & 23.0 & 1426.0 & 25.7 & 1426.0 & 25.7 & 94.6 \\
\hline SYN14-13_Run1- 92 & 1462.1 & 18.4 & 1462.6 & 17.8 & 1463.3 & 34.3 & 1463.3 & 34.3 & 99.9 \\
\hline SYN14-13_Run1- 38 & 1295.1 & 43.1 & 1363.1 & 31.8 & 1471.4 & 40.2 & 1471.4 & 40.2 & 88.0 \\
\hline SYN14-13_Run1- 99 & 1502.3 & 54.1 & 1492.4 & 32.9 & 1478.3 & 22.0 & 1478.3 & 22.0 & 101.6 \\
\hline SYN14-13_Run2- 184 & 1331.0 & 26.4 & 1394.1 & 19.7 & 1492.0 & 26.4 & 1492.0 & 26.4 & 89.2 \\
\hline SYN14-13_Run1- 58 & 1498.0 & 27.8 & 1496.6 & 20.9 & 1494.6 & 31.5 & 1494.6 & 31.5 & 100.2 \\
\hline SYN14-13_Run2- 180 & 1482.8 & 28.8 & 1489.0 & 19.7 & 1497.9 & 24.4 & 1497.9 & 24.4 & 99.0 \\
\hline SYN14-13_Run2- 211 & 1494.1 & 24.1 & 1500.2 & 19.4 & 1508.7 & 31.9 & 1508.7 & 31.9 & 99.0 \\
\hline SYN14-13_Run1- 33 & 1536.8 & 25.1 & 1526.4 & 17.7 & 1511.9 & 24.2 & 1511.9 & 24.2 & 101.6 \\
\hline SYN14-13_Run2- 166 & 1438.7 & 34.1 & 1477.6 & 23.6 & 1533.9 & 28.1 & 1533.9 & 28.1 & 93.8 \\
\hline SYN14-13_Run1- 55 & 1524.0 & 30.0 & 1531.8 & 22.9 & 1542.6 & 35.3 & 1542.6 & 35.3 & 98.8 \\
\hline SYN14-13_Run1- 93 & 1382.0 & 21.4 & 1447.2 & 17.1 & 1544.3 & 26.5 & 1544.3 & 26.5 & 89.5 \\
\hline SYN14-13_Run1- 65 & 1489.9 & 29.3 & 1515.5 & 23.4 & 1551.4 & 37.6 & 1551.4 & 37.6 & 96.0 \\
\hline SYN14-13_Run2- 172 & 1419.0 & 60.9 & 1475.4 & 39.6 & 1557.5 & 33.1 & 1557.5 & 33.1 & 91.1 \\
\hline SYN14-13_Run2- 118 & 1342.0 & 28.6 & 1432.2 & 23.6 & 1568.8 & 36.9 & 1568.8 & 36.9 & 85.5 \\
\hline SYN14-13_Run1- 24 & 1411.0 & 27.1 & 1476.3 & 23.7 & 1571.4 & 40.7 & 1571.4 & 40.7 & 89.8 \\
\hline SYN14-13_Run2-138 & 1285.7 & 31.6 & 1405.7 & 22.2 & 1592.6 & 19.7 & 1592.6 & 19.7 & 80.7 \\
\hline SYN14-13_Run1- 70 & 1561.0 & 42.4 & 1576.2 & 27.5 & 1596.5 & 29.1 & 1596.5 & 29.1 & 97.8 \\
\hline SYN14-13_Run2- 144 & 1583.8 & 64.6 & 1592.9 & 38.2 & 1604.9 & 21.9 & 1604.9 & 21.9 & 98.7 \\
\hline SYN14-13_Run1-90 & 1590.7 & 28.5 & 1597.3 & 20.1 & 1605.9 & 27.5 & 1605.9 & 27.5 & 99.1 \\
\hline SYN14-13_Run2- 176 & 1583.9 & 21.6 & 1594.8 & 16.7 & 1609.3 & 26.1 & 1609.3 & 26.1 & 98.4 \\
\hline SYN14-13_Run2- 203 & 1584.0 & 29.7 & 1596.7 & 22.5 & 1613.5 & 34.3 & 1613.5 & 34.3 & 98.2 \\
\hline SYN14-13_Run2- 186 & 1618.8 & 37.9 & 1619.9 & 24.4 & 1621.4 & 26.7 & 1621.4 & 26.7 & 99.8 \\
\hline SYN14-13_Run2- 159 & 1418.7 & 20.4 & 1503.5 & 15.9 & 1625.2 & 23.2 & 1625.2 & 23.2 & 87.3 \\
\hline
\end{tabular}




\begin{tabular}{|c|c|c|c|c|c|c|c|c|c|}
\hline SYN14-13_Run1- 31 & 1320.9 & 29.6 & 1444.6 & 21.8 & 1631.5 & 25.4 & 1631.5 & 25.4 & 81.0 \\
\hline SYN14-13_Run2- 163 & 1601.7 & 40.4 & 1614.7 & 24.4 & 1631.6 & 18.4 & 1631.6 & 18.4 & 98.2 \\
\hline SYN14-13_Run2- 122 & 1661.3 & 36.8 & 1648.7 & 23.6 & 1632.6 & 26.6 & 1632.6 & 26.6 & 101.8 \\
\hline SYN14-13_Run2- 147 & 1607.4 & 27.9 & 1618.4 & 20.4 & 1632.8 & 29.2 & 1632.8 & 29.2 & 98.4 \\
\hline SYN14-13_Run1- 81 & 1613.8 & 21.0 & 1622.5 & 17.3 & 1633.8 & 28.7 & 1633.8 & 28.7 & 98.8 \\
\hline SYN14-13_Run2- 194 & 1627.1 & 30.0 & 1630.3 & 21.2 & 1634.5 & 29.3 & 1634.5 & 29.3 & 99.6 \\
\hline SYN14-13_Run1- 5 & 1618.7 & 44.0 & 1625.6 & 27.6 & 1634.6 & 27.2 & 1634.6 & 27.2 & 99.0 \\
\hline SYN14-13_Run1- 32 & 1639.6 & 21.6 & 1639.3 & 16.7 & 1638.9 & 26.2 & 1638.9 & 26.2 & 100.0 \\
\hline SYN14-13_Run2- 156 & 1603.1 & 32.0 & 1619.5 & 22.1 & 1641.0 & 28.5 & 1641.0 & 28.5 & 97.7 \\
\hline SYN14-13_Run1- 80 & 1639.4 & 28.3 & 1641.7 & 21.8 & 1644.7 & 33.8 & 1644.7 & 33.8 & 99.7 \\
\hline SYN14-13_Run1- 4 & 1498.7 & 73.5 & 1563.0 & 45.1 & 1650.9 & 24.5 & 1650.9 & 24.5 & 90.8 \\
\hline SYN14-13_Run1- 106 & 1639.9 & 25.6 & 1645.1 & 17.6 & 1651.8 & 23.1 & 1651.8 & 23.1 & 99.3 \\
\hline SYN14-13_Run2- 187 & 1379.9 & 23.7 & 1491.4 & 17.6 & 1653.7 & 22.0 & 1653.7 & 22.0 & 83.4 \\
\hline SYN14-13_Run2- 115 & 1617.0 & 20.9 & 1633.6 & 17.7 & 1654.9 & 30.0 & 1654.9 & 30.0 & 97.7 \\
\hline SYN14-13_Run2- 121 & 1648.2 & 34.1 & 1659.0 & 22.7 & 1672.8 & 27.8 & 1672.8 & 27.8 & 98.5 \\
\hline SYN14-13_Run2-173 & 1542.7 & 25.4 & 1598.6 & 16.9 & 1673.0 & 18.5 & 1673.0 & 18.5 & 92.2 \\
\hline SYN14-13_Run2- 157 & 1494.4 & 55.7 & 1572.8 & 35.0 & 1679.5 & 24.5 & 1679.5 & 24.5 & 89.0 \\
\hline SYN14-13_Run2- 136 & 1568.8 & 31.1 & 1617.0 & 25.9 & 1680.3 & 42.3 & 1680.3 & 42.3 & 93.4 \\
\hline SYN14-13_Run2- 137 & 1447.0 & 48.5 & 1554.0 & 31.9 & 1702.6 & 26.7 & 1702.6 & 26.7 & 85.0 \\
\hline SYN14-13_Run2- 160 & 1645.2 & 39.7 & 1671.3 & 25.3 & 1704.3 & 26.4 & 1704.3 & 26.4 & 96.5 \\
\hline SYN14-13_Run2- 219 & 1712.8 & 23.2 & 1709.6 & 18.3 & 1705.6 & 29.2 & 1705.6 & 29.2 & 100.4 \\
\hline SYN14-13_Run2- 205 & 1389.1 & 23.1 & 1521.4 & 20.0 & 1710.6 & 32.0 & 1710.6 & 32.0 & 81.2 \\
\hline SYN14-13_Run1- 52 & 1730.9 & 61.4 & 1723.1 & 35.5 & 1713.6 & 25.7 & 1713.6 & 25.7 & 101.0 \\
\hline SYN14-13_Run2- 162 & 1698.1 & 29.3 & 1705.6 & 18.6 & 1714.8 & 20.4 & 1714.8 & 20.4 & 99.0 \\
\hline SYN14-13_Run2- 140 & 1654.4 & 28.6 & 1682.1 & 19.4 & 1716.8 & 24.0 & 1716.8 & 24.0 & 96.4 \\
\hline SYN14-13_Run1- 10 & 1732.9 & 36.2 & 1735.7 & 23.4 & 1739.0 & 27.6 & 1739.0 & 27.6 & 99.6 \\
\hline SYN14-13_Run1- 102 & 1551.0 & 61.1 & 1642.5 & 38.6 & 1761.6 & 30.4 & 1761.6 & 30.4 & 88.0 \\
\hline SYN14-13_Run2- 185 & 1686.8 & 34.7 & 1721.6 & 21.9 & 1764.2 & 22.4 & 1764.2 & 22.4 & 95.6 \\
\hline SYN14-13_Run1- 85 & 1726.0 & 33.1 & 1745.1 & 21.2 & 1768.0 & 24.0 & 1768.0 & 24.0 & 97.6 \\
\hline SYN14-13_Run2- 206 & 1777.8 & 34.3 & 1777.6 & 21.7 & 1777.3 & 24.5 & 1777.3 & 24.5 & 100.0 \\
\hline SYN14-13_Run1- 57 & 1789.9 & 30.3 & 1791.6 & 19.8 & 1793.5 & 24.2 & 1793.5 & 24.2 & 99.8 \\
\hline SYN14-13_Run1- 108 & 1782.9 & 27.1 & 1793.3 & 19.6 & 1805.3 & 28.1 & 1805.3 & 28.1 & 98.8 \\
\hline SYN14-13_Run2- 142 & 1749.9 & 40.8 & 1782.1 & 24.2 & 1820.0 & 19.7 & 1820.0 & 19.7 & 96.1 \\
\hline SYN14-13_Run2- 171 & 1510.6 & 17.4 & 1652.4 & 13.2 & 1837.8 & 17.7 & 1837.8 & 17.7 & 82.2 \\
\hline SYN14-13_Run2- 189 & 1821.5 & 45.5 & 1834.8 & 27.4 & 1849.8 & 26.8 & 1849.8 & 26.8 & 98.5 \\
\hline SYN14-13_Run2- 192 & 1801.8 & 35.7 & 1824.7 & 23.2 & 1850.9 & 27.8 & 1850.9 & 27.8 & 97.3 \\
\hline SYN14-13_Run2- 210 & 1551.9 & 28.1 & 1696.2 & 19.4 & 1879.5 & 21.4 & 1879.5 & 21.4 & 82.6 \\
\hline SYN14-13_Run1- 61 & 1847.1 & 28.3 & 1869.0 & 20.5 & 1893.4 & 29.4 & 1893.4 & 29.4 & 97.6 \\
\hline SYN14-13_Run2- 200 & 1709.0 & 21.8 & 1805.5 & 17.3 & 1918.7 & 25.9 & 1918.7 & 25.9 & 89.1 \\
\hline
\end{tabular}




\begin{tabular}{|l|l|l|l|l|l|l|l|l|l|} 
SYN14-13_Run1- 9 & 1910.8 & 26.6 & 1935.9 & 17.6 & 1962.7 & 22.3 & 1962.7 & 22.3 & 97.4 \\
\hline SYN14-13_Run2- 182 & 1843.2 & 32.2 & 1910.5 & 22.5 & 1984.4 & 29.7 & 1984.4 & 29.7 & 92.9 \\
\hline SYN14-13_Run1- 103 & 1935.6 & 42.1 & 1961.5 & 25.0 & 1988.9 & 24.7 & 1988.9 & 24.7 & 97.3 \\
\hline SYN14-13_Run2- 214 & 1899.6 & 35.4 & 1945.4 & 22.9 & 1994.6 & 27.3 & 1994.6 & 27.3 & 95.2 \\
\hline SYN14-13_Run2-120 & 2054.7 & 28.7 & 2041.0 & 17.2 & 2027.2 & 19.0 & 2027.2 & 19.0 & 101.4 \\
\hline SYN14-13_Run1- 109 & 2217.2 & 40.3 & 2197.6 & 22.7 & 2179.3 & 22.9 & 2179.3 & 22.9 & 101.7 \\
\hline SYN14-13_Run2-175 & 2025.7 & 37.7 & 2137.8 & 22.0 & 2247.4 & 20.1 & 2247.4 & 20.1 & 90.1 \\
\hline SYN14-13_Run1- 40 & 1879.6 & 63.6 & 2078.0 & 35.3 & 2280.8 & 14.2 & 2280.8 & 14.2 & 82.4 \\
\hline SYN14-13_Run1-44 & 2243.2 & 37.4 & 2267.0 & 23.5 & 2288.5 & 29.1 & 2288.5 & 29.1 & 98.0 \\
\hline SYN14-13_Run1-12 & 2528.5 & 50.8 & 2523.7 & 29.6 & 2519.8 & 34.5 & 2519.8 & 34.5 & 100.3 \\
\hline SYN14-13_Run2-135 & 2360.9 & 34.6 & 2484.2 & 21.2 & 2586.6 & 24.6 & 2586.6 & 24.6 & 91.3 \\
\hline SYN14-13_Run1- 83 & 2492.4 & 43.0 & 2593.6 & 22.3 & 2673.7 & 19.3 & 2673.7 & 19.3 & 93.2 \\
\hline SYN14-13_Run2-168 & 2582.2 & 46.2 & 2635.7 & 24.4 & 2677.0 & 23.7 & 2677.0 & 23.7 & 96.5 \\
\hline SYN14-13_Run2- 201 & 2639.5 & 49.6 & 2669.1 & 24.4 & 2691.6 & 20.1 & 2691.6 & 20.1 & 98.1 \\
\hline SYN14-13_Run2-127 & 2681.5 & 46.4 & 2694.8 & 25.6 & 2704.7 & 27.9 & 2704.7 & 27.9 & 99.1 \\
\hline SYN14-13_Run2-153 & 2682.2 & 61.2 & 2700.8 & 29.9 & 2714.8 & 24.5 & 2714.8 & 24.5 & 98.8 \\
\hline SYN14-13_Run2-129 & 2579.8 & 92.5 & 2663.2 & 42.1 & 2727.1 & 16.4 & 2727.1 & 16.4 & 94.6 \\
\hline SYN14-13_Run1- 11 & 2883.5 & 67.7 & 2860.9 & 30.9 & 2845.0 & 22.8 & 2845.0 & 22.8 & 101.4 \\
\hline SYN14-13_Run2-197 & 2794.1 & 36.9 & 2831.4 & 20.6 & 2858.1 & 23.1 & 2858.1 & 23.1 & 97.8 \\
\hline SYN14-13_Run2-181 & 2662.9 & 39.5 & 2783.7 & 19.5 & 2872.4 & 15.6 & 2872.4 & 15.6 & 92.7 \\
\hline SYN14-13_Run1- 56 & 2709.2 & 78.2 & 2882.8 & 35.6 & 3006.5 & 19.0 & 3006.5 & 19.0 & 90.1 \\
\hline SYN14-13_Run1- 51 & 3078.2 & 53.6 & 3219.7 & 27.0 & 3309.1 & 26.7 & 3309.1 & 26.7 & 93.0 \\
\hline
\end{tabular}

\begin{tabular}{|c|c|c|c|c|c|c|c|c|c|}
\hline Analysis & $206 \mathrm{~Pb}^{*}$ & \pm & $207 \mathrm{~Pb}^{*}$ & \pm & $206 \mathrm{~Pb}^{*}$ & \pm & Best age & \pm & Conc \\
\hline & $238 U^{*}$ & (Ma) & $235 \mathrm{U}$ & (Ma) & 207Pb* & (Ma) & (Ma) & (Ma) & $(\%)$ \\
\hline & & & & & & & & & \\
\hline $\begin{array}{l}\text { SYN14-14 Run1- } 2 \text { - } \\
58\end{array}$ & 305.7 & 6.8 & 307.1 & 9.5 & 317.5 & 62.7 & 305.7 & 6.8 & NA \\
\hline $\begin{array}{l}\text { SYN14-14 Run1- } 2 \text { - } \\
86\end{array}$ & 316.4 & 7.8 & 320.3 & 10.9 & 348.7 & 69.1 & 316.4 & 7.8 & NA \\
\hline SYN14-14 Run1-1- 42 & 317.4 & 6.9 & 331.2 & 9.6 & 428.6 & 57.8 & 317.4 & 6.9 & NA \\
\hline SYN14-14 Run1-1- 18 & 330.4 & 20.6 & 362.3 & 23.0 & 571.9 & 87.2 & 330.4 & 20.6 & NA \\
\hline $\begin{array}{l}\text { SYN14-14 Run1- } 2 \text { - } \\
63\end{array}$ & 334.3 & 7.1 & 319.9 & 10.6 & 215.9 & 74.1 & 334.3 & 7.1 & NA \\
\hline $\begin{array}{l}\text { SYN14-14 Run1- } 2 \text { - } \\
64\end{array}$ & 342.7 & 6.5 & 344.3 & 6.6 & 355.4 & 24.6 & 342.7 & 6.5 & NA \\
\hline $\begin{array}{l}\text { SYN14-14 Run1- } 2 \text { - } \\
72\end{array}$ & 346.2 & 7.5 & 351.9 & 15.3 & 389.8 & 104.6 & 346.2 & 7.5 & NA \\
\hline $\begin{array}{l}\text { SYN14-14 Run1- } 2 \text { - } \\
85\end{array}$ & 409.0 & 7.2 & 414.6 & 8.7 & 445.9 & 39.8 & 409.0 & 7.2 & 91.7 \\
\hline
\end{tabular}




\begin{tabular}{|c|c|c|c|c|c|c|c|c|c|}
\hline SYN14-14 Run1-1- 17 & 423.8 & 8.8 & 434.1 & 12.4 & 489.0 & 60.8 & 423.8 & 8.8 & 86.7 \\
\hline $\begin{array}{l}\text { SYN14-14 Run1- } 2 \text { - } \\
53\end{array}$ & 515.6 & 14.2 & 619.0 & 33.1 & 1018.0 & 132.5 & 515.6 & 14.2 & 50.6 \\
\hline $\begin{array}{l}\text { SYN14-14 Run1- } 2 \text { - } \\
67\end{array}$ & 516.0 & 10.1 & 521.7 & 13.1 & 546.7 & 54.4 & 516.0 & 10.1 & 94.4 \\
\hline $\begin{array}{l}\text { SYN14-14 Run1- } 2 \text { - } \\
80\end{array}$ & 531.4 & 10.9 & 525.8 & 13.0 & 502.0 & 51.5 & 531.4 & 10.9 & 105.8 \\
\hline $\begin{array}{l}\text { SYN14-14 Run1- } 2 \text { - } \\
69\end{array}$ & 544.0 & 6.7 & 575.5 & 14.6 & 702.3 & 65.2 & 544.0 & 6.7 & 77.5 \\
\hline $\begin{array}{l}\text { SYN14-14 Run1- } 2 \text { - } \\
98\end{array}$ & 545.6 & 10.0 & 552.6 & 15.5 & 581.6 & 67.2 & 545.6 & 10.0 & 93.8 \\
\hline $\begin{array}{l}\text { SYN14-14 Run1- } 2 \text { - } \\
77\end{array}$ & 567.2 & 8.6 & 566.6 & 8.2 & 564.4 & 22.7 & 567.2 & 8.6 & 100.5 \\
\hline SYN14-14 Run1-1- 6 & 569.4 & 12.4 & 581.2 & 15.4 & 627.7 & 56.6 & 569.4 & 12.4 & 90.7 \\
\hline $\begin{array}{l}\text { SYN14-14 Run1- } 2 \text { - } \\
96\end{array}$ & 586.0 & 13.1 & 577.1 & 14.8 & 542.0 & 52.6 & 586.0 & 13.1 & 108.1 \\
\hline SYN14-14 Run1-1- 10 & 598.4 & 10.2 & 601.7 & 13.5 & 614.1 & 51.1 & 598.4 & 10.2 & 97.4 \\
\hline $\begin{array}{l}\text { SYN14-14 Run1- } 2 \text { - } \\
83\end{array}$ & 599.7 & 8.8 & 603.4 & 11.0 & 617.5 & 40.4 & 599.7 & 8.8 & 97.1 \\
\hline SYN14-14 Run1-1- 45 & 623.9 & 20.2 & 656.2 & 24.5 & 768.9 & 79.4 & 623.9 & 20.2 & 81.1 \\
\hline SYN14-14 Run1-1- 7 & 630.9 & 9.9 & 635.2 & 10.3 & 650.9 & 30.4 & 630.9 & 9.9 & 96.9 \\
\hline $\begin{array}{l}\text { SYN14-14 Run1- } 2 \text { - } \\
62\end{array}$ & 856.4 & 18.7 & 872.9 & 15.7 & 915.0 & 27.4 & 856.4 & 18.7 & 93.6 \\
\hline SYN14-14 Run1-1- 9 & 969.9 & 14.6 & 961.6 & 19.5 & 942.4 & 55.0 & 942.4 & 55.0 & 102.9 \\
\hline $\begin{array}{l}\text { SYN14-14 Run1- } 2 \text { - } \\
65\end{array}$ & 965.6 & 42.7 & 971.1 & 31.6 & 983.7 & 34.6 & 983.7 & 34.6 & 98.2 \\
\hline $\begin{array}{l}\text { SYN14-14 Run1- } 2 \text { - } \\
75\end{array}$ & 943.2 & 24.4 & 956.7 & 18.8 & 987.7 & 24.6 & 987.7 & 24.6 & 95.5 \\
\hline $\begin{array}{l}\text { SYN14-14 Run1- } 2 \text { - } \\
91\end{array}$ & 942.3 & 16.5 & 959.4 & 14.6 & 998.7 & 28.3 & 998.7 & 28.3 & 94.4 \\
\hline SYN14-14 Run1-1- 15 & 932.2 & 15.8 & 959.2 & 21.9 & 1021.7 & 61.2 & 1021.7 & 61.2 & 91.2 \\
\hline $\begin{array}{l}\text { SYN14-14 Run1- } 2 \text { - } \\
78\end{array}$ & 1012.0 & 17.0 & 1015.3 & 18.6 & 1022.5 & 45.5 & 1022.5 & 45.5 & 99.0 \\
\hline SYN14-14 Run1-1- 3 & 1029.8 & 26.5 & 1029.9 & 25.9 & 1030.1 & 58.4 & 1030.1 & 58.4 & 100.0 \\
\hline $\begin{array}{l}\text { SYN14-14 Run1- } 2 \text { - } \\
84\end{array}$ & 940.6 & 18.7 & 973.5 & 19.4 & 1048.4 & 45.1 & 1048.4 & 45.1 & 89.7 \\
\hline $\begin{array}{l}\text { SYN14-14 Run1- } 2 \text { - } \\
60\end{array}$ & 1017.2 & 34.7 & 1030.9 & 26.3 & 1060.2 & 34.2 & 1060.2 & 34.2 & 95.9 \\
\hline $\begin{array}{l}\text { SYN14-14 Run1- } 2 \text { - } \\
92\end{array}$ & 1013.6 & 18.0 & 1039.1 & 19.7 & 1093.2 & 47.0 & 1093.2 & 47.0 & 92.7 \\
\hline $\begin{array}{l}\text { SYN14-14 Run1- } 2 \text { - } \\
66\end{array}$ & 1017.0 & 31.8 & 1042.2 & 24.4 & 1095.5 & 32.7 & 1095.5 & 32.7 & 92.8 \\
\hline SYN14-14 Run1-1- 5 & 1070.4 & 19.9 & 1079.0 & 22.2 & 1096.5 & 53.2 & 1096.5 & 53.2 & 97.6 \\
\hline SYN14-14 Run1-1- 4 & 1147.4 & 17.6 & 1147.6 & 16.0 & 1147.8 & 32.2 & 1147.8 & 32.2 & 100.0 \\
\hline SYN14-14 Run1- 2 - & 1131.7 & 16.0 & 1142.9 & 20.1 & 1164.0 & 49.6 & 1164.0 & 49.6 & 97.2 \\
\hline
\end{tabular}




\begin{tabular}{|c|c|c|c|c|c|c|c|c|c|}
\hline 61 & & & & & & & & & \\
\hline SYN14-14 Run1-1- 41 & 1203.0 & 18.9 & 1199.7 & 17.9 & 1193.6 & 37.0 & 1193.6 & 37.0 & 100.8 \\
\hline SYN14-14 Run1-1- 13 & 1338.5 & 20.0 & 1332.2 & 15.9 & 1322.1 & 26.6 & 1322.1 & 26.6 & 101.2 \\
\hline SYN14-14 Run1-1- 12 & 1240.2 & 36.6 & 1278.3 & 25.5 & 1343.0 & 26.2 & 1343.0 & 26.2 & 92.3 \\
\hline $\begin{array}{l}\text { SYN14-14 Run1- } 2 \text { - } \\
87\end{array}$ & 1299.7 & 24.5 & 1325.2 & 20.1 & 1366.8 & 33.6 & 1366.8 & 33.6 & 95.1 \\
\hline $\begin{array}{l}\text { SYN14-14 Run1- } 2 \text { - } \\
76\end{array}$ & 1352.4 & 23.4 & 1362.2 & 21.8 & 1377.5 & 42.1 & 1377.5 & 42.1 & 98.2 \\
\hline $\begin{array}{l}\text { SYN14-14 Run1- } 2 \text { - } \\
97\end{array}$ & 830.1 & 16.6 & 999.4 & 16.9 & 1392.1 & 30.4 & 1392.1 & 30.4 & 59.6 \\
\hline $\begin{array}{l}\text { SYN14-14 Run1- } 2 \text { - } \\
100\end{array}$ & 1322.2 & 33.5 & 1352.8 & 23.0 & 1401.6 & 24.0 & 1401.6 & 24.0 & 94.3 \\
\hline $\begin{array}{l}\text { SYN14-14 Run1- } 2 \text { - } \\
88\end{array}$ & 1399.5 & 25.7 & 1407.0 & 19.8 & 1418.3 & 30.6 & 1418.3 & 30.6 & 98.7 \\
\hline SYN14-14 Run1-1- 14 & 1449.0 & 21.7 & 1442.0 & 14.0 & 1431.8 & 13.5 & 1431.8 & 13.5 & 101.2 \\
\hline $\begin{array}{l}\text { SYN14-14 Run1- } 2 \text { - } \\
52\end{array}$ & 1299.0 & 22.1 & 1353.6 & 17.6 & 1440.8 & 27.1 & 1440.8 & 27.1 & 90.2 \\
\hline $\begin{array}{l}\text { SYN14-14 Run1- } 2 \text { - } \\
90\end{array}$ & 1336.5 & 27.0 & 1381.3 & 19.4 & 1451.0 & 23.9 & 1451.0 & 23.9 & 92.1 \\
\hline SYN14-14 Run1-1- 48 & 1442.4 & 21.6 & 1447.6 & 16.1 & 1455.3 & 23.7 & 1455.3 & 23.7 & 99.1 \\
\hline SYN14-14 Run1-1- 11 & 1416.6 & 16.4 & 1437.3 & 13.5 & 1468.1 & 22.5 & 1468.1 & 22.5 & 96.5 \\
\hline $\begin{array}{l}\text { SYN14-14 Run1- } 2 \text { - } \\
93\end{array}$ & 1486.0 & 39.4 & 1478.9 & 25.1 & 1468.7 & 23.9 & 1468.7 & 23.9 & 101.2 \\
\hline $\begin{array}{l}\text { SYN14-14 Run1- } 2 \text { - } \\
74\end{array}$ & 1327.1 & 31.3 & 1403.5 & 21.2 & 1521.4 & 18.2 & 1521.4 & 18.2 & 87.2 \\
\hline $\begin{array}{l}\text { SYN14-14 Run1- } 2 \text { - } \\
81\end{array}$ & 1467.8 & 20.7 & 1495.7 & 15.8 & 1535.5 & 23.8 & 1535.5 & 23.8 & 95.6 \\
\hline $\begin{array}{l}\text { SYN14-14 Run1- } 2 \text { - } \\
59\end{array}$ & 1291.6 & 32.3 & 1404.9 & 23.3 & 1581.3 & 24.7 & 1581.3 & 24.7 & 81.7 \\
\hline SYN14-14 Run1-1- 19 & 1596.7 & 27.2 & 1598.2 & 17.8 & 1600.1 & 20.2 & 1600.1 & 20.2 & 99.8 \\
\hline $\begin{array}{l}\text { SYN14-14 Run1- } 2 \text { - } \\
99\end{array}$ & 1459.0 & 41.2 & 1519.8 & 28.1 & 1605.6 & 30.9 & 1605.6 & 30.9 & 90.9 \\
\hline SYN14-14 Run1-1- 49 & 1615.8 & 20.0 & 1614.3 & 16.5 & 1612.2 & 27.5 & 1612.2 & 27.5 & 100.2 \\
\hline SYN14-14 Run1-1- 20 & 1667.8 & 18.4 & 1647.7 & 13.8 & 1622.2 & 21.1 & 1622.2 & 21.1 & 102.8 \\
\hline SYN14-14 Run1-1- 16 & 1578.3 & 26.2 & 1602.6 & 19.5 & 1634.6 & 28.4 & 1634.6 & 28.4 & 96.6 \\
\hline SYN14-14 Run1-1- 44 & 1617.1 & 29.3 & 1634.1 & 20.5 & 1656.1 & 27.2 & 1656.1 & 27.2 & 97.6 \\
\hline SYN14-14 Run1-1- 47 & 1654.0 & 27.8 & 1655.0 & 19.7 & 1656.3 & 27.3 & 1656.3 & 27.3 & 99.9 \\
\hline $\begin{array}{l}\text { SYN14-14 Run1- } 2 \text { - } \\
71\end{array}$ & 1544.1 & 33.6 & 1598.3 & 22.1 & 1670.5 & 22.9 & 1670.5 & 22.9 & 92.4 \\
\hline SYN14-14 Run1-1- 50 & 1602.0 & 22.4 & 1633.8 & 19.8 & 1675.0 & 34.4 & 1675.0 & 34.4 & 95.6 \\
\hline $\begin{array}{l}\text { SYN14-14 Run1- } 2 \text { - } \\
79\end{array}$ & 1496.7 & 34.8 & 1577.6 & 22.9 & 1687.5 & 21.9 & 1687.5 & 21.9 & 88.7 \\
\hline $\begin{array}{l}\text { SYN14-14 Run1- } 2 \text { - } \\
55\end{array}$ & 1742.6 & 28.9 & 1725.3 & 19.0 & 1704.3 & 23.5 & 1704.3 & 23.5 & 102.2 \\
\hline
\end{tabular}




\begin{tabular}{|c|c|c|c|c|c|c|c|c|c|}
\hline $\begin{array}{l}\text { SYN14-14 Run1- } 2 \text { - } \\
54\end{array}$ & 1319.1 & 21.9 & 1492.5 & 15.6 & 1748.3 & 14.5 & 1748.3 & 14.5 & 75.4 \\
\hline $\begin{array}{l}\text { SYN14-14 Run1- } 2 \text { - } \\
73\end{array}$ & 1673.0 & 44.7 & 1717.7 & 27.7 & 1772.6 & 25.9 & 1772.6 & 25.9 & 94.4 \\
\hline $\begin{array}{l}\text { SYN14-14 Run1- } 2 \text { - } \\
95\end{array}$ & 1736.5 & 24.7 & 1755.7 & 18.7 & 1778.6 & 28.1 & 1778.6 & 28.1 & 97.6 \\
\hline $\begin{array}{l}\text { SYN14-14 Run1- } 2 \text { - } \\
94\end{array}$ & 1810.4 & 42.8 & 1837.8 & 28.7 & 1869.0 & 36.3 & 1869.0 & 36.3 & 96.9 \\
\hline SYN14-14 Run1-1- 43 & 1846.2 & 30.9 & 1859.0 & 21.8 & 1873.4 & 30.3 & 1873.4 & 30.3 & 98.6 \\
\hline $\begin{array}{l}\text { SYN14-14 Run1- } 2 \text { - } \\
56\end{array}$ & 1917.3 & 25.2 & 1905.4 & 14.9 & 1892.4 & 15.1 & 1892.4 & 15.1 & 101.3 \\
\hline SYN14-14 Run1-1- 2 & 1898.5 & 40.0 & 1928.3 & 23.1 & 1960.4 & 19.9 & 1960.4 & 19.9 & 96.8 \\
\hline SYN14-14 Run1-1- 46 & 2603.8 & 31.9 & 2634.2 & 18.9 & 2657.6 & 22.4 & 2657.6 & 22.4 & 98.0 \\
\hline $\begin{array}{l}\text { SYN14-14 Run1- } 2 \text { - } \\
68\end{array}$ & 2562.6 & 43.1 & 2630.9 & 21.5 & 2683.9 & 17.3 & 2683.9 & 17.3 & 95.5 \\
\hline $\begin{array}{l}\text { SYN14-14 Run1- } 2 \text { - } \\
89\end{array}$ & 2681.4 & 43.3 & 2711.1 & 22.1 & 2733.4 & 20.6 & 2733.4 & 20.6 & 98.1 \\
\hline $\begin{array}{l}\text { SYN14-14 Run1- } 2 \text { - } \\
82\end{array}$ & 2639.0 & 26.3 & 2721.5 & 17.4 & 2783.3 & 22.6 & 2783.3 & 22.6 & 94.8 \\
\hline $\begin{array}{l}\text { SYN14-14 Run1- } 2 \text { - } \\
51\end{array}$ & 2768.5 & 36.7 & 2792.3 & 21.5 & 2809.5 & 25.5 & 2809.5 & 25.5 & 98.5 \\
\hline $\begin{array}{l}\text { SYN14-14 Run1- } 2 \text { - } \\
57\end{array}$ & 2738.8 & 36.7 & 2782.2 & 18.7 & 2813.8 & 17.7 & 2813.8 & 17.7 & 97.3 \\
\hline $\begin{array}{l}\text { SYN14-14 Run1- } 2 \text { - } \\
70\end{array}$ & 2806.6 & 45.9 & 2845.7 & 23.4 & 2873.5 & 22.8 & 2873.5 & 22.8 & 97.7 \\
\hline SYN14-14 Run1-1- 8 & 2851.1 & 80.6 & 2903.9 & 36.9 & 2940.7 & 26.0 & 2940.7 & 26.0 & 97.0 \\
\hline
\end{tabular}


Bibliography

Allmendinger, R. W. 2015. Stereonet 9:

http://www.geo.cornell.edu/geology/faculty/RWA/programs/stereonet.html (accessed August 2015).

Armstrong, R. L. 1968. Sevier Orogenic Belt in Nevada and Utah: Geological Society of America Bulletin, v. 79 p. 429-485.

Armstrong, R. L., and P. L. Ward. 1993. Late Triassic to earliest Eocene magmatism in the North American Cordillera: Implications for the western interior basin." Evolution of the western interior basin: Geological Association of Canada Special Paper, v. 39, p. 49-72.

Aschoff, Jennifer L., and Ron J. Steel. 2011. Anatomy and development of a low-accommodation clastic wedge, upper Cretaceous, Cordilleran Foreland Basin, USA: Sedimentary Geology v. 236.1, p. 1-24.

Aschoff, Jennifer, and Ron Steel. 2011. Anomalous clastic wedge development during the Sevier-Laramide transition, North American Cordilleran foreland basin, USA: Geological Society of America Bulletin, v. 123.9-10, p. 1822-1835.

Balgord, E. A., Yonkee, W. A., Link, P. K., \& Fanning, C. M. 2013. Stratigraphic, geochronologic, and geochemical record of the Cryogenian Perry Canyon Formation, northern Utah: Implications for Rodinia rifting and snowball Earth glaciation: Geological Society of America Bulletin, v. 125.9-10, p. 1442-1467.

Barbeau, D. L., Olivero, E. B., Swanson-Hysell, N. L., Zahid, K. M., Murray, K. E., \& Gehrels, G. E. 2009. Detrital-zircon geochronology of the eastern Magallanes foreland basin: Implications for Eocene kinematics of the northern Scotia Arc and Drake Passage: Earth and Planetary Science Letters, v. 284.3, p. 489-503.

Bennett, Victoria C., and Donald J. DePaolo. 1987. Proterozoic crustal history of the western United States as determined by neodymium isotopic mapping: Geological Society of America Bulletin, v. 99.5, p. 674-685.

Benvenuti, M., and I. P. Martini. 2002. Analysis of terrestrial hyperconcentrated flows and their deposits: Flood and Megaflood Processes and Deposits: Recent and Ancient Examples; International Association of Sedimentologists special publication, Ames, lowa State University Press, v. 167-193. 
Bickford, M. E., P. A. Mueller, George D. Kamenov, and Barbara M. Hil. 2008. Crustal evolution of southern Laurentia during the Paleoproterozoic: Insights from zircon $\mathrm{Hf}$ isotopic studies of ca. 1.75 Ga rocks in central Colorado: Geology, v. 36.7, p. 555-558.

Bonde, J. W., Hilton, R. P., Jackson, F. D., \& Druschke, P. A. 2015. Fauna of the Newark Canyon Formation (Lower Cretaceous), East-Central Nevada: in Pennell, W. and Garside, L. eds., 2015 Geological Society of Nevada Symposium Volume, p. 139-150.

Braun, Jean, Peter Van Der Beek, and Geoffrey Batt. 2006. Quantitative thermochronology: numerical methods for the interpretation of thermochronological data. Cambridge University Press.

Brown, Roger E., and Bruce H. Wilkinson. 1981. The Draney Limestone; Early Cretaceous lacustrine carbonate deposition in western Wyoming and southeastern Idaho: Rocky Mountain Geology, v. 20.1, p. 23-31.

Bryant, Bruce. 1988. Geology of the Farmington Canyon Complex, Wasatch Mountains, Utah: United States Geological Survey, special paper no. 1476.

Burchfiel, B.C., Cowan, D.S., Davis, G.A.,. 1992. Tectonic overview of the Cordilleran orogen in the western United States. In: Burchfiel, B. C., Lipman, W., Zoback, M. I. (Eds.), The Cordilleran Orogen, Conterminous United States: Geological Society of America, The Geology of North America G-3 4.7-479.

Burtner, R. L., Nigrini, A., \& Donelick, R. A. 1994. Thermochronology of Lower Cretaceous source rocks in the Idaho-Wyoming thrust belt: AAPG Bulletin, v. 78.10, p. 1613-1636.

Camilleri, P.A., Yonkee, W.A., DeCelles, G., Coogan, J.C., McGrew, A., Wells, M. 1997. Hinterland to foreland transect through the Sevier orogen, northeast Nevada to northcentral Utah: structural style, metamorphism, and kinematic history of a large contractional orogenic wedge: Brigham Young University Studies, v. 42.1, p. 355-380.

Camilleri, Phyllis A. 1998. Prograde metamorphism, strain evolution, and collapse of footwalls of thick thrust sheets: a case study from the Mesozoic Sevier hinterland USA: Journal of Structural Geology, v. 20.8, p. 1023-1042.

Coogan, James C. 1992. Structural evolution of piggyback basins in the Wyoming-Idaho-Utah thrust belt: Geological Society of America Memoirs, v. 179, p. 55-82.

Crittenden, Max D. 1972. Willard thrust and the Cache allochthon, Utah: Geological Society of America Bulletin, v. 83, p. 2871. 
Cruz-Uribe, A. M., Hoisch, T. D., Wells, M. L., Vervoort, J. D., \& Mazdab, F. K. 2015. Linking thermodynamic modelling, Lu-Hf geochronology and trace elements in garnet: new PT-t paths from the Sevier hinterland: Journal of Metamorphic Geology, v. 33.7, p. 763781.

Currie, Brian S. 1998. Upper Jurassic-Lower Cretaceous Morrison and Cedar Mountain Formations, Ne Utah-Nw Colordao: Relationships between Nonmarine Deposition and Early Cordilleran Foreland-Basin Development: Journal of Sedimentary Research, v. 68.4.

Currie, Brian S. 2002. Structural Configuration of the Early Cretaceous Cordilleran ForelandBasin System and Sevier Thrust Belt, Utah and Colorado: The Journal of Geology, v. 110.6 , p. 697-718.

DeCelles, G. 2004. Late Jurassic to Eocene evolution of the Cordilleran thrust belt and foreland basin system, western U.S.A: American Jounral of Science, p. 105-168.

DeCelles, P. G., \& Burden, E. T. 1992. Non-marine sedimentation in the overfilled part of the Jurassic-Cretaceous Cordilleran foreland basin: Morrison and Cloverly Formations, central Wyoming, USA: Basin Research, v. 4.3-4, p. 291-313.

DeCelles, P. G., and B. S. Currie. 1996. Long-term sediment accumulation in the Middle Jurassicearly Eocene Cordilleran retroarc foreland-basin system: Geology, v. 24.7, p. 591-594.

DeCelles, P. G., Ducea, M. N., Kapp, P., \& Zandt, G. 2009. Cyclicity in Cordilleran orogenic systems: Nature Geoscience, v. 2.4, p. 251-257.

DeCelles, P. G., Pile, H. T., \& Coogan, J. C. 1993. DeCelles, P. G., H. T. Pile, and J. C. Coogan. Kinematic history of the Meade thrust based on provenance of the Bechler conglomerate at Red Mountain, Idaho, Sevier thrust belt: Tectonics, v. 12.6, p. 1436 1450.

DeCelles, Peter G. 1994. Late Cretaceous-Paleocene synorogenic sedimentation and kinematic history of the Sevier thrust belt, northeast Utah and southwest Wyoming: Geological Society of America Bulletin, v. 106.1, p. 32-56.

DeCelles, Peter G., and James C. Coogan. 2006. Regional structure and kinematic history of the Sevier fold-and-thrust belt, central Utah: Geological Society of America Bulletin, v. 118.7-8, p. 841-864.

DeCelles, Peter G., and Katherine A. Giles. 1996. Foreland basin systems: Basin research, v. 8.2, p. 105-123. 
DeCelles, Peter G., Timothy F. Lawton, and Gautam Mitra. 1995. Thrust timing, growth of structural culminations, and synorogenic sedimentation in the type Sevier orogenic belt, western United States: Geology, v. 23.8, p. 699-702.

Dickinson, W.R. 2004. Evolution of the North American Cordillera: Annual Reviews Earth Planet, v. 32, p. $13-45$.

Dickinson, William R., and George E. Gehrels. 2009. Use of U-Pb ages of detrital zircons to infer maximum depositional ages of strata: a test against a Colorado Plateau Mesozoic database: Earth and Planetary Science Letters, v. 288.1, p. 115-125.

Dickinson, William R., and Timothy F. Lawton. 2001. Tectonic setting and sandstone petrofacies of the Bisbee basin (USA-Mexico): Journal of South American Earth Sciences, v. 14.5, p. 475-504.

Dodson, Martin H. 1973. Closure temperature in cooling geochronological and petrological systems: Contributions to Mineralogy and Petrology, v. 40.3, p. 259-274.

Drummond, Carl N., Bruce H. Wilkinson, and Kyger C. Lohmann. 1996. Climatic control of fluviallacustrine cyclicity in the Cretaceous Cordilleran Foreland Basin, western United States: Sedimentology, v. 43.4, p. 677-689.

Eleogram, Bryan. 2014. The Application of Zircon (U-Th)/He Thermochronology to Determine the Timing and Slip Rate on the Willard Thrust, Sevier Fold and Thrust Belt, Northern Utah [M.S. thesis]: Las Vegas, University of Nevada Las Vegas.

Engebretson, David C. 1985. Relative motions between oceanic and continental plates in the Pacific basin: Geological Society of America Special Paper, v. 206, p. 1-60.

Eriksson, K. A., Campbell, I. H., Palin, J. M., \& Allen, C. M. 2003. Predominance of Grenvillian magmatism recorded in detrital zircons from modern Appalachian rivers: The Journal of Geology, v. 111.6, p. 707-717.

Eyer, Jerome A. 1969. Gannett Group of western Wyoming and southeastern Idaho: AAPG Bulletin, v. 53.7, p. 1368-1390.

Fan, Majie, and Barbara Carrapa. 2014. Late Cretaceous-early Eocene Laramide uplift, exhumation, and basin subsidence in Wyoming: Crustal responses to flat slab subduction: Tectonics, v. 33.4, p. 509-529.

Fan, Majie, Peter G. DeCelles, George E. Gehrels, David L. Dettman, Jay Quade, and S. Lynn Peyton. 2011. Sedimentology, detrital zircon geochronology, and stable isotope 
geochemistry of the lower Eocene strata in the Wind River Basin, central Wyoming: Geological Society of America Bulletin, v. 123.5-6, p. 979-996.

Farley, Kenneth A. 2002. (U-Th)/He dating: Techniques, calibrations, and applications: Reviews in Mineralogy and Geochemistry, v. 47.1, p. 819-844.

Fleming, R. Farley. 1994. Cretaceous pollen in Pliocene rocks: Implications for Pliocene climate in the southwestern United States: Geology, v. 22.9, p. 787-790.

Flemings, Peter B., and Teresa E. Jordan. 1990. Stratigraphic modeling of foreland basins: Interpreting thrust deformation and lithosphere rheology: Geology, v. 18.5, p. 430-434.

Frost, C. D., Fruchey, B. L., Chamberlain, K. R., \& Frost, B. R. 2006. Archean crustal growth by lateral accretion of juvenile supracrustal belts in the south-central Wyoming Province: Canadian Journal of Earth Sciences, v. 43.10, p. 1533-1555.

Furer, Lloyd C. 1970. Petrology and stratigraphy of nonmarine Upper Jurassic-Lower Cretaceous rocks of western Wyoming and southeastern Idaho: AAPG Bulletin, v. 54.12, p. 22822302.

Gehrels, G. E., Dickinson, W. R., Riley, B. C., Finney, S. C., \& Smith, M. T. 2000. Detrital zircon geochronology of the Roberts Mountains allochthon, Nevada: Special Papers-Geological Society of America, p. 19-42.

Gehrels, G.E., Valencia, V.A., and Ruiz, J. 2008. Enhanced precision, accuracy, efficiency, and spatial resolution of U-Pb ages by laser ablation-multicollector-inductively coupled plasma-mass spectrometry: Geochemistry, Geophysics, Geosystems, v. 9, p. 1-13.

Gehrels, George, Victor Valencia, and A. L. E. X. Pullen. 2006. Detrital zircon geochronology by laser-ablation multicollector ICPMS at the Arizona LaserChron Center: Paleontological Society Papers, v. 12, p. 67.

Gehrels, N., Norris, J.P., Barthelmy, S.D., Granot, J., Kaneko, Y., Kouveliotou, C., and Markwardt, C.B. 2006. A new \&ggr; 060614, -ray burst classification scheme from GRB: Nature, v. 7122, p. 1044-1046.

Giallorenzo, Michael. 2013. Application of (U-Th)/He and 40Ar/39Ar Thermochronology to the Age of Thrust Faulting in the Sevier Orogenic Belt [Ph.D dissertation]: Las Vegas: University of Nevada, Las Vegas.

Graham, David, and Nicholas Midgley. 2015. Tri-plot: Ternary diagram plotting software. March 9. http://www.lboro.ac.uk/microsites/research/phys-geog/tri-plot/index.html (accessed January 2016). 
Gurnis, Michael. 1992. Rapid continental subsidence following the initiation and evolution of subduction: Science, v. 255.5051, p. 1556-1558.

Harris, C. R., T. D. Hoisch, and M. L. Wells. 2007. Construction of a composite pressuretemperature path: revealing the synorogenic burial and exhumation history of the Sevier hinterland, USA: Journal of Metamorphic Geology, v. 25.8, p. 915-934.

Heller, L., Bowler, S.S., Chambers, H., Coogan, J.C., Hagen, E.S., Shuster, M.W., Winslow, N. S., Lawton, T. F. 1986. Time of initial thrusting in the Sevier orogenic belt, Idaho, Wyoming and Utah: Geology, v. 14, p. 388-391.

Heller, P. L., Angevine, C. L., Winslow, N. S., \& Paola, C. 1988. Two-phase stratigraphic model of foreland-basin sequences: Geology, v. 16.6, p. 501-504.

Heller, Paul L., and Chris Paola. 1989. The paradox of Lower Cretaceous gravels and the initiation of thrusting in the Sevier orogenic belt, United States Western Interior: Geological Society of America Bulletin, v. 101.6, p. 864-975.

Heller, Paul L., Kenneth Dueker, and Margaret E. McMillan. 2003. Post-Paleozoic alluvial gravel transport as evidence of continental tilting in the US Cordillera: Geological Society of America Bulletin, v. 115.9, p. 1122-1132.

Humphreys, Eugene D. 1995. Post-Laramide removal of the Farallon slab, western United States: Geology, v. 23.11, p. 987-990.

Hunt, G. J., T. F. Lawton, and J. I. Kirkland. 2011. Detrital zircon U-Pb geochronological provenance of Lower Cretaceous strata, foreland basin, Utah: Sevier Thrust Belt: Northern and Central Utah and Adjacent Areas: Utah Geological Association, Publication, v. 40, p. 193-211.

Imlay, R.W. 1967. Twin Creek Limestone (Jurassic) in the Western Interior of the United States: a description of the stratigraphic and faunal succession in the Twin Creek limestone and regional comparisons with contemporary formations: US Geological Survey Professional Paper 540.

Ingersoll, R. V., Bullard, T. F., Ford, R. L., Grimm, J. P., Pickle, J. D., \& Sares, S. W. 1984. The effect of grain size on detrital modes: a test of the Gazzi-Dickinson point-counting method: Journal of Sedimentary Research, v. 54.1, p. 103-116.

Irwin, W. P., \& Wooden, J. L. 2001. Map showing plutons and accreted terranes of the Sierra Nevada, California with a tabulation of U/Pb isotopic ages. US Geological Survey, No. 2001-229. 
Isakson, Vincent Hans. 2012. Constraining the Origin and Evolution of the Basement Rocks in the Raft River, Albion, Grouse Creek Core Complex, Northwestern Utah and Southern Idaho [Ph.D dissertation]: Pullman, Washington State University.

Johnston, Scott, and Clark Kylander. 2016. Rapid Ophiolite Exhumation and Arc Thickening in the Southern California Late Cretaceous Convergent Margin as Defined by Nacimiento Block Forearc Detrital Zircon Geochronology and Geochemistry, in Proceedings, GSA Cordilleran Section - 112th Annual Meeting. Denver: Geological Society of America.

Karlstrom, Karl E., and Samuel A. Bowring. 1988. Early Proterozoic assembly of tectonostratigraphic terranes in southwestern North America: The Journal of Geology, p. 561-576.

Kelly, Eric D., Thomas D. Hoisch, Michael L. Wells, Jeffrey D. Vervoort, and Mengesha A. Beyene. 2015. An Early Cretaceous garnet pressure-temperature path recording synconvergent burial and exhumation from the hinterland of the Sevier orogenic belt, Albion Mountains, Idaho: Contributions to Mineralogy and Petrology, v. 170.2, p. 1-22.

Konstantinou, A., Strickland, A., Miller, E. L., \& Wooden, J. P. 2012. Multistage Cenozoic extension of the Albion-Raft River-Grouse Creek metamorphic core complex: Geochronologic and stratigraphic constraints: Geosphere, v. 8, p. 1429-1466.

Kowallis, B. J., Christiansen, E. H., Deino, A. L., Peterson, F., Turner, C. E., Kunk, M. J., \& Obradovich, J. D. 1998. The age of the Morrison Formation: Modern Geology, v. 22.1-4, p. 235-260.

Kowallis, Bart J., Eric H. Christiansen, and Alan L. Deino. 1991. Age of the Brushy Basin Member of the Morrison Formation, Colorado Plateau, western USA: Cretaceous Research, $p$. 483-493.

Laskowski, Andrew K., Peter G. DeCelles, and George E. Gehrels. 2013. Detrital zircon geochronology of Cordilleran retroarc foreland basin strata, western North America: Tectonics, v. 32.5, p. 1027-1048.

Lawton, Timothy F., Gary J. Hunt, and George E. Gehrels. 2010. Detrital zircon record of thrust belt unroofing in Lower Cretaceous synorogenic conglomerates, central Utah: Geology, v. 38.5 , p. $463-466$.

Leier, A. L., and G. E. Gehrels. 2011. Continental-scale detrital zircon provenance signatures in Lower Cretaceous strata, western North America: Geology, v. 39.4, p. 399-402. 
Linde, G. M., Trexler, J. H., Cashman, P. H., Gehrels, G., \& Dickinson, W. R. 2016. Detrital zircon $\mathrm{U}-\mathrm{Pb}$ geochronology and $\mathrm{Hf}$ isotope geochemistry of the Roberts Mountains allochthon: New insights into the early Paleozoic tectonics of western North America: Geosphere, v. GES01252-1, p. 1-16.

Liu, Shaofeng, and Dag Nummedal. 2004. Late Cretaceous subsidence in Wyoming: Quantifying the dynamic component: Geology, v. 32.5, p. 397-400.

Liu, Shaofeng, Dag Nummedal, and Lijun Liu. 2011. Migration of dynamic subsidence across the Late Cretaceous United States Western Interior Basin in response to Farallon plate subduction: Geology, v. 39.6, p. 555-558.

Liu, Shaofeng, Dag Nummedal, and Michael Gurnis. 2014. Dynamic versus flexural controls of Late Cretaceous Western Interior Basin, USA: Earth and Planetary Science Letters, v. 389, p. 221-229.

Ludwig, K. 2008. User's manual for Isoplot 3.6: Geo chronological toolkit for Microsoft.

Ludwig, K. R., and R. Mundil. 2002. Extracting reliable U-Pb ages and errors from complex populations of zircons from Phanerozoic tuffs. Vol. 66, in Geochimica et Cosmochimica Acta. KIDLINGTON, OXFORD, ENGLAND: PERGAMON-ELSEVIER SCIENCE LTD.

Madsen, J.K., Thorkelson, D.J., Friedman, R.M., Marhall, D. 2006. Cenozoic to Recent plate configurations in the Pacific Basin: Ridge subduction and slab window magmatism in western North America: Geosphere, v. 2.1, p. 11-34.

Mansfield, George R. 1952. Geography, geology, and mineral resources of the Ammon and Paradise Valley quadrangles, Idaho. Geological Survey Professional Paper 238, Washington D.C.: United States Geologic Survey.

May, S. R., Gray, G. G., Summa, L. L., Stewart, N. R., Gehrels, G. E., \& Pecha, M. E. 2013. Detrital zircon geochronology from the Bighorn Basin, Wyoming, USA: Implications for tectonostratigraphic evolution and paleogeography: Geological Society of America Bulletin, v. 125.9-10, p. 1403-1422.

M'Gonigle, John W., and James H. Dover. 1992. Geologic map of the Kemmerer 30'x 60'Quadrangle, Lincoln, Uinta, and Sweetwater counties, Wyoming: U.S. Geological Survey no. 2079.

Miall, Andrew D. 1977. Lithofacies types and vertical profile models in braided river deposits: a summary: Geological Survey of Canada, p. 597-604. 
Mitra, Gautam. 1994. Strain variation in thrust sheets across the Sevier fold-and-thrust belt (Idaho-Utah-Wyoming): Implications for section restoration and wedge taper evolution: Journal of Structural Geology, v. 16.4, p. 585-602.

Mueller, P. A., Wooden, J. L., Mogk, D. W., \& Foster, D. A. 2011. Mueller, Paul A., et al. Paleoproterozoic evolution of the Farmington zone: Implications for terrane accretion in southwestern Laurentia: Lithosphere, v. 3.6, p. 401-408.

Mueller, Paul A., and Carol D. Frost. 2006. The Wyoming Province: a distinctive Archean craton in Laurentian North America: Canadian Journal of Earth Sciences, v. 43.10, p. 1391-1397.

Nadon, G. C. 1994. The genesis and recognition of anastomosed fluvial deposits: data from the St. Mary River Formation, southwestern Alberta, Canada: Journal of Sedimentary Research, v. 64.4.

Naeser, C. W., Bryant, B., Crittenden, M. D., \& Sorensen, M. L. 1983. Fission-track ages of apatite in the Wasatch Mountains, Utah: An uplift study: Geological Society of America Memoirs, v. 157, p. 29-36.

Oldow, J. S., Bally, A. W., Lallemant, H. G. A., \& Leeman, W. P. 1989. Phanerozoic evolution of the North American Cordillera; United States and Canada: The Geology of North America-Vol. A: An Overview, p. 139-232.

Painter, C. S., Carrapa, B., DeCelles, P. G., Gehrels, G. E., \& Thomson, S. N. 2014. Exhumation of the North American Cordillera revealed by multi-dating of Upper Jurassic-Upper Cretaceous foreland basin deposits: Geological Society of America Bulletin, pp.B309991.

Painter, Clayton S., and Barbara Carrapa. 2013. Flexural versus dynamic processes of subsidence in the North American Cordillera foreland basin: Geophysical Research Letters: v. 40.16, p. 4249-4253.

Paola, Chris. 2000. Quantitative models of sedimentary basin filling: Sedimentology, v. 47.s1, p. 121-178.

Peppe, D. J., Hickey, L. J., Miller, I. M., \& Green, W. A. 2008. A morphotype catalogue, floristic analysis and stratigraphic description of the Aspen Shale flora (Cretaceous-Albian) of southwestern Wyoming: Bulletin of the Peabody Museum of Natural History, v. 49.2, p. 181-208. 
Pierson, Thomas C. 2005. Hyperconcentrated flow-transitional process between water flow and debris flow, Debris-flow hazards and related phenomena, Berlin Heidelberg: Springer 159-202.

Pipiringos, G.N., and Imlay, R.W., 1979, Lithology and subdivisions of the Jurassic Stump Formation in southeastern Idaho and adjoining areas, IN Unconformities, correlations, and nomenclature of some Triassic and Jurassic rocks, Western Interior United States: U.S. Geological Survey Professional Paper, 1035-C, p. C1-C25.

Price, R.A., Mountjoy, E.W. 1970. Geologic structure of the Canadian Rocky Mountains between Bow and Athabasca Rivers: A progress report. Structure of the southern Canadian Cordillera: Geological Association of Canada Special Paper, v. 6, p. 7-25.

Protzman, Gretchen M., and Gautam Mitra. 1990. Strain fabric associated with the Meade thrust sheet: implications for cross-section balancing: Journal of Structural Geology, v. 12.4 , p. $403-417$

Reiners, Peter W. 2005. Zircon (U-Th)/He thermochronometry: Reviews in Mineralogy and Geochemistry, v. 58.1, p. 151-179.

Rogers, Raymond R. 1998. Sequence analysis of the Upper Cretaceous Two Medicine and Judith River formations, Montana: nonmarine response to the Claggett and Bearpaw marine cycles: Journal of Sedimentary Research, v. 68.4, p. 615-631.

Schmitt, James G., and James R. Steidtmann. 1990. Interior ramp-supported uplifts: Implications for sediment provenance in foreland basins: Geological Society of America Bulletin, v. 102.4, p. 494-501.

Schumm, Stanley A. 1981. Evolution and response of the fluvial system, sedimentologic implications: The Society of Economic Paleontologists and Mineralogists Special Publication, v. 31, p. 19-29.

Schwanke, Garrett A., Steve Robison, Laurel Krumenacker, Evan Staples, and Rod Sheetz. 2008. Idaho's First Dinosaur Tracks and a U-Pb Detrital Zircon Age for the Early Cretaceous Draney Limestone: Cordilleran Section and Rocky Mountain Section Joint GSA Meeting Abstracts with Programs. v. 58.

Seton, M., Müller, R.D., Zahirovic, S., Gaina, C., Torsvik, T., Shephard, G., Talsma, A., Gurnis, M., Turner, M., Maus, S. and Chandler, M. 2012. Global continental and ocean basin reconstructions since 200Ma: Earth-Science Reviews, v. 113.3, p. 212-270. 
Shufeldt, O. P., Karlstrom, K. E., Gehrels, G. E., \& Howard, K. E. 2010. Archean detrital zircons in the Proterozoic Vishnu Schist of the Grand Canyon, Arizona: Implications for crustal architecture and Nuna supercontinent reconstructions: Geology, v. 38.12, p. 1099-1102.

Smith, D.L., Wyld, S.J., Miller, E.L., Wright, J.E. 1993. Progression and timing of Mesozoic crustal shortening in the northern Great Basin, western USA. In: Dunn, G., McDougall (Eds.), Mesozoic paleogeography of the western United States II: The Society of Economic Paleontologists and Mineralogists Book, v. 71, p. 389-406.

Snell, K. E., Koch, P. L., Druschke, P., Foreman, B. Z., \& Eiler, J. M. 2014. High elevation of the 'Nevadaplano' during the Late Cretaceous: Earth and Planetary Science Letters, v. 386, p. 52-63.

Stewart, J. H., Gehrels, G. E., Barth, A. P., Link, P. K., Christie-Blick, N., \& Wrucke, C. T. 2001. Detrital zircon provenance of Mesoproterozoic to Cambrian arenites in the western United States and northwestern Mexico: Geological Society of America Bulletin, v. 113.10, p. 1343-1356.

Strickland, Ariel, Elizabeth L. Miller, and Joseph L. Wooden. 2011. The timing of Tertiary metamorphism and deformation in the Albion-Raft River-Grouse Creek metamorphic core complex, Utah and Idaho: The Journal of Geology, v. 119.2, p. 185-206.

Suarez, C. A., González, L. A., Ludvigson, G. A., Cifelli, R. L., \& Tremain, E. 2012. Suarez, Celina A., et al. Water utilization of the Cretaceous Mussentuchit Member local vertebrate fauna, Cedar Mountain Formation, Utah, USA: Using oxygen isotopic composition of phosphate: Palaeogeography, Palaeoclimatology, Palaeoecology, v. 313, p. 78-92.

Torsvik, T.H.,Müller, R.D., Van der Voo, R., Steinberger, B., Gaina, C. 2008. Global plate motion frames: toward a unified model: Reviews of Geophysics, v. 46.3.

Vandervoort, D. S. 1987. Sedimentology, provenance, and tectonic implications of the Cretaceous Newark Canyon Formation, east-central Nevada [Ph.D dissertation], Bozeman: Montana State University, College of Letters \& Science.

Vandervoort, D. S., \& Schmitt, J. G. 1990. Cretaceous to early Tertiary paleogeography in the hinterland of the Sevier thrust belt, east-central Nevada: Geology, v. 18.6, p. 567-570.

Weil, Arlo Brandon, and W. Adolph Yonkee. 2012. Layer-parallel shortening across the Sevier fold-thrust belt and Laramide foreland of Wyoming: spatial and temporal evolution of a complex geodynamic system: Earth and Planetary Science Letters, v. 357, p. 405-420. 
Wooden, J. L., and D. M. Miller. 1990. Chronologic and isotopic framework for Early Proterozoic crustal evolution in the eastern Mojave Desert region, SE California: Journal of Geophysical Research: Solid Earth, v. 95.B12, p. 20133-20146.

Wright, James E., and Arthur W. Snoke. 1993. Tertiary magmatism and mylonitization in the Ruby-East Humboldt metamorphic core complex, northeastern Nevada: U-Pb geochronology and $\mathrm{Sr}, \mathrm{Nd}$, and $\mathrm{Pb}$ isotope geochemistry: Geological Society of America Bulletin, v. 105.7, p. 935-952.

Wyld, Sandra J. 1996. Early Jurassic deformation in the Pine Forest Range, northwest Nevada, and implications for Cordilleran tectonics: Tectonics, v. 15.3, p. 566-583.

Xie, Xiangyang, and Paul L. Heller. 2009. Plate tectonics and basin subsidence history: Geological Society of America Bulletin, v. 121.1-2, p. 55-64.

Yonkee, Adolph, and Arlo Brandon Weil. 2010. Reconstructing the kinematic evolution of curved mountain belts: Internal strain patterns in the Wyoming salient, Sevier thrust belt, USA: Geological Society of America Bulletin, v. 122.1-2, p. 24-49.

Yonkee, W. A. 2005. Strain patterns within part of the Willard thrust sheet, Idaho-UtahWyoming thrust belt: Journal of Structural Geology, v. 27, p. 1315-1343.

Yonkee, W. A., C. D. Dehler, Paul K. Link, E. A. Balgord, Joshua A. Keeley, D. S. Hayes, M. L. Wells, C. M. Fanning, and S. M. Johnston. 2014. Tectono-stratigraphic framework of Neoproterozoic to Cambrian strata, west-central US: Protracted rifting, glaciation, and evolution of the North American Cordilleran margin: Earth-Science Reviews, v. 136, p. 59-95.

Yonkee, W. A., P. G. DeCelles, and J. Coogan. 1997. Part 5: Kinematics and Synorogenic Sedimentation of the Eastern Frontal Part of the Sevier Orogenic Wedge, Northern Utah: Brigham Young University Geology Studies, v. 42, p. 355-380.

Yonkee, W. A., Parry, W. T., Bruhn, R. L., \& Cashman, P. H. 1989. Thermal models of thrust faulting: Constraints from fluid-inclusion observations, Willard thrust sheet, Idaho-UtahWyoming thrust belt: Geological Society of America Bulletin, v. 101.2, p. 304-313.

Yonkee, W. Adolph, and Arlo Brandon Weil. 2015. Tectonic evolution of the Sevier and Laramide belts within the North American Cordillera orogenic system: Earth-Science Reviews, v. 150, p. 531-593. 
Zaleha, Michael J. 2006. Sevier orogenesis and nonmarine basin filling: Implications of new stratigraphic correlations of Lower Cretaceous strata throughout Wyoming, USA: Geological Society of America Bulletin, v. 118.7-8, p. 866-896. 
Curicculum Vitae

\title{
AMANDA GENTRY
}

6530 Annie Oakley Dr. Apt. 2421, Henderson, NV 89014

303-319-0695

Amanda.L.Gentry@gmail.com

University of Nevada, Las Vegas

\section{Education}

August 2014 - present

Graduate program - M.S. emphasis on sedimentary basins- $2^{\text {nd }}$ year teaching assistant; GPA 3.8

Weber State University

January 2010 - December 2012

Bachelor of Science in Geology, Minor in Geospatial Analysis GPA - 3.74 -departmental honors

\section{Geoscience Courses}

Tectonics of Orogenic Belts

Sedimentary Basins

Environmental Systems Change

Evolution of Western North America

Deformation Crystalline Materials

Field Camp

Awards and Scholarships

Excellence in Service Award

AWG Susan Ekdale Memorial Scholarship

SGE W.A. National Tarr Award

Norman and Barbara Tanner Scholarship

Questar Scholarship

\author{
Carbonate Depositional Systems \\ Tectonics and Structure \\ Field Methods \\ Plate Tectonics \\ Structural Geology \\ Petrology \\ Earth Materials \\ Geochemistry \\ Sedimentology and Stratigraphy Remote Sensing I \& II \\ GIS I \& II
}

\begin{abstract}
$\underline{\text { Research }}$
Patterns of Synorogenic Sedimentation Associated with the Unroofing of the Willard-Paris-Meade Thrust Sheets, Sevier Fold-Thrust Belt

Synorogenic basin development associated with emplacement of the Willard-Paris-Meade thrust sheet in northeast Utah, southeast Idaho, and southwest Wyoming during the Early Cretaceous as it relates to the early deformation history of the Sevier orogenic belt and its bearing on whether the Sevier orogen experienced a two-stage or continuous shortening history.
\end{abstract}

\section{Honors Affiliations and Achievements}

- Fundraising and Silent Auction committee chair, UNLV GeoSymposium

- UNLV Geoscience GPSA representative, Activities Committee member

- Secretary and Event Coordinator, UNLV AAPG student chapter

- Past-president, Association of Women Geoscientists Salt Lake Chapter

- Past-treasurer, nationally recognized honors society: Sigma Gamma Epsilon, Eta Gamma Chapter 
- Directed, designed, and implemented a science-based trail system modeled on community hiking programs and professional input that was in partnership with Weber State University, Ogden City, and the USDA Forest Service 